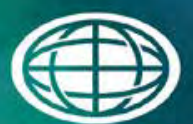

Savannah River

National Laboratory ${ }^{m}$

OPERATED BY SAVANNAH RIVER NUCLEAR SOLUTIONS

HANFORD DOUBLE SHELL WASTE TANK CORROSION STUDIES FINAL REPORT FY2014
R. E. Fuentes
B. J. Wiersma
K. R. Hicks

December 2014

SRNL-STI-2014-00616, Revision 0 
SRNL-STI-2014-00616

Revision 0

\section{DISCLAIMER}

This work was prepared under an agreement with and funded by the U.S. Government. Neither the U.S. Government or its employees, nor any of its contractors, subcontractors or their employees, makes any express or implied:

1. warranty or assumes any legal liability for the accuracy, completeness, or for the use or results of such use of any information, product, or process disclosed; or

2. representation that such use or results of such use would not infringe privately owned rights; or

3. endorsement or recommendation of any specifically identified commercial product, process, or service.

Any views and opinions of authors expressed in this work do not necessarily state or reflect those of the United States Government, or its contractors, or subcontractors.

Printed in the United States of America

Prepared for

U.S. Department of Energy 
Keywords: Hanford, double-shell tanks, vapor space corrosion, liquid air interface corrosion, pitting protocol, waste buffering

Retention: Permanent

\title{
HANFORD DOUBLE SHELL WASTE TANK CORROSION STUDIES - FINAL REPORT FY2014
}

\author{
R.E. Fuentes \\ B. J. Wiersma \\ K. R. Hicks
}

December 2014

Prepared for the U.S. Department of Energy under

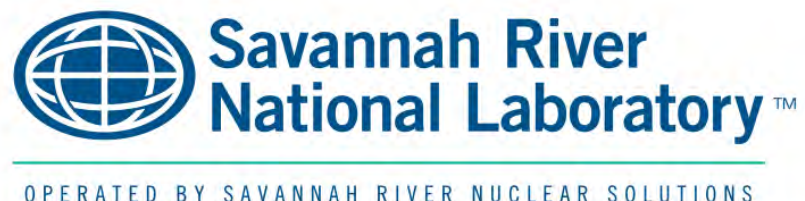
contract number DE-AC09-08SR22470. 


\section{REVIEWS AND APPROVALS}

\section{AUTHORS:}

R. E. Fuentes, Materials Science and Technology

Date

B. J. Wiersma, Materials Science and Technology

Date

K. R. Hicks, Materials Science and Technology

Date

\section{TECHNICAL REVIEW:}

R. B. Wyrwas, Materials Science and Technology

Date

\section{APPROVALS:}

K. E. Zeigler, Manager, Savannah River National Laboratory

Date

K. D. Boomer, Manager, Washington River Protection Solutions

Date

J. L. Castleberry, Manager, Washington River Protection Solutions

Date 


\section{EXECUTIVE SUMMARY}

SRNL tasks for FY14 included studies to evaluate the susceptibility of carbon steel to vapor space corrosion (VSC), liquid-air interface (LAI) corrosion, and pitting corrosion. Additionally, SRNL evaluated the susceptibility of carbon steel to pitting corrosion under buffered waste conditions, with the objective of determining the adequate amount of inhibitor (e.g., nitrite) necessary to mitigate pitting corrosion. Other CPP experiments were performed in historical waste simulants and the results were compared to previously gathered results. The results of these activities were utilized to assess the robustness of the standardized CPP protocol.

For FY2014, four task activities were performed and the results were arranged in five parts and discussed in section 5. Below is a summary and conclusions for every part:

\section{Secondary Wall of AY-102 Tank Corrosion Studies}

Liquid air interface (LAI) and vapor space (VS) corrosion tests were performed using Leak Detection Pit (LDP) residue (Solutions 12 and 13) and Groundwater (GW) simulants (Solutions 14 and 15). For LAI samples after two months, aggressive corrosion was sustained for all samples. For the partially immersed samples after two months of exposure, the corrosion occurred at the water line and below. By the fourth month, the corrosion increased above the water line of the coupon as well. The corrosion rate was steady for the 4 month test at approximately $10 \mathrm{mpy}$. A similar corrosion rate was observed for samples that were totally immersed in the LDP and GW simulants. For VS samples, corrosion was more prominent for the coupons exposed to GW simulant (Solution 8) than the coupons exposed to LDP residue (Solution 7). More aggressive attack was observed on the samples closest to the liquid level (Level $1>$ Level $2>$ Level 3) for the coupons from GW simulant.

\section{Testing in Waste Buffering Simulant from DST AN-102}

Electrochemical testing using waste buffering simulants based on waste from tank AN-102 at different hydroxide, nitrate and nitrite concentrations was performed. The results demonstrated that hydroxide concentrations as low as $0.032 \mathrm{M}$ can still offer inhibition for corrosion in carbon steel provided sufficient nitrite is present. The four month LAI tests showed no significant corrosion. These results concur with electrochemical testing in that the solutions studied inhibited the development of pits when carbon steel is immersed and creates very small pits that do not seem to grow or increase in quantity over time.

\section{Vapor Space Corrosion tests at new SCC limits with different concentrations of Ammonia gas in Air}

VSC tests were executed with three simulants based on the new SCC corrosion control guideline. The simulants contained the minimum nitrite/nitrate ratio of 0.15 and nitrate concentrations of $0.4,2$ and 4.5 M. The vapor space above each simulant had either 50 or $550 \mathrm{ppm}$ ammonia. The samples after fourmonths of exposure showed no indications of VSC at levels 3 and 2 with minor corrosion areas at Level 1 for the $550 \mathrm{ppm}$ ammonia in each of the three simulants. The same was true at $50 \mathrm{ppm}$, as there was no significant VSC. When the cold mount was removed from the coupons, some crevice attack had occurred in some instances. Although crevice attack did occur on several of the samples, the results tend to indicate that even at $50 \mathrm{ppm}$ ammonia with solutions comprised of the new SCC control limits, VSC can be inhibited. 


\section{Liquid Air Interface tests at new SCC limits}

Eight different solutions were prepared with compositions that were at or near the new SCC control limits. After two and fourth months of exposure none of the samples exhibited LAI corrosion or any attack in the immersed area. Typically any corrosion seemed to initiate at the top of the coupon and develop an area of general corrosion and that continues to spread above the LAI. The smallest ratio of nitrite to nitrite $(0.12)$ was solution 8 and during the contact with the carbon steel coupon it developed a film that completely covered the coupon and protected it from corrosion attack even though the ratio is less than the new minimum SCC control limits. The corrosion appeared to be more severe for the more dilute solutions at a given nitrite/nitrate ratio.

\section{Pitting Corrosion studies using the standardized CPP protocol}

CPP tests were conducted to compare historical data with data that was collected using the new standardized CPP protocol. The purpose was to determine the effect of the CPP parameters on the results and also compare the results from the new CPP test protocol with long-term coupon tests. Forty test conditions were selected for testing during FY2014 from the more than 900 historical test conditions. In cases where either a clear-cut pitting or no pitting case, there was $100 \%$ agreement between the historical CPP results and the present testing. On the other hand if the environment was a borderline condition (i.e., transition from pitting to not pitting) agreement was not as good. Both the CPP test parameters and the microstructure of the material may have had a role in these contrary results. The historical data and new CPP protocol still remain useful as in both cases the behavior at the borderline condition was consistent. Both the historical data and the new CPP test protocol were consistent with coupon results that indicated either clear-cut pitting or no pitting results and borderline conditions. 


\section{TABLE OF CONTENTS}

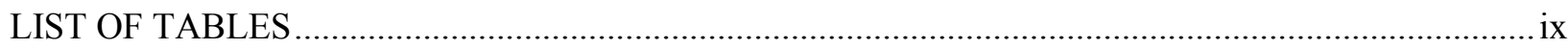

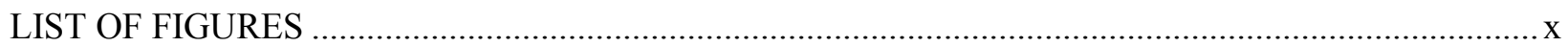

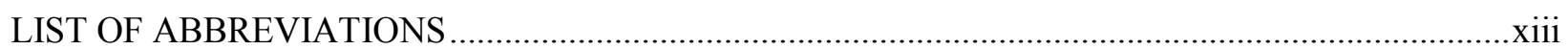

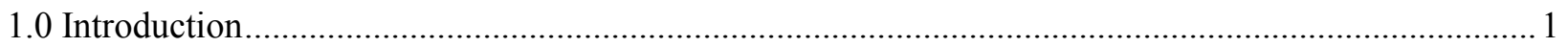

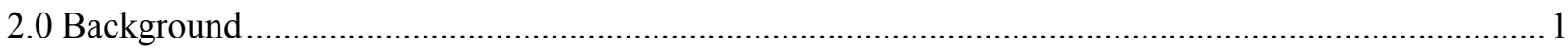

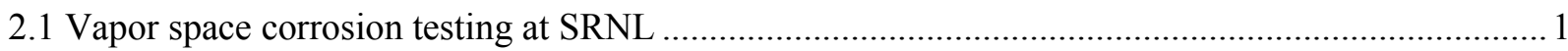

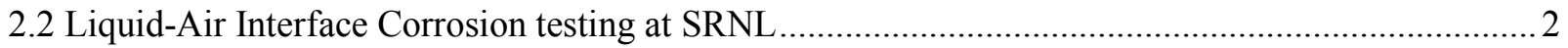

2.3 AY-102 Leak Detection Pit and Secondary Tank Corrosion Studies.............................................. 3

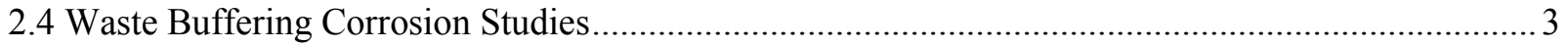

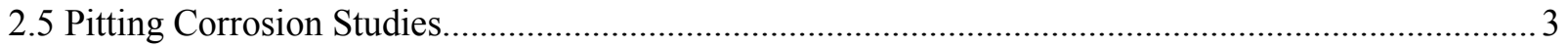

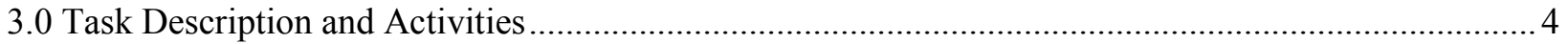

3.1 Task 1: Vapor space Corrosion Studies for Hanford Double Shell Tanks ...................................... 4

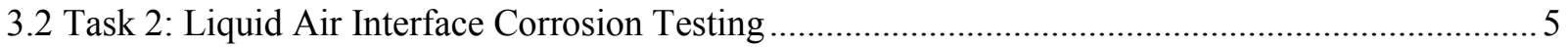

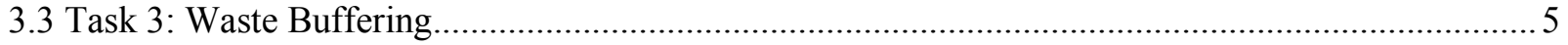

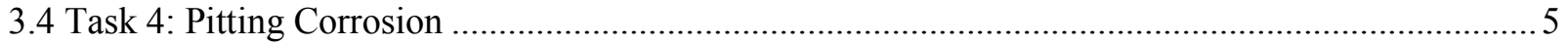

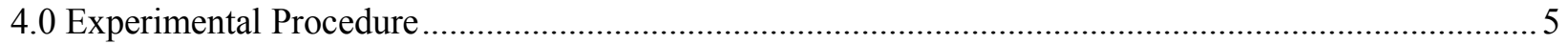

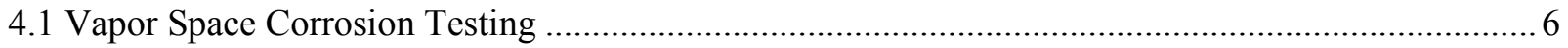

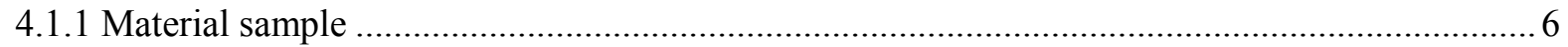

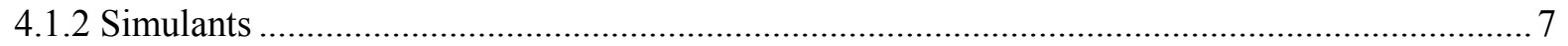

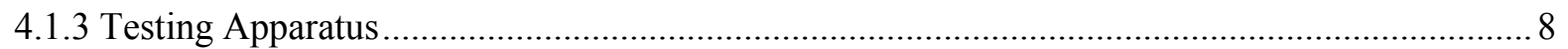

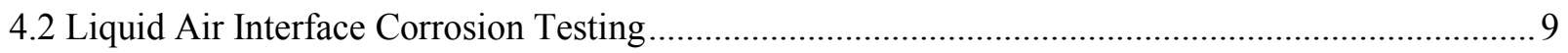

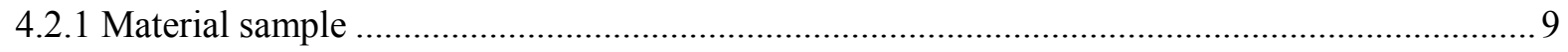

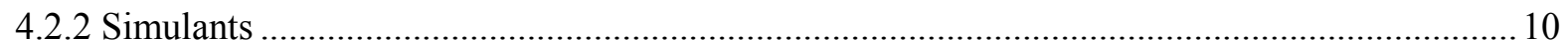

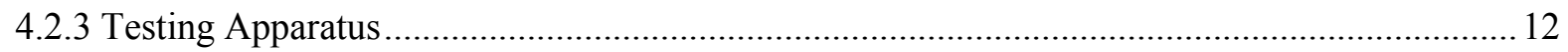

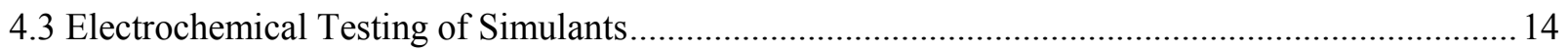

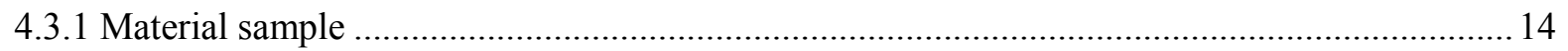

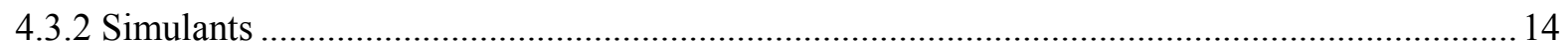

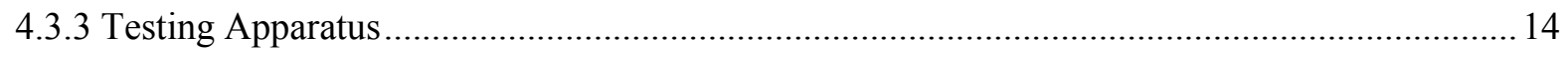

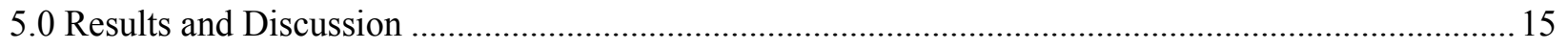

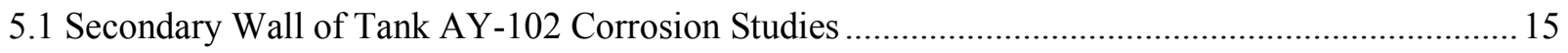

5.1.1 Liquid Air Interface and Total Immersion tests................................................................... 15

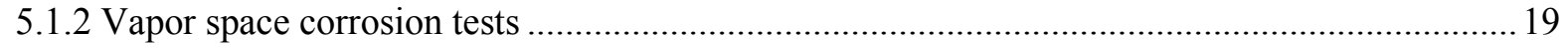

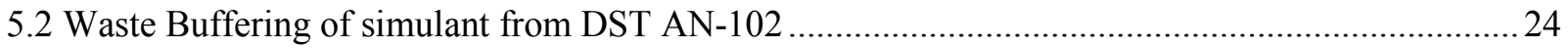




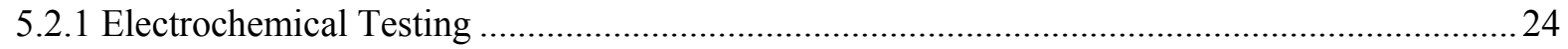

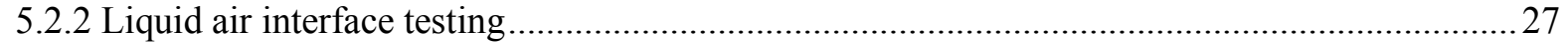

5.3 Vapor Space Corrosion tests at new SCC limits at different ammonia concentrations.................... 30

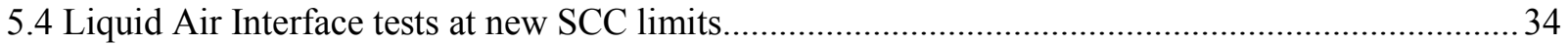

5.5 Pitting Corrosion studies using the standardized CPP protocol .....................................................40

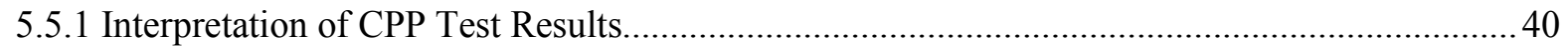

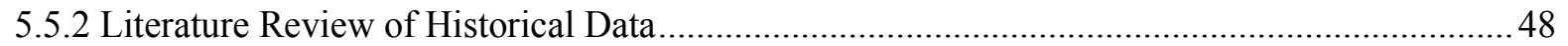

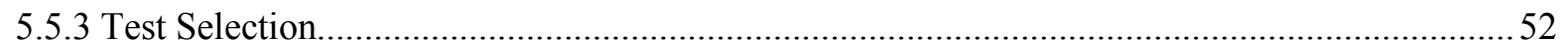

5.5.4 Distributions of Variables for the 40 Tests ....................................................................................5

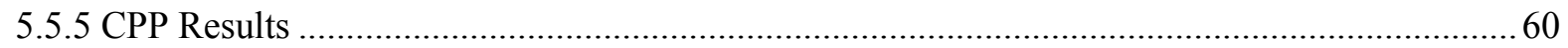

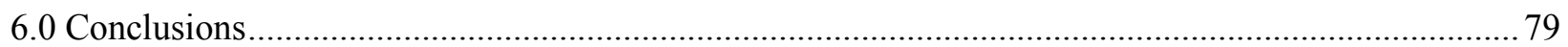

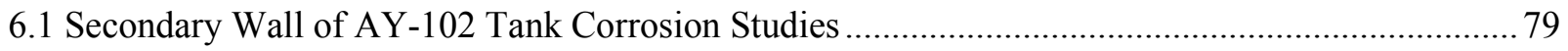

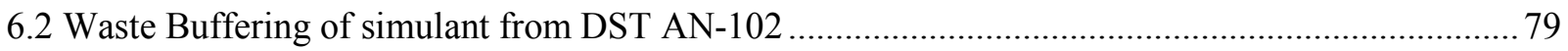

6.3 Vapor Space Corrosion tests at new SCC limits with different concentrations of Ammonia gas in

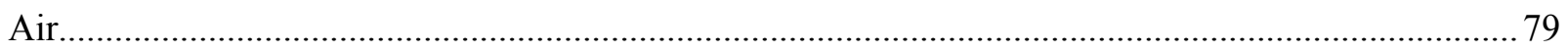

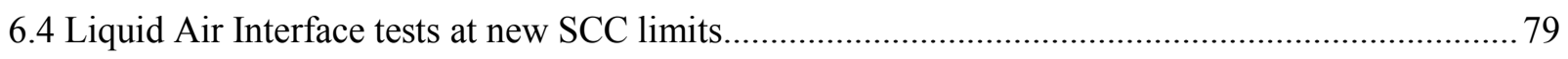

6.5 Pitting Corrosion studies using the standardized CPP protocol .................................................. 80

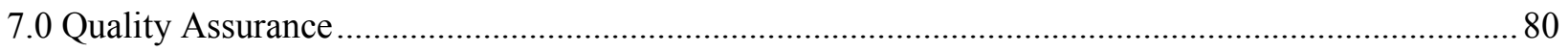

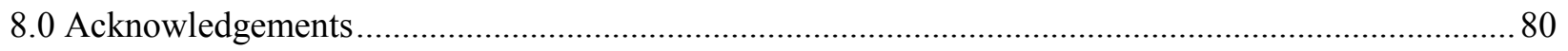

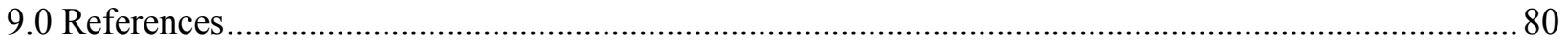

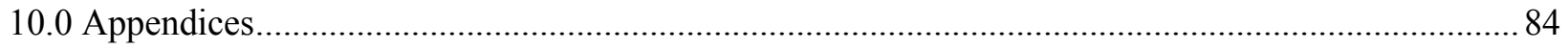




\section{LIST OF TABLES}

Table 2-1 Proposed Specifications for the Control of SCC in Nitrate Ion Wastes in DSTs....................... 2

Table 2-2 CPP protocol with the parameters utilized for testing .......................................................... 4

Table 4-1 Chemical Composition of AART128 Rail Car Steel.............................................................. 5

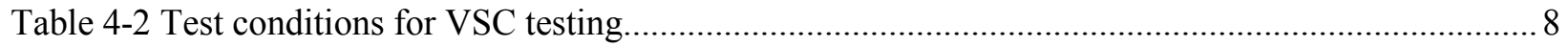

Table 4-3 Nitrate and nitrite concentrations for LAI corrosion test simulants. ....................................... 11

Table 4-4 Nitrate, nitrite and hydroxide concentrations for LAI corrosion test simulants from Waste

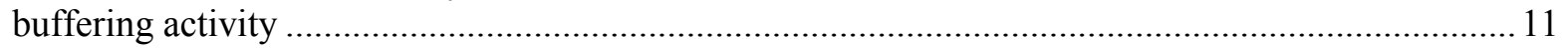

Table 5-1 Weight losses and corrosion rates for carbon steel coupons exposed to LDP and GW simulants 17

Table 5-2 Pitting diameter and depth range of solutions from LDP and GW simulants .......................... 19

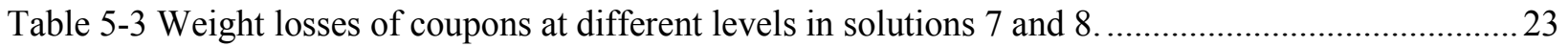

Table 5-4 Electrochemical test conditions for waste buffering simulants based from actual waste from

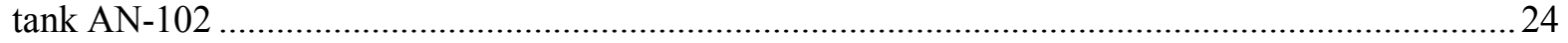

Table 5-5 Pitting diameter and depth range of solutions simulating AN-102 waste ...............................29

Table 5-6 Weight losses and corrosion rates for carbon steel coupons exposed to Solutions 1 to 8 for 2

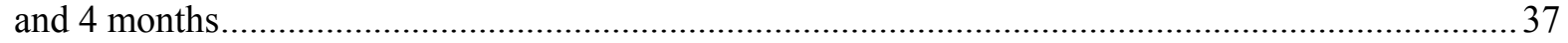

Table 5-7 Pitting diameter and depth range of solutions using the new SCC limits. Percentages were obtained by a qualitative assesment........................................................................................... 39

Table 5-8 CPP Test Solution Composition and Temperature …............................................................5

Table 5-9 CPP Test Solution Composition and Temperature (continued) ……......................................55

Table 5-10 Comparison of Chemistry for FY2014 Test Condition \#15 and Historical Test Condition .... 61

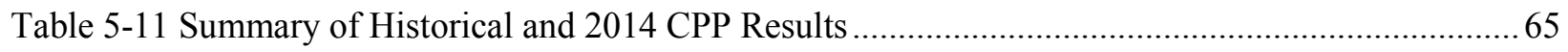

Table 5-12 Summary of Historical and 2014 CPP Results (continued 1)...............................................66

Table 5-13 Summary of Historical and 2014 CPP Results (continued 2) ................................................ 67

Table 5-14 Summary of Historical and 2014 CPP Results (continued 3).............................................. 68

Table 5-15 Summary of Historical and 2014 CPP Results (continued 4)............................................... 69

Table 5-16 Summary of Historical and 2014 CPP Results (continued 5)............................................. 70

Table 5-17 Summary of Historical and 2014 CPP Results (continued 6).............................................. 71

Table 5-18 Summary of Historical and 2014 CPP Results (continued 7) ............................................... 72 
Table 5-19 Summary of Historical and 2014 CPP Results (continued 8),................................................ 73

Table 5-20 Composition of Post-1993 test and FY2014 Test 16 (M)...................................................... 73

Table 5-21 Comparison of Post 1993 CPP Test Protocol and New Standardized CPP Protocol. .............. 74

\section{LIST OF FIGURES}

Figure 4-1 Coupon mounted in cold mount with wire ..................................................................... 6

Figure 4-2 Picture of the rod with four coupons hanged at each level..................................................... 7

Figure 4-3 Picture of the Vapor Space Corrosion setup inside the walk-in hood. ..................................... 9

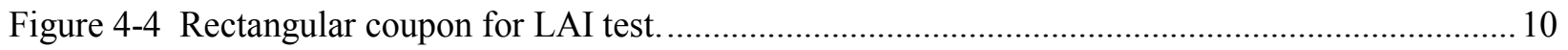

Figure 4-5 Modified cap of containers for LAI corrosion studies ........................................................... 12

Figure 4-6 LAI corrosion setup in hood showing (a) the bath on top of hot plates and (b) showing the

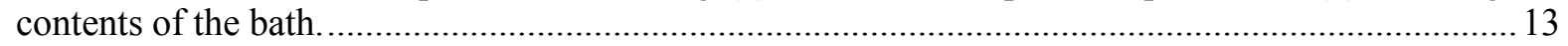

Figure 4-7 Side picture of the bullet (left) and frontal picture of the bullet (right). ............................... 14

Figure 5-1 Coupons from LAI and TI corrosion test using LDP simulant ............................................ 16

Figure 5-2 Coupons from LAI and TI corrosion test using GW simulant ........................................... 16

Figure 5-3 OCP vs. time of carbon steel coupons exposed to LDP and GW simulants for four months ... 18

Figure 5-4 Pictures after VS exposure in simulant 7 at three different levels ...................................... 20

Figure 5-5 Pictures after VS exposure in simulant 8 at three different levels ....................................2 21

Figure 5-6 Cyclic potentiodynamic polarization scans for sample and duplicate using conditions described

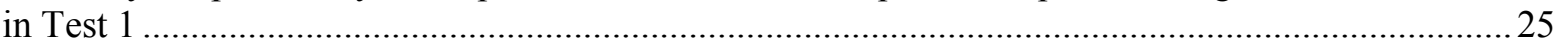

Figure 5-7 Cyclic potentiodynamic polarization scans for sample and duplicate using conditions described

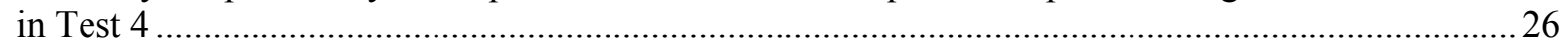

Figure 5-8 Cyclic potentiodynamic polarization scans for sample and duplicate using conditions described

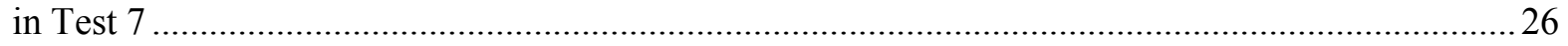

Figure 5-9 Cyclic potentiodynamic polarization scans for sample and duplicate using conditions described

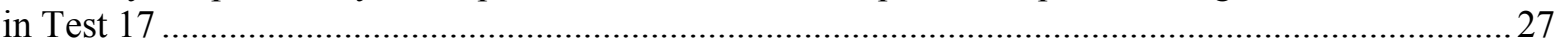

Figure 5-10 LAI coupons immersed in simulants developed for waste buffering from actual AN-102

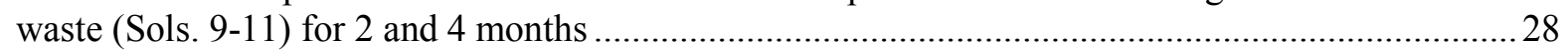

Figure 5-11 OCP measurements at different periods during four month testing of LAI for Solutions 9, 10, 11. 30

Figure 5-12 Fourth-month exposure at VS conditions of carbon steel in Vessels 1, 2 and 3 at 50 ppm Ammonia 
Figure 5-13 Fourth-month exposure at VS conditions of carbon steel in Vessels 4, 5 and 6 at 550 ppm Ammonia

Figure 5-14 Pictures of coupons after two months and four months exposure during LAI testing for solutions 1,2, 3 and 4 . The dash line represents the interface.

Figure 5-15 Pictures of coupons after two months and four months exposure during LAI testing for solutions 5, 6, 7 and 8 . The dash line represents the interface. 36

Figure 5-16 OCP measurements at different periods during four month testing of LAI for Solutions 1 to 8. 38

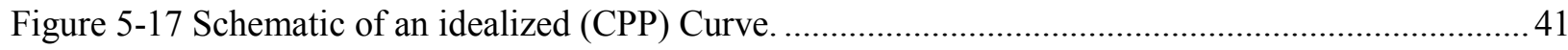

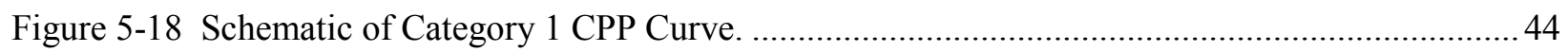

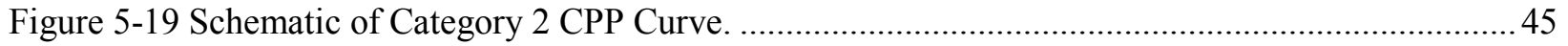

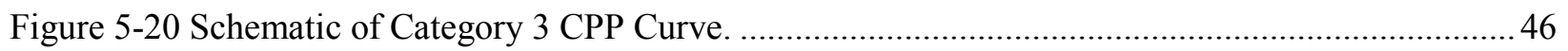

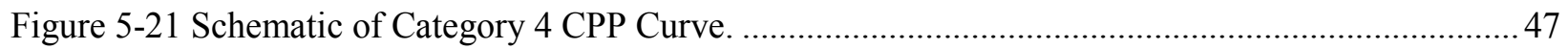

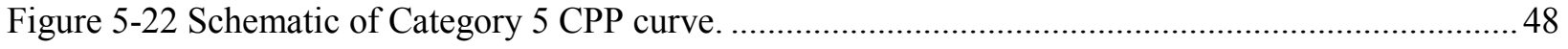

Figure 5-23 Log-log plot for SRS dilute waste simulant that shows the minimum nitrite required to prevent pit initiation as a function of nitrate concentration [36].

Figure 5-24 Borderline behavior in a dilute, SRS waste simulant [28]. The CPP scan exhibited negative hysteresis, however, small pits were detected on the electrode surface. Magnification was approximately $10 \mathrm{X}$.

Figure 5-25 CPP curve in dilute SRS waste simulant that illustrates the vertex threshold potential of $1.0 \mathrm{~V}$ [45].

Figure 5-26 CPP curve in dilute SRS waste simulant that illustrates the vertex threshold current of 1 $\mathrm{mA} / \mathrm{cm}^{2}$.

Figure $5-27 \mathrm{pH}$ distribution for total distribution and 40 test matrix......................................................5 56

Figure 5-28 Temperature distribution for total distribution and 40 test matrix. .......................................57

Figure 5-29 Temperature distribution for total distribution and 40 test matrix. ........................................ 58

Figure 5-30 Nitrite concentration distribution for total distribution and 40 test matrix............................59

Figure 5-31 Nitrite/Nitrate ratio distribution for total distribution and 40 test matrix.............................. 60

Figure 5-32 CPP curve from historical test data [35]. Note potential is with respect to SCE reference electrode......

Figure 5-33 CPP curve from FY2014 Test 15.

Figure 5-34 Micrographs (a), (b), and (c) are the longitudinal, transverse, and short directions for the A537 that was tested, while micrographs (d) and (e) are the longitudinal and transverse direction for the rail car steel that was tested. 
Figure 5-35 Open circuit potential transients for the rail car and A537 steel exposed to test solution 16.. 76

Figure 5-36 CPP curves for the rail car and A537 steel exposed to test solution 16.............................. 77

Figure 5-37 Post-CPP test observation of samples after tests in solution 16 (a) A537 steel and (b) Rail Car

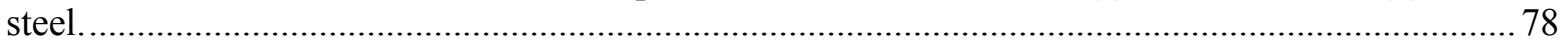




\section{LIST OF ABBREVIATIONS}

$\begin{array}{ll}\text { ASTM } & \text { American Society for Testing and Materials } \\ \text { CPP } & \text { Cyclic Potentiodynamic Polarization } \\ \text { DNV-GL } & \text { Det Norske Veritas-Germanisher Lloyd } \\ \text { DST } & \text { Double-Shell Tank } \\ \text { DWPF } & \text { Defense Waste Processing Facility } \\ \text { EPOC } & \text { External Panel Oversight Committee } \\ \text { GW } & \text { Ground Water } \\ \text { LAI } & \text { Liquid-Air Interface } \\ \text { LDP } & \text { Leak Detection Pit } \\ \text { LPR } & \text { Linear Polarization Resistance } \\ \text { OCP } & \text { Open Circuit Potential } \\ \text { PNNL } & \text { Pacific Northwest National Laboratory } \\ \text { SCC } & \text { Stress Corrosion Cracking } \\ \text { SCE } & \text { Saturated Calomel Electrode } \\ \text { SRNL } & \text { Savannah River National Laboratory } \\ \text { SRS } & \text { Savannah River Site } \\ \text { SST } & \text { Single-Shell Tank } \\ \text { TI } & \text { Total Immersion } \\ \text { VS } & \text { Vapor Space } \\ \text { VSC } & \text { Vapor Space Corrosion } \\ \text { WRPS } & \text { Washington River Protection Solutions } \\ & \end{array}$


SRNL-STI-2014-00616

Revision 0

\subsection{Introduction}

Radioactive waste at the Hanford Site is being retrieved from the single-shell tanks (SSTs) and transferred to newer double-shell tanks (DSTs) for storage prior to pretreatment and eventual vitrification at the Waste Treatment Plant (WTP). The DSTs have a detailed corrosion control program developed over the years. The program includes special construction requirements; waste chemistry controls and systematic non-destructive (NDE) tank wall inspections. On the other hand, the composition of the wastes in SSTs was never controlled to the chemistry standards required for the DSTs, and generally does not meet the DST waste specifications. Thus, it is likely that some of the wastes transferred to the DSTs will not comply with DST waste specifications and hence would be vulnerable to corrosion attack.

Since 2004, the Expert Panel for Hanford Double-Shell Tank Waste Chemistry Optimization Oversight Committee (EPOC) has advised the Hanford site on matters regarding laboratory testing that support corrosion control for the DSTs as the waste is being transitioned between the SSTs and the vitrification facility [1]. Three laboratories have been involved in the corrosion testing: Det Norske Veritas-Germanischer Lloyd (DNV-GL), Savannah River National Laboratory (SRNL), and the 222-S facility at Hanford currently operated by Washington River Protection Solutions (WRPS). SRNL has primarily been focused on laboratory studies related to vapor space corrosion (VSC), liquid-air interface (LAI) corrosion, and pitting corrosion, particularly for waste supernates that have a relatively dilute chemistry. SRNL also recently participated in a round robin test program with the other two laboratories that established a standardized test protocol for the performance of cyclic potentiodynamic polarization (CPP) tests [2]. This accelerated test is utilized to determine the susceptibility of a material (e.g., carbon steel) to a given environment (e.g., waste supernate, interstitial liquid, etc.).

The SRNL tasks for FY14 were also aligned within these general categories. Laboratory tests were conducted to evaluate the susceptibility of carbon steel to VS and LAI corrosion in supernate chemistries that are at the recently recommended requirements for inhibition of stress corrosion cracking [3]. Additionally, SRNL evaluated the susceptibility of carbon steel to pitting corrosion under buffered waste conditions, with the objective of determining the adequate amount of inhibitor (e.g., nitrite) necessary to mitigate corrosion. As a secondary objective, the results of these activities were utilized to assess the robustness of the standardized CPP protocol.

\subsection{Background}

The SRNL Task Plan included five testing programs [4]. A brief description of the previous investigations for each is given in the sections that follow.

\subsection{Vapor space corrosion testing at SRNL}

Vapor space corrosion tests were performed to investigate the likelihood of this type of attack in the Hanford DSTs. Until now, there have been no consequential incidents of uniform or localized corrosion in the DSTs, but there have been instances of unexplained corrosion in equipment that were suspended above the waste. In the vapor space, the chemistry of condensate that can form on the tank walls is complex and is constantly evolving. As the condensate evaporates from the tank wall, it influences the formation of corrosion products and the corrosion of the carbon steel. Previous explorations of the mechanism of VSC involved: the chemical composition of the liquid 
that condensed on the carbon steel in the vapor space [5] and corrosion above simulated waste environments [6],[7].

Six Hanford DST supernates were selected for testing in order to evaluate the impact of vapor space corrosion. These were: AY-101 (Segment 3), AY-101 (Segment 8), AN-102, AY-102, SY102 (high nitrate) and SY-102 (high chloride). Thermodynamic studies by Pacific Northwest National Laboratory (PNNL) were done to be able to predict the chemistry of adsorbed surface condensates based on equilibrium of major vapor space constituents and condensate and it was verified with experimental results [4]. Ammonia and carbon dioxide were determined to be the dominant species in vapor space that are most likely to impact tank corrosion.

Ammonia is produced predominantly in the supernate through thermal and radiolytically induced reactions between organic waste components and nitrate and nitrite ions. The concentration in the vapor space varies but it can be as high as $550 \mathrm{ppm}$ obtained in Tank SY-102. Through limited laboratory testing, VSC has been observed to be inhibited by ammonia [8],[9].

Corrosion chemistry limits were recommended to minimize SCC in the DST [3]. Table 2-1 lists the different specifications and the maximum or minimum requirements needed for SCC control. The specific limit of interest for current testing is the minimum nitrate/nitrate ion ratio of 0.15 with minimum nitrate concentration of $0.05 \mathrm{M}$. For nitrate concentrations of $0.4,2$ and $4.5 \mathrm{M}$ the minimum ratio of 0.15 was not able to prevent pitting corrosion in the VS [7].

Table 2-1 Proposed Specifications for the Control of SCC in Nitrate Ion Wastes in DSTs.

\begin{tabular}{|c|c|}
\hline Maximum Temperature & $50^{\circ} \mathrm{C}$ \\
\hline Maximum Concentration of Nitrate Ion & $6.0 \mathrm{M}$ \\
\hline Maximum Concentration of Hydroxide Ion & $6.0 \mathrm{M}$ \\
\hline Minimum pH & 11 \\
\hline Minimum Concentration of Nitrite Ion & $0.05 \mathrm{M}$ \\
\hline Minimum Nitrite Ion/Nitrate Ion Ratio & 0.15 \\
\hline
\end{tabular}

\subsection{Liquid-Air Interface Corrosion testing at SRNL}

LAI corrosion can occur when the liquid level remains stagnant for a long period of time and develops as pits or a localized general corrosion that initiates at the interface. At Hanford, only tank AY-101 has shown evidence of this type of corrosion [10]. During laboratory testing, DNVGL also observed LAI corrosion from a simulant of waste in Hanford DST AP-105 [11]. Several experimental approaches have been attempted during previous years to understand the phenomenon [11]-[13]. However, a clear understanding of the mechanism has not being achieved. Several findings of the results of LAI test were able to identify that LAI corrosion cannot be fully simulated and can be challenging to develop a test that can be reliable.

SRNL studied the effects of LAI corrosion in solutions at the boundary of the new corrosion control limits for SCC during long term testing for four months [14]. The tests demonstrated that the minimum ratio of nitrate to nitrate of 0.15 was insufficient to prevent corrosion at LAI. Results from these tests also indicated that both the ratio of nitrite to nitrate and the amount of nitrite influence the corrosion susceptibility at the LAI [14]. Long-term (i.e., 4 months) partial immersion coupon tests will once again be employed for this activity. These tests will cover 
compositions at the boundary of the new corrosion chemistry limits that will prevent SCC in the DST [3].

\subsection{AY-102 Leak Detection Pit and Secondary Tank Corrosion Studies}

Waste from Tank AY-102 has leaked into the annulus region of the tank [15]-[17]. The secondary liner of the tank provides the barrier between the waste and the environment. The Leak Detection Pit (LDP) provides a means of detecting a leak in the secondary liner. The exterior of the secondary wall for Tank AY-102 is potentially exposed to liquid and/or vapors from the solutions contained in the LDP or the drain line to the pit that is beneath the tank. The rate of corrosion of the steel due to this exposure is unknown. LAI corrosion and VS corrosion testing for carbon steel exposed to very dilute solutions was needed to quantify this attack. It is also possible that the underliner is completely immersed in the liquid. Therefore, coupons that are fully immersed in anticipated LDP liquid were tested as well. These tests were merged within the VSC and LAI corrosion activities.

\subsection{Waste Buffering Corrosion Studies}

Grab samples of actual waste from double shell tank 241-AN-102 (AN-102) were obtained in 2012 to determine if the waste was within the corrosion control chemistry requirements [18],[19]. The corrosion chemistry for supernate samples were taken at six different levels beneath the surface. While sufficient nitrite inhibitor was present, the hydroxide concentration was near or below the minimum requirement.

Electrochemical testing was performed by WRPS at the 222-S laboratory to determine if these solutions were corrosive towards carbon steel [18]. Testing was performed in four of the actual wastes at temperatures between 30 and $50{ }^{\circ} \mathrm{C}$. The Cyclic Potentiodynamic Polarization (CPP) test results indicated that carbon steel was not susceptible to pitting corrosion in the actual waste environment.

In parallel with the WRPS tests, CPP tests were performed at DNV-GL on carbon steel exposed to AN-102 waste simulants [20]. Tests were conducted with simulant chemistries that were adjusted such that the hydroxide concentrations were significantly less than that for the actual waste and the corrosion chemistry requirements (i.e., $\mathrm{pH}$ 10.3-13.6). The composition of the minor constituents for the waste simulant also differed from that of the actual waste. The CPP test results indicated that carbon steel was mildly susceptible to pitting corrosion in these environments. The minimum nitrite/nitrate ratio and or minimum nitrite concentration necessary to mitigate pitting at these lower $\mathrm{pH}$ values was not determined.

Both of these test programs were conducted prior to the round robin testing that was performed to develop a standardized CPP test protocol [2]. Therefore, these tests were repeated with the new standardized test protocol.

\subsection{Pitting Corrosion Studies}

Electrochemical techniques have been utilized as an accelerated means for assessment of the pitting susceptibility of waste tank materials in simulated supernates for several years. Variations 
on the CPP technique, coupled with long term immersion tests, have been performed to provide a basis for corrosion chemistry control.

The three laboratories (SRNL, DNV-GL and WRPS) that have supported Hanford corrosion testing, recently conducted a round robin test program to establish a standardized test protocol for the performance of CPP tests [2]. Table 2-2 compares the test parameters used recently by each laboratory to the new standardized pitting protocol. The major changes to the protocol were the establishing of a potential stabilization to be limited to 2 hours, the surface preparation prior to testing of a 600-grit finish and the use of the bullet geometry. This protocol will be used for all subsequent testing related to Hanford DST wastes.

Table 2-2 CPP protocol with the parameters utilized for testing

\begin{tabular}{|c|c|c|c|c|}
\hline Parameters & SRNL & WRPS & DNV & $\begin{array}{c}\text { Standardized } \\
\text { CPP } \\
\text { Protocol }\end{array}$ \\
\hline Potential Stabilization (hrs.) & 2 & $18^{*}$ & 2 & 2 \\
\hline Start Potential (V vs. OCP) & -0.05 & -0.2 & -0.1 & -0.05 \\
\hline Scan Rate (mV/s) & 0.5 & 0.167 & 0.167 & 0.167 \\
\hline Vertex Threshold (mA/cm $\left.\mathbf{2}^{2}\right)$ & 1 & 5 & 1 & 1 \\
\hline Finish Potential (V vs. OCP) & 0 & 0 & -0.1 & 0 \\
\hline Sample geometry & Disk & cylinder & bullet & bullet \\
\hline Surface Preparation & 600 grit & None & None & 600 grit \\
\hline
\end{tabular}

* potential was stabilized for 18 hours and then LPR was performed; Electrode was allowed to rest for 1 hour after LPR and then CPP scan was performed.

\subsection{Task Description and Activities}

The tasks are described in the sections below with the activities performed during FY14.

\subsection{Task 1: Vapor space Corrosion Studies for Hanford Double Shell Tanks}

Long-term exposure experiments were conducted for four months to investigate ammonia inhibition of vapor space corrosion at the new SCC control limits. The tests were also conducted above simulated LDP water and above zone groundwater [21],[22]. In general, Hanford groundwater is calcium bicarbonate dominated water with a $\mathrm{pH}$ that typically ranges from 7.5 to 8.5. Other prominent major ions are sodium, chloride, sulfate and magnesium. The LDP water has the same components although at more dilute concentrations. The dilution likely occurs to condensation of water vapor within the LDP system. Coupons of carbon steel were located at three different levels above the simulant to mimic different conditions inside the DST. These conditions were (1) the carbon steel is exposed to a wet/dry cycle with the waste; (2) the carbon steel was wetted at some point but now is only exposed to humid air and (3) carbon steel that was never wetted by the waste and therefore only exposed to humid air. The results for this task are presented in subsection 5.1.2 for the conditions near the secondary liner of Tank AY-102 and 
section 5.3 for the simulants with the new control limits for SCC with ammonia in the vapor phase.

\subsection{Task 2: Liquid Air Interface Corrosion Testing}

Carbon steel at the LAI was examined to determine the susceptibility for corrosion at simulants with the new SCC control limits and in the environment at the exterior of the secondary wall of AY-102. The coupons in rectangular form were immersed at approximately $50 \%$ into solution. Complete immersion was also performed in several coupons to study corrosive conditions at this environment beneath the secondary wall of AY-102. The results obtained for this Task are shown in subsections 5.1.1, 5.2.2 and section 5.4.

\subsection{Task 3: Waste Buffering}

Simulants based on samples of actual waste from Tank AN-102 were utilized to perform electrochemical tests. The tests were used to determine minimum hydroxide concentration necessary to prevent pitting in high nitrate solutions that also have a high nitrite concentration. These results, which were gathered with the new standardized CPP protocol, were compared with data gathered previously at DNV-GL and 222-S. The results and discussion are presented in section 5.4 .

\subsection{Task 4: Pitting Corrosion}

CPP experiments were performed in historical waste simulants with the standardized CPP protocol and the results were compared to the previously gathered. This activity was designed to understand the test parameters that may have an influence in the results and to assess the applicability of the previous tests. The results are organized in section 5.5.

\subsection{Experimental Procedure}

Carbon steel coupons were used for corrosion testing and analysis. They were fabricated from AART128 Rail Car Steel. This steel was selected for testing since it approximates the chemistry and microstructure of American Society for Testing and Materials (ASTM) A515 Grade, Grade 60 carbon steel, the steel from which the tanks were fabricated [23]. The rail car steel was also chosen because it was of the same vintage as the tank steel. The chemical composition of the steel is shown in Table 2.

Table 4-1 Chemical Composition of AART128 Rail Car Steel.

\begin{tabular}{|c|c|c|c|c|c|c|}
\hline & $\mathbf{C}$ & $\mathbf{M n}$ & $\mathbf{P}$ & $\mathbf{S}$ & $\mathbf{S i}$ & $\mathbf{F e}$ \\
\hline $\begin{array}{c}\text { Specification } \\
\text { (wt\%) }\end{array}$ & $\begin{array}{c}0.24 \\
\text { (max.) }\end{array}$ & 0.9 (max.) & $\begin{array}{c}0.035 \\
(\max .)\end{array}$ & $\begin{array}{c}0.04 \\
\text { (max.) }\end{array}$ & 0.13 to 0.33 & Balance \\
\hline $\begin{array}{c}\text { Measured } \\
\text { (wt\%) }\end{array}$ & 0.212 & 1.029 & 0.012 & 0.013 & 0.061 & Balance \\
\hline
\end{tabular}


Below are the experimental details and conditions in which the carbon steel was used and prepared for VS corrosion, LAI corrosion and electrochemical corrosion testing.

\subsection{Vapor Space Corrosion Testing}

\subsubsection{Material sample}

Circular coupons that are 5/8 inch diameter and 1/8 inch thick carbon steel (Metal samples) were mounted using a two part acrylic solution (Buehler) so that one face of the coupon was exposed. While mounting, a wire was placed in a lateral position to be able to hang the coupons. After cold mounting the coupons, they were polished to a 600 grit finish and rinsed with distilled water and ethanol. Clear nail polish was utilized around the edges of the disk to minimize crevice corrosion. A sample of the coupon can be seen in Figure 4-1.

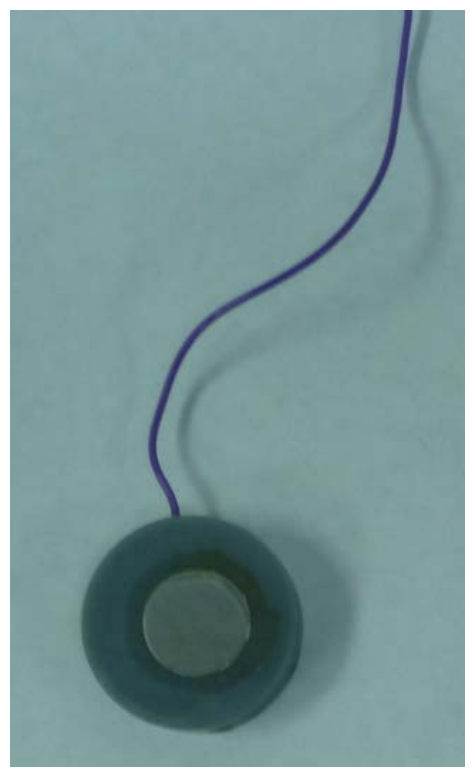

Figure 4-1 Coupon mounted in cold mount with wire

Twelve coupons were suspended in rings attached to stainless steel rods (four at each level) as shown in Figure 4-2. As shown in the figure, three rings at different locations were welded onto the rod. 
SRNL-STI-2014-00616

Revision 0

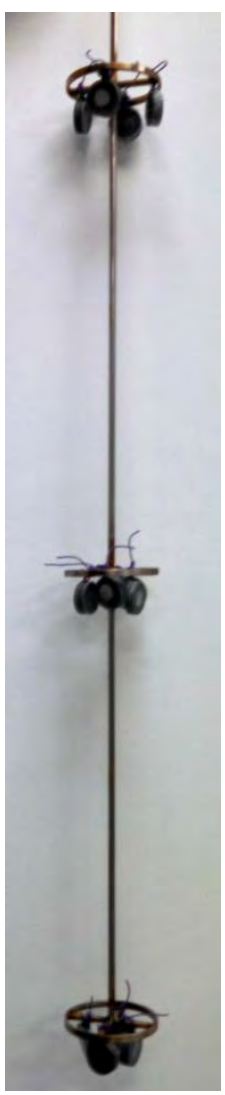

Figure 4-2 Picture of the rod with four coupons hanged at each level.

\subsubsection{Simulants}

VSC tests were conducted at conditions described in Table 4-2. Chemical compositions for each vessel are presented in Appendix A. Six vessels contained simulants that had a composition representing the new SCC control limits which is a nitrite/nitrate ratio of 0.15 . Nitrate concentrations were selected from possible supernate concentrations and nitrite was calculated using this ratio. The $\mathrm{pH}$ of the simulants was selected as 12 to be slightly above the minimum requirement of 11 . The composition of the minor constituents in the simulant is consistent with values from samples of the waste supernates that were utilized during previous testing [7]. The tests were conducted at VS ammonia levels of $50 \mathrm{ppm}$ for three vessels and $550 \mathrm{ppm}$ for three vessels. The remaining two vessels had a chemical composition representing the LDP residue and GW found close to the secondary liner in the tank, respectively. These tests were conducted with humid air passing through the glass column.

During the test, it was desired to establish equilibrium between the ammonia gas and the dissolved ammonia in the liquid. By relating the concentrations to Henry's Law [24], the concentrations of dissolved ammonia were calculated in the liquid and were added in the simulants using ammonium nitrate to achieve equilibrium. The ammonium ion from ammonium nitrate and ammonia achieve this equilibrium by the following equation [25] :

$$
\mathrm{NH}_{4}^{+}+\mathrm{OH}^{-}+(\mathrm{n}-1) \mathrm{H}_{2} \mathrm{O}_{(\mathrm{l})} \leftrightarrow \mathrm{NH}_{3} \cdot \mathrm{nH}_{2} \mathrm{O}_{(\mathrm{aq})}
$$


where $\mathrm{n}$ is stoichiometric number. A previous report explains in detail more about the ammonia addition to the solution [7].

Table 4-2 Test conditions for VSC testing

\begin{tabular}{|c|c|c|c|}
\hline Vessel & Initial $\mathbf{p H}$ & $\begin{array}{c}\text { Ammonia gas } \\
\text { concentration in Air } \\
(\mathbf{p p m})\end{array}$ & Temperature ${ }^{\circ} \mathbf{C}$ ) \\
\hline 1 & 12 & 50 & 40 \\
\hline 2 & 12 & 50 & 40 \\
\hline 3 & 12 & 50 & 40 \\
\hline 4 & 12 & 550 & 40 \\
\hline 5 & 12 & 550 & 40 \\
\hline 6 & 12 & 550 & 40 \\
\hline 7 & 7.6 & 0 & 45 \\
\hline 8 & 7.6 & 0 & 45 \\
\hline
\end{tabular}

\subsubsection{Testing Apparatus}

The VSC apparatus is shown in Figure 4-3. Eight glass columns prepared by the SRNL glass shop were placed inside a walk-in hood and mounted in an aluminum frame. The columns have dimensions of $1 \mathrm{~m}$ by $15 \mathrm{~cm}$ and consisted of a jacketed glass vessel connected to a glass tube and closed at the top with a glass cap. 1 Liter of simulant was added to each vessel and the temperature was monitored with a temperature reader (Omega). The gas cylinders provided ammonia (50 and $550 \mathrm{ppm}$ ) and were located at the right side of the configuration. The cylinders were connected to a mass flow controller at each gas concentration to maintain a flow of $15 \mathrm{sccm}$. The mass flow was diverted for each vessel by means of a flow meter. Three flowmeters at each concentration of ammonia in air maintained flow to each vessel at $5 \mathrm{sccm}$. The first six vessels (from right to left) were connected in parallel to a water circulator that maintained the simulant temperature at $40{ }^{\circ} \mathrm{C}$. The last two vessels (from right to left) were connected to a water circulator in parallel to maintain a simulant temperature of $45^{\circ} \mathrm{C}$. The ammonia gas was bubbled through a bottle filled with the corresponding simulant to humidify the gas before it entered the column.

The rods containing the coupons were placed inside the vessels. They represent different levels above the simulant. These levels are described below.

Level 3: This set of coupons was not exposed to the solution prior to testing. The coupons were suspended approximately 36 inches above the simulant. This level is representative of a vapor space region that is only exposed to the humidified air, the ammonia, and any volatile species from the solution. 
Level 2: The coupons were dipped in simulant for five minutes prior to test. The coupons were hung at the middle fixed ring. These coupons were approximately 18 inches above the liquid. This level is representative of a vapor space region of the tank that at one time was exposed to waste, but now has infrequent or no contact with the waste. However, this region is exposed to the humidified air and the ammonia gas.

Level 1: The coupons were dipped in simulant for five minutes prior to test. The coupons were hung at the bottom fixed ring. These coupons were suspended approximately 1 inch above the liquid level of the simulant. Once every two weeks the coupons were lowered to the solution to be dipped in the simulant for 5 minutes. This level is representative of a vapor space region of the tank that experiences periodic wetting/drying. This sequence could occur due to: a) waste transfers into and out of the tank, b) splashing due to flushing operations, and/or c) solution "creep" above the liquid air interface.

Testing of the coupons in vapor space environment was performed for four months with monthly specimens taken at each level for each vessel (i.e., 3 coupons each month for each vessel).

Coupons were cleaned using ASTM G1 Clark's solution [26] and weight losses were recorded.

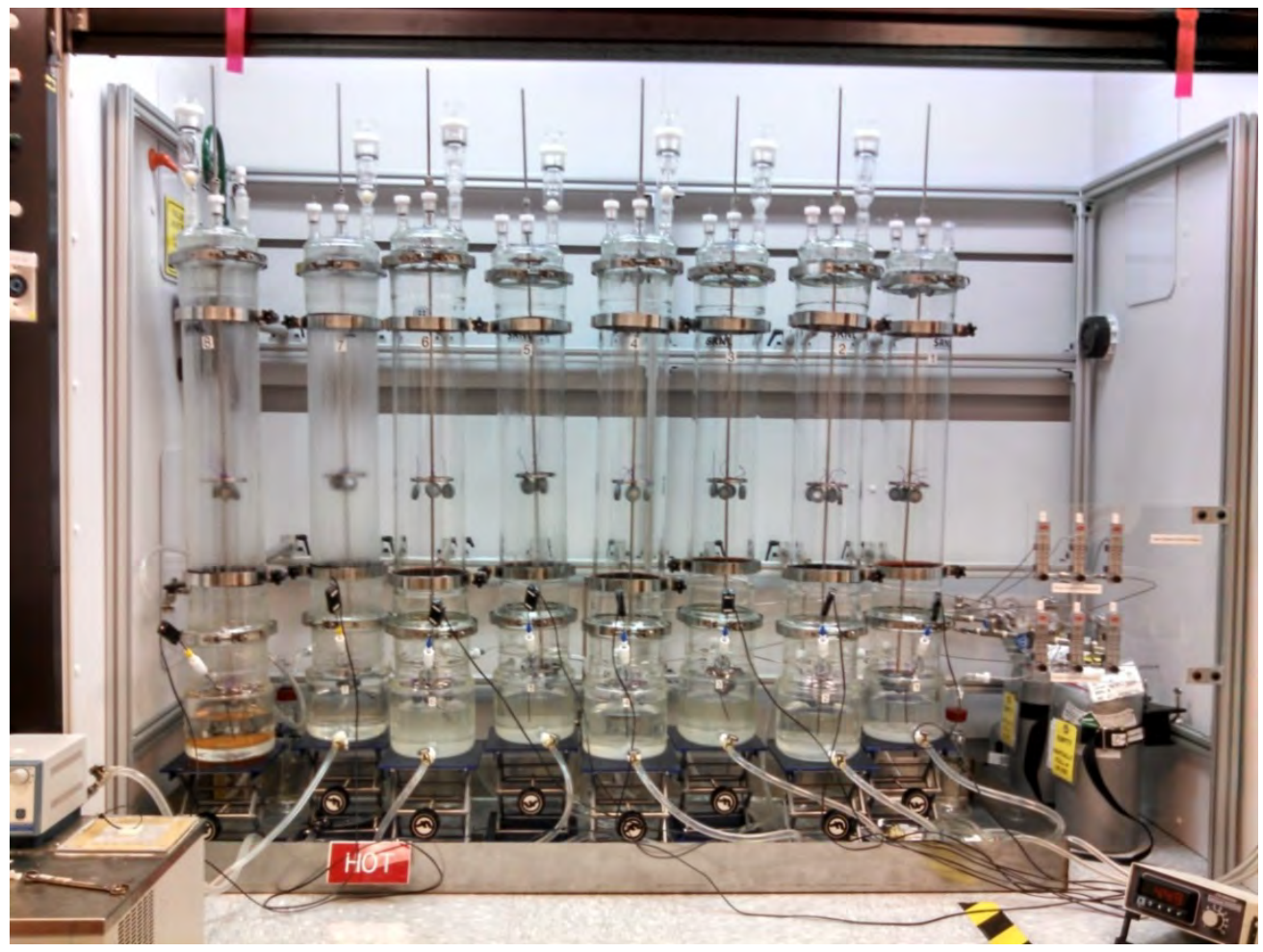

Figure 4-3 Picture of the Vapor Space Corrosion setup inside the walk-in hood.

\subsection{Liquid Air Interface Corrosion Testing}

\subsubsection{Material sample}


Rectangular carbon steel coupons, that were 1 inch by 2 inches and $1 / 4$ inch thick, were positioned in solution so that approximately 50\% of the coupon was immersed. Prior to the test, the surface was polished on 600 grit paper and rinsed with distilled water and acetone. Figure 4-4 shows an example of the coupon. A stainless steel rod was used to connect to the coupon and fixed it in place for long term testing. The stainless steel rod was insulated with Teflon tape to minimize contact with the coupon.

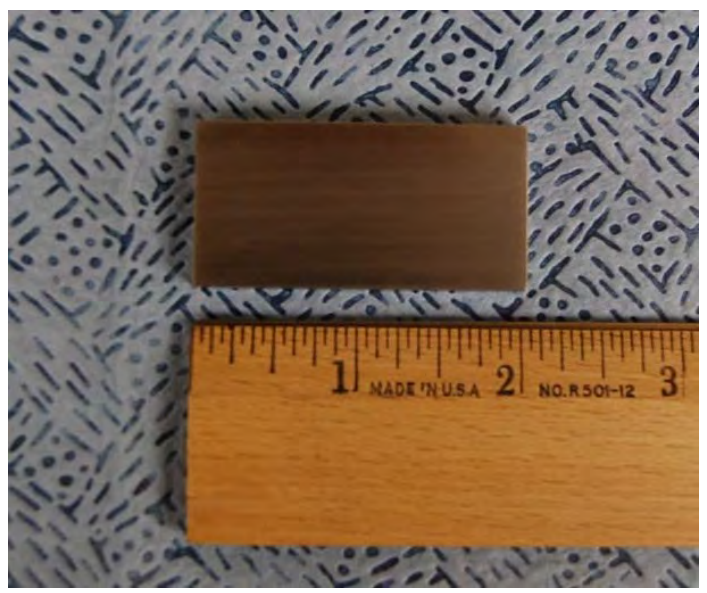

Figure 4-4 Rectangular coupon for LAI test.

\subsubsection{Simulants}

LAI corrosion tests were conducted using simulants with different chemical compositions. A total of 15 containers were utilized consisting of 13 different formulations of simulants. The first 8 containers have simulant compositions shown in Table 4-3. At the comments section a basis for the selection of each composition is explained. The initial $\mathrm{pH}$ for these simulants was 12 . A detailed chemical composition for all containers can be found in Appendix C. 
Table 4-3 Nitrate and nitrite concentrations for LAI corrosion test simulants.

\begin{tabular}{|c|c|c|c|l|}
\hline Container & $\begin{array}{c}\text { Nitrate } \\
\text { (M) }\end{array}$ & $\begin{array}{c}\text { Nitrite } \\
\text { (M) }\end{array}$ & $\begin{array}{c}\text { Nitrite/Nitrate } \\
\text { Ratio }\end{array}$ & \multicolumn{1}{|c|}{ Comments } \\
\hline 1 & 0.1 & 0.05 & 0.5 & $\begin{array}{l}\text { Dilute solution; Minimum nitrite } \\
\text { allowed by new SCC requirement; } \\
\text { Ratio is greater than that required by } \\
\text { new SCC limits, but less than that } \\
\text { required by Zapp's law [27] }\end{array}$ \\
\hline 2 & 0.1 & 0.166 & 1.66 & $\begin{array}{l}\text { Dilute solution; Zapp's law minimum } \\
\text { required nitrite/nitrate ratio. }\end{array}$ \\
\hline 3 & 0.5 & 0.075 & 0.15 & $\begin{array}{l}\text { At approximately this concentration of } \\
\text { nitrate, Hoffman observed that the } \\
\text { addition of more nitrite was not } \\
\text { necessarily beneficial [28]; Minimum } \\
\text { nitrite/nitrate ratio for new SCC limit. }\end{array}$ \\
\hline 5 & 1 & 0.15 & 0.15 & $\begin{array}{l}\text { Zapp's law minimum required nitrite to } \\
\text { nitrate ratio. }\end{array}$ \\
\hline 6 & 1 & 0.6 & $\begin{array}{l}\text { Minimum nitrite to nitrate ratio for } \\
\text { new SCC limits }\end{array}$ \\
\hline 7 & 1 & 1.66 & 1.66 & $\begin{array}{l}\text { Hoffman results suggest that this } \\
\text { amount of nitrite is sufficient to } \\
\text { prevent pitting. }\end{array}$ \\
\hline 5 & 0.6 & $\begin{array}{l}\text { Zapp's law minimum required nitrite to } \\
\text { nitrate ratio. }\end{array}$ \\
\hline
\end{tabular}

Three additional containers were added from the waste buffering activity (Task 3 ) for LAI corrosion testing. They were performed to asses a relationship between the results of electrochemical test and an alternate approach for testing pitting corrosion. Table 4-4 describes the nitrate, nitrite and hydroxide concentration for each test.

Table 4-4 Nitrate, nitrite and hydroxide concentrations for LAI corrosion test simulants from Waste buffering activity

\begin{tabular}{|c|c|c|c|}
\hline Container & Nitrate (M) & Nitrite (M) & Hydroxide (M) \\
\hline 9 & 3.11 & 1.95 & 0.26 \\
\hline 10 & 3.06 & 1.91 & 0.07 \\
\hline 11 & 3 & 1 & 0.03 \\
\hline
\end{tabular}


LAI corrosion tests were performed in the LDP and GW simulants, which simulated the environment on the exterior of the AY-102 secondary liner. Two LAI corrosion tests (containers 12 and 14) were performed with a partially immersed coupon and the other two tests (containers 13 and 15) were performed with a totally immersed coupon.

\subsubsection{Testing Apparatus}

The tests were performed in 1 Liter polycarbonate bottles. The black cap was modified as shown in Figure 4-5. Orange rubber stoppers were placed in four of the holes. Two connectors were attached to flexible Tygon tubing to provide an inlet and outlet flow of air and the other two stabilized the stainless steel rods that held the rectangular coupons in position (white Teflon tape covered rods). The stoppers were sealed with silicone to prevent air leakage. A hole in the middle was used to provide access to a $\mathrm{pH}$ probe, a thermocouple, and a reference electrode. In the picture this hole has a black rubber stopper.

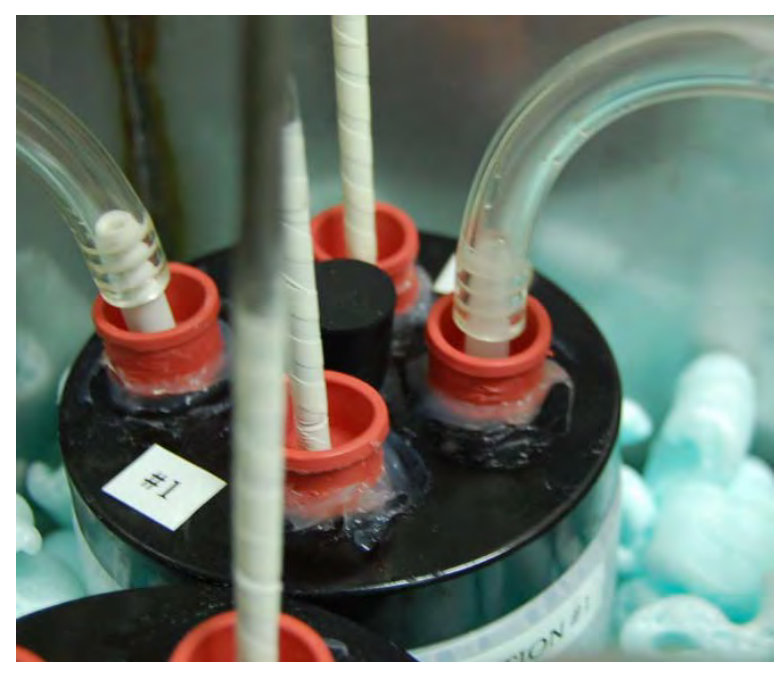

Figure 4-5 Modified cap of containers for LAI corrosion studies

Eleven containers (containers 1 to 11 ) were placed in a water bath at $40{ }^{\circ} \mathrm{C}$ and four containers (containers 12 to 15 ) were placed in another water bath at $45^{\circ} \mathrm{C}$. A picture of the bath is shown in Figure 4-6. The water bath consists of a stainless steel box on top of two hotplates. The temperature of the bath was controlled by placing each individual thermocouples from the hotplate into the water surrounding the plastic containers. These containers were connected in series with Tygon tubing to provide the flow of air from a gas humidifier that was connected to a flowmeter for a flow rate of $5 \mathrm{sccm}$. The evaporation of water from the bath was minimized by placing packaging styrofoam pellets above the water. 


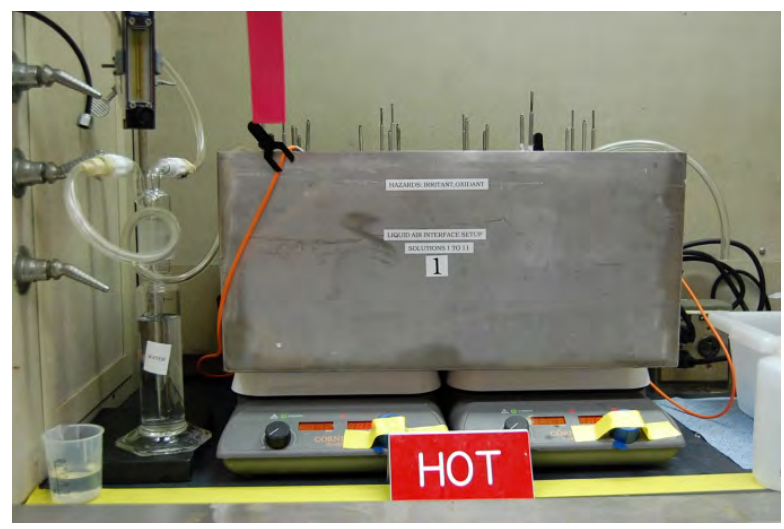

(a)

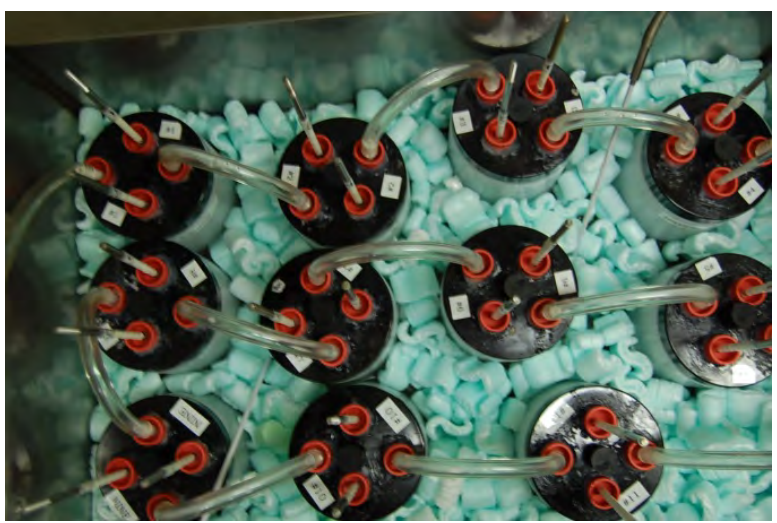

(b)

Figure 4-6 LAI corrosion setup in hood showing (a) the bath on top of hot plates and (b) showing the contents of the bath.

To each container $500 \mathrm{~mL}$ of the specified simulant was added. The rods that hold the coupons were lowered to reach the desired position within the liquid. The containers were placed in the bath and water was added to reach similar level as the liquid inside the container. This level inside was marked outside the container to account for losses during testing. Water was added periodically to the bath to maintain the same level. Make-up distilled water was added in some instances to the containers to also maintain the LAI level, although on a less frequent basis.

At the beginning of testing $\mathrm{pH}$, temperature and $\mathrm{OCP}$ was measured. The temperature of the solution in each container and $\mathrm{pH}$ was continued to be taken daily and OCP was measured two times per day when possible during working days. The coupons were maintained at these conditions for four months. One coupon was removed from each container after a two month interval.

At the end of testing the coupons were removed and cleaned using ASTM G1 Clark's solution [26] and weight losses were recorded. 


\subsection{Electrochemical Testing of Simulants}

\subsubsection{Material sample}

For electrochemical testing, carbon steel in the form of "bullets" with dimensions 0.188 inch diameter and 1.25 inches long (Metal Samples) were used. Before testing, a drill was used to polish the sample to a 600-grit finish. The electrodes were examined with a stereomicroscope for any defect and to ensure that the sample had a homogeneous surface. Then they were rinsed with distilled water and acetone. Figure 4-7 shows a picture of the sample after being polished and rinsed. It shows the surface of the shank and nose of the bullet. The bullet was attached to a stainless steel rod protected by a glass holder. A Teflon fixture was used to prevent liquid contact with the stainless steel rod and ensure electrical isolation.
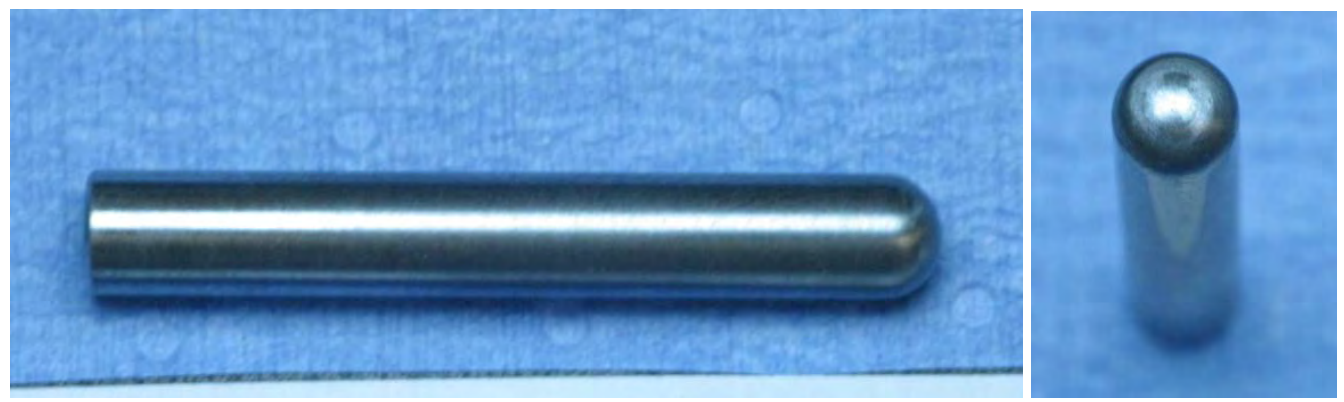

Figure 4-7 Side picture of the bullet (left) and frontal picture of the bullet (right).

\subsubsection{Simulants}

Simulants were prepared for two tasks (Task 3 and Task 4) from the task activities. For Task 3 simulants were made based on waste buffering from actual waste of tank AN-102. Task 4 simulants were obtained by performing a statistical test matrix covering data of corrosion activities from several years. Additional details of this assessment will be covered in section 5.5. A detailed chemical composition of each simulant is found in Appendix F for Task 3 and Appendix G for Task 4 as well as the corresponding results.

\subsubsection{Testing Apparatus}

Approximately $700 \mathrm{~mL}$ of simulant was added to a cell made by the SRNL glass shop that is similar to the cell for corrosion studies designed by Princeton Applied Research. At temperatures of testing greater than $50{ }^{\circ} \mathrm{C}$, a condenser was used to minimize evaporation. Two carbon graphite rods served as the counter electrode. A saturated calomel electrode (SCE) was used as the reference electrode. Prior to each test, the electrode was checked against a standard before testing (a SCE in $1 \mathrm{M} \mathrm{KCl}$ solution not used for testing). The SCE was placed in a bridge containing $0.1 \mathrm{NaNO}_{3}$ solution. The cell was placed on top of a hotplate with temperature control. REF 600 (Gamry) and VERSASTAT 3 (Ametek) potentiostats were used in this study and prior of using them ASTM G5 [29] was performed for quality assurance. The standardized pitting protocol was used to gather the data. The open circuit potential (OCP) was measured during the sample stabilization for two hours. The cyclic potentiodynamic polarization (CPP) test was conducted by applying a cyclic potential ramp from $-50 \mathrm{mV}$ vs. OCP up to a vertex threshold current of $1 \mathrm{~mA} / \mathrm{cm}^{2}$ at a scan rate of $0.167 \mathrm{mV} / \mathrm{s}$. The potential was finally returned back to the OCP to complete the test. 
SRNL-STI-2014-00616

Revision 0

\subsection{Results and Discussion}

Pictures of the samples after exposure of the different conditions are presented in Appendix B and $\mathrm{D}$ for task activity 1 and 2 . For Task activity 3 and 4 electrochemical results and pictures obtained after testing are shown in Appendix F and G, respectively. The discussion of results for the task activities are organized in sections to correlate all data obtained with the particular study.

\subsection{Secondary Wall of Tank AY-102 Corrosion Studies}

Corrosion studies were undertaken to assess the likelihood of a breach to the secondary liner of Tank AY-102. This testing will assess the susceptibility of this secondary liner to degradation by the leaked waste inside the primary containment and on the exterior of the secondary due to interactions with humid air or ground water.

The simulants for the LDP residue and the GW were recommended by the EPOC. LAI, Total Immersion (TI) and VS corrosion were performed and results are presented in the following subsections.

\subsubsection{Liquid Air Interface and Total Immersion tests}

LAI and TI tests were performed to determine the susceptibility of carbon steel to corrode in simulants corresponding to LDP and GW. Photographs of the coupons after two months and four months of exposures are presented in Figure 5-1 for LDP and in Figure 5-2 for GW for 50\% and $100 \%$ immersion.

Aggressive corrosion behavior was observed on the carbon steel exposed to LDP and GW. At $50 \%$ immersion, the corrosion attack concentrated at the water line and below for the two simulants. A clear distinction in the degree of corrosion that occurred in each simulant could not be seen visually. It did appear however, that during the first two months of exposure the majority of the coupon above the LAI did not corrode. Table 5-1 shows the weight losses of the coupons. For the LDP simulant, LAI coupon experienced approximately half the weight loss of TI coupon for the two month period. However, very similar weight losses were observed with this simulant after four months of exposure. Observing the pictures for LDP 50\% immersion at four months, general attack in the vapor space above the LAI results in a weight loss that was similar to the sample that was $100 \%$ immersed. This result indicates that corrosion during the first two months was focused at the waterline and below but after longer time exposure, the corrosion increased to the vapor space area of the coupon. The results for coupon completely immersed in LDP simulant (solution 13) were similar to 50\% immersion and TI in GW simulant (Solution 14 and 15, respectively). The general corrosion rate remained fairly constant at approximately 10 mils/yr during the 4 month test. 
LDP $50 \%$ immersion

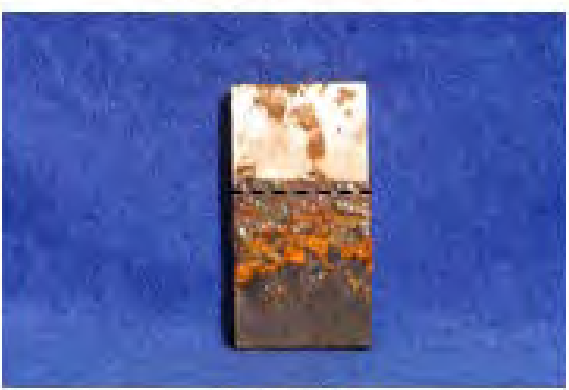

2 months

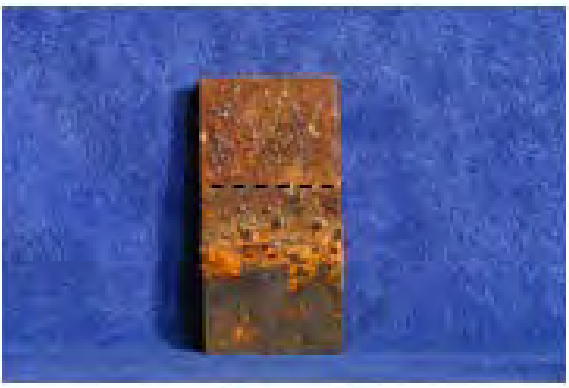

4 months
LDP $100 \%$ immersion

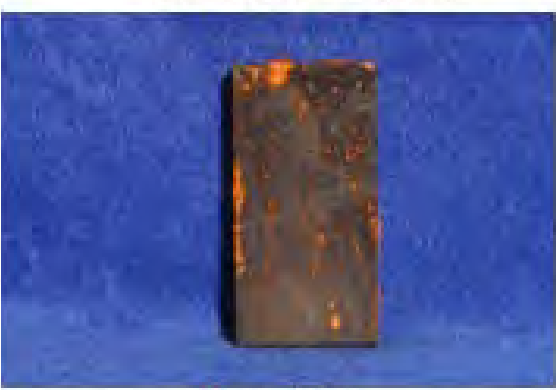

2 months

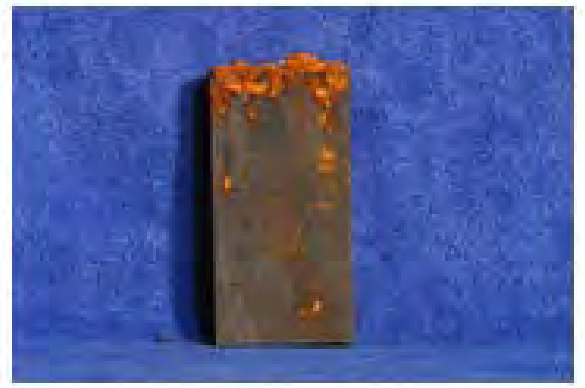

4 months

Figure 5-1 Coupons from LAI and TI corrosion test using LDP simulant

GW $50 \%$ immersion

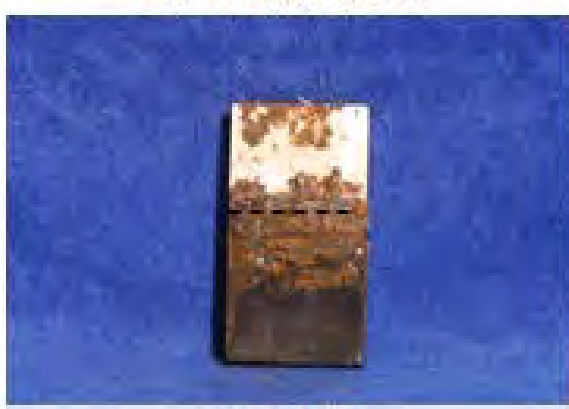

2 months

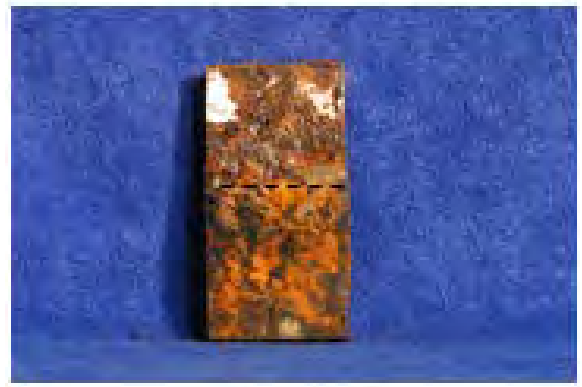

4 months
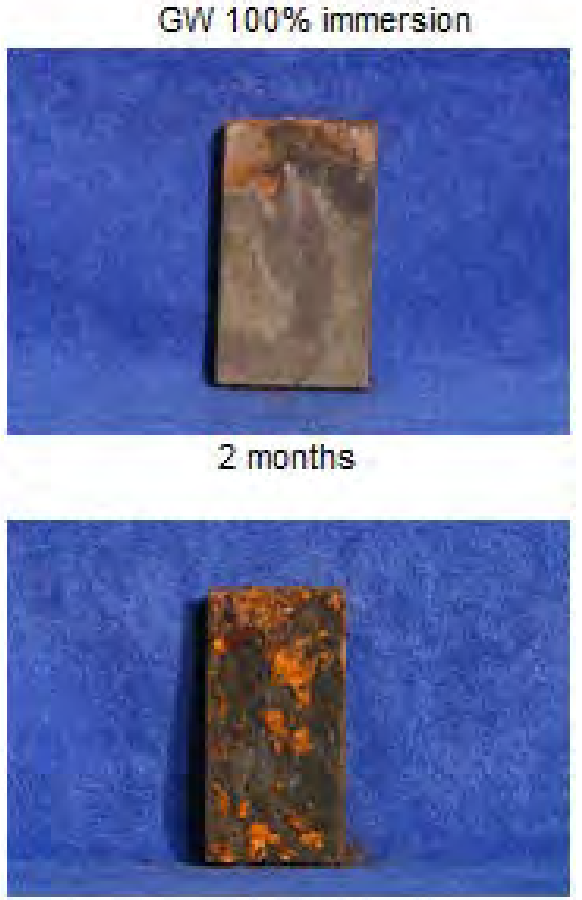

4 months

Figure 5-2 Coupons from LAI and TI corrosion test using GW simulant 
SRNL-STI-2014-00616

Revision 0

Table 5-1 Weight losses and corrosion rates for carbon steel coupons exposed to LDP and GW simulants

\begin{tabular}{|l|c|c|c|c|}
\hline \multirow{2}{*}{ Simulants } & \multicolumn{4}{|c|}{ Months Exposure } \\
\cline { 2 - 5 } & \multicolumn{2}{|c|}{ Two months } & \multicolumn{2}{c|}{ Four months } \\
\cline { 2 - 5 } & $\begin{array}{c}\text { weight loss } \\
\text { (g) }\end{array}$ & $\begin{array}{c}\text { Corrosion rate } \\
\text { (mpy) }\end{array}$ & $\begin{array}{c}\text { weight loss } \\
\text { (g) }\end{array}$ & $\begin{array}{c}\text { Corrosion rate } \\
\text { (mpy) }\end{array}$ \\
\hline $\begin{array}{l}\text { Solution 12 } \\
\text { LDP simulant 50\% } \\
\text { immersion }\end{array}$ & 0.5797 & 5.34 & 2.2387 & 10.3 \\
\hline $\begin{array}{l}\text { Solution 13 } \\
\text { LDP simulant 100\% } \\
\text { immersion }\end{array}$ & 0.9999 & 9.2 & 2.3817 & 10.96 \\
\hline $\begin{array}{l}\text { Solution 14 } \\
\text { GW simulant 50\% } \\
\text { immersion }\end{array}$ & 1.0397 & 9.57 & 2.3651 & 10.88 \\
\hline $\begin{array}{l}\text { Solution 15 } \\
\text { GW simulant 100\% } \\
\text { immersion }\end{array}$ & 1.0780 & 9.92 & 2.5246 & 11.62 \\
\hline
\end{tabular}

Figure 5-3 shows the OCP transients that were measured during the course of the long term test of the coupons immersed at 50\% and $100 \%$ in LDP and GW simulants. For all the cases the OCP started at a potential of -630 to $-675 \mathrm{mV}$ vs. $\mathrm{Ag} / \mathrm{AgCl}$ and increased to more noble values over time. Furthermore, there was no significant difference in OCP for the partially immersed and totally immersed coupons since for both simulants the OCP are comparable during the 4 months for the coupons in GW simulant; and after 1000 hours for the coupons in LDP simulant. During the first 24 hours the OCP stabilized and reached similar values for the four coupons. Shortly thereafter the OCP for the coupons exposed in LDP increased rapidly which may indicate the formation of corrosion products on the surface. The OCP started to stabilize around 560 hours for the coupon 50\% immersed and around 1100 hours for the coupon 100\% immersed in which reached a range of -130 to $-230 \mathrm{mV}$ vs. $\mathrm{Ag} / \mathrm{AgCl}$. The coupons immersed in $\mathrm{GW}$ continued gradually to increase OCP during the 4 month period and it appears that the voltage started to stabilize after 2600 hours close to $-350 \mathrm{mV}$ vs. $\mathrm{Ag} / \mathrm{AgCl}$ on average. 


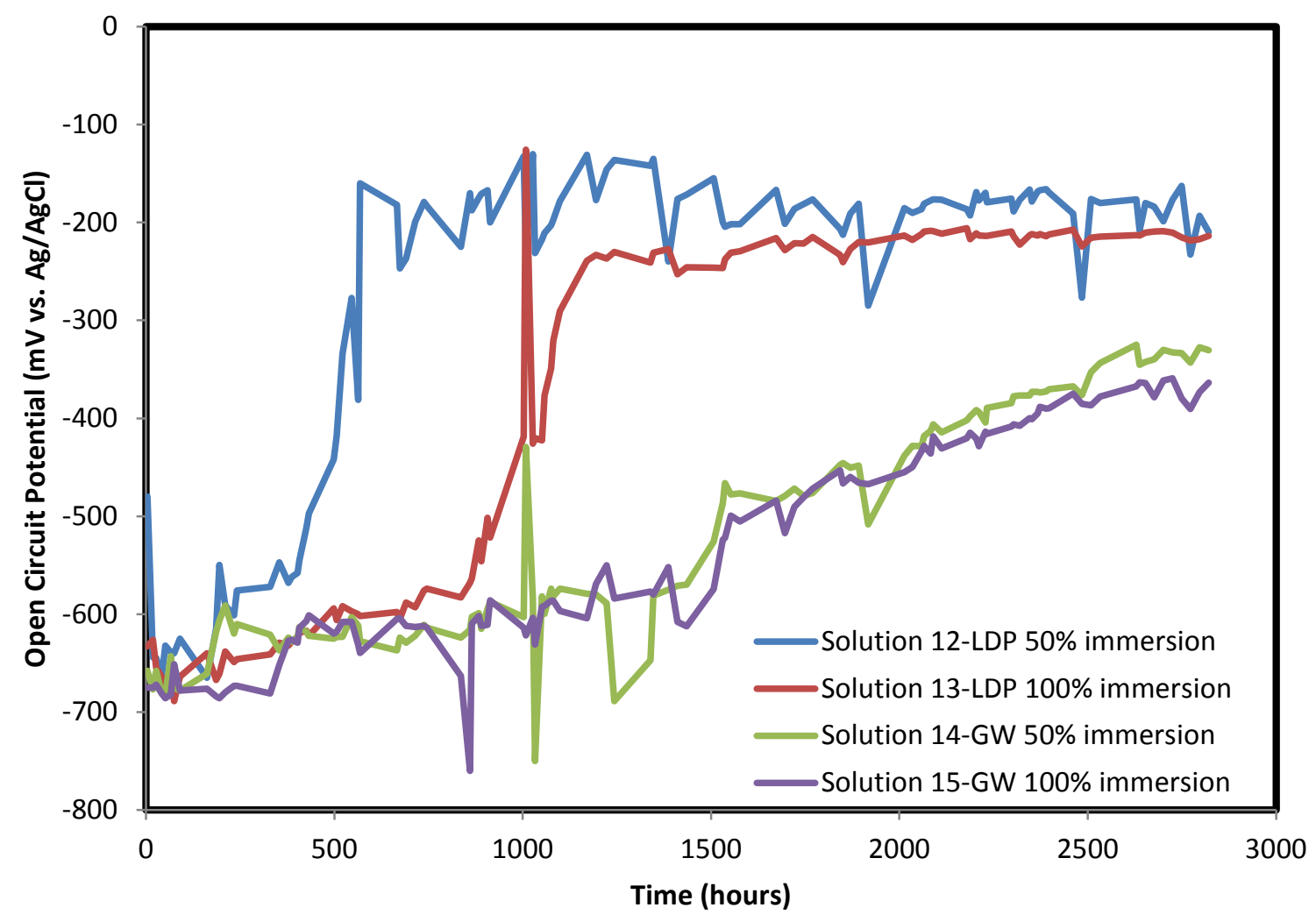

Figure 5-3 OCP vs. time of carbon steel coupons exposed to LDP and GW simulants for four months

Pits sizes were measured utilizing an optical microscope and the results are displayed in Table 52 along with qualitative assessments of the surface. The pits tended to be broad, shallow depressions within an area of general attack. For the samples immersed at 50\% in LDP and GW simulant the pits sizes were the largest at the water line and below with pits increasing in size. Pits were most uniform and with depths ranging from 1 to 2 mils above LAI after 2 months, but increased in size after 4 months of exposure to sizes similar to the pits at the waterline and below. However the attack was not as strong as observed at the waterline and below. For the totally immersed samples general corrosion with broad shallow depressions were observed. 
Table 5-2 Pitting diameter and depth range of solutions from LDP and GW simulants

\begin{tabular}{|c|c|c|c|c|}
\hline Solution & $\begin{array}{c}\text { Time } \\
\text { exposed } \\
\text { (months) }\end{array}$ & $\begin{array}{l}\text { diameter } \\
\text { range } \\
\text { (mils) }\end{array}$ & $\begin{array}{l}\text { depth } \\
\text { range } \\
\text { (mils) }\end{array}$ & Remarks \\
\hline \multirow{2}{*}{$\begin{array}{c}12 \\
(\mathrm{LDP})\end{array}$} & 2 & $1.1-24.2$ & $0.1-3.1$ & $\begin{array}{c}\text { Strong GA at waterline and below. Some areas } \\
\text { with weak GA above the waterline. Large pits } \\
\text { found at water line and decreasing in size from } \\
\text { waterline to below. }\end{array}$ \\
\hline & 4 & $1.8-37.8$ & $1.1-5.0$ & $\begin{array}{l}\text { Strong GA in the entire sample with large pits } \\
\text { found around the waterline and decreasing in size } \\
\text { from waterline to below. }\end{array}$ \\
\hline \multirow{2}{*}{$\begin{array}{c}13 \\
(\mathrm{LDP})\end{array}$} & 2 & $1.6-6.7$ & $0.6-2.6$ & $\begin{array}{c}\text { GA with broad, shallow depressions were } \\
\text { observed in all sample. }\end{array}$ \\
\hline & 4 & $0.9-25.9$ & $0.9-8.6$ & $\begin{array}{c}\text { GA with large pits observed in the entire sample } \\
\text { with very strong attack at the top area. }\end{array}$ \\
\hline \multirow{2}{*}{$\begin{array}{c}14 \\
(\mathrm{GW})\end{array}$} & 2 & $1.9-21.7$ & $0.6-3.6$ & $\begin{array}{c}\text { Strong GA at waterline and below. Some areas } \\
\text { with weak GA above waterline. Large } \\
\text { depressions found at waterline and below }\end{array}$ \\
\hline & 4 & $0.6-19.7$ & $0.5-7.8$ & $\begin{array}{l}\text { Strong GA in } 95 \% \text { of sample with large pits } \\
\text { found around waterline and below }\end{array}$ \\
\hline \multirow{2}{*}{$\begin{array}{c}15 \\
(\mathrm{GW})\end{array}$} & 2 & $1.2-4.1$ & $1.1-1.8$ & $\begin{array}{l}\text { GA with broad, shallow depressions observed in } \\
\text { the entire sample. }\end{array}$ \\
\hline & 4 & $0.6-12.5$ & $0.6-6.0$ & $\begin{array}{c}\text { GA with broad, shallow depressions observed in } \\
\text { the entire sample. }\end{array}$ \\
\hline
\end{tabular}

GA - General Attack

\subsubsection{Vapor space corrosion tests}

Vapor space corrosion testing with the LDP and GW simulants was conducted for four months at the three different levels. A coupon was removed each month from each level. Figure 5-4 and 5-5 shows pictures of the coupons after exposure for Vessel 7 and Vessel 8, respectively. Pictures of the coupons before and after cleaning are presented in Appendix B.

Generally, VS corrosion was more prevalent on the coupons above the GW simulant (Solution 8), than LDP residue simulant (Solution 7). Not surprising, VSC was greater for the samples that experienced the wet/dry cycle. As seen in Figure 5-5, the surface corroded more for the coupons located in Level 1, followed by the coupons located at Level 2 and finally little to no surface corrosion at Level 3. The coupons from LDP did not reflect the same tendency as a similar degree of corrosion was observed at every level (Figure 5-4). Additionally, the degree of attack on the coupons above the LDP simulant did not appear to increase with exposure time. For example, at each level the coupons do not identify a pattern of more VSC as the time progresses from 1 month to 4 months. These results likely reflect the higher concentrations of aggressive species (chloride and sulfate) present in the GW simulant. 
1 month

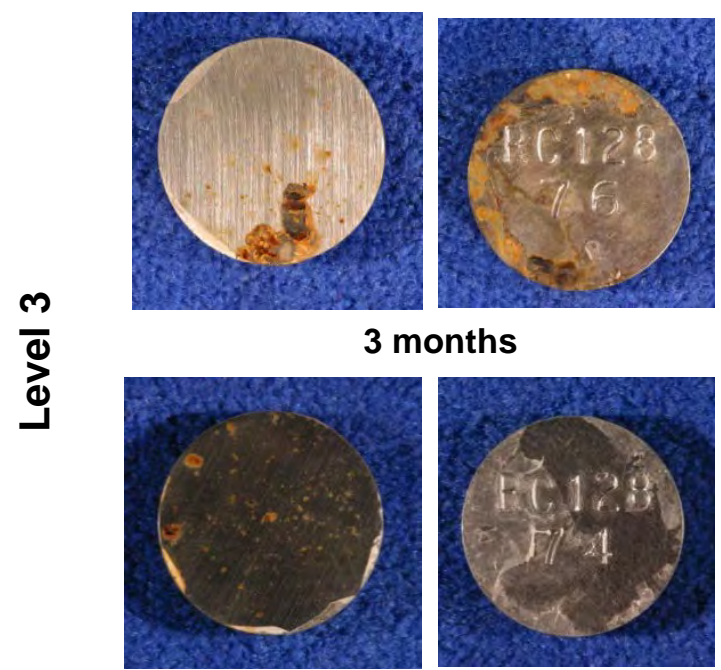

1 month

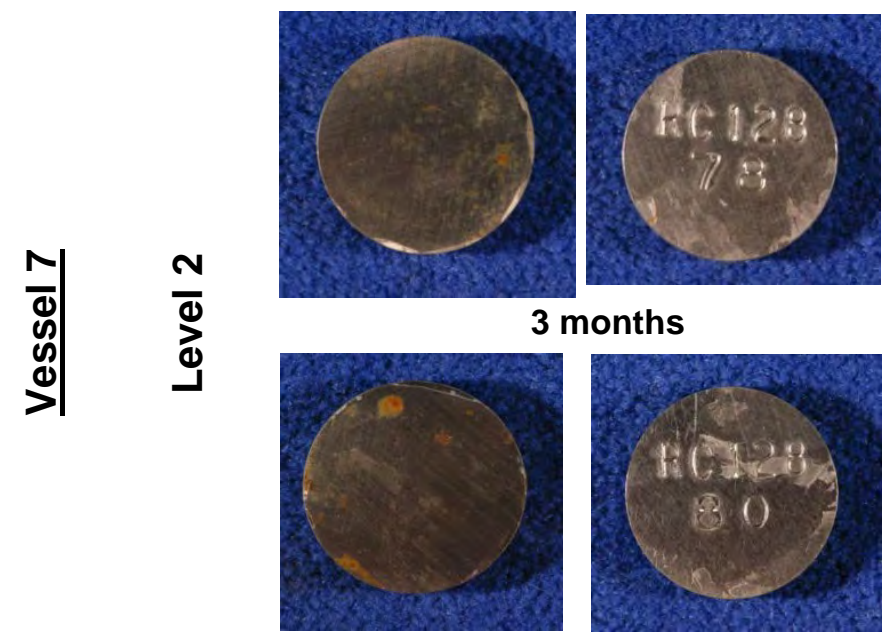

1 month

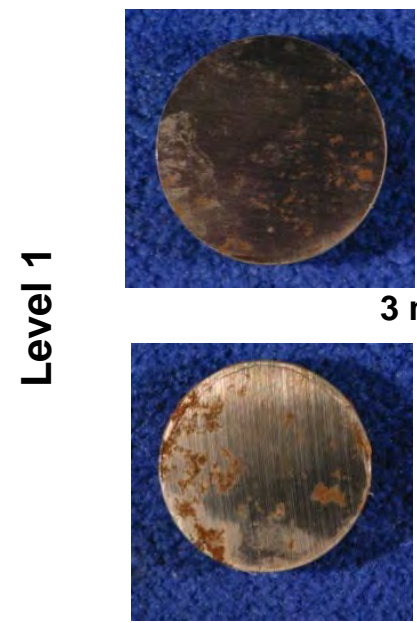

Front

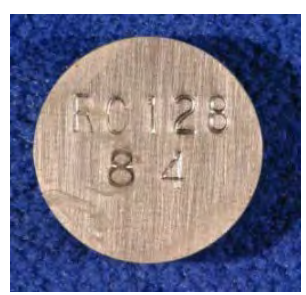

3 months

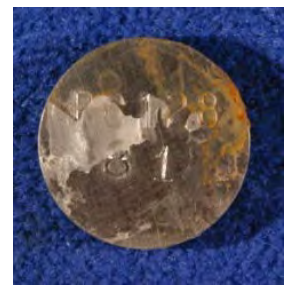

Back

2 months

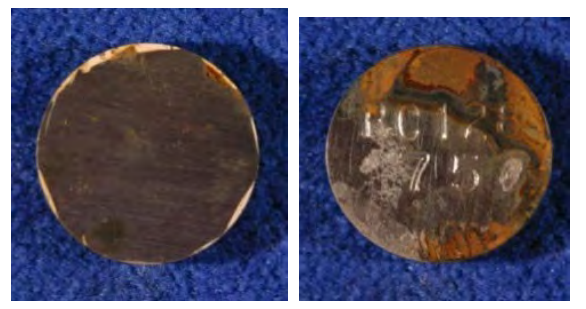

4 months

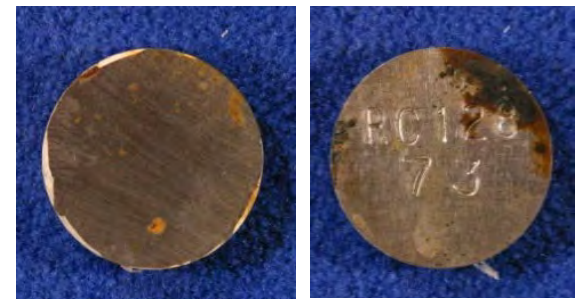

2 months

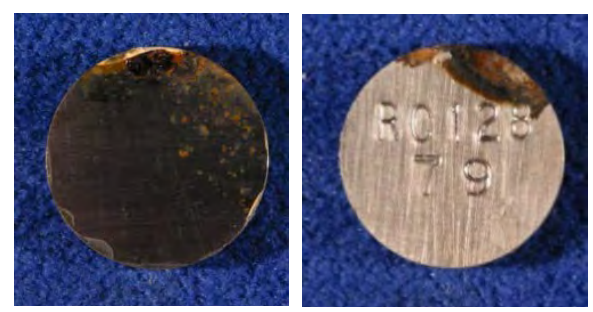

4 months

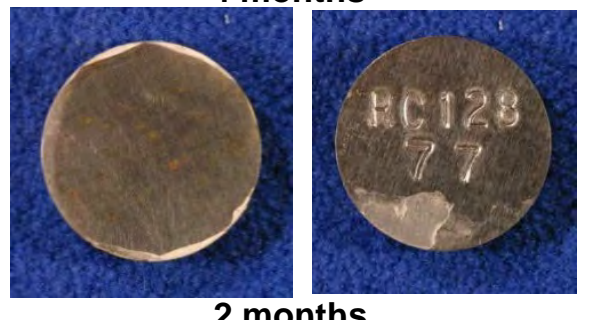

2 months

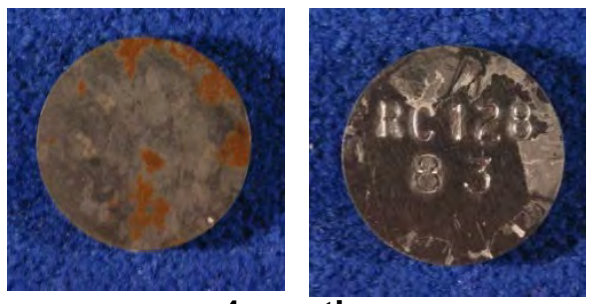

4 months

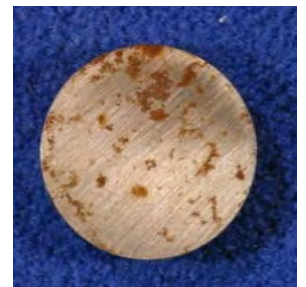

Front

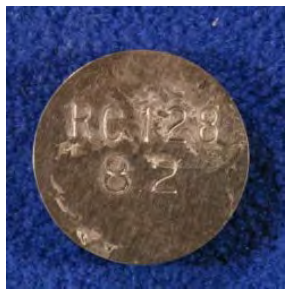

Back

Figure 5-4 Pictures after VS exposure in simulant 7 at three different levels 
1 month

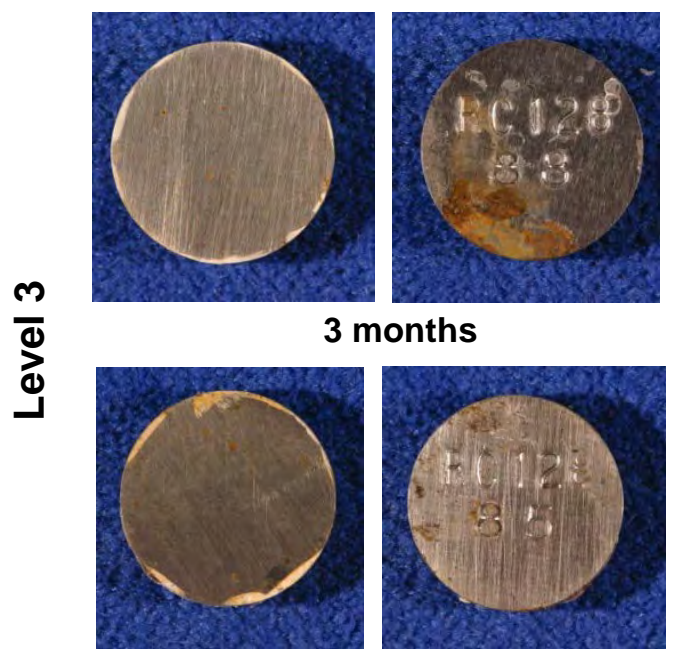

1 month

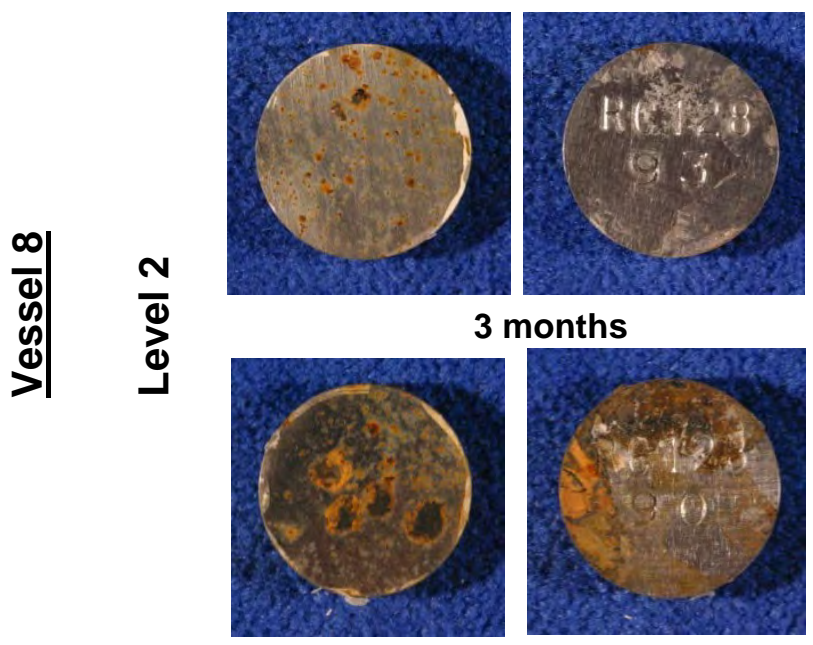

1 month

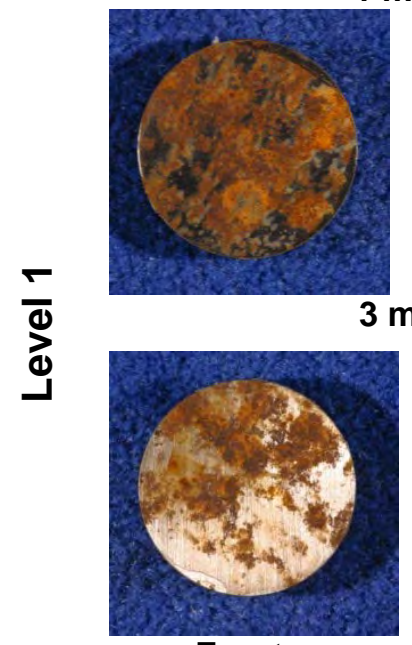

Front

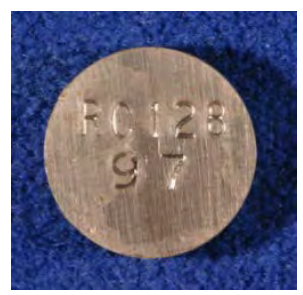

months

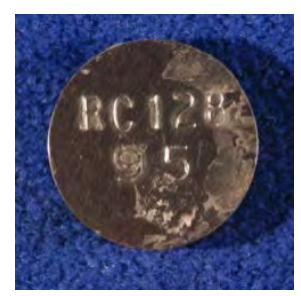

Back
2 months

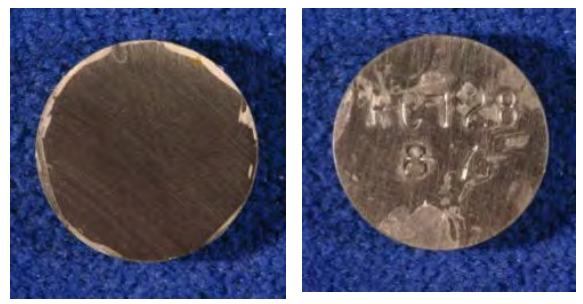

4 months

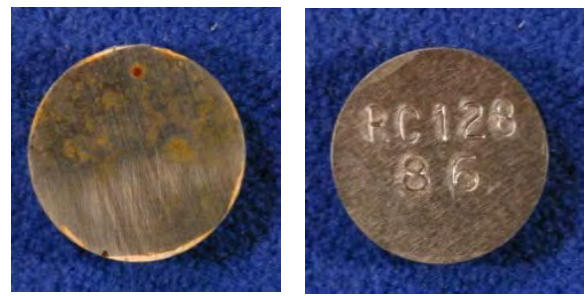

2 months

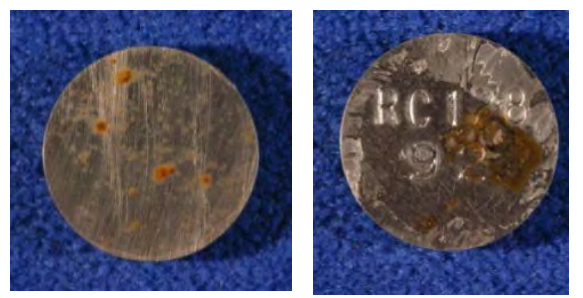

4 months

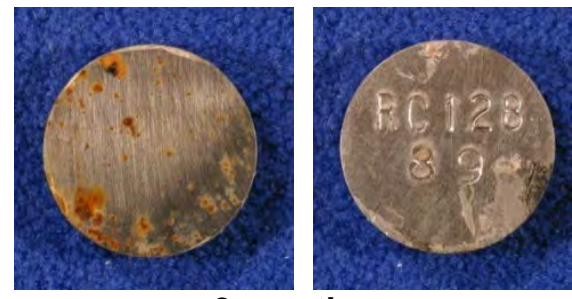

2 months

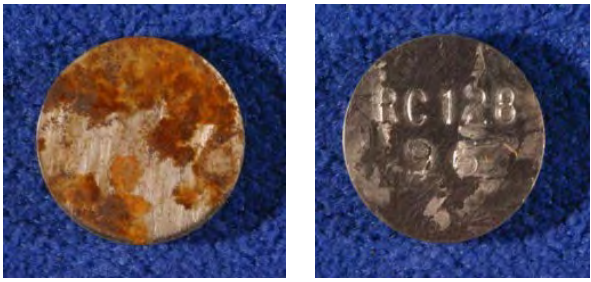

4 months

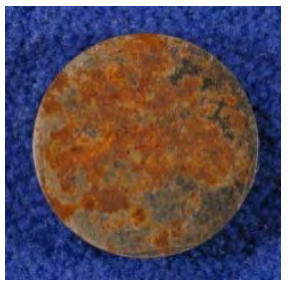

Front

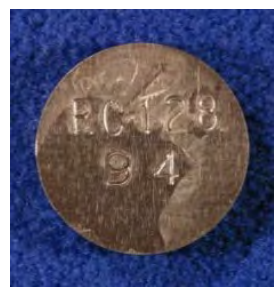

Back

Figure 5-5 Pictures after VS exposure in simulant 8 at three different levels 
An issue encountered after removing the coupons from the cold mount was crevice corrosion. The occurrence of the attack varied as it occurred in some samples more than in others, and was independent of the time that the coupon was subjected to corrosive conditions. It is evident that the nail polish was not as effective as in previous studies. Other techniques for the prevention of crevice corrosion, such as other stronger and more durable coatings, that can withstand humid environments, if cold mounted samples are being used.

Weight losses for the coupons were obtained for each sample and are presented in Table 5-3. As seen the weight losses were not representative of the amount of corrosion seen in each sample. For example, coupons from level 1 of Vessel 8 were observed to have a great amount of corrosion so it is expected high weight losses but after comparing to coupons at level 3 for this vessel that they appear to have minimal corrosion, however, the weight losses are higher. The weight losses to determine the VS corrosion rate were confounded somewhat due to the degree of attack in the crevices that do not reflect VSC. It is recommended than methods to prevent crevice corrosion can be developed and cold mounted preparation to maintain accuracy in weight loss from VSC. However, the visual evidence suggests that vapor space attack would be most significant if the surface of the steel was periodically wetted. 
Table 5-3 Weight losses of coupons at different levels in solutions 7 and 8.

\begin{tabular}{|c|c|c|c|}
\hline $\begin{array}{l}\text { Vessel } \\
\text { number }\end{array}$ & Level in vessel & $\begin{array}{c}\text { Time } \\
\text { exposure } \\
\text { (month) }\end{array}$ & Weight loss (g) \\
\hline \multirow{12}{*}{7} & High (Level 3) & 1 & 0.0174 \\
\hline & High (Level 3) & 2 & 0.0111 \\
\hline & High (Level 3) & 3 & 0.0110 \\
\hline & High (Level 3) & 4 & 0.0286 \\
\hline & Middle (Level 2) & 1 & 0.0109 \\
\hline & Middle (Level 2) & 2 & 0.0062 \\
\hline & Middle (Level 2) & 3 & 0.0061 \\
\hline & Middle (Level 2) & 4 & 0.0020 \\
\hline & Low (Level 1) & 1 & 0.0341 \\
\hline & Low (Level 1) & 2 & 0.0477 \\
\hline & Low (Level 1) & 3 & 0.0045 \\
\hline & Low (Level 1) & 4 & 0.0090 \\
\hline \multirow{12}{*}{8} & High (Level 3) & 1 & 0.0155 \\
\hline & High (Level 3) & 2 & 0.0147 \\
\hline & High (Level 3) & 3 & 0.0439 \\
\hline & High (Level 3) & 4 & 0.0282 \\
\hline & Middle (Level 2) & 1 & 0.0311 \\
\hline & Middle (Level 2) & 2 & 0.0147 \\
\hline & Middle (Level 2) & 3 & 0.0137 \\
\hline & Middle (Level 2) & 4 & 0.0077 \\
\hline & Low (Level 1) & 1 & 0.0101 \\
\hline & Low (Level 1) & 2 & 0.0121 \\
\hline & Low (Level 1) & 3 & 0.0065 \\
\hline & Low (Level 1) & 4 & 0.0049 \\
\hline
\end{tabular}




\subsection{Waste Buffering of simulant from DST AN-102}

Previous electrochemical testing in actual waste from tank 241-AN-102 was performed to determine the susceptibility of carbon steel to corrode [18]. The test results indicated that the carbon steel was not vulnerable to pitting corrosion in the actual waste. The electrochemical testing and LAI long term testing of carbon steel in waste buffering simulants of Hanford DST AN-102 supernate is discussed below.

\subsubsection{Electrochemical Testing}

Using a full simulant based in an actual waste for tank AN-102 with different concentrations of nitrate, nitrite and hydroxide, electrochemical experiments were performed. Table 5-4 shows the tests with molar concentration of nitrate, nitrite and hydroxide. The tests were ordered from the highest hydroxide concentration to the lowest. Tests 1,2 and 3 have similar hydroxide concentration to the actual waste from tank AN-102 $(0.30 \mathrm{M})$. The temperatures of the tests varied from 30 to $50{ }^{\circ} \mathrm{C}$.

Table 5-4 Electrochemical test conditions for waste buffering simulants based from actual waste from tank AN-102

\begin{tabular}{|c|c|c|c|c|c|c|}
\hline Test & $\begin{array}{c}\text { Temperature } \\
\left({ }^{\circ} \mathbf{C}\right)\end{array}$ & $\begin{array}{c}\text { Hydroxide } \\
(\mathbf{M})\end{array}$ & $\begin{array}{c}\text { Nitrite } \\
(\mathbf{M})\end{array}$ & $\begin{array}{c}\text { Nitrate } \\
(\mathbf{M})\end{array}$ & Hysteresis & $\begin{array}{c}\text { Pitting on } \\
\text { Sample? }\end{array}$ \\
\hline 1 & 40 & 0.262 & 1.00 & 3.00 & Negative & None \\
\hline 2 & 50 & 0.262 & 2.00 & 3.00 & Negative & None \\
\hline 3 & 50 & 0.262 & 2.50 & 3.00 & Negative & None \\
\hline 4 & 50 & 0.162 & 1.00 & 3.00 & Negative & None \\
\hline 5 & 30 & 0.072 & 1.95 & 3.11 & Negative & None \\
\hline 6 & 40 & 0.072 & 1.95 & 3.11 & Negative & None \\
\hline 7 & 50 & 0.072 & 1.95 & 3.11 & Negative & None \\
\hline 8 & 50 & 0.062 & 1.94 & 2.90 & Negative & None \\
\hline 9 & 30 & 0.052 & 2.24 & 3.77 & Negative & None \\
\hline 10 & 40 & 0.052 & 2.24 & 3.77 & Negative & None \\
\hline 11 & 50 & 0.052 & 2.24 & 3.77 & Negative & None \\
\hline 12 & 30 & 0.032 & 1.91 & 3.06 & Negative & None \\
\hline 13 & 30 & 0.032 & 2.13 & 3.40 & Negative & None \\
\hline 14 & 40 & 0.032 & 1.91 & 3.06 & Negative & None \\
\hline 15 & 40 & 0.032 & 2.13 & 3.40 & Negative & None \\
\hline 16 & 50 & 0.032 & 1.91 & 3.06 & Negative & None \\
\hline 17 & 50 & 0.032 & 2.13 & 3.40 & Negative & None \\
\hline
\end{tabular}

*Conditions for LAI corrosion testing are highlighted in yellow. 
Table 5-4 contains a summary of the behavior of the CPP curve and a description of the post-test condition of the sample. All the tests in the matrix indicated negative hysteresis and no pitting on the sample. Appendix F shows each of the simulant compositions followed by the CPP curves for the sample and a duplicate test. Post-test pictures of nose and shank at 10X and 20X magnification, respectively, are also exhibited. Figure 5-6 shows CPP curves of sample and duplicate of carbon steel for tests 1,2 and 3. These tests had the maximum hydroxide concentration with a variation in nitrite concentration. Similar curve patterns were observed for Tests 1 and 2 (also shown in Appendix F). The open circuit potential was approximately $-0.600 \mathrm{~V}$ vs. SCE in both cases. This was followed by was an extended passive region for approximately 1 $\mathrm{V}$ before transpassive behavior was observed. Negative hysteresis was observed on the return scan. Almost all of the tests showed a CPP curve as described, except the duplicate of test 16 in which the open circuit was obtained close to $-0.800 \mathrm{~V}$ vs. SCE so the transpassive region started at a lower potential. Tests at lower nitrite and hydroxide concentrations produced CPP curves similar to that shown in Figure 5-7. No significant change in the corrosion behavior was observed.

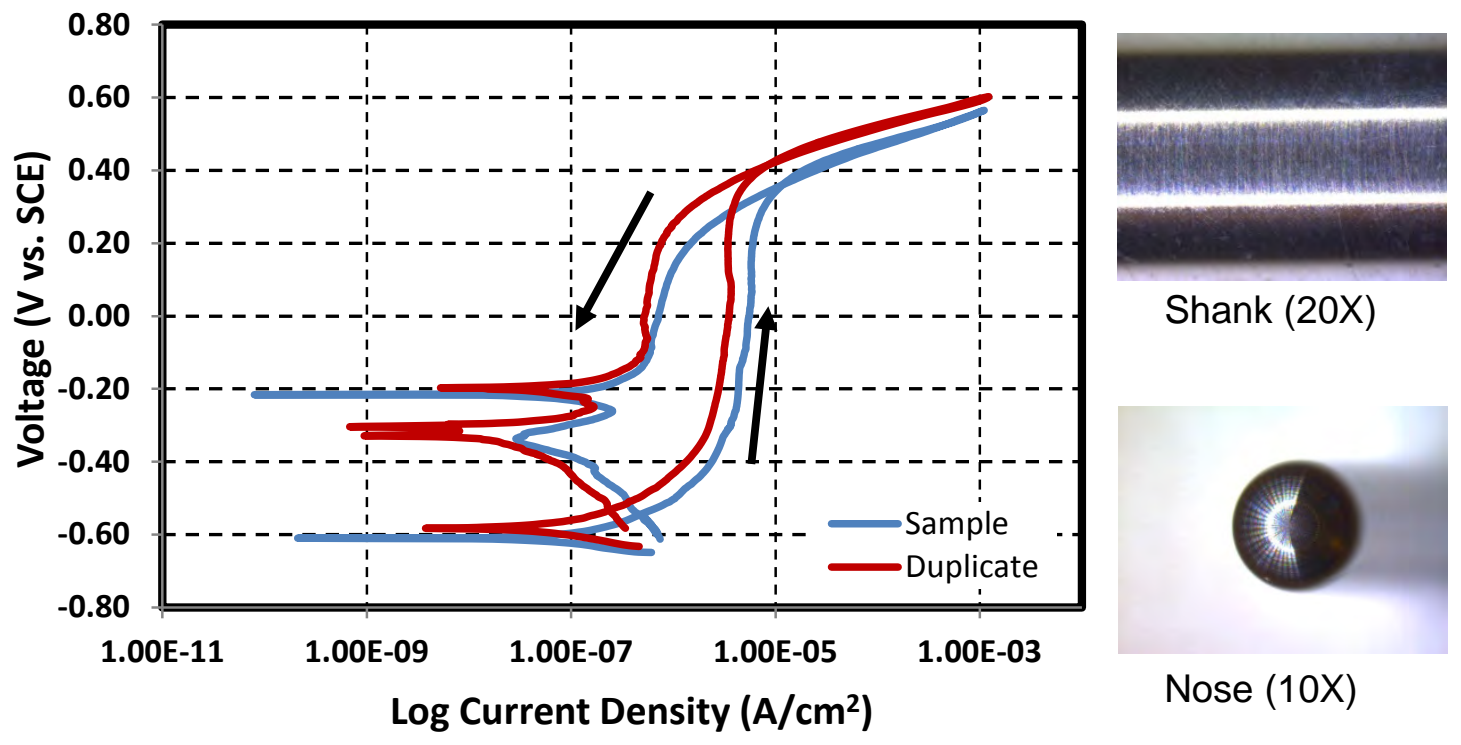

Figure 5-6 Cyclic potentiodynamic polarization scans for sample and duplicate using conditions described in Test 1 


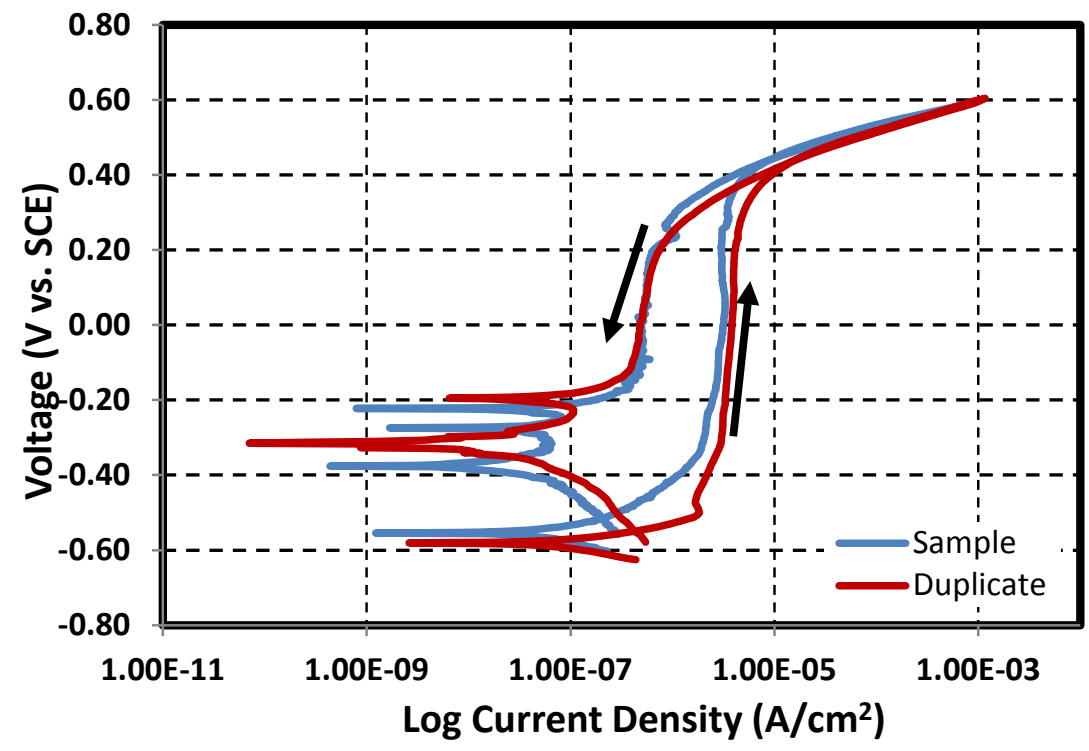

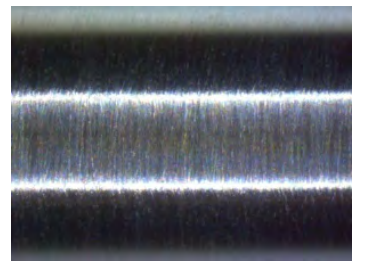

Shank (20X)

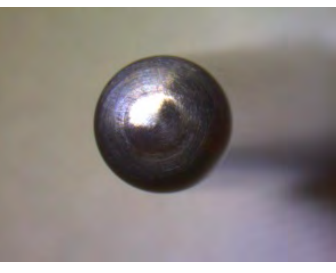

Nose $(10 X)$

Figure 5-7 Cyclic potentiodynamic polarization scans for sample and duplicate using conditions described in Test 4

Tests 5, 6, and 7 had similar nitrate, nitrite and hydroxide concentrations with temperature as the only variable. The CPP results for the highest temperature at $50{ }^{\circ} \mathrm{C}$ (Test 7) are presented in Figure 5-8. The open circuit potential was again approximately $-0.600 \mathrm{~V}$ vs. SCE and the passive region again extended for approximately $1.0 \mathrm{~V}$. The electrode surfaces were again free of attack. Similar curves were obtained for tests 5 and 6 indicating no strong temperature dependence within this range of temperatures.

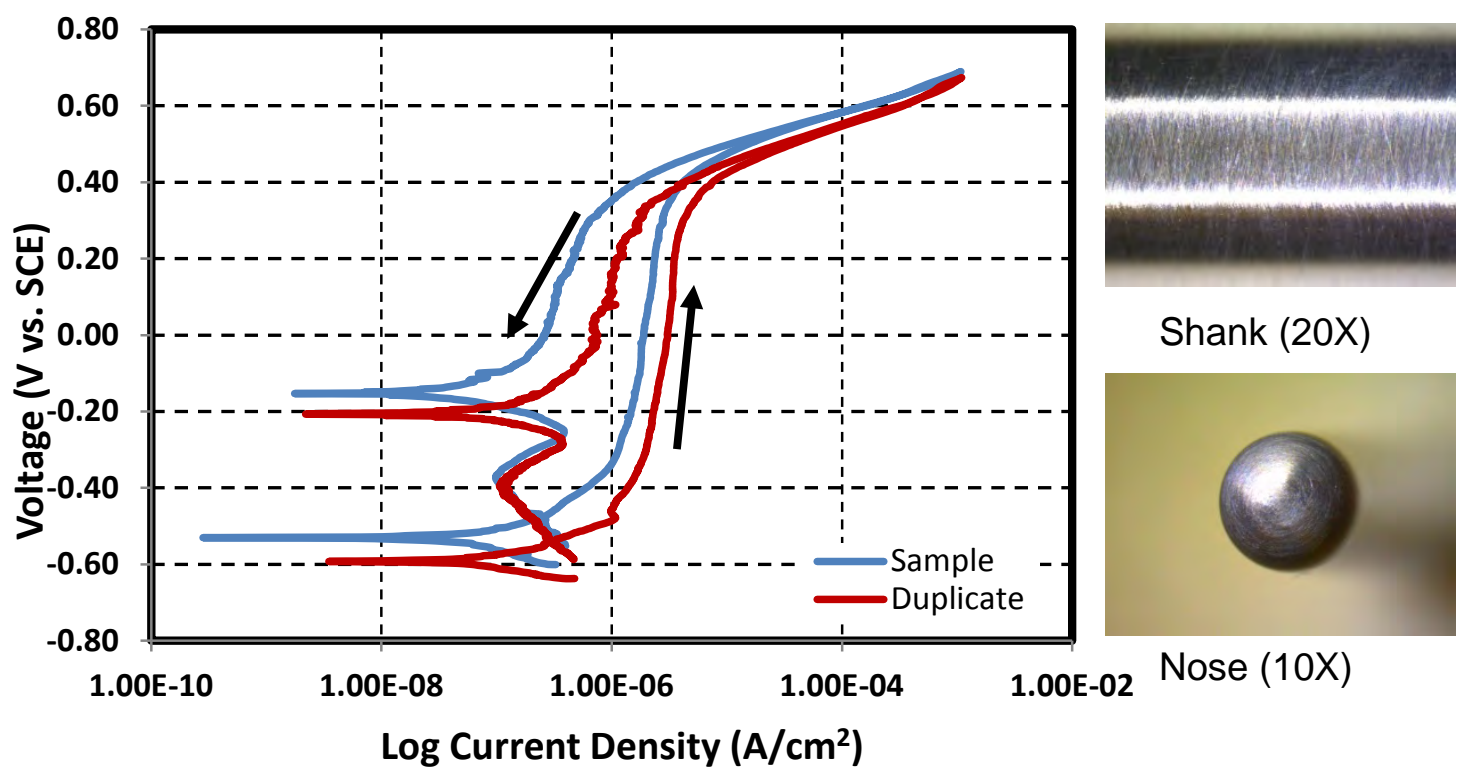

Figure 5-8 Cyclic potentiodynamic polarization scans for sample and duplicate using conditions described in Test 7 
At very low hydroxide concentrations $(0.032 \mathrm{M})$ no evidence of pitting was observed on the electrode as shown in the pictures in Figure 13 for test 17. The CPP curve was similar to the previous cases, but the degree of negative hysteresis was less.

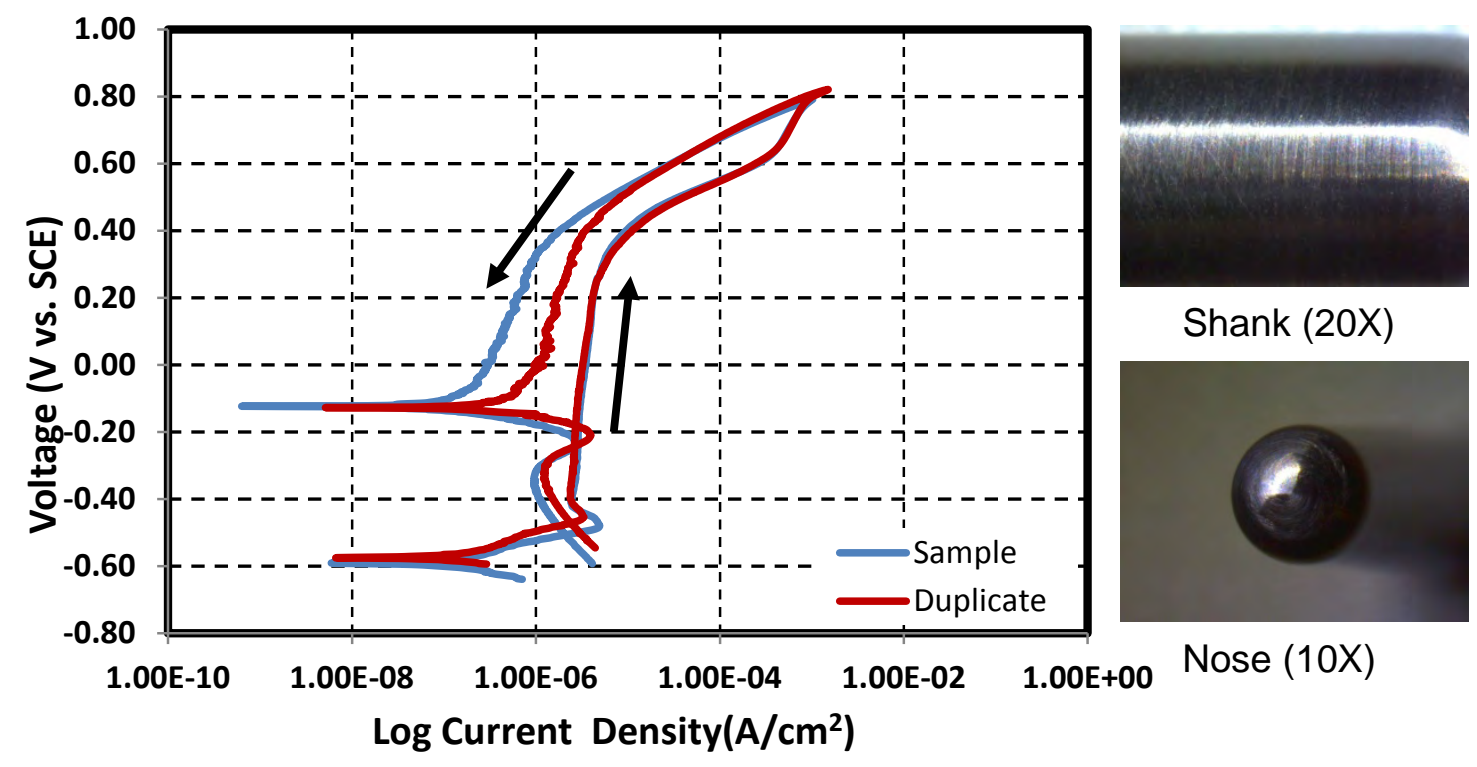

Figure 5-9 Cyclic potentiodynamic polarization scans for sample and duplicate using conditions described in Test 17

The results demonstrated that hydroxide concentrations as low as $0.032 \mathrm{M}$ can still offer inhibition at these high nitrate concentrations provided sufficient nitrite is also present.

\subsubsection{Liquid air interface testing}

LAI corrosion testing was performed for four months for tests 1, 6 and 14 from Table 5-4, which corresponds to solutions 9,10, and 11, respectively. Figure 5-10 shows the pictures of the carbon steel coupons after two and four months of exposure. As seen in the pictures no general corrosion developed on any of the specimens and coupons appeared visually to be independent of the amount of time exposed to the simulant. A salt film developed above the LAI (white residue at the top of coupon) during condensation in this area. The salt film appeared to be thicker for Solutions 9 and 10 than for solution 11. Close to the LAI and below the carbon steel in solution showed no signs of localized corrosion or any corrosion attack. Weight loss results for all the samples showed negligible weight loss indicating no dissolution of the metal during exposure. 
Solution 9

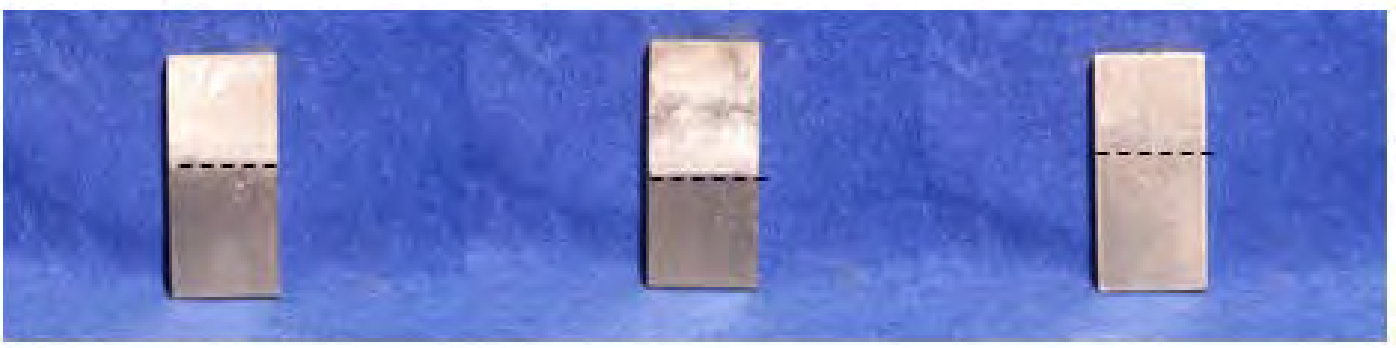

2 months

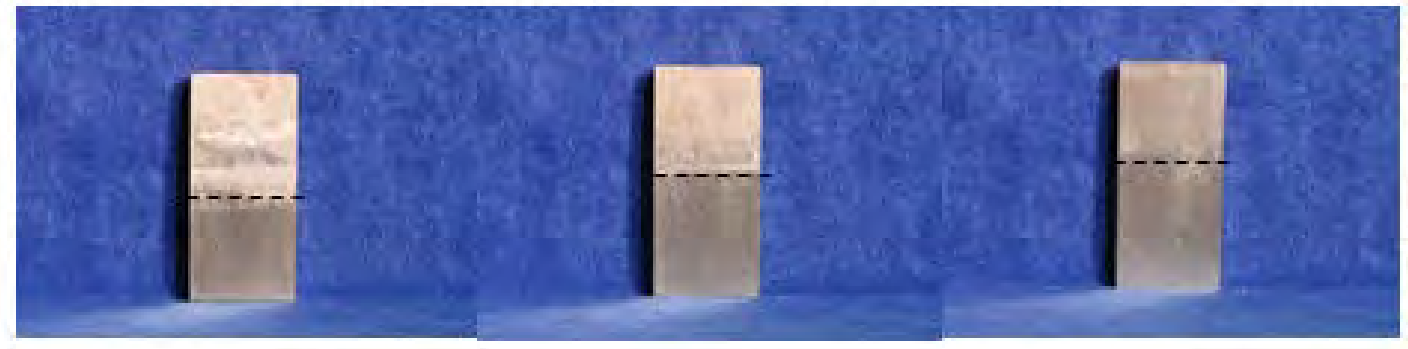

4 months

\section{Solution 11}

Solution 10 
Table 5-5 Pitting diameter and depth range of solutions simulating AN-102 waste

\begin{tabular}{|c|c|c|c|c|}
\hline Solution & $\begin{array}{c}\text { Time } \\
\text { exposed } \\
\text { (month) }\end{array}$ & $\begin{array}{c}\text { diameter } \\
\text { range } \\
\text { (mils) }\end{array}$ & $\begin{array}{c}\text { depth } \\
\text { range } \\
\text { (mils) }\end{array}$ & Remarks \\
\hline \multirow{2}{*}{9} & 2 & $0.6-1.0$ & $0.7-0.9$ & $\begin{array}{c}\text { Salt deposition above LAI with small shallow } \\
\text { pics observed at LAI. }\end{array}$ \\
\cline { 2 - 6 } & 4 & $1.1-4.3$ & $0.6-1.3$ & $\begin{array}{c}\text { Salt deposition above LAI with small shallow } \\
\text { pics observed at LAI. }\end{array}$ \\
\hline \multirow{2}{*}{10} & 2 & $0.5-1.6$ & $0.6-0.7$ & $\begin{array}{c}\text { Salt deposition above LAI with small shallow } \\
\text { pics observed at LAI }\end{array}$ \\
\cline { 2 - 5 } & 4 & $0.7-3.4$ & $0.7-1.1$ & $\begin{array}{c}\text { Salt deposition above LAI with small shallow } \\
\text { pics observed at LAI. }\end{array}$ \\
\cline { 2 - 5 } & 2 & $0.6-1.1$ & $0.5-0.7$ & $\begin{array}{c}\text { Salt deposition above LAI with minor } \\
\text { deposition below LAI. Small shallow pics } \\
\text { observed above and at LAI. }\end{array}$ \\
\cline { 2 - 5 } & 4 & $0.6-2.1$ & $0.3-0.8$ & $\begin{array}{c}\text { Salt deposition above LAI with minor } \\
\text { deposition below LAI. Small shallow pics } \\
\text { observed above and at LAI. }\end{array}$ \\
\hline
\end{tabular}

The OCP was measured during the LAI testing to examine trends in open circuit potential that can identify periods of active corrosion and passivation. Figure 5-11 shows an OCP vs. time curve for Solutions 9, 10 and 11. The general trend shows that below 100 hours the OCP increases as the oxide film grows and become stable. The long term stability of the OCP suggests that the steel surface is passive and minimal corrosion is occurring. 


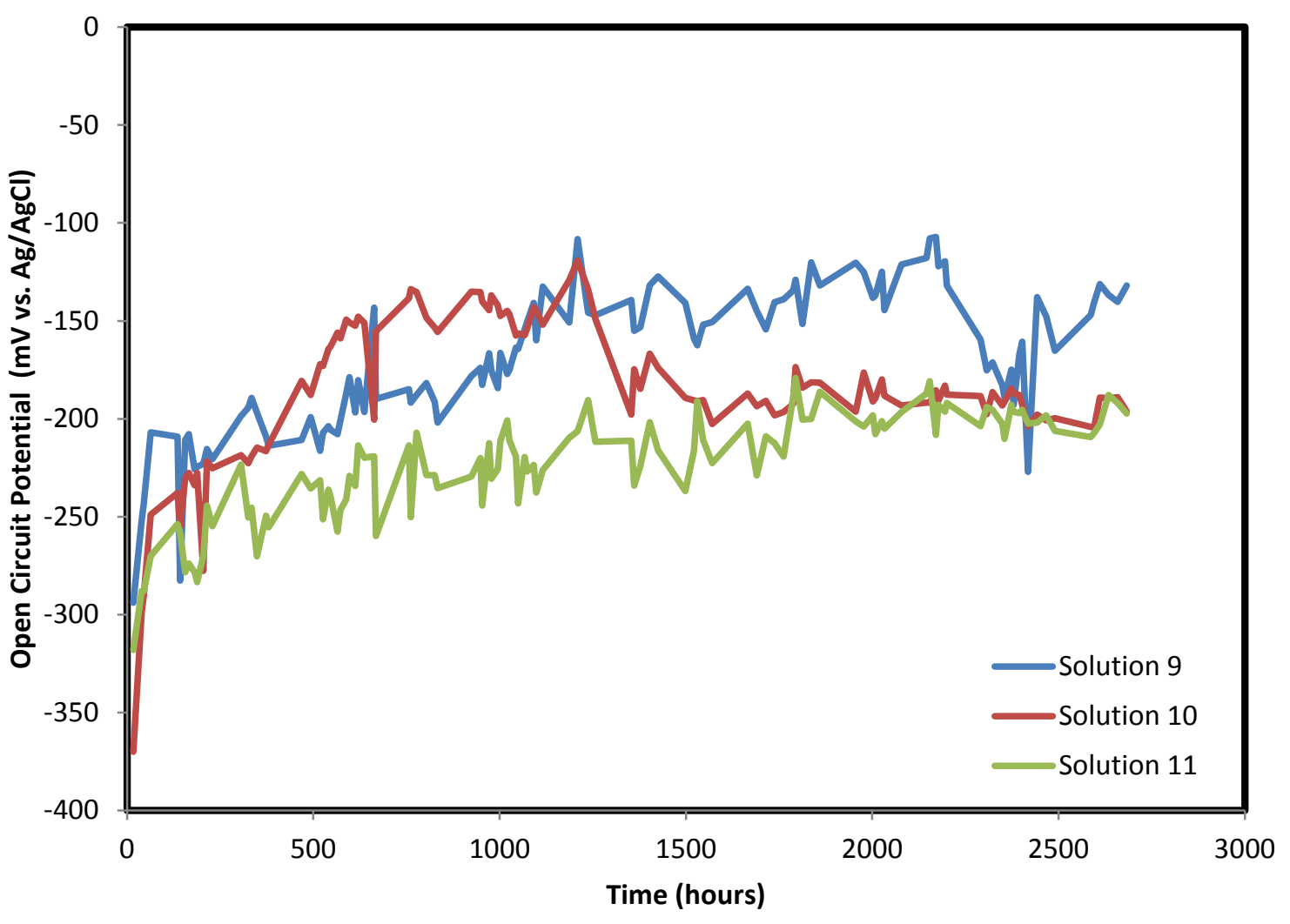

Figure 5-11 OCP measurements at different periods during four month testing of LAI for Solutions 9, 10, 11.

\section{3 $\underline{\text { Vapor Space Corrosion tests at new SCC limits at different ammonia concentrations }}$}

Three different simulants, at the borderline of the new SCC corrosion limits were utilized for VSC tests. The specific limit of testing is the minimum nitrite/nitrate ratio of 0.15 and the nitrate concentrations that were tested were 0.4, 2 and 4.5 M. Previously, it was found that these concentrations of nitrate were insufficient to prevent pitting in the vapor space [7]. Ammonia gas at two concentrations (50 and 550ppm) was selected based on previous results that indicated that $550 \mathrm{ppm}$ mitigates vapor space corrosion for a simulant with a high nitrate concentration [7].

Coupon tests were conducted for four months in each environment with coupons being removed on monthly intervals. Figures 5-12 and 5-13 correspond to fourth month testing at $50 \mathrm{ppm}$ ammonia (Vessels 1,2 and 3) and 550ppm ammonia (Vessels 4, 5 and 6), respectively. The complete arrays of images for all coupons are shown in Appendix B. At $550 \mathrm{ppm}$ of ammonia in the VS (Figure 5-12), the front surface for all of the simulants indicated no sign of VSC at Levels 3 and 2 with very minor general corrosion regions at Level 1. Even at $50 \mathrm{ppm}$ (Figure 5-13), there was no appararent VSC at the surface for Levels 3 and 2. Al Level 1 for the samples exposed at $50 \mathrm{ppm}$ Ammonia, minor corrosion can be observed around the edges of the circular coupon. However, is still falls below of what was previously seen in previous experiments [7]. This confirms that as little as $50 \mathrm{ppm}$ of ammonia can be effective to inhibit VSC of carbon steel at wastes covered by the new SCC control specifications. 
When the back of the coupons were examined, evidence of crevice corrosion was observed. As explained in section 5.1.2, the occurrence of crevice attack was variable and did not depend on the exposure time or the height above the liquid level. The nail polish, utilized to prevent crevice attack, appeared to degrade as it turned from clear to white in most cases. Other prevention methods for crevice corrosion should be utilized in the future. An interesting observation of the corrosion on the back of the coupons is that it appears that this type of corrosion affected more coupons subjected at $550 \mathrm{ppm}$ than $50 \mathrm{ppm}$ of ammonia.

The weight losses for coupons were not shown in the report because they were not representative of the VSC on each sample due to the crevice attack. Moreover, the weight losses for VSC in this case are essentially not needed since very minor or almost no corrosion was observed on the exposed surface of the coupon. 

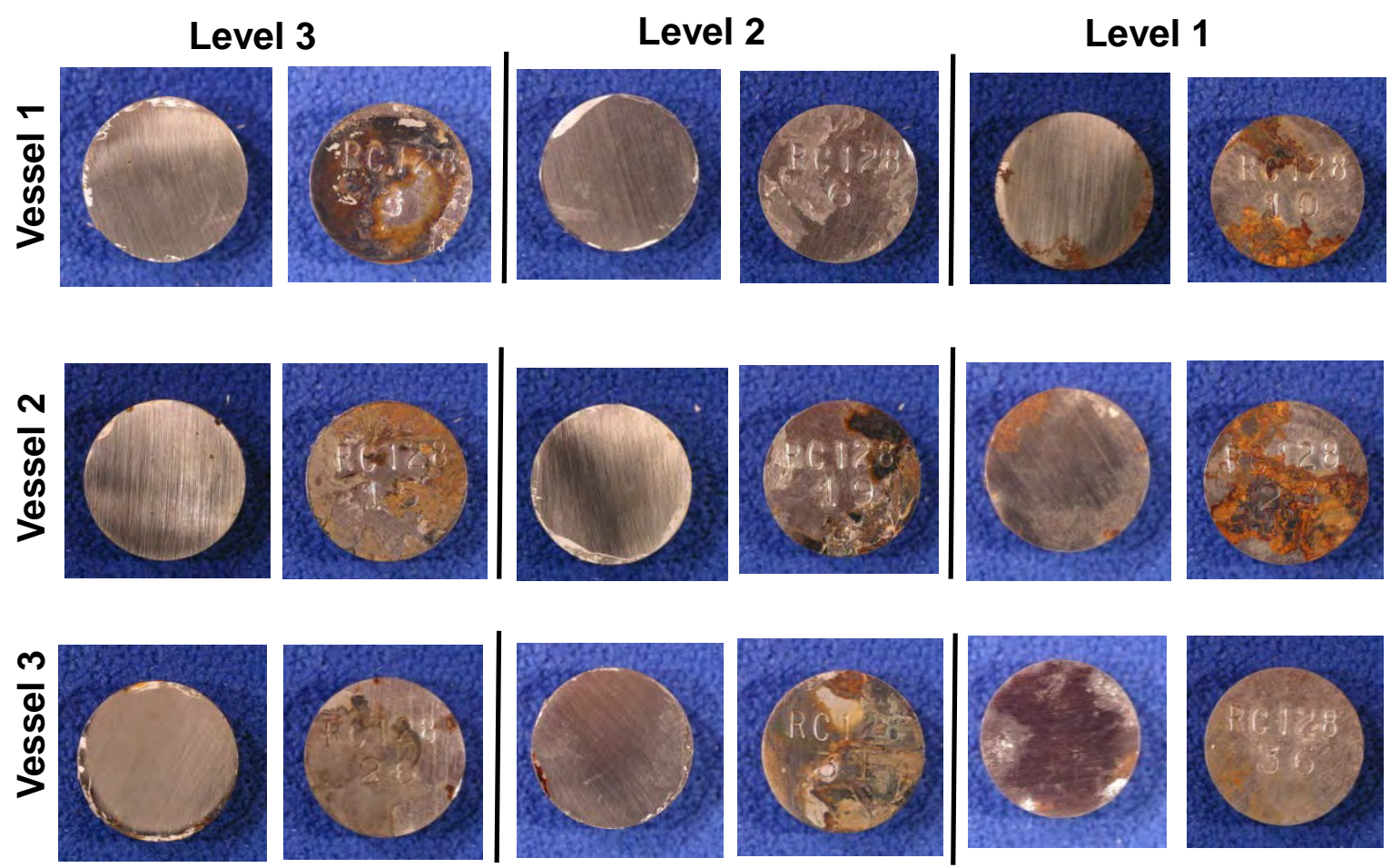

Front

Back

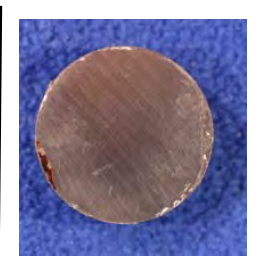

Front

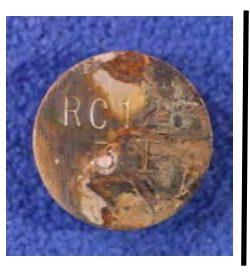

Back

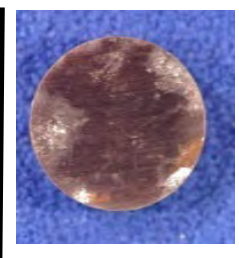

Front

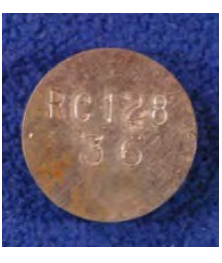

Back

Figure 5-12 Fourth-month exposure at VS conditions of carbon steel in Vessels 1, 2 and 3 at 50 ppm Ammonia 

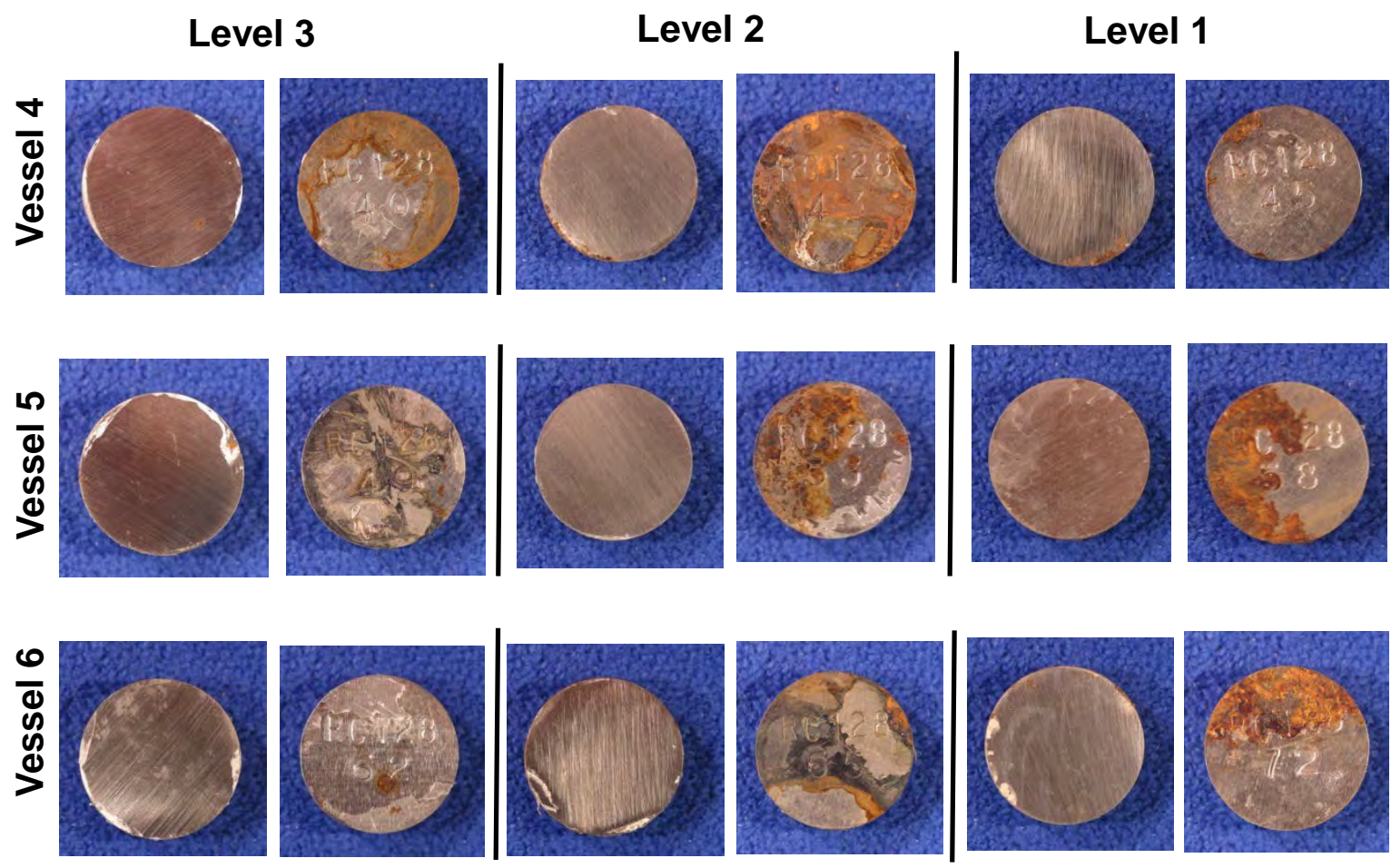

Front

Back

Front

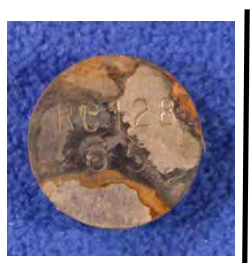

Back

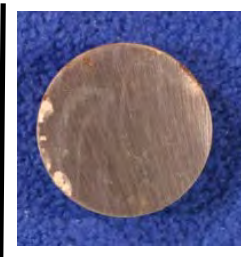

Front

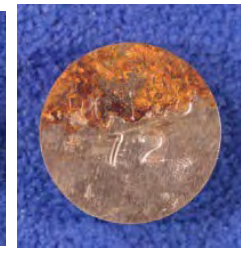

Back

Figure 5-13 Fourth-month exposure at VS conditions of carbon steel in Vessels 4, 5 and 6 at 550 ppm Ammonia 


\subsection{Liquid Air Interface tests at new SCC limits}

For these tests, the ratio of nitrite to nitrate was varied shown in Table 4-3. The solution chemistries for the eight tests are shown in Appendix C. Coupons were exposed for two months and four months using the LAI setup.

Figure 5-14 and 5-15 shows the pictures for carbon steel coupons in solutions 1-4 and 5-8, respectively. None of these coupons exhibited significant corrosion attack at LAI or below. This confirms previous results obtained from Zapp [27] and Hoffman [28] that observed no pitting corrosion at the conditions outline in Table 4-3. Varying degrees of attack were observed in the VS above the liquid level, with the worst case being solution 3 . This condition was at a relatively low nitrate concentration $(0.5 \mathrm{M})$ and at the minimum nitrite/nitrate ratio for the new SCC limits (0.15). The coupons exposed to solution 7 appeared almost exactly as they did prior to test. This solution had a higher nitrate concentration and the highest nitrite/nitrate ratio (1.66). 

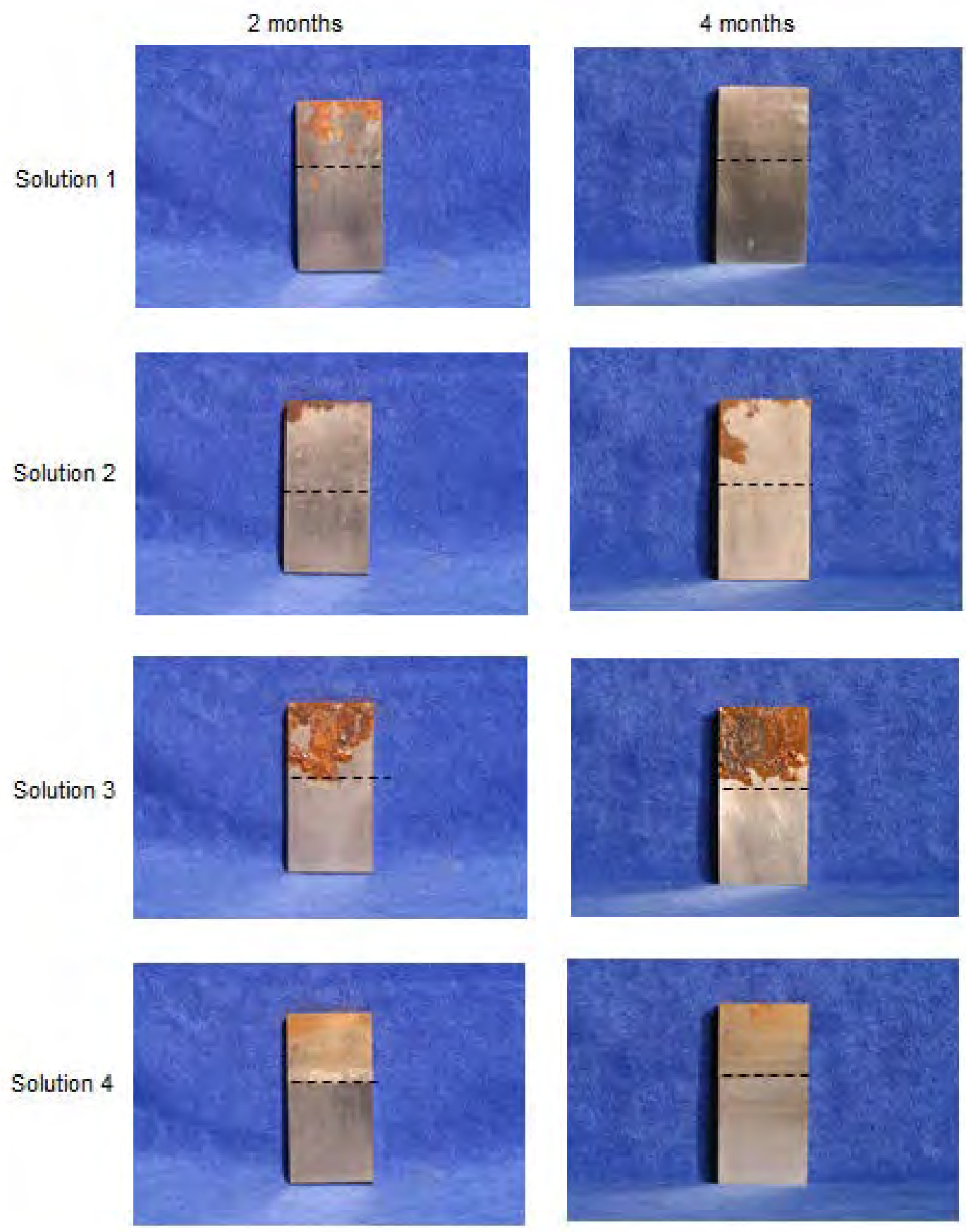

Figure 5-14 Pictures of coupons after two months and four months exposure during LAI testing for solutions $1,2,3$ and 4 . The dash line represents the interface. 

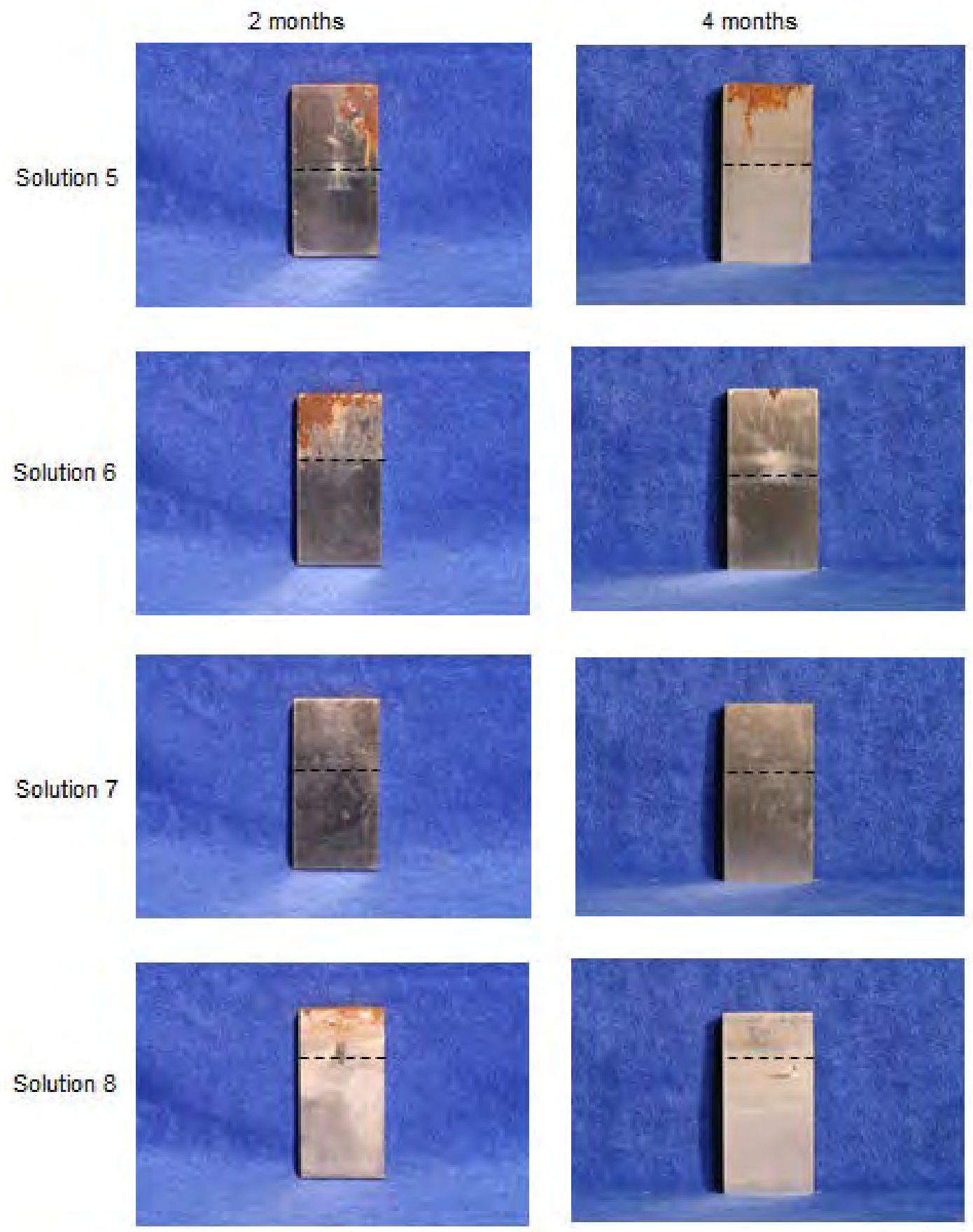

Figure 5-15 Pictures of coupons after two months and four months exposure during LAI testing for solutions $5,6,7$ and 8 . The dash line represents the interface. 
SRNL-STI-2014-00616

Revision 0

Table 5-6 Weight losses and corrosion rates for carbon steel coupons exposed to Solutions 1 to 8 for 2 and 4 months

\begin{tabular}{|c|c|c|c|c|}
\hline \multirow{2}{*}{ Solutions } & \multicolumn{4}{|c|}{ Months Exposure } \\
\cline { 2 - 5 } & \multicolumn{2}{|c|}{ Two months } & \multicolumn{2}{c|}{ Four months } \\
\cline { 2 - 5 } & weight loss (g) & $\begin{array}{c}\text { Corrosion rate } \\
(\mathbf{m} \text { py) }\end{array}$ & weight loss (g) & $\begin{array}{c}\text { Corrosion rate } \\
\text { (mpy) }\end{array}$ \\
\hline 1 & 0.0329 & 0.30 & 0.0000 & 0.00 \\
\hline 2 & 0.0386 & 0.36 & 0.0180 & 0.08 \\
\hline 3 & 0.0988 & 0.91 & 0.1723 & 0.79 \\
\hline 4 & 0.0166 & 0.15 & 0.0003 & 0.00 \\
\hline 5 & 0.0282 & 0.26 & 0.0041 & 0.02 \\
\hline 6 & 0.0097 & 0.09 & 0.0022 & 0.01 \\
\hline 7 & 0.0028 & 0.03 & 0.0002 & 0.00 \\
\hline 8 & 0.0000 & 0.00 & 0.0002 & 0.00 \\
\hline
\end{tabular}

Weight losses after two months and four months are presented in Table 5-6 and confirm corrosion rates are less than 1 mpy and in some cases close to $0 \mathrm{mpy}$. The highest corrosion rate occurred in carbon steel coupons exposed to solution 3. All the corrosion on this sample occurred in the VS. Typically any corrosion seemed to initiate at the top of the coupon and develop an area of general corrosion and that continues to spread above the LAI. The smallest ratio of nitrite to nitrite $(0.12)$ was solution 8 and during the contact with the carbon steel coupon it developed a film that completely covered the coupon and protected it from corrosion attack even though the ratio is less than the new minimum SCC control limits. The corrosion appeared to be more severe for the more dilute solutions at a given nitrite/nitrate ratio.

OCP measurements for solutions 1 to 8 are presented in Figure 5-16. The OCP for the solutions in general gradually increased from an initial value to a relatively constant value after 2-3 days. The potential transient does oscillate slightly, which indicates some changes in the oxide film. For most of the tests, there was a period of OCP instability during four months indicated active corrosion for short periods of time. The most negative OCP was for carbon steel in contact with solution 3 and 4. Solutions 3, and 5 exhibited the most activity (i.e., the largest variance in OCP values) during the course of the test. For the carbon steel exposed in solution 7 the OCP was the most stable during the fourth month period and indicates a stable oxide condition in that particular solution. 


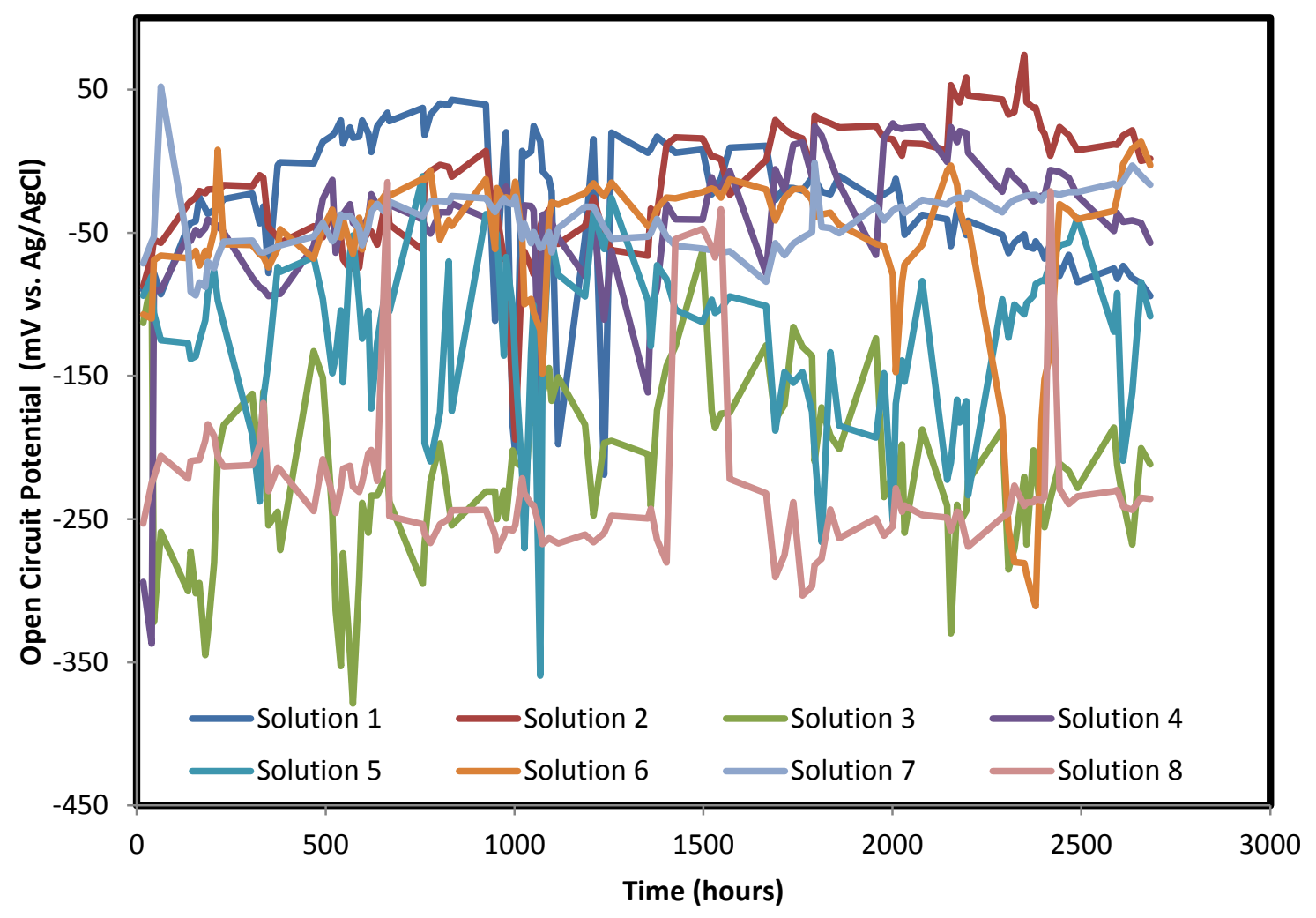

Figure 5-16 OCP measurements at different periods during four month testing of LAI for Solutions 1 to 8.

The pit diameter and depth for each of the coupons are summarized in Table 5-7. The qualitative assessments of the sample are also listed in the table. Small, broad, shallow pits were observed on most of the coupons and were located inside the areas of general attack. The largest and deepest pits were observed on coupons exposed to solution 3. The range of the pit diameter was 0.5-12.3 mils, while the range for the pit depths was 0.4-4.5 mils. As in other cases, it appears that the pits do not get deeper during time but spread and increase the general attack area. There were some special cases that less general corrosion was observed for the four month exposure than the two month exposure (Solution 1 and 6) which tends to indicate a borderline condition. Weak LAI corrosion attack was observed on coupons exposed to solutions 4 and 8 . Pits were present in this area, but were no larger than the pit sizes found above the interface. The ranking observed for corrosion of these samples based on the degree and amount of corrosion attack from higher to lower was $3,2,1,5,6,4,8$ and 7 . 
Table 5-7 Pitting diameter and depth range of solutions using the new SCC limits. Percentages were obtained by a qualitative assesment.

\begin{tabular}{|c|c|c|c|c|}
\hline Solution & $\begin{array}{c}\text { Time } \\
\text { exposed } \\
\text { (months) }\end{array}$ & $\begin{array}{l}\text { diameter } \\
\text { range } \\
\text { (mils) }\end{array}$ & $\begin{array}{l}\text { depth } \\
\text { range } \\
\text { (mils) }\end{array}$ & Remarks \\
\hline \multirow{2}{*}{1} & 2 & $0.6-8.1$ & $0.7-2.7$ & $\begin{array}{c}\text { GA with small pits observed above LAI on top area. } \\
\text { Less than } 20 \% \text { corrosion. No LAI corrosion } \\
\text { observed. }\end{array}$ \\
\hline & 4 & $0.8-3.4$ & $0.2-1.6$ & $\begin{array}{c}\text { GA observed in less than } 2 \% \text { of coupon. Very small } \\
\text { pits observed above LAI. No LAI corrosion } \\
\text { observed. }\end{array}$ \\
\hline \multirow{2}{*}{2} & 2 & $0.5-3.8$ & $1.2-2.7$ & $\begin{array}{c}\text { GA with small pits observed on top area of coupon. } \\
\text { Less than } 10 \% \text { corrosion. No LAI corrosion } \\
\text { observed. }\end{array}$ \\
\hline & 4 & $0.3-1.9$ & $0.4-0.9$ & $\begin{array}{c}\text { GA with small pits observed above LAI on top area. } \\
\text { Less than } 20 \% \text { corrosion. No LAI corrosion } \\
\text { observed. }\end{array}$ \\
\hline \multirow{2}{*}{3} & 2 & $1.0-11.7$ & $0.4-3.9$ & $\begin{array}{l}\text { Large GA with pits observed above LAI on top area. } \\
\text { Around } 30 \% \text { to } 40 \% \text { corrosion. No distinctive LAI } \\
\text { corrosion observed. }\end{array}$ \\
\hline & 4 & $0.5-12.3$ & $0.6-4.5$ & $\begin{array}{l}\text { Large GA with pits observed above LAI on top area. } \\
\text { Around } 40 \% \text { corrosion. No distinctive LAI corrosion } \\
\text { observed. }\end{array}$ \\
\hline \multirow{2}{*}{4} & 2 & $0.7-7.3$ & $0.8-1.1$ & $\begin{array}{c}\text { GA with shallow pits above LAI. Weak LAI } \\
\text { corrosion attack. }\end{array}$ \\
\hline & 4 & $0.8-5.3$ & $0.5-0.9$ & $\begin{array}{c}\text { GA with shallow pits above LAI. Weak LAI } \\
\text { corrosion attack. }\end{array}$ \\
\hline \multirow{2}{*}{5} & 2 & $0.1-3.6$ & $0.7-1.8$ & $\begin{array}{c}\text { GA with small pits observed above LAI on top area. } \\
\text { Less than } 10 \% \text { corrosion. No LAI corrosion } \\
\text { observed. }\end{array}$ \\
\hline & 4 & $0.7-7.6$ & $0.7-1.5$ & $\begin{array}{l}\text { GA with small pits observed above LAI on top area. } \\
\text { Less than } 10 \% \text { corrosion. No LAI corrosion } \\
\text { observed. GA small area below LAI. }\end{array}$ \\
\hline \multirow[t]{2}{*}{6} & 2 & $0.7-3.7$ & $0.9-1.8$ & $\begin{array}{c}\text { GA with shallow pits observed on top area of } \\
\text { coupon. Less than } 20 \% \text { corrosion. No LAI corrosion } \\
\text { observed. }\end{array}$ \\
\hline & 4 & $1.2-5.6$ & $1.1-1.8$ & $\begin{array}{l}\text { GA with small pits observed above LAI on top area. } \\
\text { Less than } 4 \% \text { corrosion. No LAI corrosion observed. }\end{array}$ \\
\hline \multirow{2}{*}{7} & 4 & $0.9-2.2$ & $0.5-1.0$ & $\begin{array}{l}\text { Very small pits were observed above LAI. No LAI } \\
\text { corrosion observed. }\end{array}$ \\
\hline & 2 & $0.9-2.9$ & $1.0-1.5$ & $\begin{array}{c}\text { Very small pits were observed above LAI. No LAI } \\
\text { corrosion observed. }\end{array}$ \\
\hline \multirow{2}{*}{8} & 2 & $0.8-1.4$ & $0.6-1.1$ & $\begin{array}{l}\text { GA with shallow pits observed above LAI on top } \\
\text { area. Salt deposition below and above LAI. Weak } \\
\text { LAI corrosion observed. }\end{array}$ \\
\hline & 4 & $0.4-5.2$ & $0.5-1.4$ & $\begin{array}{l}\text { GA with shallow pits observed above LAI on top } \\
\text { area. Salt deposition below and above LAI. Weak } \\
\text { LAI corrosion observed. }\end{array}$ \\
\hline
\end{tabular}


SRNL-STI-2014-00616

Revision 0

\subsection{Pitting Corrosion studies using the standardized CPP protocol}

The CPP technique has been utilized for over 30 years in the DOE weapons complex as a means for evaluating the pitting susceptibility of waste tank materials (i.e., carbon steel) exposed to the waste environments. The results of these tests, coupled with long-term (i.e., 4 month) coupon immersion testing, have provided a basis for corrosion control requirements for pitting corrosion at both the SRS and the Hanford site (Corrosion Control Program). These tests were performed over a wide range of environmental conditions (i.e., composition and temperature) and at several different laboratories (e.g., SRNL, DNV-GL, WRPS, etc.).

During FY13, DOE requested that WRPS demonstrate that the outcome of the tests were reproducible irrespective of the laboratory that performed the test. As a result, a round robin test program between three laboratories was conducted [2]. Prior to initiating the round robin, a review of the CPP techniques at each of the laboratories was performed. Differences between the laboratories were noted for test set-up and CPP parameters (e.g., sample geometry, sample preparation, pre-test exposure, threshold current, etc.). The outcomes although similar were different, particularly at environmental conditions where the response transitions from "pitting" to "no-pitting". The round robin program eventually led to the development of a standardized CPP protocol [2]. This standardized CPP protocol was utilized will be utilized for all future testing related to the Hanford DST.

A correlation between previous CPP test data (i.e., prior to the standardized CPP protocol development), and data gathered utilizing the standardized CPP protocol was performed.

\subsubsection{Interpretation of CPP Test Results}

During 2014, the EPOC also standardized an approach for interpreting the results of the CPP tests [31]. Important aspects of this approach are summarized as a reference here since they will be utilized in the discussion of the results. Figure 5-17 shows a schematic of an idealized CPP curve along with experimental parameters that are measured from the curve. 


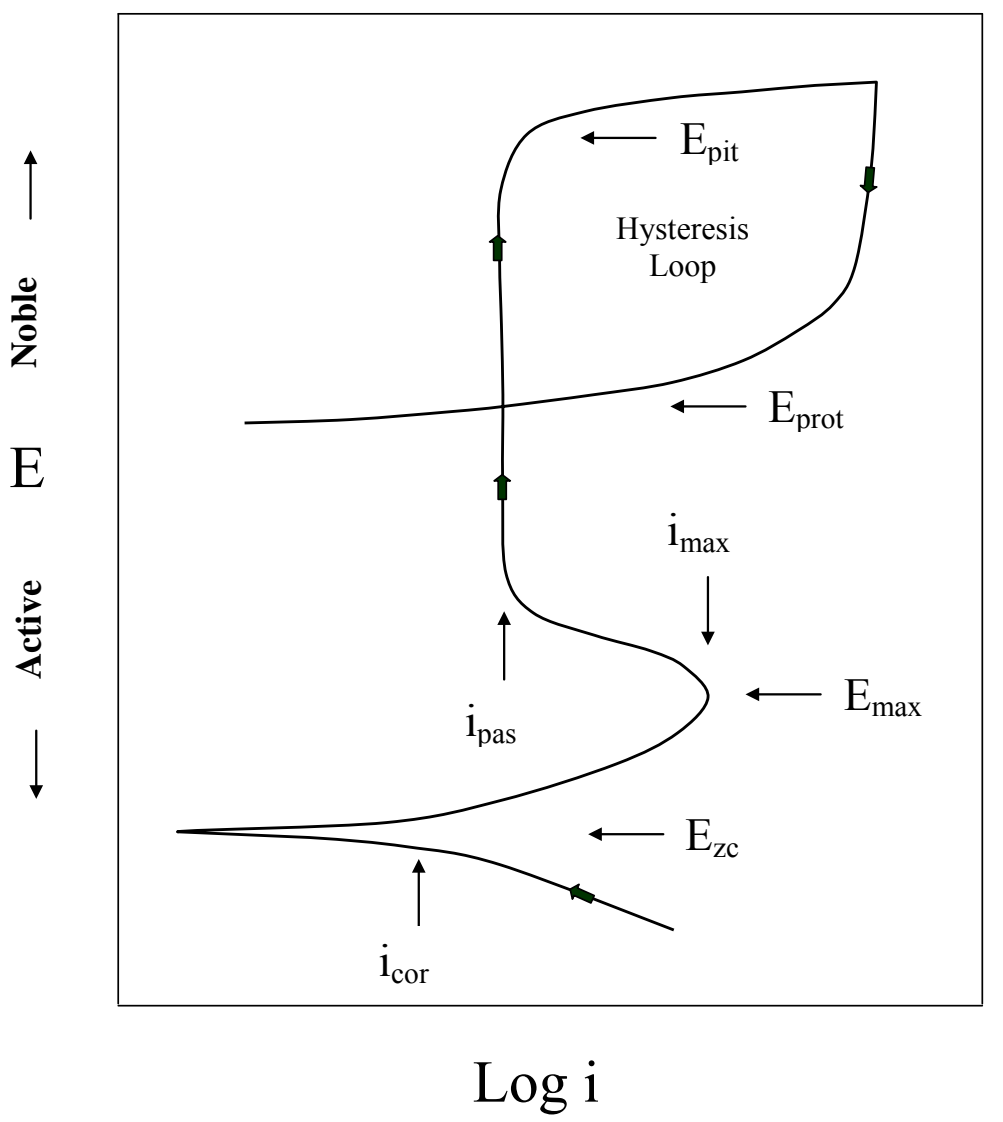

Figure 5-17 Schematic of an idealized (CPP) Curve.

Definitions for these polarization parameters:

$\mathrm{E}_{\mathrm{zc}}=$ Zero Current Potential:

$\mathrm{E}_{\max }=$ Peak Current Potential:

$\mathrm{E}_{\mathrm{pit}}=$ Pitting Potential:

$\mathrm{E}_{\text {prot }}=$ Protection Potential:

$\mathrm{i}_{\text {cor }}=$ Corrosion Current Density:

$\mathrm{i}_{\max }=$ Peak Current Density:

$\mathrm{i}_{\text {pas }}=$ Passive Current Density:
The potential at zero current, measured on the forward scan.

The potential at the active peak prior to passivation.

The potential at which stable pits initiate on the forward scan. The increase in current at this potential may not be associated with pitting. The potential may be the result of other anodic reactions (e.g., oxygen evolution). In that case the potential may be referred to as the transpassive potential $\left(\mathrm{E}_{\text {trans }}\right)$. A transpassive potential is often observed for samples that have negative hysteresis.

The potential at which pits (if they occur) passivate and stop growing on the reverse scan.

The corrosion current density, which is related to the corrosion rate by Faraday's law.

The current density at the active peak prior to passivation.

The current density in the passive range. 
The zero current potential, $\mathrm{E}_{\mathrm{zc}}$, taken from CPP curves is the potential at which the current changes polarity from negative to positive on the forward scan. The corrosion potential, $\mathrm{E}_{\text {cor }}$, also sometimes referred to as the open circuit potential, is the potential of a specimen measured under open circuit conditions where the specimen is connected solely to a high impedance voltmeter. In a CPP test, $\mathrm{E}_{\text {cor }}$ is measured for a short time period (e.g., 2 hours) prior to starting the scan and the scan is started at a fixed voltage $\left(\mathrm{e} . \mathrm{g}, 100 \mathrm{mV}\right.$ ) below the measured $\mathrm{E}_{\text {cor }}$ The $\mathrm{E}_{\mathrm{zc}}$ may not be the same potential as $E_{\text {cor }}$ measured before starting the scan. $E_{\text {cor }}$ typically moves in the noble direction with exposure time for passive alloys. Therefore, the $\mathrm{E}_{\text {cor }}$ value measured prior to starting a CPP scan and $\mathrm{E}_{\mathrm{zc}}$ typically are more negative than $\mathrm{E}_{\mathrm{cor}}$ values measured after longer exposure times.

If the sample is corroding actively at $\mathrm{E}_{\mathrm{zc}}$, the current will increase exponentially as the potential is scanned upwards from $\mathrm{E}_{\mathrm{zc}}$, exhibiting a straight line in the semi-log plot. Samples susceptible to pitting must be passive, so an active/passive transition resulting in a peak current density, $\mathrm{i}_{\max }$, will be observed for such samples. Under conditions where the alloy is spontaneously passive, the current reaches a relatively constant value just above $E_{z c}$, so that $i_{\max }$ is not observed. In the passive region, the current, $i_{\mathrm{pas}}$, is usually almost constant, with little dependence on potential.

The pitting potential is the value at which the current increases rapidly owing to the onset of stable pitting. In most instances, pitting potentials are reasonably easy to define by a change in slope and a sharp increase in the corrosion current. The occurrence of positive hysteresis, where the current on the reverse (downward) scan is higher than during the forward scan, is usually indicative of the occurrence of localized corrosion such as pitting or crevice corrosion. For steel samples that do not exhibit localized corrosion, the current will eventually increase above $i_{\text {pas }}$ at high applied potentials owing to oxygen evolution by water oxidation. In such a case, during the reverse scan, the current will trace back along the increasing part of the forward scan with no evidence of hysteresis. Often, a negative hysteresis is observed where the passive current on the reverse scan is lower than that on the forward scan. Pitting and crevice corrosion are almost never found in association with such a CPP curve. The potential in this case is referred to as a transpassive potential $\left(\mathrm{E}_{\text {trans }}\right)$ rather than the pitting potential.

For a sample exhibiting pitting and a positive hysteresis, the pits will eventually repassivate during the reverse scan as the potential is lowered. The potential at which this happens is called the protection or repassivation potential $\left(\mathrm{E}_{\text {prot }}\right)$. This is a critical parameter in the assessment of localized corrosion susceptibility because a conservative approach for designing against localized corrosion would be to determine that the corrosion potential would remain well below this value. $E_{\text {prot }}$ is often defined as the potential at which the current on the reverse scan falls below that observed on the forward scan. In other words, it is the potential at which the reverse scan crosses the forward scan as shown in Figure 5-17. However, in some cases, the passive current on the reverse scan is higher than that on the forward scan. In that case, the protection potential is taken as the point at which the current exhibits a sharp decrease. In other cases, the protection potential is below the $E_{z c}$ observed on the forward scan. If the original $E_{z c}$ was used as the final limit for the reverse scan, then the protection potential cannot be definitively determined in this situation.

The severity of pitting corrosion can be ranked based on the shape of the CPP curve according to five categories:

Category 1: Negative hysteresis and no evidence of pitting.

Category 2: Positive hysteresis, but with pitting and protection potentials well above the zero current potential $\left(\mathrm{E}_{\text {prot }}>>\mathrm{E}_{\mathrm{zc}}\right)$. 
Category 3: Positive hysteresis with a noble pitting potential, but with the protection potential relatively near the zero current potential $\left(\mathrm{E}_{\text {prot }}\right.$ near $\left.\mathrm{E}_{\mathrm{zc}}\right)$.

Category 4: Positive hysteresis with the protection potential lower than the zero current potential $\left(\mathrm{E}_{\text {prot }}<\mathrm{E}_{\mathrm{zc}}\right)$.

Category 5: Spontaneous pitting at the zero current potential so that the current increases rapidly upon polarization to potentials above the zero current potential.

These categories are shown graphically in Figures 5-18 to 5-22. For these figures, the metal is assumed to be passive at the free corrosion potential so no active-passive transition is shown.

The Category 1 ranking (Figure 5-18) is the most desirable because it indicates that the environment is not capable of promoting pitting of the alloy. This should be confirmed by a posttest examination of the specimen. Note that the potential associated with the significant increase in current on the forward scan is not called a pitting potential $\left(\mathrm{E}_{\mathrm{pit}}\right)$ for Category 1 because it is not associated with pitting corrosion. The increase in current is associated with water breakdown or transpassive behavior and the potential is referred to as the transpassive potential $\left(\mathrm{E}_{\text {trans }}\right)$ in Figure 5-18. This case is defined as a "pass" condition and no additional testing is required; the environment is considered to be benign with respect to pitting.

For Categories 3 through 5 (Figures 5-19 through 5-22) localized corrosion is likely to occur in service. In the presence of pitting on the sample, these categories are considered a "fail" condition; the environment is considered to be aggressive with regard to pitting.

All other outcomes require additional testing. Examples of other outcomes include:

- Category 1 behavior with pitting on the sample;

- Category 2 behavior (Figure 5-19) with or without pitting;

- Category 3 through 5 with no pitting;

- Undefined hysteresis with or without pitting; this type of behavior is typified by the reverse scan following close to the forward scan or crossing it several times.

Additional tests include ASTM G192 [32] long-term coupon immersion testing, and in-tank reference electrode measurements to determine $E_{\text {cor }}$. The ASTM G192 protocol is being modified for carbon steels in waste simulants by DNV-GL[33]. 
SRNL-STI-2014-00616

Revision 0

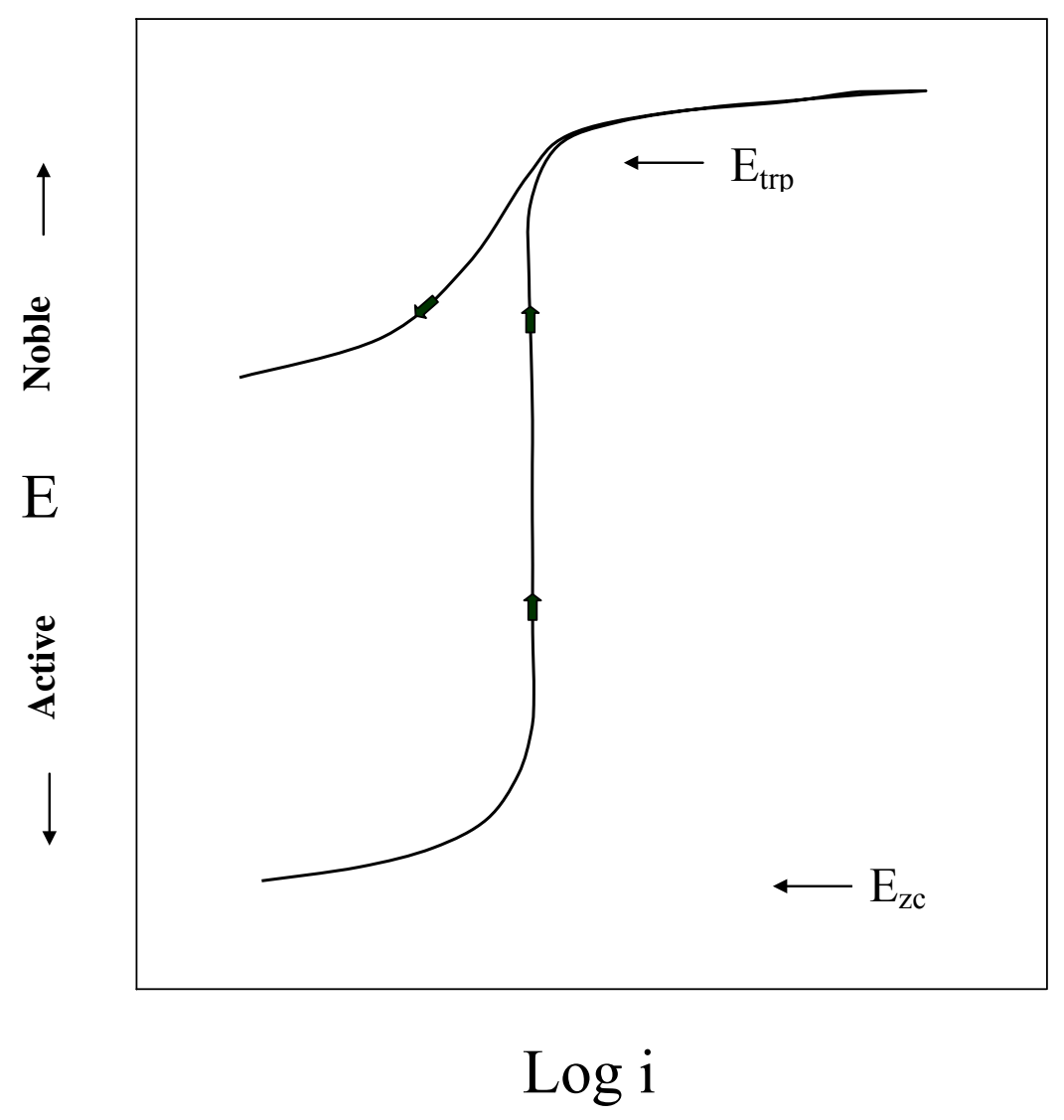

Figure 5-18 Schematic of Category 1 CPP Curve. 
SRNL-STI-2014-00616

Revision 0

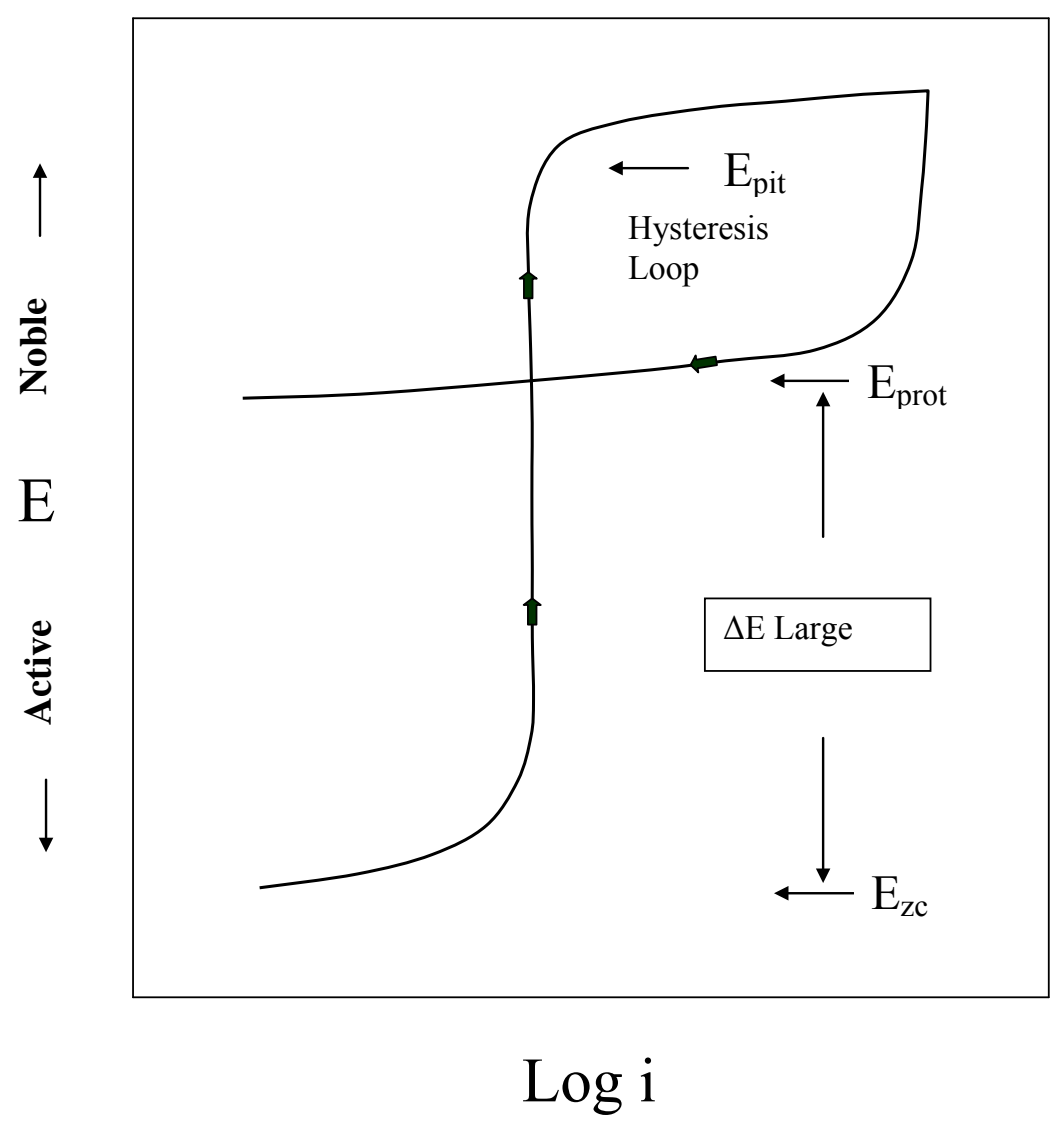

Figure 5-19 Schematic of Category 2 CPP Curve. 
SRNL-STI-2014-00616

Revision 0

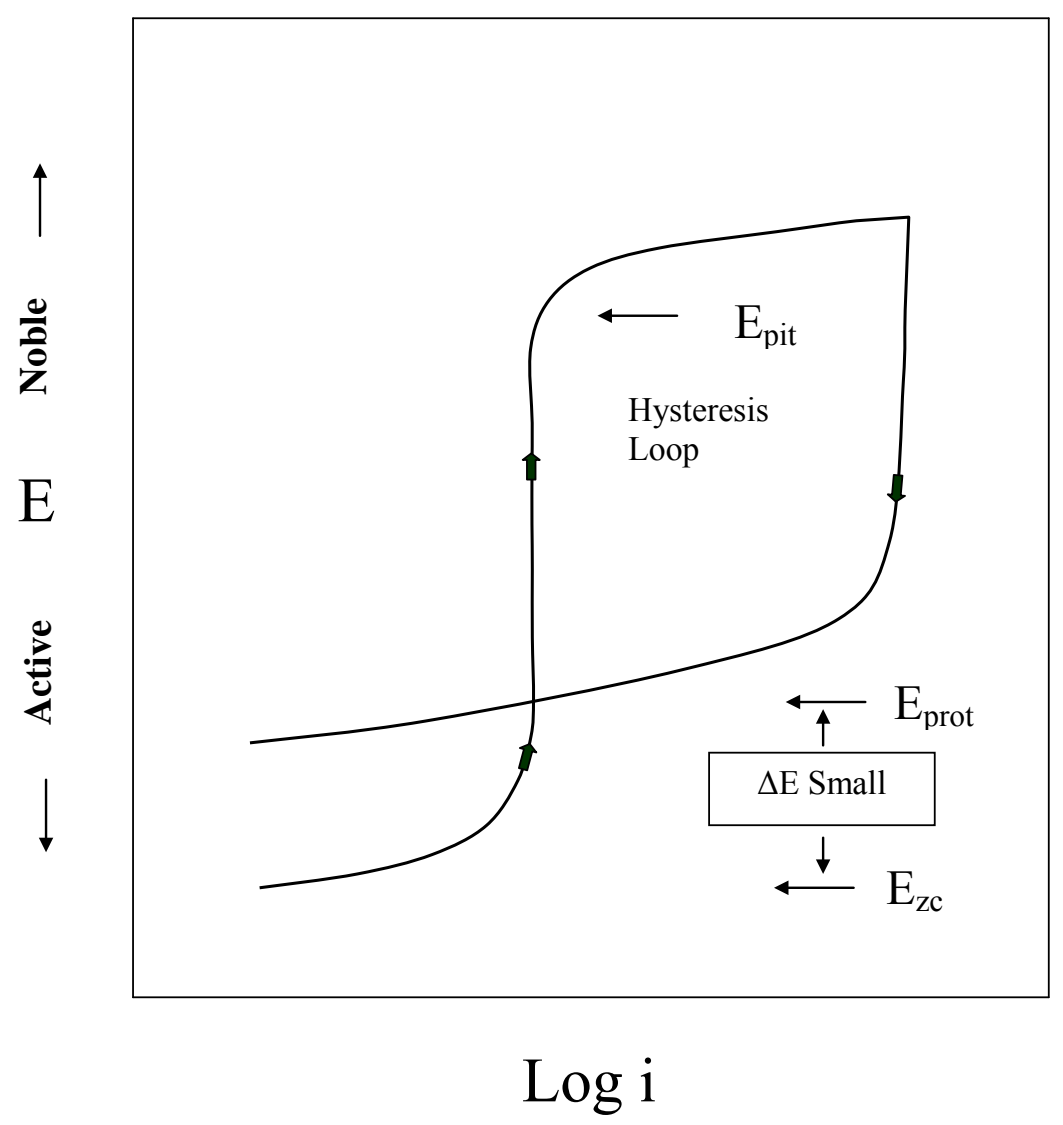

Figure 5-20 Schematic of Category 3 CPP Curve. 
SRNL-STI-2014-00616

Revision 0

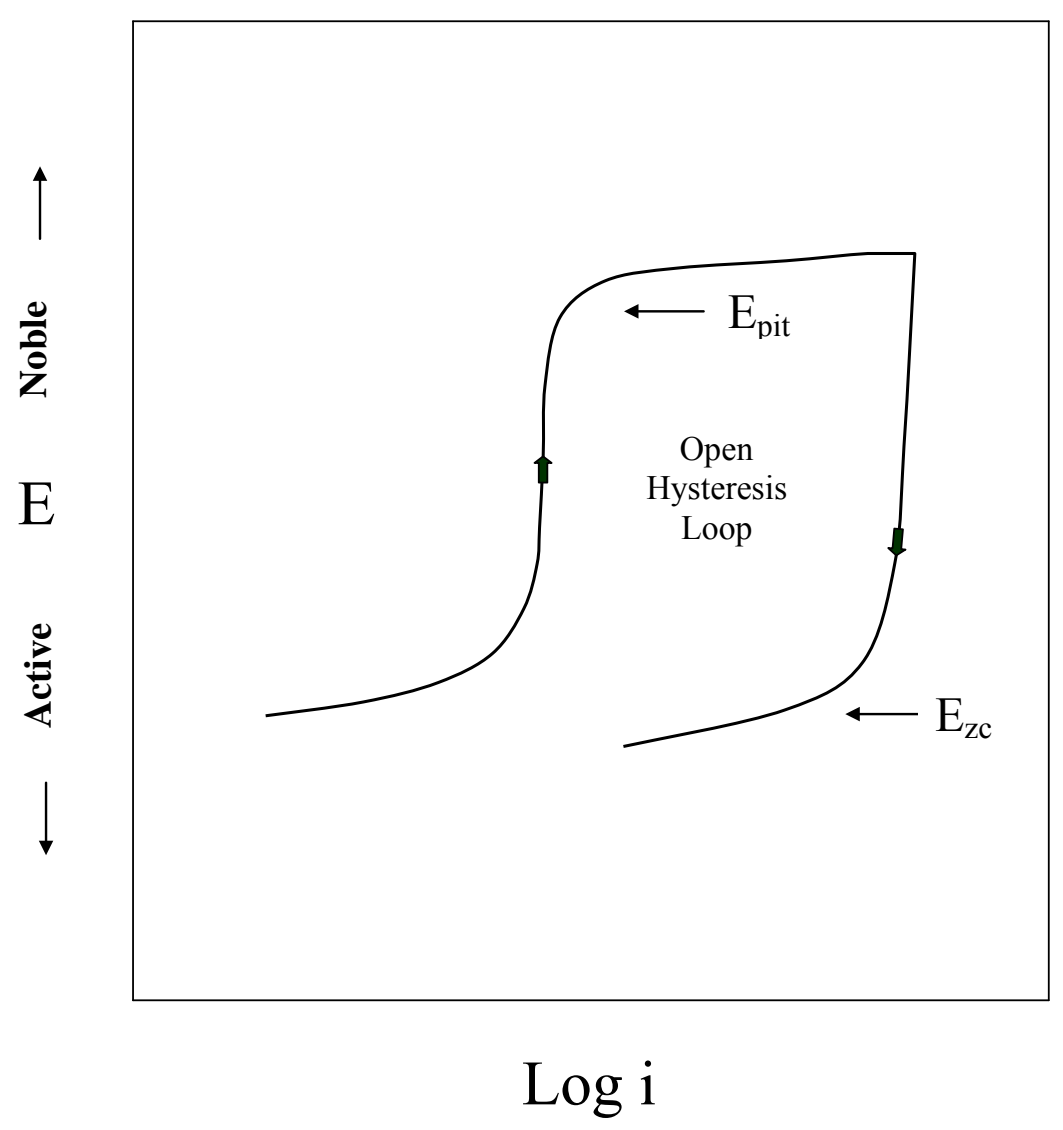

Figure 5-21 Schematic of Category 4 CPP Curve. 
SRNL-STI-2014-00616

Revision 0

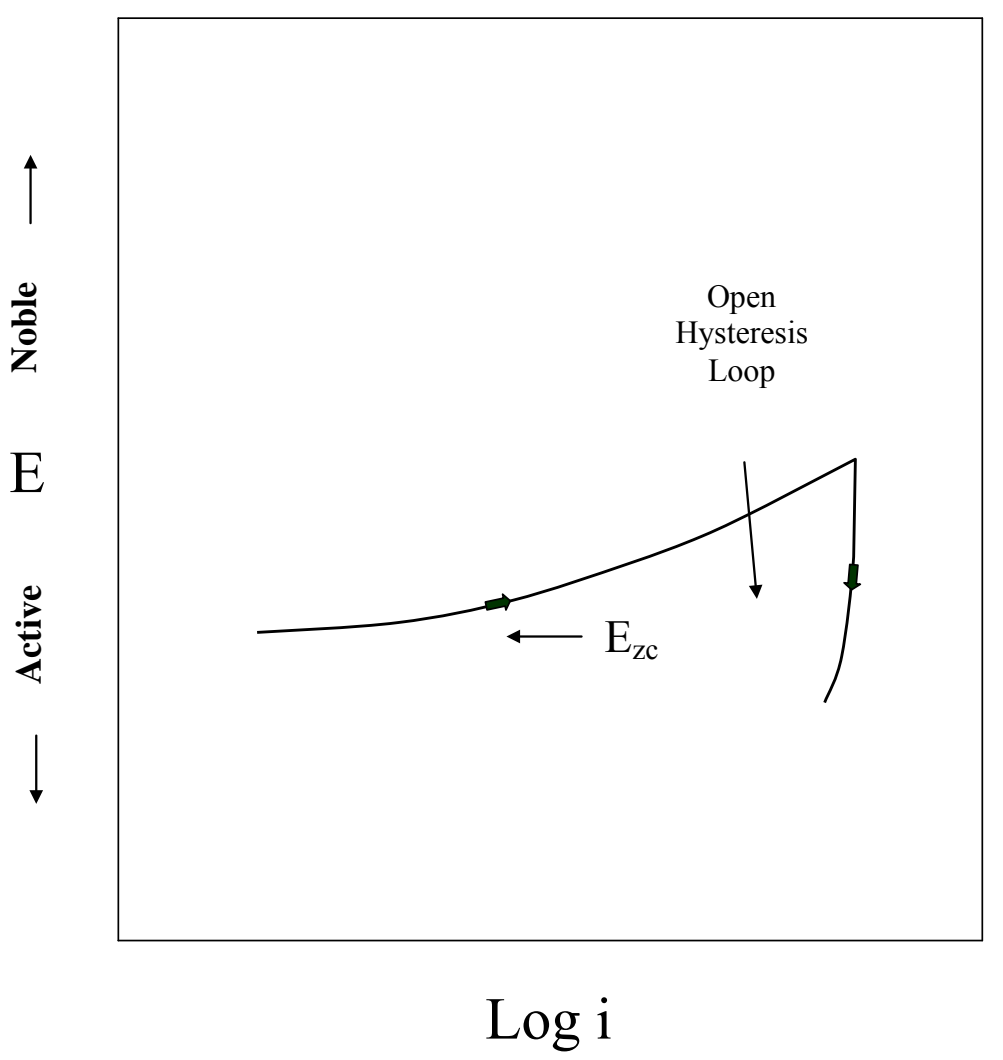

Figure 5-22 Schematic of Category 5 CPP curve.

\subsubsection{Literature Review of Historical Data}

Data from 22 literature sources were compiled for this activity [28],[33]-Error! Reference source not found. More than $1800 \mathrm{CPP}$ data points, which represents 920 test conditions, were catalogued and classified (See Appendix H). Of these test conditions, 814 tests were performed in SRS waste simulants and 106 tests were performed in Hanford simulants. Pitting was observed in $479(52 \%)$ of the tests. Further observations of the data indicated that it could be classified based on when the test was performed, that is, pre-1993 and post-1993. CPP tests were performed on 518 test conditions (56\%) pre-1993. Pitting was observed on $242(47 \%)$ of these CPP tests. On the other hand, of the 402 CPP test conditions examined post-1993, 237 (59\%) indicated pitting susceptibility.

There were three major differences in how test matrices were selected for the two data classifications. First, all the pre-1993 data focused on anticipated feed preparation streams for the Defense Waste Processing Facility (DWPF) at SRS. These were low sodium dilute waste streams (i.e., less than $1 \mathrm{M}$ nitrate) typically at temperatures lower than $40{ }^{\circ} \mathrm{C}$. Secondly, the test conditions (i.e., compositions with various inhibitor anion/aggressive anion ratios) were selected by a "best guess" process based on the previous test results rather than using a pre-determined test matrix of compositions [36]. This selection process minimized the number of scans required, yet still resulted in a very exact determination of the pit-no pit boundary. Finally, records of the pre1993 CPP data are sparse. A few examples of CPP curves exist in reports, however, a majority of the data in the reports is shown in log-log plots of the aggressive species vs. the inhibitor species. 
These plots show qualitative assessments of the CPP results such as, "no pits", "occasional pitting", or "pits".

In contrast, the post-1993 data has focused on compositions related to present waste storage or waste retrieval conditions. As such, there is typically a greater range in composition (e.g., up to 7 $\mathrm{M}$ nitrate) and temperature (e.g., up to $77^{\circ} \mathrm{C}$ ) and waste simulants from both the Hanford site and SRS have been considered. Also, rather than a "best-guess" approach, typically the test compositions were based on either a statistical matrix that covered a broad envelope of anticipated compositions or on waste samples taken from the tank. As a result, there is a more even distribution of data for these tests. Finally, records for the CPP data are more readily available and can be compared.

Due to the lack of pre-1993 records, the definition of a borderline or marginal test condition is also different for the two data classifications. Figure 5-23 shows an example of a log-log plot taken from a report. In this case, the nitrate is the aggressive species and nitrite is the inhibitor species. The line that is drawn represents the minimum nitrite that prevented the initiation of pitting. Open squares above the line were conditions that no pits were observed, while partially filled and filled squares represented conditions where occasional pitting and pits were observed, respectively. The borderline condition was drawn at the transition between occasional pitting and no pits. As a result of the "best-guess" approach, a majority of the test conditions are located at the boundary between the pit-no pit regime. Therefore, in analyzing the present CPP tests, the location (i.e., borderline condition) of the historical data will be taken into account.

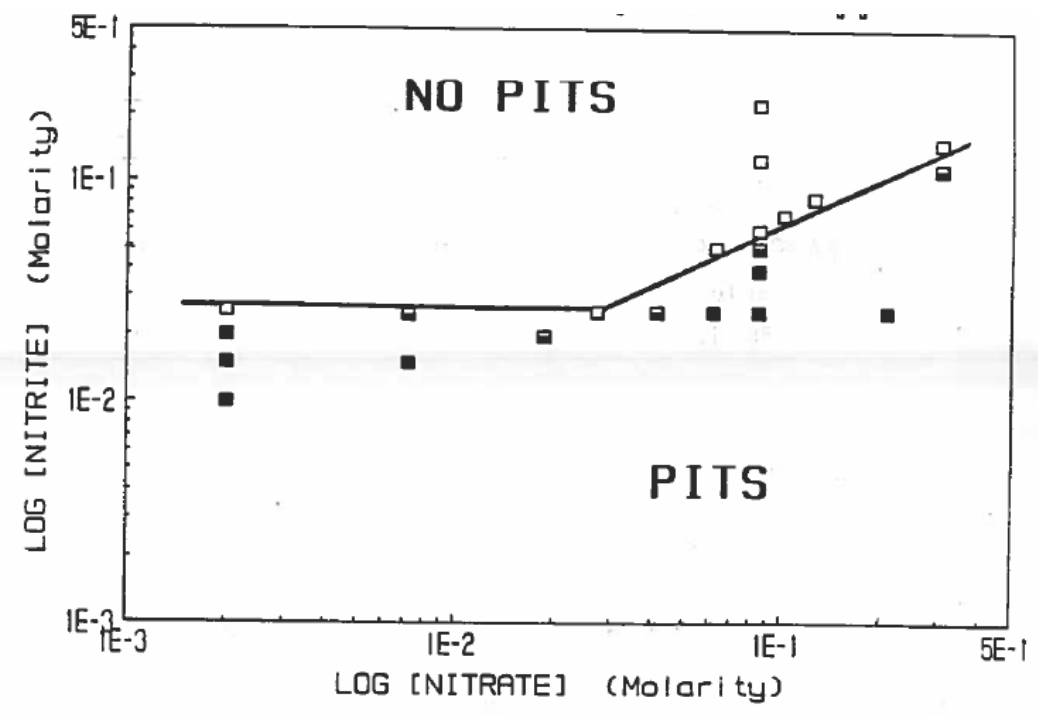

KEY:

NO PITTING

OCCASIONAL PITTING

PITTING OR GENERAL CORROSION

Figure 5-23 Log-log plot for SRS dilute waste simulant that shows the minimum nitrite required to prevent pit initiation as a function of nitrate concentration [36]. 
Although no CPP curves were shown, one pre-1993 report suggests that borderline of marginal results were observed during tests [55]. The author noted, "The scans...almost always corroborated the specimen appearance when the hysteresis, either positive or negative, was of the order of an order of magnitude in current density...With hysteresis less than an order of magnitude, the scans did not correlate as well with the specimen appearance." In a separate report [45] another author observed, "Samples were run in duplicate and in cases where the results differed the more aggressive corrosion behavior was reported." It is not known whether either of these cases are associated with all the occasional pitting data, but this may be a reasonable assumption.

In contrast, the CPP curves can be utilized to define borderline behavior for the post-1993 tests. Figure 5-24 shows an example of borderline behavior for a dilute, SRS waste simulant. For this test, the nitrate concentration was $0.85 \mathrm{M}$, the nitrite concentration was $0.43 \mathrm{M}$, the $\mathrm{pH}$ was 10 and the temperature was $40{ }^{\circ} \mathrm{C}$. Negative hysteresis was observed for the CPP curve, however, small pits were observed on the electrode. Given the priority placed on the post-test condition of the sample, this result was considered to be a fail. Based on the pre-1993 log-log plots, this test condition would fall slightly below the line de-lineating the pit regime from the no-pit regime. In this region, "occasional pitting" data might be expected.
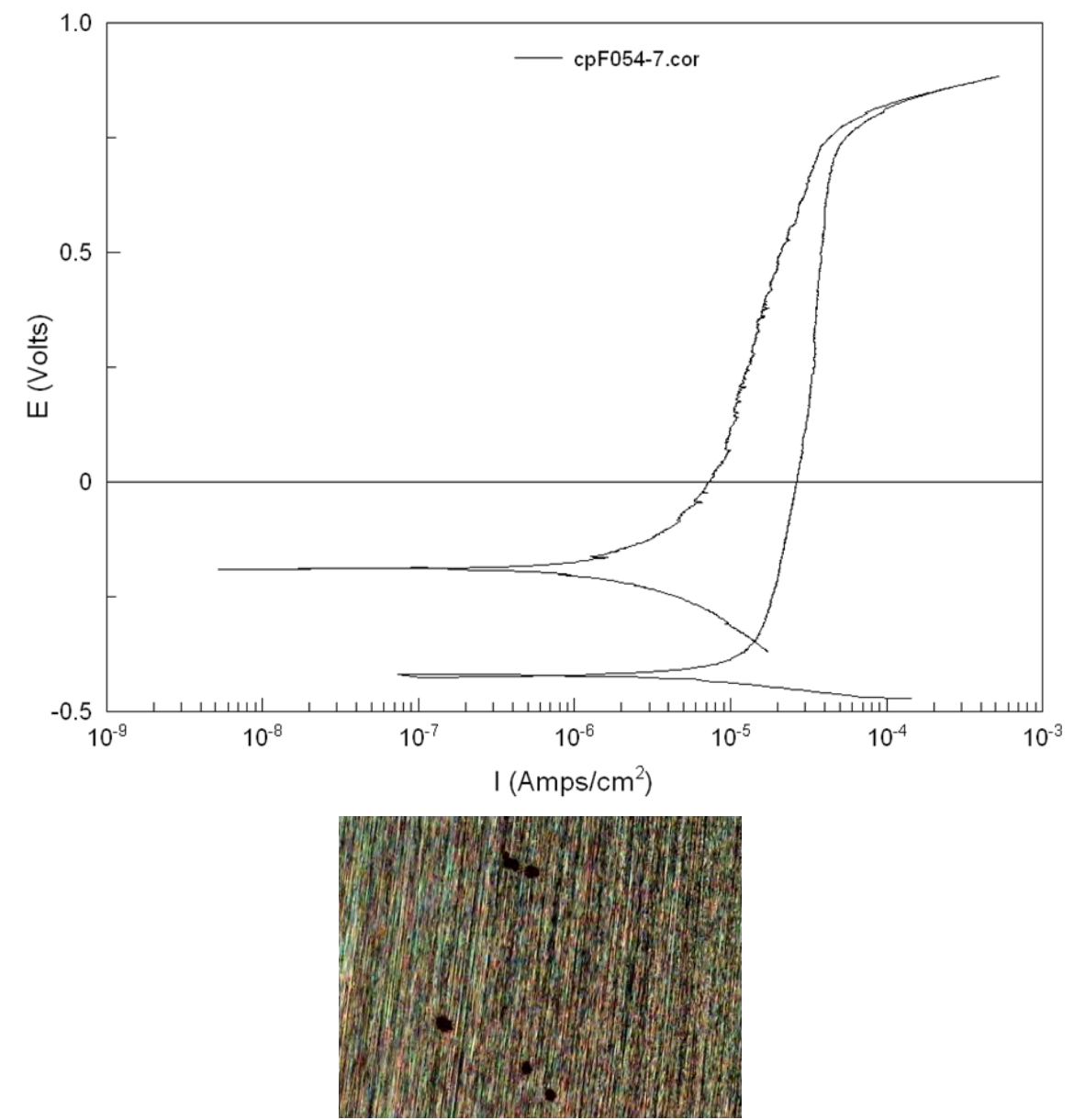

Figure 5-24 Borderline behavior in a dilute, SRS waste simulant [28]. The CPP scan exhibited negative hysteresis, however, small pits were detected on the electrode surface. Magnification was approximately $10 X$. 
The third difference between the two data set classifications lies in the manner in which the tests were conducted. A full catalog of the protocol that was used for each of the 21 tests programs is shown in Appendix H. There were primarily 2 differences between the pre-1993 test protocol and the post-1993 test protocol: 1) the potential stabilization time and 2) the scan vertex threshold. For the pre-1993 tests, potential stabilization time was typically 30 minutes, whereas for the post1993 tests the potential stabilization time was typically 2 hours or more. The longer stabilization time likely ensured a stable $\mathrm{E}_{\text {corr }}$ value at the initiation of the test, which is indicative of an equilibrated system.

The vertex threshold (i.e., the potential or current at which the CPP scan is reversed) for the pre1993 tests was based on a threshold potential, typically around 1.0 V. Figure 5-25 shows an example of a pre-1993 CPP curve in a dilute SRS waste simulant that exhibited positive hysteresis and pitting [45]. Given that the sample was approximately $2 \mathrm{~cm}^{2}$, the current density at the vertex potential was greater than $15 \mathrm{~mA} / \mathrm{cm}^{2}$. There are no pictures of the sample from the pre-1993 test, therefore the size and density of the pitting is unknown, however, relatively low current density and small degree of hysteresis suggests that pitting was minimal. In the case of the post-1993, a threshold current of approximately 1 to $5 \mathrm{~mA} / \mathrm{cm}^{2}$ was utilized.

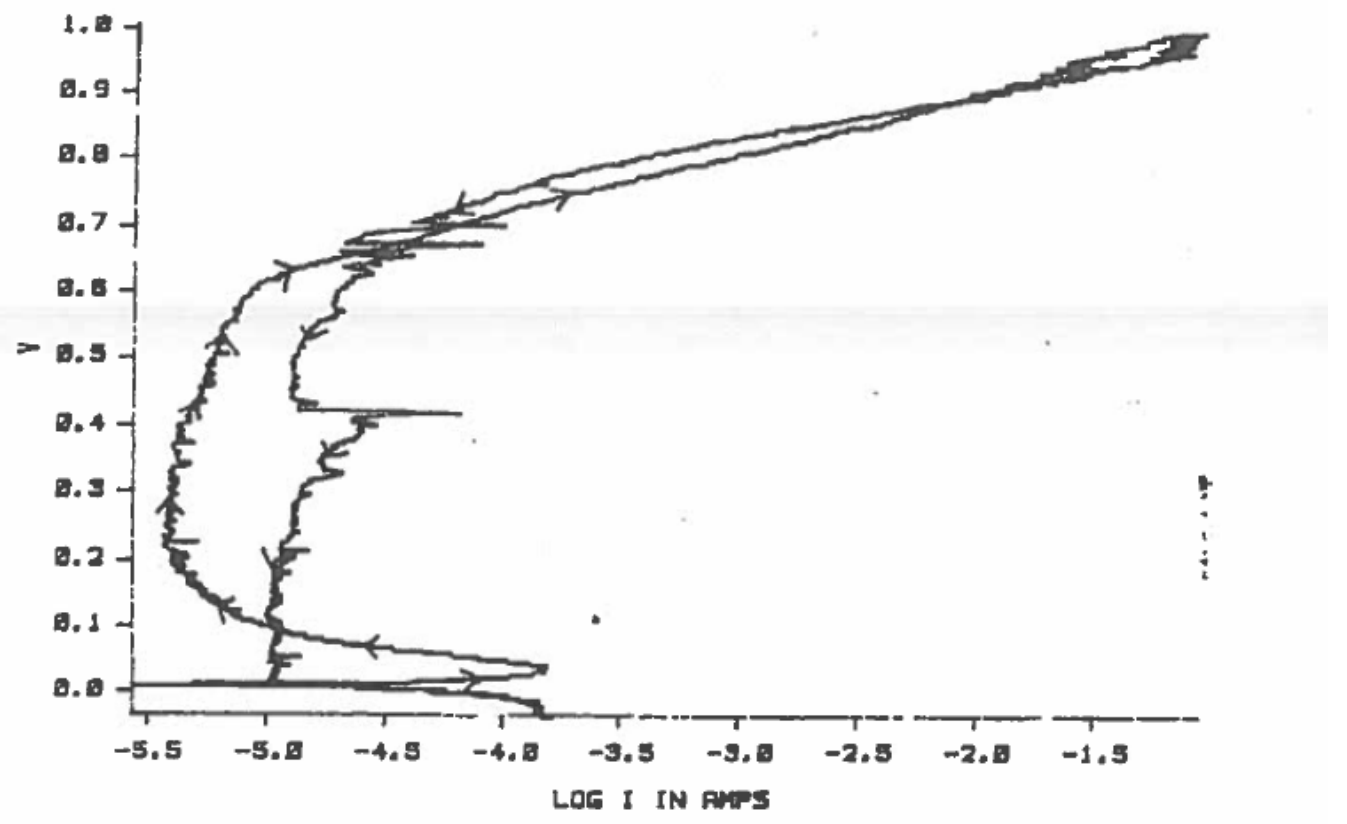

Figure 5-25 CPP curve in dilute SRS waste simulant that illustrates the vertex threshold potential of $1.0 \mathrm{~V}$ [45].

Figure 5-26 shows an example of a CPP curve taken from the FY2014 tests with a threshold current of $1 \mathrm{~mA} / \mathrm{cm}^{2}$. The CPP curve exhibited negative hysteresis and the electrode did not pit. The difference between the simulants is that the $\mathrm{pH}$ of the pre-1993 test solution was 10.07, while for the post-1993 test the $\mathrm{pH}$ was 10.18. It cannot be stated for certain, but it is possible that by scanning to a higher threshold current, that small pits were initiated at an overly conservative condition. At a borderline condition, this could be the difference between interpreting the CPP test as pass or fail. (Note: The post-1993 test is a repeat of a pre-1993 test. The result of the test 
in 1988 was a pass as no pitting was observed. Thus, the test shown in Figure 5-19 would have been considered a borderline condition.)

Coupon tests were used in the past to confirm the results of CPP tests, particularly near the borderline conditions. A review of this data indicates that coupon testing was more common pre1993. The tests were set-up so that the coupon was partially immersed, enabling the evaluation of LAI and VSC as well as corrosion of the immersed portion of the sample. The tests were typically performed for four months. The tests typically corroborated the results of the CPP tests. Conditions that resulted in pitting during a CPP test would produce pitting during a coupon test. Of interest was the observation that borderline CPP results oftentimes produced mixed results for the coupon tests. That is, a coupon test may indicate pitting on one sample, but not on a duplicate sample. Thus, the results of the coupon tests increased the confidence in the results observed for the CPP tests.

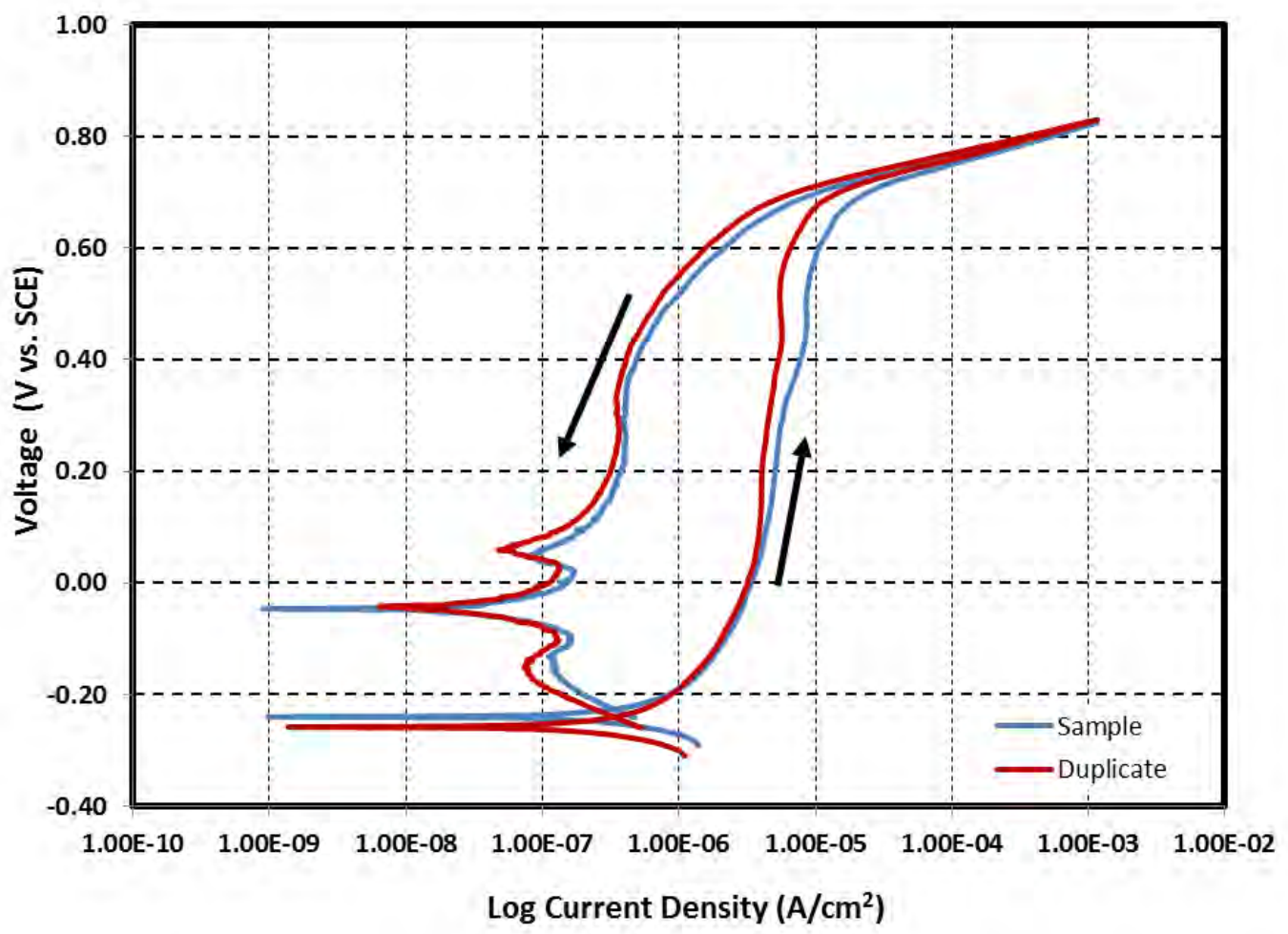

\section{Figure 5-26 CPP curve in dilute SRS waste simulant that illustrates the vertex threshold current of $1 \mathrm{~mA} / \mathrm{cm}^{2}$.}

\subsubsection{Test Selection}

A random sample of 40 tests was selected from the historical database for testing by the new CPP protocol. The sample was not completely random as the EPOC requested that additional tests be performed at higher $\mathrm{pH}$ [4] in order to ensure that the Hanford site waste chemistry was adequately addressed. The 40 tests that were selected are shown in Table 5-8 and 5-9. The composition and temperature for each test is listed. Characteristics of the test matrix included: 
- $\quad 35$ of the 40 CPP tests (87.5\%) were performed in SRS waste simulants, which leaves only 5 tests performed in Hanford waste simulants. This proportion is slightly less than the proportion for the total database $(88.5 \%)$.

- 26 of the 40 CPP tests (65\%) indicated pitting susceptibility. This proportion is higher than what was observed for the total database (52\%).

- 21 of the 40 CPP tests were replicates of pre-1993 tests. This proportion is slightly less than the proportion for the total database (53\% vs. 56\%). The proportion is likely less because the distribution was skewed slightly to include more tests at a higher $\mathrm{pH}$ [4]. Since the post-1993 tests contained the higher $\mathrm{pH}$ tests, there would be an increase in the proportion of these CPP tests.

- $\quad 12$ of the 21 (57\%) pre-1993 tests indicated pitting susceptibility. This proportion is slightly higher than that observed for the total distribution of pre-1993 tests.

- $\quad 16$ of the 21 pre-1993 tests were considered borderline conditions. This proportion seems a high given that Figure 5-23 suggests that a little over half of the data would be expected to be borderline conditions. However, as mentioned before, the "best-guess" approach would tend to cluster more of the data near the borderline defined by the minimum inhibitor concentration.

- $\quad 14$ of the 19 post-1993 (74\%) tests indicated pitting susceptibility. This proportion is slightly higher than that observed for the total distribution of pre-1993 tests.

- 4 of the 19 post-1993 tests were considered borderline conditions. This lower proportion is not as surprising given that the selection process produced a test matrix that was more evenly distributed across the envelope.

The quasi-random sample appears to have adequately represented the tested conditions in the total database as well as the test conditions for the two classifications. The next section will review the distributions for the variables that were tested. 
Table 5-8 CPP Test Solution Composition and Temperature

\begin{tabular}{|c|c|c|c|c|c|c|c|c|c|c|c|c|}
\hline \multirow{2}{*}{ Reference } & \multirow{2}{*}{ Test } & \multirow{2}{*}{$\begin{array}{c}\text { Temperature } \\
\left({ }^{\circ} \mathrm{C}\right)\end{array}$} & \multicolumn{9}{|c|}{ Concentration of Species (M) } & \multirow{2}{*}{ pH } \\
\hline & & & $\mathrm{CO}_{3}{ }^{=}$ & $\mathrm{HCO}_{3}{ }^{=}$ & Citrate & $\mathrm{PO}_{4}{ }^{3-}$ & $\mathrm{Cl}^{-}$ & $F^{-}$ & $\mathrm{SO}_{4}{ }^{2-}$ & $\mathrm{NO}_{3}{ }^{-}$ & $\mathrm{NO}_{2}^{-}$ & \\
\hline 42 & 1 & 40 & 0.0015 & NA & NA & 0.000084 & 0.00032 & 0.000153 & 0.0014 & 0.04 & 0.015 & 12.5 \\
\hline 50 & 2 & 50 & 1.118 & NA & NA & 0.009 & 0.046 & 0.084 & 0.028 & 1.635 & 1.27 & 14.5 \\
\hline 48 & 3 & 50 & 0.1 & NA & NA & 0.05 & 0.1 & NA & 0.1 & 7 & 0.1 & 13 \\
\hline 48 & 4 & 50 & 0.1 & NA & NA & 0.05 & 0.1 & NA & 0.1 & 5.5 & 0.2 & 13.8 \\
\hline 42 & 5 & 40 & 0.0015 & NA & NA & 0.000084 & 0.01 & 0.000153 & 0.0014 & 0.04 & 0.325 & 12.5 \\
\hline 42 & 8 & 40 & 0.0015 & NA & NA & 0.000084 & 0.00032 & 0.000153 & 0.1 & 0.04 & 0.12 & 12.5 \\
\hline 49 & 9 & 40 & 0.02 & NA & 0.6 & 0.0005 & 0.4 & 0.05 & 0.005 & 0.1 & 0.5 & 12.12 \\
\hline 42 & 10 & 40 & 0.0015 & NA & NA & 0.000084 & 0.00032 & 0.000153 & 0.1 & 0.04 & 0.18 & 12.5 \\
\hline 42 & 11 & 40 & 0.0015 & NA & NA & 0.000084 & 0.00032 & 0.000153 & 0.02 & 0.04 & 0.04 & 12.5 \\
\hline 49 & 12 & 40 & 0.2 & NA & 0.04 & 0.05 & 0.06 & 0.02 & 0.1 & 0.7 & 0.02 & 11.7 \\
\hline 46 & 16 & 40 & 0.0526 & 0.098 & NA & NA & 0.0037 & NA & 0.0451 & 0.2 & 0.15 & 10 \\
\hline 45 & 17 & 40 & 0.236 & 0.438 & NA & $\mathrm{NA}$ & 0.0115 & NA & 0.186 & 0.9 & 0.675 & 10 \\
\hline 35 & 18 & 40 & 0.013 & 0.058 & NA & 0.00039 & 0.0011 & 0.00073 & 0.0059 & 0.06 & 0.04 & 9.6 \\
\hline 46 & 19 & 40 & 0.0789 & 0.147 & NA & NA & 0.0051 & NA & 0.0731 & 0.45 & 0.225 & 10 \\
\hline 36 & 20 & 40 & 0.0042 & 0.019 & NA & 0.00013 & 0.00036 & 0.00024 & 0.13 & 0.0284 & 0.2 & 9.6 \\
\hline
\end{tabular}


Table 5-9 CPP Test Solution Composition and Temperature (continued)

\begin{tabular}{|c|c|c|c|c|c|c|c|c|c|c|c|c|}
\hline \multirow{2}{*}{ Reference } & \multirow{2}{*}{ Test } & \multirow{2}{*}{$\begin{array}{c}\text { Temperature } \\
\left({ }^{\circ} \mathrm{C}\right)\end{array}$} & \multicolumn{9}{|c|}{ Concentration of Species (M) } & \multirow{2}{*}{$\mathbf{p H}$} \\
\hline & & & $\mathrm{CO}_{3}{ }^{=}$ & $\mathrm{HCO}_{3}{ }^{=}$ & Citrate & $\mathrm{PO}_{4}{ }^{3-}$ & $\mathrm{Cl}^{-}$ & $\mathbf{F}^{-}$ & $\mathrm{SO}_{4}{ }^{2-}$ & $\mathrm{NO}_{3}^{-}$ & $\mathrm{NO}_{2}^{-}$ & \\
\hline 35 & 21 & 40 & 0.013 & 0.058 & NA & 0.00039 & 0.0011 & 0.00073 & 0.0059 & 0.05 & 0.025 & 9.6 \\
\hline 35 & 22 & 40 & 0.013 & 0.058 & NA & 0.00039 & 0.0011 & 0.00073 & 0.0085 & 0.085 & 0.1 & 9.6 \\
\hline 46 & 23 & 40 & 0.3158 & 0.586 & NA & NA & 0.0142 & NA & 0.3806 & 0.9 & 0.9 & 10 \\
\hline 28 & 24 & 40 & 0.1491 & 0.277 & NA & NA & 0.010625 & NA & 0.0425 & 0.85 & 0.425 & 10 \\
\hline 33,34 & 25 & 40 & 0.011 & 0.035 & NA & 0.00016 & 0.0006 & 0.0003 & 0.0026 & 0.039 & 0.022 & 9.66 \\
\hline 28 & 28 & 40 & 0.193 & 0.358 & $\mathrm{NA}$ & NA & 0.006875 & NA & 0.0275 & 0.55 & 0.55 & 10 \\
\hline 36 & 29 & 40 & 0.0042 & 0.019 & NA & 0.00013 & 0.004 & 0.00024 & 0.002 & 0.028 & 0.07 & 9.6 \\
\hline 28 & 30 & 40 & 0.6316 & 1.173 & $\mathrm{NA}$ & NA & 0.0175 & NA & 0.0375 & 0.25 & 1.8 & 10 \\
\hline 36 & 31 & 40 & 0.013 & 0.058 & $\mathrm{NA}$ & 0.00039 & 0.0011 & 0.00073 & 0.0059 & 0.06 & 0.06 & 9.6 \\
\hline 44 & 32 & 80 & 0.337 & 0.243 & NA & NA & 0.0009 & 0.00267 & 0.00264 & 0.0098 & 0.022 & 10.18 \\
\hline 47 & 36 & 40 & 0.1754 & 0.326 & NA & NA & 0.032 & NA & 0.121 & 0.8 & 0.5 & 10 \\
\hline 35 & 37 & 40 & 0.013 & 0.058 & NA & 0.00039 & 0.0015 & 0.00073 & 0.0059 & 0.085 & 0.04 & 9.6 \\
\hline 50 & 38 & 77 & 1.028 & NA & NA & 0.012 & 0.004 & 0.003 & 0.018 & 0.002 & 0 & 11 \\
\hline 35 & 39 & 40 & 0.013 & 0.058 & $\mathrm{NA}$ & 0.00039 & 0.0011 & 0.0011 & 0.0059 & 0.085 & 0.06 & 9.6 \\
\hline 36 & 40 & 40 & 0.0042 & 0.019 & $\mathrm{NA}$ & 0.00013 & 0.00036 & 0.00024 & 0.03 & 0.0284 & 0.02 & 9.6 \\
\hline
\end{tabular}




\subsubsection{Distributions of Variables for the 40 Tests}

The distribution of $\mathrm{pH}$, temperature, nitrate concentration, nitrite concentration, and the nitrite/nitrate ratio associated with the total distribution and the proposed test matrix were examined. In general, the proposed test matrix appears to represent the total distribution of these key corrosion variables well.

\subsubsection{1 pH}

Figure 5-27 shows the distribution for the $\mathrm{pH}$ that was observed for the total database (blue diamonds) and the proposed test matrix (red squares). Nearly $80 \%$ of the tests from the total database were at a $\mathrm{pH}$ of $\sim 10$ or slightly less. This is the equilibrium $\mathrm{pH}$ that is established due to the reaction of carbon dioxide from the air with the hydroxide in the waste [56]. Most of the SRS tests were performed at this condition. The proposed test distribution deviates slightly from the total database distribution. As mentioned previously, the EPOC requested that the matrix include a representative number of tests that were performed at a higher $\mathrm{pH}$. Thus, a higher proportion of the 40 tests were performed at a $\mathrm{pH}$ greater than 10 than would have been dictated by a random selection from the total distribution.

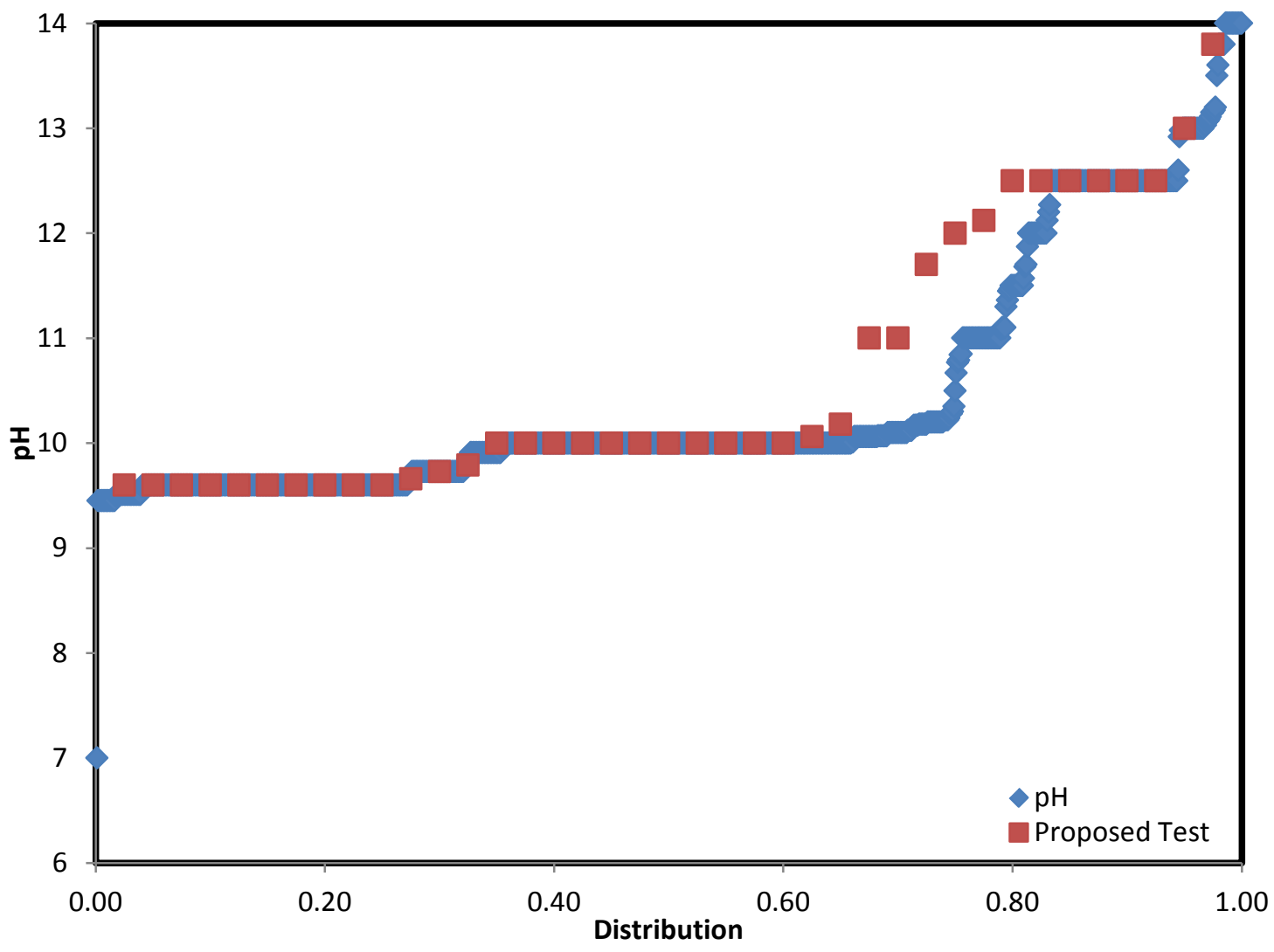

Figure 5-27 pH distribution for total distribution and 40 test matrix. 


\subsubsection{Temperature}

Figure 5-28 shows the distribution for the temperature that was observed for the total data base (blue diamonds) and the proposed test matrix (red squares). Approximately $80 \%$ of the tests from the total database were at a temperature of $40{ }^{\circ} \mathrm{C}$ or less. This temperature is the maximum expected value during feed preparation for the DWPF at SRS. Seven tests were performed at temperatures of $50{ }^{\circ} \mathrm{C}$ or higher. Three of these were performed in Hanford waste simulants, most notably the present interstitial liquid simulant for Tank AY-102.

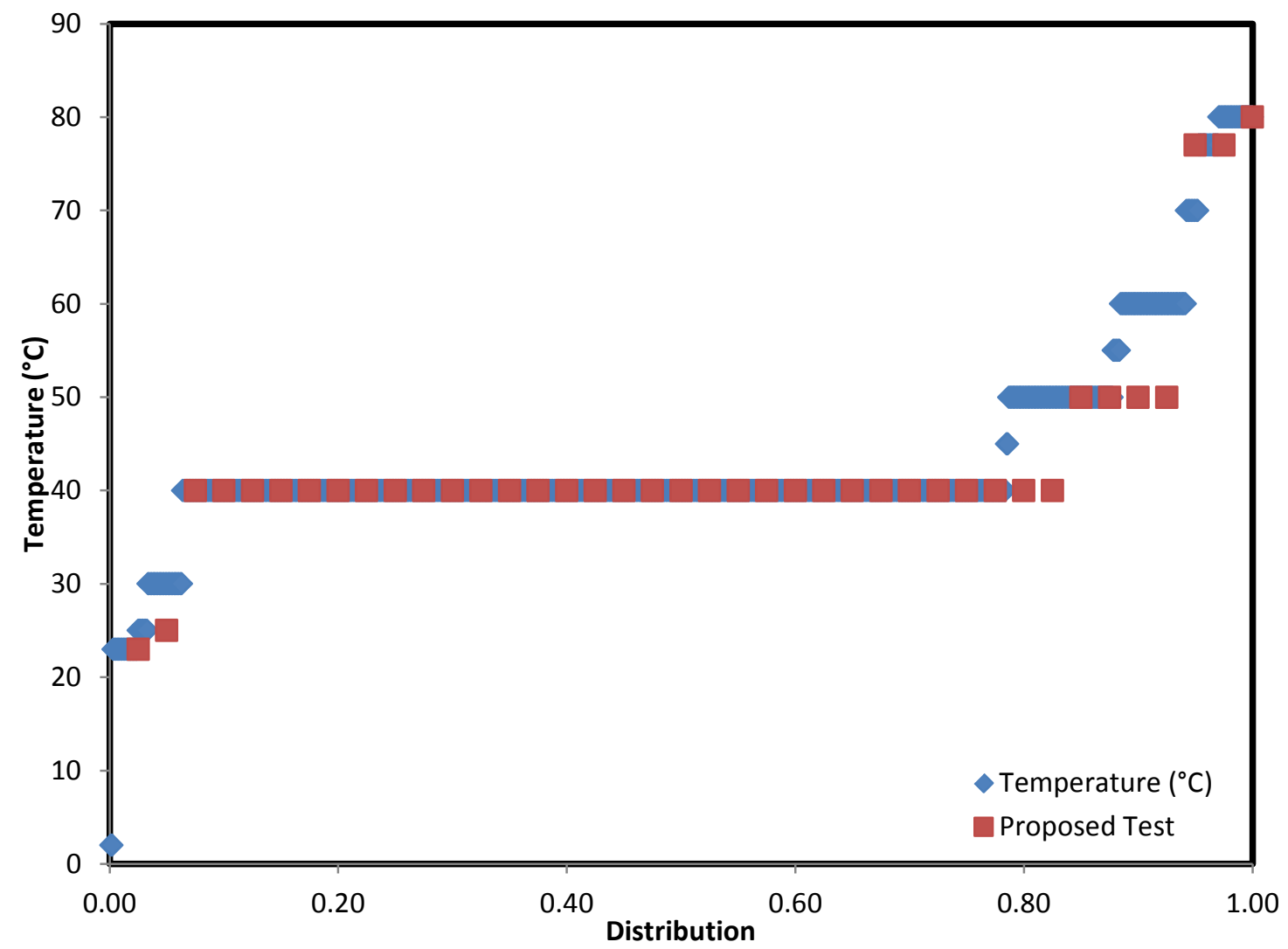

Figure 5-28 Temperature distribution for total distribution and 40 test matrix.

\subsubsection{Nitrate Concentration}

Figure 5-29 shows the distribution for the nitrate concentration that was observed for the total data base (blue diamonds) and the proposed test matrix (red squares). Approximately $90 \%$ of the tests from the total database were at a nitrate concentration of $1 \mathrm{M}$ or less. These nitrate concentrations occur during the washing process for feed preparation to the DWPF at SRS. The large source of data may enable the Hanford site to investigate the possibility of nitrite inhibition in dilute wastes. A limited amount of data exists at nitrate concentrations greater than $1 \mathrm{M}$. Most of this data is related to either Hanford simulants or SRS dissolved salt solutions. 


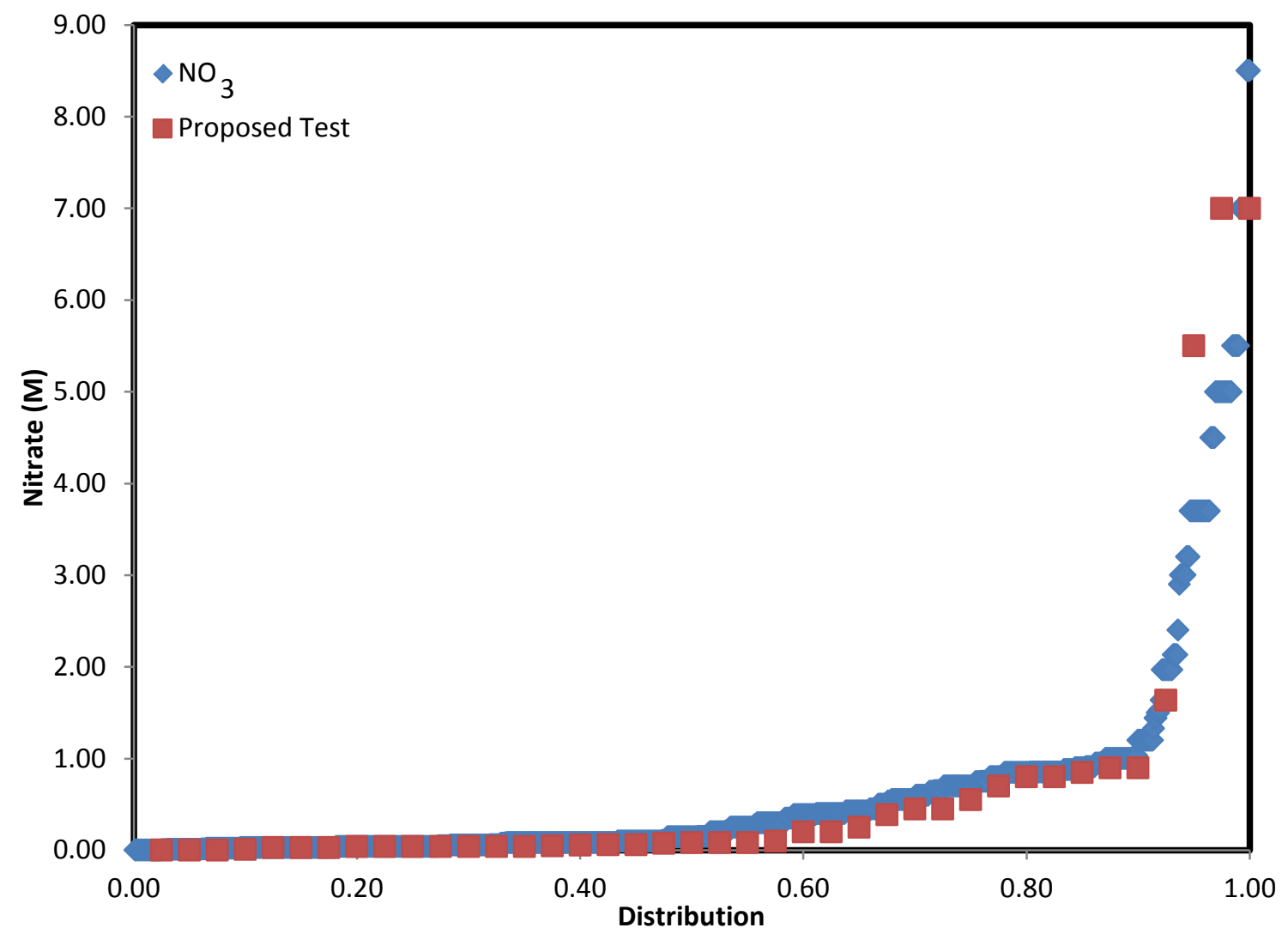

Figure 5-29 Temperature distribution for total distribution and 40 test matrix.

\subsubsection{Nitrite Concentration}

Figure 5-30 shows the distribution for the nitrite concentration that was observed for the total data base (blue diamonds) and the proposed test matrix (red squares). Again approximately $90 \%$ of the tests from the total database were at a nitrite concentration of $1 \mathrm{M}$ or less. These nitrite concentrations occur during the washing process for feed preparation to the DWPF at SRS. A limited amount of data exists at nitrite concentrations greater than $1 \mathrm{M}$. Most of this data is related to Hanford waste simulants. 


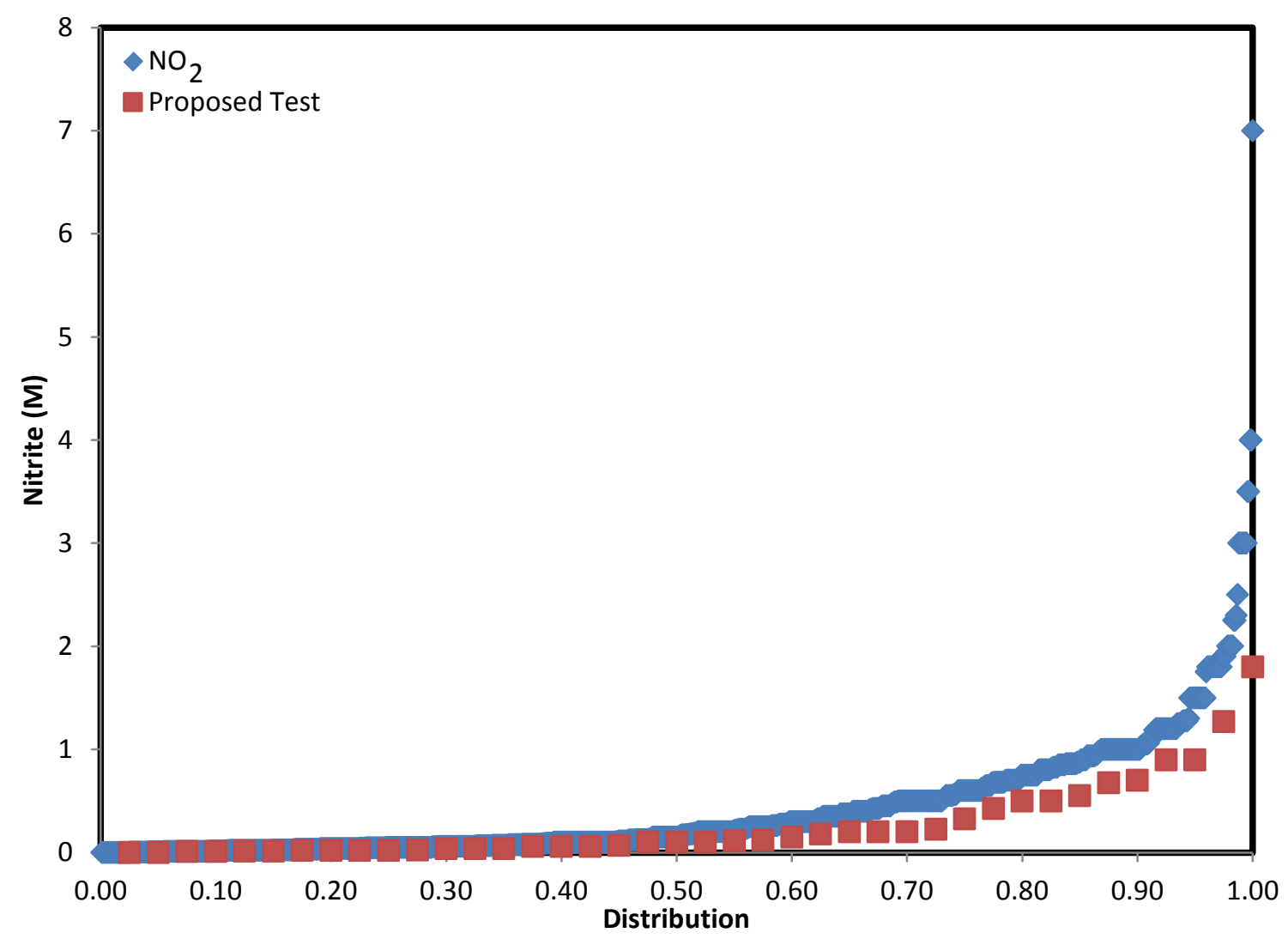

Figure 5-30 Nitrite concentration distribution for total distribution and 40 test matrix.

\subsubsection{Nitrite/Nitrate Ratio}

Figure 5-31 shows the distribution for the nitrite/nitrate ratio that was observed for the total data base (blue diamonds) and the proposed test matrix (red squares). Again approximately $80 \%$ of the tests from the total database were at a nitrite/nitrate ratio less than 1. Of particular interest to Hanford is the observation that $20 \%$ of the data was obtained at nitrite/nitrate ratios less than 0.15 . This nitrite/nitrate is the minimum required by the new SCC limits that are being established at Hanford [3]. 


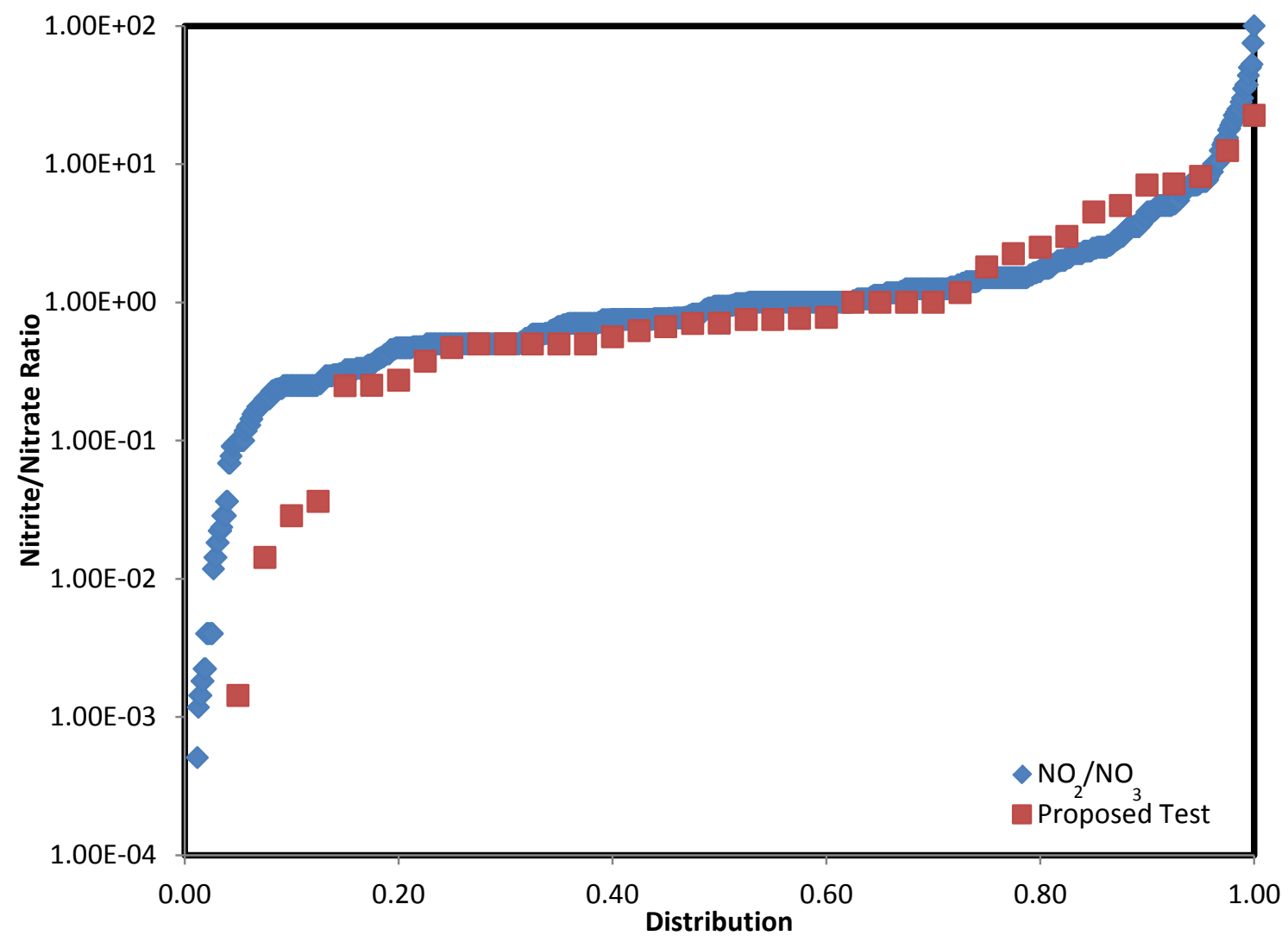

Figure 5-31 Nitrite/Nitrate ratio distribution for total distribution and 40 test matrix.

\subsubsection{CPP Results}

Results from historical CPP curves, where available, and the FY2014 testing were compared to determine how well test conditions were reproduced. No results from historical tests that line-up exactly with the FY2014 test conditions have not been located yet. However, an historical case with a similar waste composition to one of the FY2014 conditions was located in a paper [35]. The simulant compositions are shown in Table 5-10. The simulants are the same, except that the nitrite concentration for the historical case is slightly higher. The nitrite to nitrate ratio for the historical test was approximately 1, while for the FY2014 test the ratio was 0.76 . 
Table 5-10 Comparison of Chemistry for FY2014 Test Condition \#15 and Historical Test

\section{Condition}

\begin{tabular}{|c|c|c|}
\hline Simulant Source & $\begin{array}{c}\text { FY2014 } \\
\text { Concentration } \\
\text { (M) }\end{array}$ & $\begin{array}{c}\text { Historical } \\
\text { Concentration }\end{array}$ \\
\hline Sodium carbonate & 0.0263 & 0.0263 \\
\hline Sodium bicarbonate & 0.0564 & 0.0564 \\
\hline Sodium oxalate & 0.000268 & 0.000268 \\
\hline Sodium molybdate, dihydrate & 0.0000143 & 0.0000143 \\
\hline Sodium metasilicate, 5-hydrate & 0.000109 & 0.000109 \\
\hline Sodium phosphate, 12-hydrate & 0.000309 & 0.000309 \\
\hline Sodium chloride & 0.0011875 & 0.0011875 \\
\hline Sodium fluoride & 0.000573 & 0.000573 \\
\hline Sodium sulfate & 0.00503 & 0.00503 \\
\hline Sodium nitrate & 0.0758 & 0.0758 \\
\hline Sodium nitrite & 0.058 & 0.076 \\
\hline Sodium aluminate & 0.00045 & 0.00045 \\
\hline Cobalt nitrate, 6-hydrate & 0.00003 & 0.00003 \\
\hline Nickel nitrate, 6-hydrate & 0.0015 & 0.0015 \\
\hline Ferric nitrate, 9-hydrate & 0.000248 & 0.000248 \\
\hline Mercury (II) nitrate & 0.00025 & 0.00025 \\
\hline Cupric nitrate, 2.5 hydrate & 0.000043 & 0.000043 \\
\hline Manganese dioxide & 0.00575 & 0.00575 \\
\hline
\end{tabular}

The historical CPP curve is shown in Figure 5-32. No pitting was observed on the surface of the sample. The FY2014 Test 15 curve is shown in Figure 5-33. Negative hysteresis was observed, however, minor pitting was observed (i.e., Category 1 with additional testing recommended). The historical results at the Test 15 conditions concurred that this would potentially be a borderline test condition. Despite this difference, the curves illustrate similar polarization characteristics.

- $\quad$ The $\mathrm{E}_{\mathrm{zc}}$ is approximately the same, $0.1 \mathrm{~V}$ vs. SCE.

- Both curves exhibit an active/passive transition (i.e., $\mathrm{I}_{\max }, \mathrm{E}_{\max }$ ).

- The passive current density is slightly higher for Test 15 , however, they are of the same order of magnitude. 
SRNL-STI-2014-00616

Revision 0

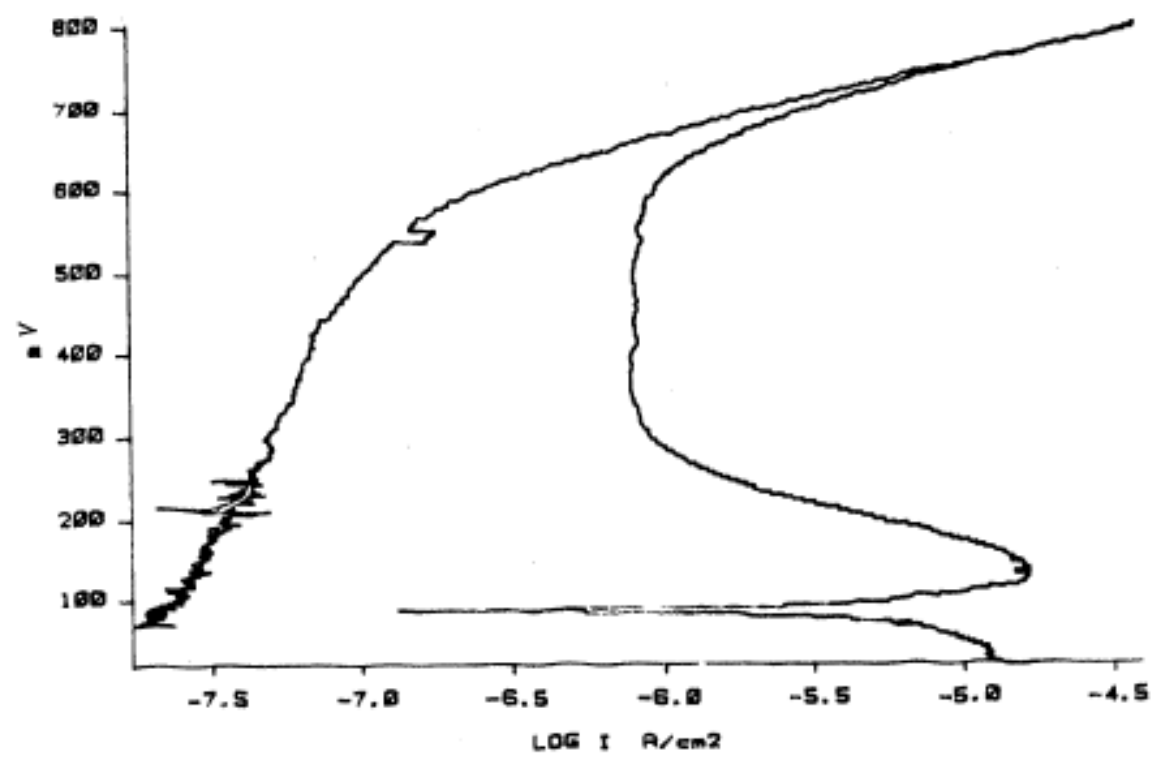

Figure 5-32 CPP curve from historical test data [35]. Note potential is with respect to SCE reference electrode.

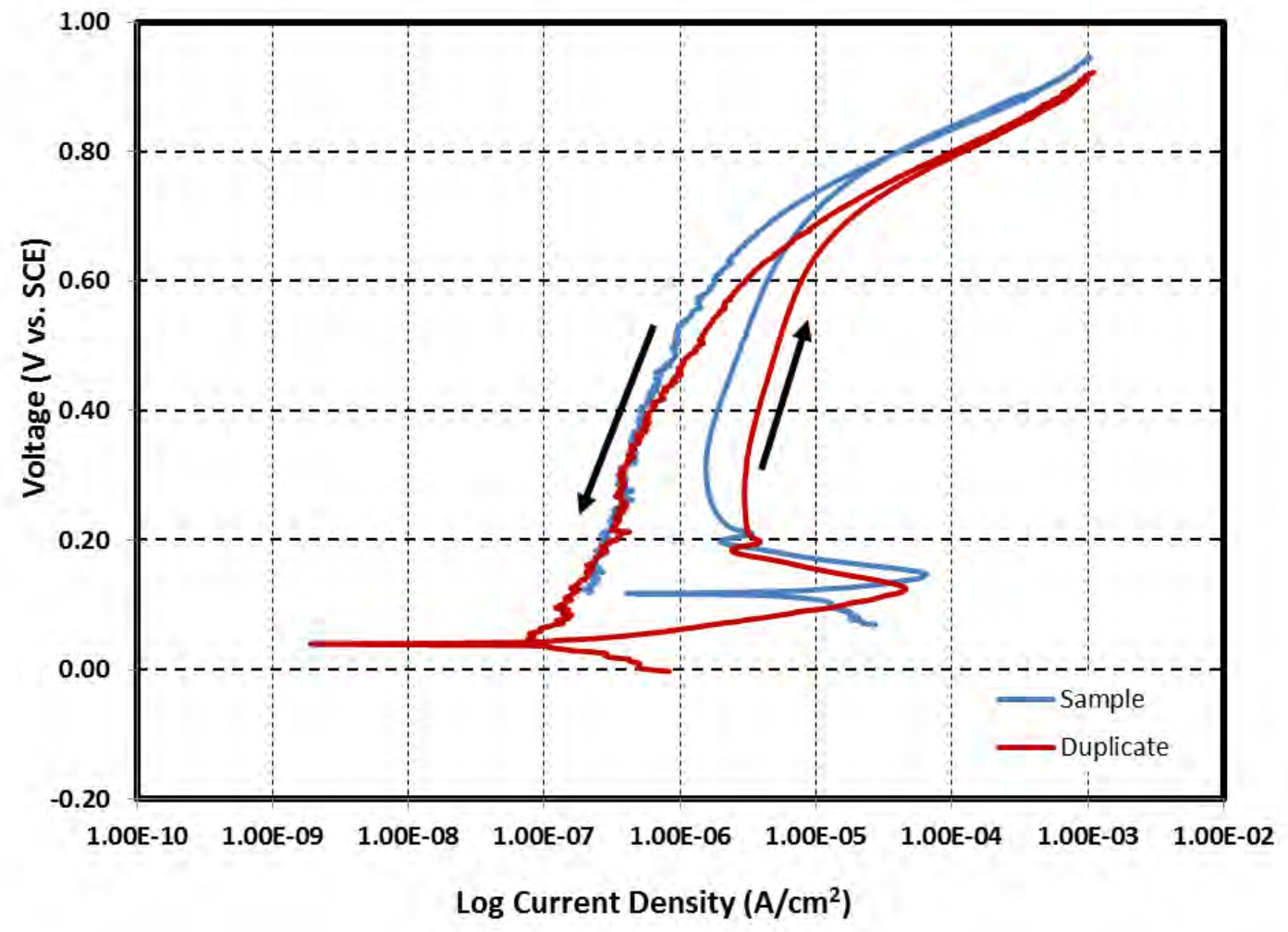

Figure 5-33 CPP curve from FY2014 Test 15. 
The CPP curves and photographs of the samples for the 40 tests are shown in Appendix G. A summary of the results is shown in Table 5-11 to 5-19. Important observations from the results included:

- $\quad 28$ of $40 \mathrm{CPP}$ tests (70\%) indicated pitting susceptibility. This proportion is higher than what was observed historically.

- 28 of the new CPP tests agreed with historical results, while there were 12 cases where there was disagreement.

- 10 of the 40 CPP tests (25\%) had companion coupon tests for comparison.

- For the FY2014 tests, the results were divided into one of four categories:

o Category 1, with no pits; a pass condition;

o Category 1, with pits; a fail condition with additional testing required;

o Undefined hysteresis with pits; a fail condition with additional testing required;

o Category 4, with pitting; a fail condition.

- No protection potentials were observed in any of the tests.

- For the pre-1993 CPP data, 16 were at borderline conditions were tested. Of the 16 borderline conditions, 10 new CPP tests were in disagreement with the historical data, while 6 were in agreement. In most cases (i.e., 7 of 10, \#10, \#15, \#20, \#22, \#29, \#31, \#39) the disagreement occurred as a previous pass result changed to a fail for the new tests. Minor pitting (i.e., small pits) was typically the cause of noting a failure even though negative hysteresis (Category 1) was observed. For all 7 conditions, the EPOC approach would recommend that additional testing be performed. For the 3 conditions where an historical fail condition changed to a pass for the new tests (\#1, \#8, \#26), it was noted from the CPP curves for the FY2014 tests that the vertex potential was much less than $1.0 \mathrm{~V}$. This observation suggests that during the historical test, where a vertex potential of $1.0 \mathrm{~V}$ was specified, the vertex current was greater than $1 \mathrm{~mA} / \mathrm{cm}^{2}$, and that pits may have been initiated at these high potentials. In these 3 cases, the FY2014 test results showed Category 1 behavior with no pitting. Therefore at these conditions, no additional testing would be required based on the FY2014 test result; however, the historical test result would suggest a fail condition or that additional testing would be necessary.

- 5 pre-1993 tests where clear cut pitting or no pitting was observed were tested with the new CPP protocol. In all 5 cases, the new CPP protocol produced identical results.

- Coupon tests were performed at 7 of the pre-1993 CPP test conditions. Four of the 7 cases were at border line conditions. For the borderline conditions, there were 2 cases in which duplicate coupons did not agree (i.e., one exhibited pitting and the other did not at conditions \#31 and \#39). In one case pitting was observed on duplicate coupons (i.e., \#40) and in the other case it was not (i.e.,\#26). The new CPP protocol results are in agreement with these coupon test results. For the 3 clear cut pitting or no pitting conditions (i.e., \#18,\# 21, and \#37), the new CPP protocol results were in agreement with the coupon test results.

- For the post-1993 tests, 4 conditions were borderline (\#16,\#17,\#24,\#36). Of the 4 borderline conditions 2 new CPP tests were in disagreement with the historical data (\#16, $\# 17)$, while 2 were in agreement (\#24,\#36). For the two cases that were in disagreement 
the historical data indicated that one CPP curve indicated no pitting, while the duplicate CPP scan indicated pitting. In both cases, the FY2014 results indicated a pass condition. In these cases, no additional testing would be required based on the FY2014 test result, however, the historical test result would suggest additional testing would be necessary. For the two cases that were in agreement, additional testing would have been recommended. An example of case where disagreement occurred will be discussed below (\#16).

- 15 post-1993 tests, where clear cut pitting of no pitting was observed, were tested with the new CPP protocol. In all 15 cases, the new CPP protocol and historical results were identical.

- Coupon tests were performed at 3 of the post-1993 CPP test conditions. All three conditions produced clear cut pitting on the coupons. The new CPP protocol accurately predicted pitting corrosion in these cases. 
Table 5-11 Summary of Historical and 2014 CPP Results

\begin{tabular}{|c|c|c|c|c|c|c|c|c|c|c|c|}
\hline .0̃ & 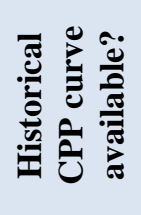 & 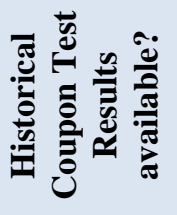 & 플 & 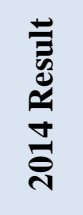 & 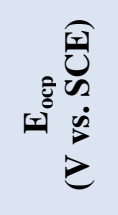 & 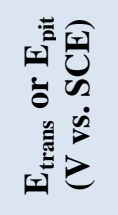 & 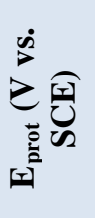 & 事 & 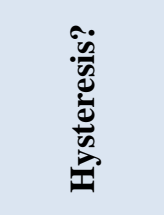 & 葛 & Comments \\
\hline 1 & No [43] & No & Fail & Pass & -0.205 & 0.618 & NA & None & Negative & 1 & $\begin{array}{l}\text { Historical CPP data indicated occasional } \\
\text { pitting; borderline condition }\end{array}$ \\
\hline 2 & Yes [51] & No & Pass & Pass & -0.570 & 0.266 & NA & None & Negative & 1 & $\begin{array}{l}\text { Historical CPP data indicated negative } \\
\text { hysteresis with not pitting. }\end{array}$ \\
\hline 3 & Yes [49] & No & Fail & Fail & -0.617 & -0.087 & NA & Major & Positive & 4 & $\begin{array}{l}\text { Historical CPP data indicated positive } \\
\text { hysteresis with major pitting. }\end{array}$ \\
\hline 4 & Yes [49] & No & Fail & Fail; & -0.628 & 0.142 & NA & Minor & Undefined & Undefined & $\begin{array}{l}\text { Historically, } 5 \text { CPP tests were performed } \\
\text { at this condition; Minor pitting was } \\
\text { observed on } 3 \text { samples, while no pitting } \\
\text { was observed on } 2 \text { samples; borderline } \\
\text { condition }\end{array}$ \\
\hline 5 & No [43] & No & Pass & Pass & -0.225 & 0.591 & NA & None & Negative & 1 & $\begin{array}{l}\text { Historical CPP data indicated no pitting; } \\
\text { line delineating pit/no pit regions passes } \\
\text { through data point; borderline condition }\end{array}$ \\
\hline 6 & No [43] & No & Fail & Fail & -0.196 & 0.406 & NA & Major & Positive & 4 & $\begin{array}{l}\text { 1.Historical CPP data indicated } \\
\text { occasional pitting; high chloride; } \\
\text { 2. New CPP curve has strange pattern }\end{array}$ \\
\hline 7 & Yes [49] & No & Fail & Fail & -0.373 & -0.070 & NA & Major & Positive & 4 & $\begin{array}{l}\text { Historical CPP data indicated positive } \\
\text { hysteresis with major pitting. }\end{array}$ \\
\hline 8 & No [43] & No & Fail & Pass & -0.235 & 0.623 & NA & None & Negative & 1 & $\begin{array}{l}\text { Historical CPP data indicated occasional } \\
\text { pitting; No pits were observed with what } \\
\text { appears to be only a small addition nitrite; } \\
\text { borderline condition. }\end{array}$ \\
\hline
\end{tabular}

AT: Additional Testing 
Table 5-12 Summary of Historical and 2014 CPP Results (continued 1)

\begin{tabular}{|c|c|c|c|c|c|c|c|c|c|c|c|}
\hline 兽 & 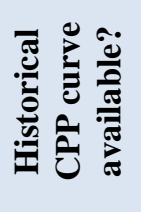 & 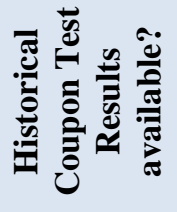 & 점 & 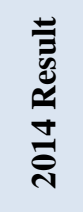 & 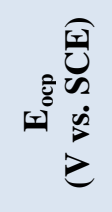 & 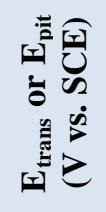 & 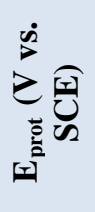 & .0. & 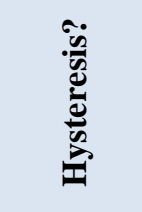 & 茪 & Comments \\
\hline 9 & No [50] & Yes [50] & Fail & Fail & -0.319 & -0.026 & NA & Major & Positive & 4 & $\begin{array}{l}\text { 1. Historical CPP data indicated pitting. } \\
\text { Positive hysteresis and pitting was } \\
\text { observed. } \\
\text { 2. Crevice corrosion was observed on } \\
\text { coupons after } 1 \text { and } 6 \text { months. General } \\
\text { corrosion was observed on the coupon } \\
\text { after } 11 \text { months of exposure. } \\
\text { 3. Active peak evident in forward scan. }\end{array}$ \\
\hline 10 & No [43] & No & Pass & $\begin{array}{c}\text { Fail; } \\
\text { AT }\end{array}$ & -0.232 & 0.602 & NA & Minor & Negative & 1 & $\begin{array}{l}\text { Historical CPP data indicated no pitting; } \\
\text { However, at a nitrite concentration that } \\
\text { appears to be about } 0.01 \mathrm{M} \text { less, } \\
\text { occasional pitting was observed; } \\
\text { borderline condition }\end{array}$ \\
\hline 11 & No [43] & No & Fail & $\begin{array}{c}\text { Fail; } \\
\text { AT }\end{array}$ & -0.224 & 0.625 & NA & Minor & Negative & 1 & $\begin{array}{l}\text { Historical CPP data indicated occasional } \\
\text { pitting; However, the line that separates } \\
\text { the pit/no pit region nearly passes through } \\
\text { the data point; borderline condition. }\end{array}$ \\
\hline 12 & No [50] & Yes [50] & Fail & Fail & -0.305 & -0.100 & NA & Major & Positive & 4 & $\begin{array}{l}\text { 1. Historical CPP data indicated pitting. } \\
\text { Positive hysteresis and pitting were } \\
\text { observed. } \\
\text { 2. Crevice corrosion on a coupon was } \\
\text { observed after } 1 \text { month, pitting corrosion } \\
\text { was observed on a coupon after } 6 \text { months, } \\
\text { and pitting and crevice corrosion were } \\
\text { observed on a coupon after } 11 \text { months. }\end{array}$ \\
\hline
\end{tabular}

AT: Additional Testing 
Table 5-13 Summary of Historical and 2014 CPP Results (continued 2)

\begin{tabular}{|c|c|c|c|c|c|c|c|c|c|c|c|}
\hline 竭 & 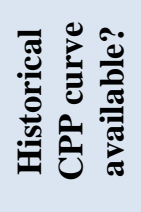 & 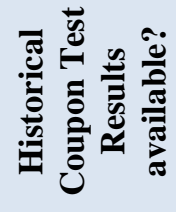 & 를 & 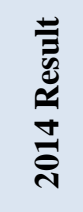 & 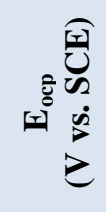 & 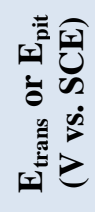 & 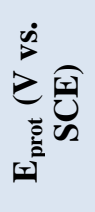 & 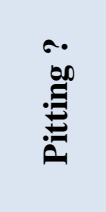 & 苞 & 范 & Comments \\
\hline 13 & No [37] & No & Fail & Fail & -0.027 & 0.672 & NA & Major & Positive & 4 & $\begin{array}{l}\text { 1. Historical CPP data indicated } \\
\text { occasional pitting; Line delineating pit/no } \\
\text { pit region passes through the data point; } \\
\text { borderline condition. } \\
\text { 2. Active peak evident in forward scan. }\end{array}$ \\
\hline 14 & $\begin{array}{c}\text { No }[34, \\
35]\end{array}$ & No & Fail & $\begin{array}{c}\text { Fail; } \\
\text { AT }\end{array}$ & 0.108 & 0.775 & NA & Minor & Negative & 1 & $\begin{array}{l}\text { 1. Historical CPP data indicated pitting; } \\
\text { Increasing the nitrite concentration by a } \\
\text { small amount eliminated pitting; } \\
\text { borderline condition. } \\
\text { 2. Active peak evident in forward scan. }\end{array}$ \\
\hline 15 & $\begin{array}{c}\text { No }[34, \\
35]\end{array}$ & No & Pass & $\begin{array}{c}\text { Fail; } \\
\text { AT }\end{array}$ & 0.078 & 0.67 & NA & Minor & Negative & 1 & $\begin{array}{l}\text { 1. Historical CPP data indicated no } \\
\text { pitting; borderline condition } \\
\text { 2. Active peak evident in forward scan; } \\
\text { 3. Coupon test results at a similar nitrate } \\
\text { concentration }(0.055 \mathrm{M}) \text { and the same } \\
\text { nitrite/nitrate ratio }(0.76) \text { exhibited no } \\
\text { pitting. }\end{array}$ \\
\hline 16 & Yes [47] & No & Fail & Pass & -0.270 & 0.719 & NA & None & Negative & 1 & $\begin{array}{l}\text { Historically, one CPP curve had positive } \\
\text { hysteresis, the other curve had negative } \\
\text { hysteresis; borderline condition. }\end{array}$ \\
\hline 17 & Yes [46] & No & Fail & Pass & -0.257 & 0.694 & NA & None & Negative & 1 & $\begin{array}{l}\text { Historically, one CPP result indicated no } \\
\text { pitting, while the second result was } \\
\text { negative hysteresis with minor pitting; } \\
\text { borderline condition. }\end{array}$ \\
\hline
\end{tabular}

AT: Additional Testing 
Table 5-14 Summary of Historical and 2014 CPP Results (continued 3)

\begin{tabular}{|c|c|c|c|c|c|c|c|c|c|c|c|}
\hline 总 & 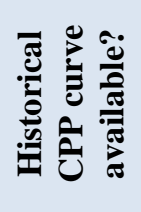 & 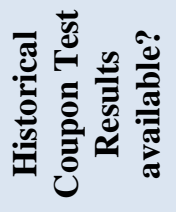 & 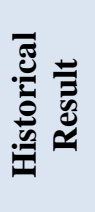 & 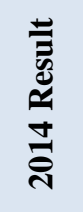 & 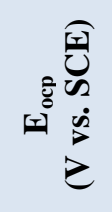 & 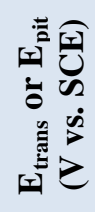 & 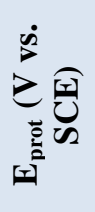 & .0. & 䓪 & 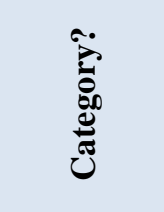 & Comments \\
\hline 18 & No [36] & Yes [57] & Fail & Fail & 0.108 & 0.78 & NA & Major & Positive & 4 & $\begin{array}{l}\text { 1. Historical CPP data indicated pitting; } \\
\text { Increasing the nitrite concentration by a } \\
\text { small amount eliminated pitting; } \\
\text { borderline condition; } \\
\text { 2. Historical coupon test indicated } \\
\text { pitting; } \\
\text { 3. Active peak evident in forward scan. }\end{array}$ \\
\hline 19 & Yes [47] & No & Fail & $\begin{array}{l}\text { Fail; } \\
\text { AT }\end{array}$ & -0.279 & 0.696 & NA & Minor & $\begin{array}{c}\text { Positive/ } \\
\text { Undefined }\end{array}$ & $\begin{array}{c}4 / \\
\text { Undefined } \\
\end{array}$ & $\begin{array}{l}\text { Historical CPP data indicated positive } \\
\text { hysteresis with moderate pitting. }\end{array}$ \\
\hline 20 & No [37] & No & Pass & Fail; & 0.100 & 0.737 & NA & Minor & Undefined & Undefined & $\begin{array}{l}\text { 1. Historical CPP data indicated no } \\
\text { pitting; Line delineating pit/no pit regions } \\
\text { passes through the data point; borderline } \\
\text { condition. } \\
\text { 2. Active peak evident in forward scan. }\end{array}$ \\
\hline 21 & No [36] & Yes [57] & Fail & Fail; & 0.128 & 0.680 & NA & Major & Undefined & Undefined & $\begin{array}{l}\text { 1. Historical CPP data indicated pitting; } \\
\text { Increasing the nitrite concentration by a } \\
\text { small amount eliminated pitting; } \\
\text { borderline condition; } \\
\text { 2. Historical coupon test indicated } \\
\text { pitting; } \\
\text { 3. Active peak evident in forward scan. }\end{array}$ \\
\hline
\end{tabular}

AT: Additional Testing 
Table 5-15 Summary of Historical and 2014 CPP Results (continued 4)

\begin{tabular}{|c|c|c|c|c|c|c|c|c|c|c|c|}
\hline & 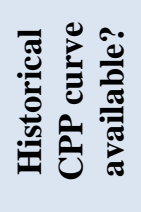 & 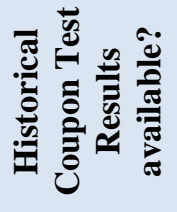 & ⿹ㅠㄹ & 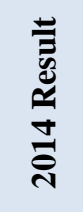 & 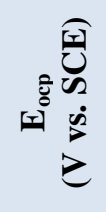 & 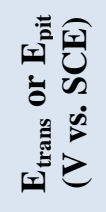 & 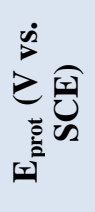 & 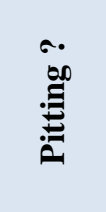 & 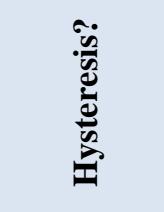 & 苛 & Comments \\
\hline 22 & No [36] & No & Pass & $\begin{array}{l}\text { Fail; } \\
\text { AT }\end{array}$ & 0.087 & 0.695 & NA & Minor & Negative & 1 & $\begin{array}{l}\text { 1. Historical CPP data indicated that no } \\
\text { pitting was observed. However, the line } \\
\text { dividing the pitting from no pitting } \\
\text { condition was drawn through this data } \\
\text { point. This is a borderline condition; } \\
\text { 2. Active peak evident in forward scan. } \\
\text { 3. Pits could not be seen with the unaided } \\
\text { eye. }\end{array}$ \\
\hline 23 & Yes [47] & No & Pass & Pass & -0.274 & 0.707 & NA & None & Negative & 1 & $\begin{array}{l}\text { Historical CPP data showed negative } \\
\text { hysteresis with no pitting. }\end{array}$ \\
\hline 24 & Yes [28] & No & Fail & $\begin{array}{l}\text { Fail; } \\
\text { AT }\end{array}$ & -0.224 & $\begin{array}{c}0.684 / \\
0.606\end{array}$ & NA & $\begin{array}{l}\text { None/ } \\
\text { Minor }\end{array}$ & $\begin{array}{l}\text { Negative/ } \\
\text { Undefined }\end{array}$ & $\begin{array}{c}1 / \\
\text { Undefined }\end{array}$ & $\begin{array}{l}\text { 1. Historical CPP data indicated negative } \\
\text { hysteresis with moderate pitting. A } \\
\text { borderline condition. This test condition } \\
\text { is that was used for the round robin } \\
\text { testing in FY2013 [2]. This condition } \\
\text { was used to explore the borderline } \\
\text { condition. } \\
2 \text {. New CPP data had mixed results. One } \\
\text { CPP curve showed negative hysteresis } \\
\text { with no pitting on the sample. The } \\
\text { second CPP curve exhibited an undefined } \\
\text { characteristic, with minor pitting. }\end{array}$ \\
\hline 25 & $\begin{array}{c}\text { No }[34, \\
35]\end{array}$ & No & Fail & $\begin{array}{l}\text { Fail; } \\
\text { AT }\end{array}$ & 0.131 & 0.739 & NA & Minor & Negative & 1 & $\begin{array}{l}\text { 1. Historically, CPP data indicated } \\
\text { occasional pitting; borderline condition } \\
\text { 2. Active peak evident in forward scan }\end{array}$ \\
\hline
\end{tabular}

AT: Additional Testing 
Table 5-16 Summary of Historical and 2014 CPP Results (continued 5)

\begin{tabular}{|c|c|c|c|c|c|c|c|c|c|c|c|}
\hline 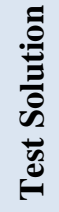 & 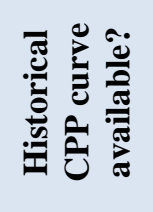 & 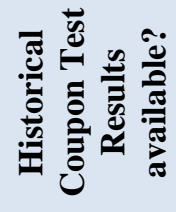 & 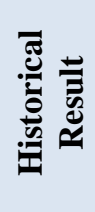 & 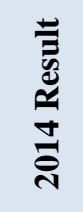 & 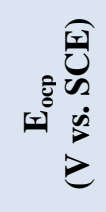 & 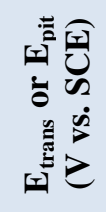 & 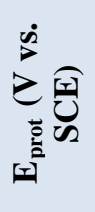 & 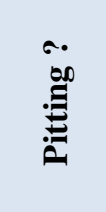 & 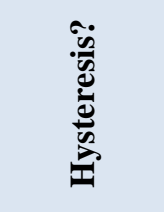 & 居 & Comments \\
\hline 26 & $\begin{array}{c}\text { No }[34, \\
35]\end{array}$ & Yes [27] & Fail & Pass & $\begin{array}{l}0.025 / \\
0.127\end{array}$ & $\begin{array}{c}0.546 / \\
0.813\end{array}$ & NA & None & Negative & 1 & $\begin{array}{l}\text { 1. Historically, } 1 \text { CPP curve indicated no } \\
\text { pitting, while the replicate indicated } \\
\text { pitting; borderline condition } \\
\text { 2. Active peak evident in forward scan; } \\
\text { 3. Test was not as reproducible as others. }\end{array}$ \\
\hline 27 & Yes [47] & No & Fail & Fail & -0.289 & 0.400 & NA & Major & Positive & 4 & $\begin{array}{l}\text { Historical CPP curves showed undefined } \\
\text { or negative hysteresis. Moderate pitting } \\
\text { was observed on the sample. }\end{array}$ \\
\hline 28 & Yes [28] & No & Pass & Pass & -0.222 & 0.692 & NA & None & Negative & 1 & $\begin{array}{l}\text { Historical CPP data indicated negative } \\
\text { hysteresis with no pitting. }\end{array}$ \\
\hline 29 & No [37] & No & Pass & $\begin{array}{c}\text { Fail; } \\
\text { AT }\end{array}$ & 0.116 & 0.787 & NA & Minor & Undefined & Undefined & $\begin{array}{l}\text { 1. Historically, no pits were observed for } \\
\text { the CPP test. However, line that } \\
\text { separates the pit/no pit region goes } \\
\text { through this data point; borderline } \\
\text { condition. } \\
\text { 2. Two active peaks evident in forward } \\
\text { scan. }\end{array}$ \\
\hline 30 & Yes [28] & No & Pass & Pass & -0.247 & 0.624 & NA & None & Negative & 1 & $\begin{array}{l}\text { Historical CPP data indicated negative } \\
\text { hysteresis and no pitting. }\end{array}$ \\
\hline 31 & No [36] & Yes [57] & Pass & $\begin{array}{c}\text { Fail; } \\
\text { AT }\end{array}$ & 0.102 & 0.705 & NA & Minor & Negative & 1 & $\begin{array}{l}\text { 1. Historical CPP data indicated no } \\
\text { pitting was observed; However, line } \\
\text { separating pit/no pit region passes } \\
\text { through the data point; borderline } \\
\text { condition. } \\
\text { 2. Historical coupon tests indicate that } 1 \\
\text { in } 4 \text { coupons showed evidence of pitting } \\
\text { at a similar simulant composition. } \\
\text { 3. Active peak evident in forward scan. }\end{array}$ \\
\hline
\end{tabular}

AT: Additional Testing 
Table 5-17 Summary of Historical and 2014 CPP Results (continued 6)

\begin{tabular}{|c|c|c|c|c|c|c|c|c|c|c|c|}
\hline 章 & 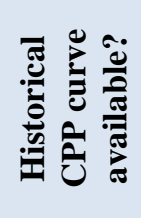 & 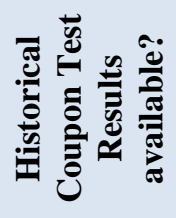 & 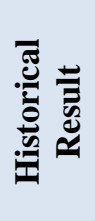 & 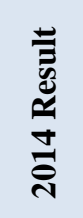 & 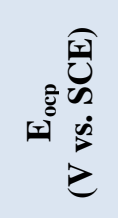 & 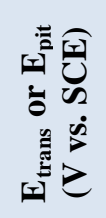 & 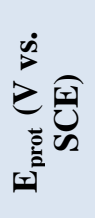 & .0. & 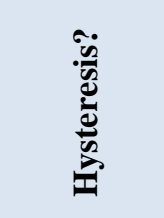 & 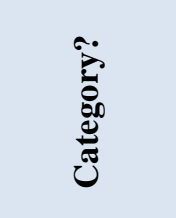 & Comments \\
\hline 32 & No [45] & No & Pass & Pass & -0.249 & 0.639 & NA & None & Negative & 1 & $\begin{array}{l}\text { Historical CPP data indicated no pitting } \\
\text { was observed. }\end{array}$ \\
\hline 33 & Yes [47] & Yes [47] & Fail & Fail & -0.200 & $\begin{array}{c}0.400 / \\
0.600\end{array}$ & NA & Minor & $\begin{array}{l}\text { Positive/ } \\
\text { Negative }\end{array}$ & $4 / 1$ & $\begin{array}{l}\text { 1. Historical CPP data indicated positive } \\
\text { hysteresis with significant pitting on the } \\
\text { sample. } \\
\text { 2. Partially immersed coupon tests were } \\
\text { conducted on as-received and heat treated } \\
\text { samples for } 19 \text { weeks. The samples } \\
\text { exhibited minor weight loss with the } \\
\text { maximum pit depths between } 18 \text { to } 27 \\
\text { mils. Pits were located near liquid air } \\
\text { interface. } \\
3 \text {. New CPP data had } 1 \text { case of positive } \\
\text { hysteresis with pitting and } 1 \text { case of } \\
\text { negative hysteresis with minor pitting. }\end{array}$ \\
\hline 34 & Yes [51] & No & Fail & Fail & $\begin{array}{l}-0.242 / \\
-0.171\end{array}$ & $\begin{array}{c}0.131 / \\
0.290\end{array}$ & NA & Major & Positive & 4 & $\begin{array}{l}\text { Historical CPP data indicated positive } \\
\text { hysteresis with major pitting. }\end{array}$ \\
\hline 35 & Yes [47] & No & Fail & Fail & $\begin{array}{l}-0.158 \\
/ 0.126\end{array}$ & $\begin{array}{c}0.663 / \\
0.740\end{array}$ & NA & Minor & $\begin{array}{l}\text { Positive/ } \\
\text { Undefined }\end{array}$ & $\begin{array}{c}4 / \\
\text { Undefined }\end{array}$ & $\begin{array}{l}\text { 1. Historical CPP curves showed positive } \\
\text { hysteresis with major pitting on the } \\
\text { sample. } \\
\text { 2. New CPP curves showed positive } \\
\text { hysteresis or undefined hysteresis with } \\
\text { pitting present on the sample. } \\
\text { 3. Reproducibility not as good for this } \\
\text { test. }\end{array}$ \\
\hline
\end{tabular}


Table 5-18 Summary of Historical and 2014 CPP Results (continued 7)

\begin{tabular}{|c|c|c|c|c|c|c|c|c|c|c|c|}
\hline 章 & 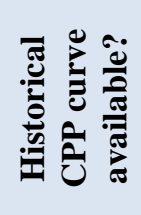 & 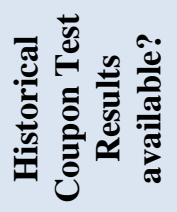 & 플 & 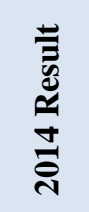 & 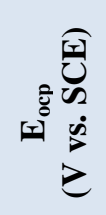 & 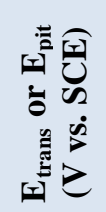 & 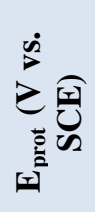 & 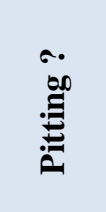 & 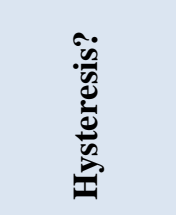 & 胥 & Comments \\
\hline 36 & Yes [48] & No & Fail & $\begin{array}{l}\text { Fail; } \\
\text { AT }\end{array}$ & -0.270 & 0.625 & NA & Minor & Negative & 1 & $\begin{array}{l}\text { 1. Historical CPP curves showed } \\
\text { undefined hysteresis with minor pitting. } \\
\text { A borderline condition. } \\
\text { 2. New CPP curves exhibited negative } \\
\text { hysteresis with minor pitting observed. }\end{array}$ \\
\hline 37 & No [36] & Yes [57] & Fail & $\begin{array}{l}\text { Fail; } \\
\text { AT }\end{array}$ & 0.093 & 0.737 & NA & Major & Undefined & Undefined & $\begin{array}{l}\text { 1. Historical CPP data indicated pitting. } \\
\text { 2. Historical coupon tests indicated } \\
\text { pitting susceptibility. } \\
\text { 3. Active peak evident in forward scan. }\end{array}$ \\
\hline 38 & Yes [51] & No & Pass & Pass & -0.300 & 0.518 & NA & None & Negative & 1 & $\begin{array}{l}\text { Historically, CPP curves had negative } \\
\text { hysteresis with no pitting on the sample. } \\
\text { This was AY-102 Present Interstitial } \\
\text { Liquid (PIL) with no nitrite. }\end{array}$ \\
\hline 39 & No [36] & Yes [57] & Pass & $\begin{array}{l}\text { Fail; } \\
\text { AT }\end{array}$ & 0.102 & 0.764 & NA & Minor & Negative & 1 & $\begin{array}{l}\text { 1. Historical CPP data indicated no } \\
\text { pitting; However, line separating pit/no } \\
\text { pit region passes through the data point; } \\
\text { A borderline condition. } \\
\text { 2. Historical coupon data indicated that } 1 \\
\text { in } 4 \text { coupons showed evidence of pitting } \\
\text { at a similar simulant composition. } \\
\text { 3. Active peak evident in forward scan. }\end{array}$ \\
\hline
\end{tabular}

AT: Additional Testing 
Table 5-19 Summary of Historical and 2014 CPP Results (continued 8)

\begin{tabular}{|c|c|c|c|c|c|c|c|c|c|c|c|}
\hline & 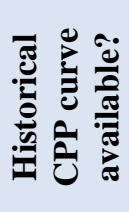 & 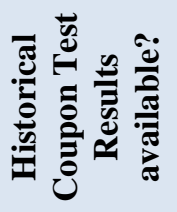 & 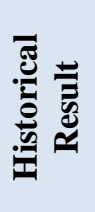 & 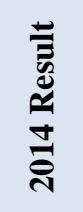 & 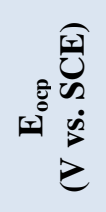 & 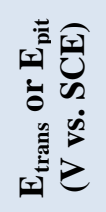 & 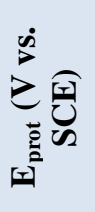 & 范 & 苞 & 葛 & Comments \\
\hline 40 & No [36] & Yes [57] & Fail & $\begin{array}{l}\text { Fail; } \\
\text { AT }\end{array}$ & 0.097 & 0.800 & NA & Minor & Undefined & Undefined & $\begin{array}{l}\text { 1. Historical CPP data indicated } \\
\text { occasional pitting; Line separating pit/no } \\
\text { pit region passes through the data point; } \\
\text { borderline condition. } \\
\text { 2. Historical coupon data indicated that } \\
\text { this may be a borderline condition. } \\
\text { Pitting was observed at nitrite } \\
\text { concentrations slightly below this } \\
\text { simulant }(0.0085 \mathrm{M}) \text { concentration, and } \\
\text { no pitting was observed at nitrite } \\
\text { concentrations slightly above this } \\
\text { simulant concentration }(0.03 \mathrm{M}) \text {. } \\
\text { 3. Active peak evident in forward scan. }\end{array}$ \\
\hline
\end{tabular}

AT: Additional Testing

Table 5-20 Composition of Post-1993 test and FY2014 Test 16 (M).

\begin{tabular}{|c|c|c|c|c|c|c|c|c|c|c|}
\hline \multirow{2}{*}{$\begin{array}{c}\text { Temperature } \\
\left({ }^{\circ} \mathrm{C}\right)\end{array}$} & \multicolumn{9}{|c|}{ Concentration of Species (M) } & \multirow{2}{*}{ pH } \\
\hline & $\mathrm{CO}_{3}{ }^{=}$ & $\mathrm{HCO}_{3}{ }^{=}$ & Citrate & $\mathrm{PO}_{4}{ }^{3-}$ & $\mathrm{Cl}^{-}$ & $\mathbf{F}^{-}$ & $\mathrm{SO}_{4}{ }^{2-}$ & $\mathrm{NO}_{3}{ }^{-}$ & $\mathrm{NO}_{2}^{-}$ & \\
\hline 40 & 0.0526 & 0.098 & NA & NA & 0.0037 & $\mathrm{NA}$ & 0.0451 & 0.2 & 0.15 & 10 \\
\hline
\end{tabular}


SRNL-STI-2014-00616

Revision 0

Table 5-21 Comparison of Post 1993 CPP Test Protocol and New Standardized CPP Protocol.

\begin{tabular}{|c|c|c|}
\hline Parameters & $\begin{array}{c}\text { Post-1993 SRNL CPP } \\
\text { protocol }\end{array}$ & $\begin{array}{l}\text { New Standardized } \\
\text { CPP protocol }\end{array}$ \\
\hline Potential Stabilization Time (hrs) & 2 & 2 \\
\hline Initial Potential (V vs. OCP) & -0.1 & -0.05 \\
\hline Scan Rate (mV/s) & 0.5 & 0.167 \\
\hline Vertex Threshold ( V vs. SCE or $\mathrm{mA} / \mathrm{cm}^{2}$ ) & $1.2 \mathrm{~V}$ & $1 \mathrm{~mA} / \mathrm{cm}^{2}$ \\
\hline Final Potential ( V vs. OCP) & 0.0 & 0.0 \\
\hline Sample Geometry & Disk & Bullet \\
\hline Surface Finish & 800 grit & 600 grit \\
\hline
\end{tabular}

An example of a case in which there was disagreement between a post-1993 test and one of the 40 tests that was performed in FY2014, is presented below. The solution that was tested had the composition shown in Table 5-20. At this nitrite to nitrate ratio, pre-1993 CPP data indicates that this may be a borderline condition [55]. A comparison of the CPP protocol that was used for the post-1993 test [47] that produced contrary results and the new CPP protocol (i.e., Test 16) is shown in Table 5-21. There were several differences in the protocol that was performed for this set of tests, the primary being the scan rate and the vertex threshold.

Another difference that existed between the tests is the steel that was tested. ASTM A537 carbon steel was used in the historical test, while the rail car steel was utilized in the current tests. Micrographs of each of the steels are shown in Figure 5-34. Tests were performed with the sample oriented in the short direction (Figure 5-26 (c)). Differences in the grain sizes (i.e., finer grain size for the A537) and manganese sulfide inclusion density (greater for the A537 steel) were noted. The higher density of manganese sulfide inclusions would tend to make a material more susceptible to pitting [59]. 


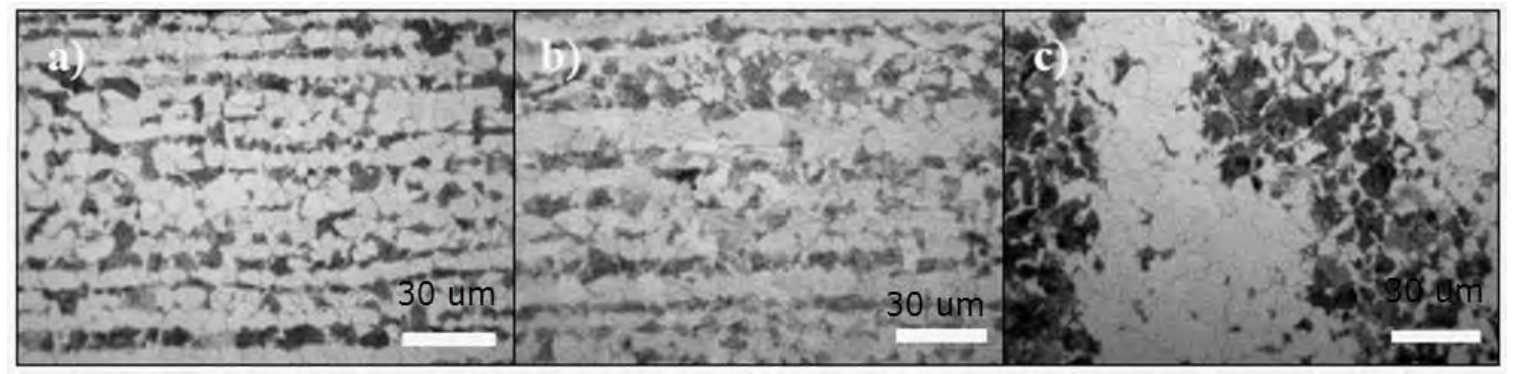

(a)

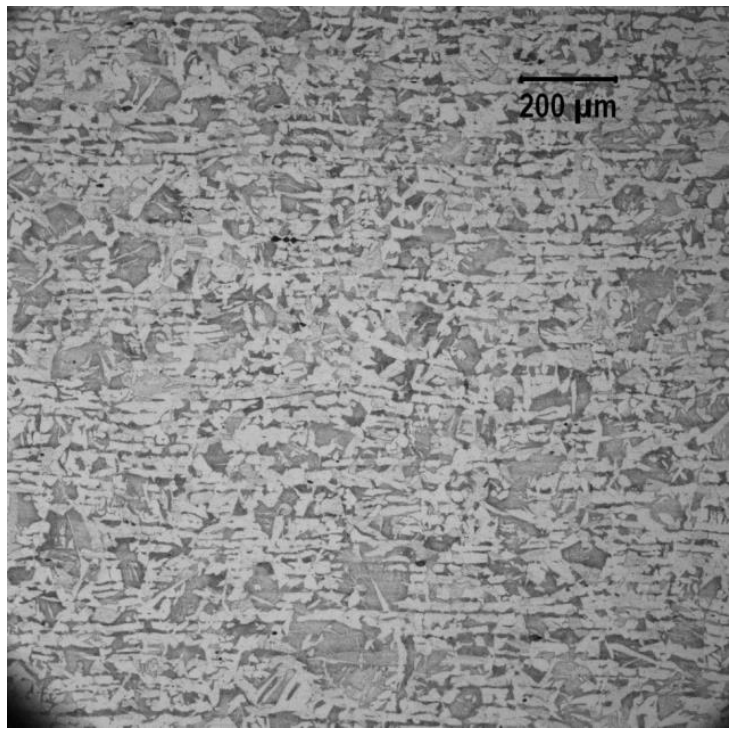

(d) (b)

(c)

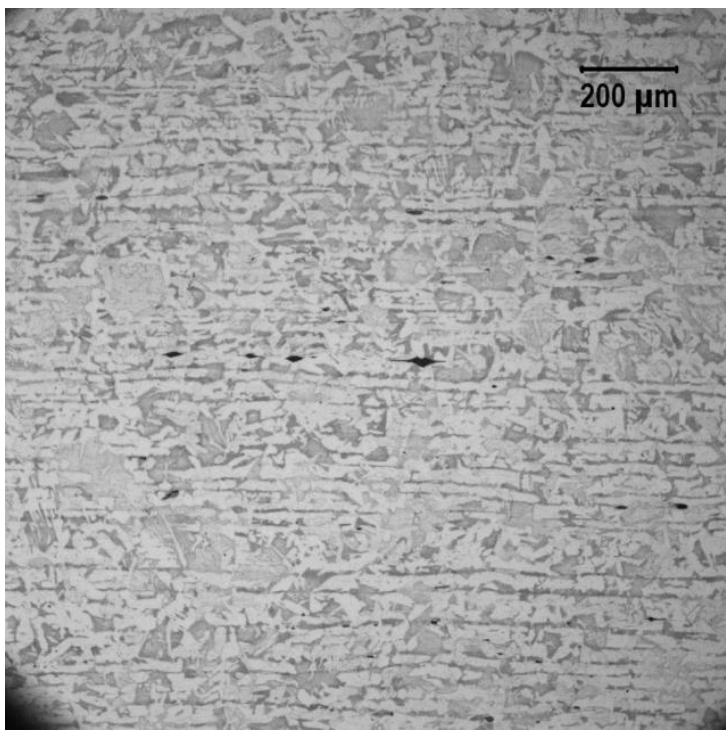

(e)

Figure 5-34 Micrographs (a), (b), and (c) are the longitudinal, transverse, and short directions for the A537 that was tested, while micrographs (d) and (e) are the longitudinal and transverse direction for the rail car steel that was tested.

Figure 5-35 compares the open circuit potential transients for the A537 and rail car steels that was measured during the potential stabilization time. Although both were exposed for two hours, the potential for the rail car steel appears to have stabilized, whereas the potential for the A537 steel continued to increase, which indicates that perhaps the oxide had not stabilized on the surface. 


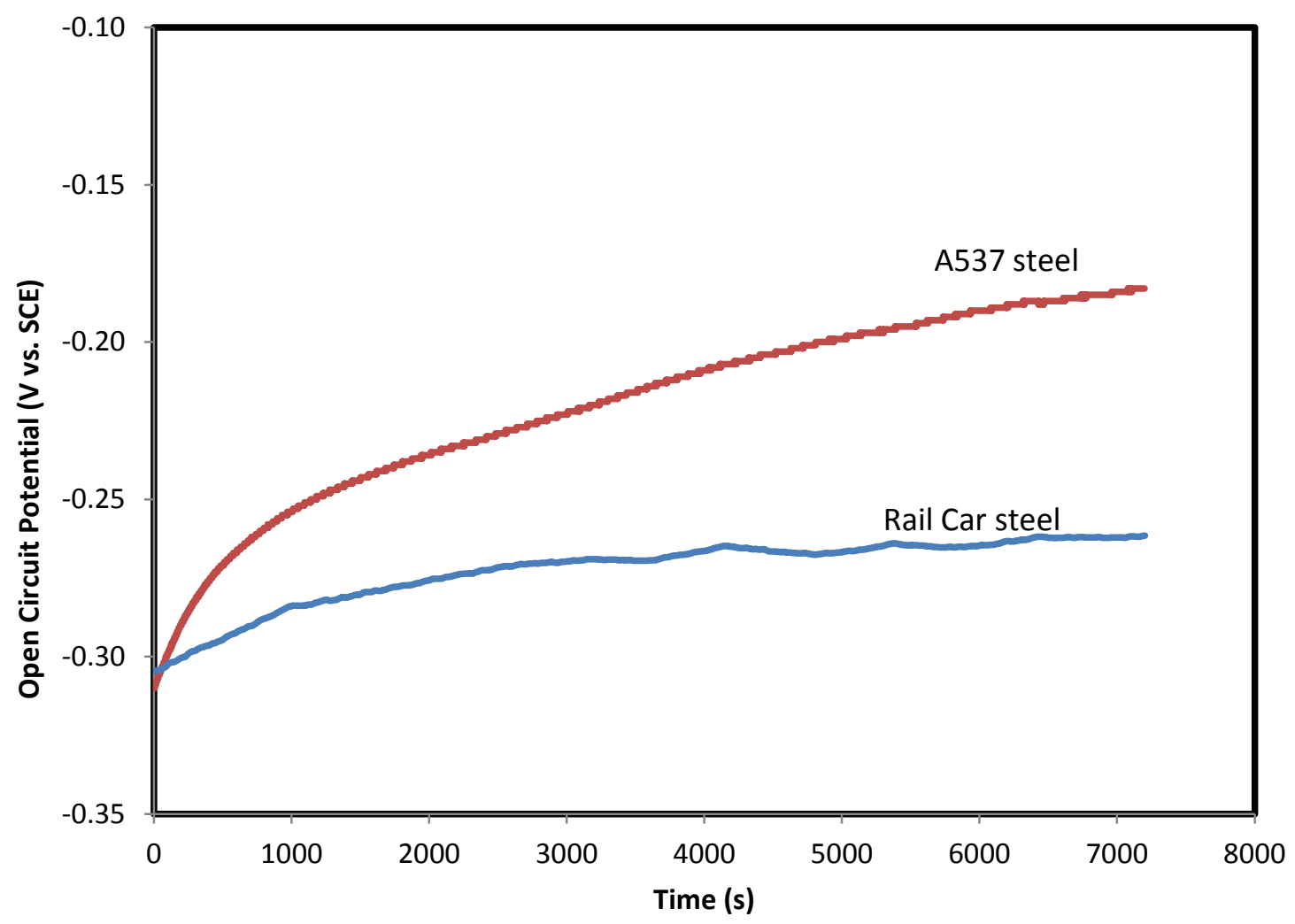

Figure 5-35 Open circuit potential transients for the rail car and A537 steel exposed to test solution 16.

Figure 5-36 compares the CPP curves for the A537 carbon steel and the rail car steel. The higher scan rate and the lack of a stable oxide film are a possible cause for the higher observed current density during the forward scan of the A537 steel compared to the rail car steel. Additionally, the high vertex potential that was utilized for the A537 resulted in a higher vertex current density. This coupled with the higher density of inclusions may have resulted in the small degree of positive hysteresis and the initiation of the small pits that were observed on the sample (see Figure 5-37). The rail car steel, on the other hand, exhibited negative hysteresis and no pits were observed as shown in Figure 5-37. 


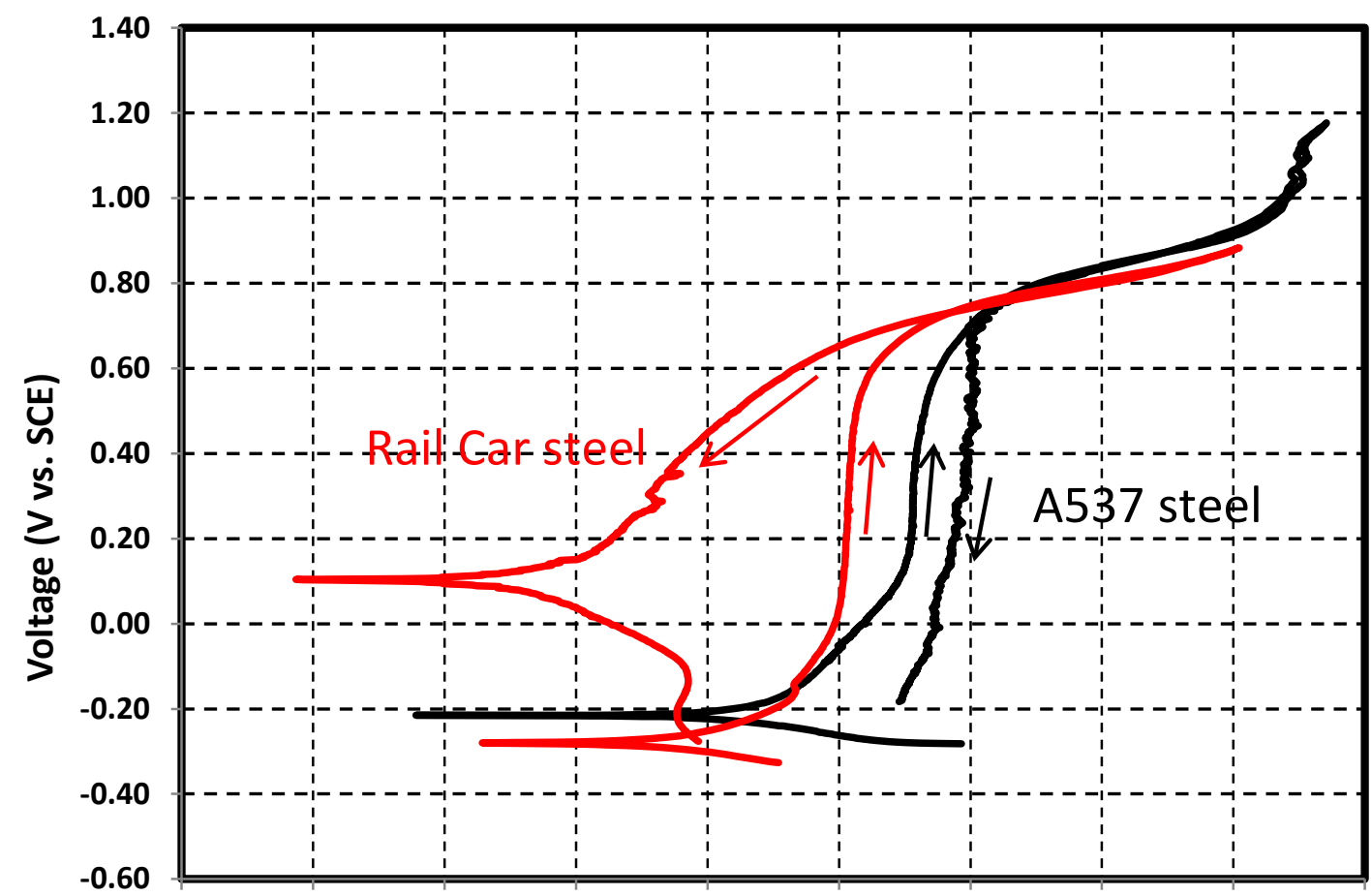

1.00E-11 1.00E-10 1.00E-09 1.00E-08 1.00E-07 1.00E-06 1.00E-05 1.00E-04 1.00E-03 1.00E-02

\section{Log Current Density $\left(\mathrm{A} / \mathrm{cm}^{2}\right)$}

Figure 5-36 CPP curves for the rail car and A537 steel exposed to test solution 16.

Two principle observations can be made from this testing. For environmental conditions where there is clear-cut pitting or no pitting on an electrode, the historical and new CPP protocol data are in agreement. The CPP curves (i.e., positive or negative hysteresis) were also in agreement with observations on the sample. Long-term coupon tests demonstrated agreement with the CPP tests. On the other hand, for environmental conditions that are borderline, agreement is not guaranteed. Borderline environmental conditions can result in contradictions between the CPP curve and the observation of pits (i.e., negative hysteresis with minor pitting) and difficulty in replicating coupon test results (i.e., one coupon exhibits pitting, while no pitting is observed on a duplicate coupon). The disagreement in the CPP test results may be a result of differences in the test protocol or differences in the microstructure of the material that was tested. Therefore, if the historical data is applied to evaluate current conditions in the tanks or future corrosion controls a careful review of the test parameters and steel utilized is required. 

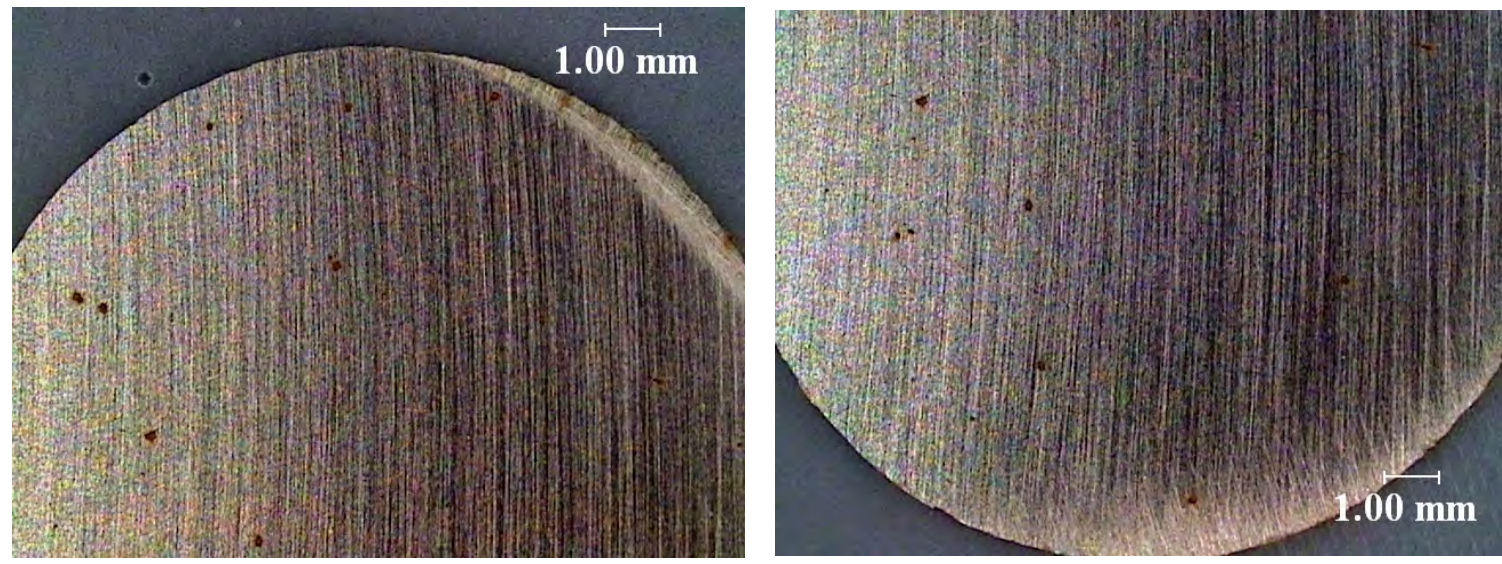

(a)
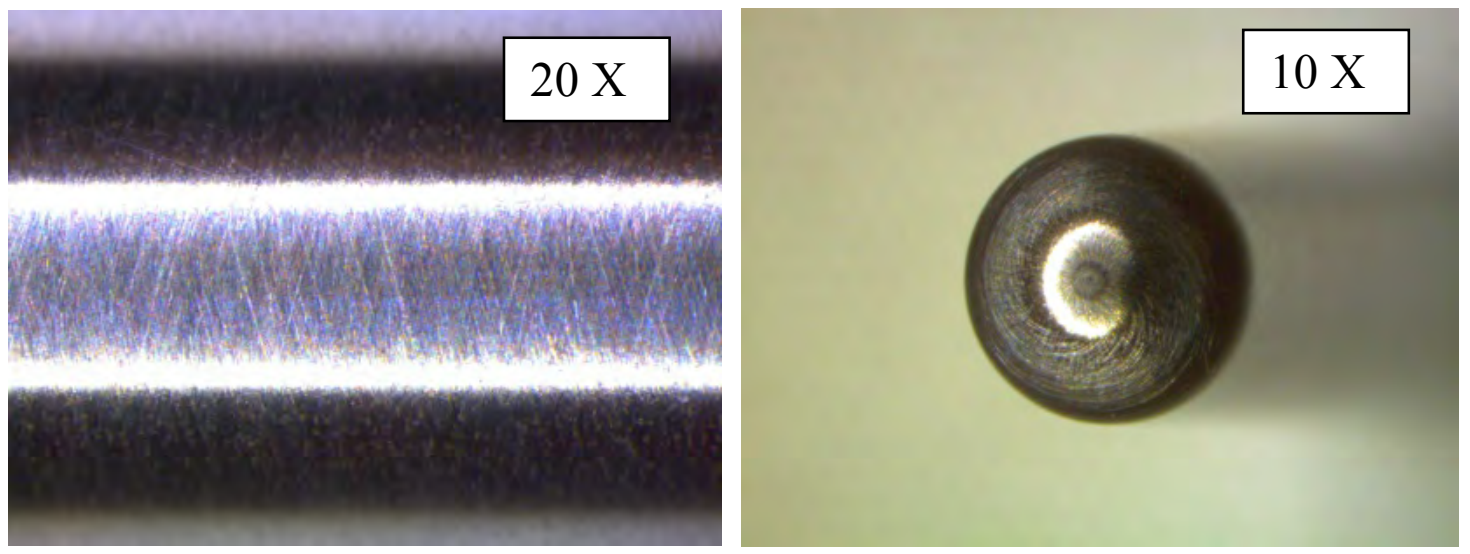

(b)

Figure 5-37 Post-CPP test observation of samples after tests in solution 16 (a) A537 steel and (b) Rail Car steel. 
SRNL-STI-2014-00616

Revision 0

\subsection{Conclusions}

For FY2014, four task activities were performed and the results were arranged in five parts and discussed in section 5. Below is a summary and conclusions for every part:

\subsection{Secondary Wall of AY-102 Tank Corrosion Studies}

LAI and VS corrosion tests were performed using LDP residue (Solutions 12 and 13) and GW simulants (Solutions 14 and 15). For LAI samples after two months, aggressive corrosion was sustained for all samples. For the partially immersed samples after two months of exposure, the corrosion occurred at the water line and below. By the fourth month, the corrosion increased above the water line of the coupon as well. The corrosion rate was steady for the 4 month test at approximately 10 mpy. A similar corrosion rate was observed for samples that were totally immersed in the LDP and GW simulants. For VS samples, corrosion was more prominent for the coupons exposed to GW simulant (Solution 8) than the coupons exposed to LDP residue (Solution 7). More aggressive attack was observed on the samples closest to the liquid level (Level $1>$ Level $2>$ Level 3) for the coupons from GW simulant.

\subsection{Waste Buffering of simulant from DST AN-102}

Electrochemical testing using waste buffering simulants based on waste from tank AN-102 at different hydroxide, nitrate and nitrite concentrations was performed. All the samples showed no pitting and the CPP curve had negative hysteresis. Also an OCP of $-0.600 \mathrm{~V}$ vs. SCE was common, except one test (test 16 duplicate). The results demonstrated that hydroxide concentrations as low as $0.032 \mathrm{M}$ can still offer inhibition for corrosion in carbon steel provided sufficient nitrite is present. The four month LAI tests showed no significant corrosion. Under the microscope pits from 0.5 to 1.3 mils in depth were seen sporadically at the LAI and above while none were found below. These results concur with electrochemical testing in that the solutions studied inhibited the development of pits when carbon steel is immersed and creates very small pits that do not seem to grow or increase in quantity over time.

6.3 Vapor Space Corrosion tests at new SCC limits with different concentrations of Ammonia gas in Air

VSC tests were executed with three simulants based on the new SCC corrosion control guideline. The simulants contained the minimum nitrite/nitrate ratio of 0.15 and nitrate concentrations of 0.4 , 2 and $4.5 \mathrm{M}$. The vapor space above each simulant had either 50 or $550 \mathrm{ppm}$ ammonia. The samples after four-months of exposure showed no indications of VSC at levels 3 and 2 with minor corrosion areas at Level 1 for the $550 \mathrm{ppm}$ ammonia in each of the three simulants. The same was true at $50 \mathrm{ppm}$, as there was no significant VSC. When the cold mount was removed from the coupons, some crevice attack had occurred in some instances. The crevice corrosion seemed more aggressive in these samples than for the samples exposed with LDP and GW simulant. The results tend to indicate that even at $50 \mathrm{ppm}$ ammonia with solutions comprised of the new SCC control limits VSC can be inhibited.

\subsection{Liquid Air Interface tests at new SCC limits}

Eight different solutions were prepared with compositions that were at or near the new SCC control limits. After two and fourth months of exposure none of the samples exhibited LAI corrosion or any attack in the immersed area. Typically any corrosion seemed to initiate at the top 
of the coupon and develop an area of general corrosion and that continues to spread above the LAI. The smallest ratio of nitrite to nitrite $(0.12)$ was solution 8 and during the contact with the carbon steel coupon it developed a film that completely covered the coupon and protected it from corrosion attack even though the ratio is less than the new minimum SCC control limits. The corrosion appeared to be more severe for the more dilute solutions at a given nitrite/nitrate ratio.

\subsection{Pitting Corrosion studies using the standardized CPP protocol}

CPP tests were conducted to compare historical data with data that was collected using the new standardized CPP protocol. The purpose was to determine the effect of the CPP parameters on the results and also compare the results from the new CPP test protocol with long-term coupon tests. Forty test conditions were selected for testing during FY2014 from the more than 900 historical test conditions. In cases where either a clear-cut pitting or no pitting case, there was $100 \%$ agreement between the historical CPP results and the present testing. On the other hand if the environment was a borderline condition (i.e., transition from pitting to not pitting) agreement was not as good. Both the CPP test parameters and the microstructure of the material may have had a role in these contrary results. The historical data and new CPP protocol still remain useful as in both cases the behavior at the borderline condition was consistent. Both the historical data and the new CPP test protocol were consistent with coupon results that indicated either clear-cut pitting or no pitting results and borderline conditions.

\subsection{Quality Assurance}

Data were recorded in the electronic laboratory notebook system, notebook number G8519-00126.

Requirements for performing reviews of technical reports and the extent of review are established in manual E7 2.60. SRNL documents the extent and type of review using the SRNL Technical Report Design Checklist contained in WSRC-IM-2002-00011, Rev. 2.

\subsection{Acknowledgements}

The authors appreciate the assistance given by H.L. Thacker Jr for taking most of the images post-test for Task 3 and 4, K.J. Kalbaugh with providing additional help with testing and disposal of simulants and R. S. Garritano in helping to collect readings for Task 2.

\subsection{References}

[1] M. T. Terry, "Expert Panel Workshop for Hanford Site Double-Shell Tank Waste Chemistry Optimization," Rpp-RPT-22126, Rev. 0, CH2MHill Hanford Group, Richland, WA, October 2004.

[2] T. Martin, "Outcomes from the August 2013 Expert Panel Oversight Committee Meeting," RPP-ASMT-56781, February, 2014.

[3] L. M. Stock, J. R. Follett, and E. C. Shallman, "Specifications for the Minimization of the Stress Corrosion Cracking Threat in Double-Shell Tank Wastes," RPP-RPT47337, Washington River Protection Solutions, Richland, WA, March 2011.

[4] R. E. Fuentes, "SRNL Task Technical And Quality Assurance Plan for Hanford Double Shell Waste Tank Corrosin Studies", SRNL-RP-2014-00213, April 30, 2014. 
[5] A.R. Felmy, O. Qafoku, B. Arey, K.D. Boomer, "Chemical Species in the Vapor Phase of Hanford Double-Shell Tanks: Potential Impacts on Waste Tank Corrosion Processes" PNNL-19767, September 2010.

[6] E. N. Hoffman, "Testing Vapor Space and Liquid-Air Interface Corrosion In Simulated Environments of Hanford Double-Shelled Tanks," SRNL-STI-2011-00494, September.

[7] J. R. Gray, B. L. Garcia-Diaz, T. H. Murphy, K. R. Hicks, "Vapor Space Corrosion Testing Simulating the Environment of Hanford Double Shell Tanks," SRNL-STI2013-00739, Savannah River National Laboratory, Aiken, SC, January 2014.

[8] R. P. Anantatmula, "DST Pitting Annual Report," WHC-SD-WM-PRS-016, Rev. 0, Westinghouse Hanford Company, Richland, WA, September, 1996.

[9] J. W. Congdon, "Evaluation of Corrosion Inhibitors for Washed Precipitate Coupon Test Results,” DPST-86-721, Savannah River Laboratory, Aiken SC, October, 1986.

[10] C. W. Stewart, et. al., "Independent Review of Tank 241-AY-101 Fitness for Service", PNNL-14176, February 2003.

[11] H. Cong, et. al, "A Study on the Interfacial Corrosion of Carbon Steel in Dilute Hanford Waste Simulants", CORROSION 2010, Paper no. 10247, NACE International, Houston, TX, 2010.

[12] H. Cong, et. al., "An Electrochemical Study of Liquid Air Interface Corrosion in Dilute Hanford Nuclear Waste", CORROSION 2011, Paper no. 11212, NACE International, Houston, TX, 2011.

[13] H. Cong, et. al, "Evaluation of Laboratory Screening Techniques for Liquid Air Interface Corrosion in Hanford Waste Simulants", CORROSION 2012, Paper no. 1562, NACE International, Houston, TX, 2012.

[14] J. R. Gray, B. L. Garcia-Diaz, T. Murphy, K. Hicks, "Liquid Air Interface Corrosion Testing Simulating the Environment of Hanford Double Shell Tanks", SRNL-STI-2013-00743, Savannah River National laboratory, Aiken, SC, January 2014.

[15] A. J. Feero, "Evaluation of the Corrosion Threats to the LDP and Secondary Liner of Tank 241-AY-102", RPP-RPT-54099, Washington River Protections Solutions, Richland, WA, February 2014.

[16] J. K. Engeman, C. L. Girardot, D. G. Harlow, C. L. Rosenkrance, "Tank 241-AY102 Leak Assessment Report", RPP-ASTM-53793, November 2012

[17] J. R. Gunte, A. J. Feero, D. G. Baide, K. D. Boomer, "Evaluation of Tank 241-AY102 Secondary Containment System", July 2014.

[18] R. B. Wyrwas and J. S. Page, "Report on Corrosion Potential Testing of Tank 241AN-102 Grab Samples in Support of Corrosion Mitigation for Fiscal Year 2012," LAB-RPT-12-00009, Rev. 0, Washington River Protection Solutions, Richland, WA, December 2012.

[19] G. P. Ritenour, "Final Report for Tank 241-AN-102 Liquid Grab Samples in Support of Corrosion Mitigation for Fiscal Year 2012," RPP-RPT-52587, Rev. 0, Washington River Protection Solutions, Richland, WA, May 2012.

[20] M. T. Terry, "Assessment of Double-Shell Tank 241-AN-102 Propensity for Pitting Under Current Waste Chemistry Composition," RPP-ASMT-53360. Rev. 0, Washington River Protection Solutions, Richland, WA, August 2012.

[21] J. K. Engeman, C. L. Girardot, D. G. Harlow, and C. L. Rosenkrance, "Tank 241AY-102 Leak Assessment Supporting Documentation: Miscellaneous Reports, Letters, Memoranda, and Data”, RPP-ASMT-53794, Rev. 0, December 2012.

[22] K. J. Cantrell, R. J. Serne, and G. V. Last, "Hanford Contaminant Distribution Coefficient Database and Users Guide", PNNL-13895, Rev. 1, June 2003. 
[23] ASTM A515, “ Standard Specification for Pressure Vessel Plates, Carbon Steel, for Intermediate- and Higher-Temperature Service", ASTM International, West Conshohocken, PA, 2003.

[24] R. J. Yarbrough, "Steady-State Flammable Gas Release Rate Calculation and Lower Flammability Level Evaluation for Hanford Tank Waste", RPP-5926, Rev. 12, September 26, 2012.

[25] R. V. Thurston, et.al, “Aqueous Ammonia Equilibrium”, EPA-600/3-79-091, August 1979.

[26] ASTM G1-03 "Standard Practice for Preparing, Cleaning, and Evaluating Corrosion Test Specimens", ASTM International, West Conshohocken, PA, 2011.

[27] P. E. Zapp, "Effect of Temperature on the Nitrite Requirement to Inhibit Washed Sludge”, WSRC-TR-90-292, Savannah River National laboratory, Aiken, SC, September 1990.

[28] E. N. Hoffman, B. L. Garcia-Diaz, T. B. Edwards, "Effect of Chloride and Sulfate Concentration on Probability Based Corrosion Control for Liquid Waste Tanks-Part IV", SRNL-STI-2011-00479, July 2012.

[29] ASTM G5-13 "Standard Reference Test Method for Making Potentiodynamic Anodic Polarization Measurements", ASTM International, West Conshohocken, PA, 2013.

[30] P. E. Zapp, "Pitting Growth Rate in Carbon Steel Exposed to Simulated Radioactive Waste", WSRC-TR-96-0024, June 1996.

[31] T. M. Martin, "Subject: Expert Panel Oversight Committee August 2014 Meeting Outcomes", RPP-ASMT-59979, Rev. 0, September 30, 2014.

[32] ASTM G192-08, "Standard Test Method for Determining the Crevice Repassivation Potential of Corrosion-Resistant Alloys Using a Potentiodynamic-GalvanostaticPotentiostatic Technique”, ASTM International, West Conshohocken, PA, 2014.

[33] J. Beavers, S. Chwala, S. Jain, X. Li, B. Padgett, B. Rollins, K. Sherer, K. Tummala, and N. Sridhar, "FY2014 DST and SST Chemistry Testing Report", RPP-RPT-58300, December 2014.

[34] T. B. Edwards, "Statistical Analysis in Support of the Study of Carbon Steel Pitting", SCS-ASG-93-0031.

[35] P. E. Zapp, "Inhibiting Pitting Corrosion in Carbon Steel Exposed to Dilute Radioactive Waste Slurries”, CORROSION 92, Paper No. 98, NACE International, Houston, TX, 1992.

[36] J. W. Congdon, “Aggressive Anions in Washed Precipitates”, DPST-86-803, November 24, 1986.

[37] J. W. Congdon, "Corrosion Inhibition of Dilute Washed Precipitate with Sodium Nitrite”, DPST-87-369, April 1, 1987.

[38] J. W. Congdon, "Inhibition of Partially Washed Precipitate", DPST-87-663, September 17, 1987.

[39] P. E. Zapp, "Final Report on Nitrite Inhibitor Concentrations for Washed Precipitate at Elevated Temperatures”, WSRC-TR-93-198, April 13, 1993.

[40] P. E. Zapp, "Interim Report on Nitrite Inhibitor Concentrations for Washed Precipitate at Elevated Temperatures", SRTC-MTS-92-3018, May 19, 1993.

[41] J. W. Congdon, "Effect of Temperature on the Nitrite Requirements for Inhibition of Washed Precipitate", DPST-87-590, July 30, 1987. 
[42] P. E. Zapp, "Effect of Temperature on Nitrite Inhibitor Requirements of Washed Precipitate Slurry", WSRC-RP-89-24, April 13, 1989.

[43] J. W. Congdon and J. S. Lozier, "Inhibition of Washed Sludge with Sodium Nitrite", DPST-87-379, April 7, 1987.

[44] P. E. Zapp, "Effect of Temperature on the Nitrite Requirement to Inhibit Tank 42H", WSRC-TR-90-115, February 26, 1990.

[45] J. D. Fritz, "Inhibitor Requirements for the DWPF Recycle Solution", DPST-88-863, September 22, 1988.

[46] E. N. Hoffman and K. H. Subramanian, "Probability Based Corrosion Control for High Level Waste Tanks: Interim Report”, WSRC-STI-2007-00712, February 2008.

[47] E. N. Hoffman, "Probability Based Corrosion Control for High Level Waste Tanks", SRNL-STI-2008-00539, February 2009.

[48] E. N. Hoffman, B. L. Garcia-Diaz, and T. B. Edwards, "Probability Based Corrosion Control for Liquid Waste Tanks - Part III”, SRNL-STI-2010-00246, September 2010.

[49] B. J. Wiersma and J. I. Mickalonis, "Determination of Corrosion Inhibitor Criteria for Type III/IIIA Tanks During Salt Dissolution”, WSRC-STI-2006-00029, September 2007.

[50] R. Haddad and R. Carranza, "Task 1.0: Tank Corrosion Chemistry Cooperation Monthly Progress Report", July 13, 2004.

[51] C. S. Brossia, C. Scott, and F. Gui, "Hanford Tanks AY-102 and AP-101: Effect of Chemistry and Other Variables on Corrosion and Stress Corrosion Cracking", RPPRPT-31932, CC Technologies a DNV company, September 7, 2007.

[52] C. S. Brossia, C. Scott, and F. Gui, "Hanford Tanks AN-107 and AN-102: Effect of Chemistry and Other Variables on Corrosion and Stress Corrosion Cracking", RPPRPT-31680, CC Technologies a DNV Company, September 8, 2006.

[53] C. S. Brossia and H. Cong, "FY2011 DNV DST Corrosion and Stress Corrosion Cracking Testing Report”, RPP-47895, Rev. 0, June 2012.

[54] R. B. Wyrwas and J. S. Page, "Report on Corrosion Potential Testing of Tank 241AN-102 Grab Samples in Support of Corrosion Mitigation for Fiscal Year 2012," LAB-RPT-12-00009, Rev. 0, Washington River Protection Solutions, Richland, WA, December 2012; M. T. Terry, "Assessment of Double-Shell Tank 241-AN-102 Propensity for Pitting Under Current Waste Chemistry Composition," RPP-ASMT53360. Rev. 0, Washington River Protection Solutions, Richland, WA, August 2012.

[55] P. E. Zapp, "Electrochemical Study of Corrosion Inhibition During Sludge Washing', DPST-88-399, March 14, 1988.

[56] D. T. Hobbs and R. M. Wallace, "Hydroxide Depletion in Waste Storage Tanks by Reaction with Carbon Dioxide", DPST-85-846, October 14, 1985.

[57] J. W. Congdon, "Corrosion Inhibition of Worst-Case Wash Precipitate", DPST-87339, March 17, 1997.

[58] J. W. Congdon, "Corrosion Inhibition of Dilute Washed Precipitate - Coupon Test Results”, DPST-87-569, July 29, 1987.

[59] J. I. Mickalonis, "Corrosion of Steel in Simulated Waste Containing Different Nitrate and Nitrite Concentrations", WSRC-TR-93-322, July 1993. 


\title{
10.0 Appendices
}

\author{
Appendix A \\ Chemical Composition of Simulants used in Vapor Space Corrosion Testing
}

Appendix B

Pictures of Vapor Space Corrosion Samples After Test

Appendix C

Chemical Composition of Simulants used in Liquid Air Interface Corrosion Testing

Appendix D

Pictures of Liquid Air Interface Corrosion Samples after Test

Appendix E

Open Circuit Potential, pH and Temperature vs. Time plots for Liquid Air Interface

Solutions

Appendix F

Chemical Composition of Simulants used in Waste Buffering (Task 3) with Electrochemical Results and After Pictures

Appendix G

Chemical Composition of Simulants used in Pitting Corrosion (Task 4) with Electrochemical Results and After Pictures

\section{Appendix $\mathbf{H}$}

Test Conditions from Previous Electrochemical Results (Task 4) 
Appendix A

Chemical Composition of Simulants used in Vapor Space Corrosion Testing 


\section{Composition of simulant for VS-Solution 1}

Volume

1.5

$\mathrm{L}$

\begin{tabular}{|c|c|c|c|c|}
\hline $\begin{array}{c}\text { Simulant } \\
\text { Source }\end{array}$ & Formula & $\begin{array}{c}\text { Molecular } \\
\text { weight (g/mol) }\end{array}$ & $\begin{array}{c}\text { Concentration } \\
\text { (M) }\end{array}$ & $\begin{array}{l}\text { Weight required } \\
(\mathrm{g})\end{array}$ \\
\hline $\begin{array}{l}\text { Aluminum } \\
\text { Nitrate }\end{array}$ & $\mathrm{Al}\left(\mathrm{NO}_{3}\right)_{3} \cdot 9 \mathrm{H}_{2} \mathrm{O}$ & 375.1314 & 0.0002 & 0.1125 \\
\hline Sodium Chloride & $\mathrm{NaCl}$ & 58.4400 & 0.01 & 0.8766 \\
\hline Sodium Fluoride & $\mathrm{NaF}$ & 41.9882 & 0.003 & 0.1889 \\
\hline Sodium Sulfate & $\mathrm{Na}_{2} \mathrm{SO}_{4}$ & 142.0400 & 0.005 & 1.0653 \\
\hline $\begin{array}{l}\text { Ammonium } \\
\text { Nitrate }\end{array}$ & $\mathrm{NH}_{4} \mathrm{NO}_{3}$ & 80.0520 & 0.0012 & 0.1441 \\
\hline $\begin{array}{c}\text { Sodium } \\
\text { Hydroxide }\end{array}$ & $\mathrm{NaOH}$ & 40.0000 & 0.01 & 0.6000 \\
\hline $\begin{array}{c}\text { Sodium } \\
\text { Phosphate }\end{array}$ & $\mathrm{Na}_{3} \mathrm{PO}_{4} 12 \mathrm{H}_{2} \mathrm{O}$ & 380.1200 & 0.0005 & 0.2851 \\
\hline $\begin{array}{c}\text { Sodium } \\
\text { Carbonate }\end{array}$ & $\mathrm{Na}_{2} \mathrm{CO}_{3}$ & 105.9885 & 0.1 & 15.8983 \\
\hline Sodium Nitrate & $\mathrm{NaNO}_{3}$ & 84.9947 & 0.400 & 50.9968 \\
\hline Sodium Nitrite & $\mathrm{NaNO}_{2}$ & 68.9953 & 0.060 & 6.2096 \\
\hline
\end{tabular}

\section{Composition of simulant for VS-Solution 2}

Volume

\begin{tabular}{|c|c|c|c|c|}
\hline $\begin{array}{c}\text { Simulant } \\
\text { Source }\end{array}$ & Formula & $\begin{array}{c}\text { Molecular } \\
\text { weight (g/mol) }\end{array}$ & $\begin{array}{c}\text { Concentration } \\
\text { (M) }\end{array}$ & $\begin{array}{c}\text { Weight required } \\
\text { (g) }\end{array}$ \\
\hline $\begin{array}{c}\text { Aluminum } \\
\text { Nitrate }\end{array}$ & $\mathrm{Al}\left(\mathrm{NO}_{3}\right)_{3} \cdot 9 \mathrm{H}_{2} \mathrm{O}$ & 375.1314 & 0.0020 & 1.1254 \\
\hline Sodium Chloride & $\mathrm{NaCl}$ & 58.4400 & 0.04 & 3.5064 \\
\hline Sodium Fluoride & $\mathrm{NaF}$ & 41.9882 & 0.01 & 0.6298 \\
\hline Sodium Sulfate & $\mathrm{Na}_{2} \mathrm{SO}_{4}$ & 142.0400 & 0.050 & 10.6530 \\
\hline $\begin{array}{c}\text { Ammonium } \\
\text { Nitrate }\end{array}$ & $\mathrm{NH}_{4} \mathrm{NO}_{3}$ & 80.0520 & 0.0012 & 0.1441 \\
\hline $\begin{array}{c}\text { Sodium } \\
\text { Hydroxide }\end{array}$ & $\mathrm{NaOH}_{\text {Sodium }}$ & 40.0000 & 0.01 & 0.6000 \\
\hline $\begin{array}{c}\text { Phosphate } \\
\text { Sodium }\end{array}$ & $\mathrm{Na}_{3} \mathrm{PO}_{4} 12 \mathrm{H}_{2} \mathrm{O}$ & 380.1200 & 0.01 & 5.7018 \\
\hline Carbonate & $\mathrm{Na}_{2} \mathrm{CO}_{3}$ & 105.9885 & 0.5 & 79.4914 \\
\hline Sodium Nitrate & $\mathrm{NaNO}_{3}$ & 84.9947 & 2 & 254.9841 \\
\hline Sodium Nitrite & $\mathrm{NaNO}_{2}$ & 68.9953 & 0.3 & 31.0479 \\
\hline
\end{tabular}




\section{Composition of simulant for VS-Solution 3}

Volume

\begin{tabular}{|c|c|c|c|c|}
\hline $\begin{array}{c}\text { Simulant } \\
\text { Source }\end{array}$ & Formula & $\begin{array}{c}\text { Molecular } \\
\text { weight (g/mol) }\end{array}$ & $\begin{array}{c}\text { Concentration } \\
\text { (M) }\end{array}$ & $\begin{array}{c}\text { Weight required } \\
\text { (g) }\end{array}$ \\
\hline $\begin{array}{c}\text { Aluminum } \\
\text { Nitrate }\end{array}$ & $\mathrm{Al}\left(\mathrm{NO}_{3}\right)_{3} .9 \mathrm{H}_{2} \mathrm{O}$ & 375.1314 & 0.0033 & 1.8569 \\
\hline Sodium Chloride & $\mathrm{NaCl}$ & 58.4400 & 0.10 & 8.7660 \\
\hline Sodium Fluoride & $\mathrm{NaF}$ & 41.9882 & 0.020 & 1.2596 \\
\hline Sodium Sulfate & $\mathrm{Na}_{2} \mathrm{SO}_{4}$ & 142.0400 & 0.100 & 21.3060 \\
\hline $\begin{array}{c}\text { Ammonium } \\
\text { Nitrate }\end{array}$ & $\mathrm{NH}_{4} \mathrm{NO}_{3}$ & 80.0520 & 0.0011 & 0.1321 \\
\hline $\begin{array}{c}\text { Sodium } \\
\text { Hydroxide }\end{array}$ & $\mathrm{NaOH}^{\text {Sodium }}$ & 40.0000 & 0.010 & 0.6000 \\
\hline $\begin{array}{c}\text { Phosphate } \\
\text { Sodium }\end{array}$ & $\mathrm{Na}_{3} \mathrm{PO}_{4} 12 \mathrm{H}_{2} \mathrm{O}$ & 380.1200 & 0.0500 & 28.5090 \\
\hline Carbonate & $\mathrm{Na}_{2} \mathrm{CO}_{3}$ & 105.9885 & 1.0 & 158.9828 \\
\hline Sodium Nitrate & $\mathrm{NaNO}_{3}$ & 84.9947 & 4.500 & 573.7142 \\
\hline Sodium Nitrite & $\mathrm{NaNO}_{2}$ & 68.9953 & 0.675 & 69.8577 \\
\hline
\end{tabular}

\section{Composition of simulant for VS-Solution 4}

Volume

1.5

\begin{tabular}{|c|c|c|c|c|}
\hline $\begin{array}{c}\text { Simulant } \\
\text { Source }\end{array}$ & Formula & $\begin{array}{c}\text { Molecular } \\
\text { weight (g/mol) }\end{array}$ & $\begin{array}{c}\text { Concentration } \\
(\mathbf{M})\end{array}$ & $\begin{array}{c}\text { Weight required } \\
\text { (g) }\end{array}$ \\
\hline $\begin{array}{c}\text { Aluminum } \\
\text { Nitrate }\end{array}$ & $\mathrm{Al}\left(\mathrm{NO}_{3}\right)_{3} \cdot 9 \mathrm{H}_{2} \mathrm{O}$ & 375.1314 & 0.0002 & 0.1125 \\
\hline Sodium Chloride & $\mathrm{NaCl}$ & 58.4400 & 0.01 & 0.8766 \\
\hline Sodium Fluoride & $\mathrm{NaF}$ & 41.9882 & 0.003 & 0.1889 \\
\hline Sodium Sulfate & $\mathrm{Na}_{2} \mathrm{SO}_{4}$ & 142.0400 & 0.005 & 1.0653 \\
\hline $\begin{array}{c}\text { Ammonium } \\
\text { Nitrate }\end{array}$ & $\mathrm{NH}_{4} \mathrm{NO}_{3}$ & 80.0520 & 0.0012 & 0.1441 \\
\hline $\begin{array}{c}\text { Sodium } \\
\text { Hydroxide }\end{array}$ & $\mathrm{NaOH}^{\text {Sodium }}$ & 40.0000 & 0.01 & 0.6000 \\
\hline $\begin{array}{c}\text { Phosphate } \\
\text { Sodium } \\
\text { Carbonate }\end{array}$ & $\mathrm{Na}_{3} \mathrm{PO}_{4} 12 \mathrm{H}_{2} \mathrm{O}$ & 380.1200 & 0.0005 & 0.2851 \\
\hline Sodium Nitrate & $\mathrm{Na}_{2} \mathrm{NO}_{3}$ & 105.9885 & 0.1 & 15.8983 \\
\hline Sodium Nitrite & $\mathrm{NaNO}_{2}$ & 84.9947 & 0.400 & 50.9968 \\
\hline
\end{tabular}




\section{Composition of simulant for VS-Solution 5}

Volume

\begin{tabular}{|c|c|c|c|c|}
\hline $\begin{array}{c}\text { Simulant } \\
\text { Source }\end{array}$ & Formula & $\begin{array}{c}\text { Molecular } \\
\text { weight (g/mol) }\end{array}$ & $\begin{array}{c}\text { Concentration } \\
\text { (M) }\end{array}$ & $\begin{array}{c}\text { Weight required } \\
\text { (g) }\end{array}$ \\
\hline $\begin{array}{c}\text { Aluminum } \\
\text { Nitrate }\end{array}$ & $\mathrm{Al}\left(\mathrm{NO}_{3}\right)_{3} .9 \mathrm{H}_{2} \mathrm{O}$ & 375.1314 & 0.0020 & 1.1254 \\
\hline Sodium Chloride & $\mathrm{NaCl}$ & 58.4400 & 0.04 & 3.5064 \\
\hline Sodium Fluoride & $\mathrm{NaF}$ & 41.9882 & 0.01 & 0.6298 \\
\hline Sodium Sulfate & $\mathrm{Na}_{2} \mathrm{SO}_{4}$ & 142.0400 & 0.050 & 10.6530 \\
\hline $\begin{array}{c}\text { Ammonium } \\
\text { Nitrate }\end{array}$ & $\mathrm{NH}_{4} \mathrm{NO}_{3}$ & 80.0520 & 0.0132 & 1.5850 \\
\hline $\begin{array}{c}\text { Sodium } \\
\text { Hydroxide }\end{array}$ & $\mathrm{NaOH}^{\text {Sodium }}$ & 40.0000 & 0.01 & 0.6000 \\
\hline $\begin{array}{c}\text { Phosphate } \\
\text { Sodium }\end{array}$ & $\mathrm{Na}_{3} \mathrm{PO}_{4} 12 \mathrm{H}_{2} \mathrm{O}$ & 380.1200 & 0.01 & 5.7018 \\
\hline Carbonate & $\mathrm{Na}_{2} \mathrm{CO}_{3}$ & 105.9885 & 0.5 & 79.4914 \\
\hline Sodium Nitrate & $\mathrm{NaNO}_{3}$ & 84.9947 & 2 & 254.9841 \\
\hline Sodium Nitrite & $\mathrm{NaNO}_{2}$ & 68.9953 & 0.3 & 31.0479 \\
\hline
\end{tabular}

\section{Composition of simulant for VS-Solution 6}

Volume

\section{Simulant Source}

Aluminum Nitrate Sodium Chloride

Sodium Fluoride Sodium Sulfate

Ammonium Nitrate Sodium Hydroxide Sodium Phosphate Sodium Carbonate Sodium Nitrate Sodium Nitrite

1.5

\begin{tabular}{|c|c|c|c|}
\hline Formula & $\begin{array}{c}\text { Molecular } \\
\text { weight (g/mol) }\end{array}$ & $\begin{array}{c}\text { Concentration } \\
(\mathbf{M})\end{array}$ & $\begin{array}{c}\text { Weight required } \\
\text { (g) }\end{array}$ \\
\hline $\mathrm{Al}\left(\mathrm{NO}_{3}\right)_{3} .9 \mathrm{H}_{2} \mathrm{O}$ & 375.1314 & 0.0033 & 1.8569 \\
\hline $\mathrm{NaCl}$ & 58.4400 & 0.10 & 8.7660 \\
\hline $\mathrm{NaF}$ & 41.9882 & 0.020 & 1.2596 \\
\hline $\mathrm{Na}_{2} \mathrm{SO}_{4}$ & 142.0400 & 0.100 & 21.3060 \\
\hline $\mathrm{NH}_{4} \mathrm{NO}_{3}$ & 80.0520 & 0.0122 & 1.4650 \\
\hline $\mathrm{NaOH}$ & 40.0000 & 0.010 & 0.6000 \\
\hline $\mathrm{Na}_{3} \mathrm{PO}_{4 .} 12 \mathrm{H}_{2} \mathrm{O}$ & 380.1200 & 0.0500 & 28.5090 \\
\hline $\mathrm{Na}_{2} \mathrm{CO}_{3}$ & 105.9885 & 1.0 & 158.9828 \\
\hline $\mathrm{NaNO}_{3}$ & 84.9947 & 4.500 & 573.7142 \\
\hline $\mathrm{NaNO}_{2}$ & 68.9953 & 0.675 & 69.8577 \\
\hline
\end{tabular}


Appendix B

Pictures of Vapor Space Corrosion Samples After Test 


\section{Vessel 1: Level 3}

1 month

RC128-1
2 months

RC128-2

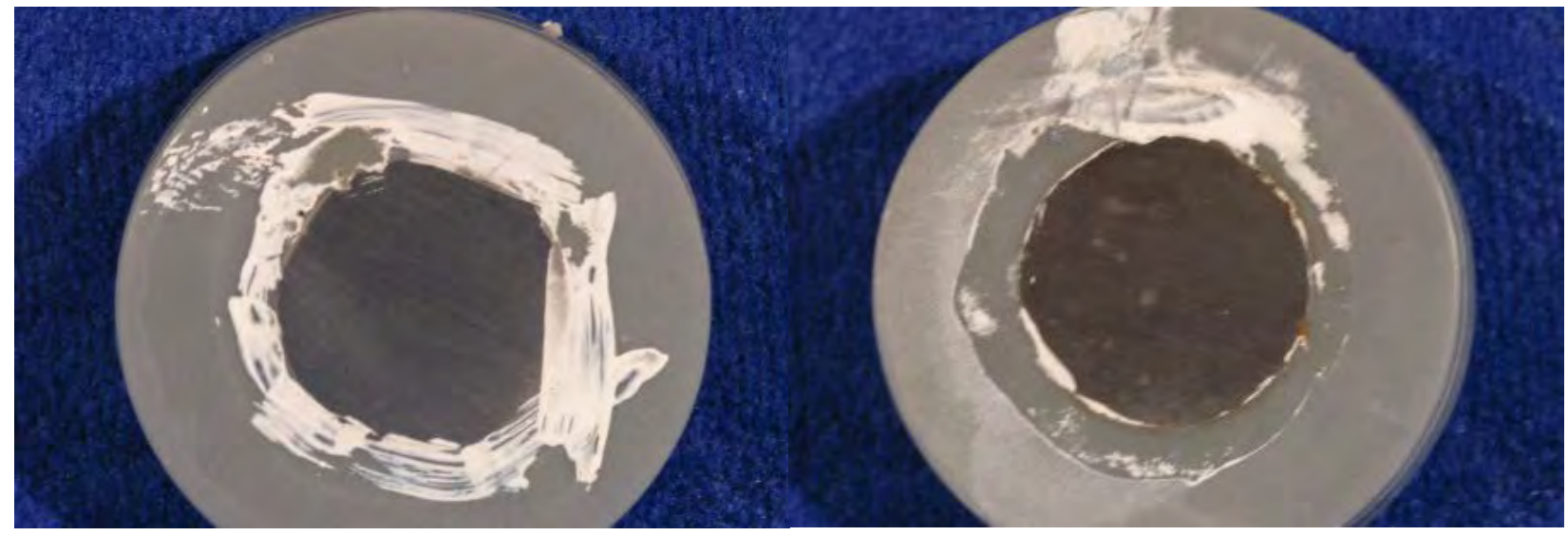

3 months

RC128-4
4 months

RC128-3

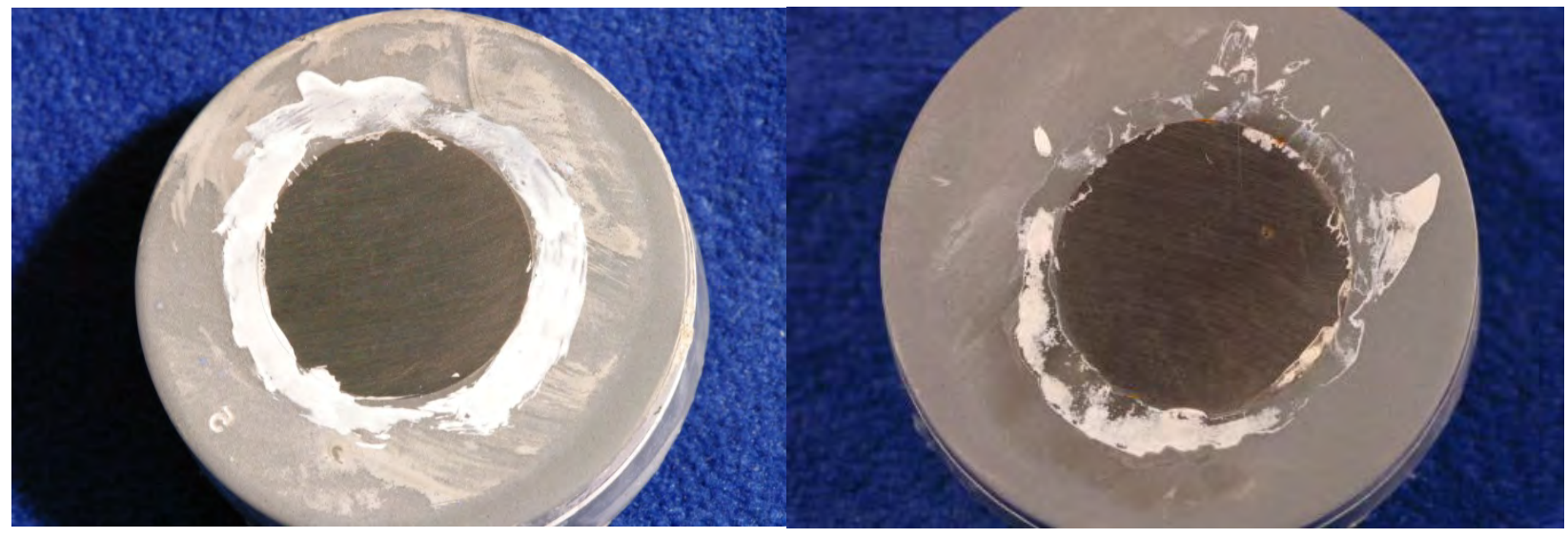


Vessel 1: Level 3

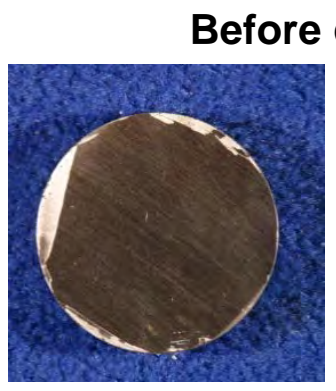

Front

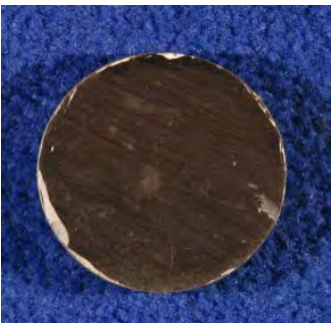

Front

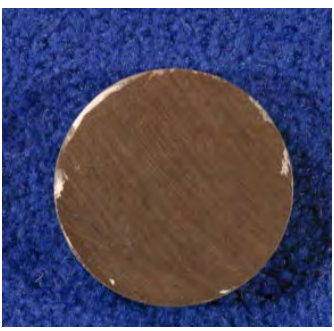

Front

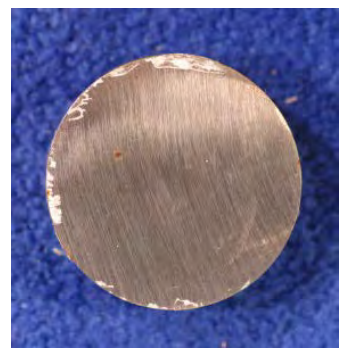

Front

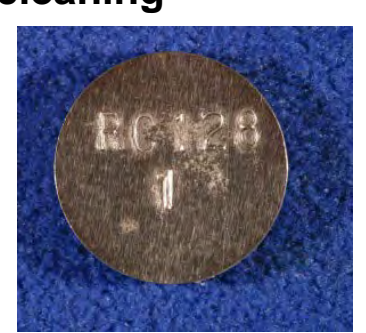

Back

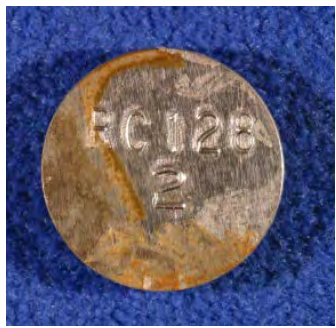

Back

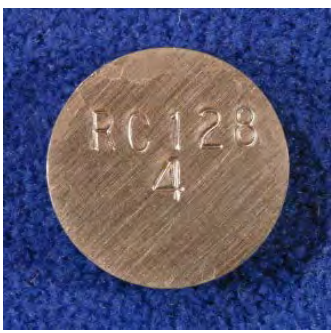

Back

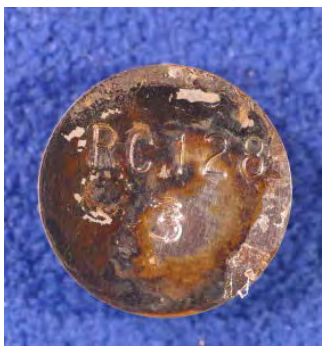

Back
1 month

RC128-1

2 months

RC128-2

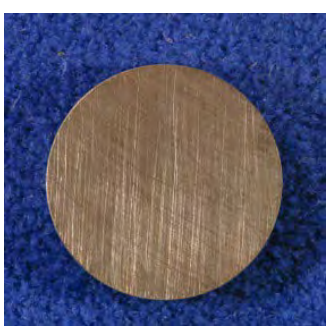

Front

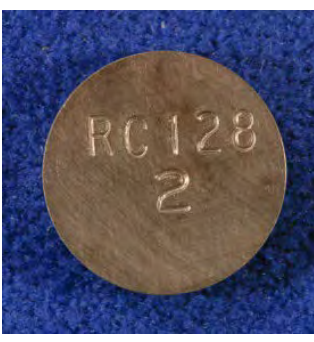

Back

3 months

RC128-4

4 months

RC128-3

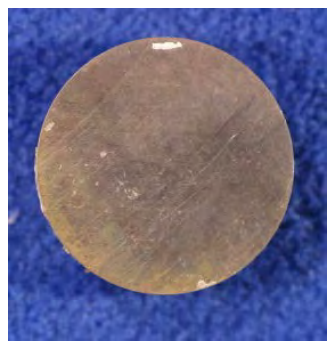

Front

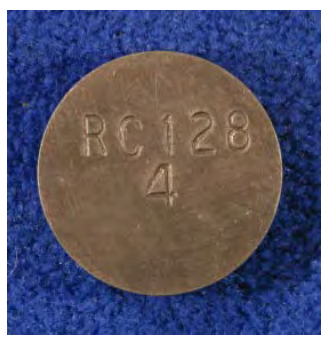

Back

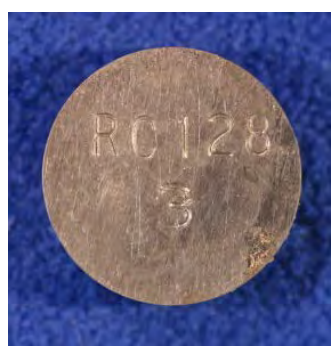

Back 


\section{Vessel 1: Level 2}

1 month

RC128-5
2 months

RC128-8

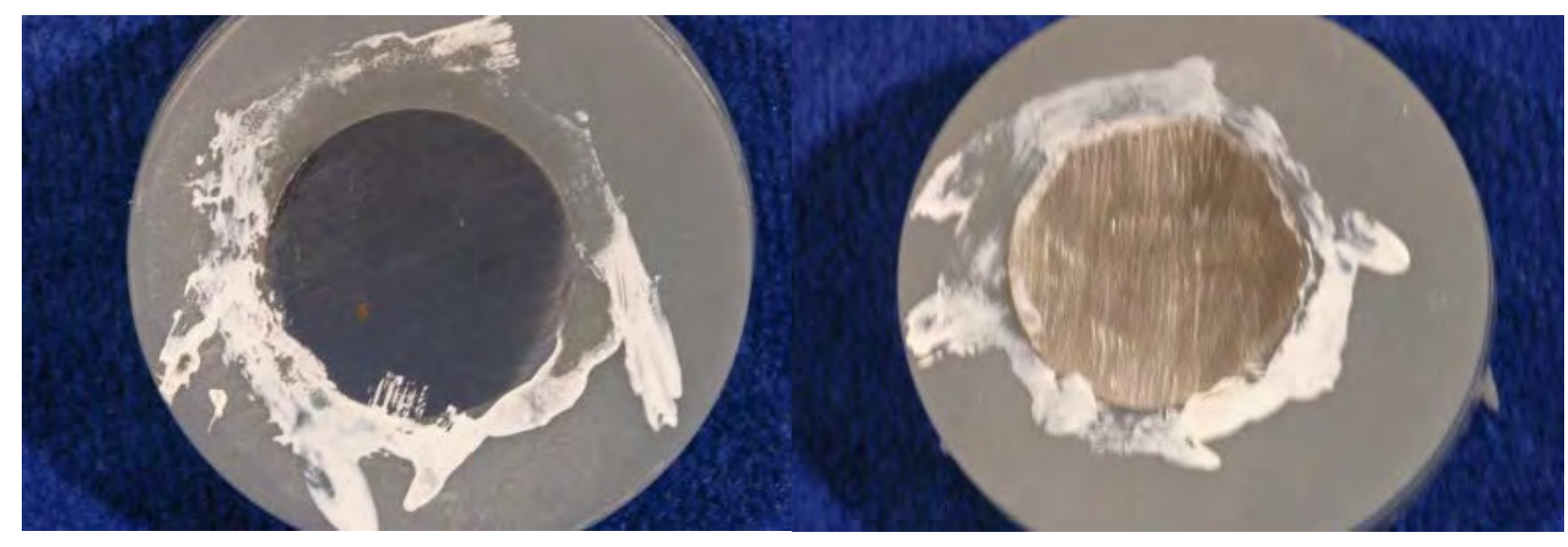

3 months

RC128-7
4 months

RC128-6

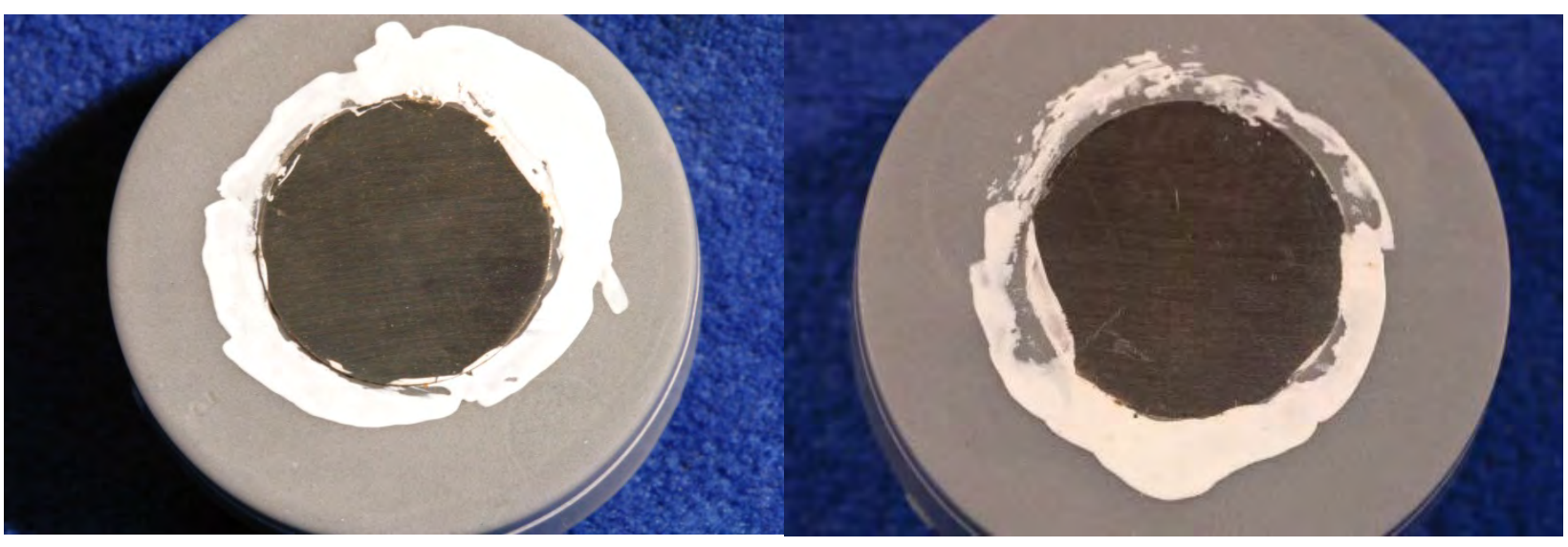




\section{Vessel 1: Level 2}

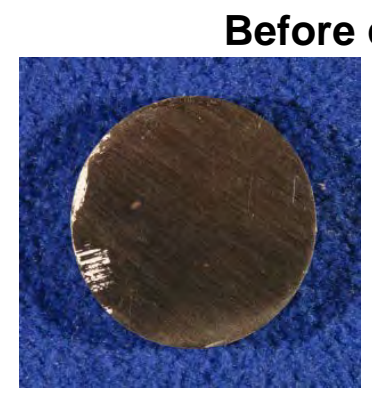

Front

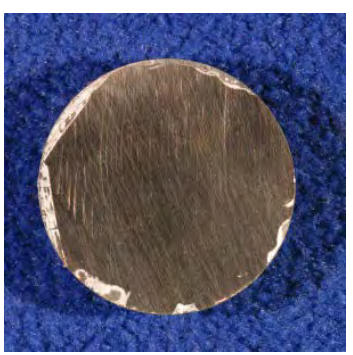

Front

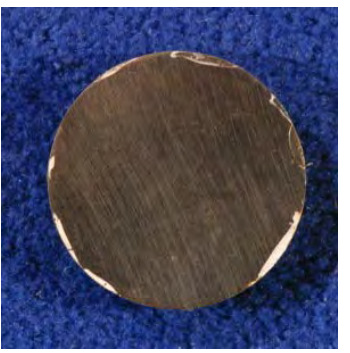

Front

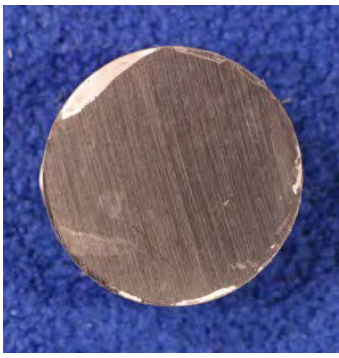

Front

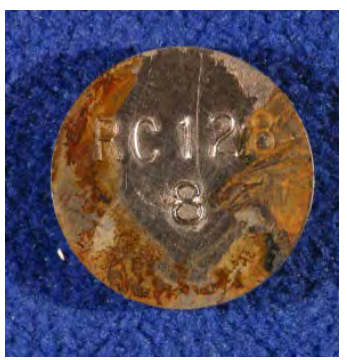

Back

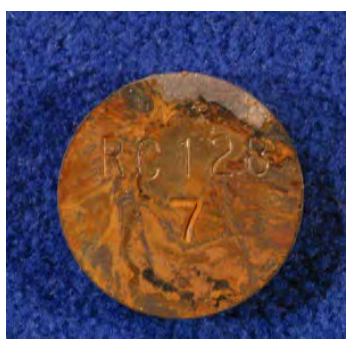

Back

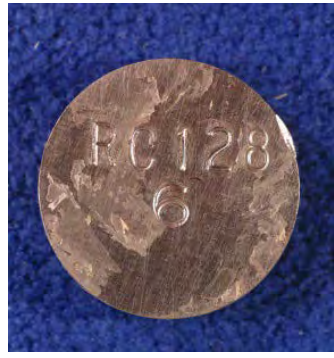

Back

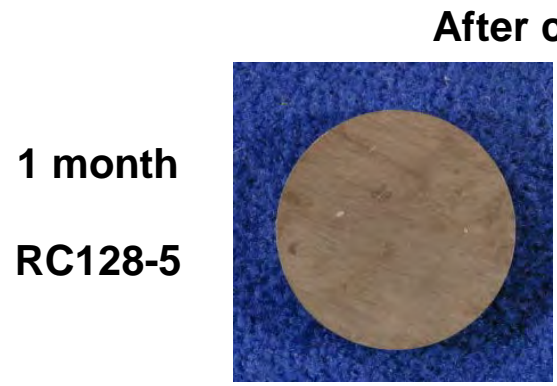

Front

2 months

RC128-8

3 months

RC128-7

4 months

RC128-6

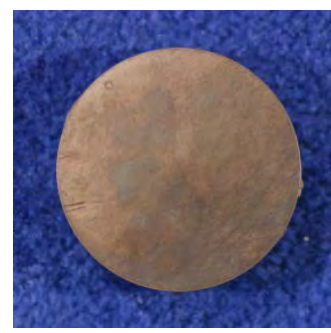

Front

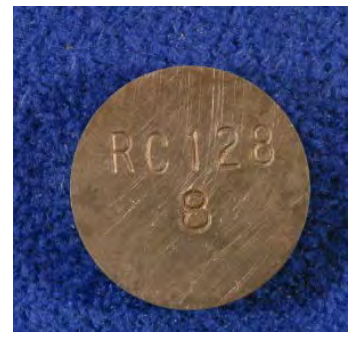

Back

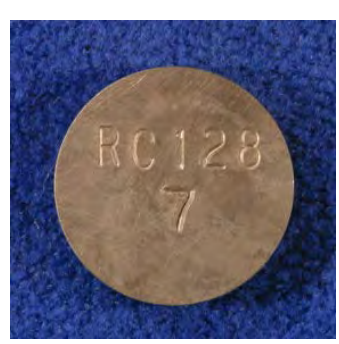

Back

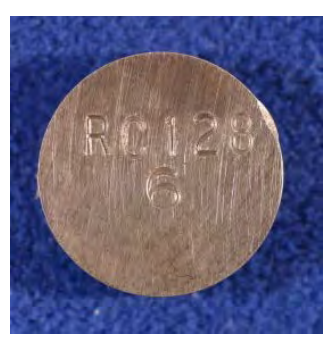

Back 


\section{Vessel 1: Level 1}

1 month

RC128-9
2 months

RC128-12

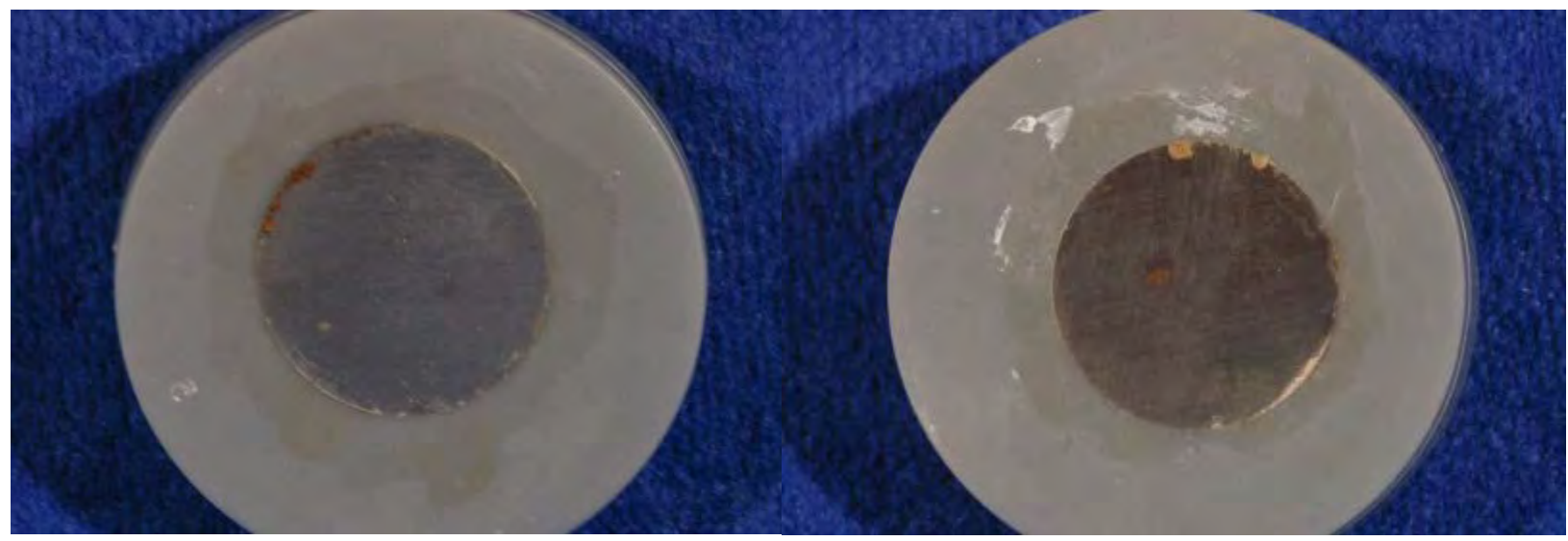

3 months

RC128-11
4 months

RC128-10

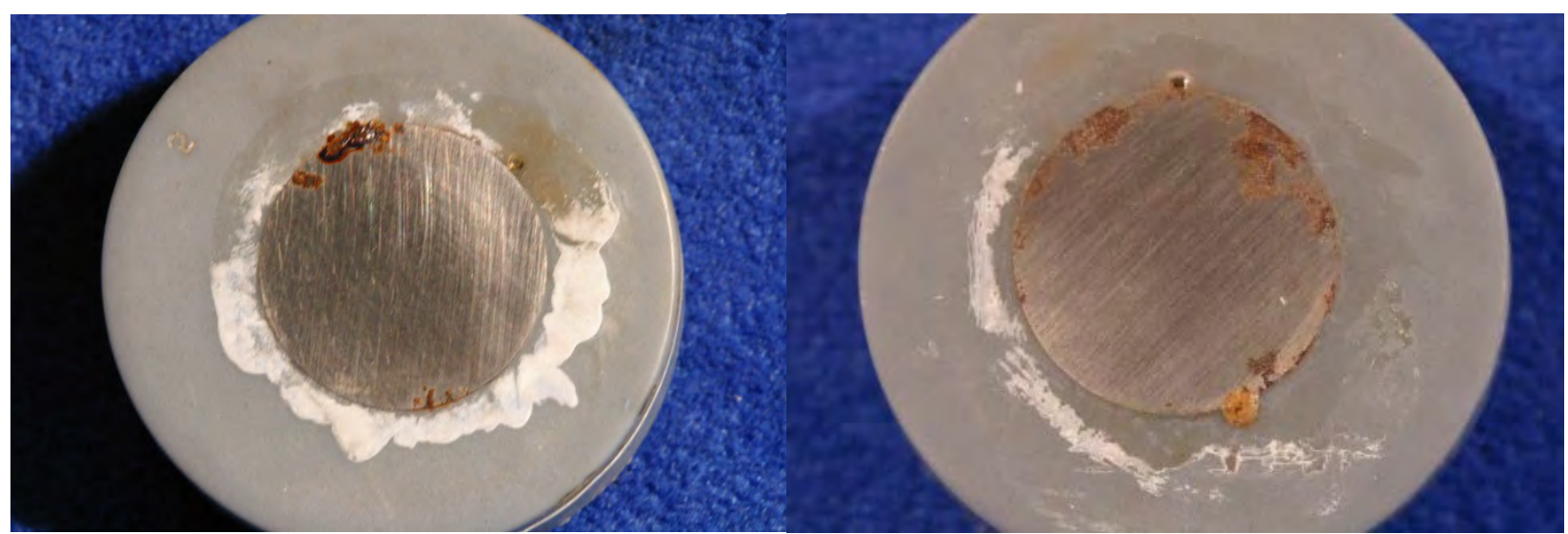


Vessel 1: Level 1

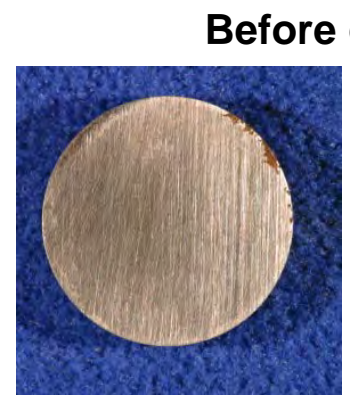

Front

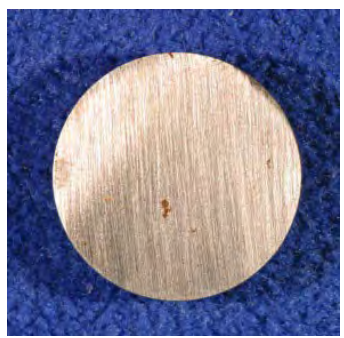

Front

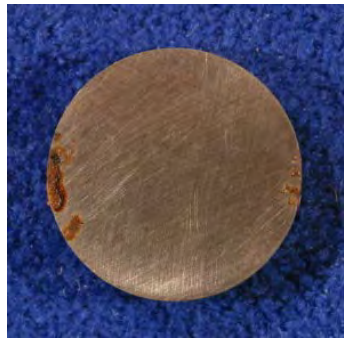

Front

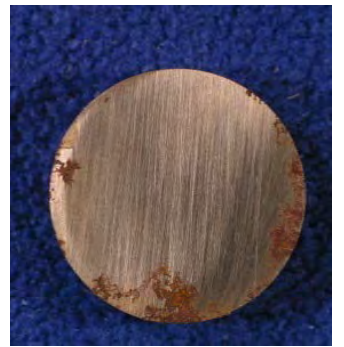

Front

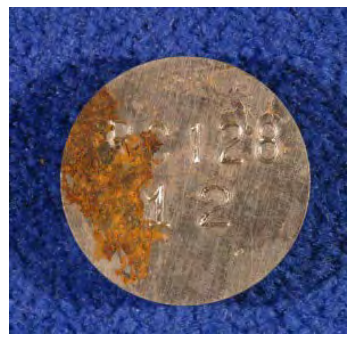

Back

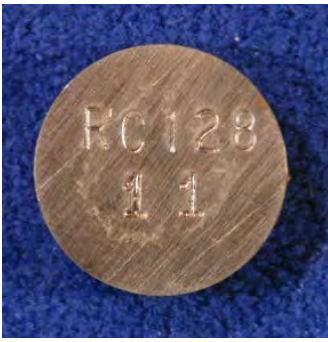

Back

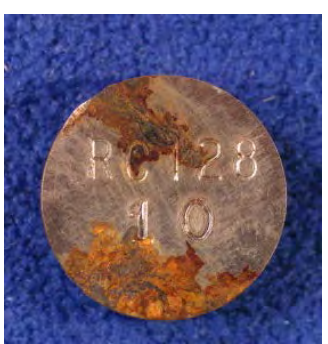

Back

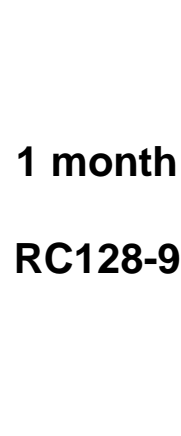

2 months

RC128-12

3 months

RC128-11

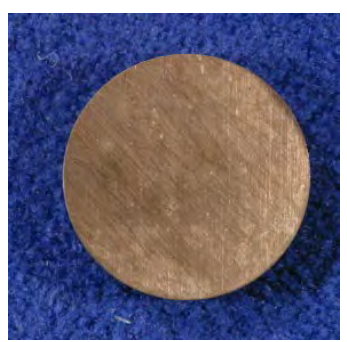

Front

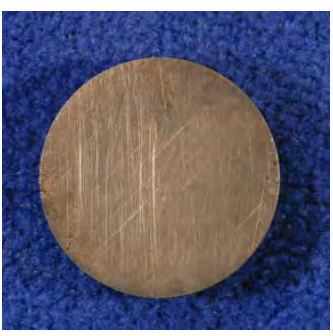

Front

4 months

RC128-10

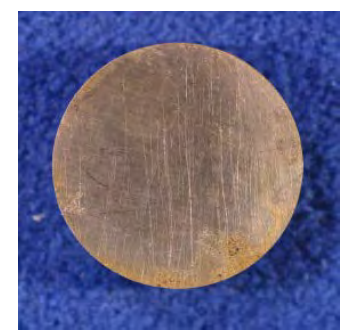

Front

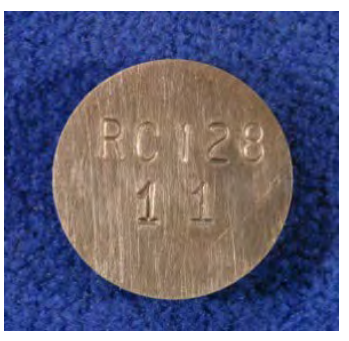

Back

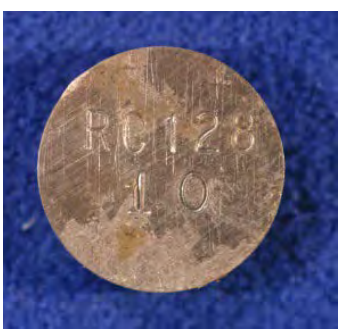

Back 


\section{Vessel 2: Level 3}

1 month

RC128-13
2 months

RC128-16

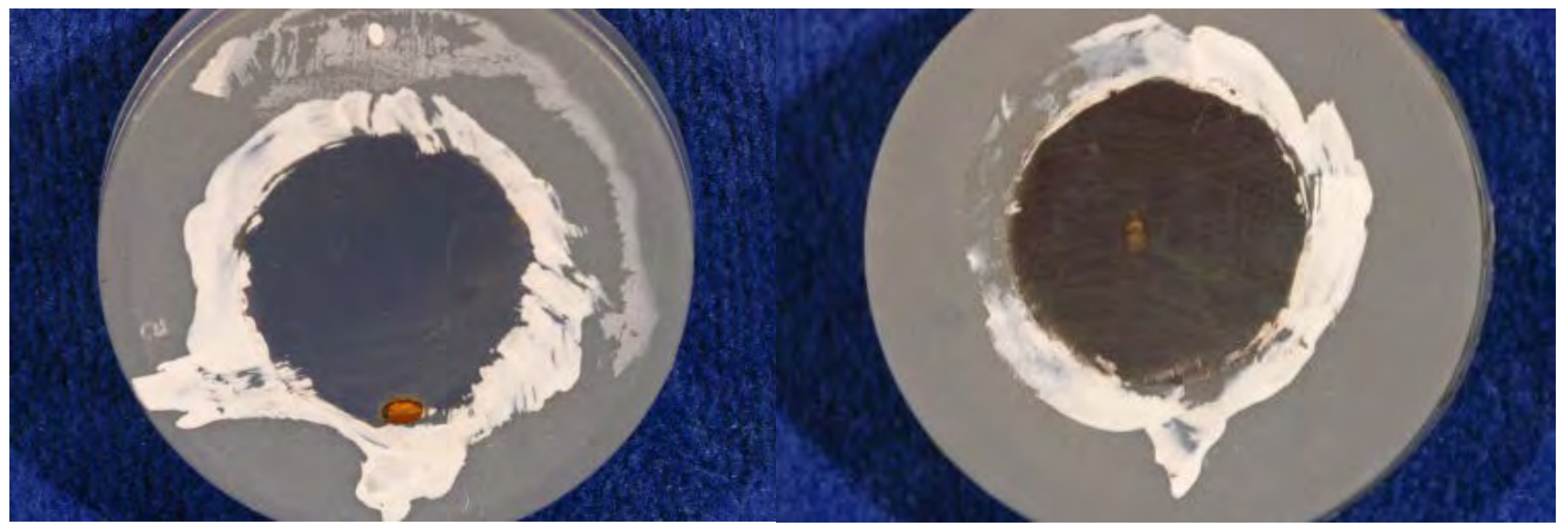

3 months

RC128-14
4 months

RC128-15

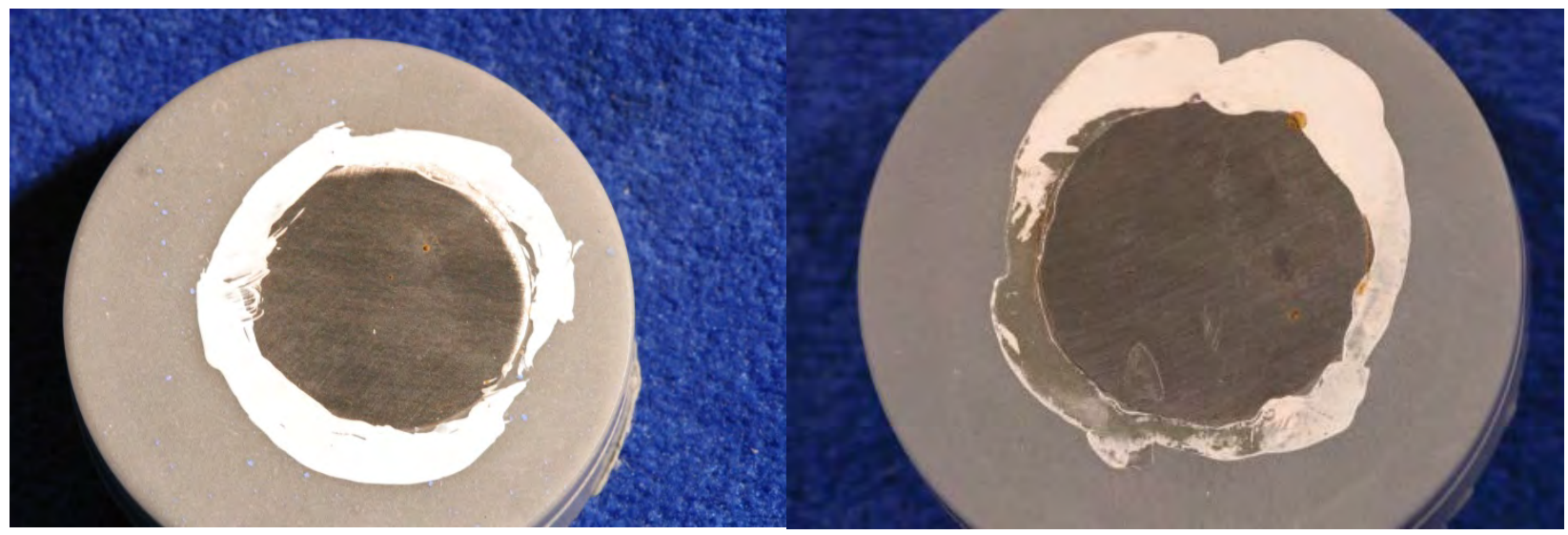




\section{Vessel 2: Level 3}

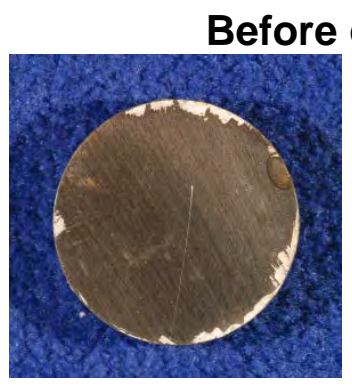

Front

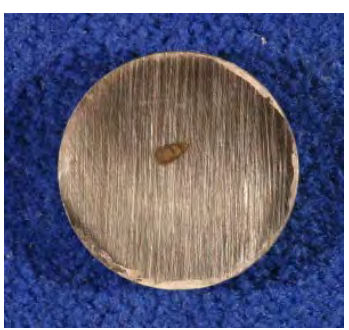

Front

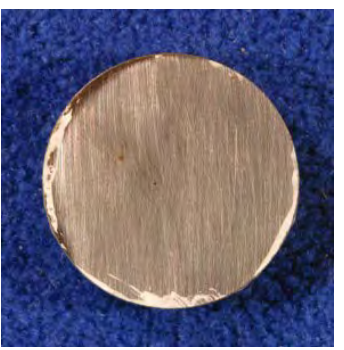

Front

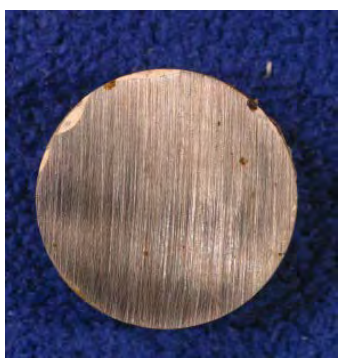

Front

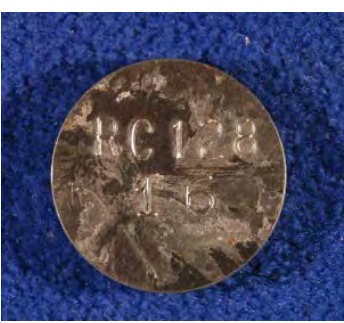

Back

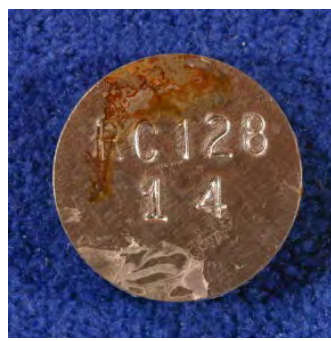

Back

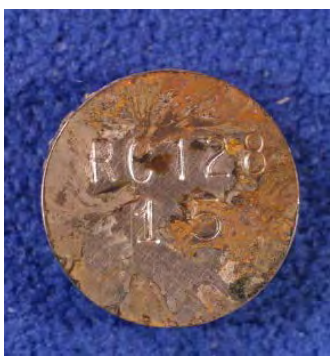

Back

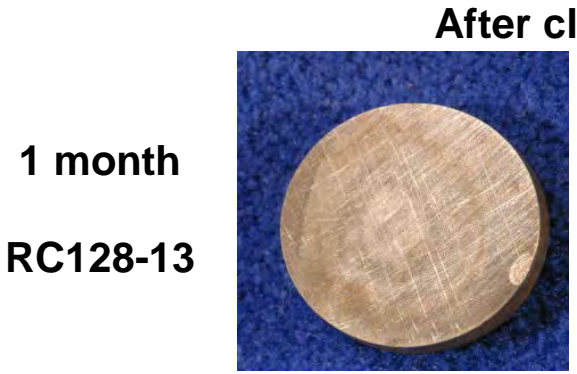

Front

2 months

RC128-16

3 months

RC128-14

4 months

RC128-15

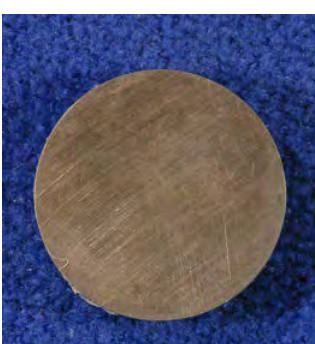

Front

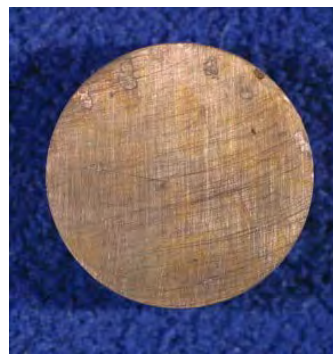

Front

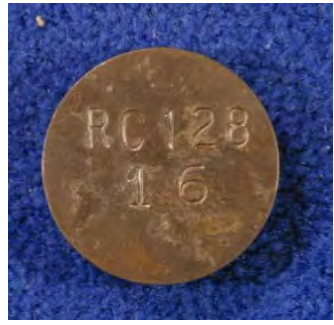

Back

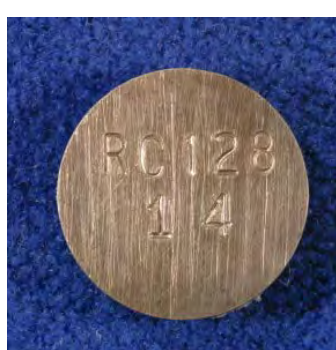

Back

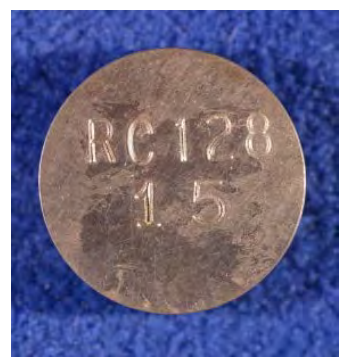

Back 


\section{Vessel 2: Level 2}

1 month

RC128-17
2 months

RC128-20

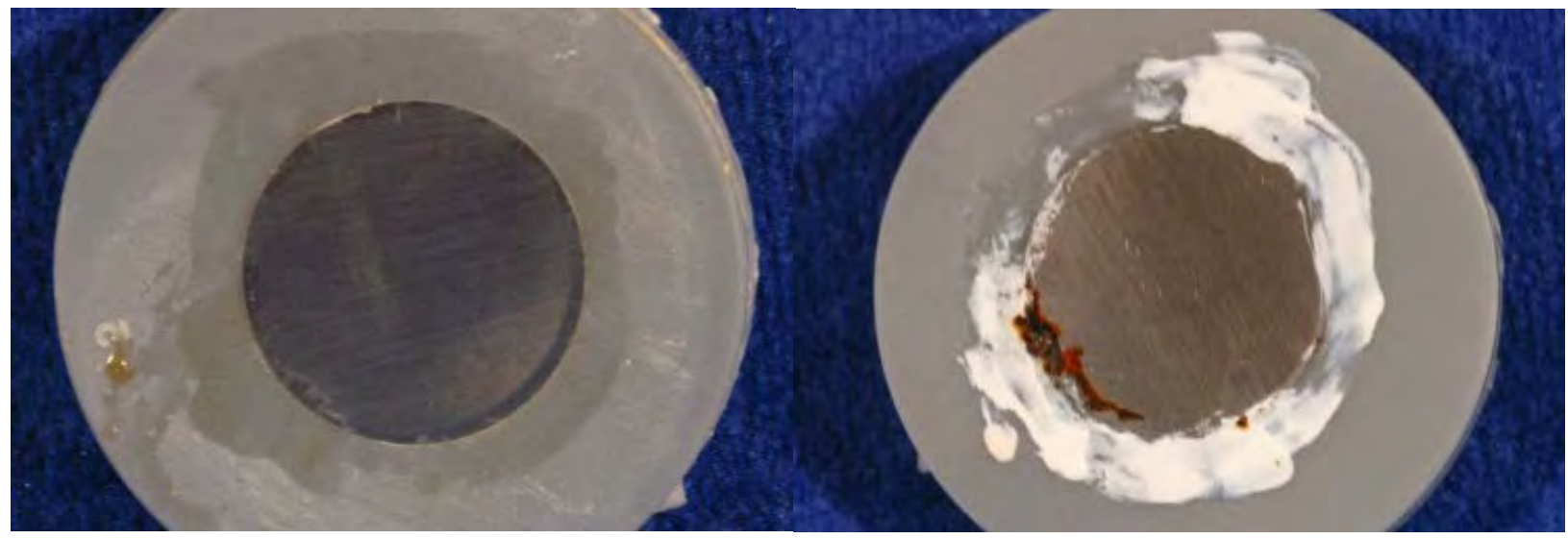

3 months

RC128-18
4 months

RC128-19

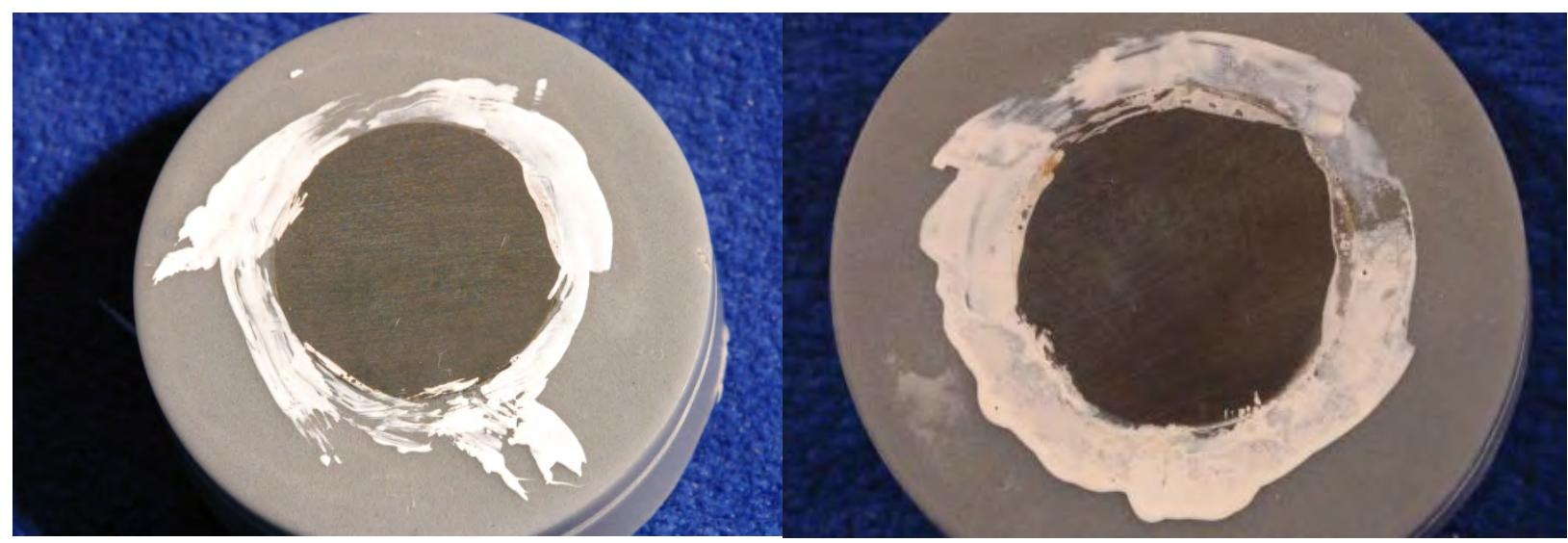


Vessel 2: Level 2

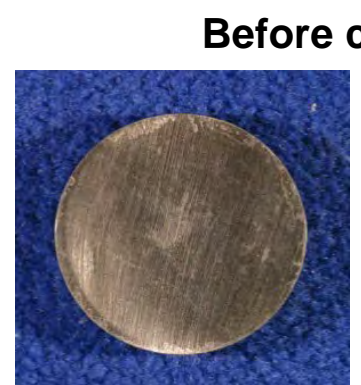

Front

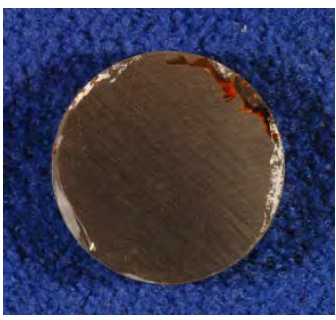

Front

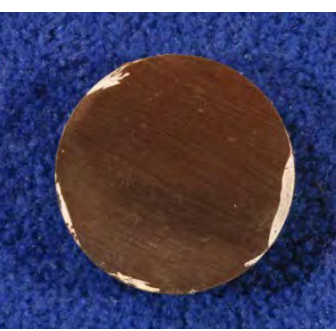

Front

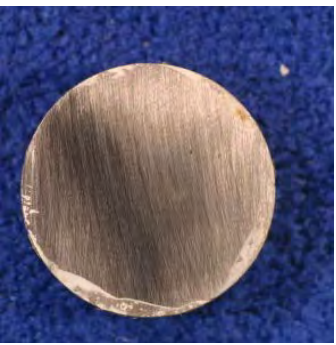

Front

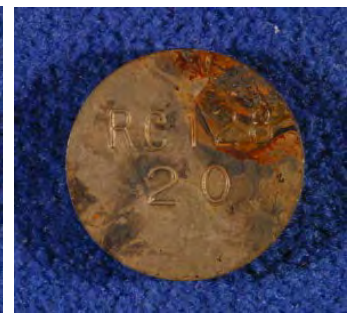

Back

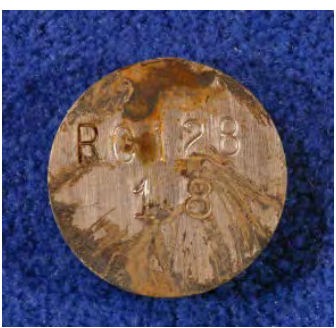

Back

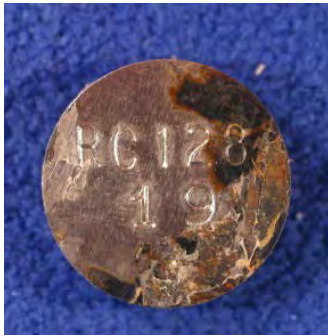

Back

1 month

RC128-17

2 months

RC128-20

3 months

RC128-18

4 months

RC128-19

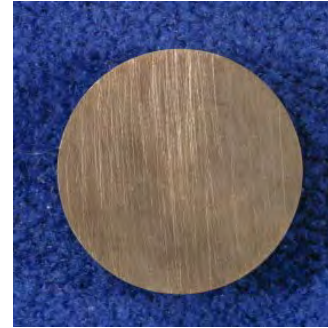

Front

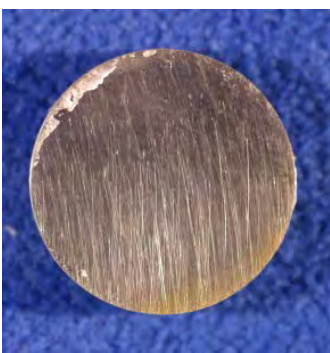

Front

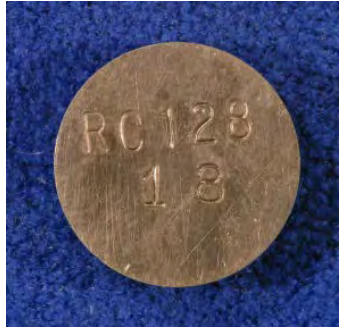

Back

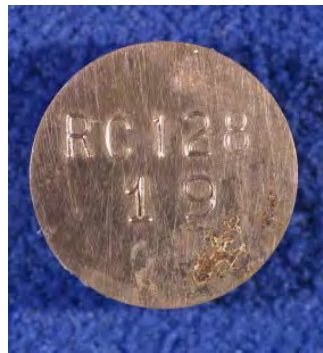

Back 
Vessel 2: Level 1

1 month

RC128-21
2 months

RC128-22

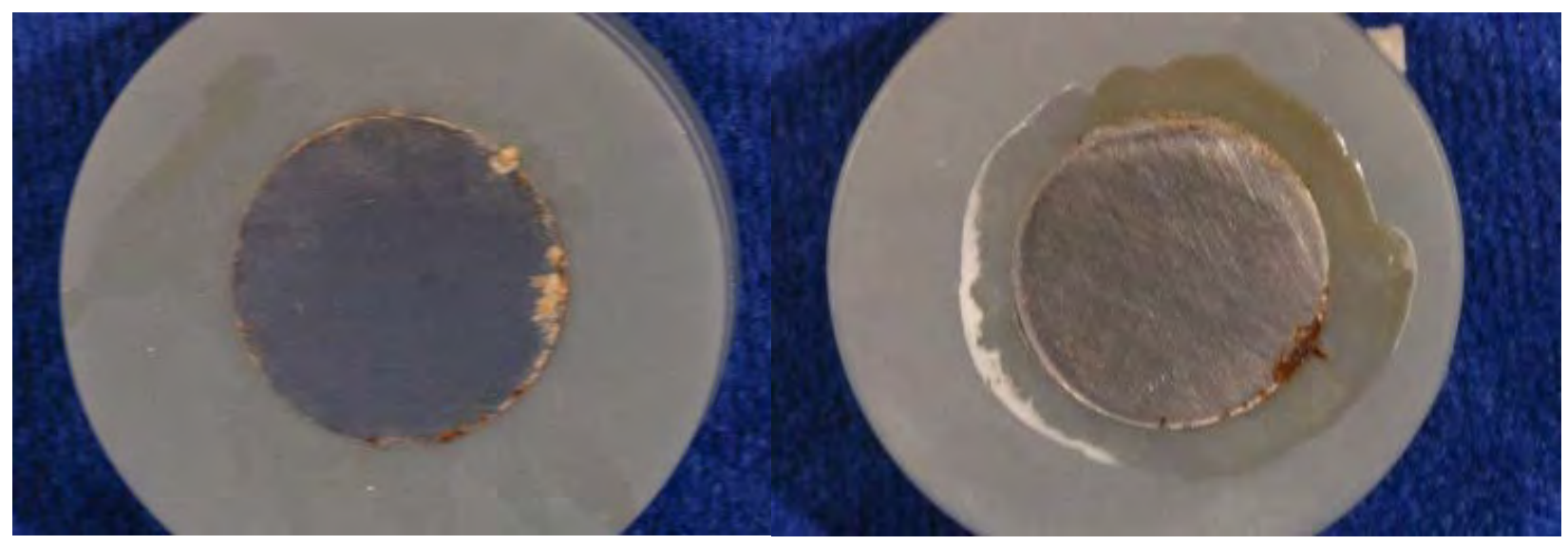

3 months

RC128-24
4 months

RC128-23

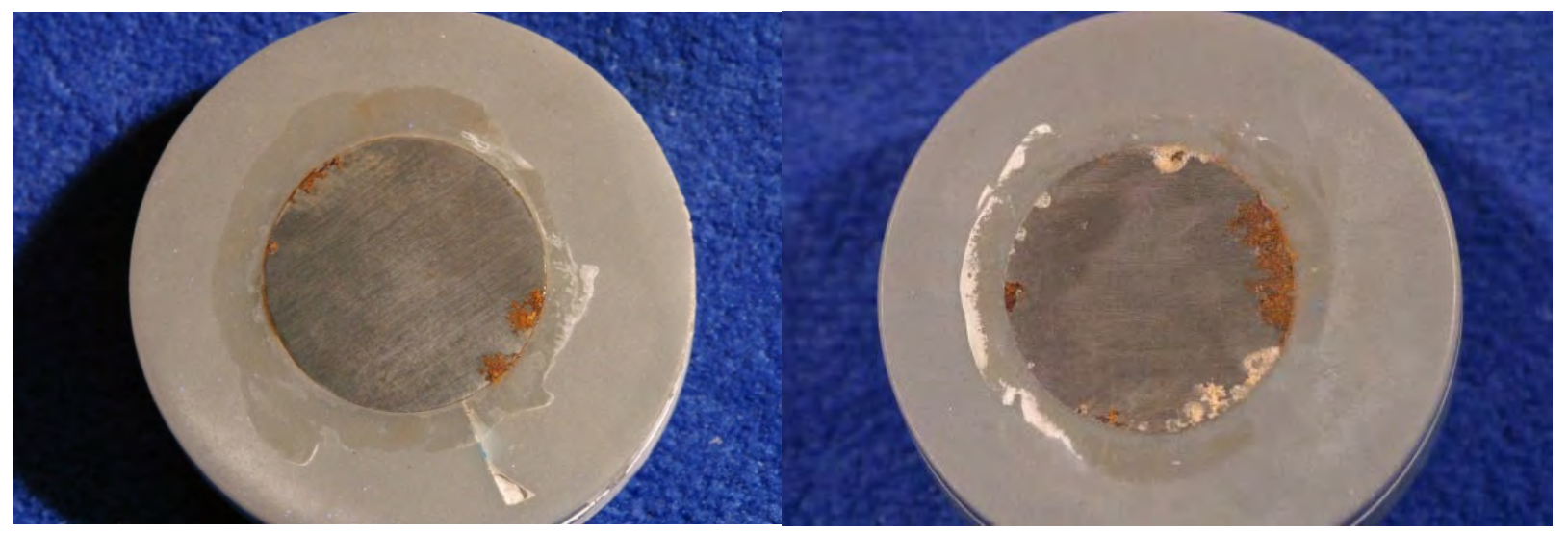




\section{Vessel 2: Level 1}

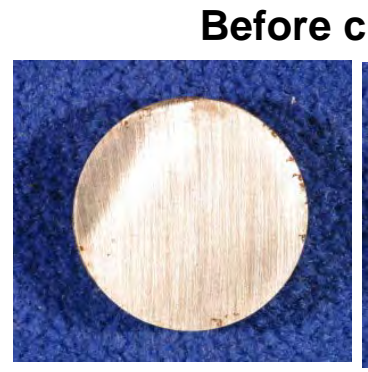

Front

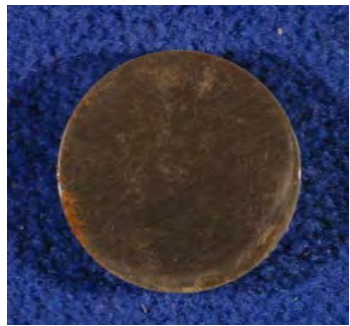

Front

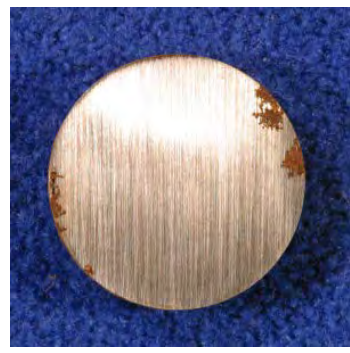

Front

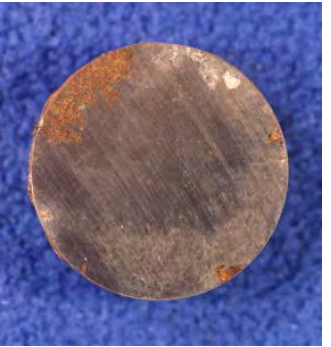

Front

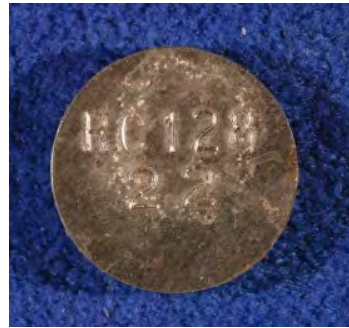

Back

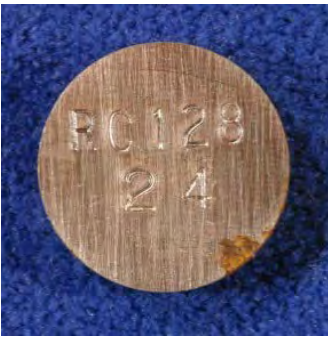

Back

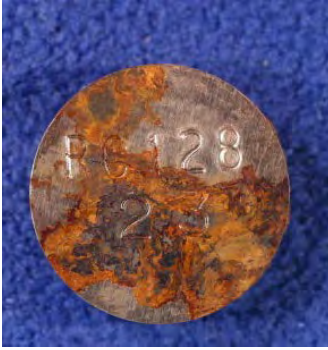

Back
2 months

RC128-22

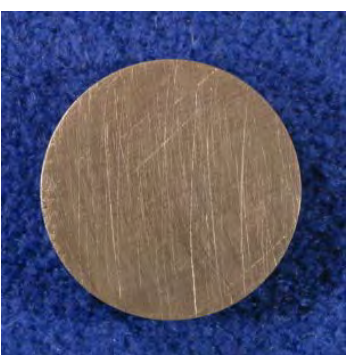

Front

3 months

RC128-24

4 months

RC128-23

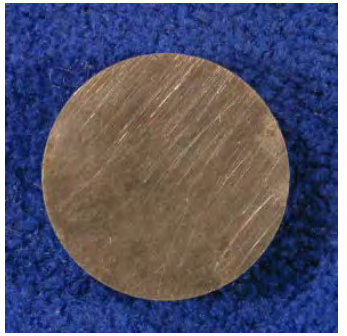

Front
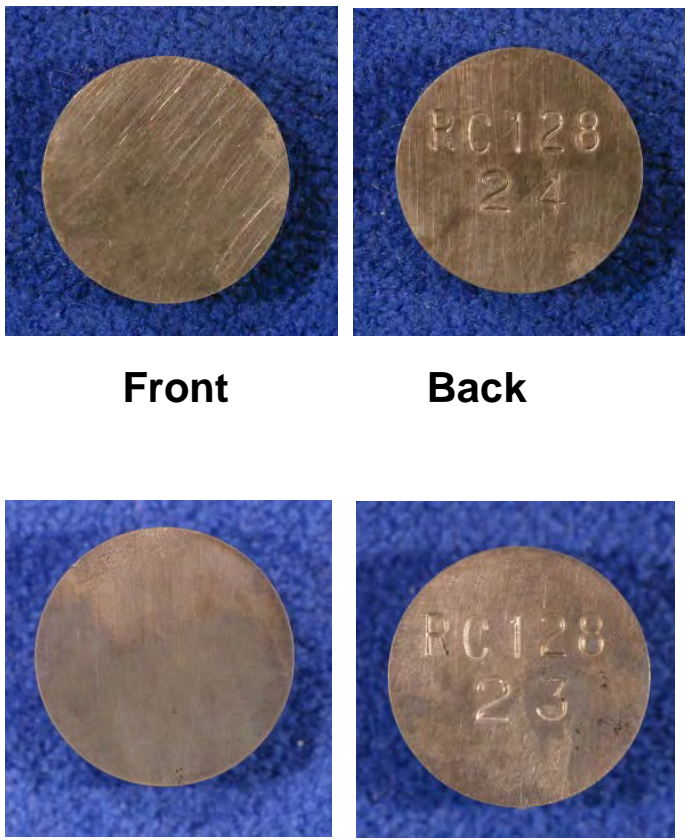

Front
Back

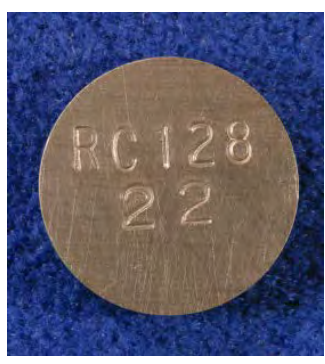

Back

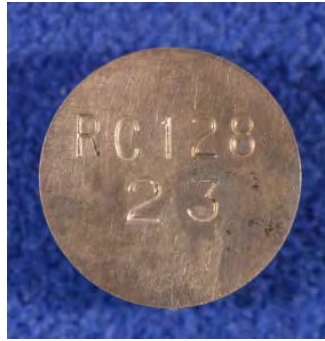

Back 


\section{Vessel 3: Level 3}

1 month

RC128-25
2 months

RC128-27

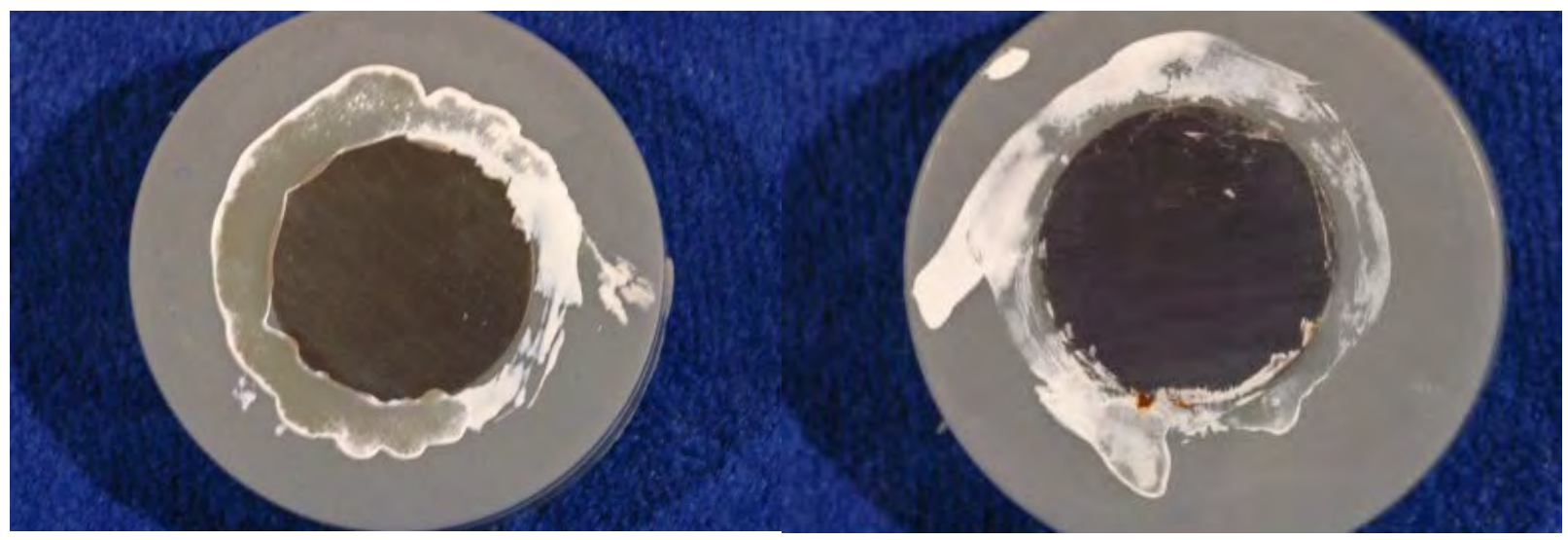

3 months

RC128-28
4 months

RC128-26

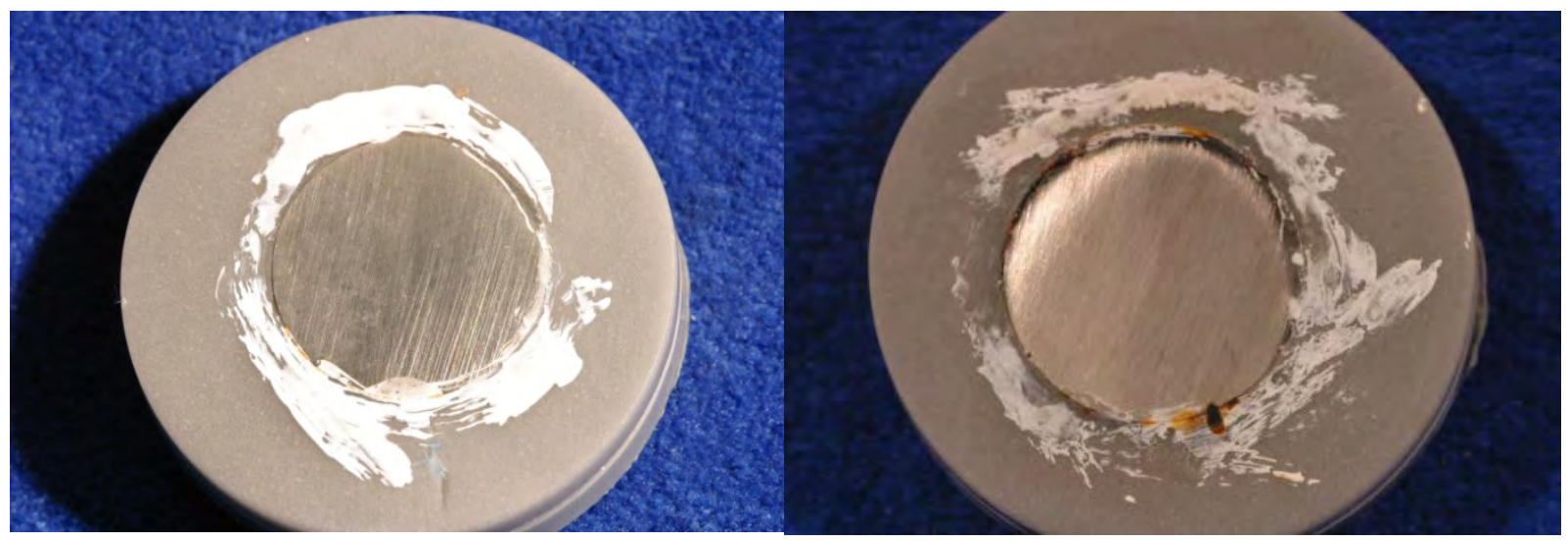




\section{Vessel 3: Level 3}

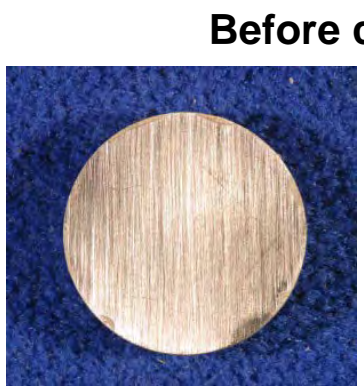

Front

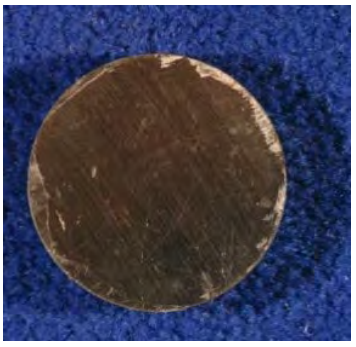

Front

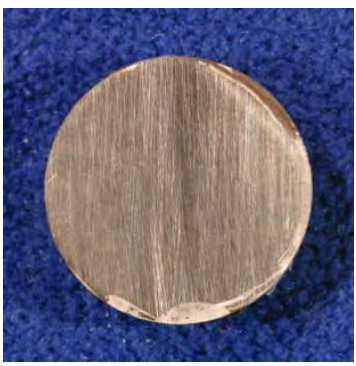

Front

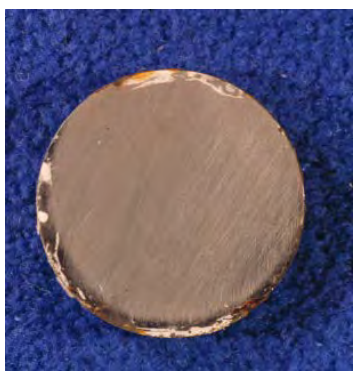

Front

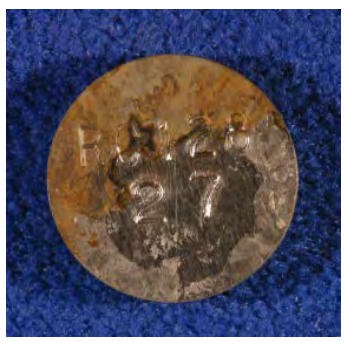

Back

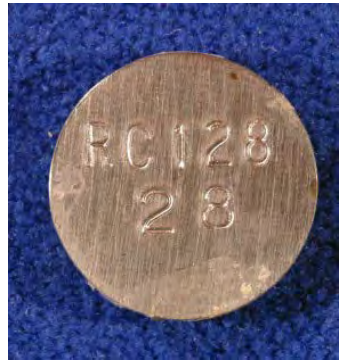

Back

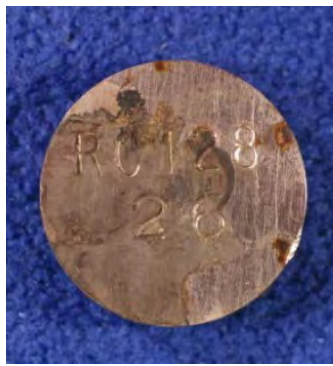

Back
2 months

RC128-27

3 months

$\mathrm{RC} 128-28$

4 months

RC128-26

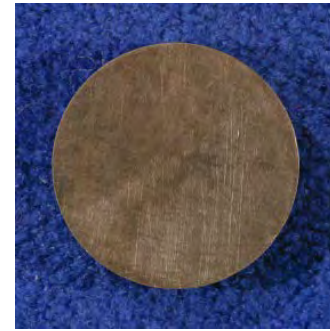

Front

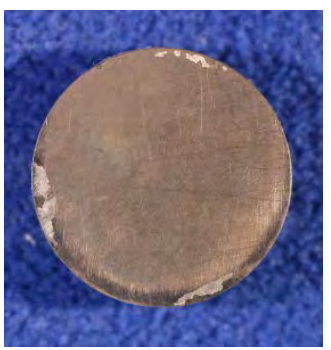

Front

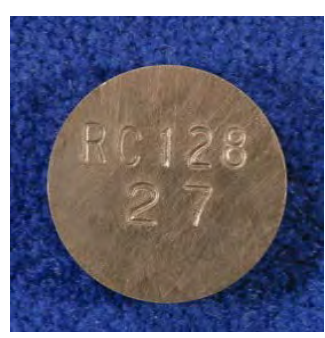

Back

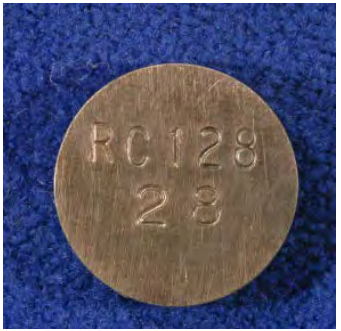

Back

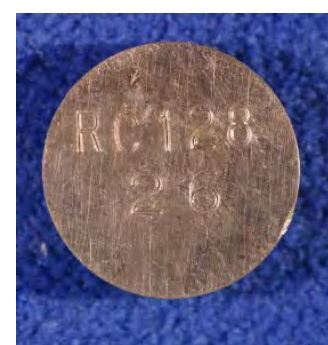

Back 


\section{Vessel 3: Level 2}

1 month

RC128-29
2 months

RC128-32

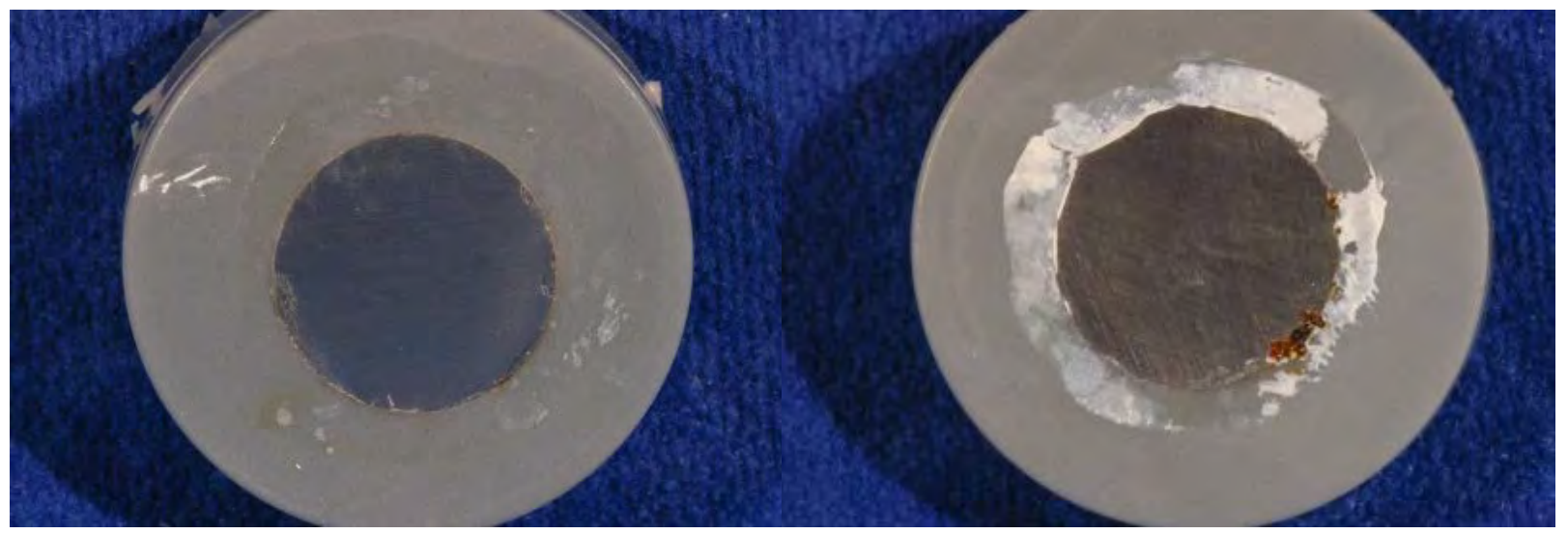

3 months

RC128-30
4 months

RC128-31

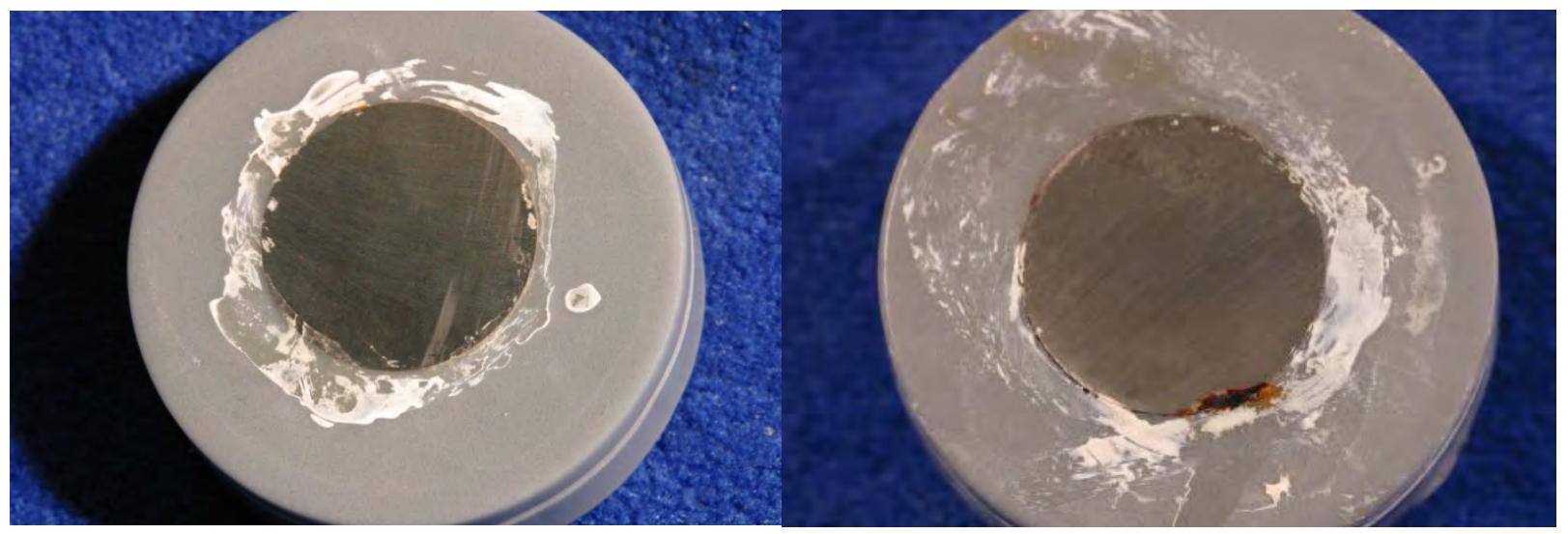




\section{Vessel 3: Level 2}

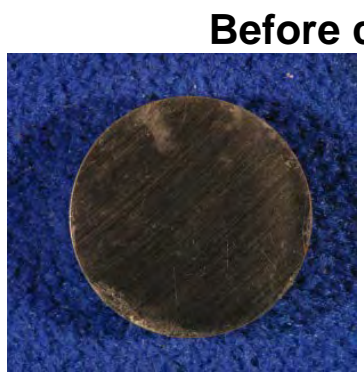

Front

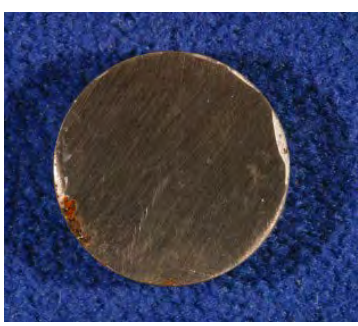

Front

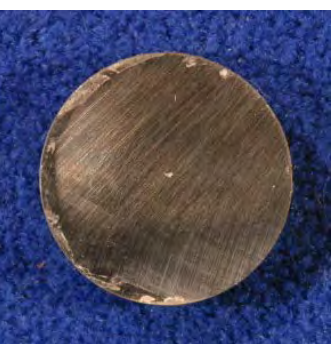

Front

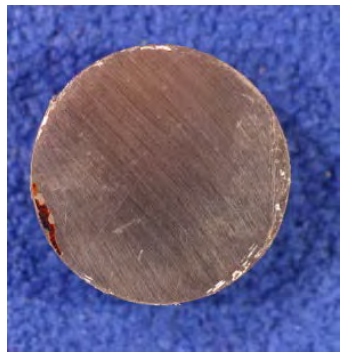

Front

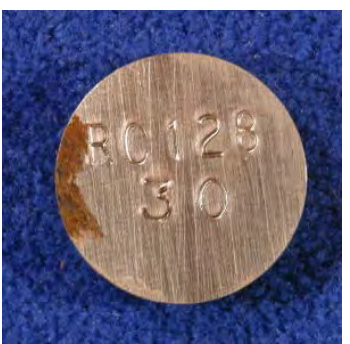

Back

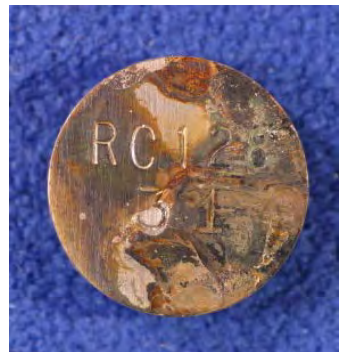

Back

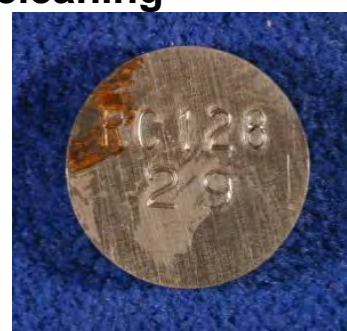

Back

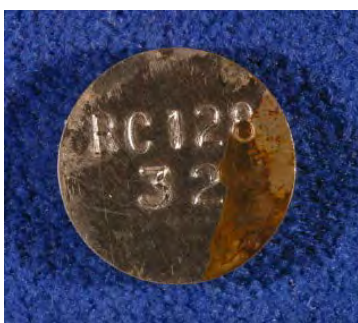

Back

3 months

RC128-30

4 months

RC128-31

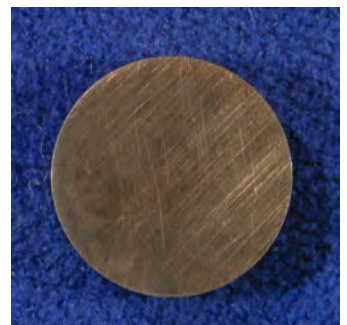

Front

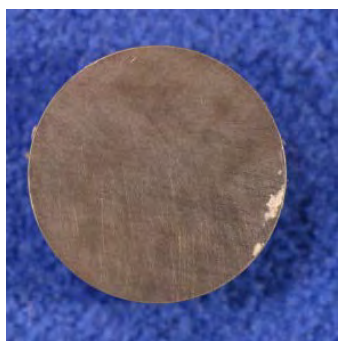

Front

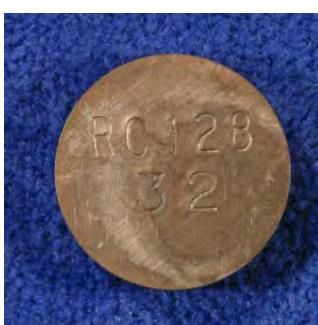

Back

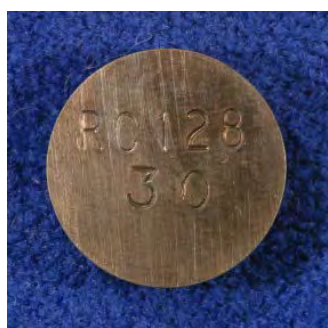

Back

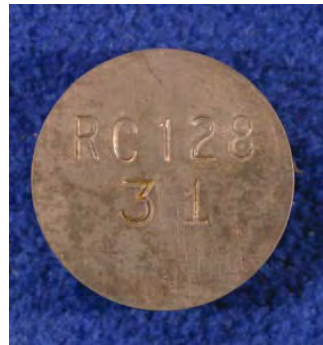

Back 


\section{Vessel 3: Level 1}

1 month

RC128-33
2 months

RC128-34

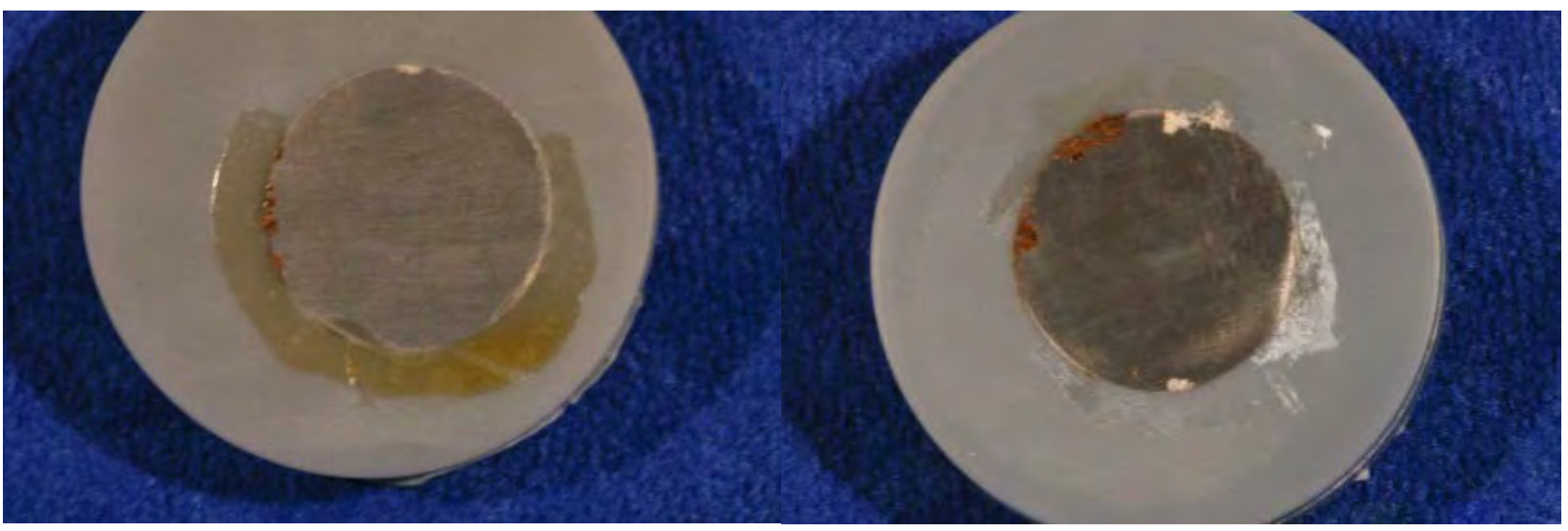

3 months

RC128-35
4 months

RC128-36

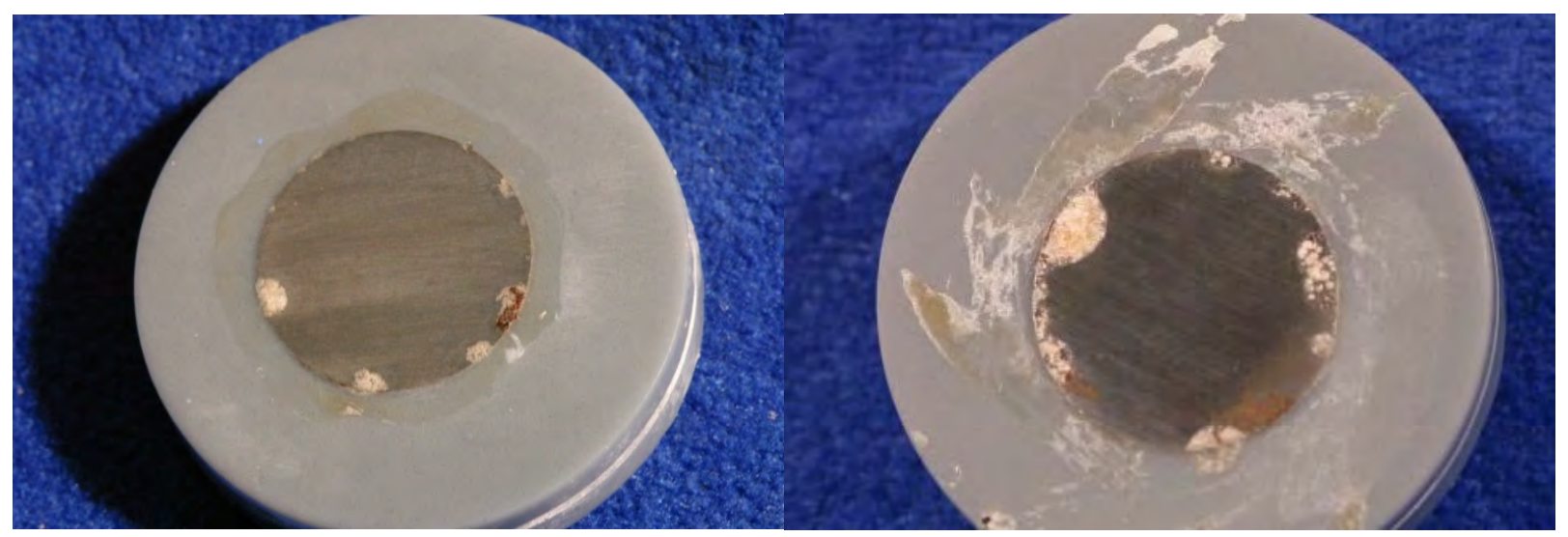


Vessel 3: Level 1

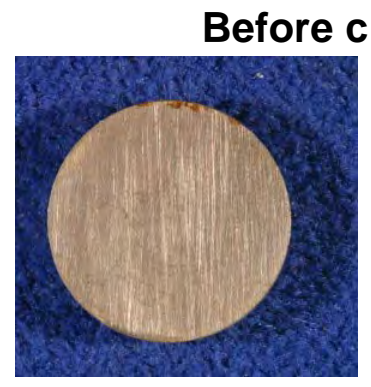

Front

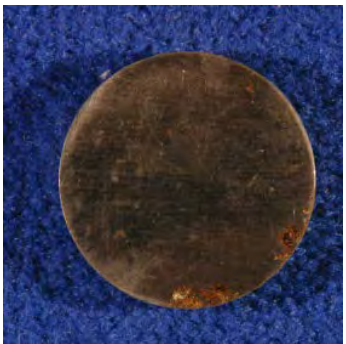

Front

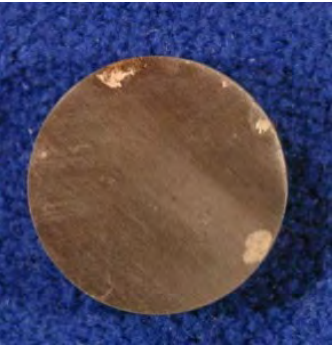

Front

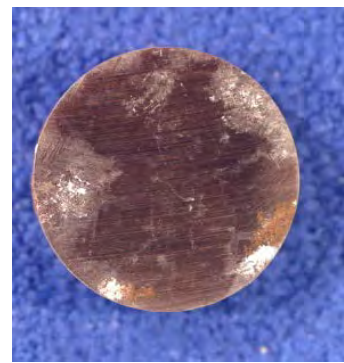

Front

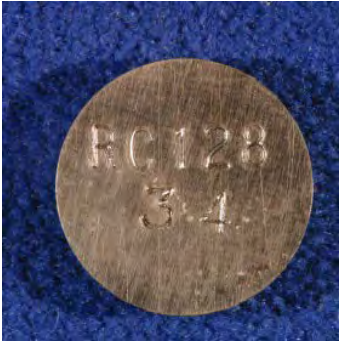

Back

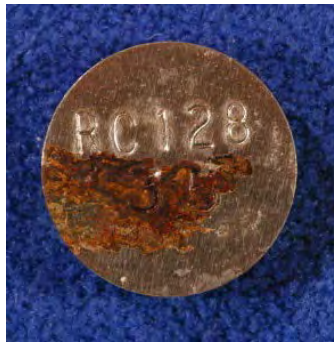

Back

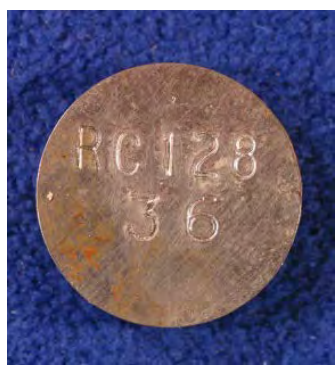

Back

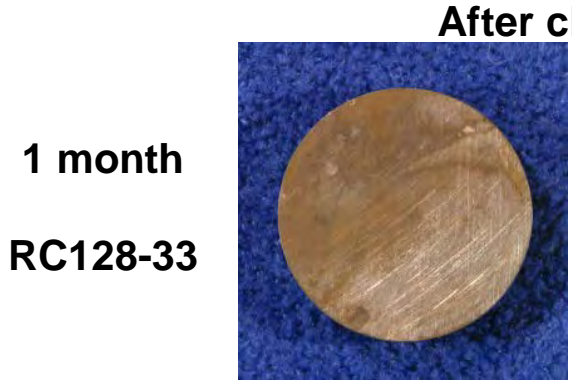

Front

2 months

RC128-34

3 months

RC128-35

4 months

RC128-36

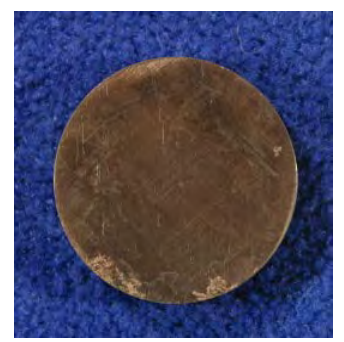

Front

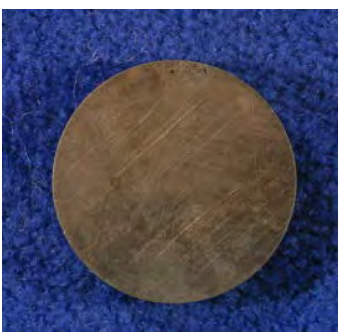

Front

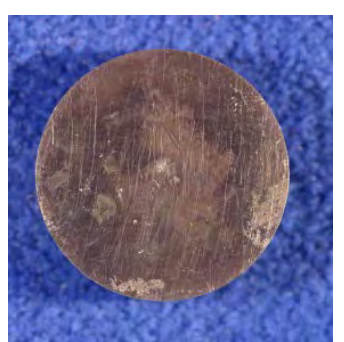

Front

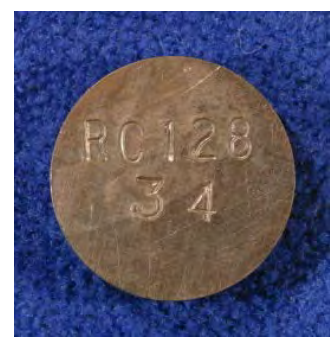

Back

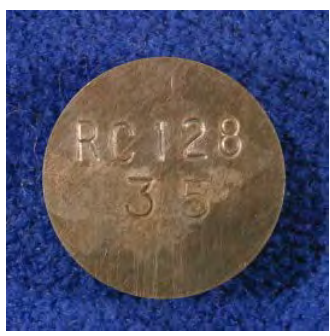

Back

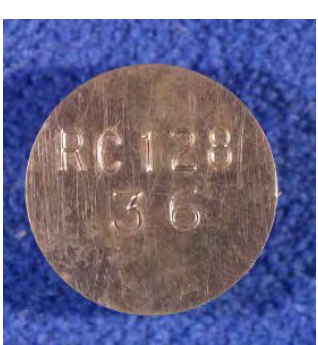

Back 


\section{Vessel 4: Level 3}

1 month

RC128-38
2 months

RC128-39

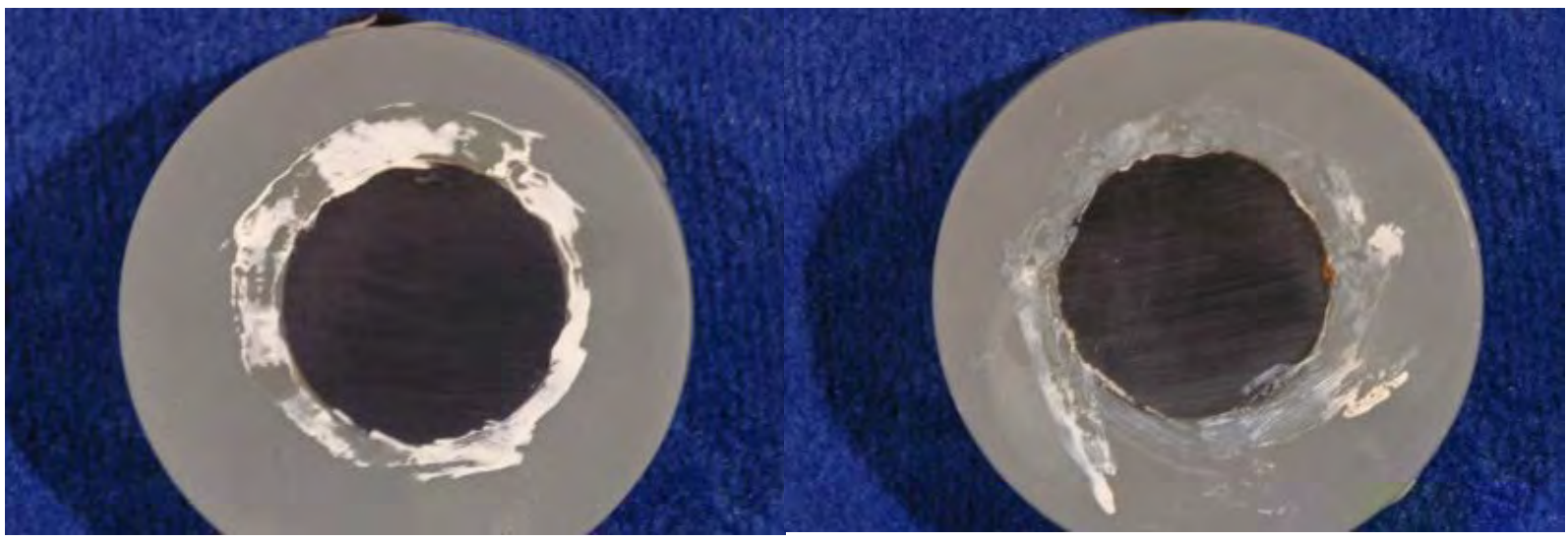

3 months

RC128-37
4 months

RC128-40

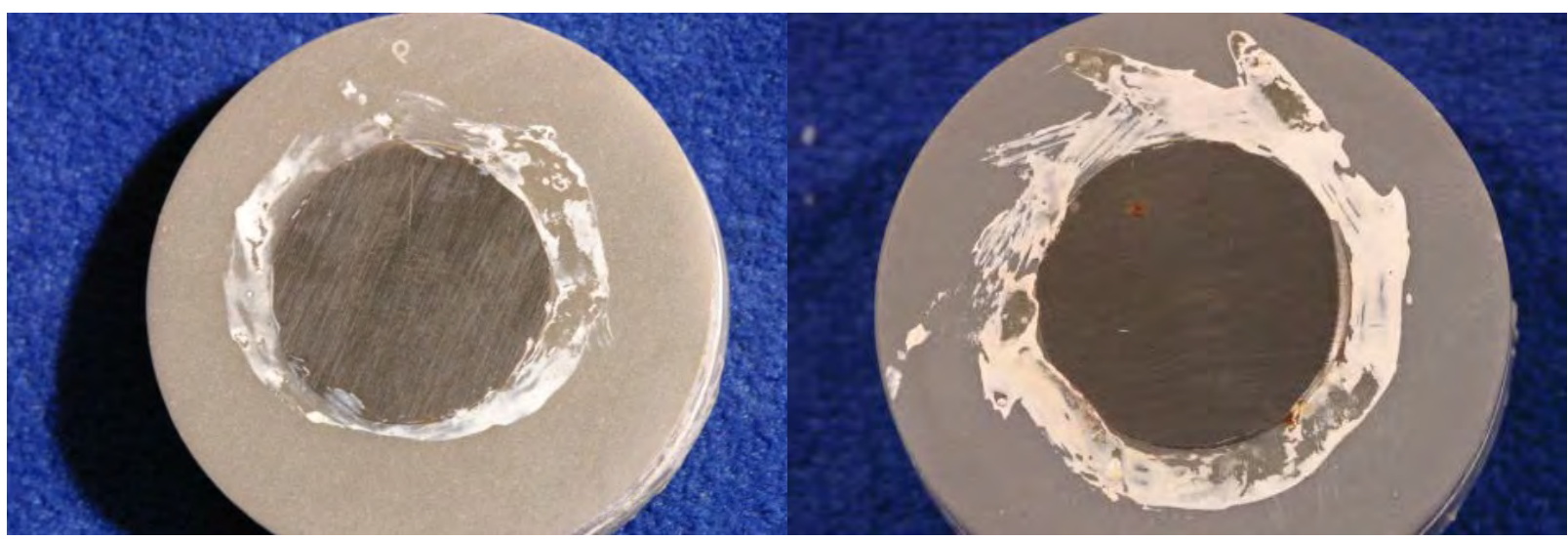




\section{Vessel 4: Level 3}

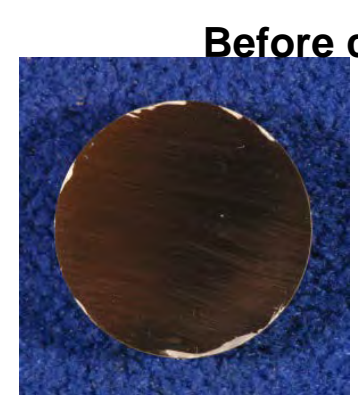

Front

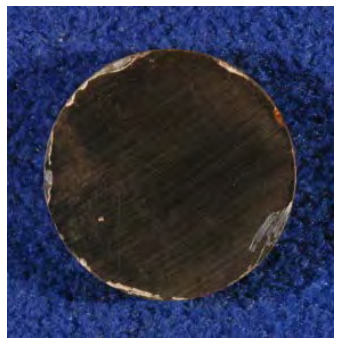

Front

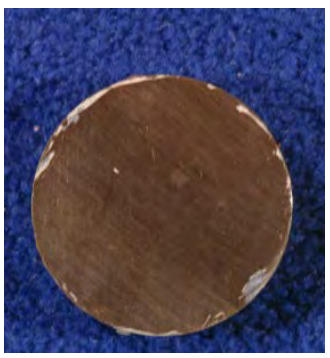

Front

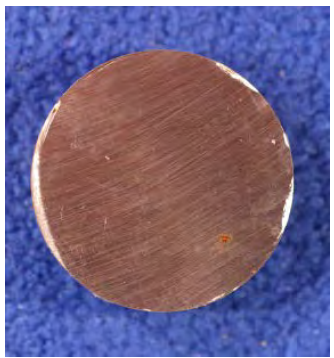

Front

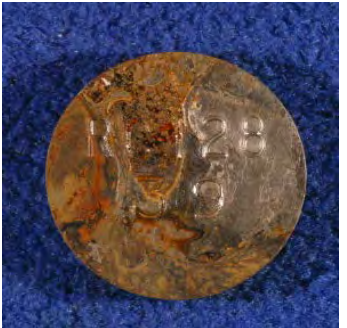

Back

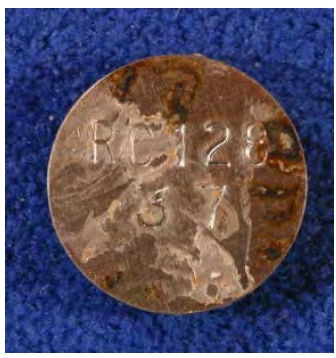

Back

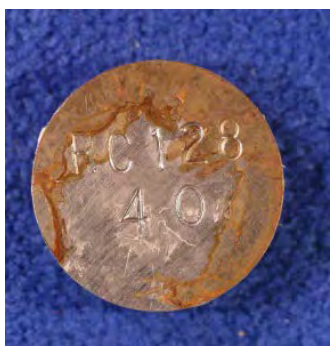

Back

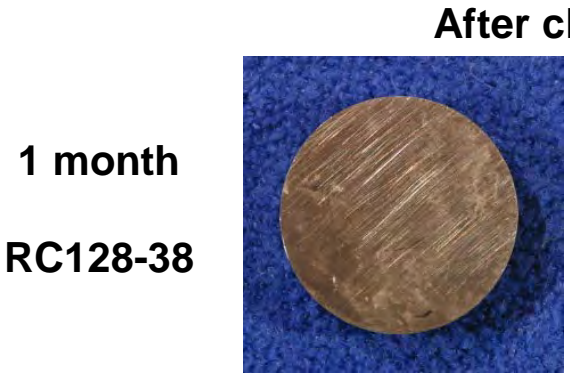

Front

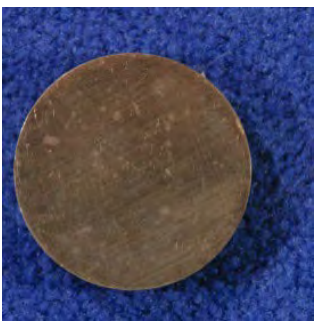

Front

3 months

RC128-37

4 months

RC128-40

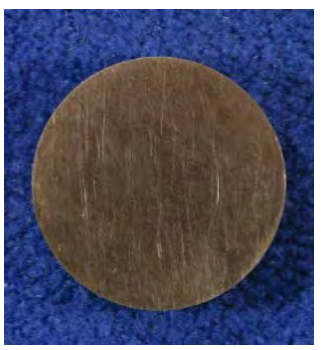

Front

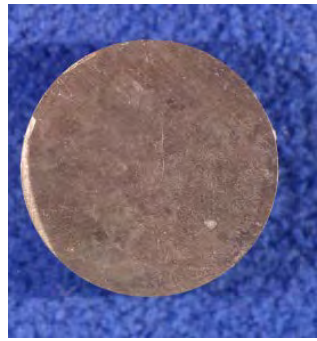

Front

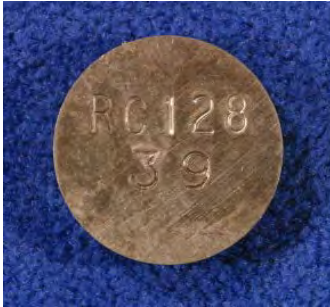

Back

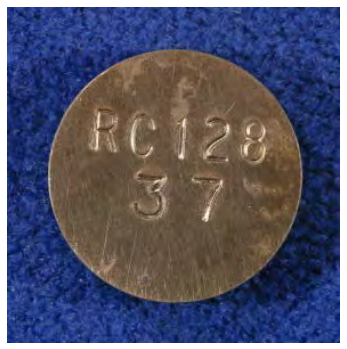

Back

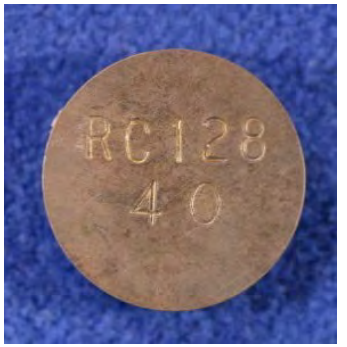

Back 
Vessel 4: Level 2

1 month

RC128-44
2 months

RC128-42

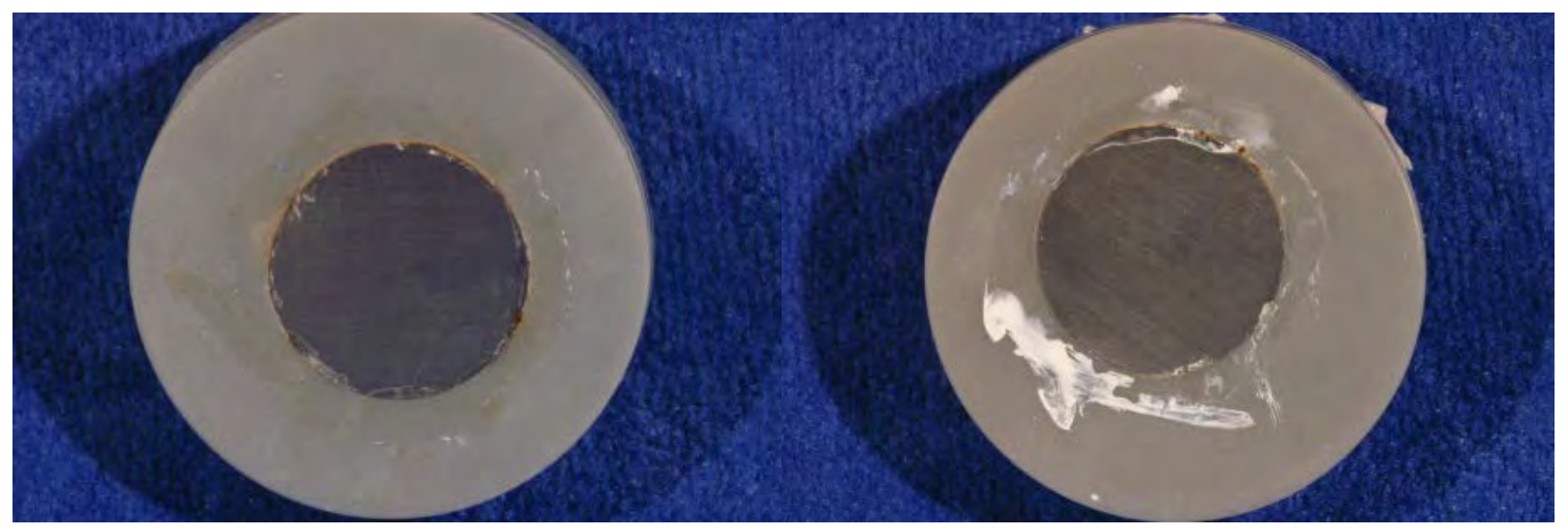

3 months

RC128-41
4 months

RC128-43

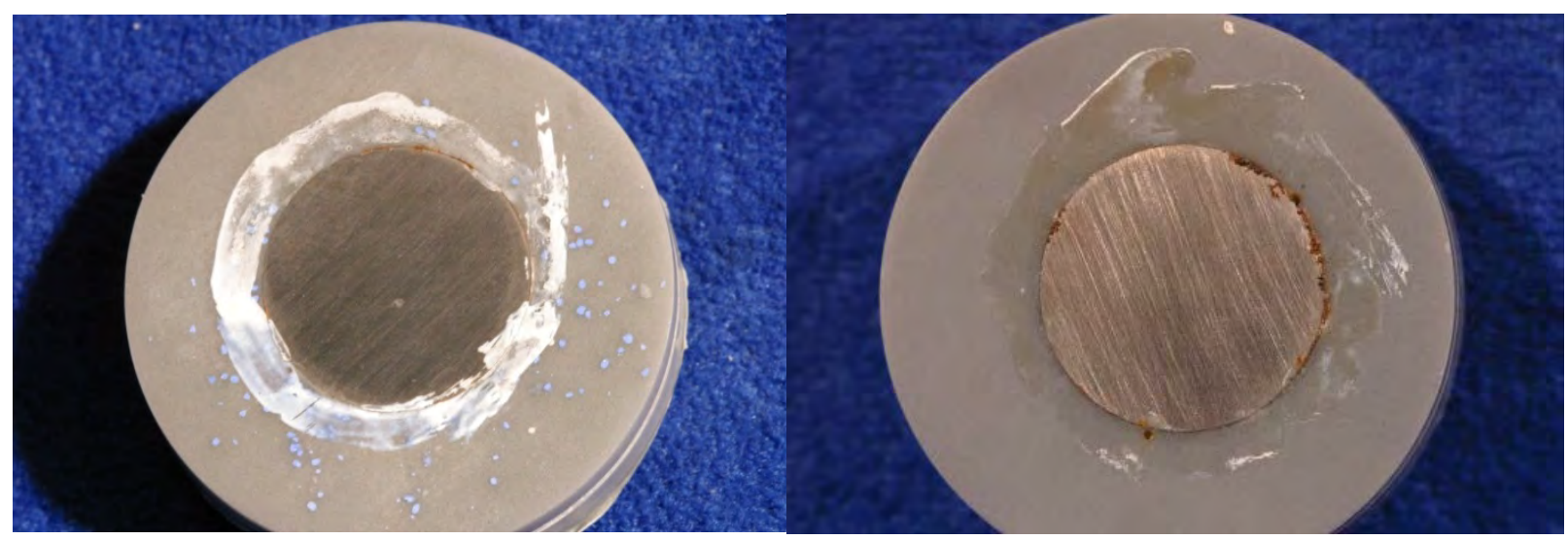




\section{Vessel 4: Level 2}

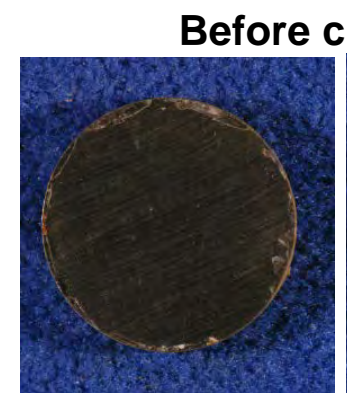

Front

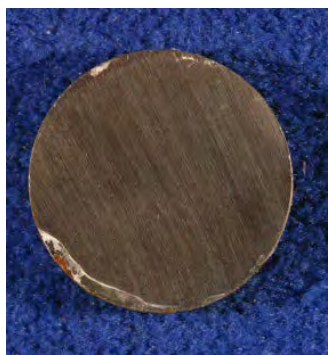

Front

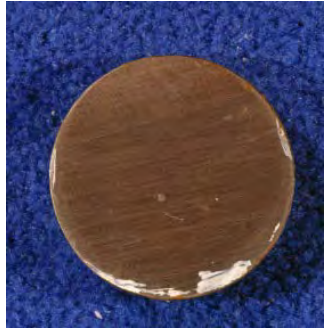

Front

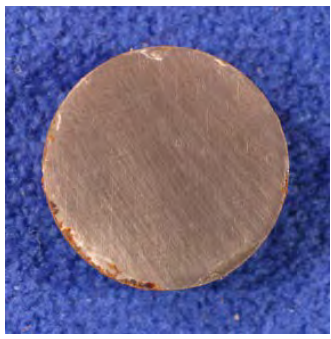

Front

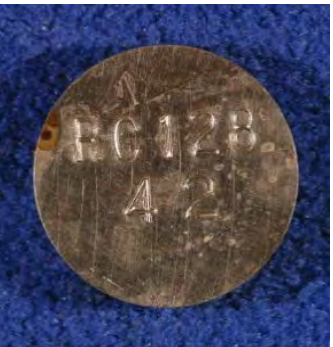

Back

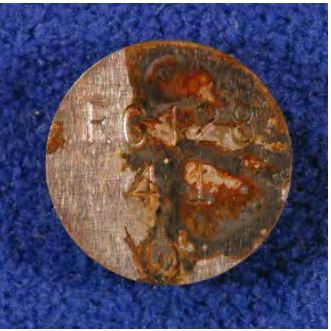

Back

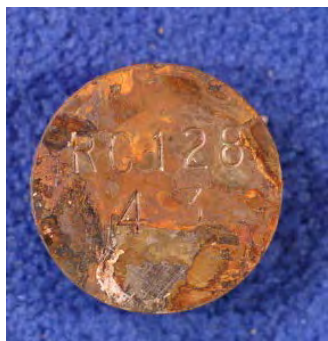

Back

\section{3 months}

RC128-41

4 months

RC128-43

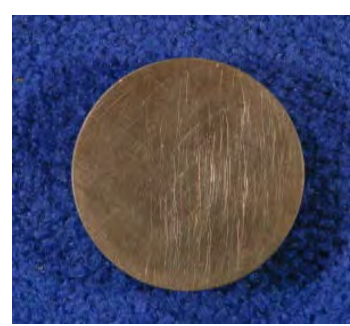

Front

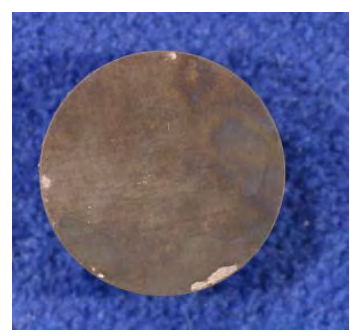

Front

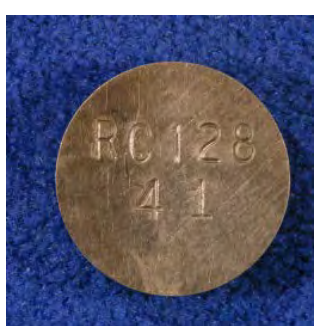

Back

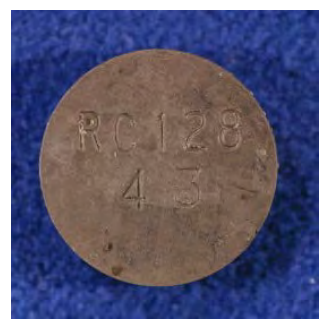

Back 
Vessel 4: Level 1

1 month

RC128-47
2 months

RC128-46

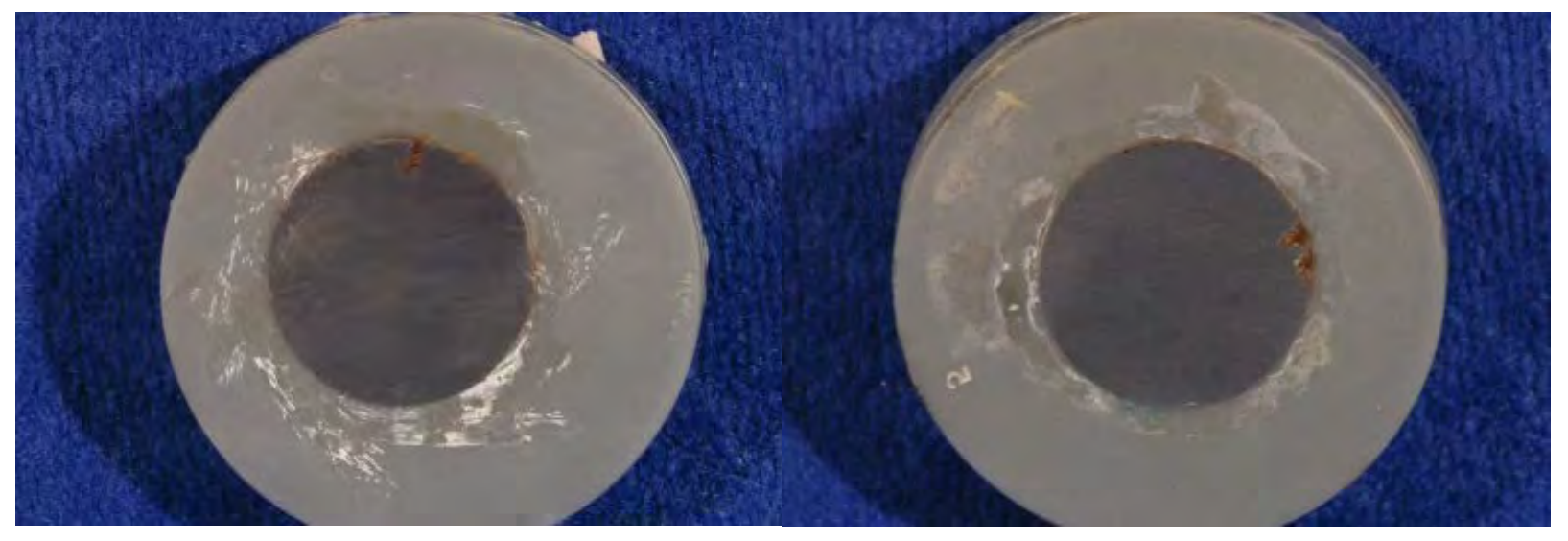

3 months

RC128-48
4 months

RC128-45

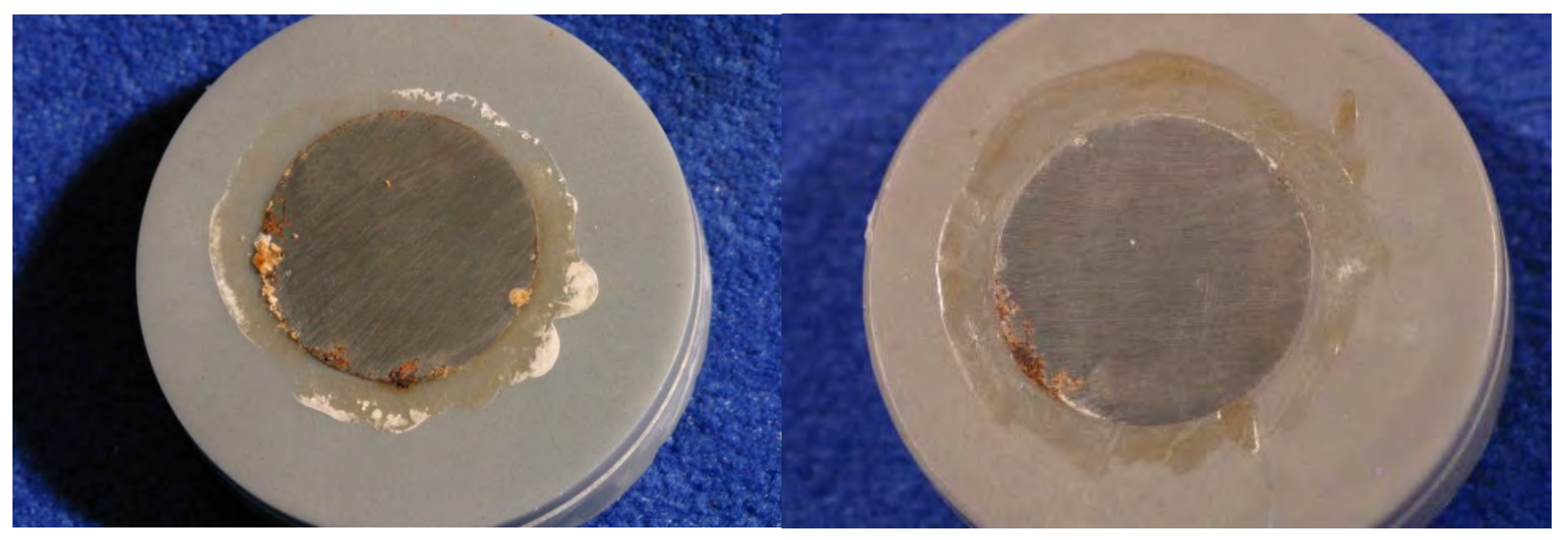


Vessel 4: Level 1

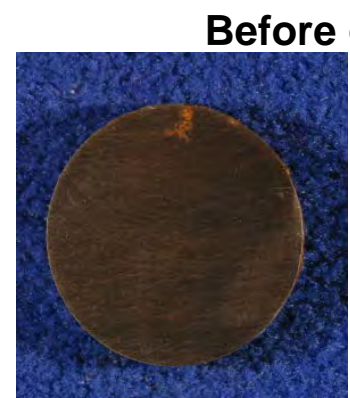

Front

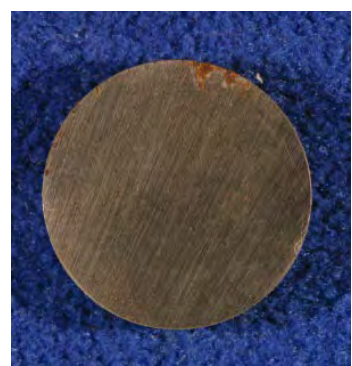

Front

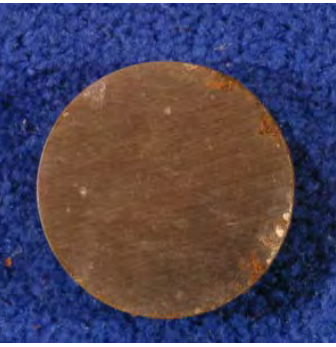

Front

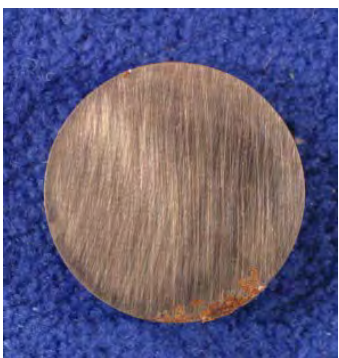

Front

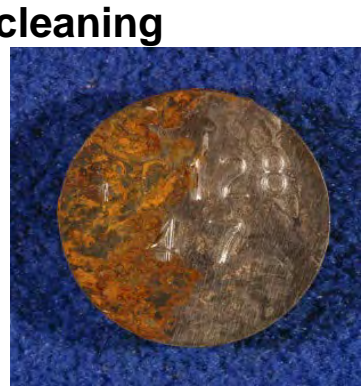

Back

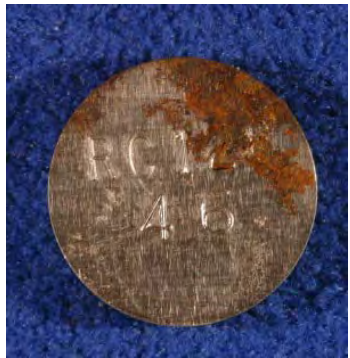

Back

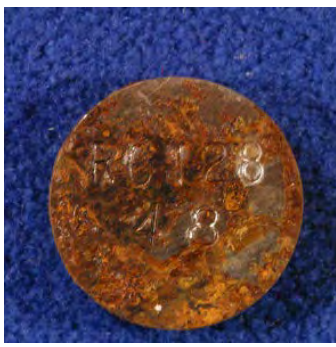

Back

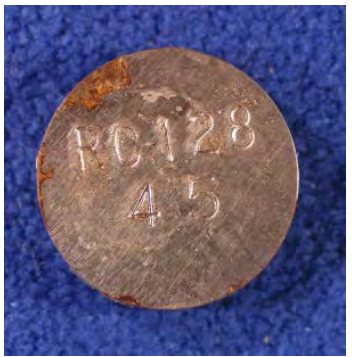

Back

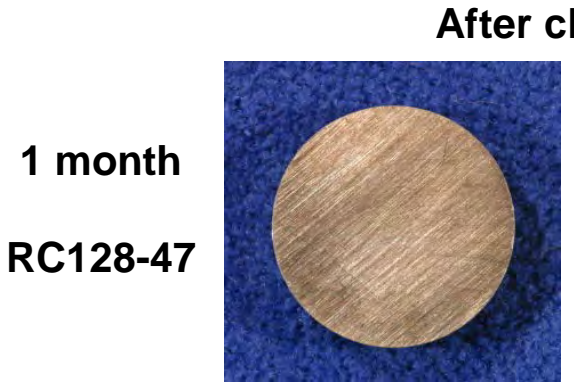

Front

2 months

RC128-46

3 months

RC128-48

4 months

RC128-45

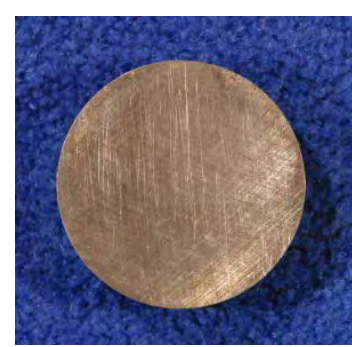

Front

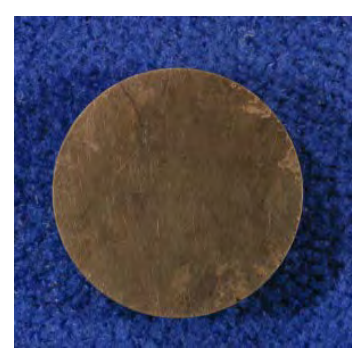

Front

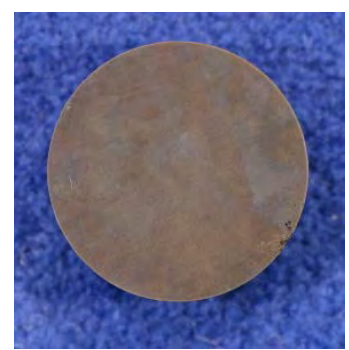

Front

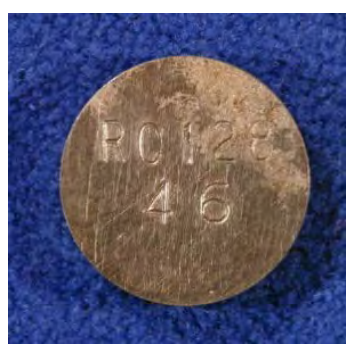

Back

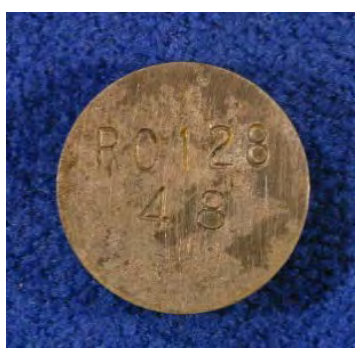

Back

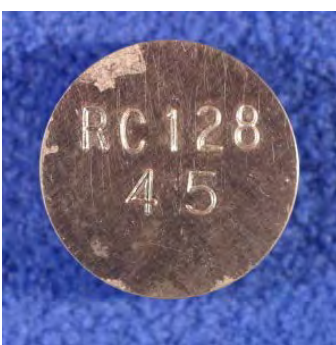

Back 


\section{Vessel 5: Level 3}

1 month

RC128-51
2 months

RC128-50

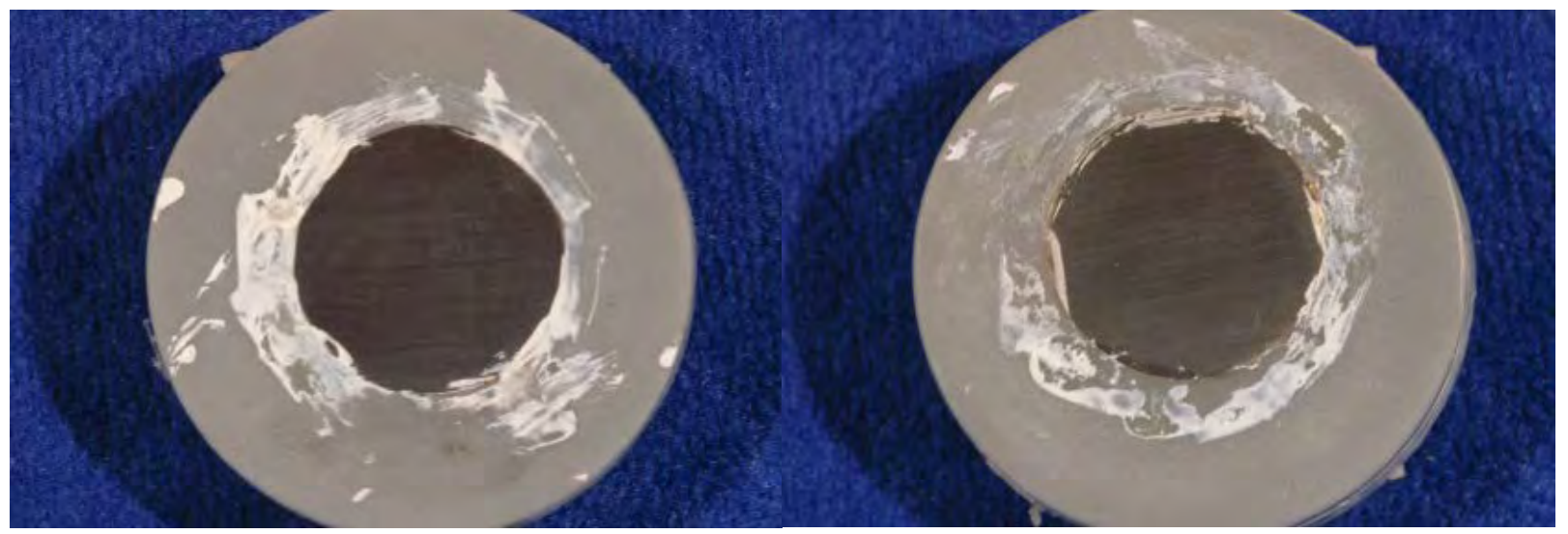

3 months

RC128-52
4 months

RC128-49

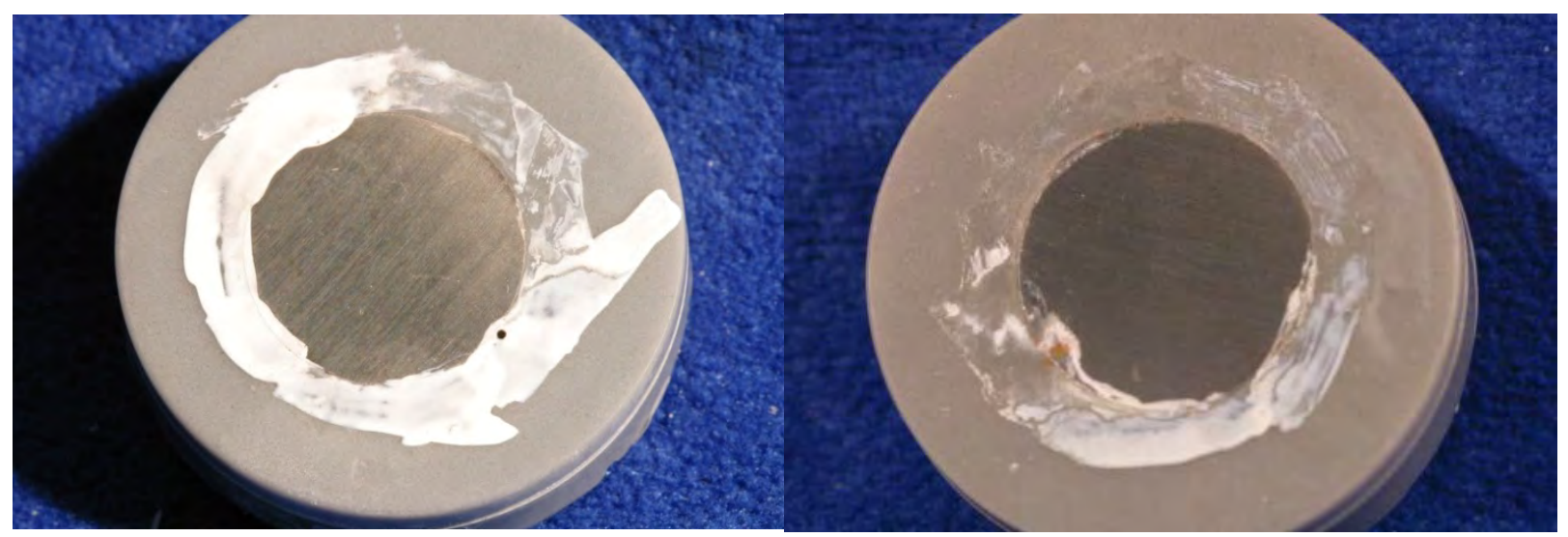




\section{Vessel 5: Level 3}

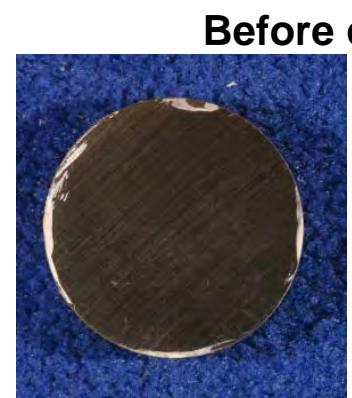

Front

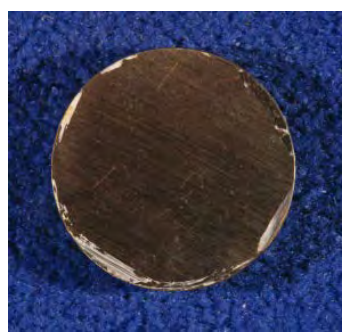

Front

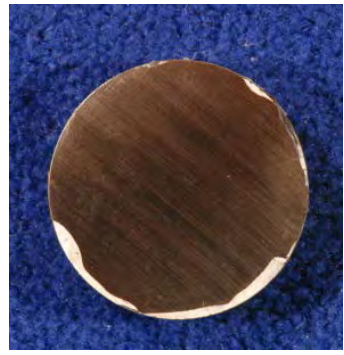

Front

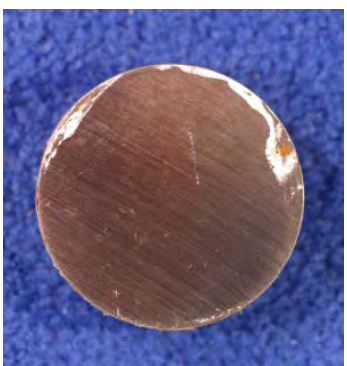

Front

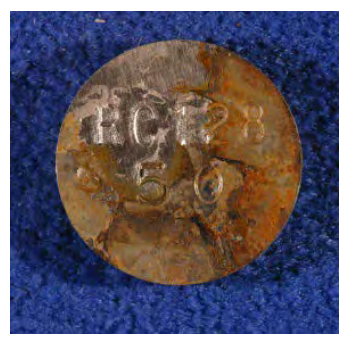

Back

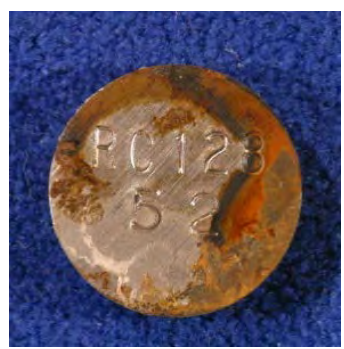

Back

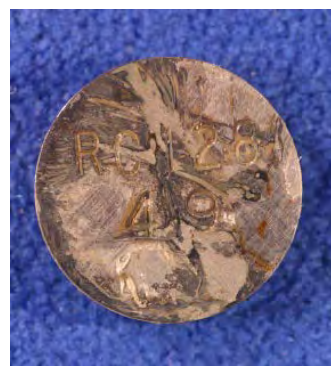

Back
2 months

RC128-50

3 months

RC128-52

4 months

RC128-49

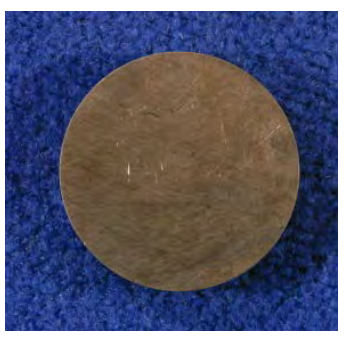

Front

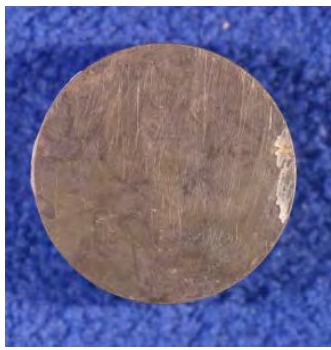

Front

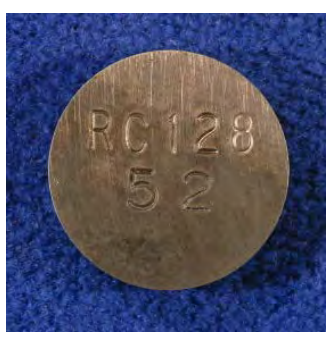

Back

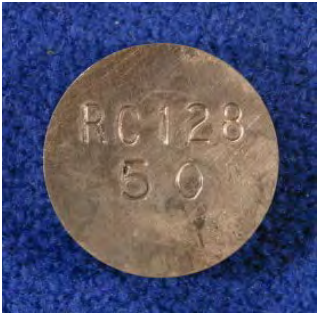

Back

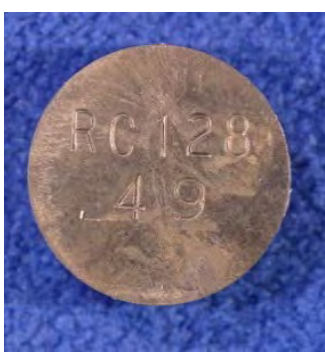

Back 


\section{Vessel 5: Level 2}

1 month

RC128-55
2 months

RC128-56

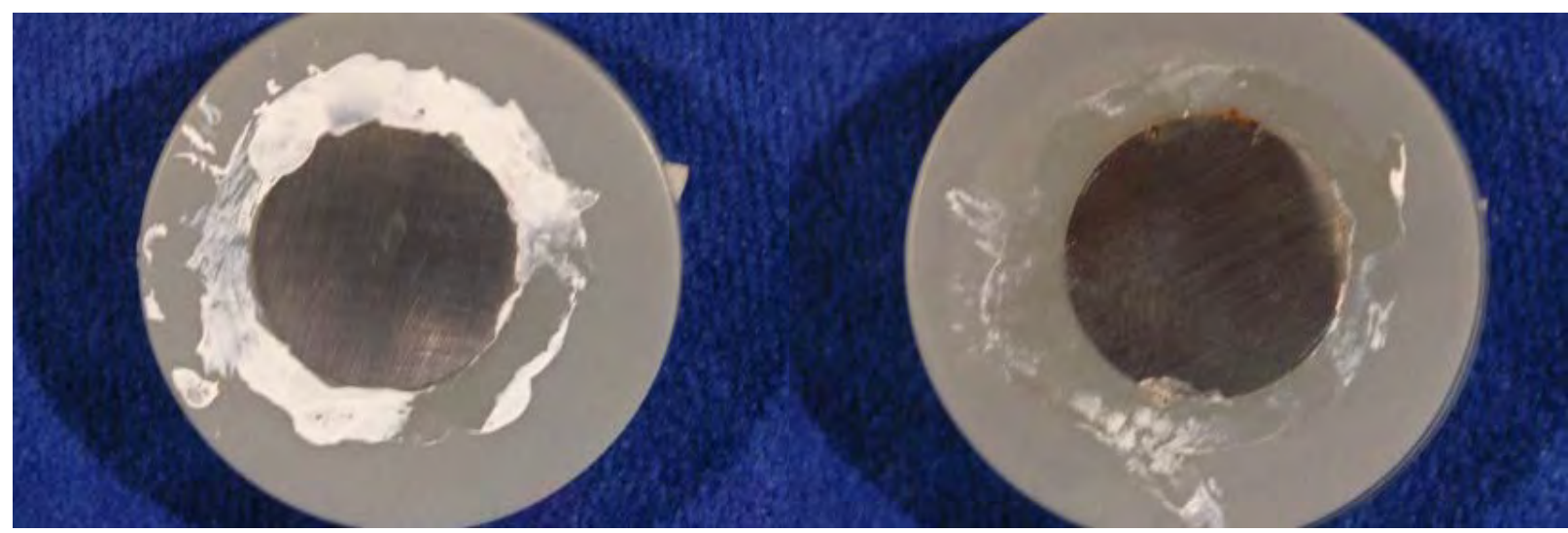

3 months

RC128-54
4 months

RC128-53

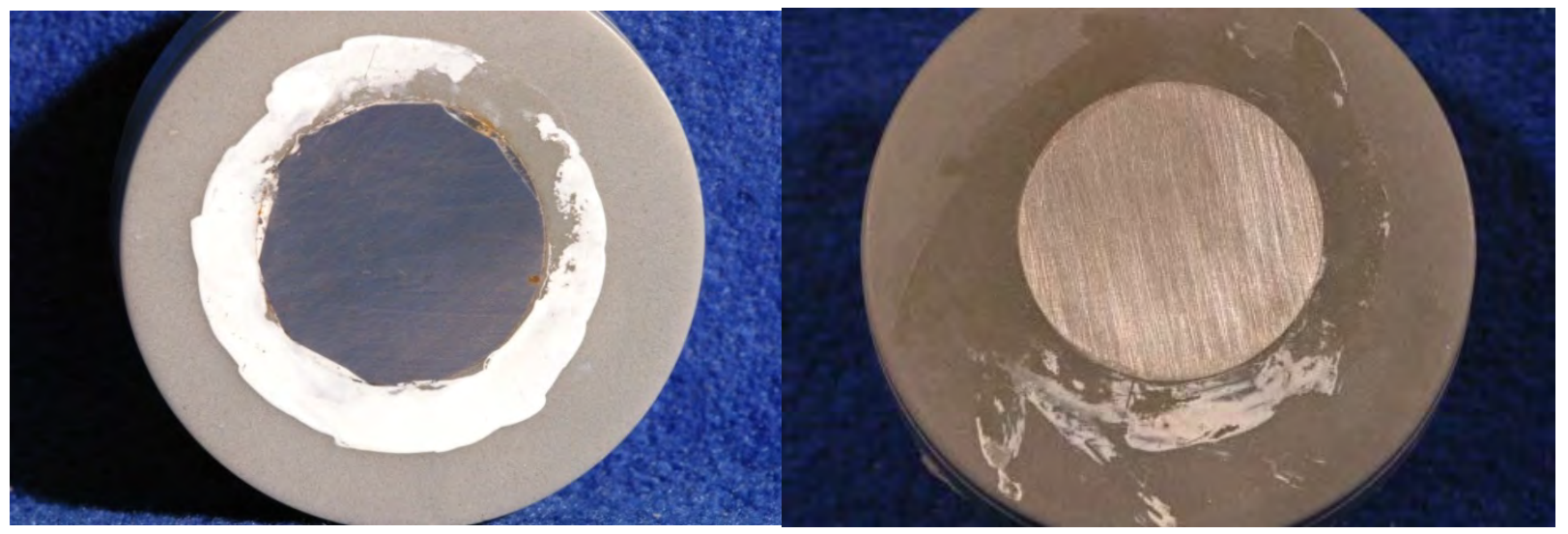


Vessel 5: Level 2

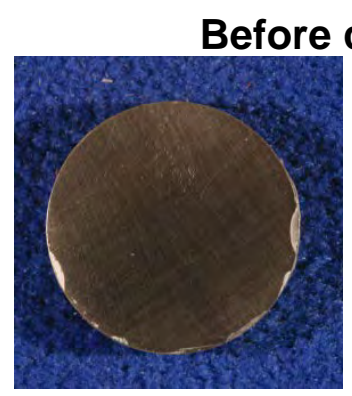

Front

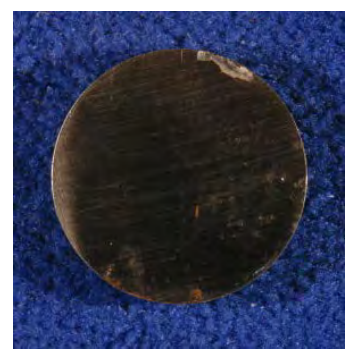

Front

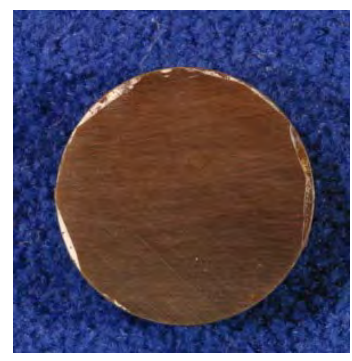

Front

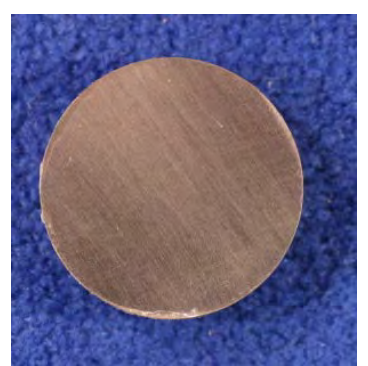

Front

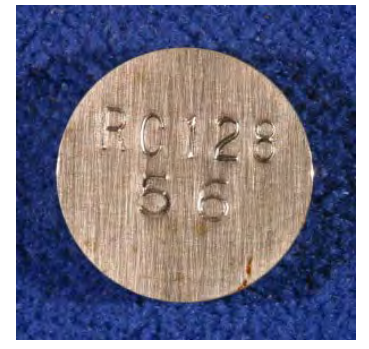

Back

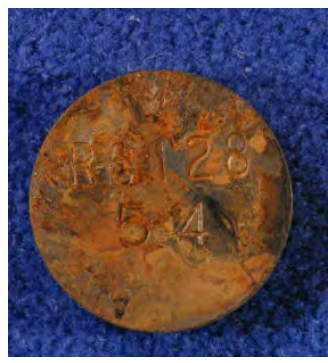

Back

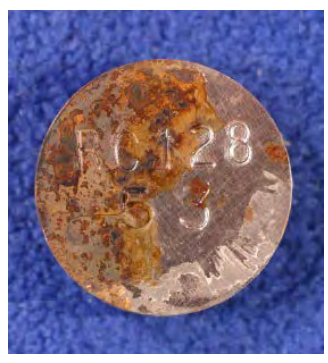

Back

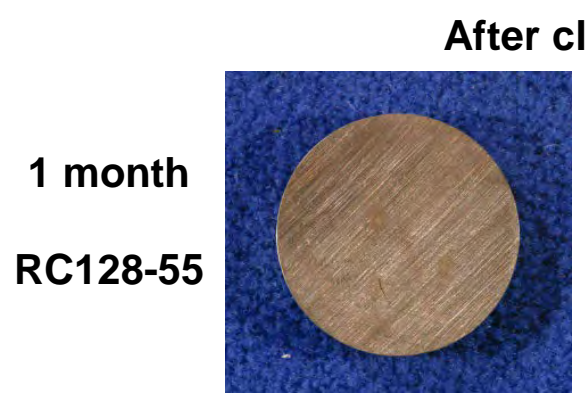

Front

2 months

RC128-56

3 months

RC128-54

4 months

RC128-53

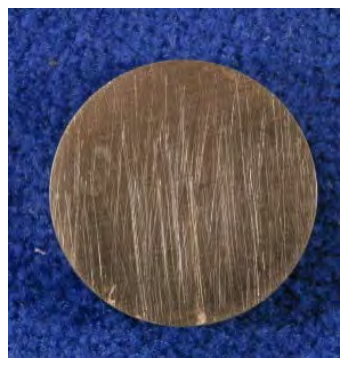

Front

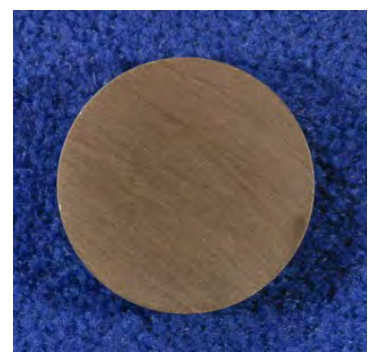

Front

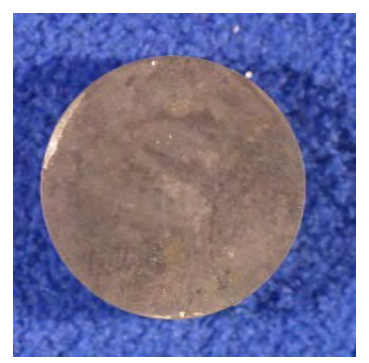

Front

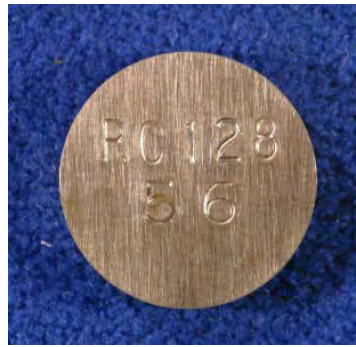

Back

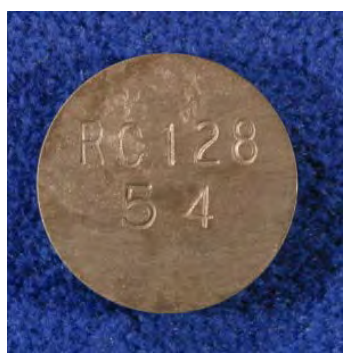

Back

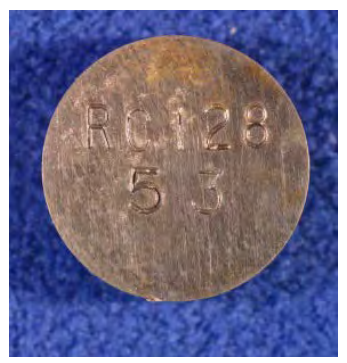

Back 


\section{Vessel 5: Level 1}

1 month

RC128-57
2 months

RC128-59

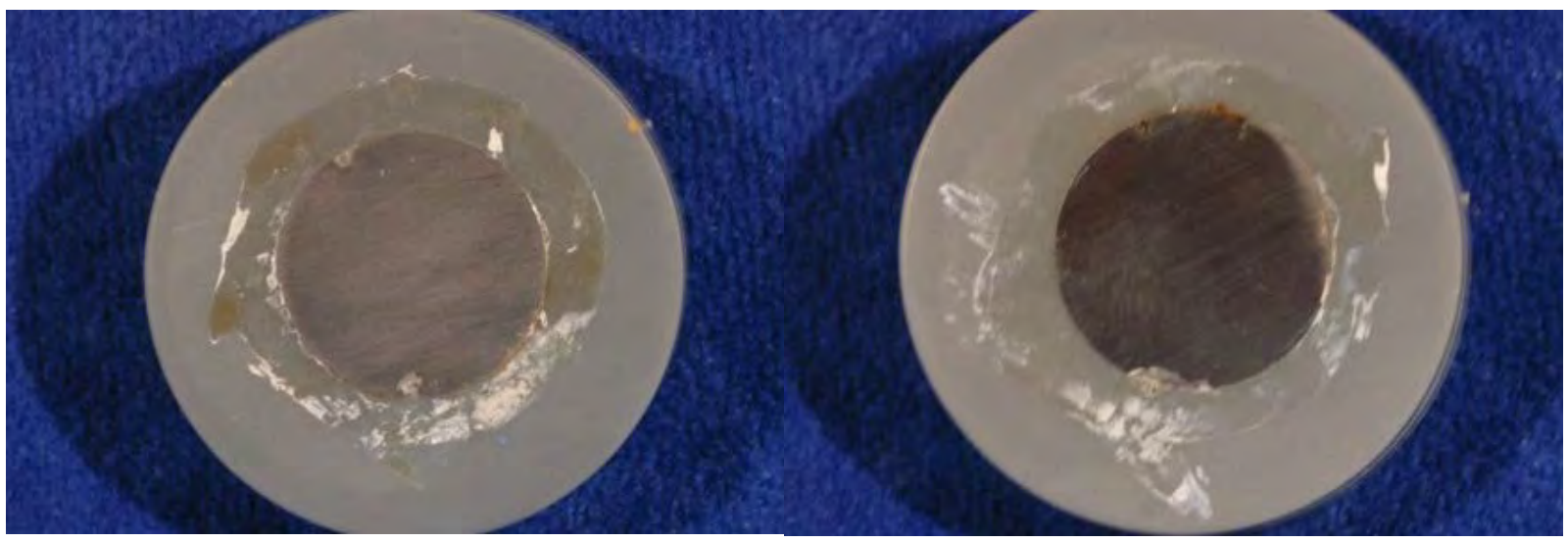

3 months

RC128-60
4 months

RC128-58

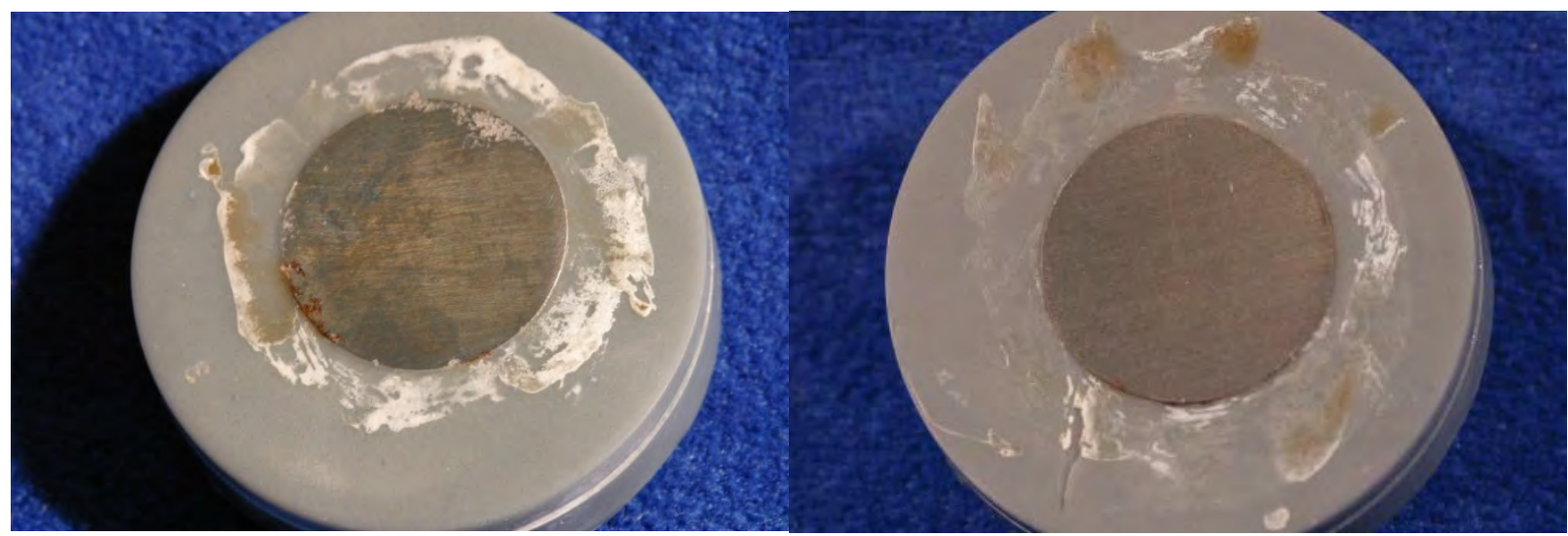


Vessel 5: Level 1

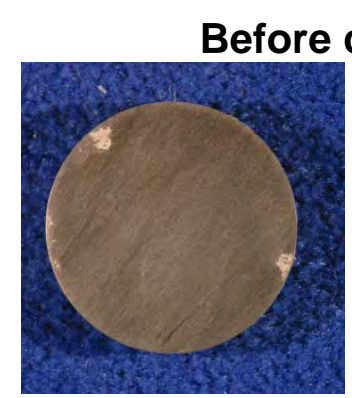

Front

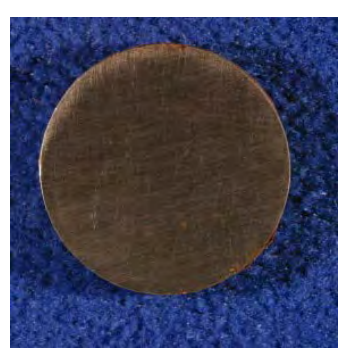

Front

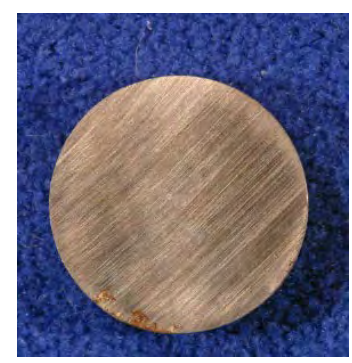

Front

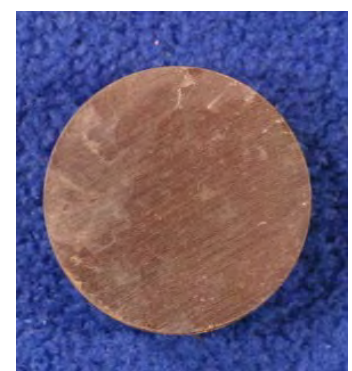

Front

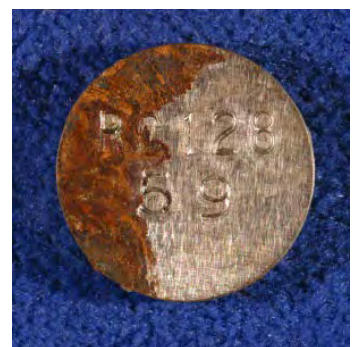

Back

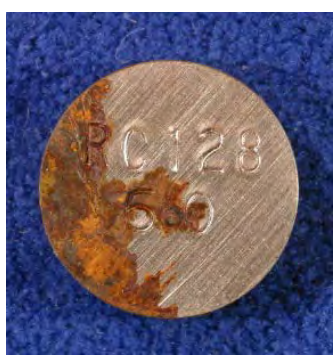

Back

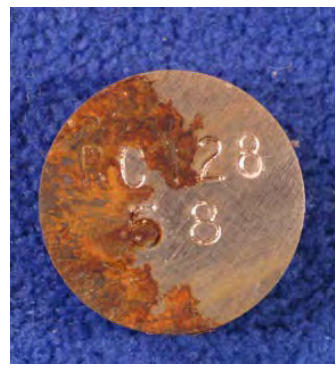

Back

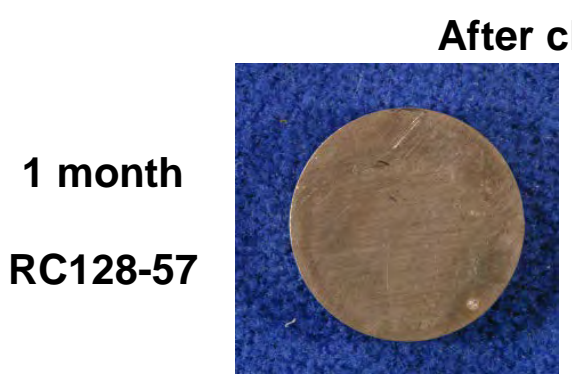

Front

2 months

RC128-59

3 months

RC128-60

4 months

RC128-58

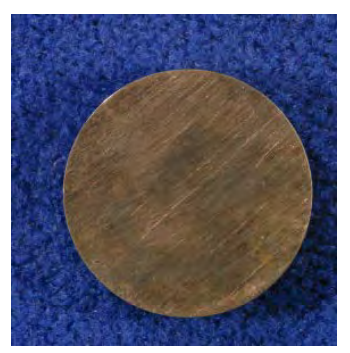

Front

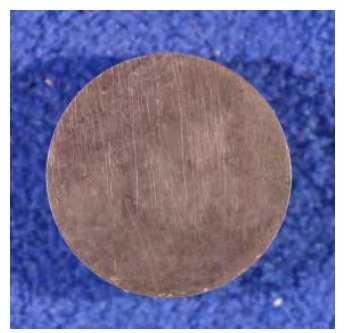

Front

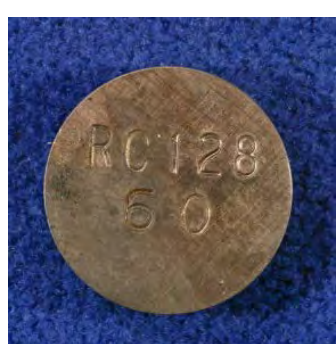

Back

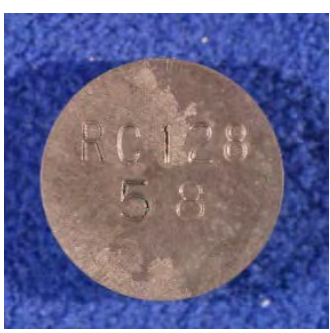

Back 


\section{Vessel 6: Level 3}

1 month

RC128-64
2 months

RC128-61

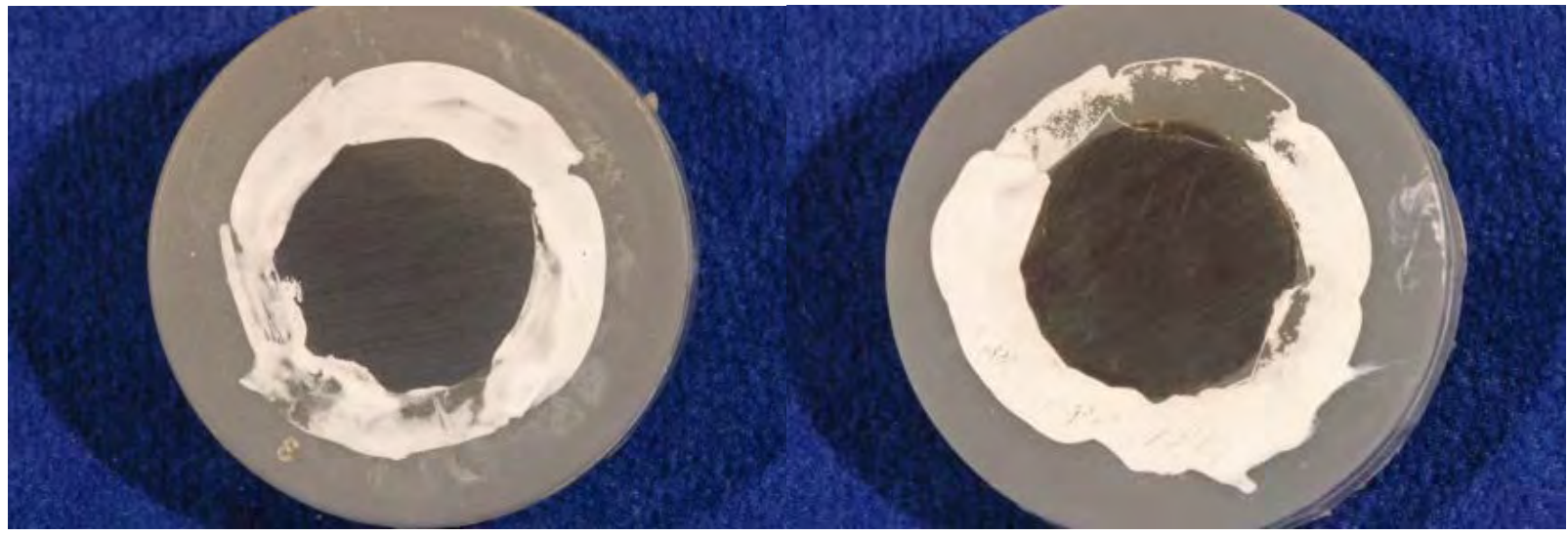

3 months

RC128-63
4 months

RC128-62

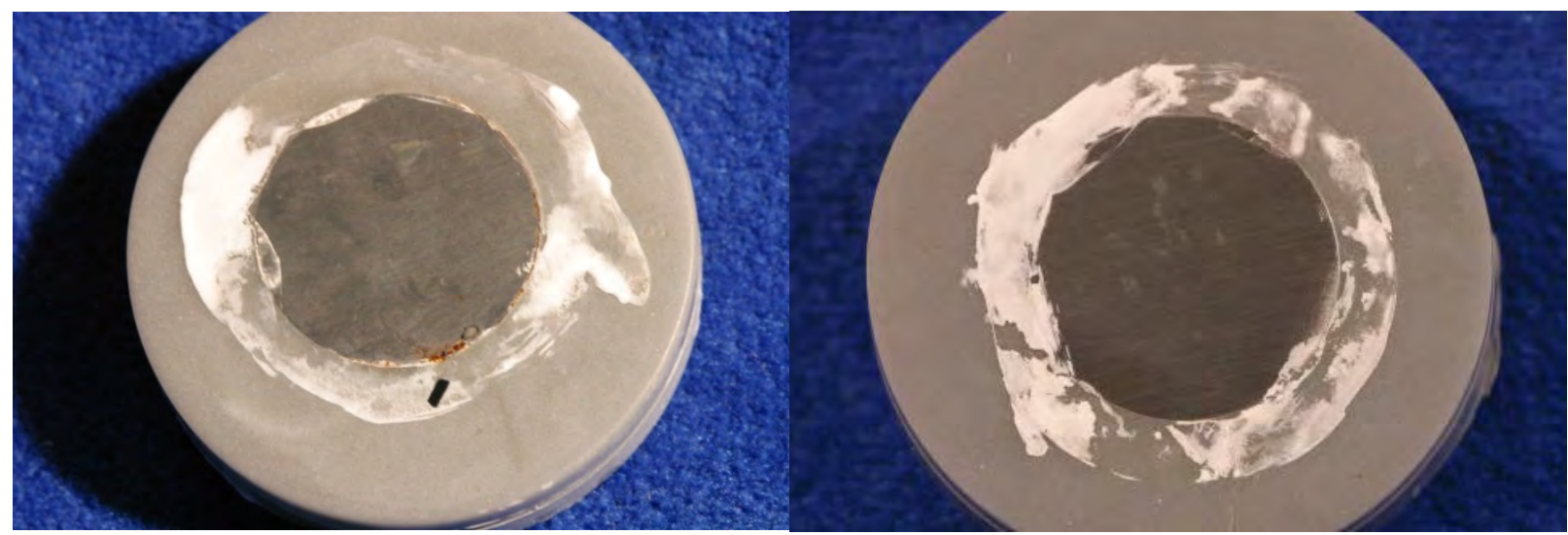




\section{Vessel 6: Level 3}

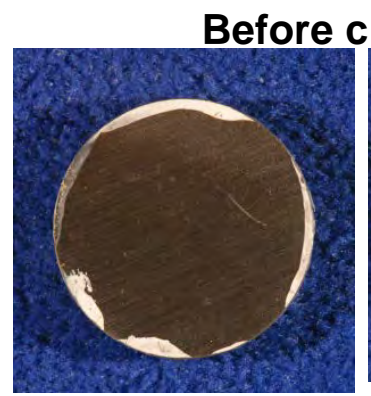

Front

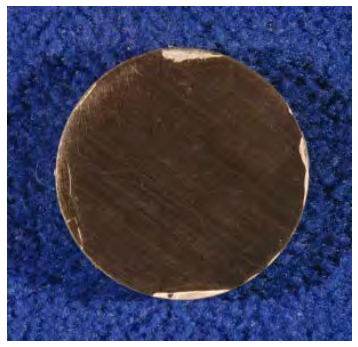

Front

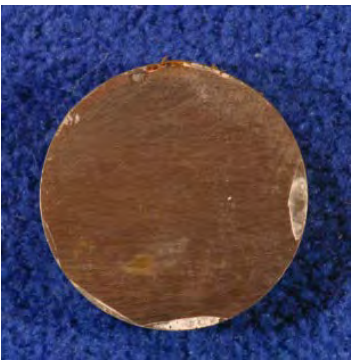

Front

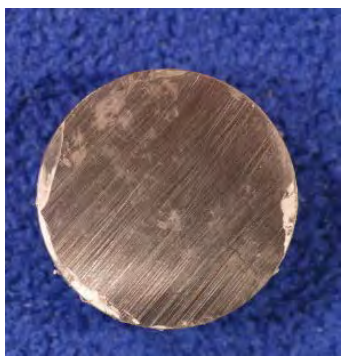

Front

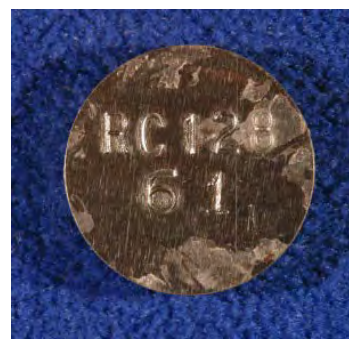

Back

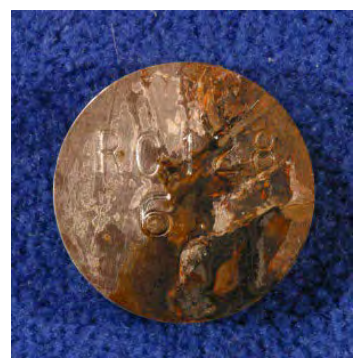

Back

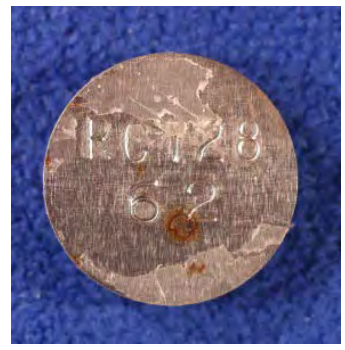

Back
2 months

RC128-61

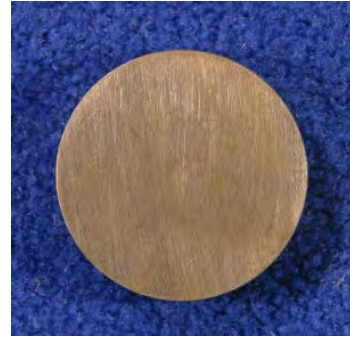

Front

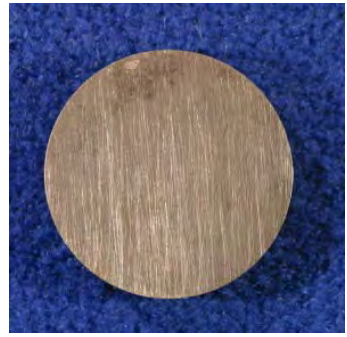

Front

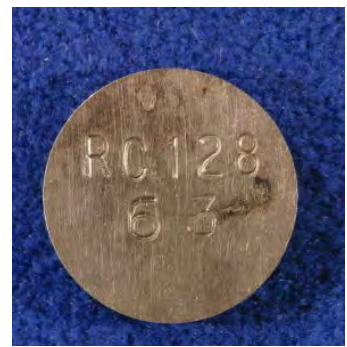

Back

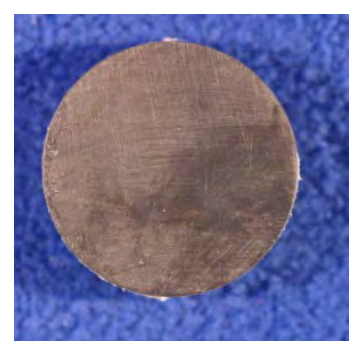

Front

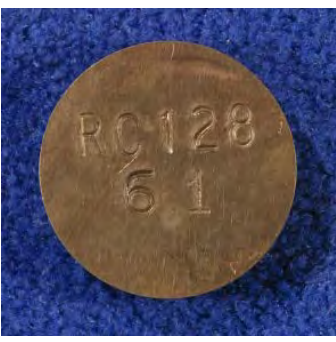

Back

4 months

RC128-62 
Vessel 6: Level 2

1 month

2 months

RC128-66

RC128-68

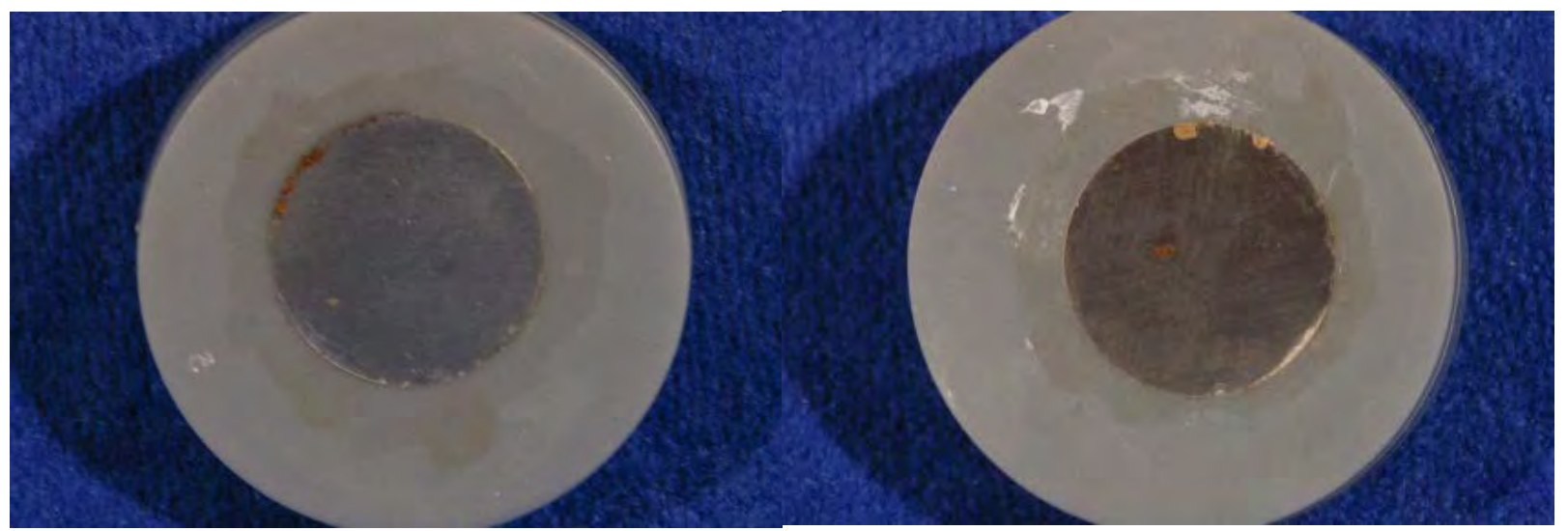

3 months

RC128-67
4 months

RC128-65

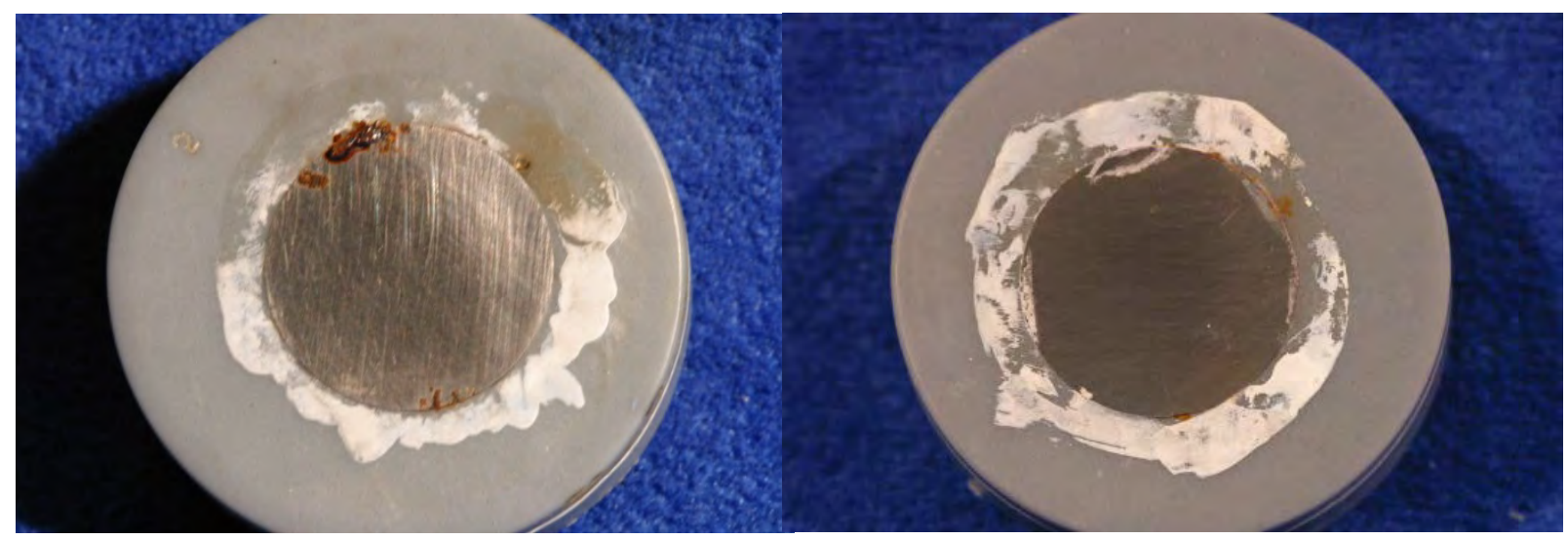


Vessel 6: Level 2

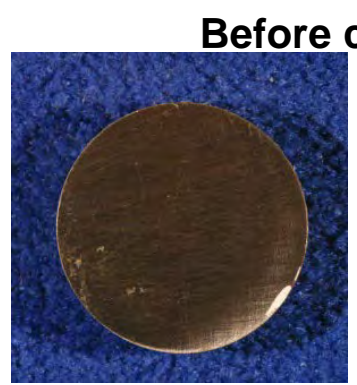

Front

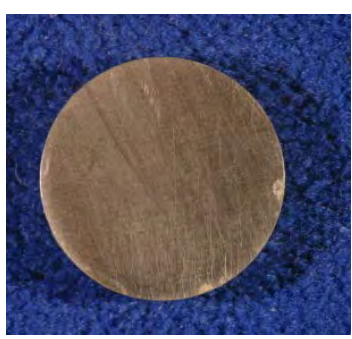

Front

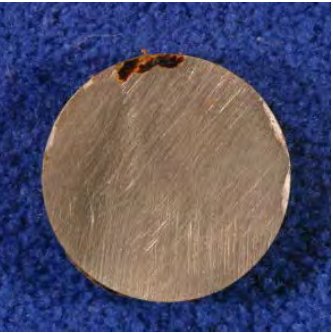

Front

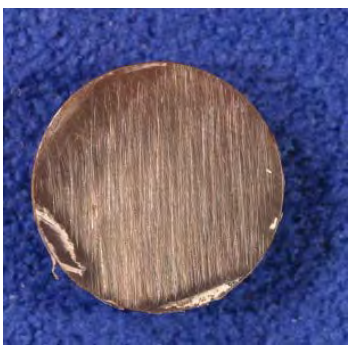

Front

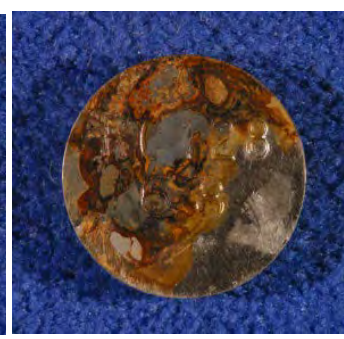

Back

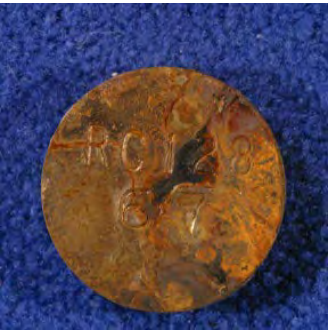

Back

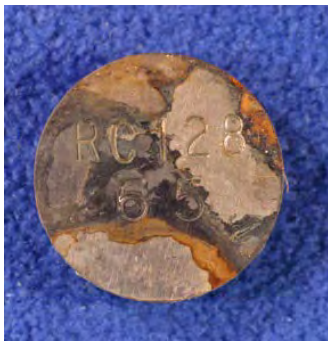

Back

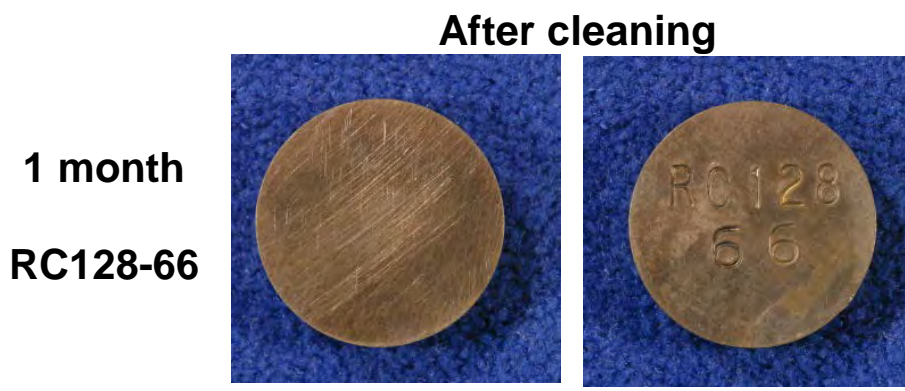

Front

Back

2 months

RC128-68

3 months

RC128-67

4 months

RC128-65

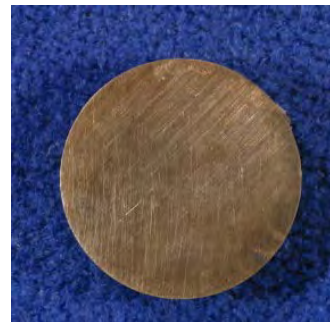

Front
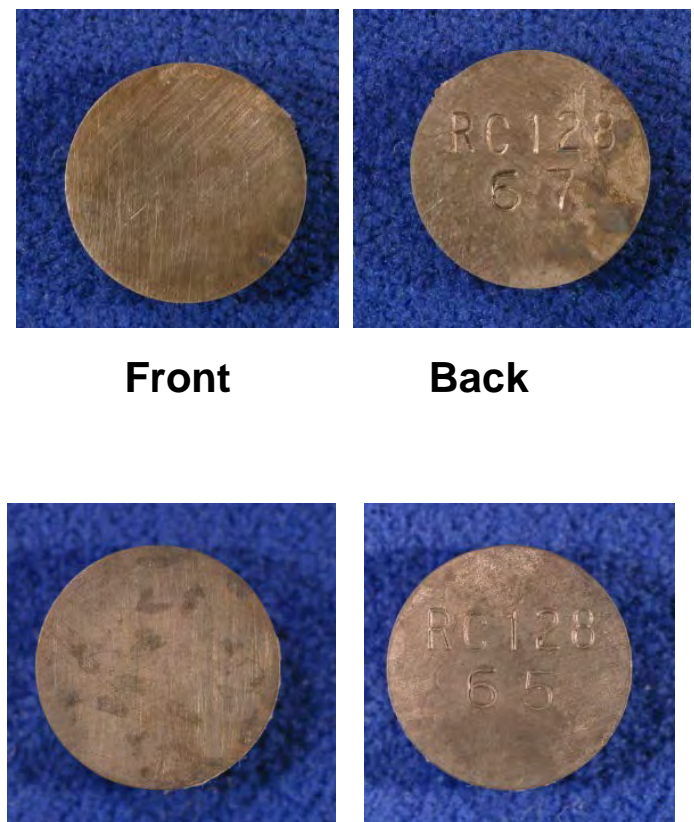

Front
Back

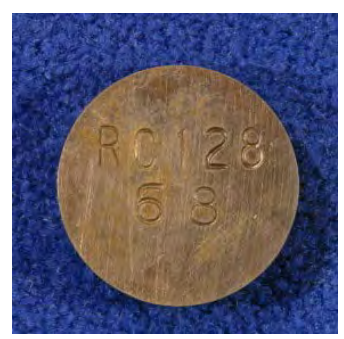

Back

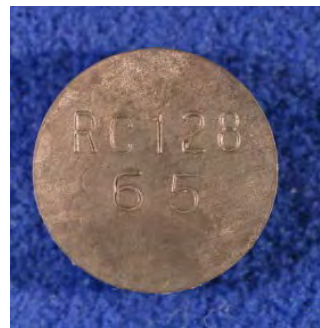

Back 
Vessel 6: Level 1

1 month

RC128-70
2 months

RC128-71

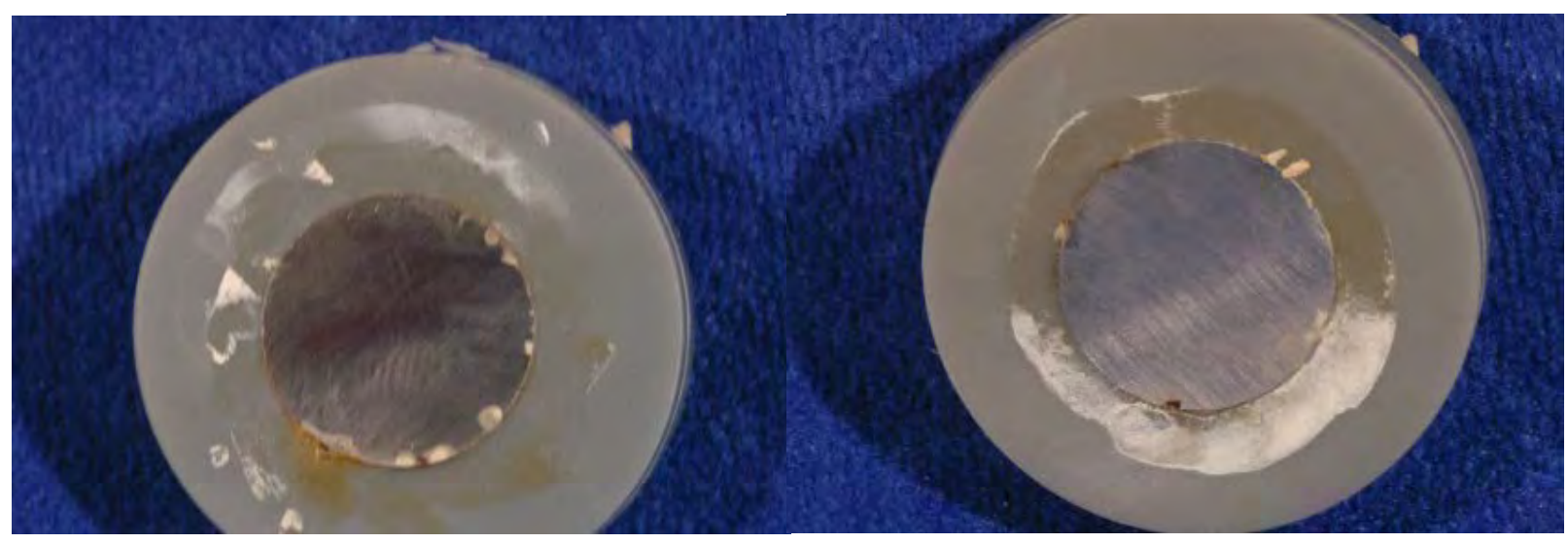

3 months

RC128-69
4 months

RC128-72

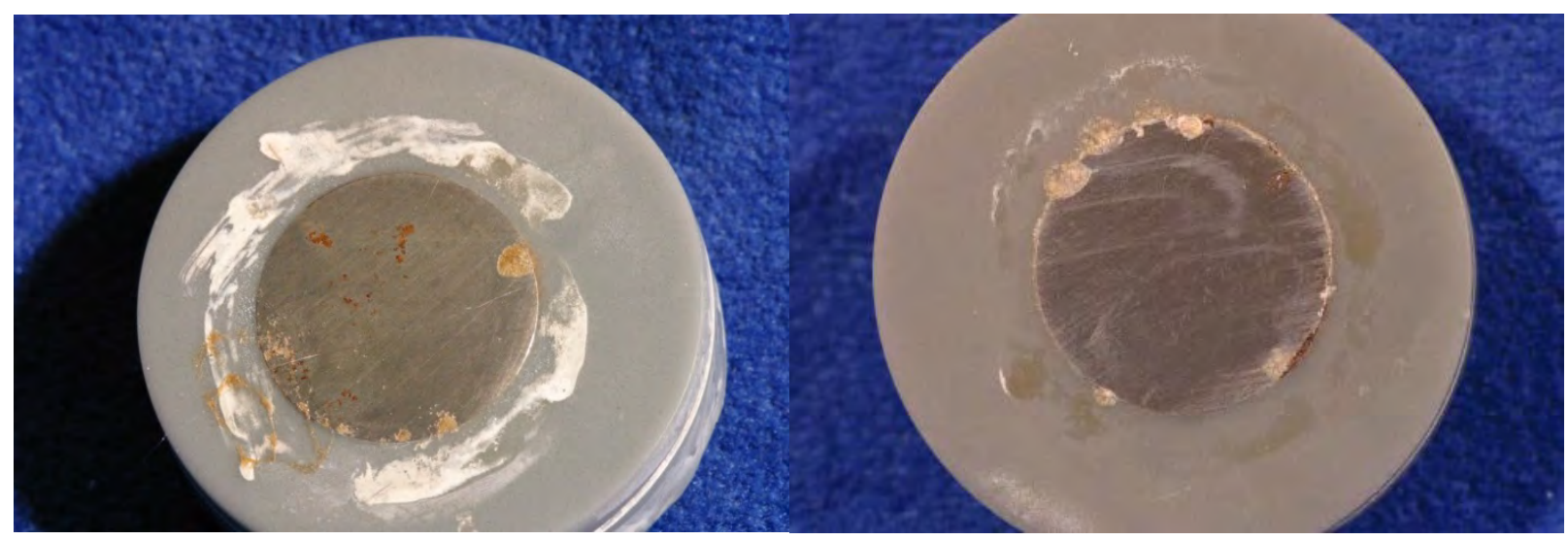


Vessel 6: Level 1

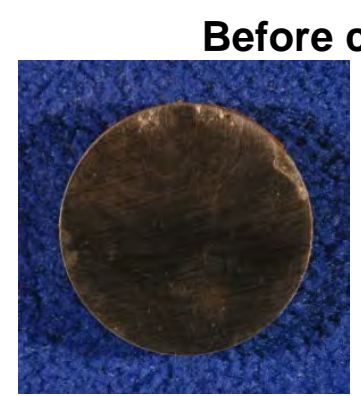

Front

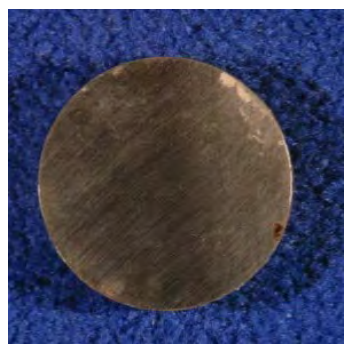

Front

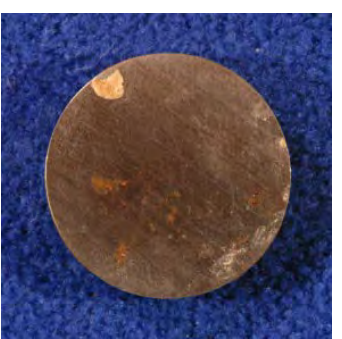

Front

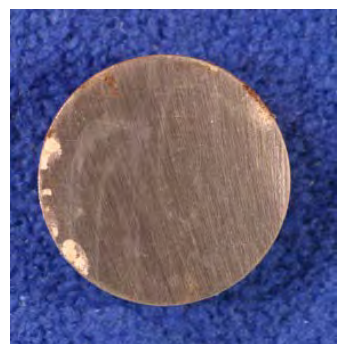

Front

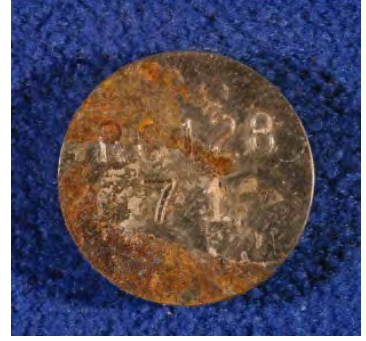

Back

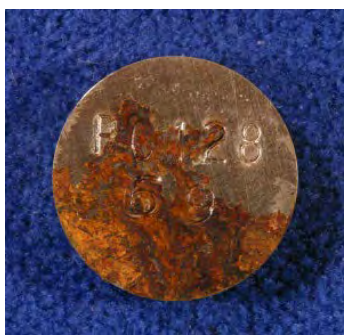

Back

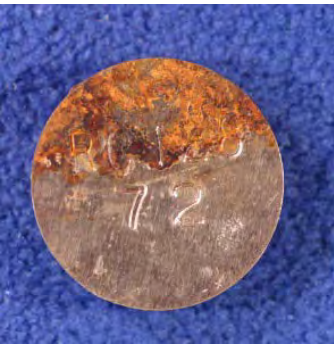

Back

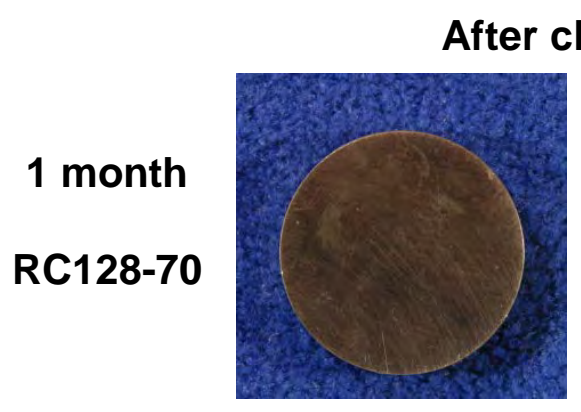

Front

2 months

RC128-71

3 months

RC128-69

4 months

RC128-72

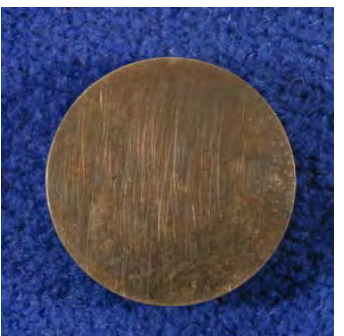

Front

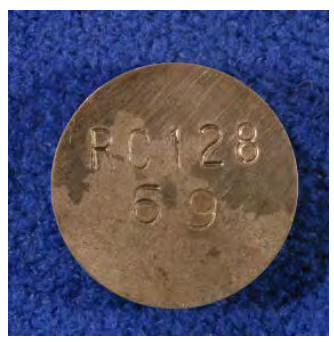

Back

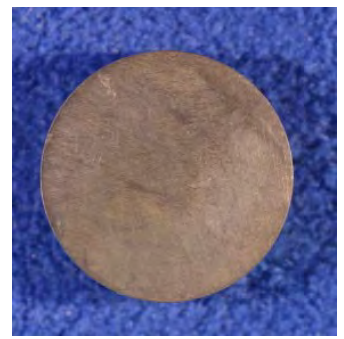

Front

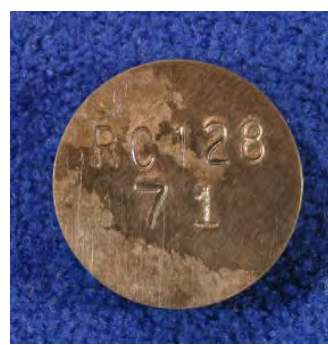

Back

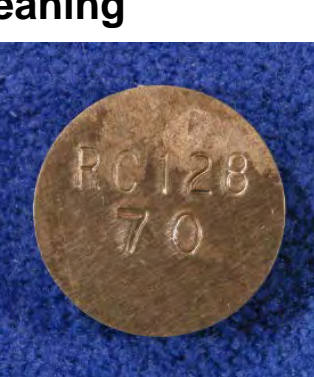

Back

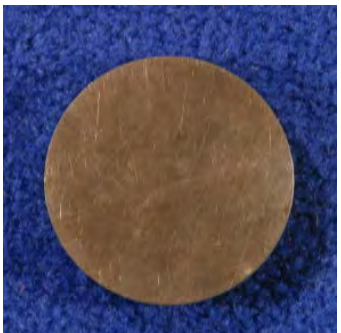

Front

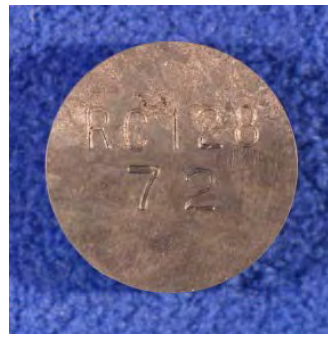

Back 


\section{Vessel 7: Level 3}

1 month

RC128-76
2 months

RC128-75

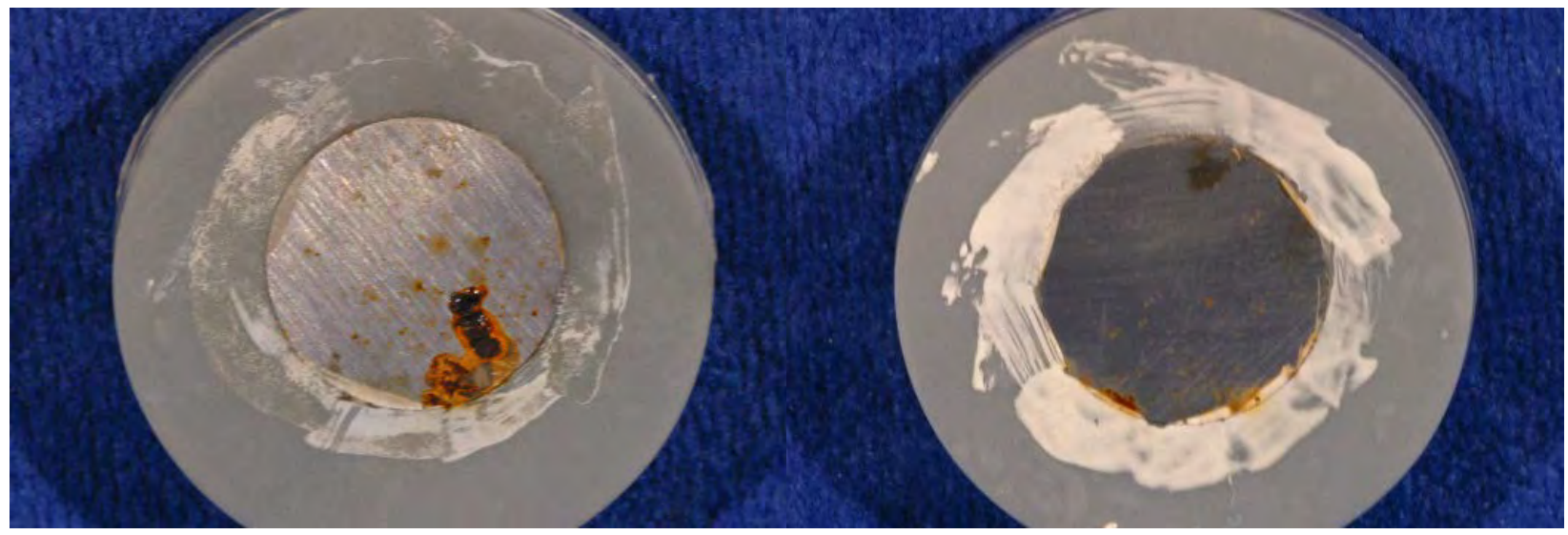

3 months

RC128-74
4 months

RC128-73

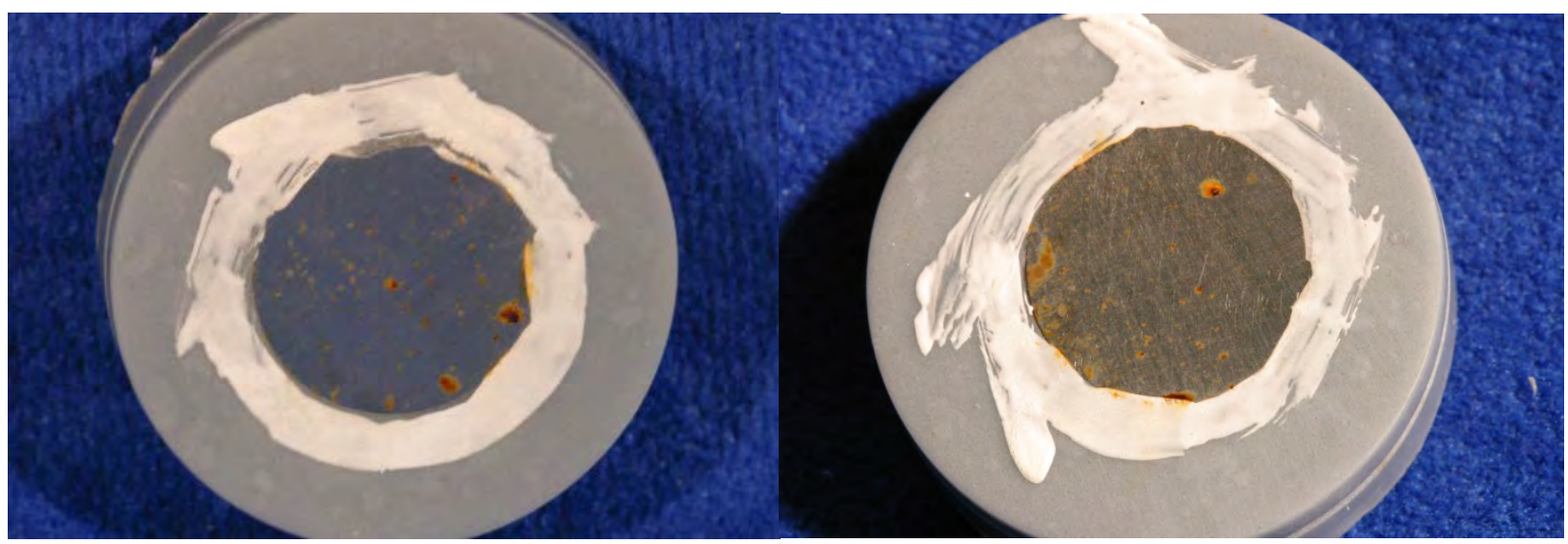




\section{Vessel 7: Level 3}

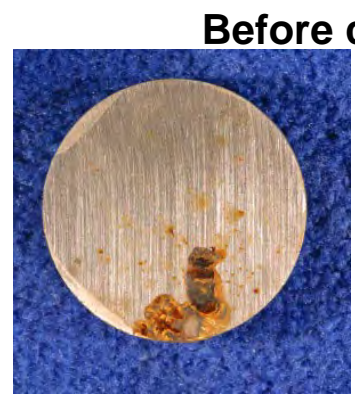

Front

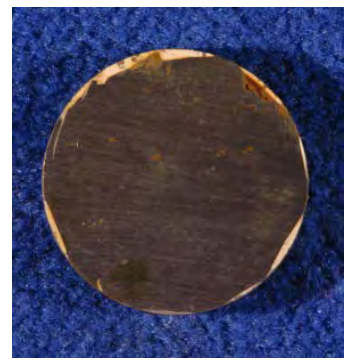

Front

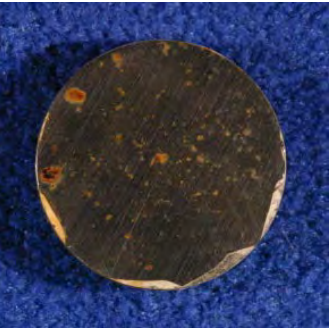

Front

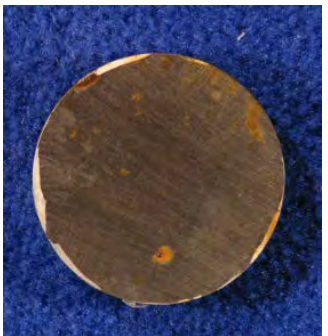

Front

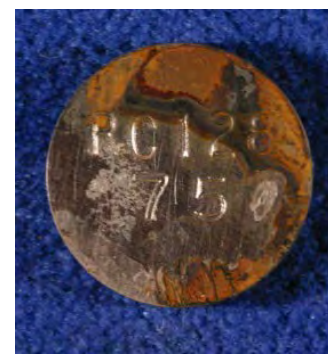

Back

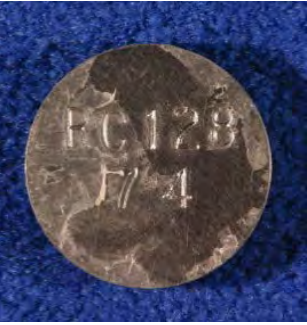

Back

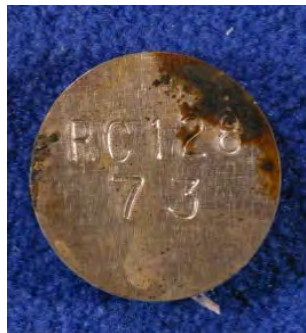

Back

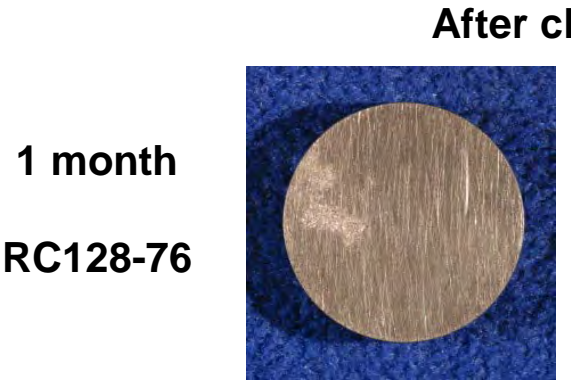

Front

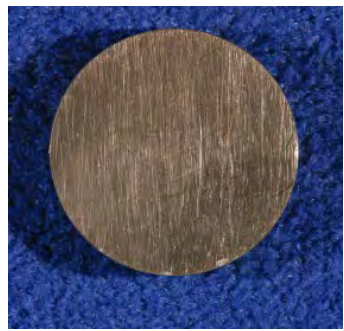

Front

3 months

RC128-74

4 months

RC128-73

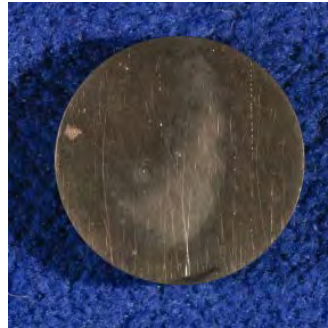

Front

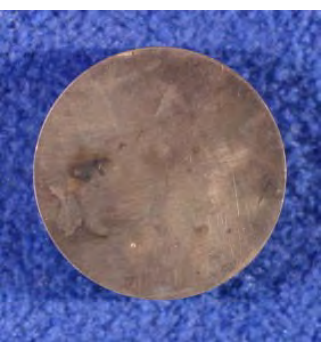

Front

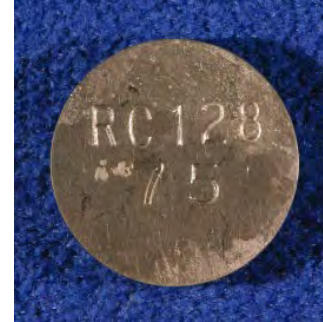

Back

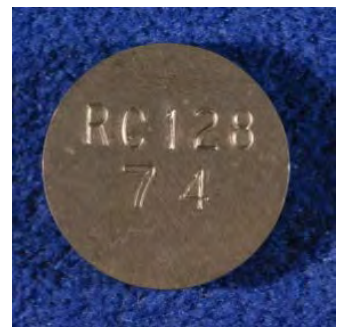

Back

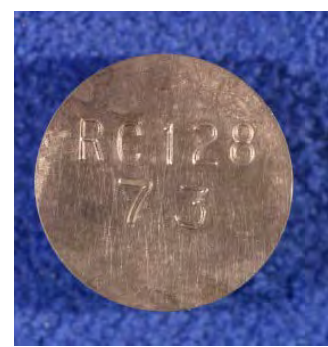

Back 


\section{Vessel 7: Level 2}

1 month

RC128-78
2 months

RC128-79

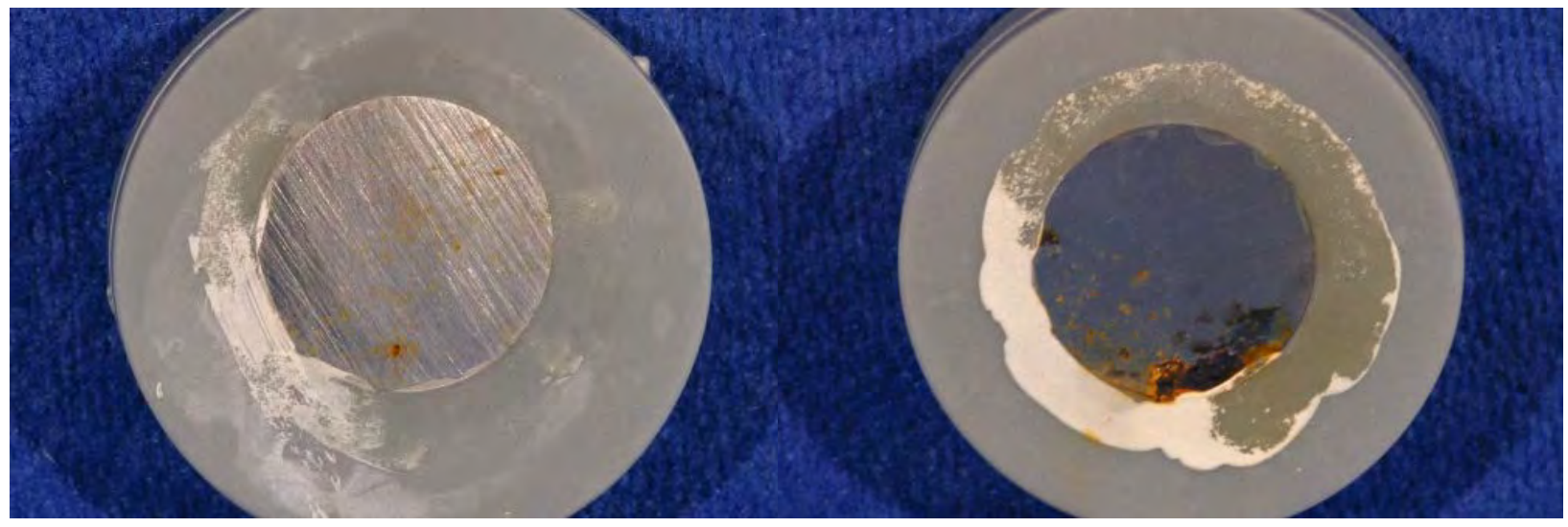

3 months

RC128-80
4 months

RC128-77

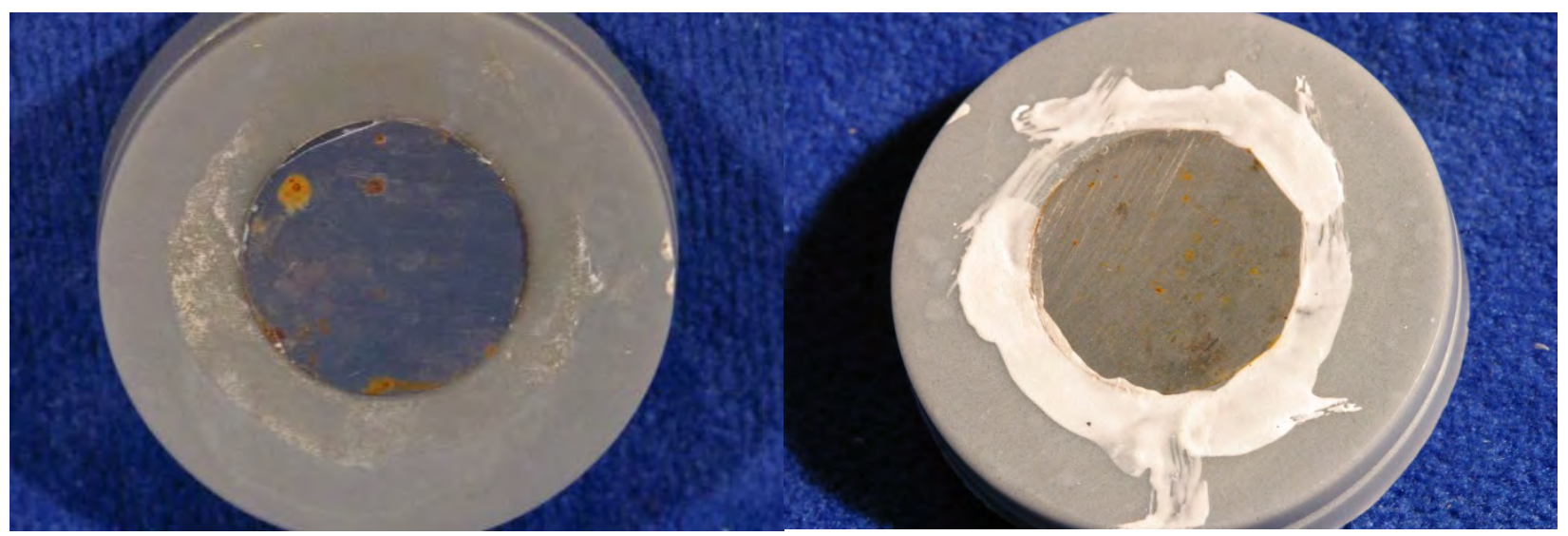


Vessel 7: Level 2

Before cleaning

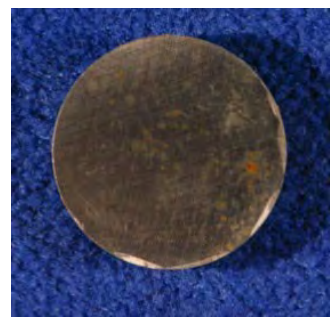

Front

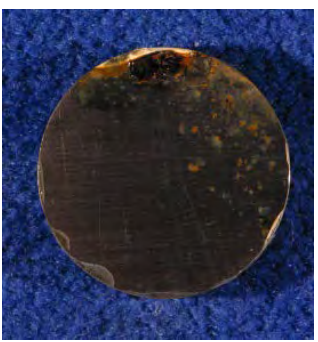

Front

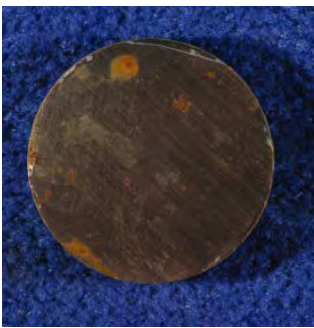

Front

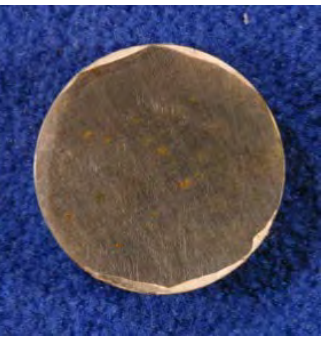

Front

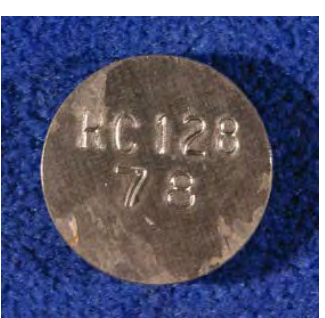

Back

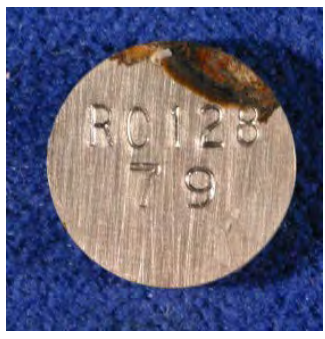

Back

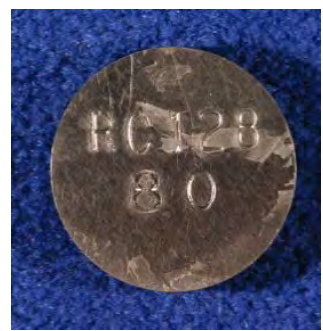

Back

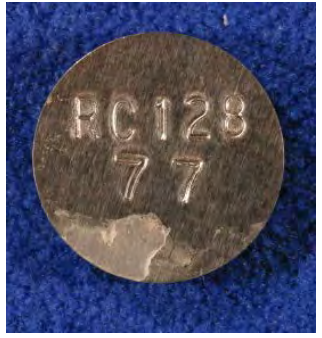

Back

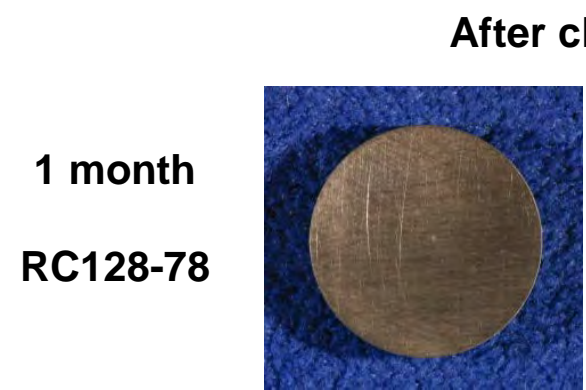

Front

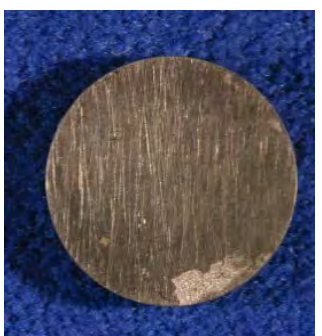

Front

3 months

RC128-80

4 months

RC128-77

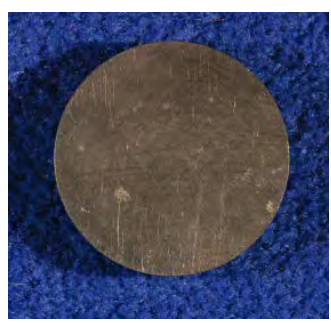

Front
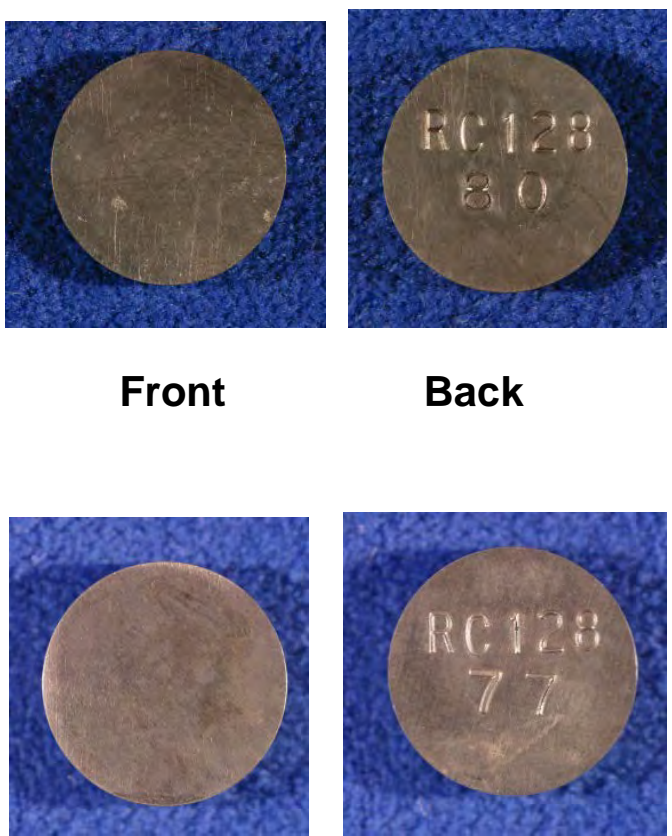

Front
Back

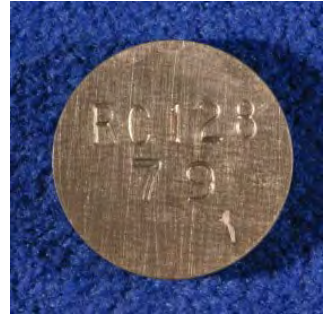

Back

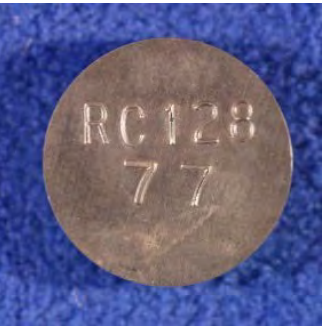

Back 


\section{Vessel 7: Level 1}

1 month

RC128-84
2 months

RC128-83

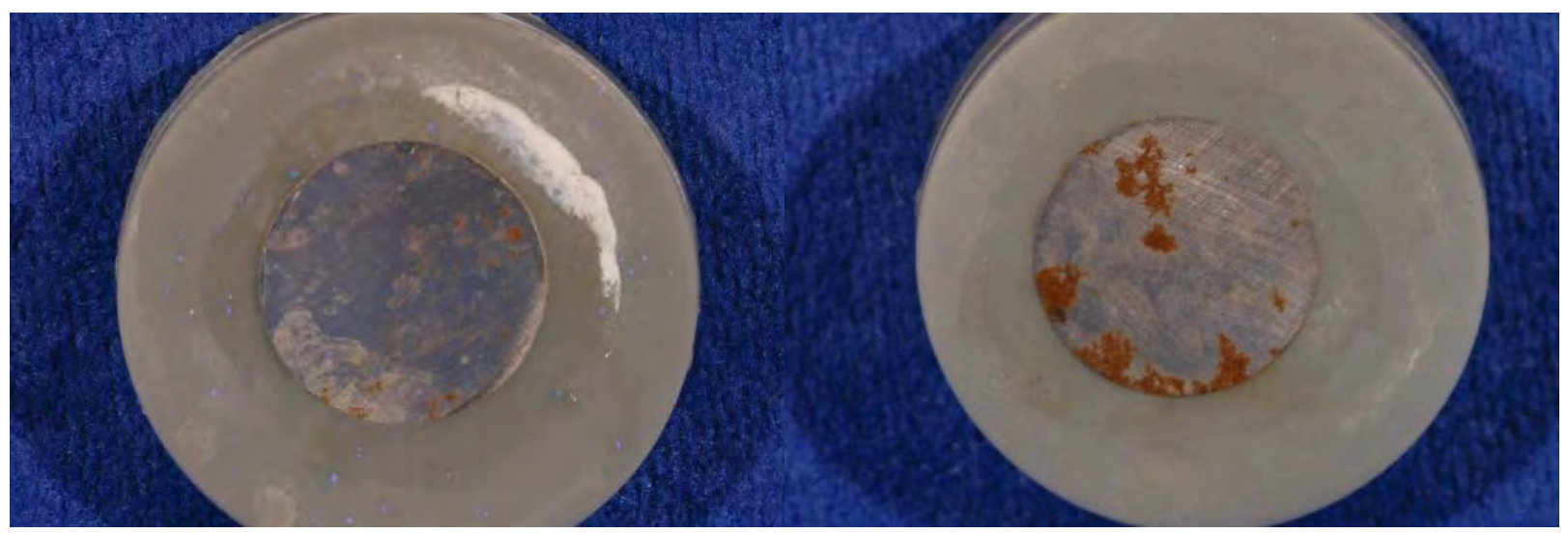

3 months

RC128-81
4 months

RC128-82

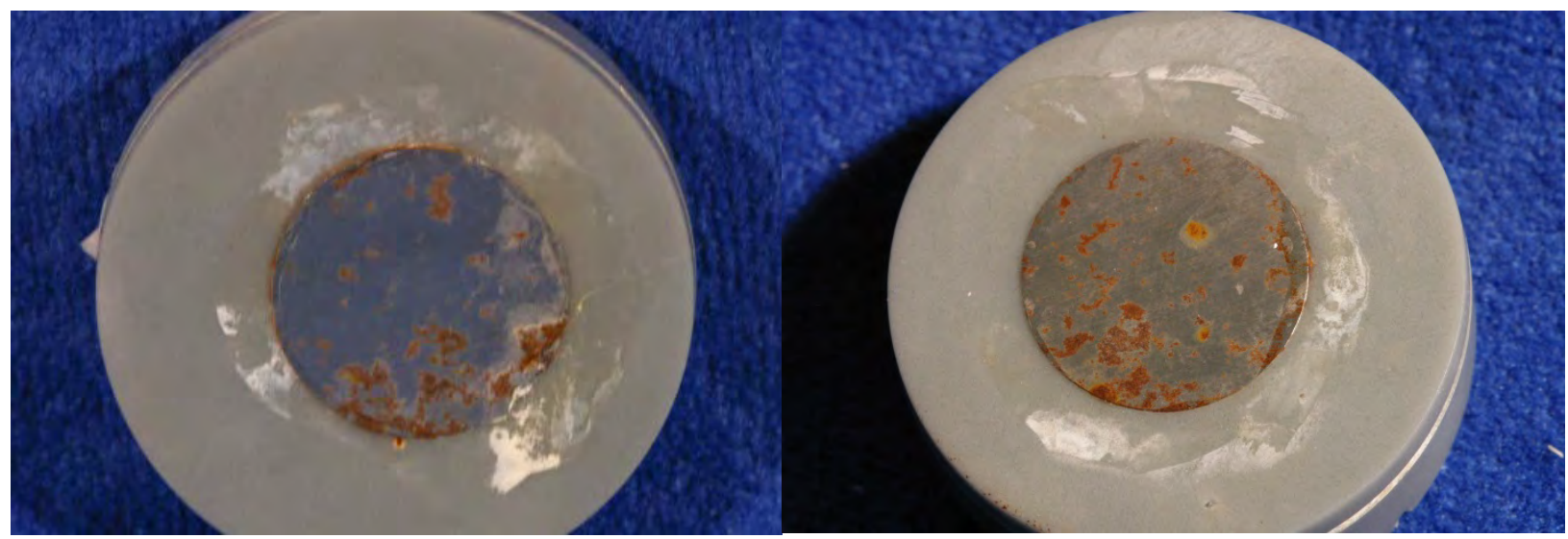


Vessel 7: Level 1

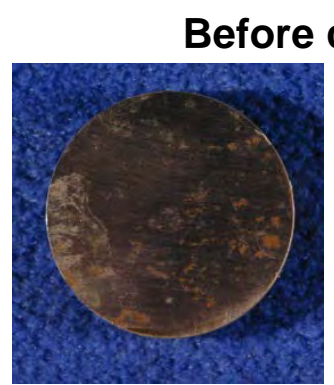

Front

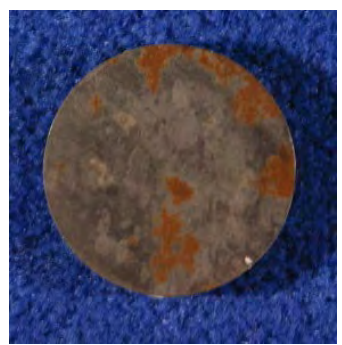

Front

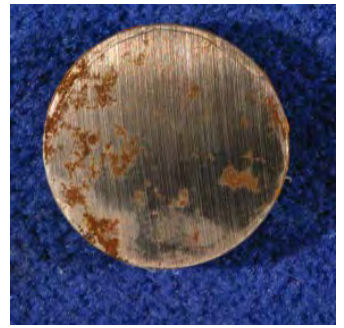

Front

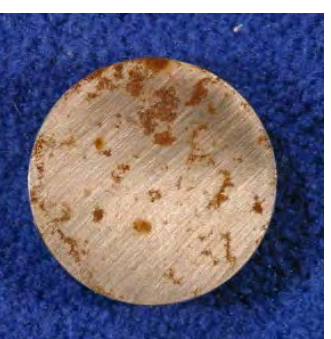

Front

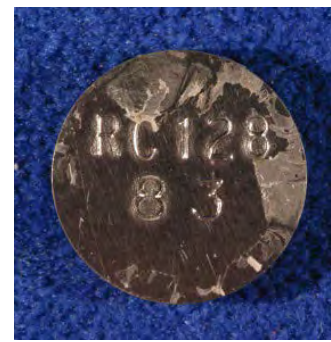

Back

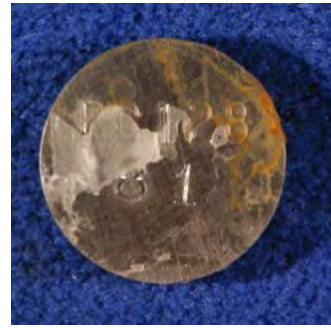

Back

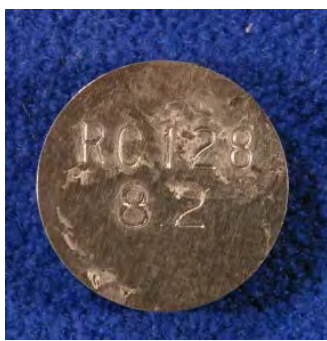

Back

1 month

RC128-84

2 months

RC128-83

3 months

RC128-81

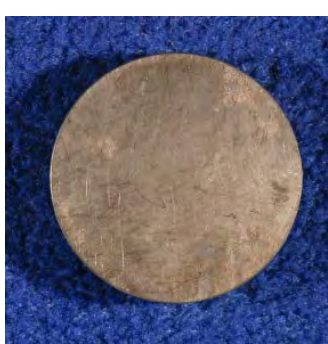

Front

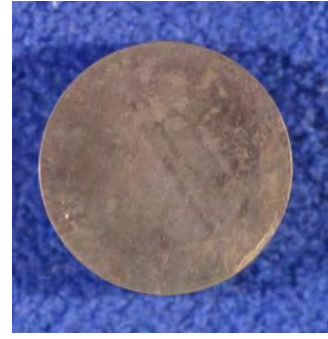

Front

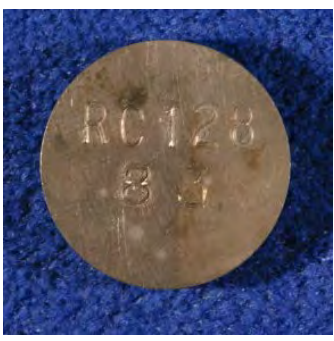

Back

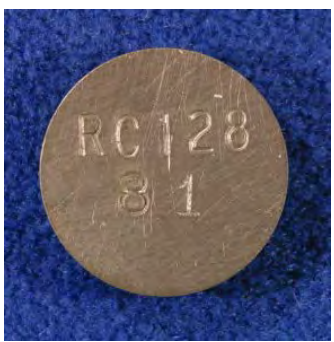

Back

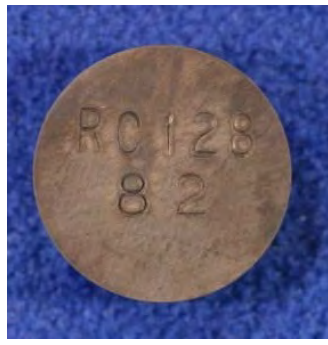

Back 


\section{Vessel 8: Level 3}

1 month

RC128-88
2 months

RC128-87

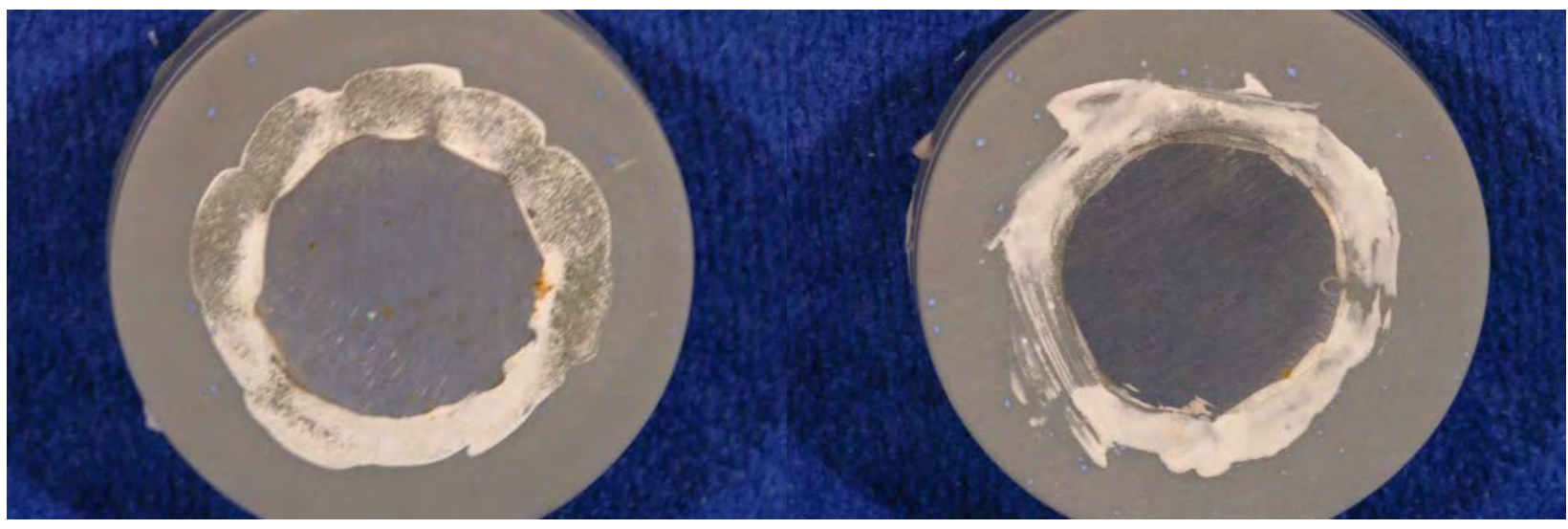

3 months

RC128-85
4 months

RC128-86

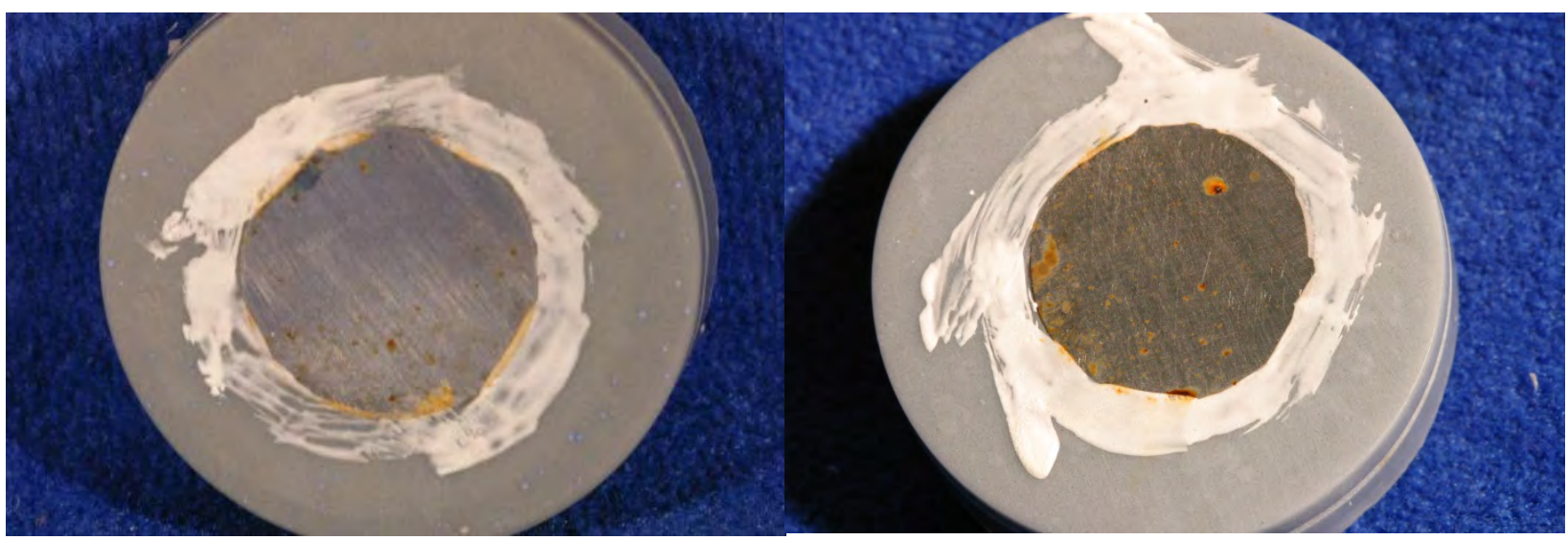




\section{Vessel 8: Level 3}

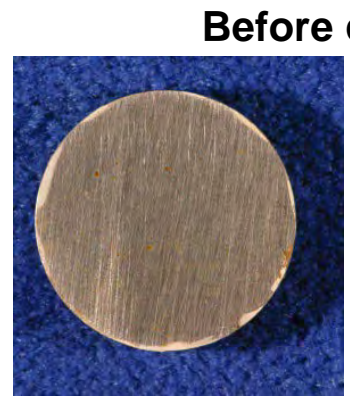

Front

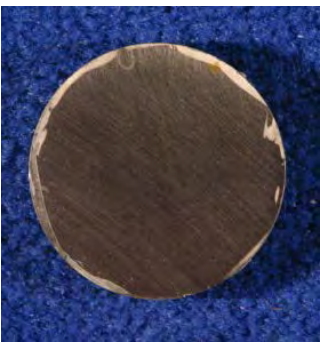

Front

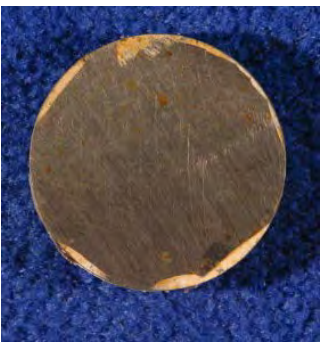

Front

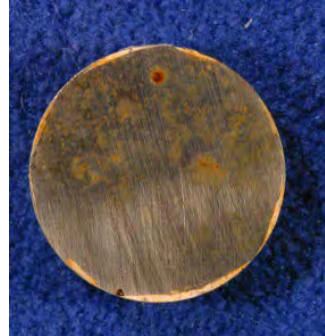

Front

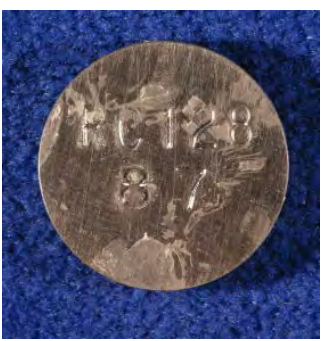

Back

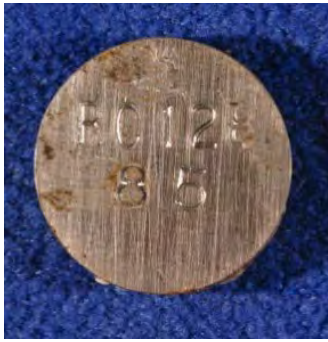

Back

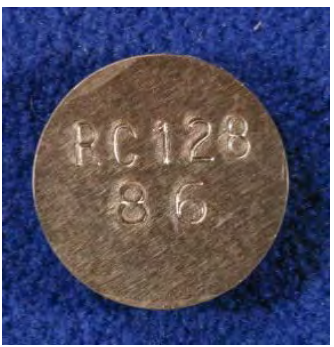

Back

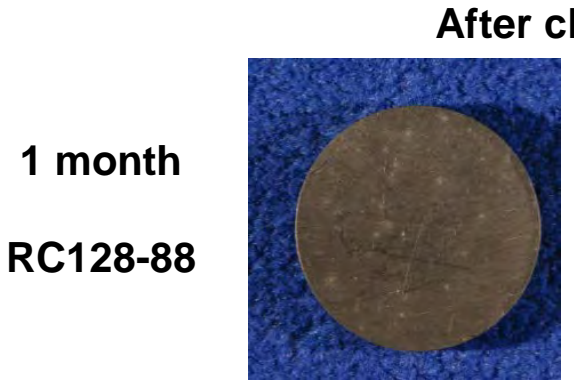

Front

2 months

RC128-87

3 months

RC128-85

4 months

RC128-86

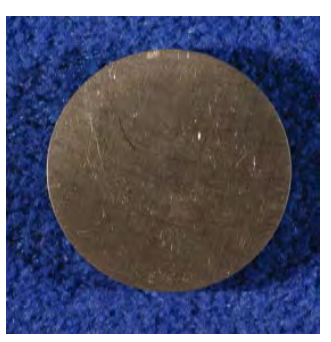

Front

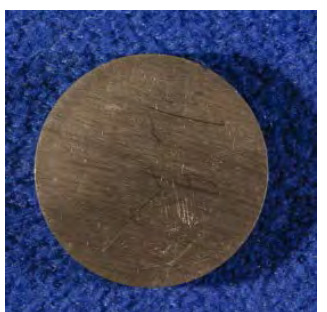

Front

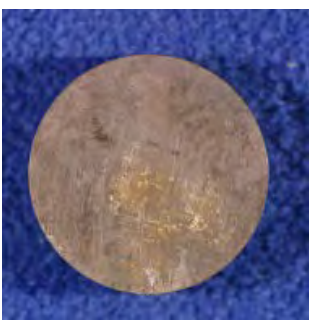

Front

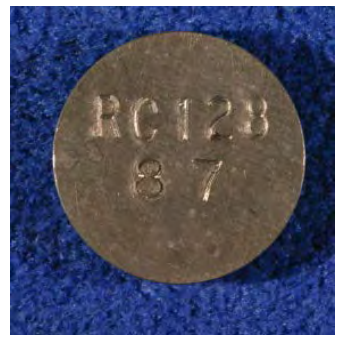

Back

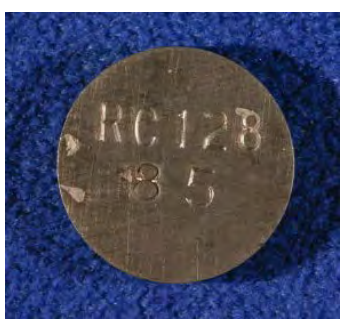

Back

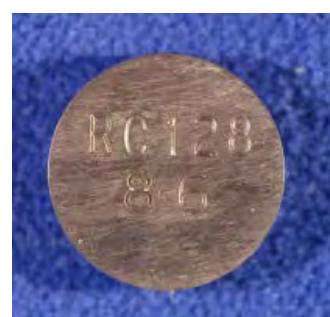

Back 


\section{Vessel 8: Level 2}

1 month

RC128-93
2 months

RC128-92

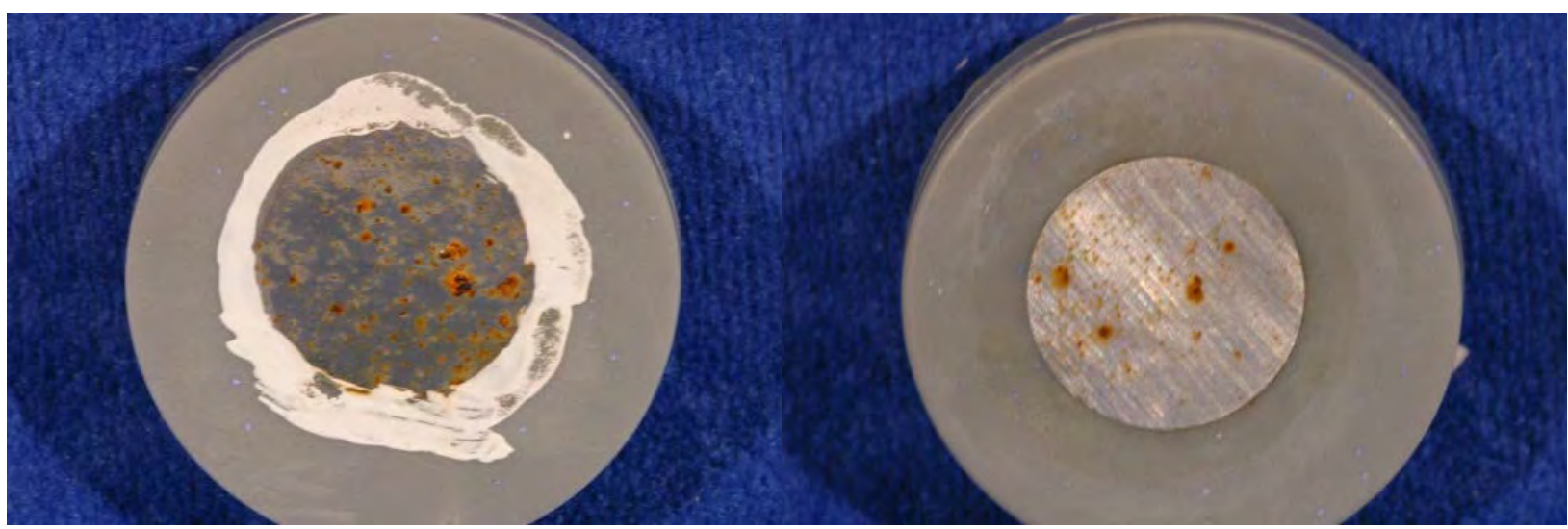

3 months

RC128-90
4 months

RC128-89

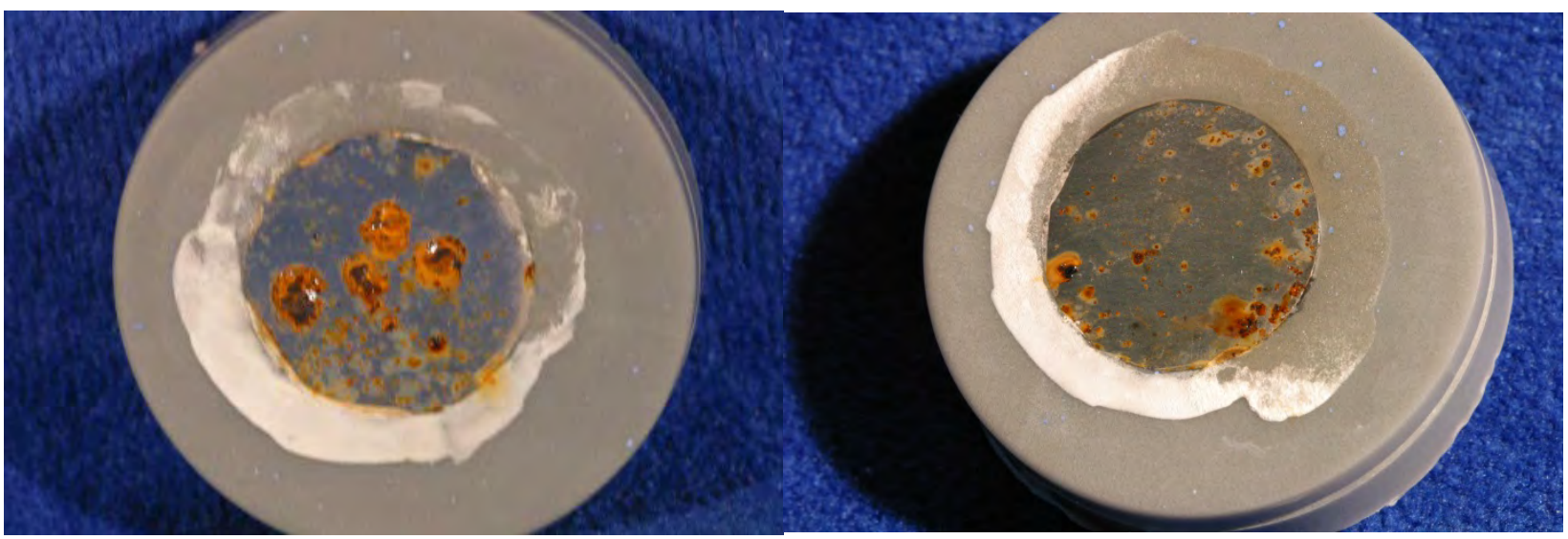


Vessel 8: Level 2

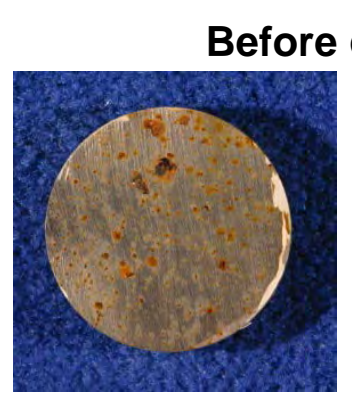

Front

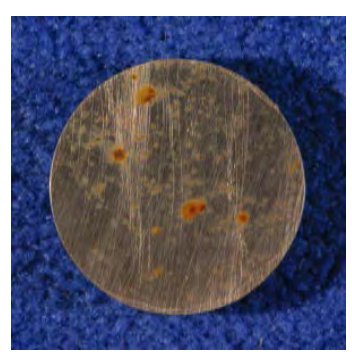

Front

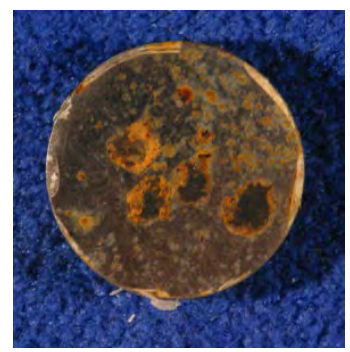

Front

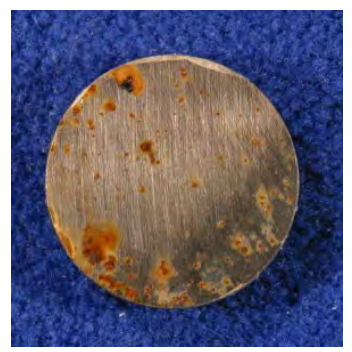

Front

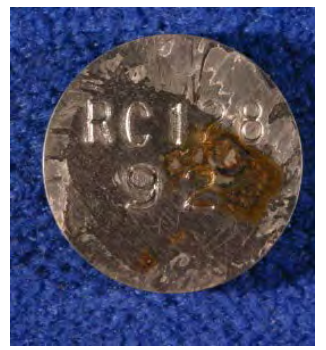

Back

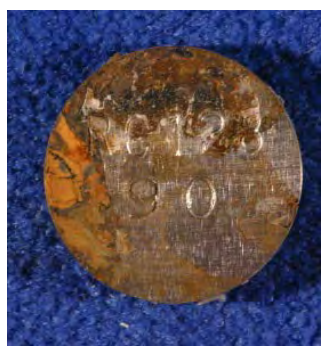

Back

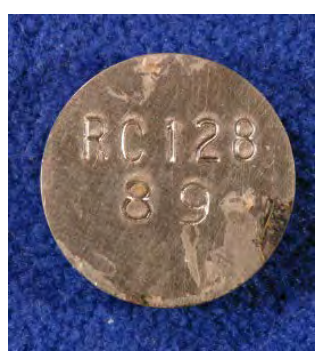

Back

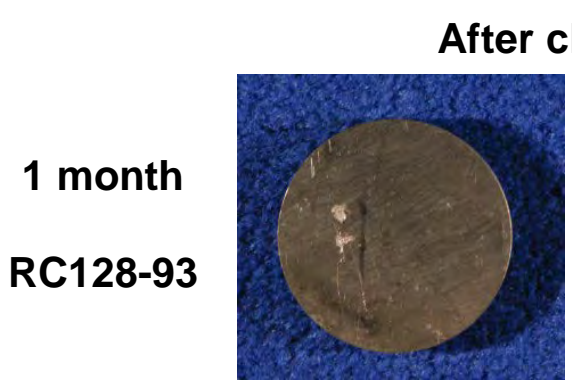

Front

2 months

RC128-92

3 months

RC128-90

4 months

RC128-89

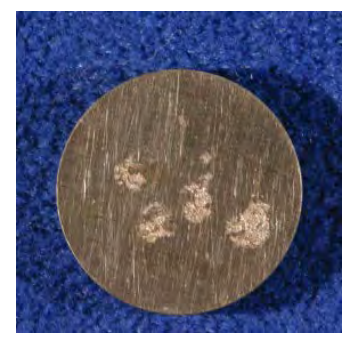

Front

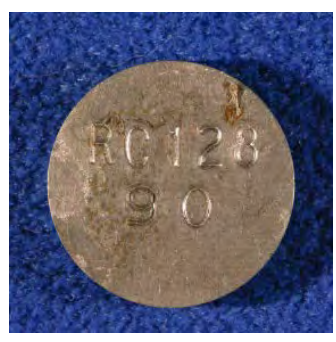

Back

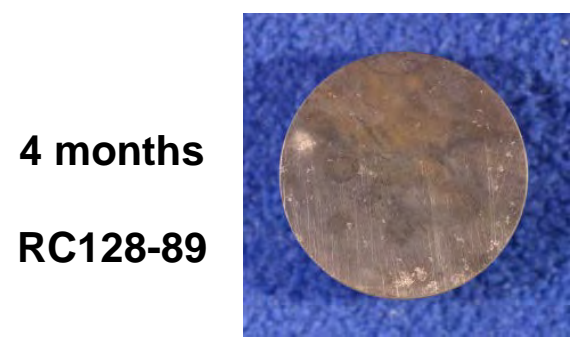

Front

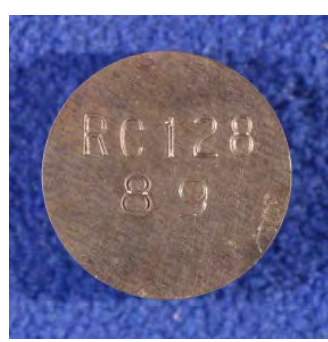

Back 
Vessel 8: Level 1

1 month

2 months

RC128-97

RC128-96

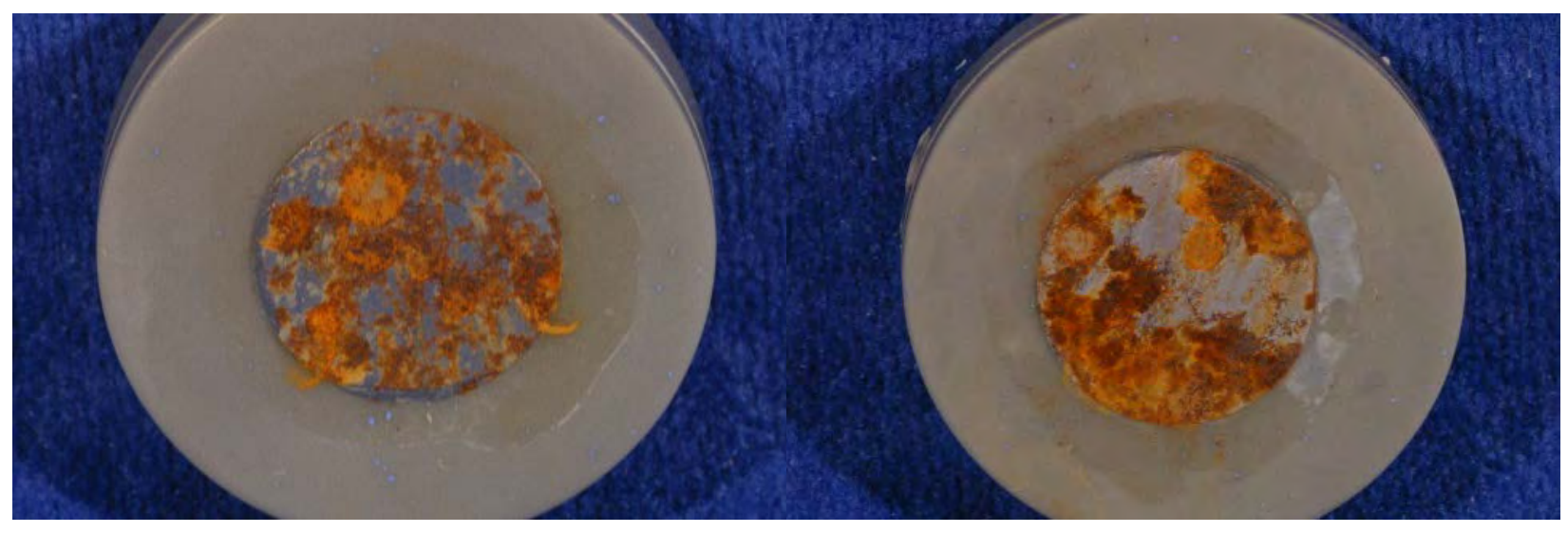

3 months

RC128-95
4 months

RC128-94

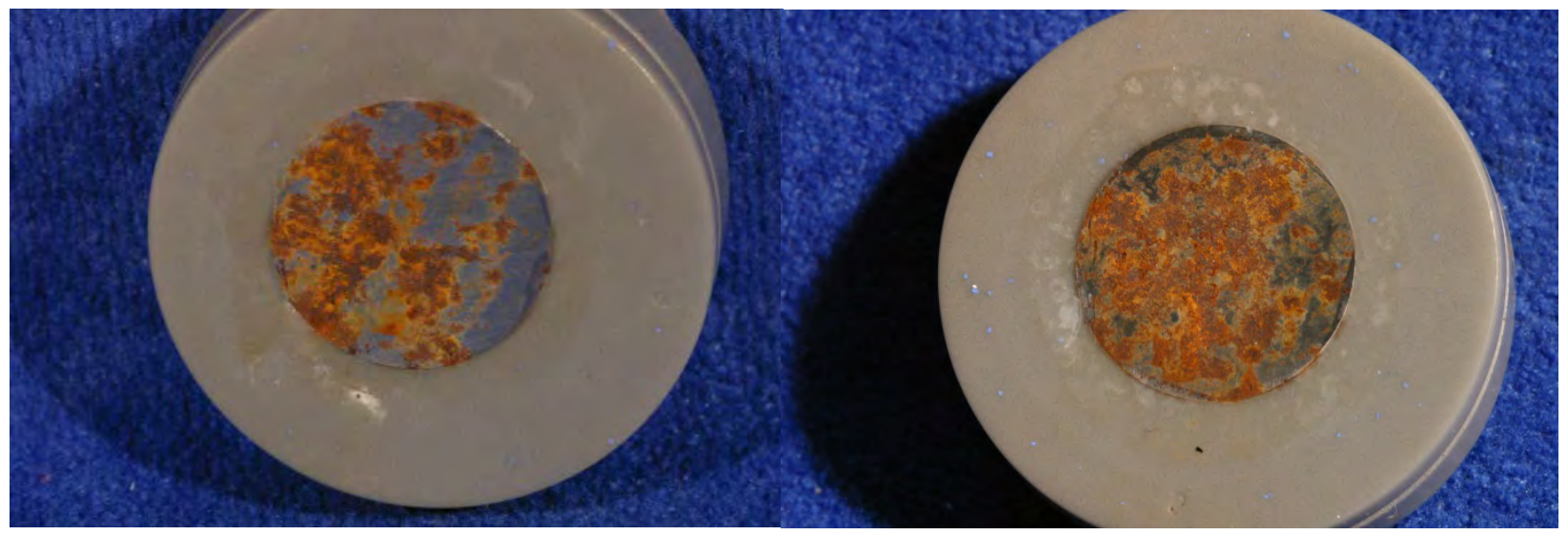


Vessel 8: Level 1

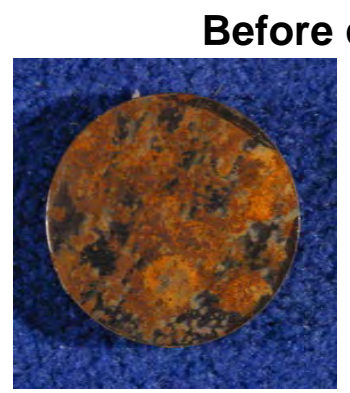

Front

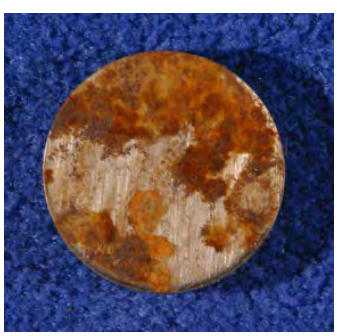

Front

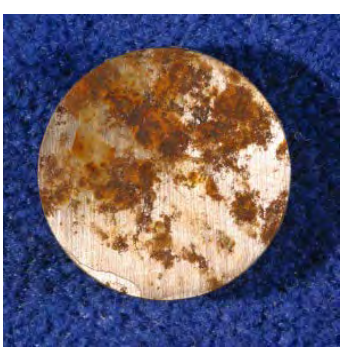

Front

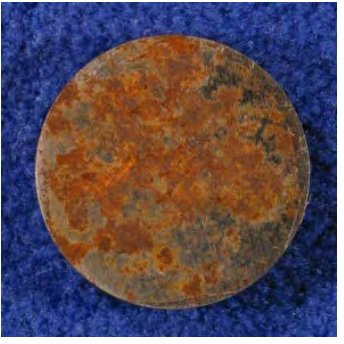

Front

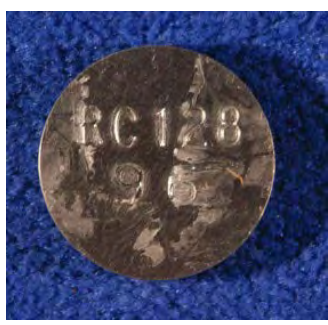

Back

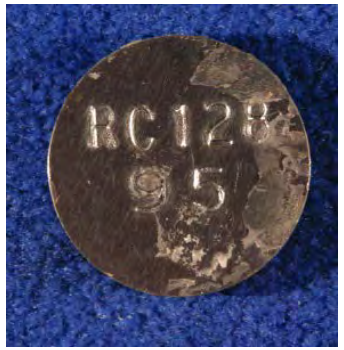

Back

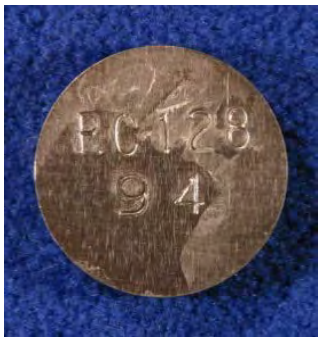

Back

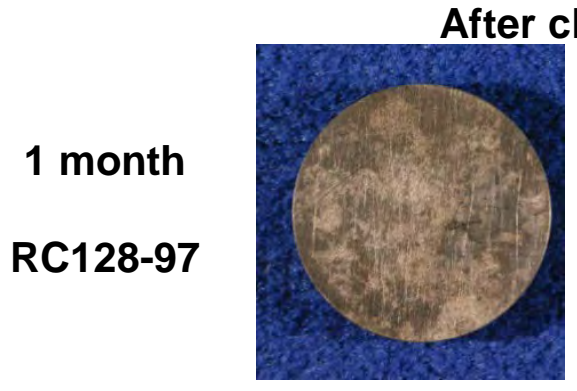

Front

2 months

RC128-96

3 months

RC128-95

4 months

RC128-94

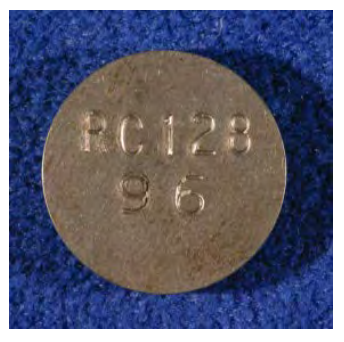

Back

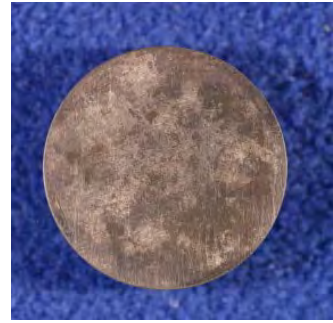

Front

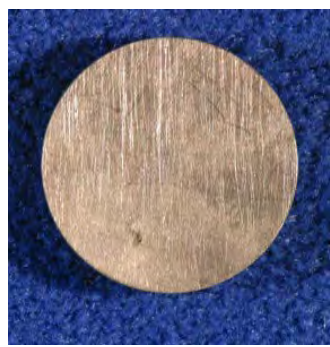

Front

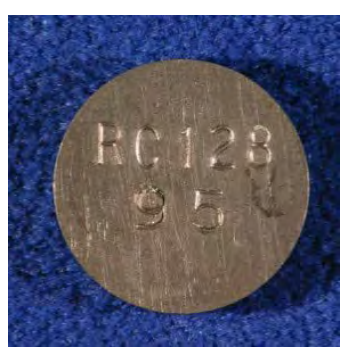

Back

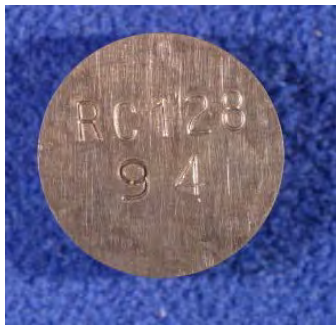

Back 
Appendix C

Chemical Composition of Simulants used in Liquid Air Interface Corrosion Testing 


\section{Composition of simulant for LAI-Solution 1}

$\begin{array}{rrr}\text { Temperature } & 40 & { }^{\circ} \mathrm{C} \\ \mathrm{pH} & 12 & \\ \text { Volume } & 0.5 \quad \mathrm{~L}\end{array}$

\begin{tabular}{|c|c|c|c|c|}
\hline Simulant Source & Formula & $\begin{array}{c}\text { Molecular weight } \\
(\mathbf{g} / \mathbf{m o l})\end{array}$ & $\begin{array}{c}\text { Concentration } \\
\text { (M) }\end{array}$ & $\begin{array}{c}\text { Weight } \\
\text { required (g) }\end{array}$ \\
\hline Aluminum nitrate, 9-hydrate & $\mathrm{Al}\left(\mathrm{NO}_{3}\right)_{3} .9 \mathrm{H}_{2} \mathrm{O}$ & 375.1314 & 0.00005 & 0.0094 \\
\hline Sodium chloride & $\mathrm{NaCl}$ & 58.4400 & 0.0025 & 0.0731 \\
\hline Sodium fluoride & $\mathrm{NaF}$ & 41.9882 & 0.00075 & 0.0157 \\
\hline Sodium sulfate & $\mathrm{Na}_{2} \mathrm{SO}_{4}$ & 142.0400 & 0.0013 & 0.0888 \\
\hline Sodium hydroxide & $\mathrm{NaOH}$ & 39.9971 & 0.01 & 0.2000 \\
\hline $\begin{array}{c}\text { Sodium phosphate, 12- } \\
\text { hydrate }\end{array}$ & $\mathrm{Na}_{3} \mathrm{PO}_{4} 12 \mathrm{H}_{2} \mathrm{O}$ & 380.1200 & 0.000125 & 0.0238 \\
\hline Sodium carbonate & $\mathrm{Na}_{2} \mathrm{CO}_{3}$ & 105.9885 & 0.1 & 5.2994 \\
\hline Sodium nitrate & $\mathrm{NaNO}_{3}$ & 84.9947 & 0.1 & 4.2497 \\
\hline Sodium nitrite & $\mathrm{NaNO}_{2}$ & 68.9953 & 0.0500 & 1.7249 \\
\hline
\end{tabular}

\section{Composition of simulant for LAI-Solution 2}

$\begin{array}{rrr}\text { Temperature } & 40 & { }^{\circ} \mathrm{C} \\ \mathrm{pH} & 12 & \\ \text { Volume } & 0.5 & \mathrm{~L}\end{array}$

\begin{tabular}{|c|c|c|c|c|}
\hline Volume & Formula & $\begin{array}{c}\text { Molecular weight } \\
\text { (g/mol) }\end{array}$ & $\begin{array}{c}\text { Concentration } \\
\text { (M) }\end{array}$ & $\begin{array}{c}\text { Weight } \\
\text { required (g) }\end{array}$ \\
\hline Aluminum nitrate, 9-hydrate & $\mathrm{Al}\left(\mathrm{NO}_{3}\right)_{3} .9 \mathrm{H}_{2} \mathrm{O}$ & 375.1314 & 0.00005 & 0.0094 \\
\hline Sodium chloride & $\mathrm{NaCl}$ & 58.4400 & 0.0025 & 0.0731 \\
\hline Sodium fluoride & $\mathrm{NaF}$ & 41.9882 & 0.00075 & 0.0157 \\
\hline Sodium sulfate & $\mathrm{Na}_{2} \mathrm{SO}_{4}$ & 142.0400 & 0.00125 & 0.0888 \\
\hline Sodium hydroxide & $\mathrm{NaOH}_{2}$ & 39.9971 & 0.01 & 0.2000 \\
\hline $\begin{array}{c}\text { Sodium phosphate, 12- } \\
\text { hydrate }\end{array}$ & $\mathrm{Na}_{3} \mathrm{PO}_{4} 12 \mathrm{H}_{2} \mathrm{O}$ & 380.1200 & 0.000125 & 0.0238 \\
\hline Sodium carbonate & $\mathrm{Na}_{2} \mathrm{CO}_{3}$ & 105.9885 & 0.1 & 5.2994 \\
\hline Sodium nitrate & $\mathrm{NaNO}_{3}$ & 84.9947 & 0.1 & 4.2497 \\
\hline Sodium nitrite & $\mathrm{NaNO}_{2}$ & 68.9953 & 0.166 & 5.7266 \\
\hline
\end{tabular}




\section{Composition of simulant for LAI-Solution 3}

$\begin{array}{rrr}\text { Temperature } & 40 & { }^{\circ} \mathrm{C} \\ \mathrm{pH} & 12 & \\ \text { Volume } & 0.5 & \mathrm{~L}\end{array}$

\begin{tabular}{|c|c|c|c|c|}
\hline Simulant Source & Formula & $\begin{array}{c}\text { Molecular weight } \\
\text { (g/mol) }\end{array}$ & $\begin{array}{c}\text { Concentration } \\
\text { (M) }\end{array}$ & $\begin{array}{c}\text { Weight } \\
\text { required (g) }\end{array}$ \\
\hline $\begin{array}{c}\text { Aluminum nitrate, 9- } \\
\text { hydrate }\end{array}$ & $\begin{array}{c}\mathrm{Al}\left(\mathrm{NO}_{3}\right)_{3} .9 \mathrm{H}_{2} \\
\mathrm{O}\end{array}$ & 375.1314 & 0.0002 & 0.0375 \\
\hline Sodium chloride & $\mathrm{NaCl}$ & 58.4400 & 0.01 & 0.2922 \\
\hline Sodium fluoride & $\mathrm{NaF}$ & 41.9882 & 0.003 & 0.0630 \\
\hline Sodium sulfate & $\mathrm{Na}_{2} \mathrm{SO}_{4}$ & 142.0400 & 0.005 & 0.3551 \\
\hline Sodium hydroxide & $\mathrm{NaOH}_{3}$ & 39.9971 & 0.01 & 0.2000 \\
\hline $\begin{array}{c}\text { Sodium phosphate, 12- } \\
\text { hydrate }\end{array}$ & $\begin{array}{c}\mathrm{Na}_{3} \mathrm{PO}_{4} 12 \mathrm{H}_{2} \\
\mathrm{O}\end{array}$ & 380.1200 & 0.0005 & 0.0950 \\
\hline Sodium carbonate & $\mathrm{Na}_{2} \mathrm{CO}_{3}$ & 105.9885 & 0.1 & 5.2994 \\
\hline Sodium nitrate & $\mathrm{NaNO}_{3}$ & 84.9947 & 0.5 & 21.2487 \\
\hline Sodium nitrite & $\mathrm{NaNO}_{2}$ & 68.9953 & 0.075 & 2.5873 \\
\hline
\end{tabular}

\section{Composition of simulant for LAI-Solution 4}

$\begin{array}{rrr}\text { Temperature } & 40 \quad{ }^{\circ} \mathrm{C} \\ \mathrm{pH} & 12 & \\ \text { Volume } & 0.5 \quad \mathrm{~L}\end{array}$

\begin{tabular}{|c|c|c|c|c|}
\hline Simulant Source & Formula & $\begin{array}{c}\text { Molecular weight } \\
\text { (g/mol) }\end{array}$ & $\begin{array}{c}\text { Concentration } \\
\text { (M) }\end{array}$ & $\begin{array}{c}\text { Weight } \\
\text { required (g) }\end{array}$ \\
\hline $\begin{array}{c}\text { Aluminum nitrate, 9- } \\
\text { hydrate }\end{array}$ & $\mathrm{Al}\left(\mathrm{NO}_{3}\right)_{3} .9 \mathrm{H}_{2} \mathrm{O}$ & 375.1314 & 0.0002 & 0.0375 \\
\hline Sodium chloride & $\mathrm{NaCl}$ & 58.4400 & 0.01 & 0.2922 \\
\hline Sodium fluoride & $\mathrm{NaF}$ & 41.9882 & 0.0030 & 0.0630 \\
\hline Sodium sulfate & $\mathrm{Na}_{2} \mathrm{SO}_{4}$ & 142.0400 & 0.0050 & 0.3551 \\
\hline Sodium hydroxide & $\mathrm{NaOH}$ & 39.9971 & 0.01 & 0.2000 \\
\hline $\begin{array}{c}\text { Sodium phosphate, 12- } \\
\text { hydrate }\end{array}$ & $\mathrm{Na}_{3} \mathrm{PO}_{4 .} 12 \mathrm{H}_{2} \mathrm{O}$ & 380.1200 & 0.0005 & 0.0950 \\
\hline Sodium carbonate & $\mathrm{Na}_{2} \mathrm{CO}_{3}$ & 105.9885 & 0.1000 & 5.2994 \\
\hline Sodium nitrate & $\mathrm{NaNO}_{3}$ & 84.9947 & 0.5 & 21.2487 \\
\hline Sodium nitrite & $\mathrm{NaNO}_{2}$ & 68.9953 & 0.83 & 28.6330 \\
\hline
\end{tabular}




\section{Composition of simulant for LAI-Solution 5}

$\begin{array}{rr}\text { Temperature } & 40 \quad{ }^{\circ} \mathrm{C} \\ \mathrm{pH} & 12 \\ \text { Volume } & 0.5 \quad \mathrm{~L}\end{array}$

\begin{tabular}{|c|c|c|c|c|}
\hline Simulant Source & Formula & $\begin{array}{c}\text { Molecular weight } \\
\text { (g/mol) }\end{array}$ & $\begin{array}{c}\text { Concentration } \\
\text { (M) }\end{array}$ & $\begin{array}{c}\text { Weight } \\
\text { required (g) }\end{array}$ \\
\hline $\begin{array}{c}\text { Aluminum nitrate, 9- } \\
\text { hydrate }\end{array}$ & $\mathrm{Al}\left(\mathrm{NO}_{3}\right)_{3} .9 \mathrm{H}_{2} \mathrm{O}$ & 375.1314 & 0.001 & 0.1876 \\
\hline Sodium chloride & $\mathrm{NaCl}$ & 58.4400 & 0.02 & 0.5844 \\
\hline Sodium fluoride & $\mathrm{NaF}$ & 41.9882 & 0.005 & 0.1050 \\
\hline Sodium sulfate & $\mathrm{Na}_{2} \mathrm{SO}_{4}$ & 142.0400 & 0.025 & 1.7755 \\
\hline Sodium hydroxide & $\mathrm{NaOH}$ & 39.9971 & 0.01 & 0.2000 \\
\hline $\begin{array}{c}\text { Sodium phosphate, } 12- \\
\text { hydrate }\end{array}$ & $\mathrm{Na}_{3} \mathrm{PO}_{4} 12 \mathrm{H}_{2} \mathrm{O}$ & 380.1200 & 0.005 & 0.9503 \\
\hline Sodium carbonate & $\mathrm{Na}_{2} \mathrm{CO}_{3}$ & 105.9885 & 0.25 & 13.2486 \\
\hline Sodium nitrate & $\mathrm{NaNO}_{3}$ & 84.9947 & 1 & 42.4974 \\
\hline Sodium nitrite & $\mathrm{NaNO}_{2}$ & 68.9953 & 0.1500 & 5.1746 \\
\hline
\end{tabular}

\section{Composition of simulant for LAI-Solution 6}

$\begin{array}{rl}\text { Temperature } & 40 \\ \mathrm{pH} & 12\end{array}$

\begin{tabular}{|c|c|c|c|c|}
\hline Volume & Formula & $\begin{array}{c}\text { Molecular weight } \\
\text { (g/mol) }\end{array}$ & $\begin{array}{c}\text { Concentration } \\
\text { (M) }\end{array}$ & $\begin{array}{c}\text { Weight } \\
\text { required (g) }\end{array}$ \\
\hline $\begin{array}{c}\text { Aluminum nitrate, 9- } \\
\text { hydrate }\end{array}$ & $\mathrm{Al}\left(\mathrm{NO}_{3}\right)_{3} .9 \mathrm{H}_{2} \mathrm{O}$ & 375.1314 & 0.001 & 0.1876 \\
\hline Sodium chloride & $\mathrm{NaCl}$ & 58.4400 & 0.02 & 0.5844 \\
\hline Sodium fluoride & $\mathrm{NaF}$ & 41.9882 & 0.005 & 0.1050 \\
\hline Sodium sulfate & $\mathrm{Na}_{2} \mathrm{SO}_{4}$ & 142.0400 & 0.025 & 1.7755 \\
\hline Sodium hydroxide & $\mathrm{NaOH}$ & 39.9971 & 0.01 & 0.2000 \\
\hline $\begin{array}{c}\text { Sodium phosphate, } 12- \\
\text { hydrate }\end{array}$ & $\mathrm{Na}_{3} \mathrm{PO}_{4} 12 \mathrm{H}_{2} \mathrm{O}$ & 380.1200 & 0.005 & 0.9503 \\
\hline Sodium carbonate & $\mathrm{Na}_{2} \mathrm{CO}_{3}$ & 105.9885 & 0.25 & 13.2486 \\
\hline Sodium nitrate & $\mathrm{NaNO}_{3}$ & 84.9947 & 1 & 42.4974 \\
\hline Sodium nitrite & $\mathrm{NaNO}_{2}$ & 68.9953 & 0.6 & 20.6986 \\
\hline
\end{tabular}




\section{Composition of simulant for LAI-Solution 7}

\begin{tabular}{|c|c|c|c|c|}
\hline Temperature & 40 & \multicolumn{3}{|l|}{${ }^{\circ} \mathrm{C}$} \\
\hline $\mathrm{pH}$ & 12 & & & \\
\hline Volume & 0.5 & $\mathrm{~L}$ & & \\
\hline Simulant Source & Formula & $\begin{array}{c}\text { Molecular weight } \\
(\mathrm{g} / \mathrm{mol})\end{array}$ & $\begin{array}{c}\text { Concentration } \\
\text { (M) }\end{array}$ & $\begin{array}{l}\text { Weight } \\
\text { required }(\mathrm{g})\end{array}$ \\
\hline $\begin{array}{c}\text { Aluminum nitrate, 9- } \\
\text { hydrate }\end{array}$ & $\mathrm{Al}\left(\mathrm{NO}_{3}\right)_{3} \cdot 9 \mathrm{H}_{2} \mathrm{O}$ & 375.1314 & 0.001 & 0.1876 \\
\hline Sodium chloride & $\mathrm{NaCl}$ & 58.4400 & 0.02 & 0.5844 \\
\hline Sodium fluoride & $\mathrm{NaF}$ & 41.9882 & 0.005 & 0.1050 \\
\hline Sodium sulfate & $\mathrm{Na}_{2} \mathrm{SO}_{4}$ & 142.0400 & 0.025 & 1.7755 \\
\hline Sodium hydroxide & $\mathrm{NaOH}$ & 39.9971 & 0.01 & 0.2000 \\
\hline $\begin{array}{l}\text { Sodium phosphate, } 12- \\
\text { hydrate }\end{array}$ & $\mathrm{Na}_{3} \mathrm{PO}_{4} .12 \mathrm{H}_{2} \mathrm{O}$ & 380.1200 & 0.005 & 0.9503 \\
\hline Sodium carbonate & $\mathrm{Na}_{2} \mathrm{CO}_{3}$ & 105.9885 & 0.25 & 13.2486 \\
\hline Sodium nitrate & $\mathrm{NaNO}_{3}$ & 84.9947 & 1 & 42.4974 \\
\hline Sodium nitrite & $\mathrm{NaNO}_{2}$ & 68.9953 & 1.66 & 57.2661 \\
\hline
\end{tabular}

Composition of simulant for LAI-Solution 8

$\begin{array}{rrr}\text { Temperature } & 40 & { }^{\circ} \mathrm{C} \\ \mathrm{pH} & 12 & \\ \text { Volume } & 0.5 & \mathrm{~L}\end{array}$

\begin{tabular}{|c|c|c|c|c|}
\hline Simulant Source & Formula & $\begin{array}{c}\text { Molecular weight } \\
\text { (g/mol) }\end{array}$ & $\begin{array}{c}\text { Concentration } \\
\text { (M) }\end{array}$ & $\begin{array}{c}\text { Weight } \\
\text { required (g) }\end{array}$ \\
\hline $\begin{array}{c}\text { Aluminum nitrate, 9- } \\
\text { hydrate }\end{array}$ & $\mathrm{Al}\left(\mathrm{NO}_{3}\right)_{3} .9 \mathrm{H}_{2} \mathrm{O}$ & 375.1314 & 0.0033 & 0.6190 \\
\hline Sodium chloride & $\mathrm{NaCl}$ & 58.4400 & 0.1 & 2.9220 \\
\hline Sodium fluoride & $\mathrm{NaF}$ & 41.9882 & 0.02 & 0.4199 \\
\hline Sodium sulfate & $\mathrm{Na}_{2} \mathrm{SO}_{4}$ & 142.0400 & 0.1 & 7.1020 \\
\hline Sodium hydroxide & $\mathrm{NaOH}$ & 39.9971 & 0.01 & 0.2000 \\
\hline $\begin{array}{c}\text { Sodium phosphate, 12- } \\
\text { hydrate }\end{array}$ & $\mathrm{Na}_{3} \mathrm{PO}_{4} 12 \mathrm{H}_{2} \mathrm{O}$ & 380.1200 & 0.05 & 9.5030 \\
\hline Sodium carbonate & $\mathrm{Na}_{2} \mathrm{CO}_{3}$ & 105.9885 & 1 & 52.9943 \\
\hline Sodium nitrate & $\mathrm{NaNO}_{3}$ & 84.9947 & 5 & 212.4868 \\
\hline Sodium nitrite & $\mathrm{NaNO}_{2}$ & 68.9953 & 0.6 & 20.6986 \\
\hline
\end{tabular}




\section{Composition of simulant for LAI-Solution 9}

Temperature

$\mathrm{pH}$

Volume

\begin{tabular}{|c|c|c|c|c|}
\hline Simulant Source & Formula & $\begin{array}{l}\text { Molecular Weight } \\
(\mathrm{g} / \mathrm{mol})\end{array}$ & $\begin{array}{l}\text { Concentration } \\
\text { (M) }\end{array}$ & $\begin{array}{l}\text { Weight } \\
\text { required }(\mathrm{g})\end{array}$ \\
\hline $\begin{array}{c}\text { Aluminum nitrate, 9- } \\
\text { hydrate }\end{array}$ & $\mathrm{Al}\left(\mathrm{NO}_{3}\right)_{3} \cdot 9 \mathrm{H}_{2} \mathrm{O}$ & 375.0000 & 0.4970 & 93.1875 \\
\hline $\begin{array}{c}\text { Cadmium nitrate, 4- } \\
\text { hydrate }\end{array}$ & $\mathrm{Cd}\left(\mathrm{NO}_{3}\right)_{2} \cdot 4 \mathrm{H}_{2} \mathrm{O}$ & 308.0000 & 0.0006 & 0.0852 \\
\hline Calcium nitrate, 4-hydrate & $\mathrm{Ca}\left(\mathrm{NO}_{3}\right)_{2} \cdot 4 \mathrm{H}_{2} \mathrm{O}$ & 236.0000 & 0.0100 & 1.1800 \\
\hline Cupric nitrate, 2.5 hydrate & $\mathrm{Cu}\left(\mathrm{NO}_{3}\right)_{2} \cdot 2.5 \mathrm{H}_{2} \mathrm{O}$ & 233.0000 & 0.0004 & 0.0437 \\
\hline Ferric nitrate, 9-hydrate & $\mathrm{Fe}\left(\mathrm{NO}_{3}\right)_{3} .9 \mathrm{H}_{2} \mathrm{O}$ & 404.0000 & 0.0006 & 0.1162 \\
\hline $\begin{array}{c}\text { Lanthanum nitrate, 6- } \\
\text { hydrate }\end{array}$ & $\mathrm{La}\left(\mathrm{NO}_{3}\right)_{3} \cdot 6 \mathrm{H}_{2} \mathrm{O}$ & 433.0000 & 0.0001 & 0.0187 \\
\hline Lead nitrate & $\mathrm{Pb}\left(\mathrm{NO}_{3}\right)_{2}$ & 331.0000 & 0.0007 & 0.1086 \\
\hline $\begin{array}{c}\text { Manganous chloride, 4- } \\
\text { hydrate }\end{array}$ & $\mathrm{MnCl}_{2} \cdot 4 \mathrm{H}_{2} \mathrm{O}$ & 198.0000 & 0.0003 & 0.0321 \\
\hline Nickel nitrate, 6-hydrate & $\mathrm{Ni}\left(\mathrm{NO}_{3}\right)_{2} \cdot 6 \mathrm{H}_{2} \mathrm{O}$ & 291.0000 & 0.0068 & 0.9821 \\
\hline Potassium nitrate & $\mathrm{K}\left(\mathrm{NO}_{3}\right)$ & 101.0000 & 0.0464 & 2.3432 \\
\hline Disodium EDTA & $\begin{array}{c}\mathrm{Na}_{2} \mathrm{C}_{10} \mathrm{H}_{14} \mathrm{O}_{8} .2 \mathrm{H}_{2} \\
\mathrm{O} \\
\end{array}$ & 372.0000 & 0.0481 & 8.9481 \\
\hline HEDTA & $\mathrm{C}_{10} \mathrm{H}_{18} \mathrm{~N}_{2} \mathrm{O}_{7}$ & 278.0000 & 0.0190 & 2.6396 \\
\hline Sodium gluconate & $\mathrm{C}_{6} \mathrm{H}_{11} \mathrm{O}_{7} \mathrm{Na}$ & 218.0000 & 0.0253 & 2.7599 \\
\hline Citric acid, 1-hydrate & $\mathrm{C}_{6} \mathrm{H}_{8} \mathrm{O}_{7} \cdot \mathrm{H}_{2} \mathrm{O}$ & 210.0000 & 0.1114 & 11.6978 \\
\hline Nitrilotriacetic Acid & $\mathrm{C}_{6} \mathrm{H}_{9} \mathrm{NO}_{6}$ & 191.0000 & 0.0076 & 0.7254 \\
\hline Iminodiacetic Acid & $\mathrm{C}_{4} \mathrm{H}_{7} \mathrm{NO}_{2}$ & 133.0000 & 0.1127 & 7.4928 \\
\hline Sodium chloride & $\mathrm{NaCl}$ & 58.4000 & 0.1060 & 3.0952 \\
\hline Sodium sulfate & $\mathrm{Na}_{2} \mathrm{SO}_{4}$ & 142.0000 & 0.1280 & 9.0880 \\
\hline Ammonium Chloride & $\mathrm{NH}_{4} \mathrm{Cl}$ & 55.4920 & 0.0050 & 0.1382 \\
\hline Glycolic acid & $\mathrm{C}_{2} \mathrm{H}_{4} \mathrm{O}_{3}$ & 76.1000 & 0.1610 & 6.1261 \\
\hline Sodium hydroxide & $\mathrm{NaOH}$ & 40.0000 & 3.0012 & 60.0246 \\
\hline $\begin{array}{c}\text { Sodium phosphate, } 12- \\
\text { hydrate }\end{array}$ & $\mathrm{Na}_{3} \mathrm{PO}_{4} \cdot 12 \mathrm{H}_{2} \mathrm{O}$ & 380.0000 & 0.0514 & 9.7660 \\
\hline Sodium formate & $\mathrm{Na}(\mathrm{CHO} 2)$ & 68.0000 & 0.2330 & 7.9220 \\
\hline $\begin{array}{c}\text { Sodium acetate, } 3- \\
\text { hydrate }\end{array}$ & $\mathrm{Na}\left(\mathrm{C}_{2} \mathrm{H}_{3} \mathrm{O}_{2}\right) \cdot 3 \mathrm{H}_{2} \mathrm{O}$ & 136.0000 & 0.0208 & 1.4144 \\
\hline Sodium carbonate & $\mathrm{Na}_{2} \mathrm{CO}_{3}$ & 106.0000 & 1.1200 & 59.3600 \\
\hline Sodium nitrate & $\mathrm{NaNO}_{3}$ & 85.0000 & 1.5362 & 65.2877 \\
\hline Sodium nitrite & $\mathrm{NaNO}_{2}$ & 69.0000 & 1.9500 & 67.2750 \\
\hline
\end{tabular}

$40{ }^{\circ} \mathrm{C}$

12

$0.5 \mathrm{~L}$ 


\section{Composition of simulant for LAI-Solution 10}

$\begin{array}{rr}\text { Temperature } & 40{ }^{\circ} \mathrm{C} \\ \mathrm{pH} & 12 \\ \text { Volume } & 0.5 \mathrm{~L}\end{array}$

\begin{tabular}{|c|c|c|c|c|}
\hline Simulant Source & Formula & $\begin{array}{c}\text { Molecular Weight } \\
\text { (g/mol) }\end{array}$ & $\begin{array}{l}\text { Concentration } \\
\text { (M) }\end{array}$ & $\begin{array}{c}\text { Weight } \\
\text { required (g) }\end{array}$ \\
\hline $\begin{array}{c}\text { Aluminum nitrate, 9- } \\
\text { hydrate }\end{array}$ & $\mathrm{Al}\left(\mathrm{NO}_{3}\right)_{3} \cdot 9 \mathrm{H}_{2} \mathrm{O}$ & 375.0000 & 0.4970 & 93.1875 \\
\hline $\begin{array}{c}\text { Cadmium nitrate, 4- } \\
\text { hydrate }\end{array}$ & $\mathrm{Cd}\left(\mathrm{NO}_{3}\right)_{2} \cdot 4 \mathrm{H}_{2} \mathrm{O}$ & 308.0000 & 0.0006 & 0.0852 \\
\hline $\begin{array}{c}\text { Calcium nitrate, } 4- \\
\text { hydrate }\end{array}$ & $\mathrm{Ca}\left(\mathrm{NO}_{3}\right)_{2} \cdot 4 \mathrm{H}_{2} \mathrm{O}$ & 236.0000 & 0.0100 & 1.1800 \\
\hline $\begin{array}{c}\text { Cupric nitrate, } 2.5 \\
\text { hydrate }\end{array}$ & $\mathrm{Cu}\left(\mathrm{NO}_{3}\right)_{2} \cdot 2 \cdot 5 \mathrm{H}_{2} \mathrm{O}$ & 233.0000 & 0.0004 & 0.0437 \\
\hline Ferric nitrate, 9-hydrate & $\mathrm{Fe}\left(\mathrm{NO}_{3}\right)_{3} \cdot 9 \mathrm{H}_{2} \mathrm{O}$ & 404.0000 & 0.0006 & 0.1162 \\
\hline $\begin{array}{c}\text { Lanthanum nitrate, 6- } \\
\text { hydrate }\end{array}$ & $\mathrm{La}\left(\mathrm{NO}_{3}\right)_{3} \cdot 6 \mathrm{H}_{2} \mathrm{O}$ & 433.0000 & 0.0001 & 0.0187 \\
\hline Lead nitrate & $\mathrm{Pb}\left(\mathrm{NO}_{3}\right)_{2}$ & 331.0000 & 0.0007 & 0.1086 \\
\hline $\begin{array}{c}\text { Manganous chloride, 4- } \\
\text { hydrate }\end{array}$ & $\mathrm{MnCl}_{2} \cdot 4 \mathrm{H}_{2} \mathrm{O}$ & 198.0000 & 0.0003 & 0.0321 \\
\hline Nickel nitrate, 6-hydrate & $\mathrm{Ni}\left(\mathrm{NO}_{3}\right)_{2} \cdot 6 \mathrm{H}_{2} \mathrm{O}$ & 291.0000 & 0.0068 & 0.9821 \\
\hline Potassium nitrate & $\mathrm{K}\left(\mathrm{NO}_{3}\right)$ & 101.0000 & 0.0464 & 2.3432 \\
\hline Disodium EDTA & $\mathrm{Na}_{2} \mathrm{C}_{10} \mathrm{H}_{14} \mathrm{O}_{8} .2 \mathrm{H}_{2} \mathrm{O}$ & 372.0000 & 0.0481 & 8.9481 \\
\hline HEDTA & $\mathrm{C}_{10} \mathrm{H}_{18} \mathrm{~N}_{2} \mathrm{O}_{7}$ & 278.0000 & 0.0190 & 2.6396 \\
\hline Sodium gluconate & $\mathrm{C}_{6} \mathrm{H}_{11} \mathrm{O}_{7} \mathrm{Na}$ & 218.0000 & 0.0253 & 2.7599 \\
\hline Citric acid, 1-hydrate & $\mathrm{C}_{6} \mathrm{H}_{8} \mathrm{O}_{7} \cdot \mathrm{H}_{2} \mathrm{O}$ & 210.0000 & 0.1114 & 11.6978 \\
\hline Nitrilotriacetic Acid & $\mathrm{C}_{6} \mathrm{H}_{9} \mathrm{NO}_{6}$ & 191.0000 & 0.0076 & 0.7254 \\
\hline Iminodiacetic Acid & $\mathrm{C}_{4} \mathrm{H}_{7} \mathrm{NO}_{2}$ & 133.0000 & 0.1127 & 7.4928 \\
\hline Sodium chloride & $\mathrm{NaCl}$ & 58.4000 & 0.1060 & 3.0952 \\
\hline Sodium sulfate & $\mathrm{Na}_{2} \mathrm{SO}_{4}$ & 142.0000 & 0.1280 & 9.0880 \\
\hline Ammonium Chloride & $\mathrm{NH}_{4} \mathrm{Cl}$ & 55.4920 & 0.0050 & 0.1382 \\
\hline Glycolic acid & $\mathrm{C}_{2} \mathrm{H}_{4} \mathrm{O}_{3}$ & 76.1000 & 0.1610 & 6.1261 \\
\hline Sodium hydroxide & $\mathrm{NaOH}$ & 40.0000 & 2.9612 & 59.2246 \\
\hline $\begin{array}{l}\text { Sodium phosphate, } 12- \\
\text { hydrate }\end{array}$ & $\mathrm{Na}_{3} \mathrm{PO}_{4} \cdot 12 \mathrm{H}_{2} \mathrm{O}$ & 380.0000 & 0.0514 & 9.7660 \\
\hline Sodium formate & $\mathrm{Na}(\mathrm{CHO} 2)$ & 68.0000 & 0.2330 & 7.9220 \\
\hline $\begin{array}{c}\text { Sodium acetate, 3- } \\
\text { hydrate }\end{array}$ & $\mathrm{Na}\left(\mathrm{C}_{2} \mathrm{H}_{3} \mathrm{O}_{2}\right) \cdot 3 \mathrm{H}_{2} \mathrm{O}$ & 136.0000 & 0.0208 & 1.4144 \\
\hline Sodium carbonate & $\mathrm{Na}_{2} \mathrm{CO}_{3}$ & 106.0000 & 1.1200 & 59.3600 \\
\hline
\end{tabular}




\begin{tabular}{|l|l|l|l|l|}
\hline Sodium nitrate & $\mathrm{NaNO}_{3}$ & 85.0000 & 1.4862 & 63.1622 \\
\hline Sodium nitrite & $\mathrm{NaNO}_{2}$ & 69.0000 & 1.9100 & 65.8950 \\
\hline
\end{tabular}

Composition of simulant for LAI-Solution 11

\begin{tabular}{|c|c|}
\hline Temperature & 40 \\
\hline $\mathrm{pH}$ & 12 \\
\hline Volume & 0.5 \\
\hline
\end{tabular}

\begin{tabular}{|c|c|c|c|c|}
\hline Simulant Source & Formula & $\begin{array}{c}\text { Molecular Weight } \\
\text { (g/mol) }\end{array}$ & $\begin{array}{l}\text { Concentration } \\
\text { (M) }\end{array}$ & $\begin{array}{l}\text { Weight } \\
\text { required }(\mathrm{g})\end{array}$ \\
\hline $\begin{array}{c}\text { Aluminum nitrate, 9- } \\
\text { hydrate }\end{array}$ & $\mathrm{Al}\left(\mathrm{NO}_{3}\right)_{3} \cdot 9 \mathrm{H}_{2} \mathrm{O}$ & 375.0000 & 0.4970 & 93.1875 \\
\hline $\begin{array}{c}\text { Cadmium nitrate, 4- } \\
\text { hydrate }\end{array}$ & $\mathrm{Cd}\left(\mathrm{NO}_{3}\right)_{2} \cdot 4 \mathrm{H}_{2} \mathrm{O}$ & 308.0000 & 0.0006 & 0.0852 \\
\hline $\begin{array}{c}\text { Calcium nitrate, 4- } \\
\text { hydrate }\end{array}$ & $\mathrm{Ca}\left(\mathrm{NO}_{3}\right)_{2} \cdot 4 \mathrm{H}_{2} \mathrm{O}$ & 236.0000 & 0.0100 & 1.1800 \\
\hline $\begin{array}{c}\text { Cupric nitrate, } 2.5 \\
\text { hydrate }\end{array}$ & $\mathrm{Cu}\left(\mathrm{NO}_{3}\right)_{2} \cdot 2 \cdot 5 \mathrm{H}_{2} \mathrm{O}$ & 233.0000 & 0.0004 & 0.0437 \\
\hline Ferric nitrate, 9-hydrate & $\mathrm{Fe}\left(\mathrm{NO}_{3}\right)_{3} \cdot 9 \mathrm{H}_{2} \mathrm{O}$ & 404.0000 & 0.0006 & 0.1162 \\
\hline $\begin{array}{c}\text { Lanthanum nitrate, 6- } \\
\text { hydrate }\end{array}$ & $\mathrm{La}\left(\mathrm{NO}_{3}\right)_{3} \cdot 6 \mathrm{H}_{2} \mathrm{O}$ & 433.0000 & 0.0001 & 0.0187 \\
\hline Lead nitrate & $\mathrm{Pb}\left(\mathrm{NO}_{3}\right)_{2}$ & 331.0000 & 0.0007 & 0.1086 \\
\hline $\begin{array}{l}\text { Manganous chloride, 4- } \\
\text { hydrate }\end{array}$ & $\mathrm{MnCl}_{2} \cdot 4 \mathrm{H}_{2} \mathrm{O}$ & 198.0000 & 0.0003 & 0.0321 \\
\hline Nickel nitrate, 6-hydrate & $\mathrm{Ni}\left(\mathrm{NO}_{3}\right)_{2} \cdot 6 \mathrm{H}_{2} \mathrm{O}$ & 291.0000 & 0.0068 & 0.9821 \\
\hline Potassium nitrate & $\mathrm{K}\left(\mathrm{NO}_{3}\right)$ & 101.0000 & 0.0464 & 2.3432 \\
\hline Disodium EDTA & $\begin{array}{c}\mathrm{Na}_{2} \mathrm{C}_{10} \mathrm{H}_{14} \mathrm{O}_{8} .2 \mathrm{H}_{2} \\
\mathrm{O}\end{array}$ & 372.0000 & 0.0481 & 8.9481 \\
\hline HEDTA & $\mathrm{C}_{10} \mathrm{H}_{18} \mathrm{~N}_{2} \mathrm{O}_{7}$ & 278.0000 & 0.0190 & 2.6396 \\
\hline Sodium gluconate & $\mathrm{C}_{6} \mathrm{H}_{11} \mathrm{O}_{7} \mathrm{Na}$ & 218.0000 & 0.0253 & 2.7599 \\
\hline Citric acid, 1-hydrate & $\mathrm{C}_{6} \mathrm{H}_{8} \mathrm{O}_{7} \cdot \mathrm{H}_{2} \mathrm{O}$ & 210.0000 & 0.1114 & 11.6978 \\
\hline Nitrilotriacetic Acid & $\mathrm{C}_{6} \mathrm{H}_{9} \mathrm{NO}_{6}$ & 191.0000 & 0.0076 & 0.7254 \\
\hline Iminodiacetic Acid & $\mathrm{C}_{4} \mathrm{H}_{7} \mathrm{NO}_{2}$ & 133.0000 & 0.1127 & 7.4928 \\
\hline Sodium chloride & $\mathrm{NaCl}$ & 58.4000 & 0.1060 & 3.0952 \\
\hline Sodium sulfate & $\mathrm{Na}_{2} \mathrm{SO}_{4}$ & 142.0000 & 0.1280 & 9.0880 \\
\hline Ammonium Chloride & $\mathrm{NH}_{4} \mathrm{Cl}$ & 55.4920 & 0.0050 & 0.1382 \\
\hline Glycolic acid & $\mathrm{C}_{2} \mathrm{H}_{4} \mathrm{O}_{3}$ & 76.1000 & 0.1610 & 6.1261 \\
\hline Sodium hydroxide & $\mathrm{NaOH}$ & 40.0000 & 3.1912 & 63.8246 \\
\hline $\begin{array}{c}\text { Sodium phosphate, } 12- \\
\text { hydrate }\end{array}$ & $\mathrm{Na}_{3} \mathrm{PO}_{4} 12 \mathrm{H}_{2} \mathrm{O}$ & 380.0000 & 0.0514 & 9.7660 \\
\hline Sodium formate & $\mathrm{Na}(\mathrm{CHO} 2)$ & 68.0000 & 0.2330 & 7.9220 \\
\hline $\begin{array}{c}\text { Sodium acetate, 3- } \\
\text { hydrate }\end{array}$ & $\mathrm{Na}\left(\mathrm{C}_{2} \mathrm{H}_{3} \mathrm{O}_{2}\right) \cdot 3 \mathrm{H}_{2} \mathrm{O}$ & 136.0000 & 0.0208 & 1.4144 \\
\hline
\end{tabular}




\begin{tabular}{|c|c|c|c|c|} 
Sodium carbonate & $\mathrm{Na}_{2} \mathrm{CO}_{3}$ & 106.0000 & 1.1200 & 59.3600 \\
\hline Sodium nitrate & $\mathrm{NaNO}_{3}$ & 85.0000 & 1.4262 & 60.6122 \\
\hline Sodium nitrite & $\mathrm{NaNO}_{2}$ & 69.0000 & 1.0000 & 34.5000 \\
\hline
\end{tabular}

\section{Composition of simulant for LAI-Solutions 12 and 13}

\begin{tabular}{|c|c|c|c|c|}
\hline Temperature & 45 & \multicolumn{3}{|l|}{${ }^{\circ} \mathrm{C}$} \\
\hline $\mathrm{pH}$ & 7.6 & & & \\
\hline Volume & 1 & $\mathrm{~L}$ & & \\
\hline Simulant Source & Formula & $\begin{array}{c}\text { Molecular Weight } \\
\text { (g/mol) }\end{array}$ & $\begin{array}{c}\text { Concentration } \\
\text { (M) }\end{array}$ & $\begin{array}{l}\text { Weight } \\
\text { required }(g)\end{array}$ \\
\hline Sodium bicarbonate & $\mathrm{NaHCO}_{3}$ & 84.0100 & $1.12 \mathrm{E}-03$ & 0.0941 \\
\hline Calcium hydroxide & $\mathrm{Ca}(\mathrm{OH})_{2}$ & 74.0930 & $1.21 \mathrm{E}-04$ & 0.0090 \\
\hline Potassium nitrate & $\mathrm{KNO}_{3}$ & 101.1032 & $6.75 \mathrm{E}-05$ & 0.0068 \\
\hline $\begin{array}{c}\text { Magnesium Nitrate, } 6 \text { - } \\
\text { hydrate }\end{array}$ & $\mathrm{Mg}\left(\mathrm{NO}_{3}\right)_{2} \cdot 6 \mathrm{H}_{2} \mathrm{O}$ & 256.4100 & $1.52 \mathrm{E}-05$ & 0.0039 \\
\hline Strontium Nitrate & $\mathrm{Sr}\left(\mathrm{NO}_{3}\right)_{2}$ & 211.6300 & 4.04E-06 & 0.0009 \\
\hline Sodium sulfate & $\mathrm{Na}_{2} \mathrm{SO}_{4}$ & 142.0400 & $1.83 \mathrm{E}-06$ & 0.0003 \\
\hline $\begin{array}{l}\text { Sodium Metasilicate, } 5- \\
\text { hydrate }\end{array}$ & $\mathrm{Na}_{2} \mathrm{SiO}_{3} .5 \mathrm{H}_{2} \mathrm{O}$ & 212.1400 & 4.57E-05 & 0.0097 \\
\hline Ferric chloride & $\mathrm{FeCl}_{3}$ & 162.2000 & 2.67E-06 & 0.0004 \\
\hline Manganese Nitrate & $\mathrm{Mn}\left(\mathrm{NO}_{3}\right)_{2}$ & 178.9500 & $3.43 \mathrm{E}-07$ & 0.0001 \\
\hline Acetic Acid & $\mathrm{C}_{2} \mathrm{H}_{4} \mathrm{O}_{2}$ & 60.0500 & $3.00 \mathrm{E}-04$ & 0.0180 \\
\hline
\end{tabular}

\section{Composition of simulant for LAI-Solutions 14 and 15}

$\begin{array}{rrr}\text { Temperature } & 45 & { }^{\circ} \mathrm{C} \\ \mathrm{pH} & 7.6 & \\ \text { Volume } & 1 & \mathrm{~L}\end{array}$

\begin{tabular}{|c|c|c|c|c|}
\hline Volume & Formula & $\begin{array}{c}\text { Molecular weight } \\
\text { (g/mol) }\end{array}$ & $\begin{array}{c}\text { Concentration } \\
\text { (M) }\end{array}$ & $\begin{array}{c}\text { Weight } \\
\text { required (g) }\end{array}$ \\
\hline Sodium bicarbonate & $\mathrm{NaHCO}_{3}$ & 84.0100 & $1.75 \mathrm{E}-03$ & 0.1470 \\
\hline Calcium hydroxide & $\mathrm{Ca}(\mathrm{OH})_{2}$ & 74.0930 & $1.50 \mathrm{E}-03$ & 0.1111 \\
\hline Potassium nitrate & $\mathrm{KNO}_{3}$ & 101.1032 & $2.40 \mathrm{E}-04$ & 0.0243 \\
\hline Ferric sulfate & $\mathrm{Fe}_{2}\left(\mathrm{SO}_{4}\right)_{3}$ & 399.8800 & $6.25 \mathrm{E}-04$ & 0.2499 \\
\hline Ferric chloride & $\mathrm{FeCl}_{3}$ & 162.2000 & $7.67 \mathrm{E}-05$ & 0.0124 \\
\hline Strontium Nitrate & $\left.\mathrm{Sr}_{(\mathrm{NO}}\right)_{2}$ & 211.6300 & $2.87 \mathrm{E}-06$ & 0.0006 \\
\hline $\begin{array}{c}\text { Sodium Metasilicate, 5- } \\
\text { hydrate }\end{array}$ & $\mathrm{Na}_{2} \mathrm{SiO}_{3} .5 \mathrm{H}_{2} \mathrm{O}$ & 212.1400 & $6.00 \mathrm{E}-04$ & 0.1273 \\
\hline Magnesium Chloride & $\mathrm{MgCl}_{2}$ & 95.2110 & $3.10 \mathrm{E}-04$ & 0.0295 \\
\hline Acetic Acid & $\mathrm{C}_{2} \mathrm{H}_{4} \mathrm{O}_{2}$ & 60.0500 & $3.00 \mathrm{E}-04$ & 0.0180 \\
\hline
\end{tabular}


Appendix D

Pictures of Liquid Air Interface Corrosion Samples after Test 


\section{Solution 1}

\section{2 month exposure}

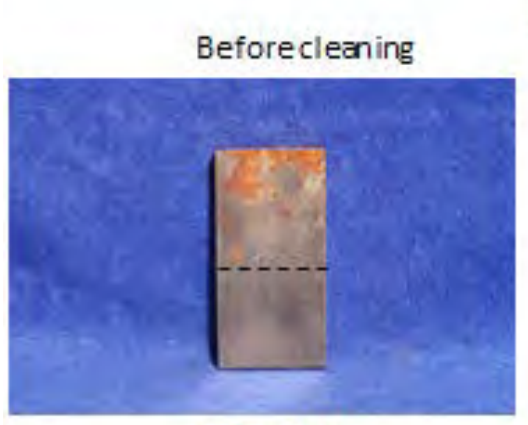

Front side

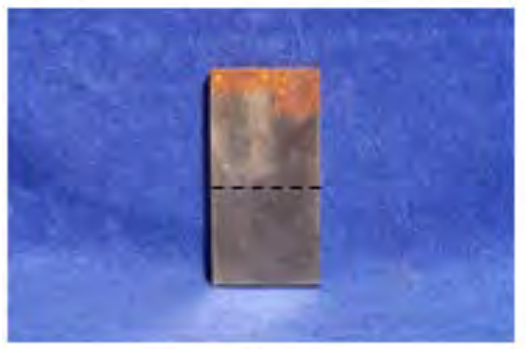

Back side
RC128-2

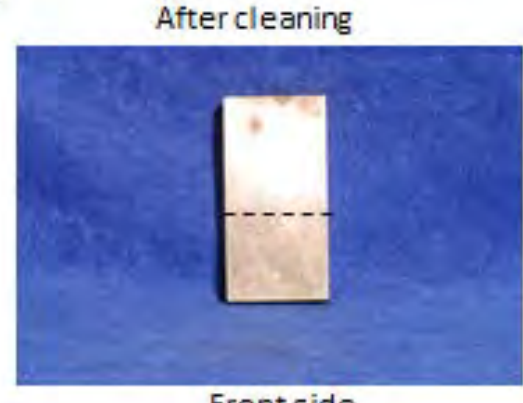

Front side

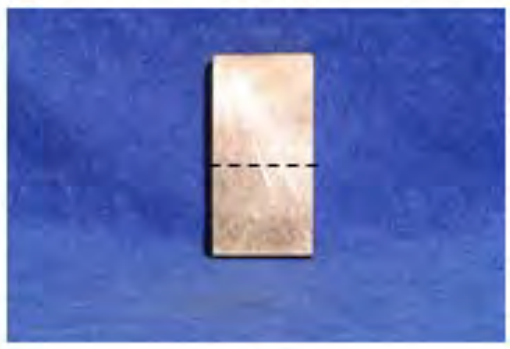

Back side

4 month exposure

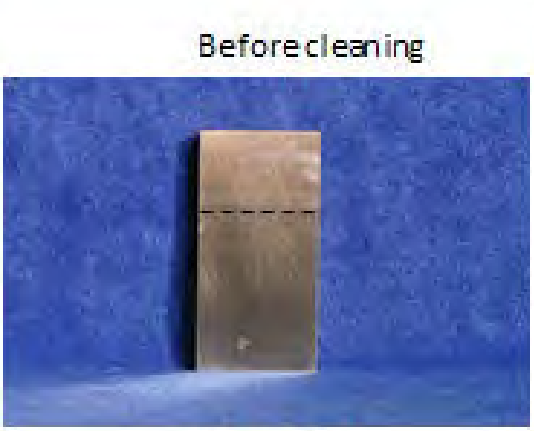

Front side

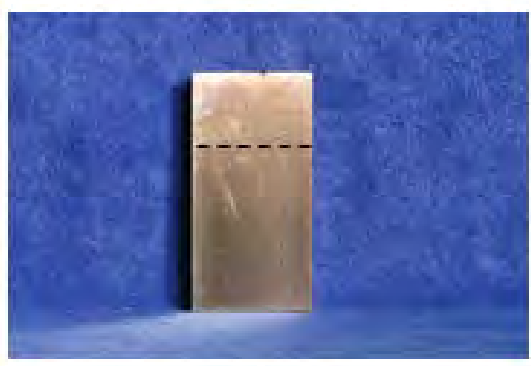

Back side
RC128-1
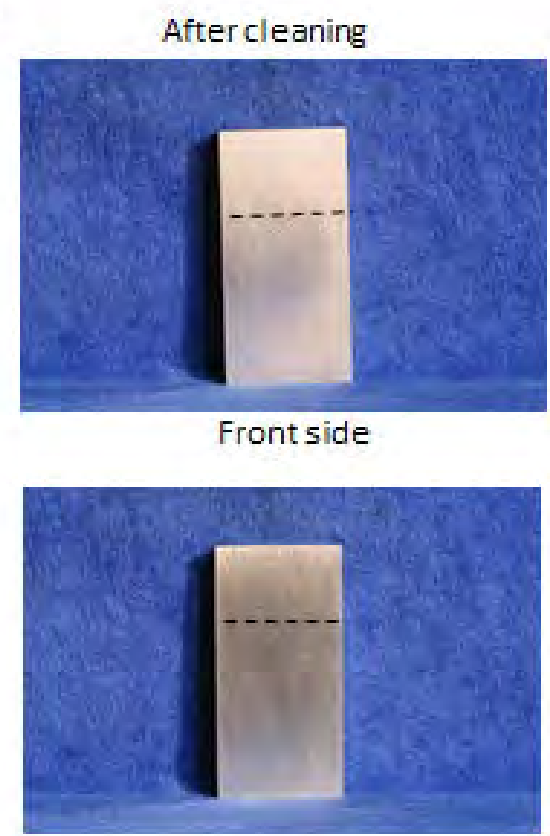

Back side 


\section{Solution 2}

\section{2 month exposure}

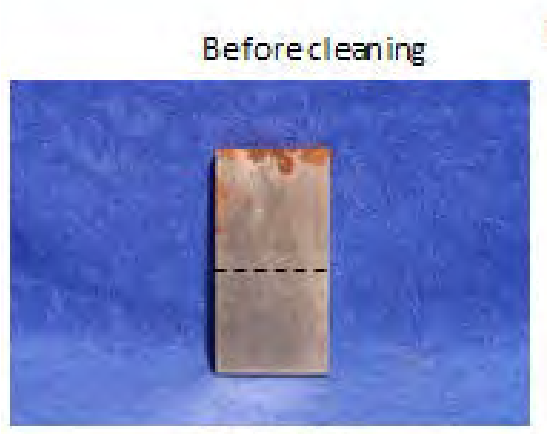

Front side

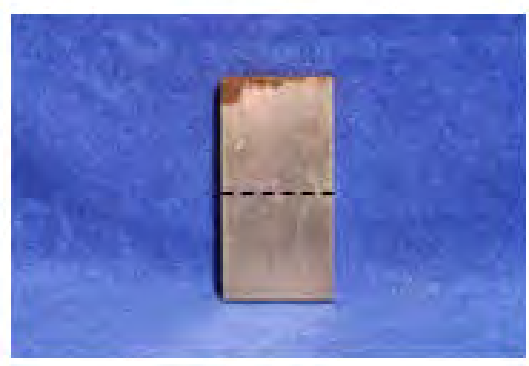

Back side
$\mathrm{RC} 128-4$

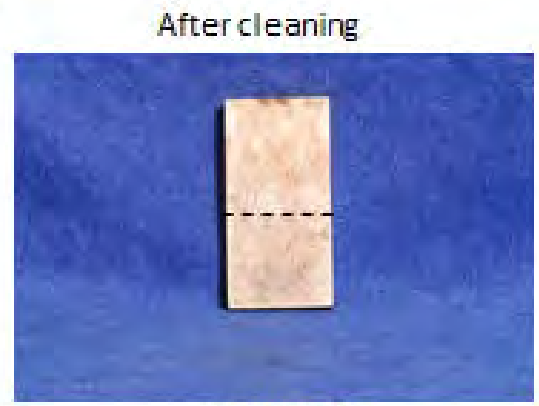

Front side

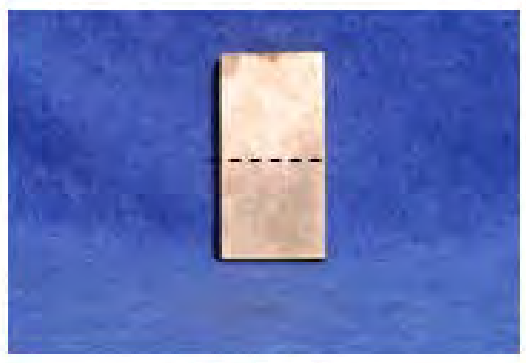

Back side

4 month exposure

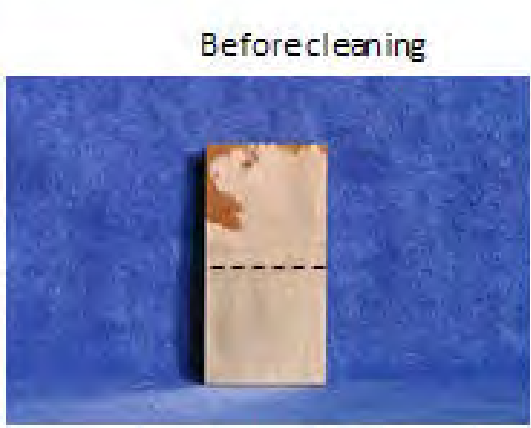

Front side

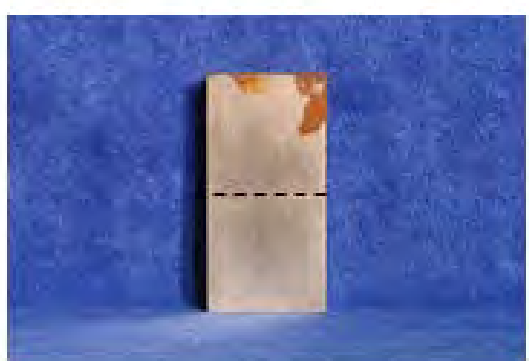

Back side
RC128-3

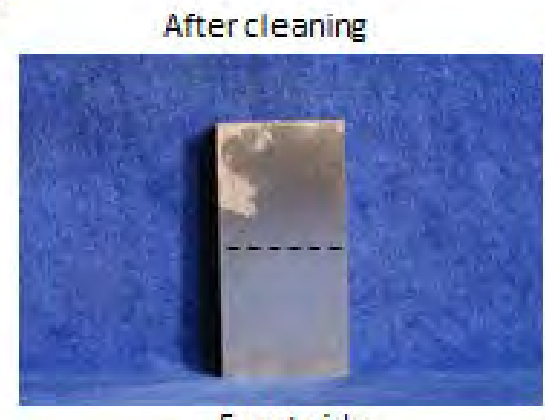

Front side

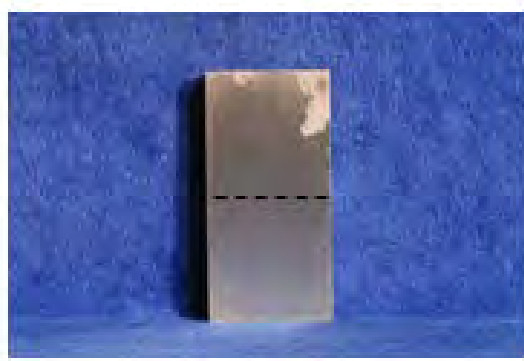

Back side 


\section{Solution 3}

\section{2 month exposure}

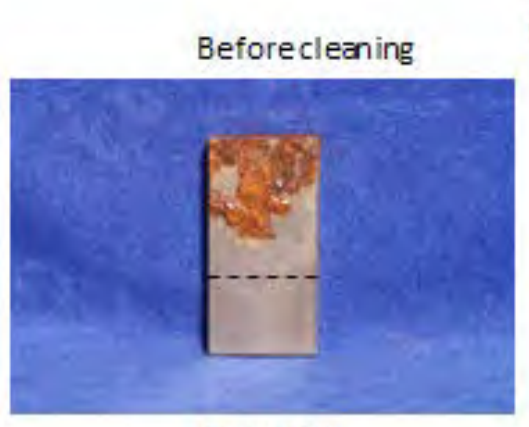

Front side

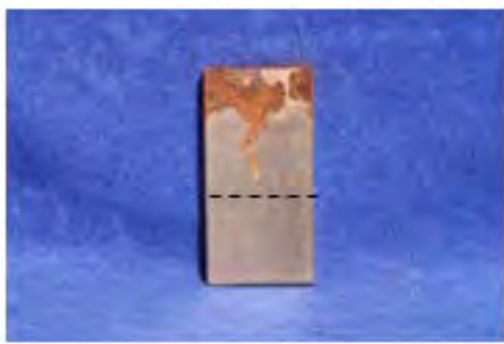

Back side
RC128-6

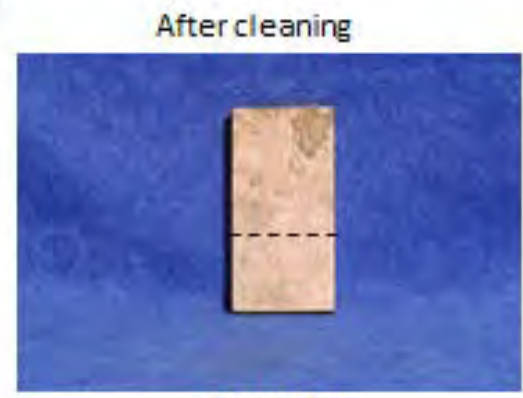

Front side

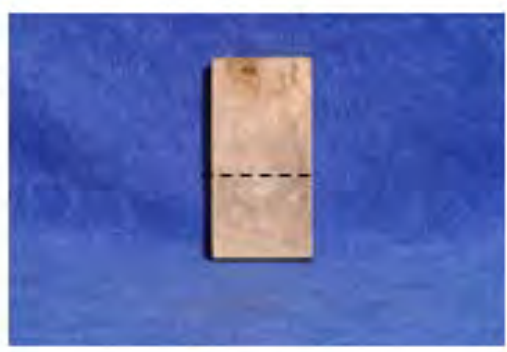

Back side

4 month exposure

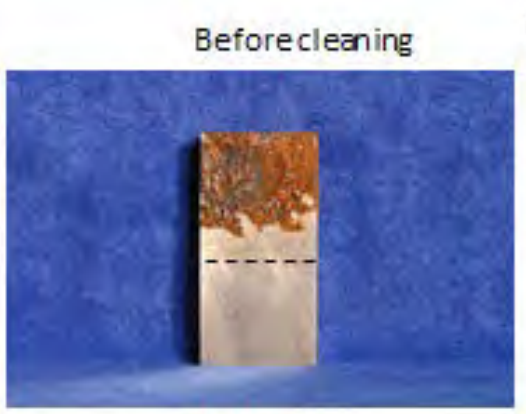

Front side

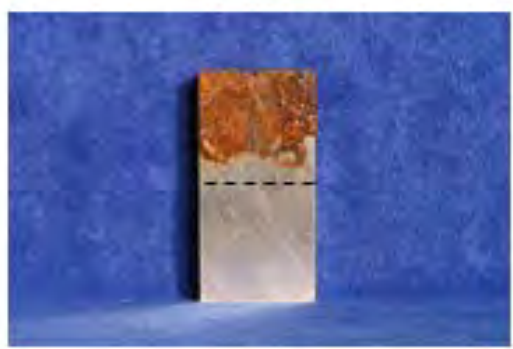

Back side
RC128-5

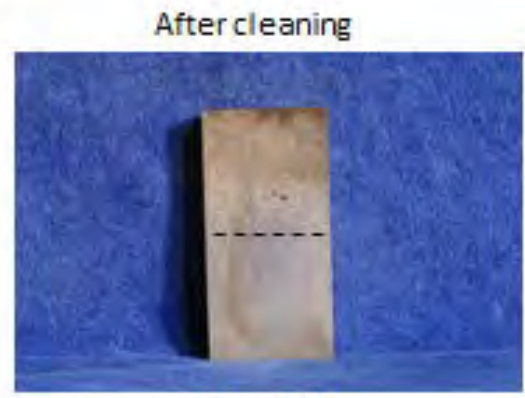

Front side

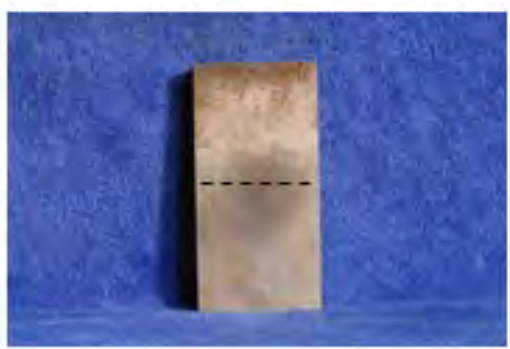

Back side 


\section{Solution 4}

\section{2 month exposure}

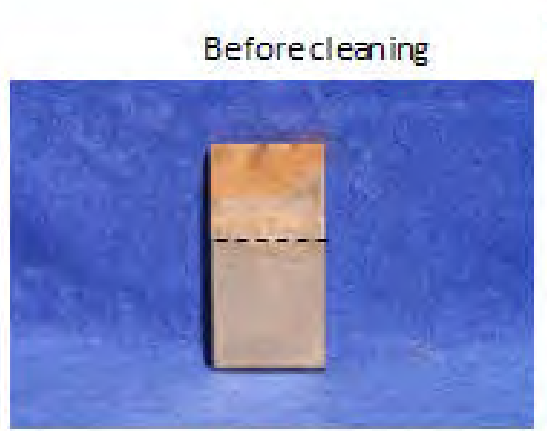

Front side

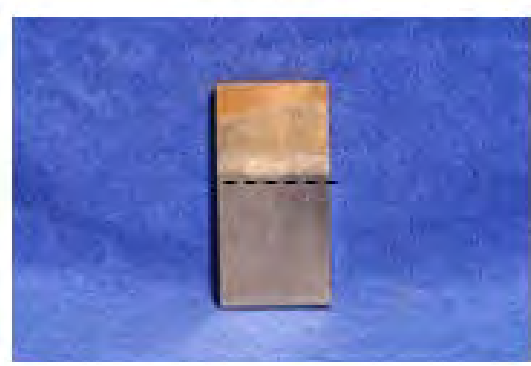

Back side
RC128-8

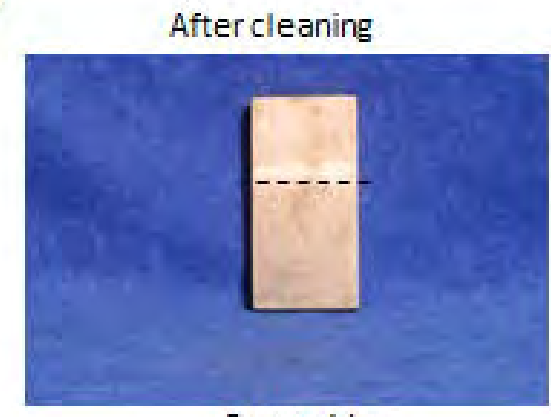

Front side

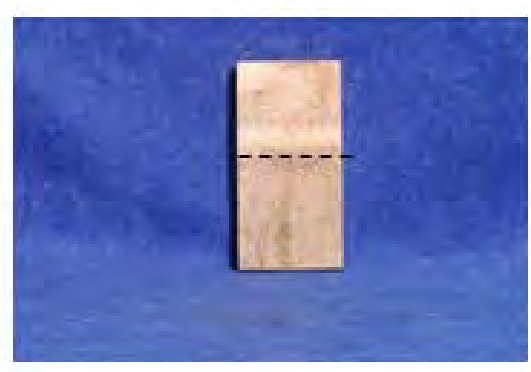

Back side

4 month exposure

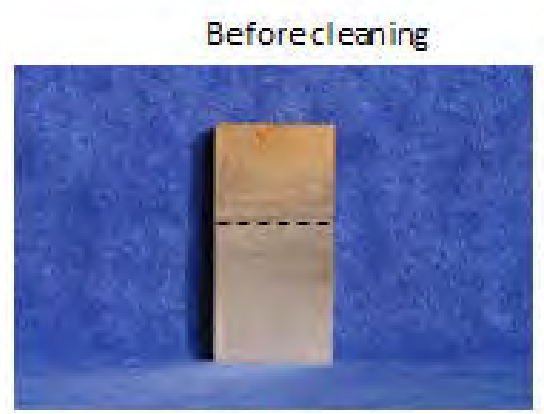

Front side

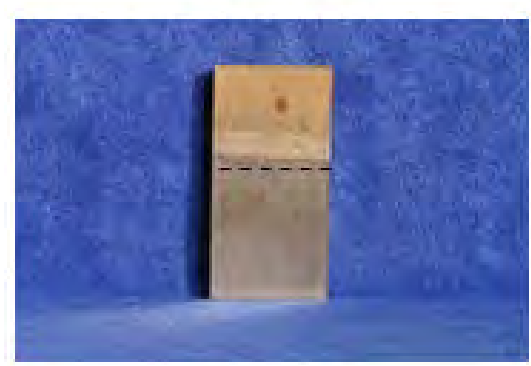

Back side
RC128-7

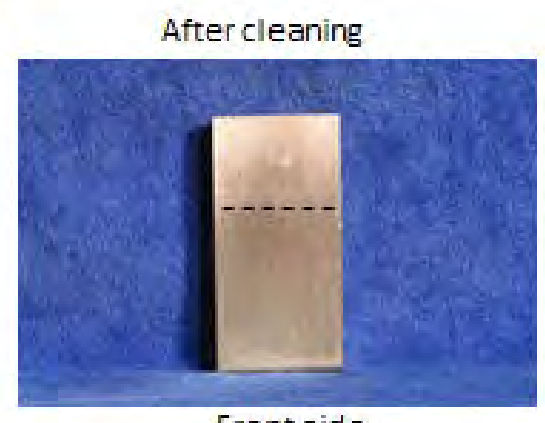

Front side

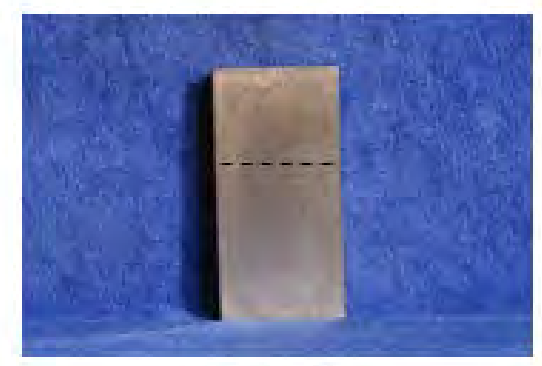

Back side 


\section{Solution 5}

\section{2 month exposure}

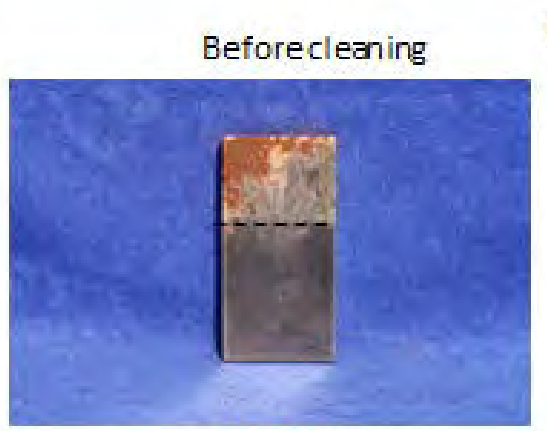

Front side

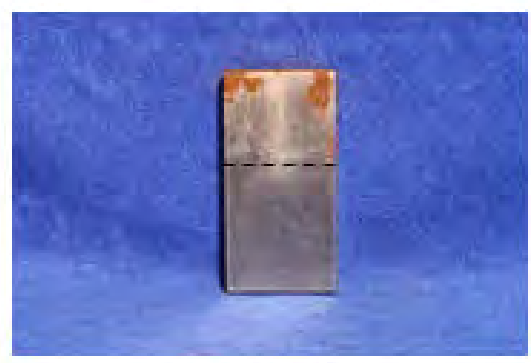

Back side
$\mathrm{RC} 128-10$

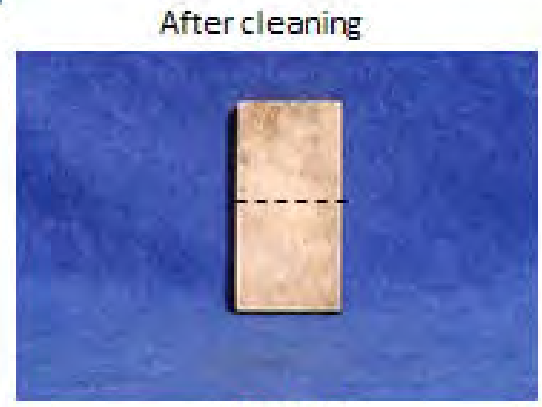

Front side

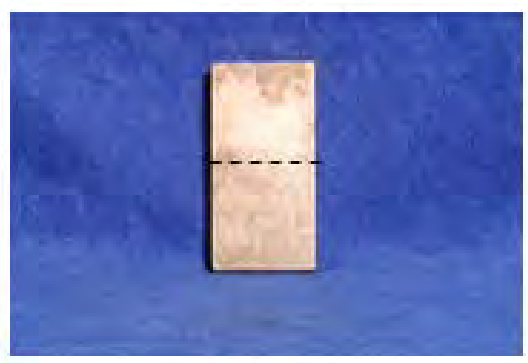

Back side

4 month exposure

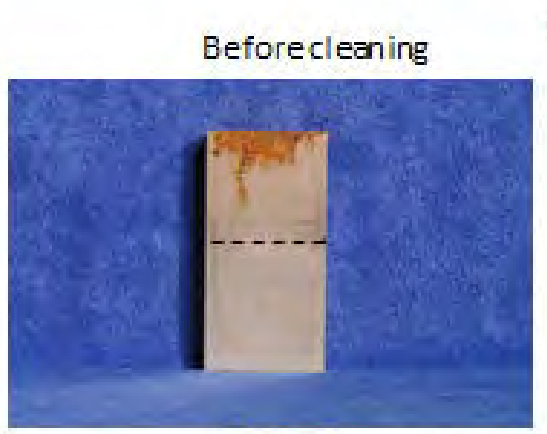

Frontside

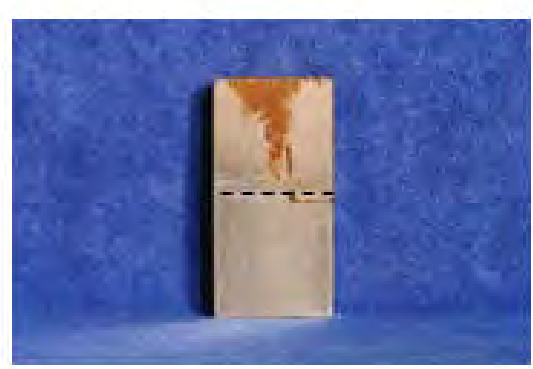

Back side
RC128-9

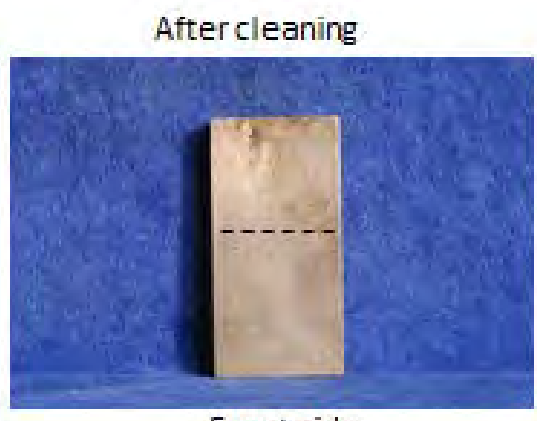

Front side

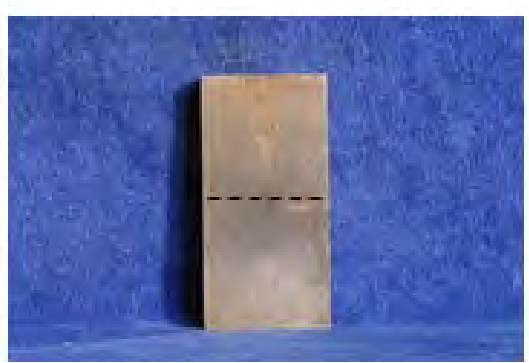

Back side 


\section{Solution 6}

\section{2 month exposure}

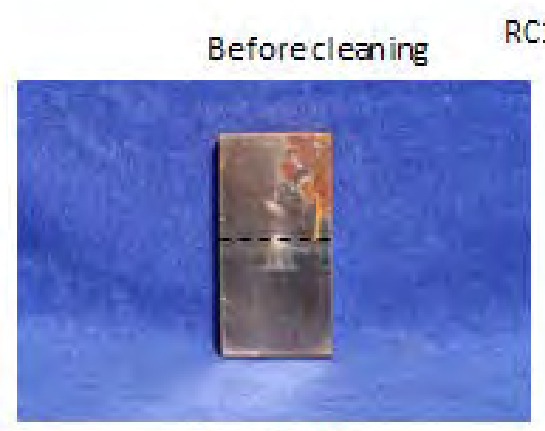

Front side

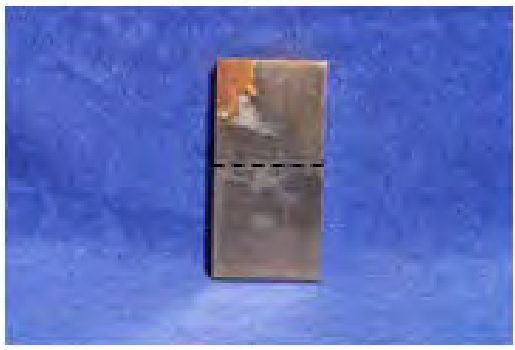

Back side
$\mathrm{RC} 128-12$

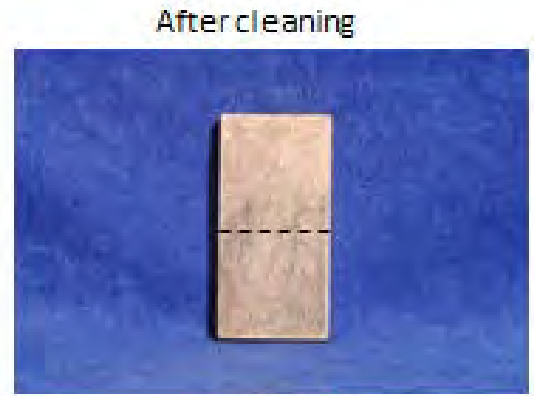

Front side

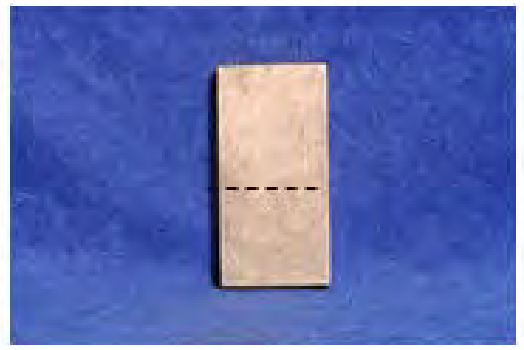

Back side

4 month exposure

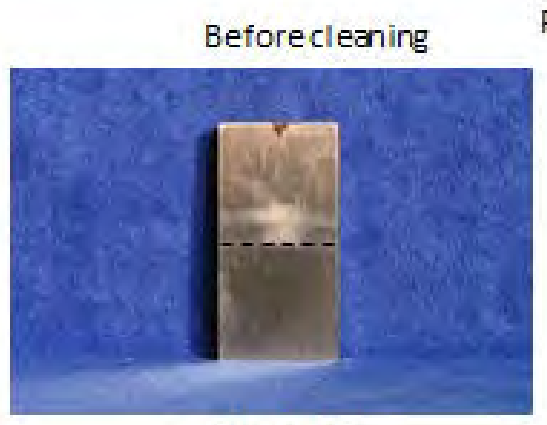

Front side

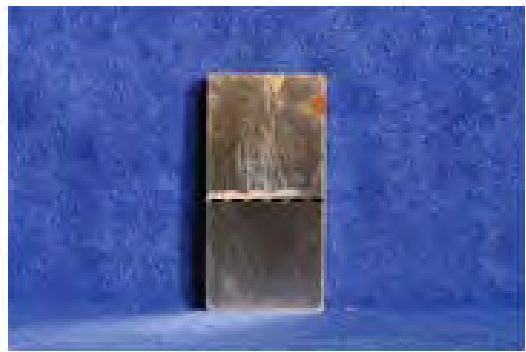

Back side
RC128-11

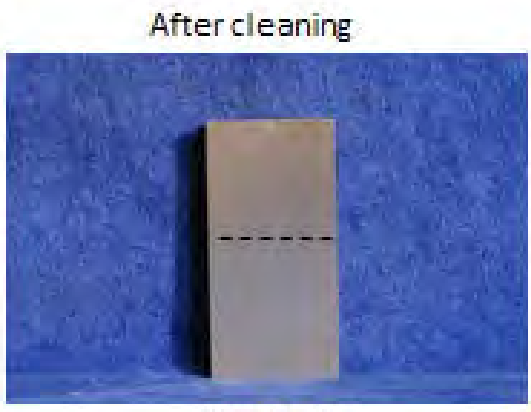

Front side

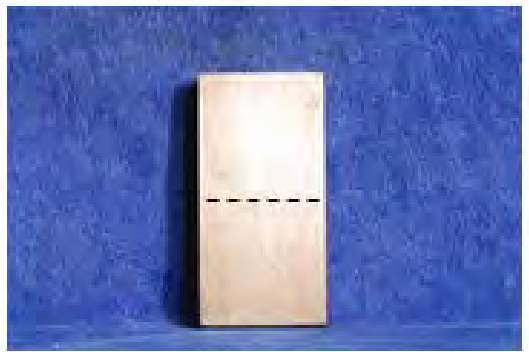

Back side 


\section{Solution 7}

\section{2 month exposure}

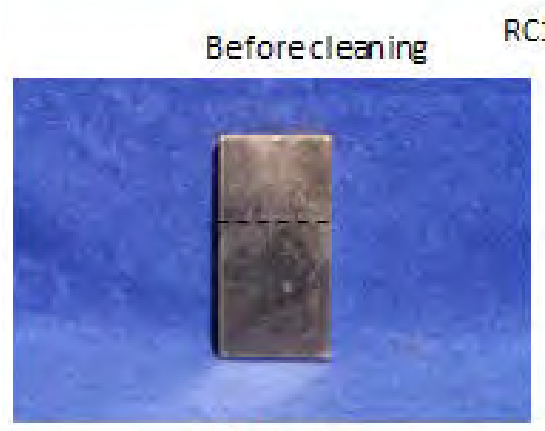

Front side

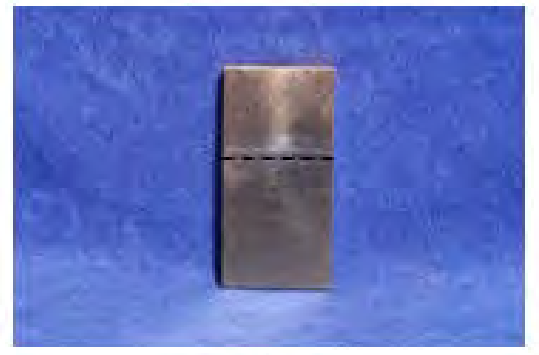

Back side
After cleaning

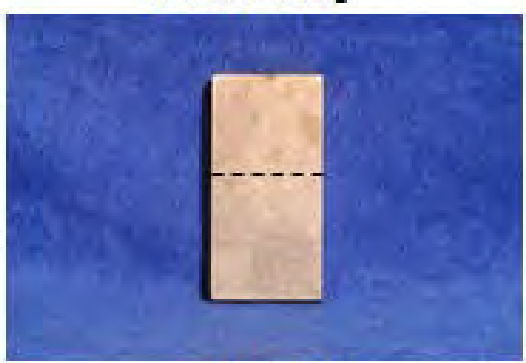

Front side

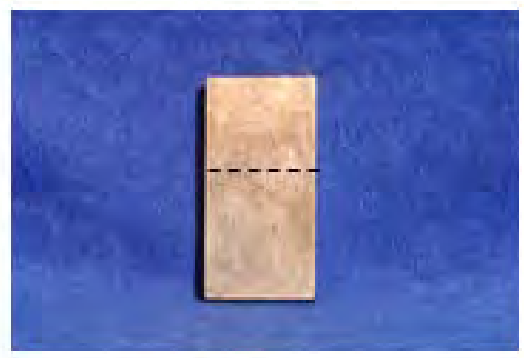

Back side

4 month exposure

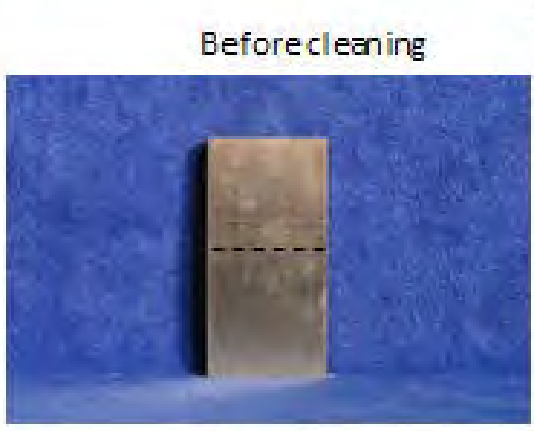

Front side

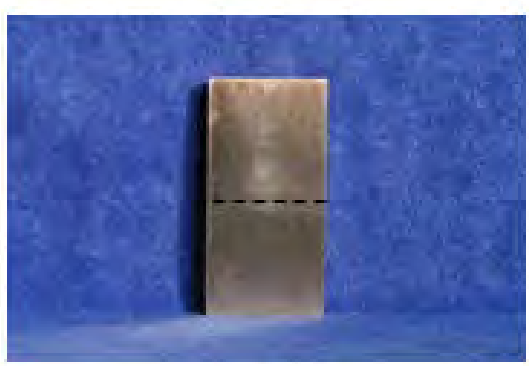

Back side
$\mathrm{RC} 128-13$

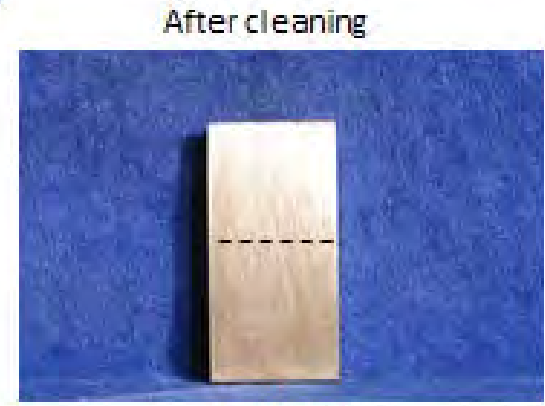

Front side

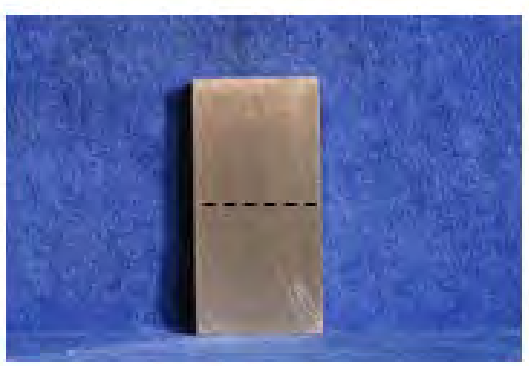

Back side 


\section{Solution 8}

\section{2 month exposure}

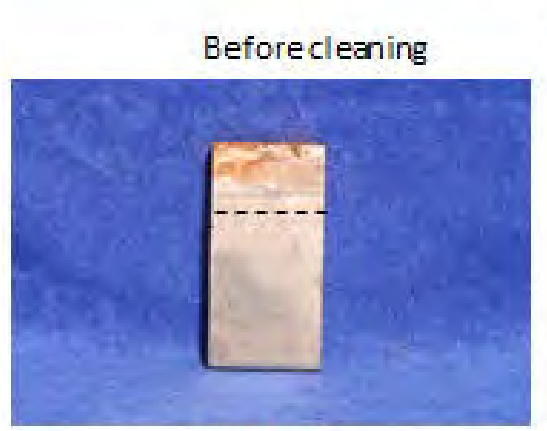

Front side

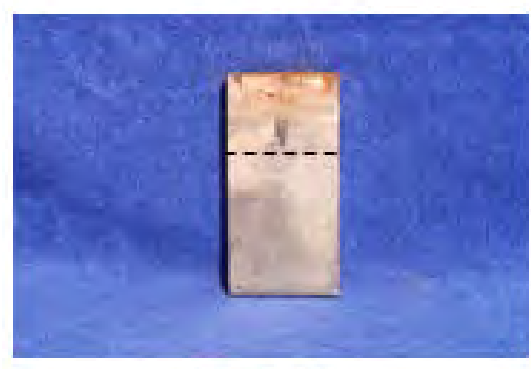

Back side

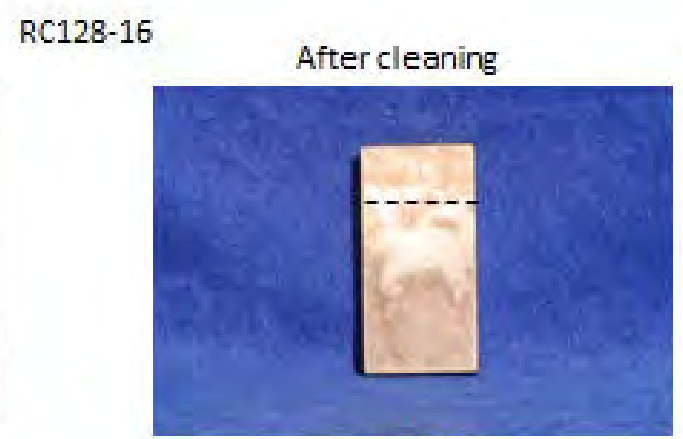

Front side

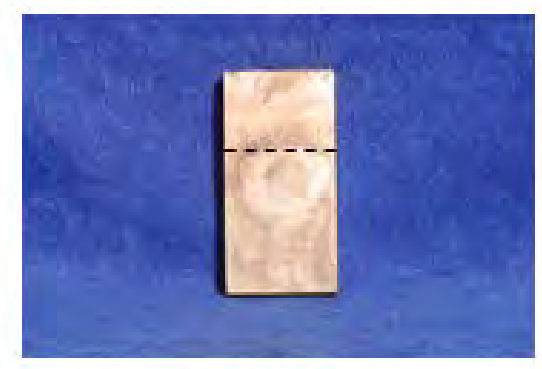

Back side

\section{4 month exposure}

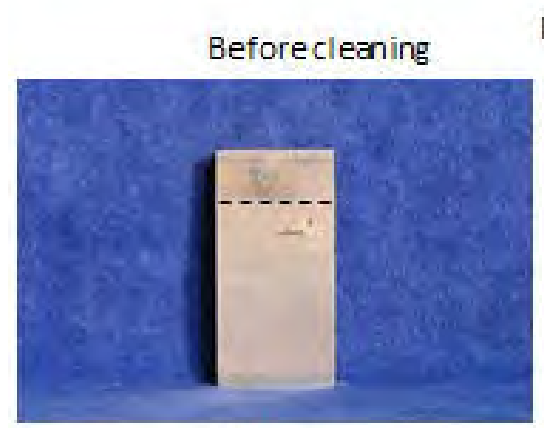

Front side

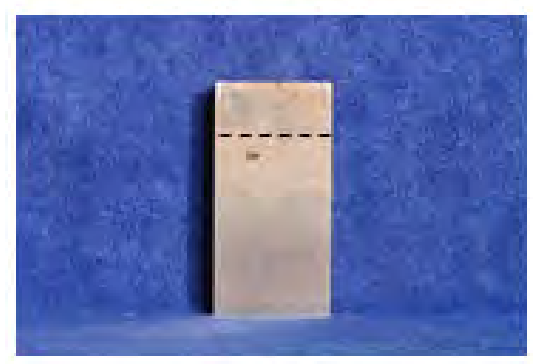

Back side
$\mathrm{RC} 128-15$

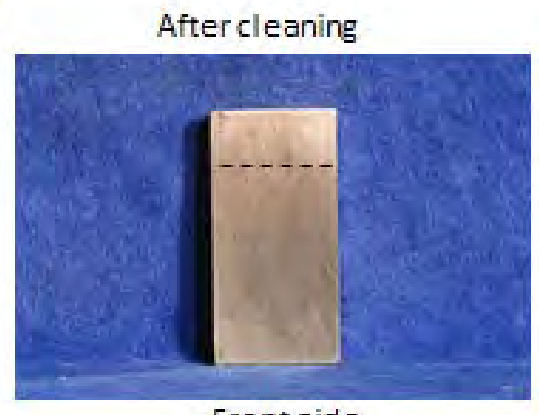

Front side

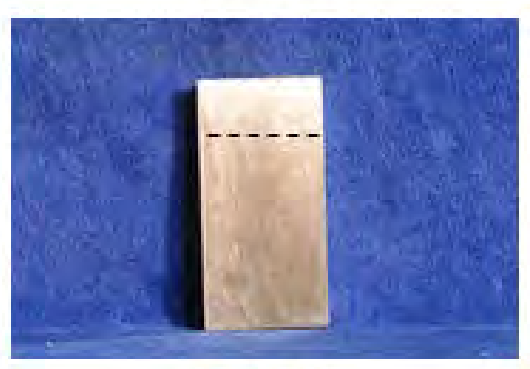

Back side 


\section{Solution 9}

\section{2 month exposure}

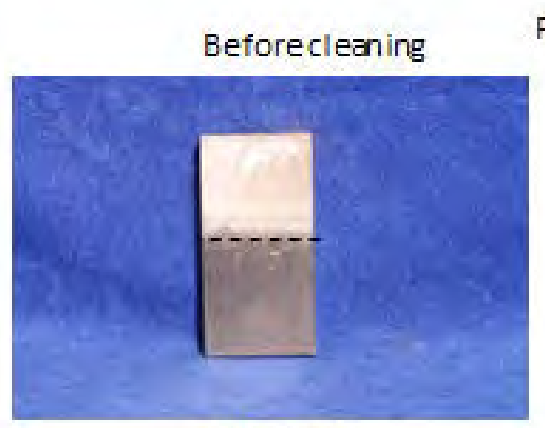

Front side

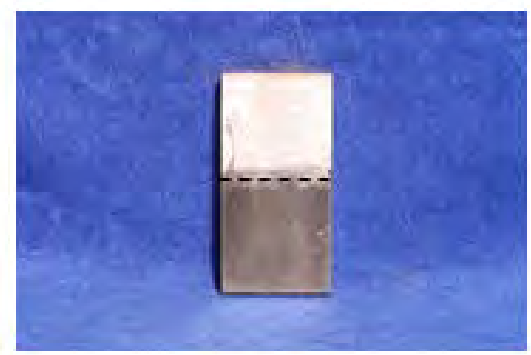

Back side
RC128-18

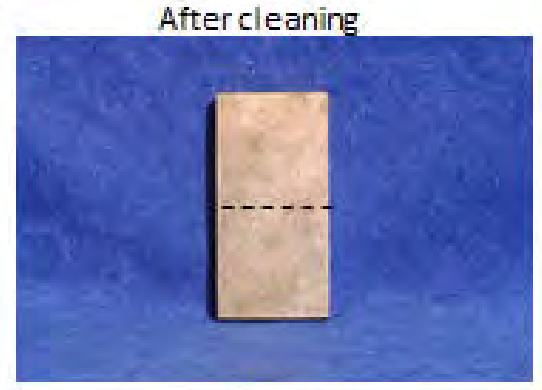

Front side

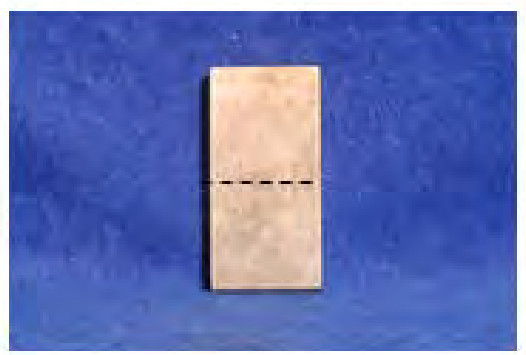

Back side

4 month exposure

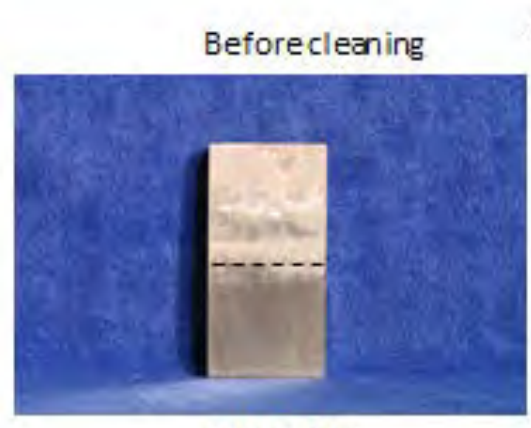

Front side

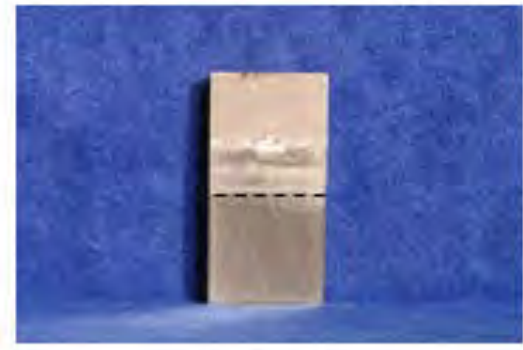

Back side
RC128-17

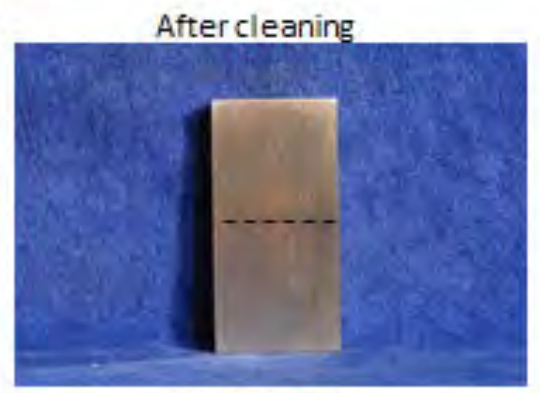

Frontside

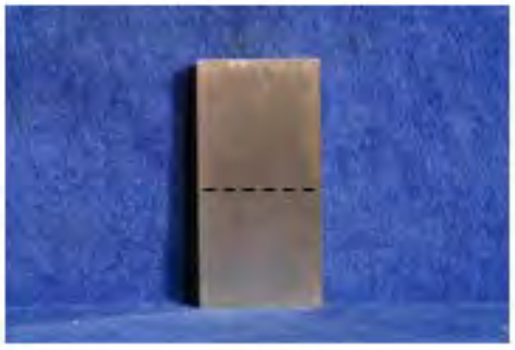

Back side 


\section{Solution 10}

\section{2 month exposure}

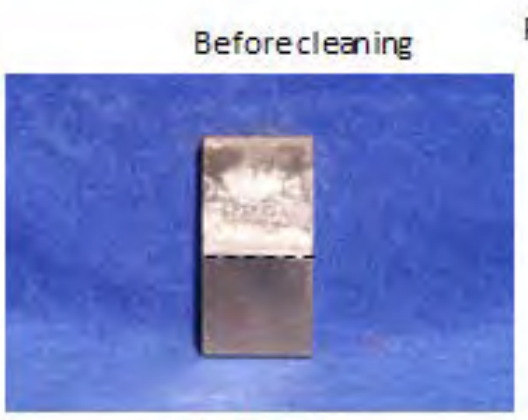

Front side

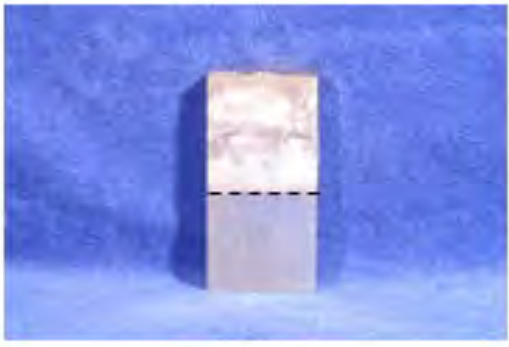

Back side
$\mathrm{RC} 128-20$

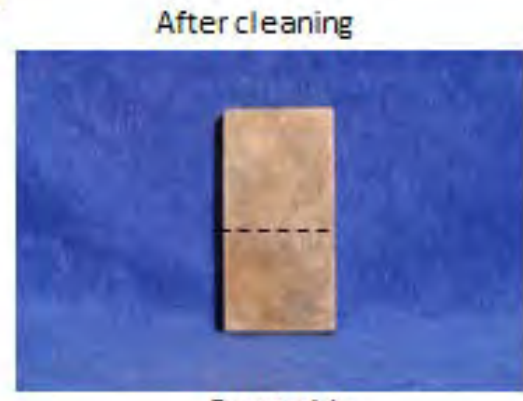

Front side

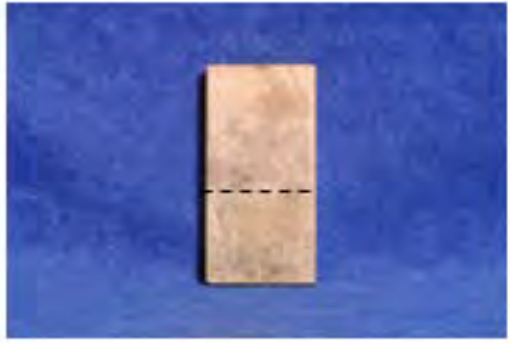

Back side

4 month exposure

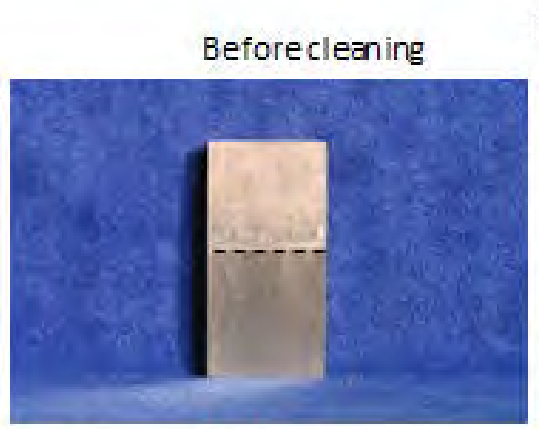

Front side

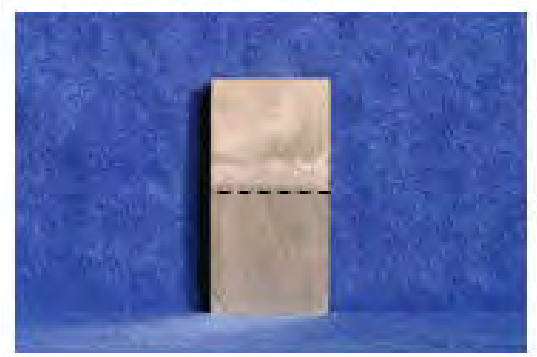

Back side
$\mathrm{RC} 128-19$
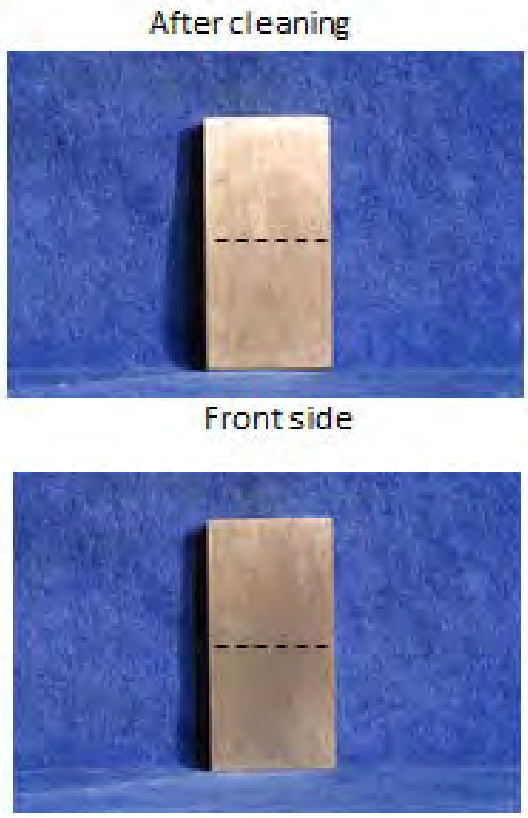

Back side 


\section{Solution 11}

\section{2 month exposure}

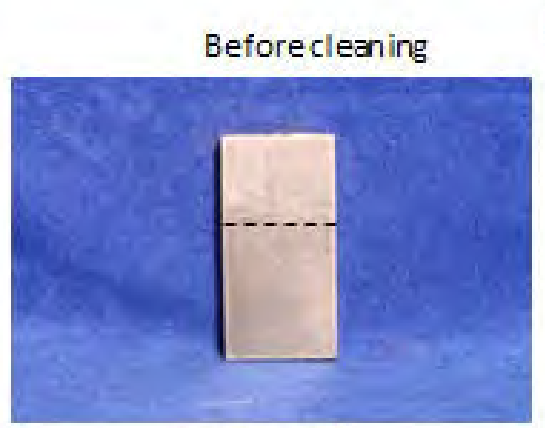

Front side

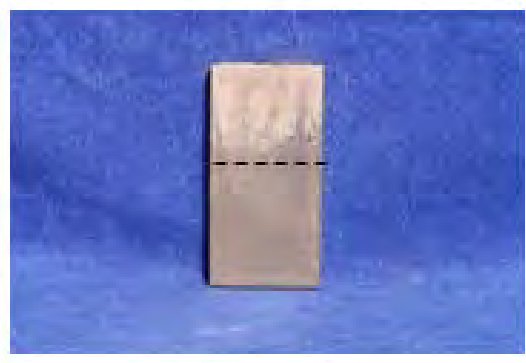

Back side
$\mathrm{RC} 128-22$

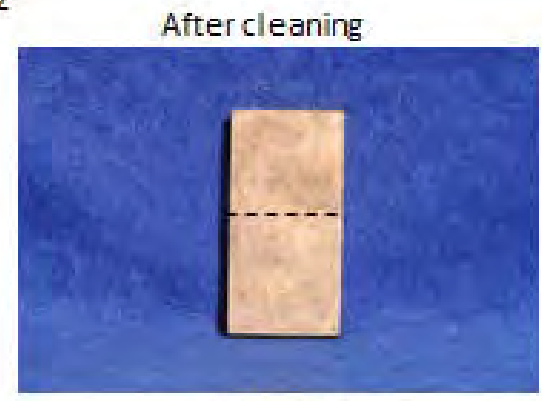

Front side

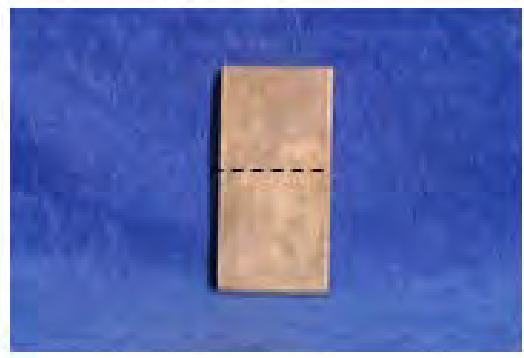

Back side

4 month exposure

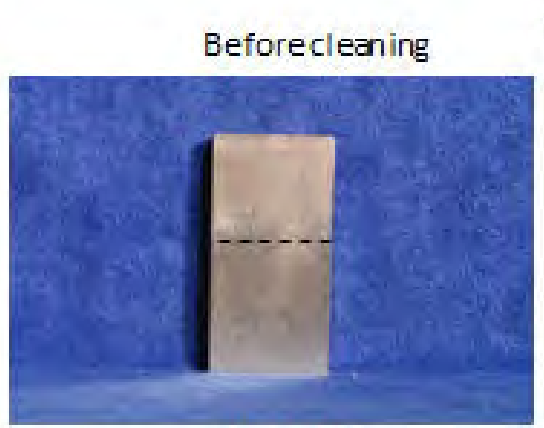

Front side

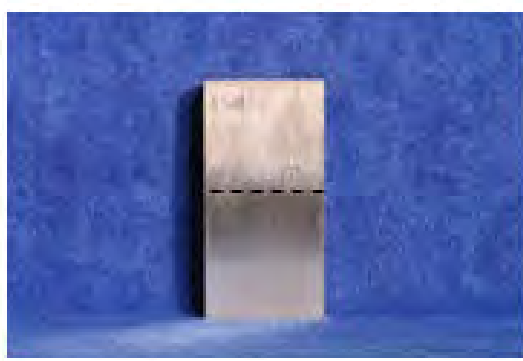

Back side
RC128-21

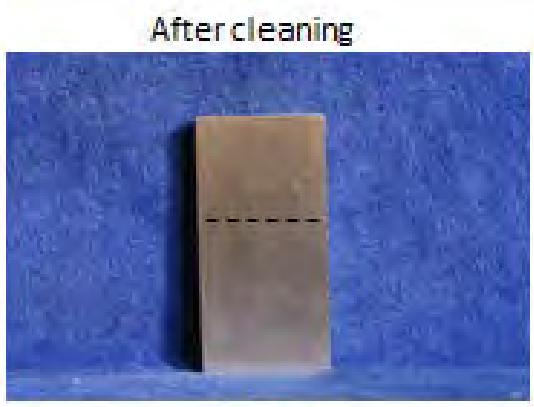

Front side

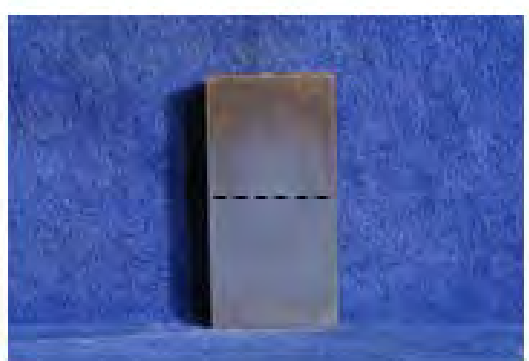

Back side 


\section{Solution 12}

\section{2 month exposure}

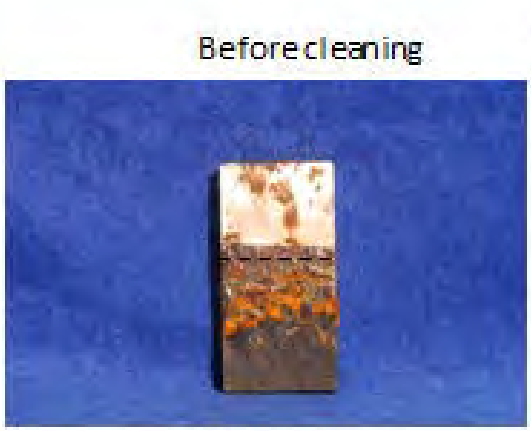

Front side

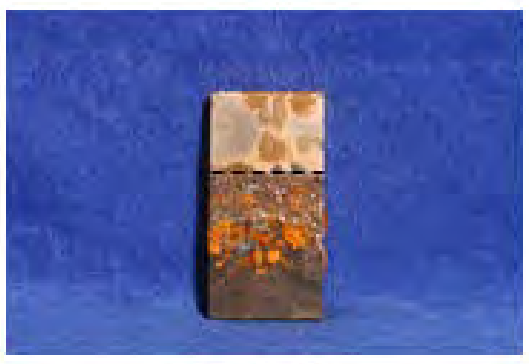

Back side
$\mathrm{RC} 128-24$

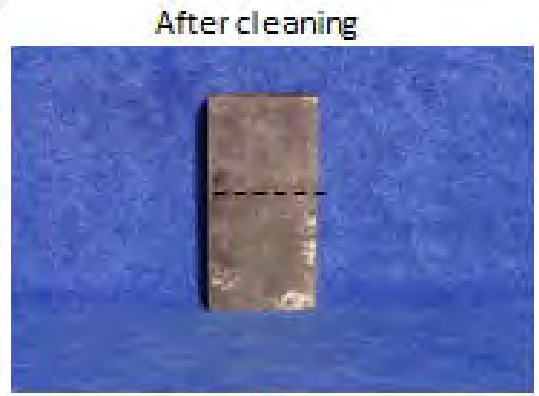

Front side

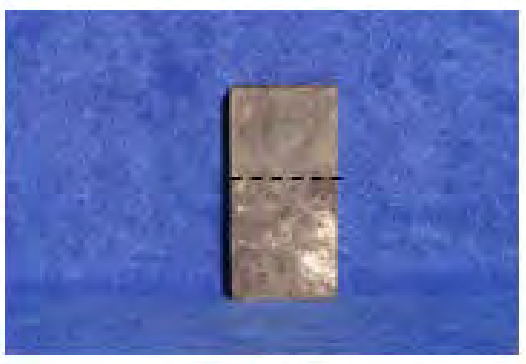

Back side

4 month exposure

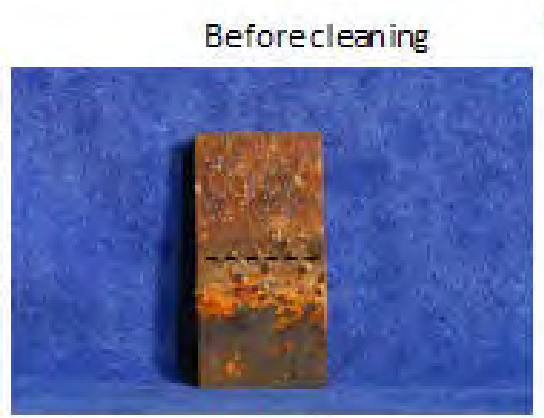

Front side

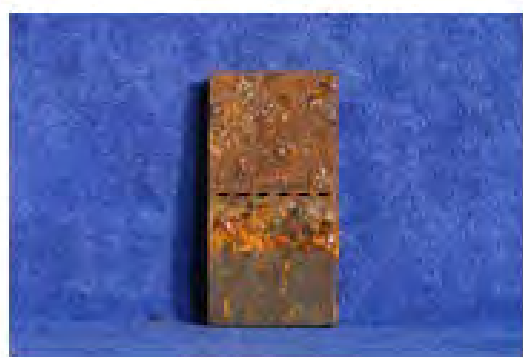

Back side
RC128-23

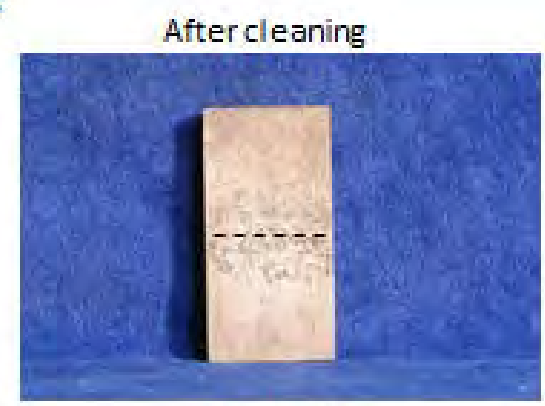

Front side

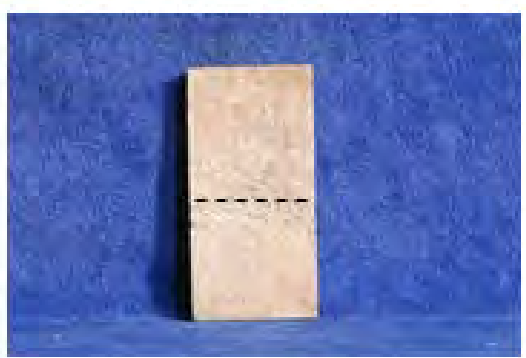

Back side 


\section{Solution 13}

\section{2 month exposure}

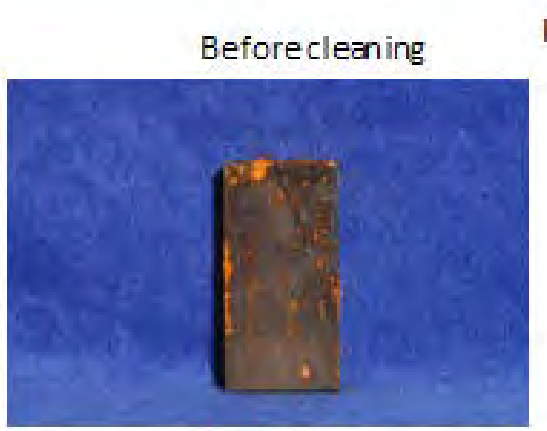

Front side

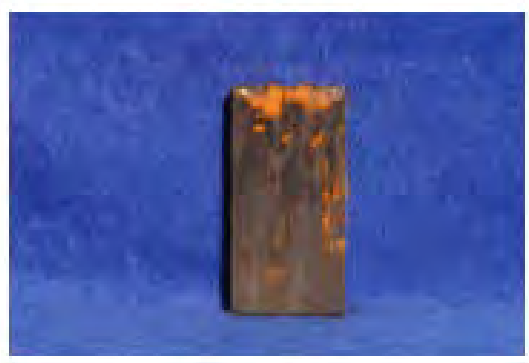

Back side
$\mathrm{RC} 128-26$

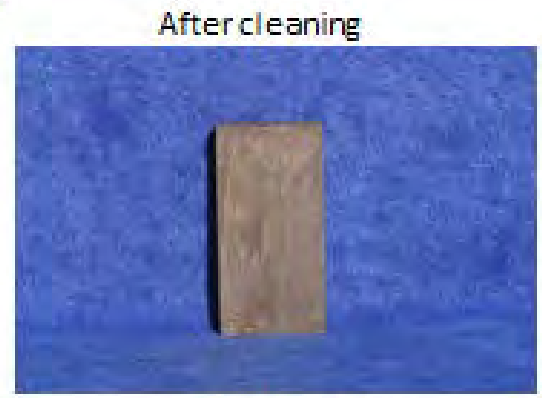

Front side

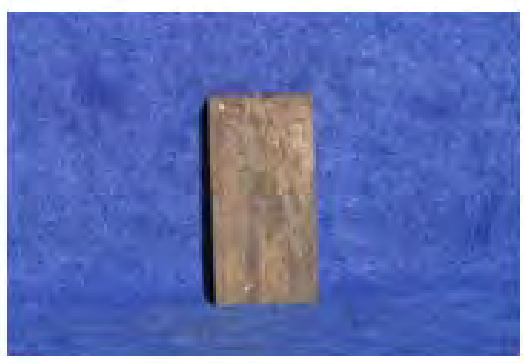

Back side

4 month exposure

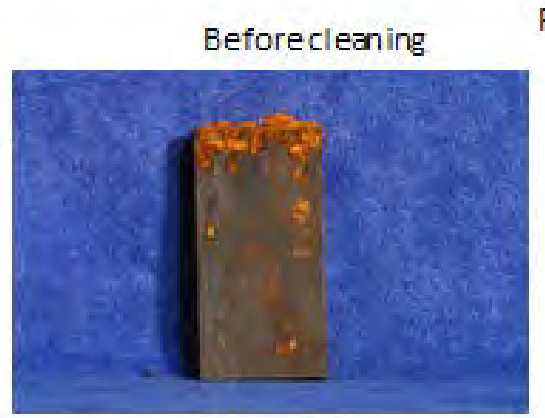

Frontside

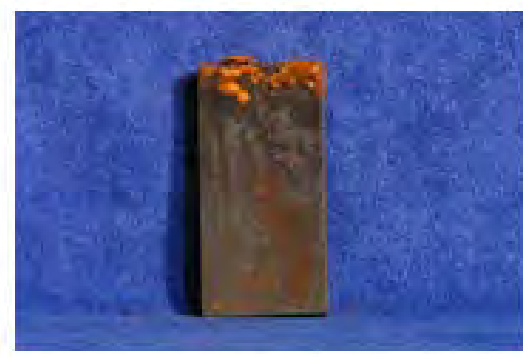

Back side
$\mathrm{RC} 128-25$

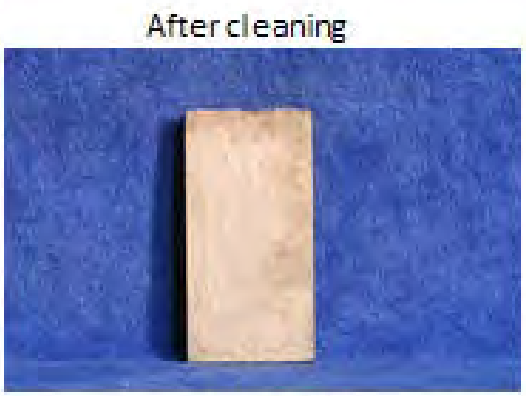

Front side

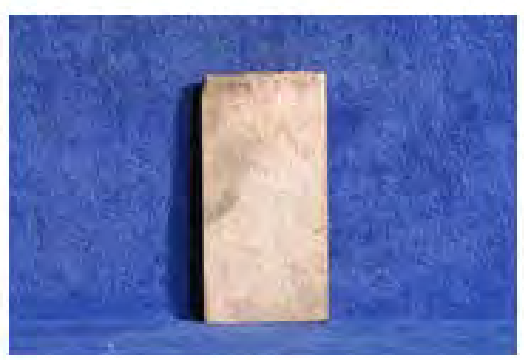

Back side 


\section{Solution 14}

\section{2 month exposure}

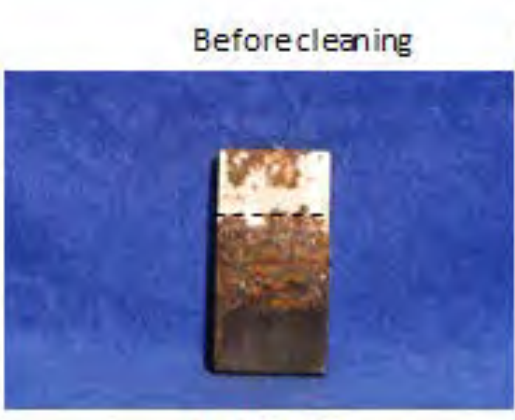

Front side

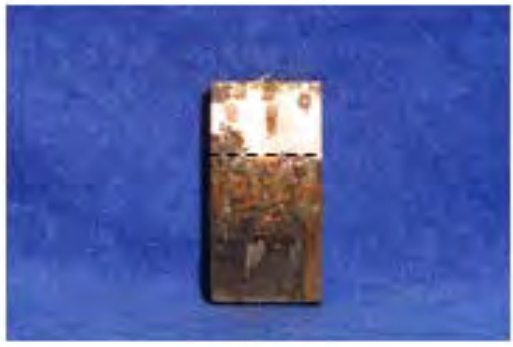

Back side
$\mathrm{RC} 128-28$

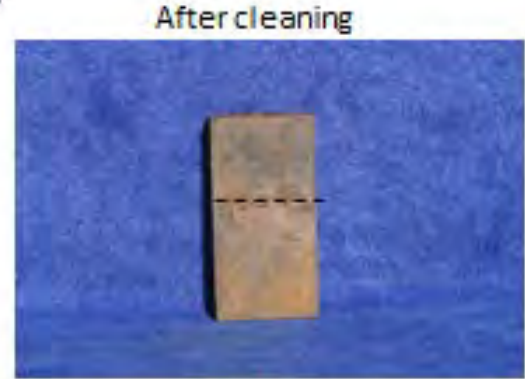

Front side

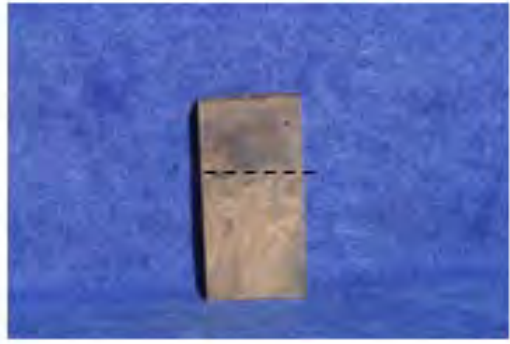

Back side

4 month exposure

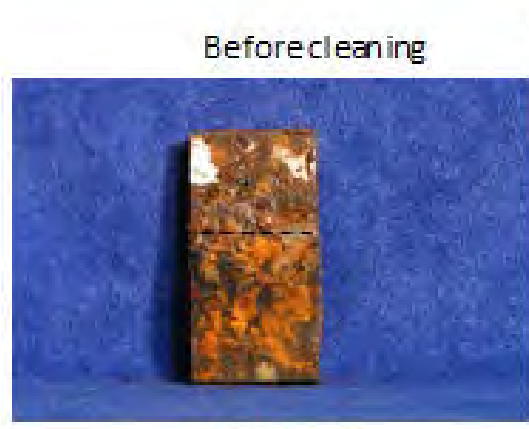

Front side

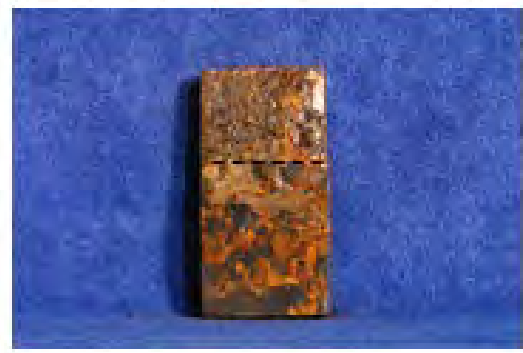

Back side
$\mathrm{RC} 128-27$

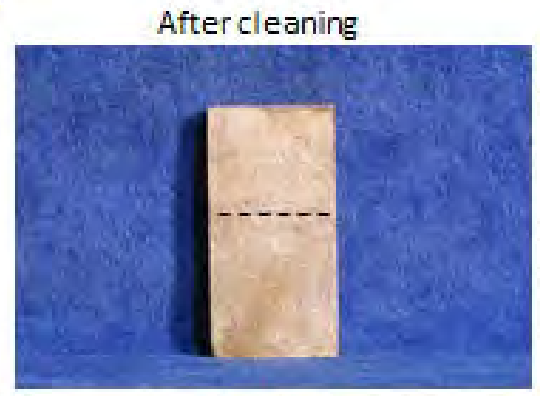

Front side

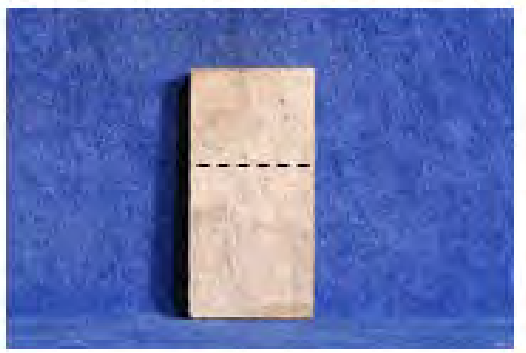

Back side 


\section{Solution 15}

\section{2 month exposure}

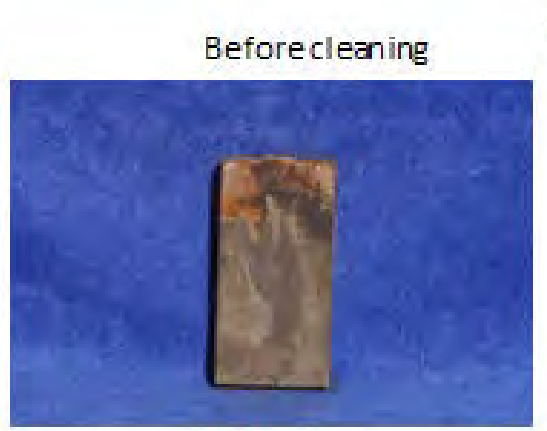

Front side

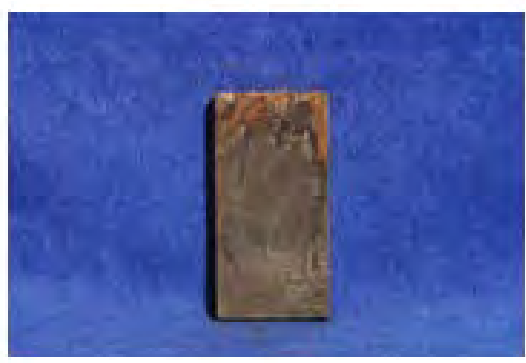

Back side
$\mathrm{RC} 128-30$

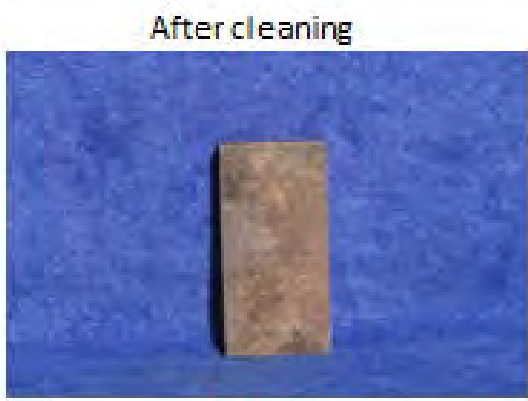

Front side

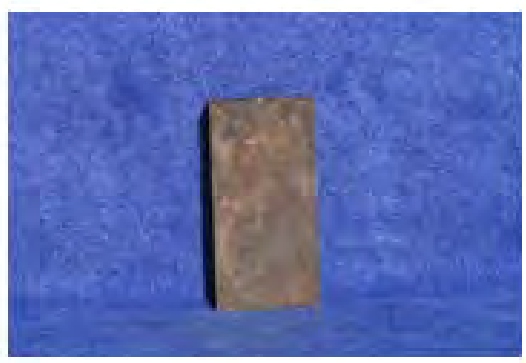

Back side

4 month exposure

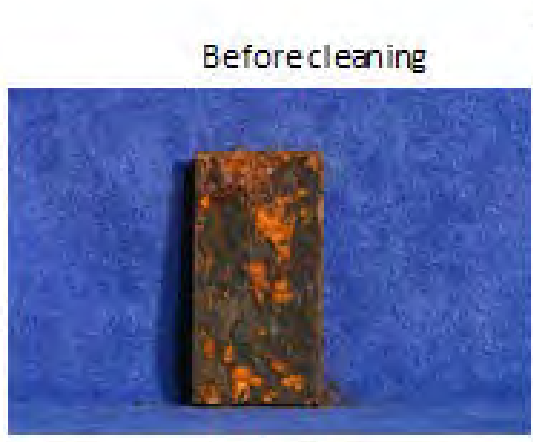

Front side

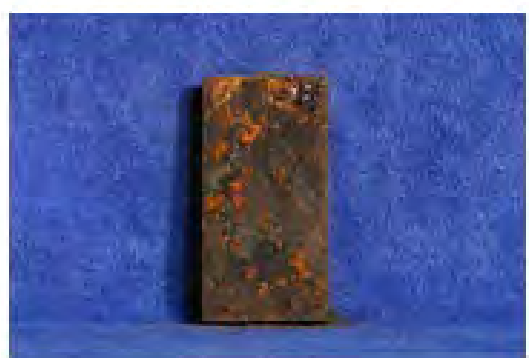

Back side
RC128-29

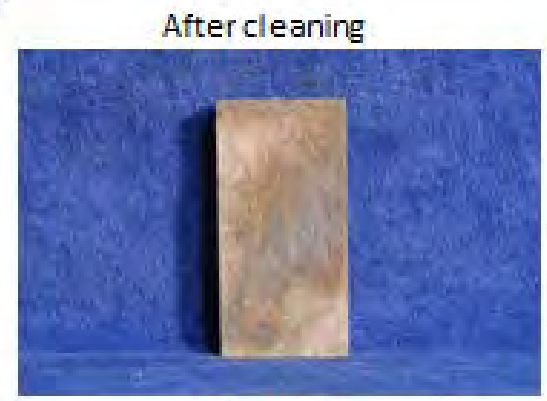

Front side

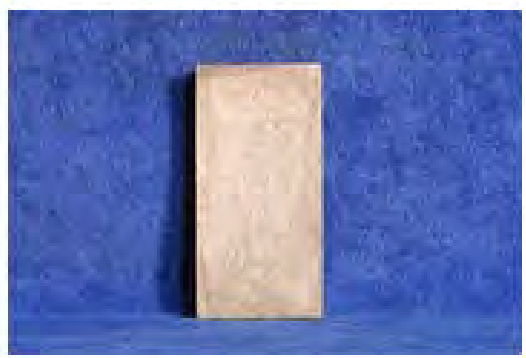

Back side 
Appendix E

Open Circuit Potential, pH and Temperature vs. Time plots for Liquid Air Interface Solutions 


\section{Solution 1 to 8}

2 months samples

Open circuit potential vs. Time

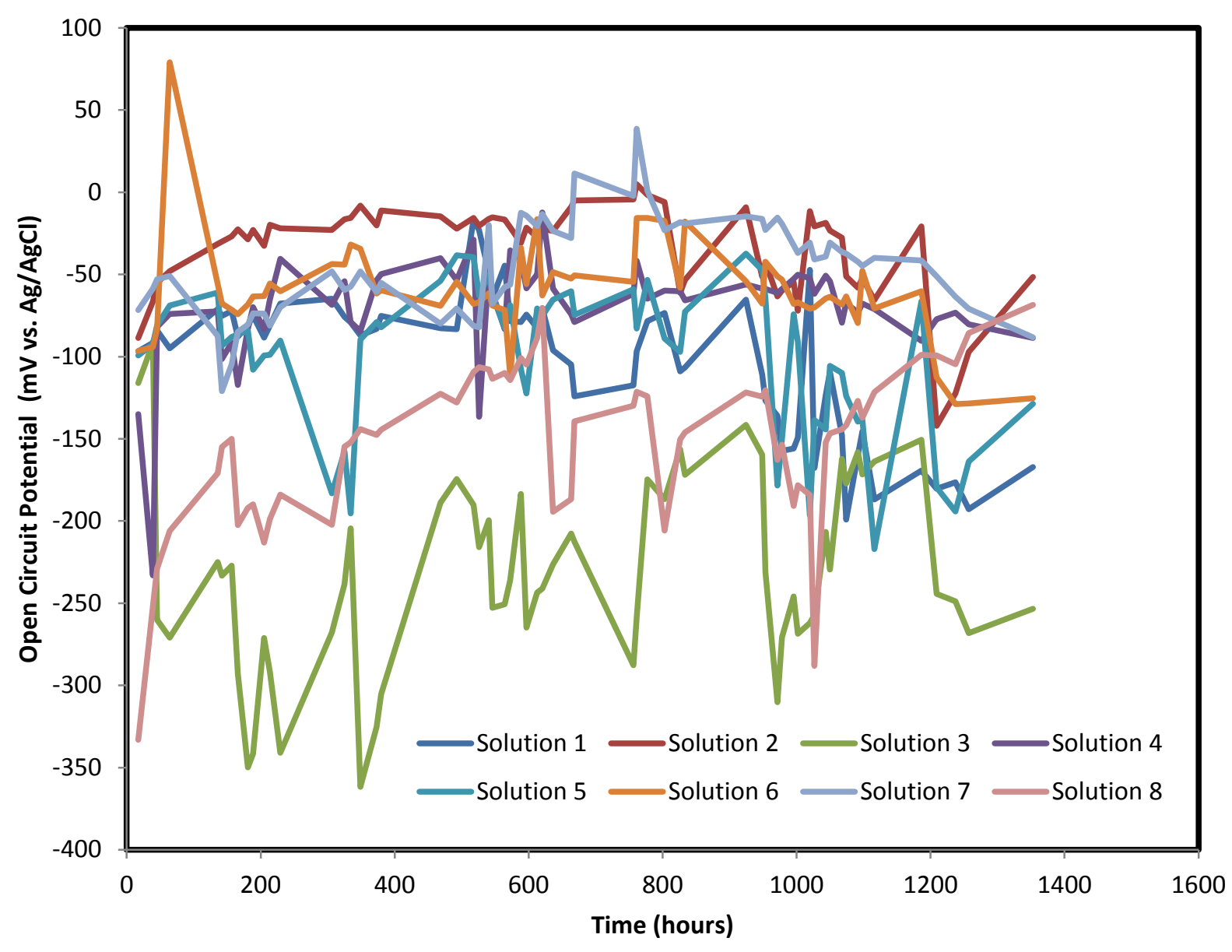


Solution 1 to 8

4 month samples

Open circuit potential vs. Time

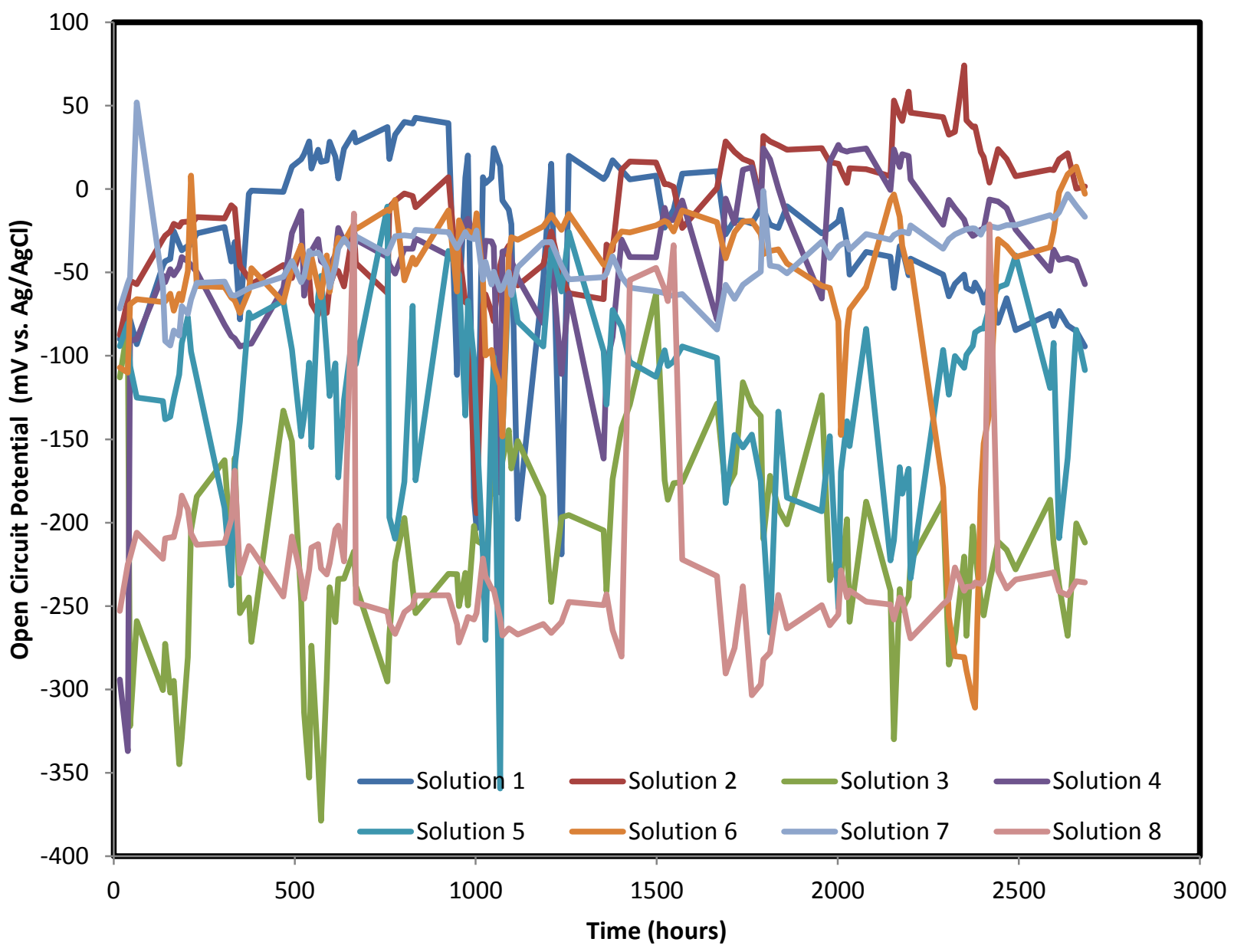




\section{Solutions 1 to 8}

pH and Temperature

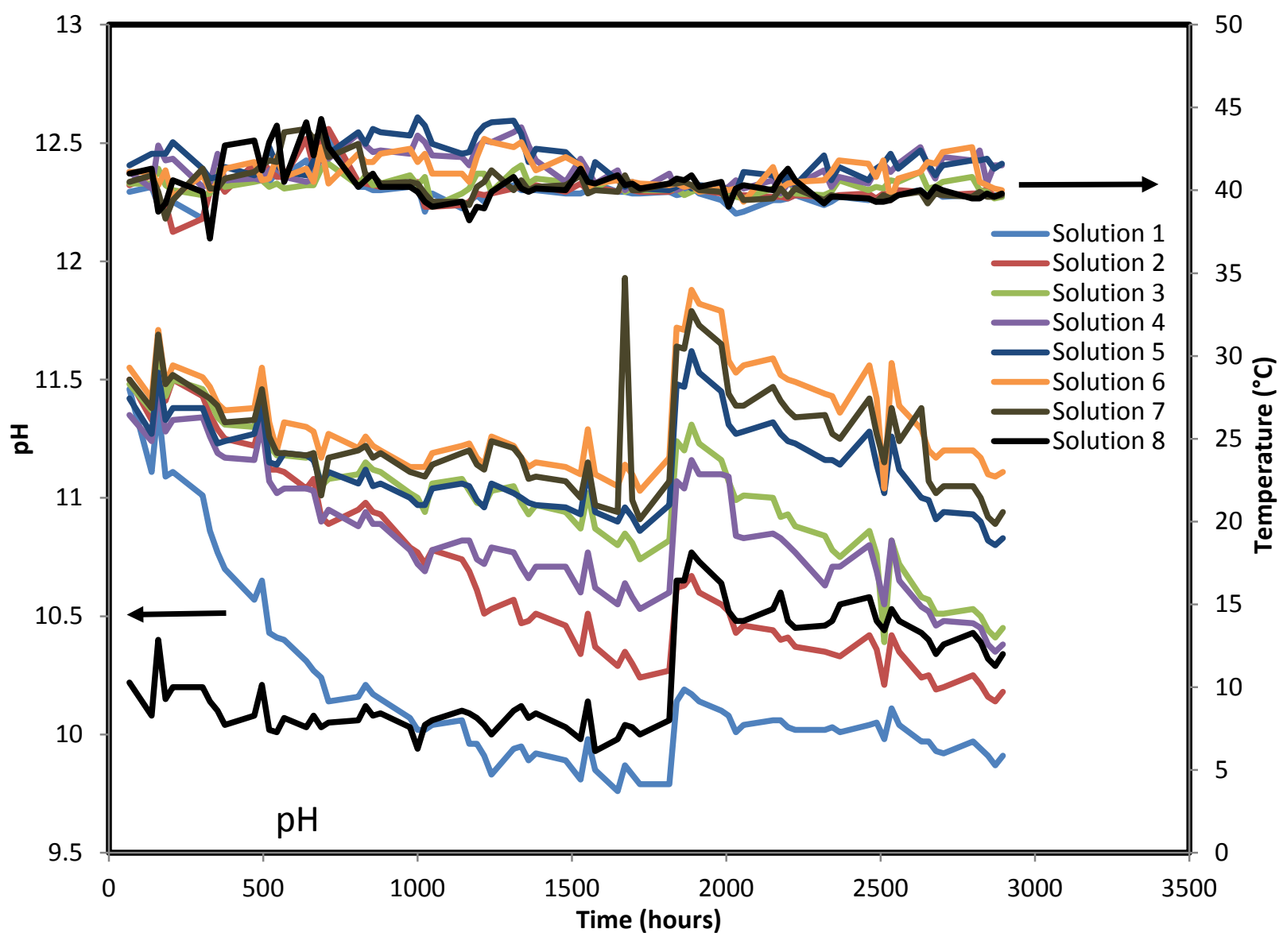




\section{Solution 9 to 11}

2 months samples

Open circuit potential vs. Time

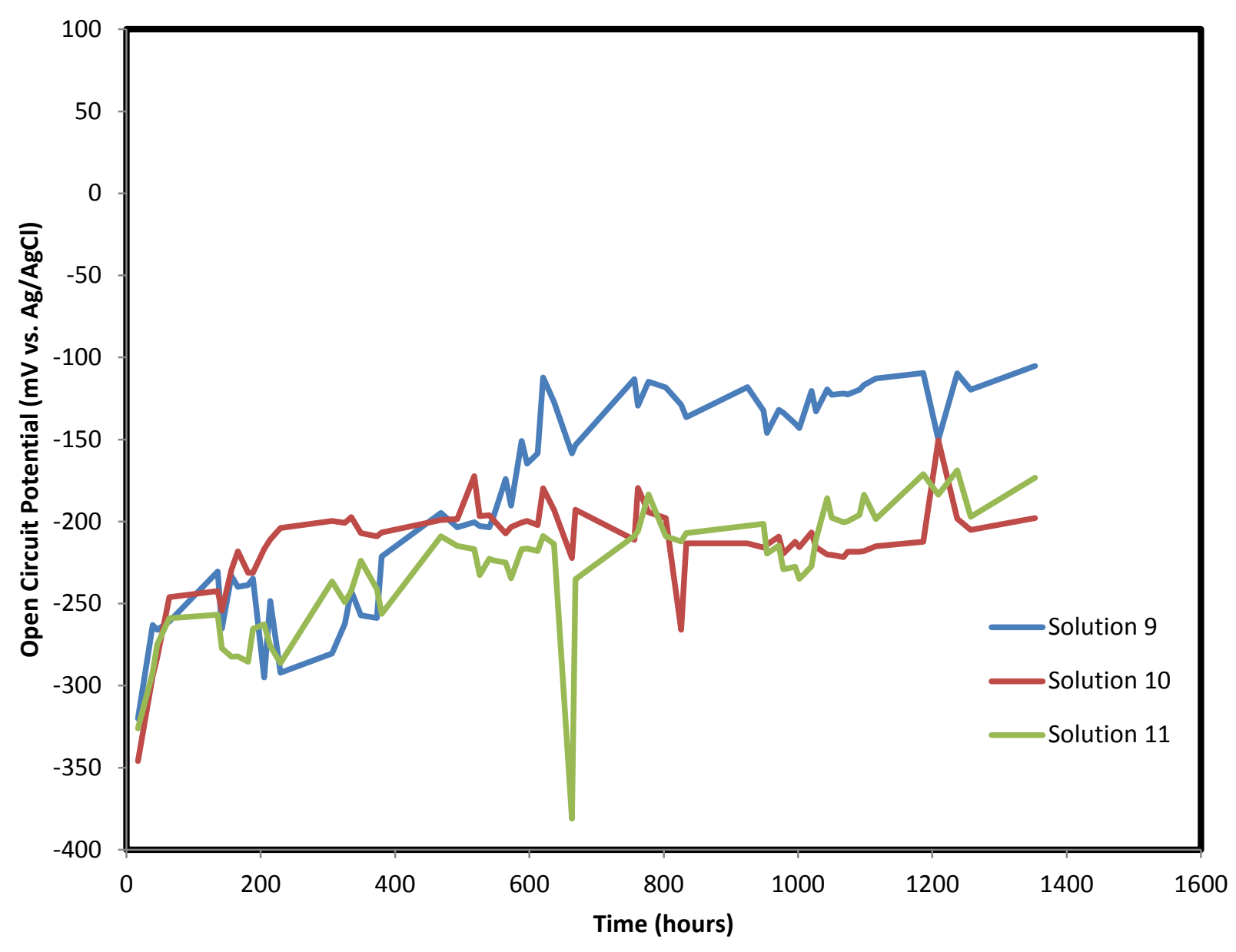




\section{Solution 9 to 11}

\section{4 month samples}

Open circuit potential vs. Time

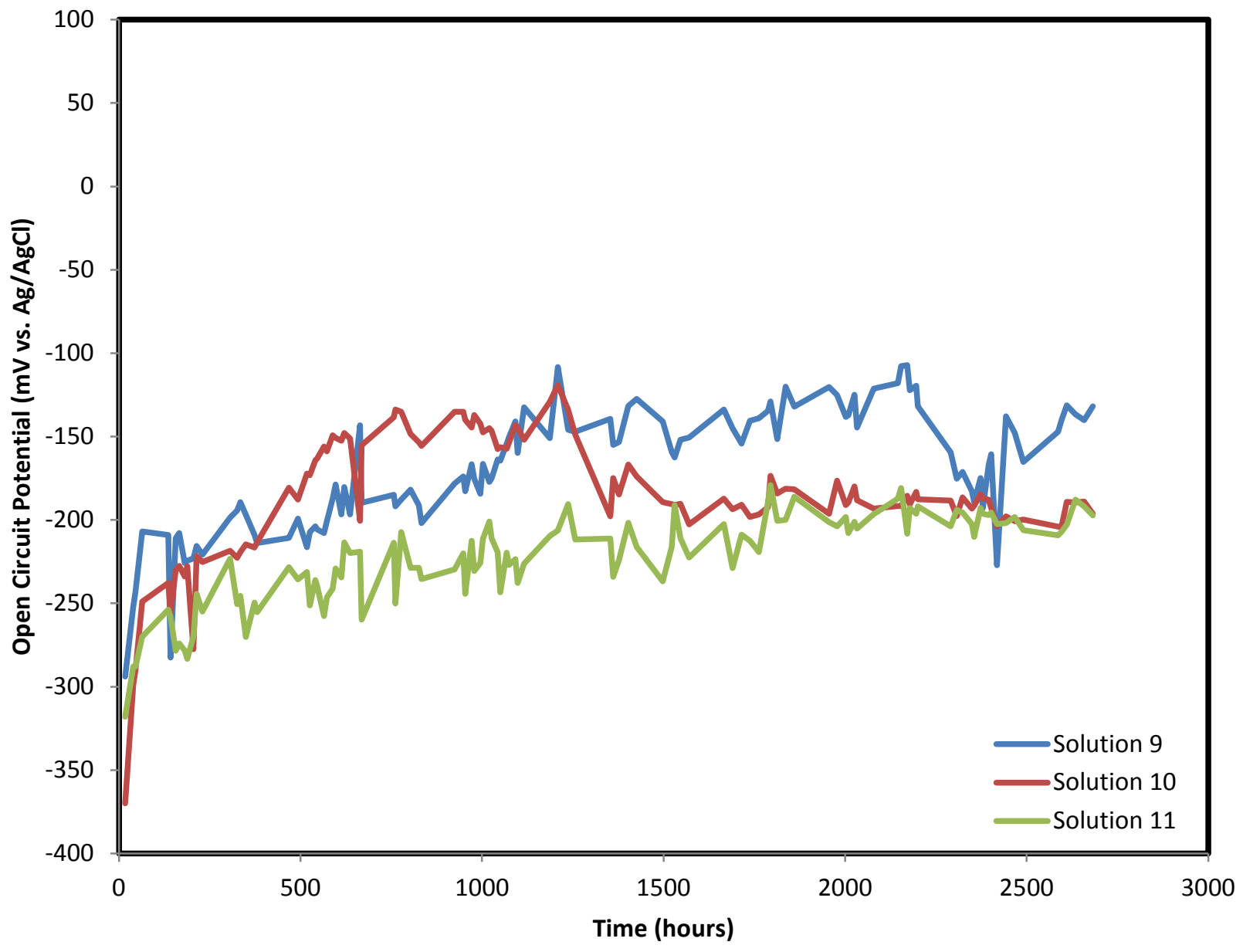




\section{Solution 9 to 11}

$\mathrm{pH}$ and Temperature

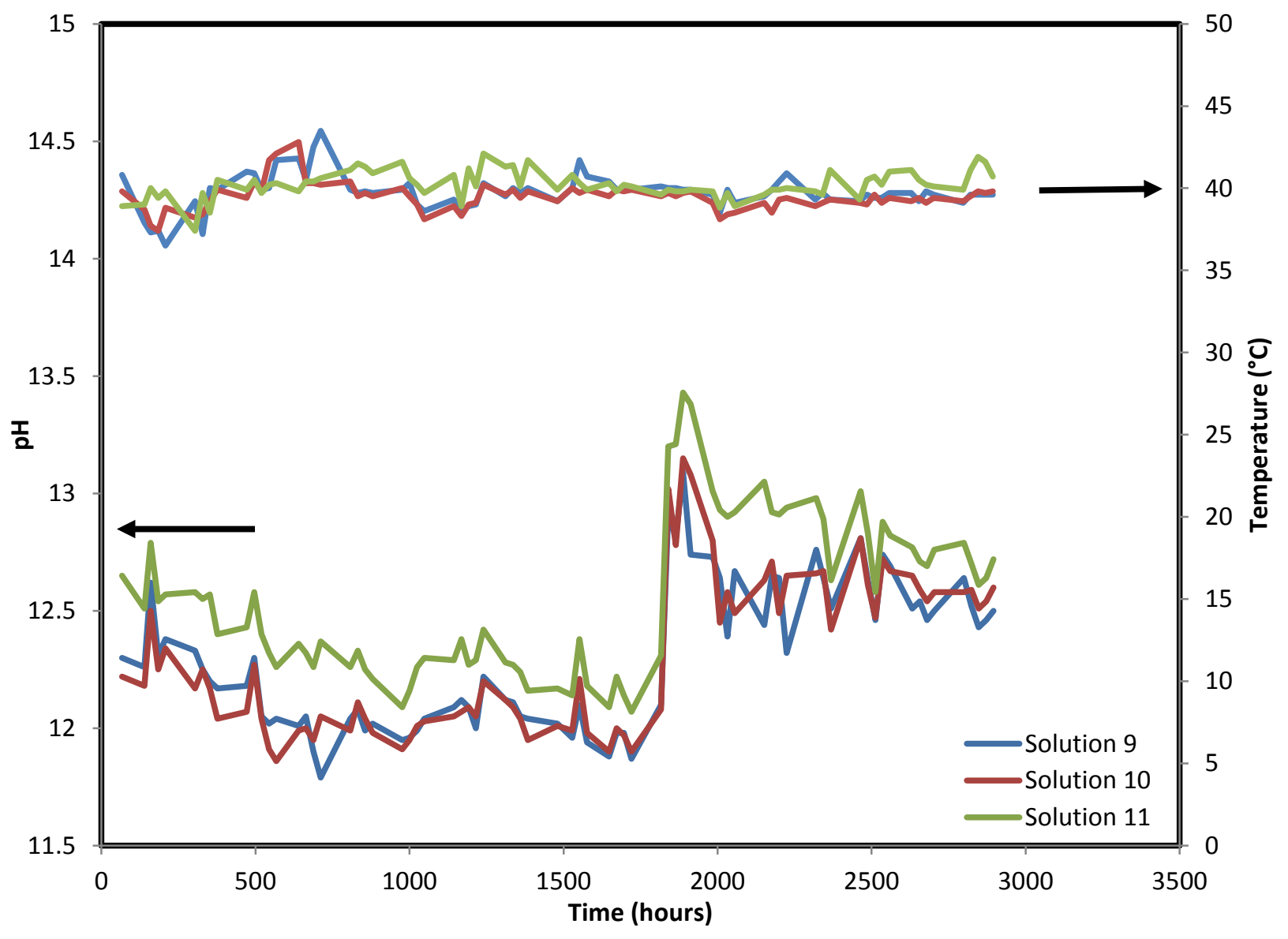




\section{Solution 12 to 15}

2 months samples

Open circuit potential vs. Time

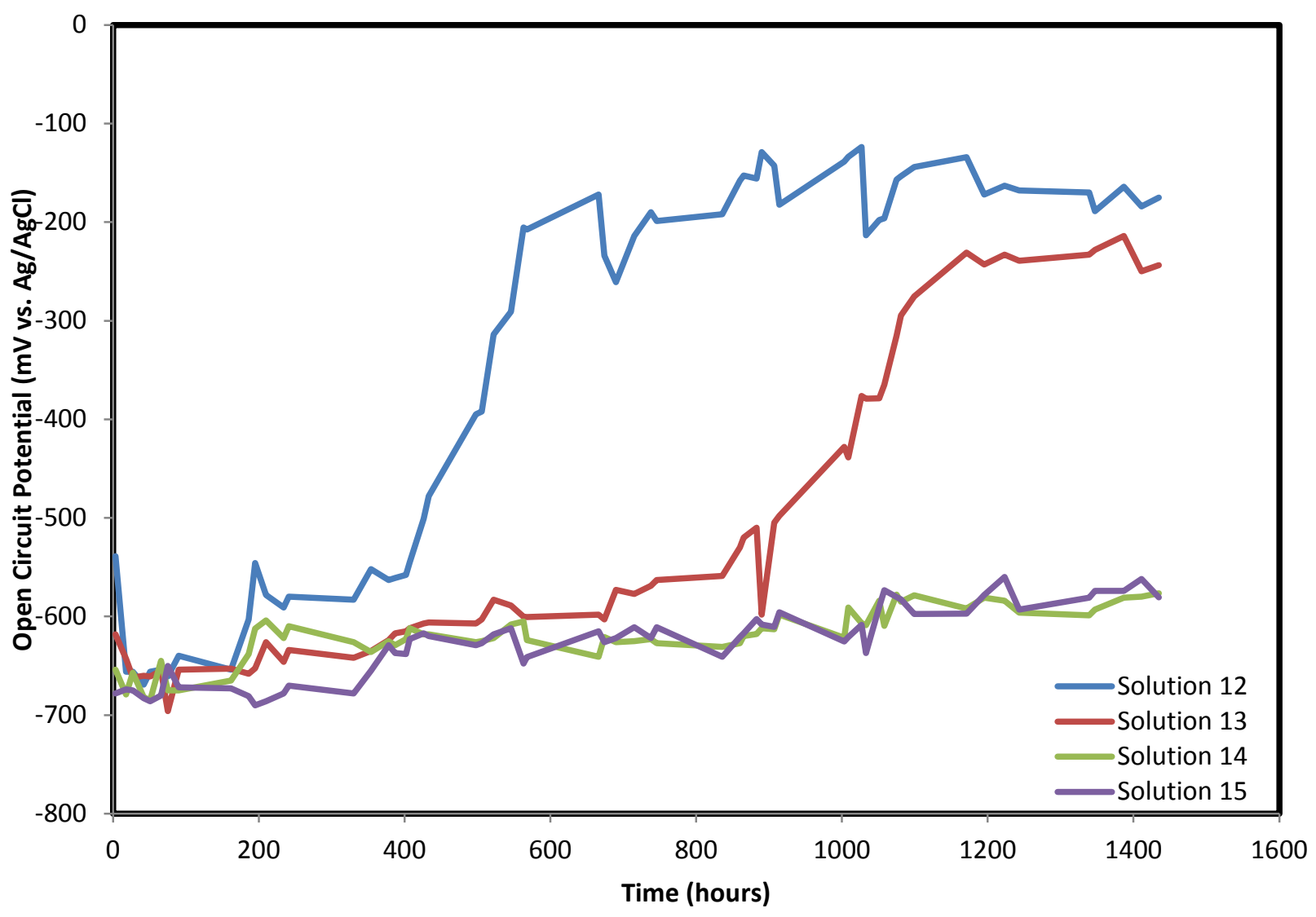




\section{Solution 12 to 15}

\section{4 month samples}

Open circuit potential vs. Time

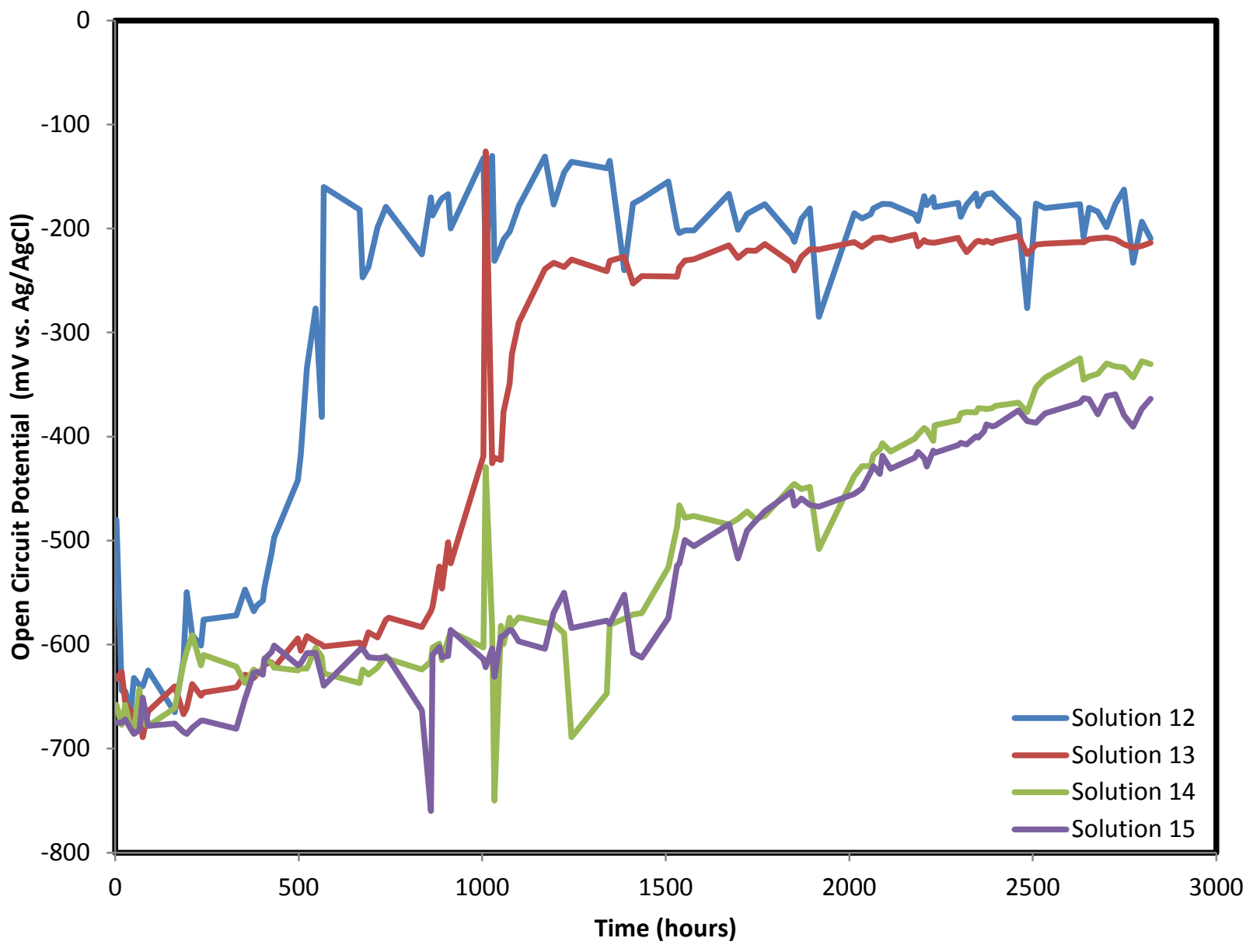


Solution 12 to 15

$\mathrm{pH}$ and Temperature

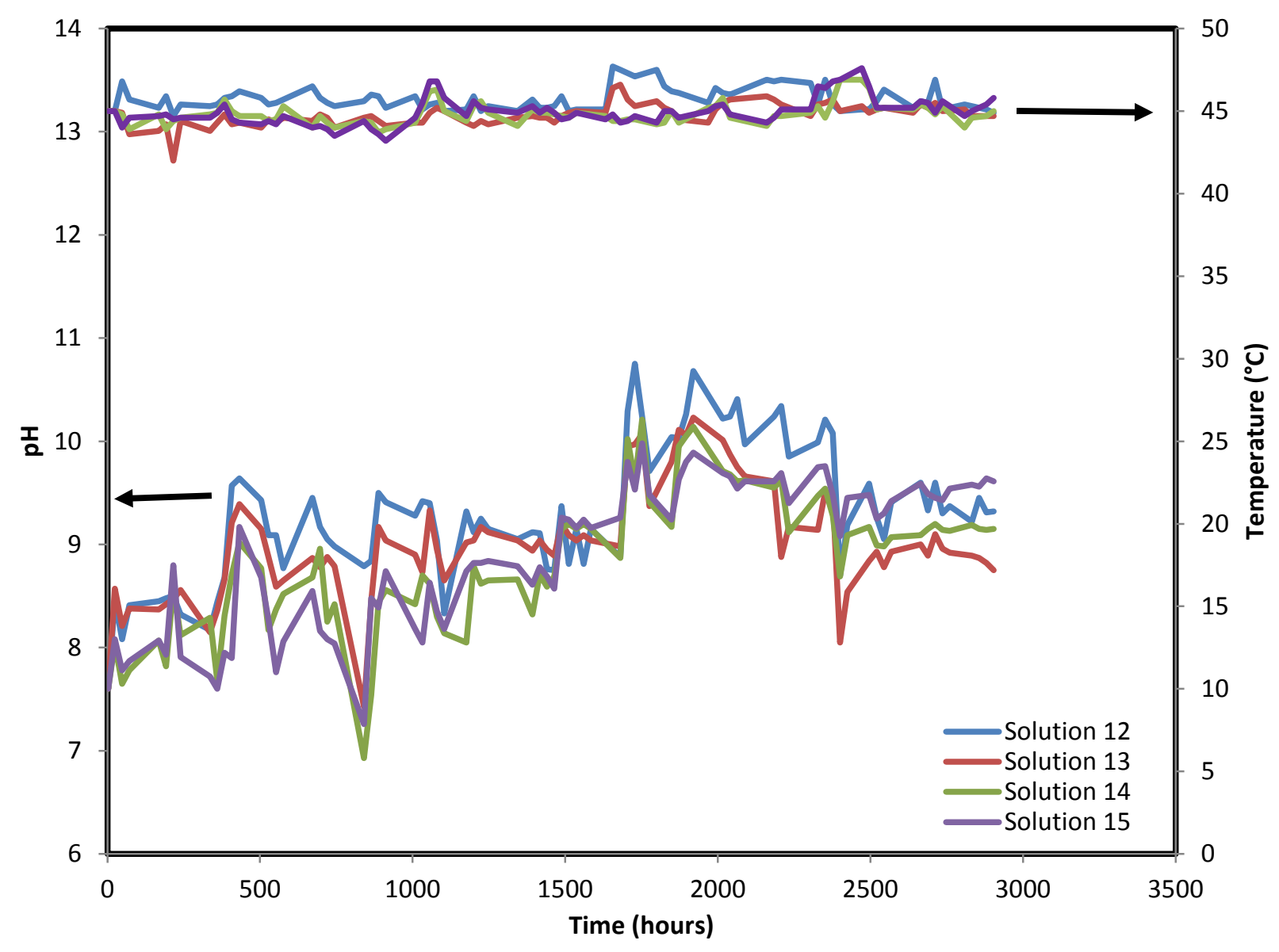




\section{Appendix F}

Chemical Composition of Simulants used in Waste Buffering (Task 3) with Electrochemical Results and After Pictures 


\section{Composition of simulant for waste buffering-Test 1}

\section{Test 1}

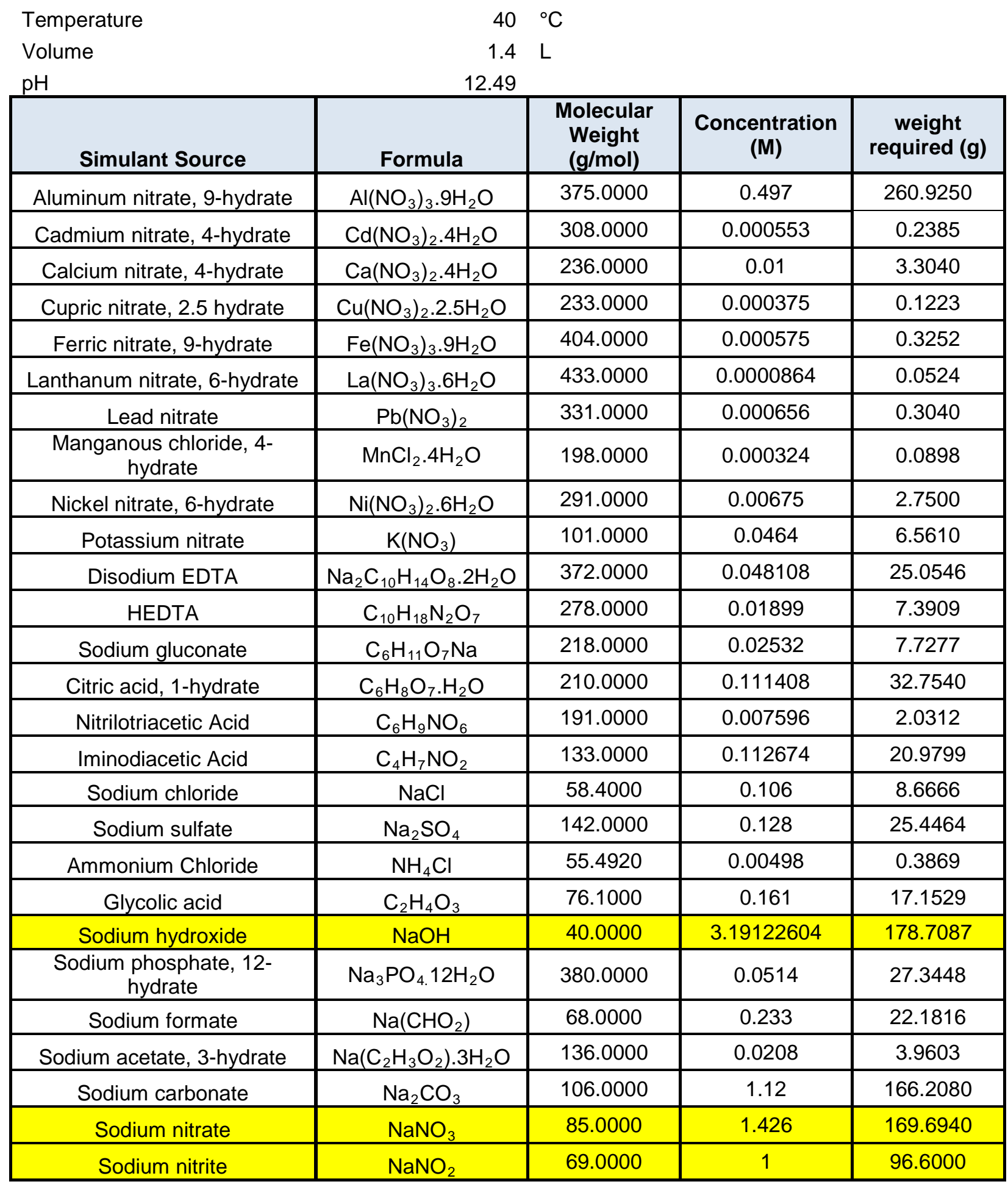




\section{Cyclic Potentiodynamic Polarization}

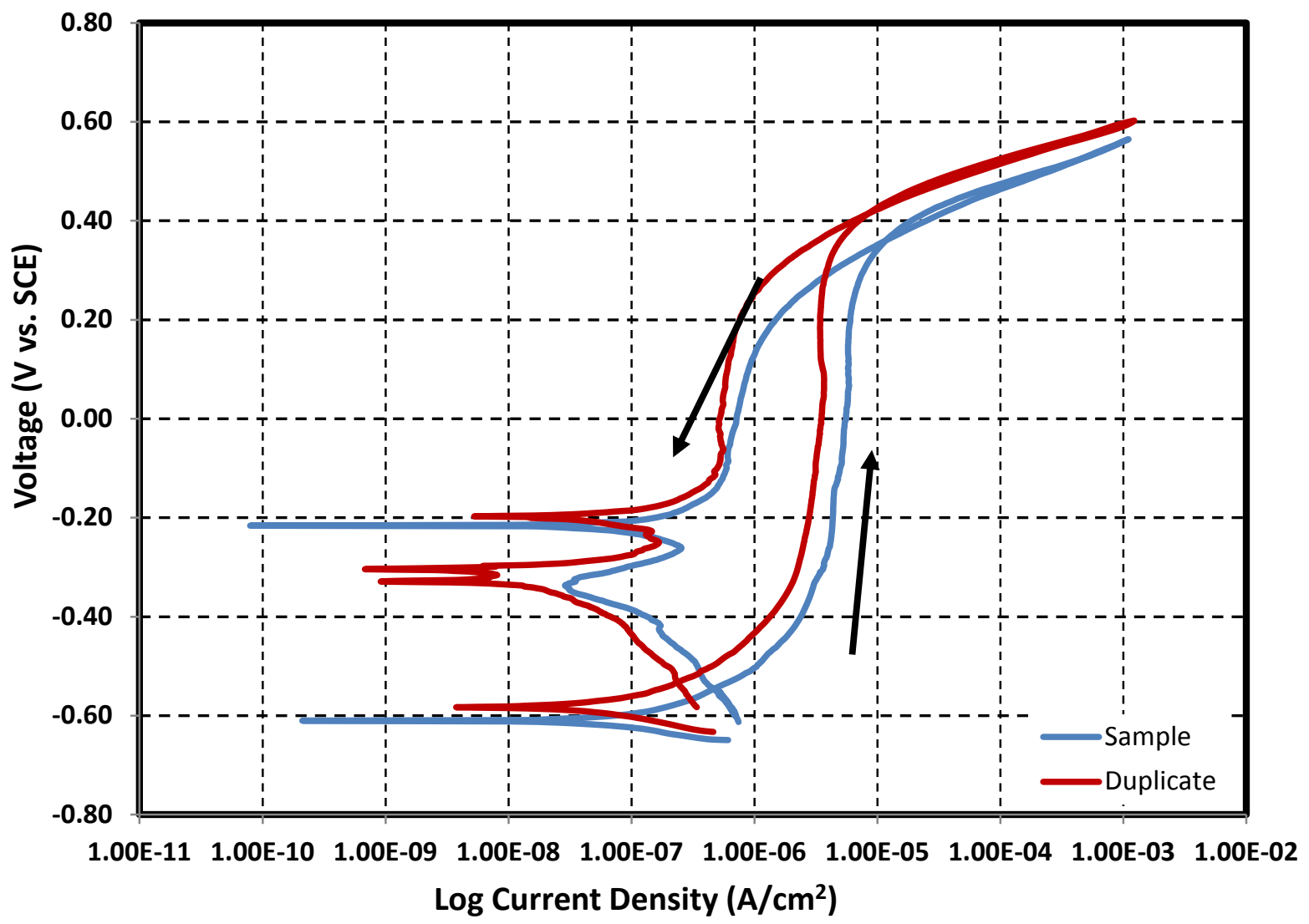

Images of bullet samples after electrochemical tests

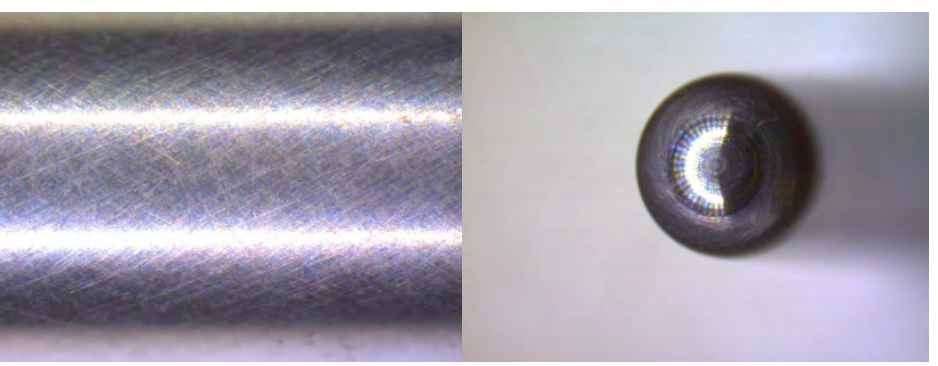

Test 1D
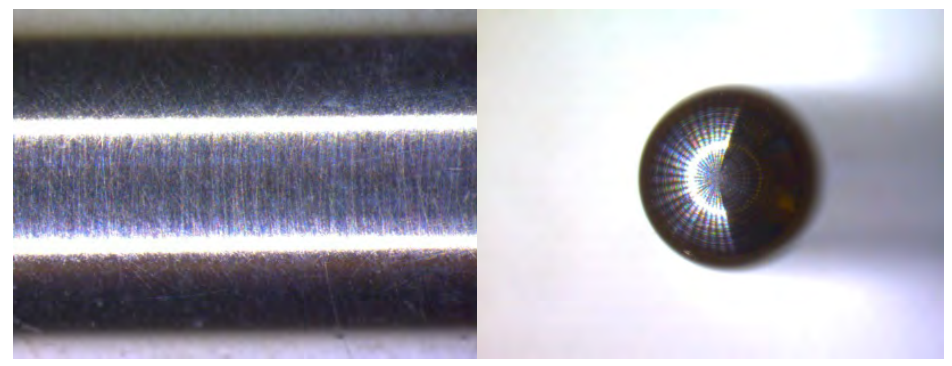

Shank (20X)

Nose (10X) 


\section{Composition of simulant for waste buffering-Test 2}

\section{Test 2}

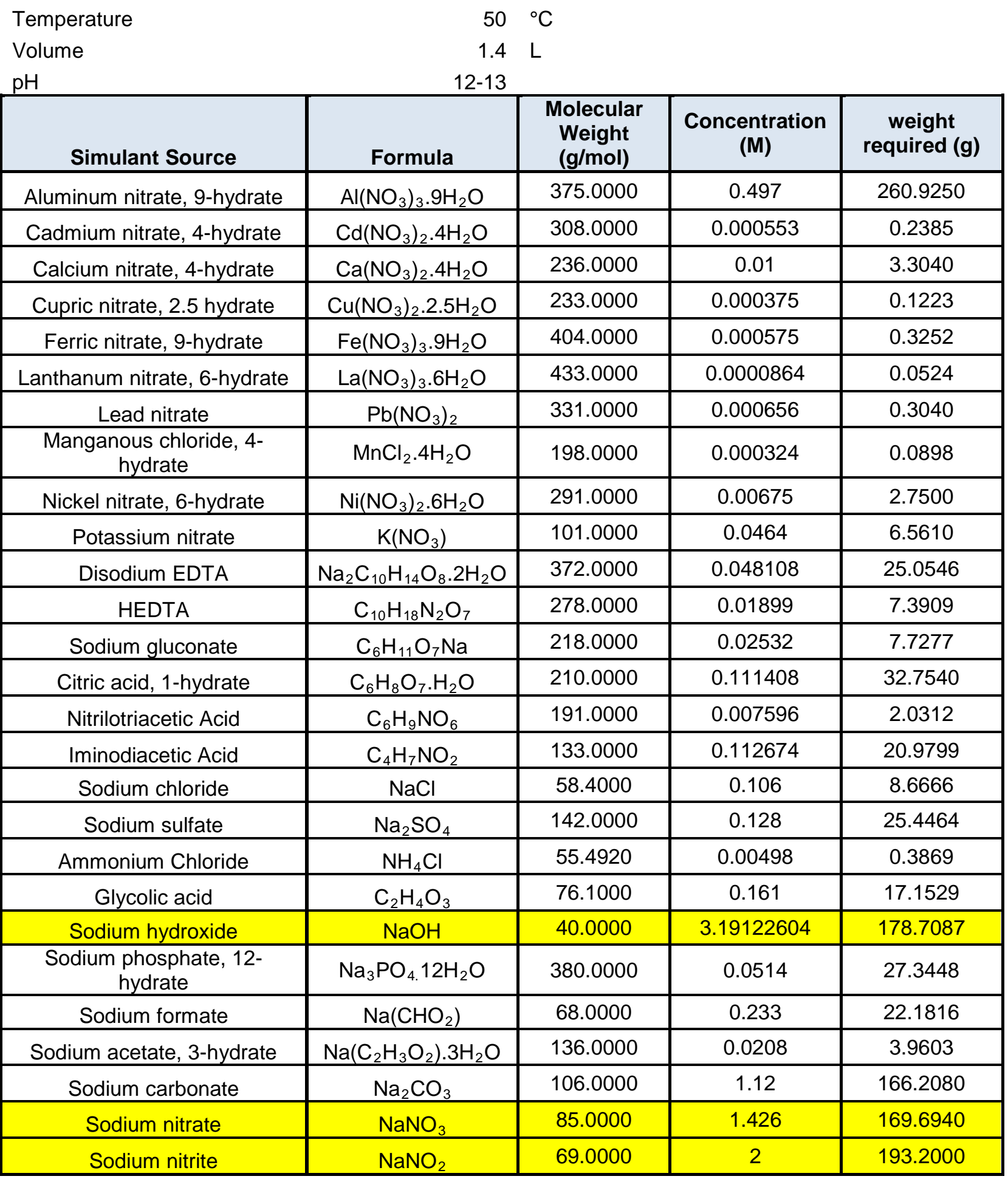




\section{Cyclic Potentiodynamic Polarization}

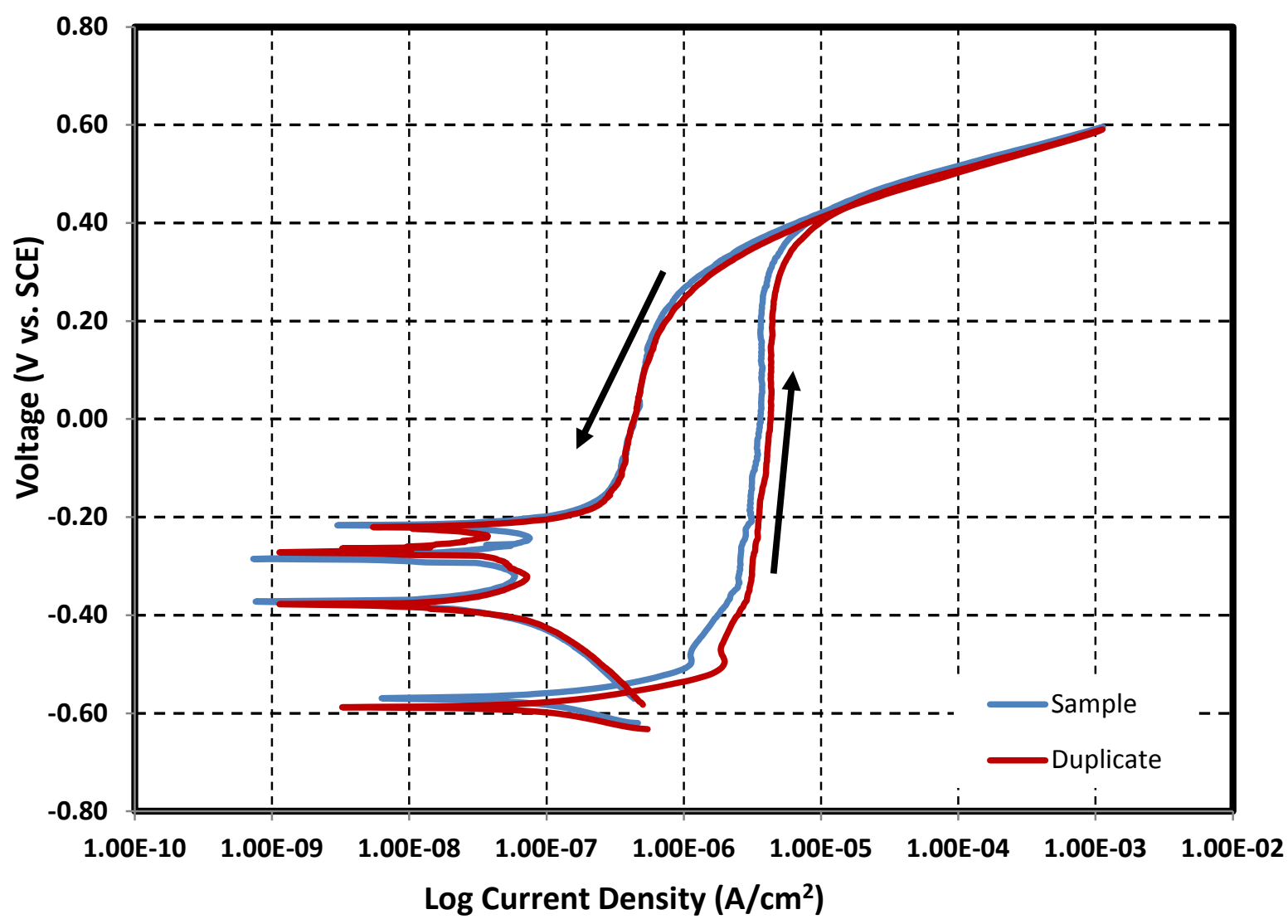

Images of bullet samples after electrochemical tests

Test 2

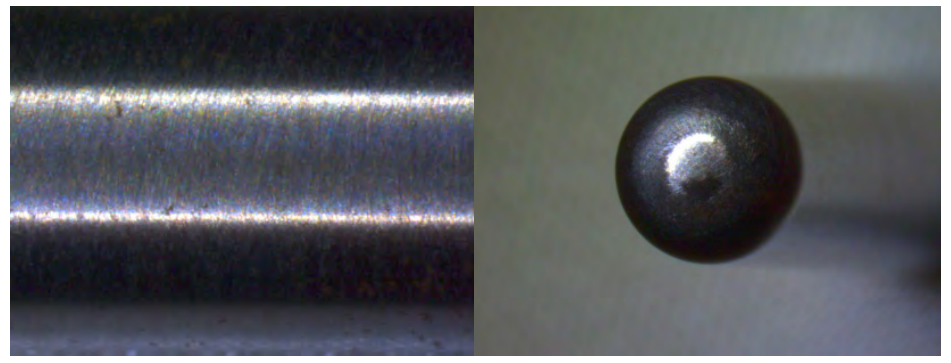

Test 2D

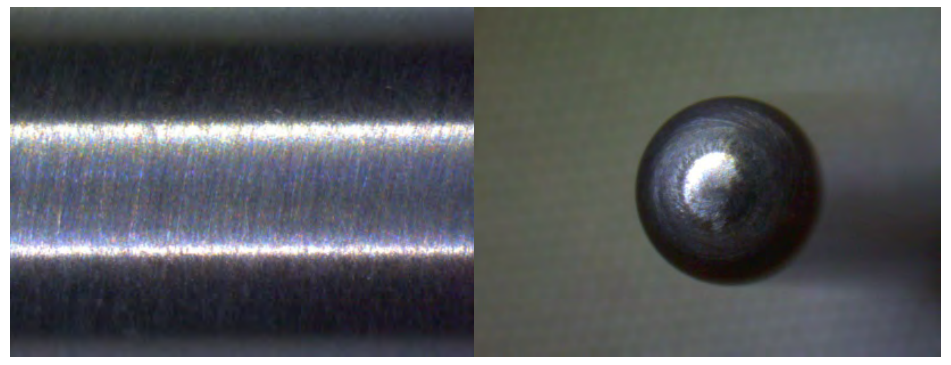

Shank (20X)

Nose (10X) 


\section{Composition of simulant for waste buffering-Test 3}

\section{Test 3}

\begin{tabular}{|c|c|c|c|c|}
\hline Temperature & & ${ }^{\circ} \mathrm{C}$ & & \\
\hline Volume & 1.4 & $\mathrm{~L}$ & & \\
\hline $\mathrm{pH}$ & $12-13$ & & & \\
\hline Simulant Source & Formula & $\begin{array}{c}\text { Molecular } \\
\text { Weight } \\
\text { (g/mol) }\end{array}$ & $\begin{array}{c}\text { Concentration } \\
\text { (M) }\end{array}$ & $\begin{array}{l}\text { weight } \\
\text { required (g) }\end{array}$ \\
\hline Aluminum nitrate, 9-hydrate & $\mathrm{Al}\left(\mathrm{NO}_{3}\right)_{3} \cdot 9 \mathrm{H}_{2} \mathrm{O}$ & 375.0000 & 0.497 & 260.9250 \\
\hline Cadmium nitrate, 4-hydrate & $\mathrm{Cd}\left(\mathrm{NO}_{3}\right)_{2} \cdot 4 \mathrm{H}_{2} \mathrm{O}$ & 308.0000 & 0.000553 & 0.2385 \\
\hline Calcium nitrate, 4-hydrate & $\mathrm{Ca}\left(\mathrm{NO}_{3}\right)_{2} \cdot 4 \mathrm{H}_{2} \mathrm{O}$ & 236.0000 & 0.01 & 3.3040 \\
\hline Cupric nitrate, 2.5 hydrate & $\mathrm{Cu}\left(\mathrm{NO}_{3}\right)_{2} \cdot 2 \cdot 5 \mathrm{H}_{2} \mathrm{O}$ & 233.0000 & 0.000375 & 0.1223 \\
\hline Ferric nitrate, 9-hydrate & $\mathrm{Fe}\left(\mathrm{NO}_{3}\right)_{3} .9 \mathrm{H}_{2} \mathrm{O}$ & 404.0000 & 0.000575 & 0.3252 \\
\hline Lanthanum nitrate, 6-hydrate & $\mathrm{La}\left(\mathrm{NO}_{3}\right)_{3} \cdot 6 \mathrm{H}_{2} \mathrm{O}$ & 433.0000 & 0.0000864 & 0.0524 \\
\hline Lead nitrate & $\mathrm{Pb}\left(\mathrm{NO}_{3}\right)_{2}$ & 331.0000 & 0.000656 & 0.3040 \\
\hline $\begin{array}{c}\text { Manganous chloride, 4- } \\
\text { hydrate }\end{array}$ & $\mathrm{MnCl}_{2} \cdot 4 \mathrm{H}_{2} \mathrm{O}$ & 198.0000 & 0.000324 & 0.0898 \\
\hline Nickel nitrate, 6-hydrate & $\mathrm{Ni}\left(\mathrm{NO}_{3}\right)_{2} \cdot 6 \mathrm{H}_{2} \mathrm{O}$ & 291.0000 & 0.00675 & 2.7500 \\
\hline Potassium nitrate & $\mathrm{K}\left(\mathrm{NO}_{3}\right)$ & 101.0000 & 0.0464 & 6.5610 \\
\hline Disodium EDTA & $\mathrm{Na}_{2} \mathrm{C}_{10} \mathrm{H}_{14} \mathrm{O}_{8} .2 \mathrm{H}_{2} \mathrm{O}$ & 372.0000 & 0.048108 & 25.0546 \\
\hline HEDTA & $\mathrm{C}_{10} \mathrm{H}_{18} \mathrm{~N}_{2} \mathrm{O}_{7}$ & 278.0000 & 0.01899 & 7.3909 \\
\hline Sodium gluconate & $\mathrm{C}_{6} \mathrm{H}_{11} \mathrm{O}_{7} \mathrm{Na}$ & 218.0000 & 0.02532 & 7.7277 \\
\hline Citric acid, 1-hydrate & $\mathrm{C}_{6} \mathrm{H}_{8} \mathrm{O}_{7} \cdot \mathrm{H}_{2} \mathrm{O}$ & 210.0000 & 0.111408 & 32.7540 \\
\hline Nitrilotriacetic Acid & $\mathrm{C}_{6} \mathrm{H}_{9} \mathrm{NO}_{6}$ & 191.0000 & 0.007596 & 2.0312 \\
\hline Iminodiacetic Acid & $\mathrm{C}_{4} \mathrm{H}_{7} \mathrm{NO}_{2}$ & 133.0000 & 0.112674 & 20.9799 \\
\hline Sodium chloride & $\mathrm{NaCl}$ & 58.4000 & 0.106 & 8.6666 \\
\hline Sodium sulfate & $\mathrm{Na}_{2} \mathrm{SO}_{4}$ & 142.0000 & 0.128 & 25.4464 \\
\hline Ammonium Chloride & $\mathrm{NH}_{4} \mathrm{Cl}$ & 55.4920 & 0.00498 & 0.3869 \\
\hline Glycolic acid & $\mathrm{C}_{2} \mathrm{H}_{4} \mathrm{O}_{3}$ & 76.1000 & 0.161 & 17.1529 \\
\hline Sodium hydroxide & $\mathrm{NaOH}$ & 40.0000 & 3.19122604 & 178.7087 \\
\hline $\begin{array}{l}\text { Sodium phosphate, } 12- \\
\text { hydrate }\end{array}$ & $\mathrm{Na}_{3} \mathrm{PO}_{4} \cdot 12 \mathrm{H}_{2} \mathrm{O}$ & 380.0000 & 0.0514 & 27.3448 \\
\hline Sodium formate & $\mathrm{Na}(\mathrm{CHO} 2)$ & 68.0000 & 0.233 & 22.1816 \\
\hline Sodium acetate, 3-hydrate & $\mathrm{Na}\left(\mathrm{C}_{2} \mathrm{H}_{3} \mathrm{O}_{2}\right) \cdot 3 \mathrm{H}_{2} \mathrm{O}$ & 136.0000 & 0.0208 & 3.9603 \\
\hline Sodium carbonate & $\mathrm{Na}_{2} \mathrm{CO}_{3}$ & 106.0000 & 1.12 & 166.2080 \\
\hline Sodium nitrate & $\mathrm{NaNO}_{3}$ & 85.0000 & 1.426 & 169.6940 \\
\hline Sodium nitrite & $\mathrm{NaNO}_{2}$ & 69.0000 & 2.5 & 241.5000 \\
\hline
\end{tabular}




\section{Cyclic Potentiodynamic Polarization}

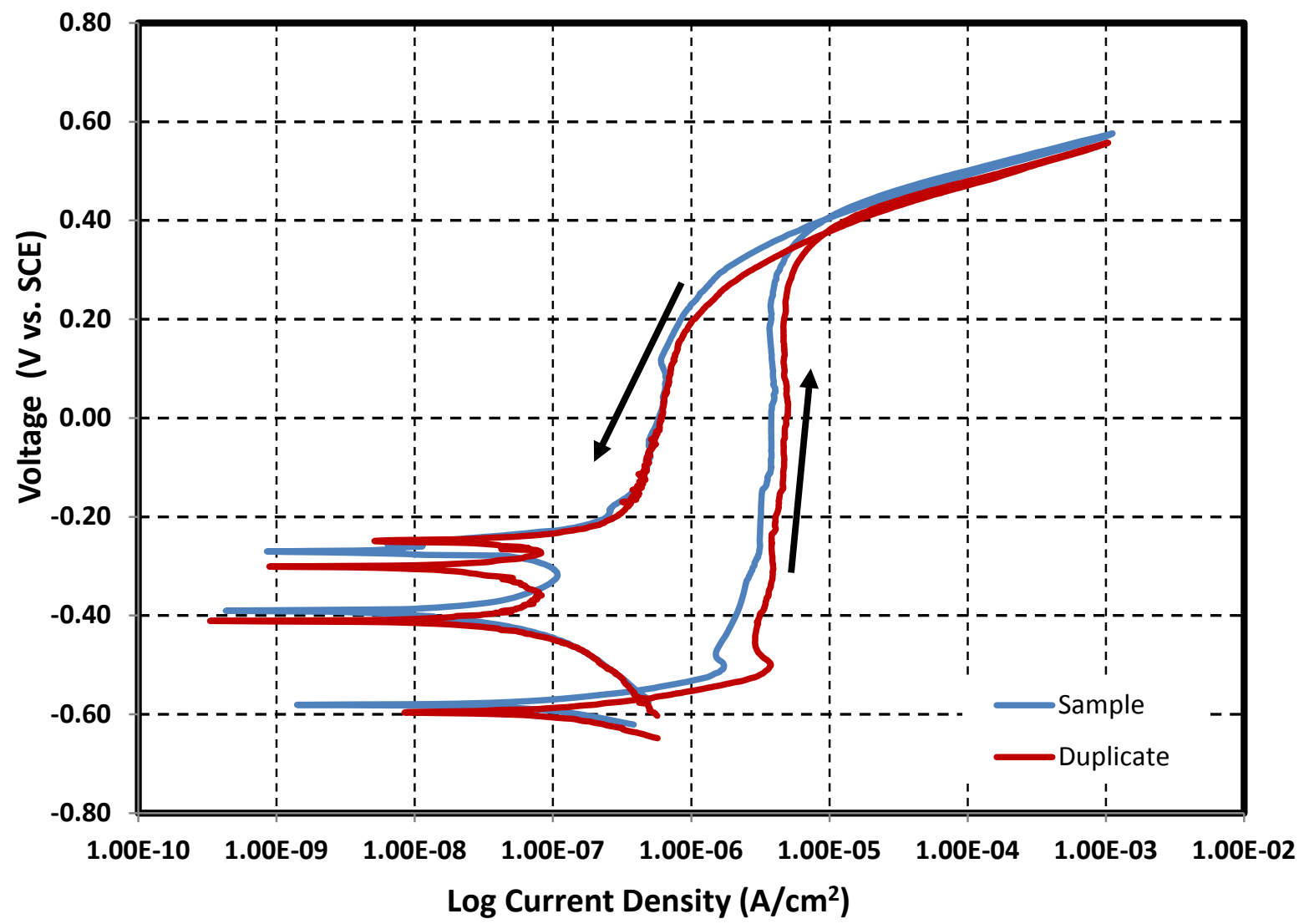

Images of bullet samples after electrochemical tests

Test 3

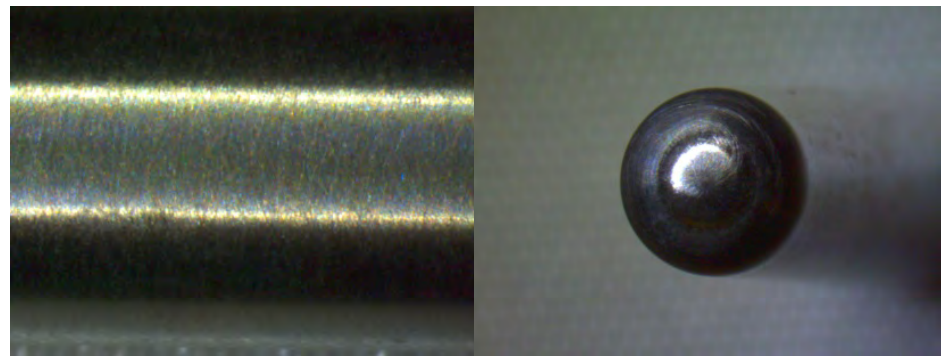

Test 3D

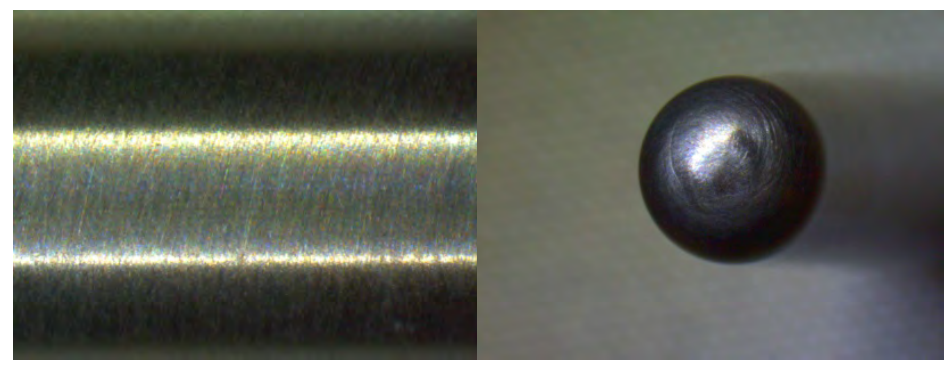

Shank $(20 X)$

Nose (10X) 


\section{Composition of simulant for waste buffering-Test 4}

\section{Test 4}

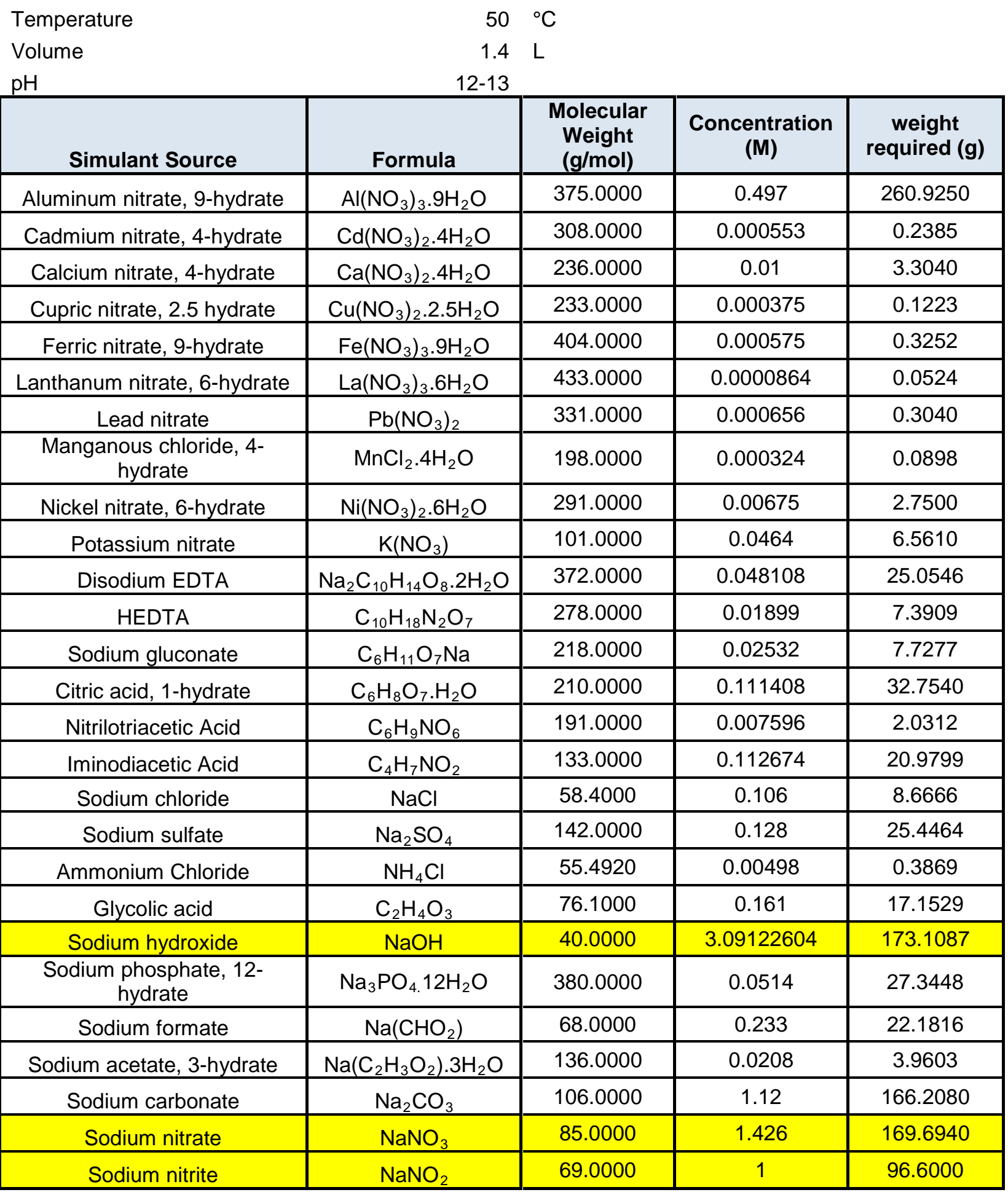




\section{Cyclic Potentiodynamic Polarization}

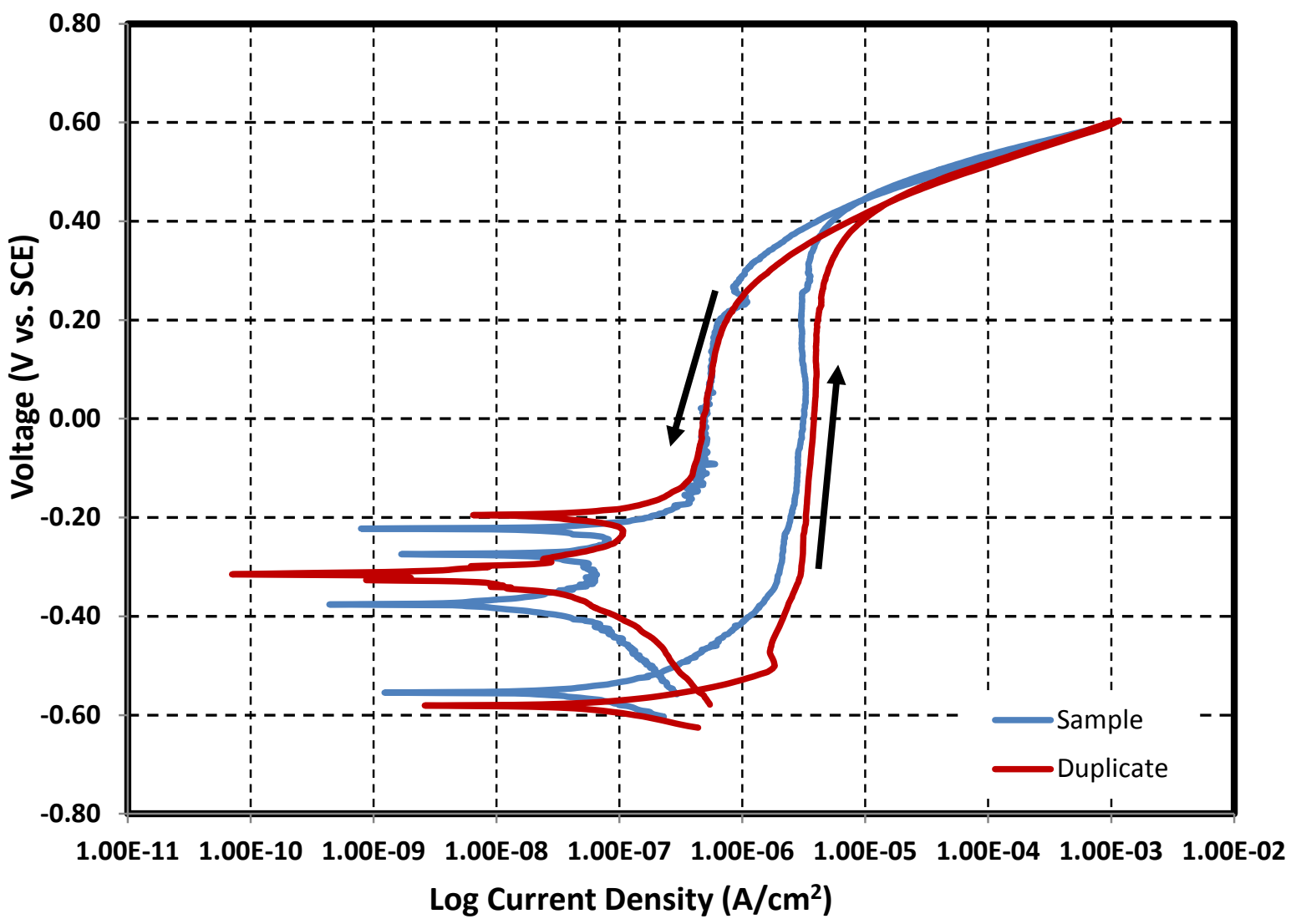

Images of bullet samples after electrochemical tests

Test 4

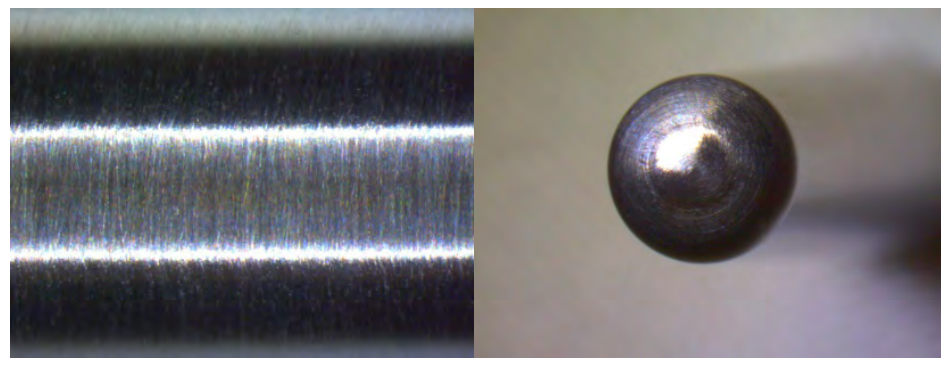

Test 4D

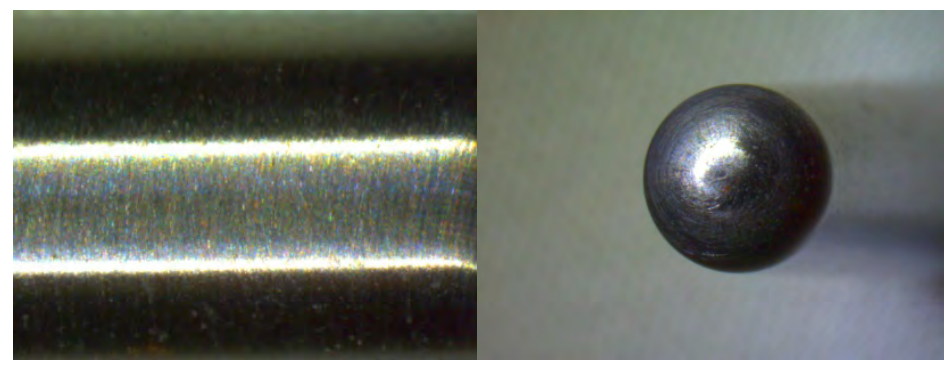

Shank (20X)

Nose (10X) 


\section{Composition of simulant for waste buffering-Test 5}

\section{Test 5}

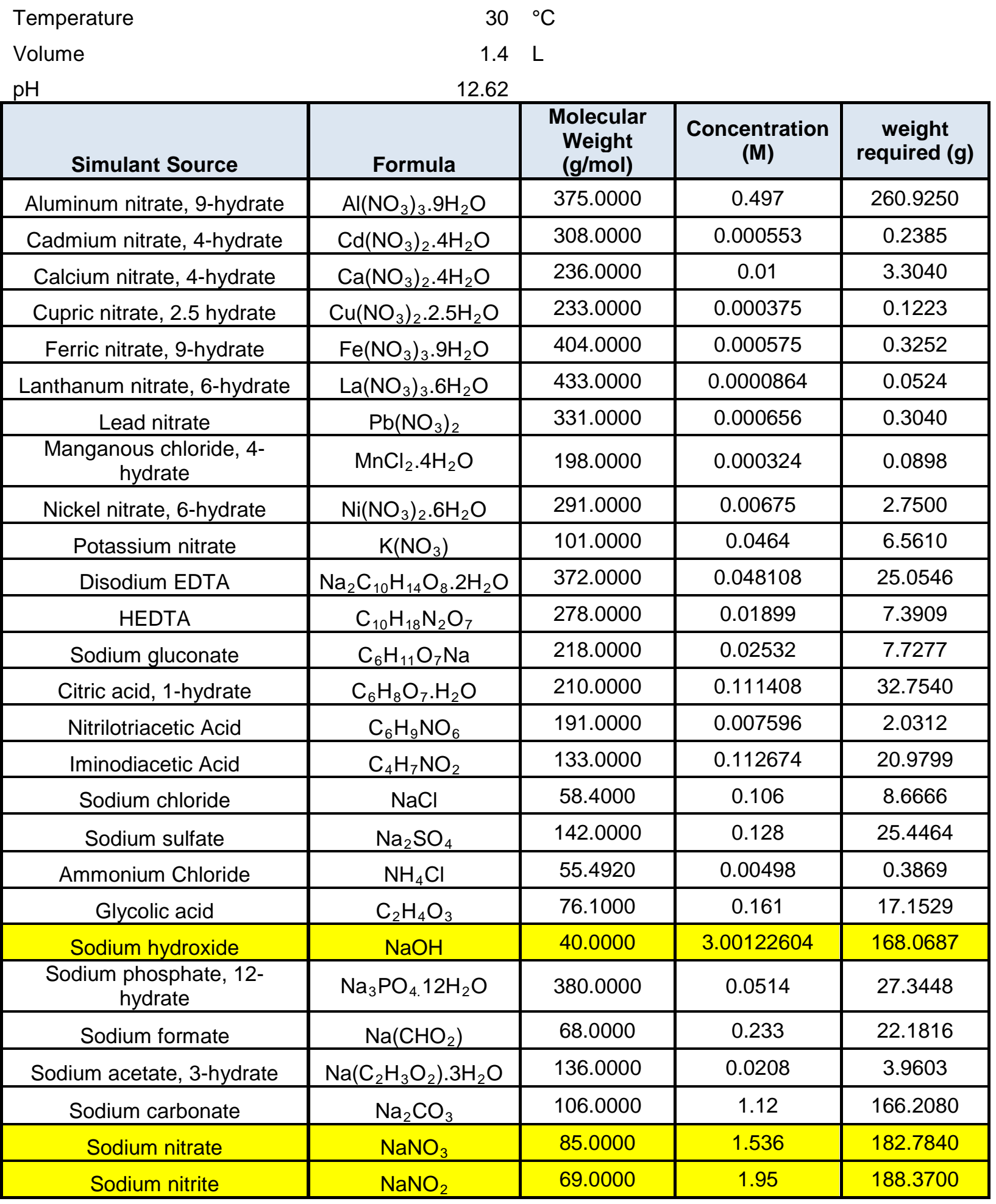




\section{Cyclic Potentiodynamic Polarization}

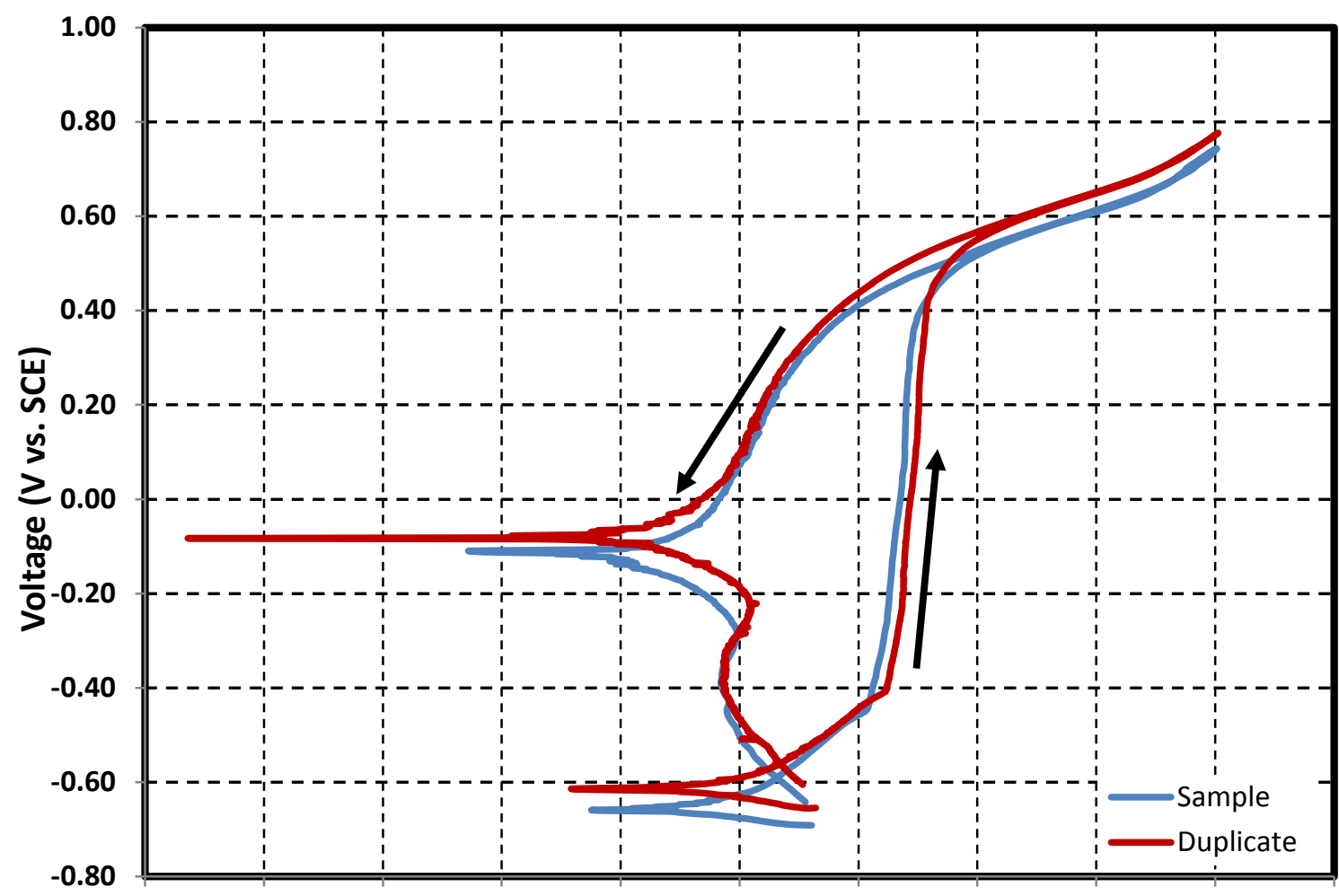

1.00E-12 1.00E-11 1.00E-10 1.00E-09 1.00E-08 1.00E-07 1.00E-06 1.00E-05 1.00E-04 1.00E-03 1.00E-02 Log Current Density $\left(\mathrm{A} / \mathrm{cm}^{2}\right)$

Images of bullet samples after electrochemical tests

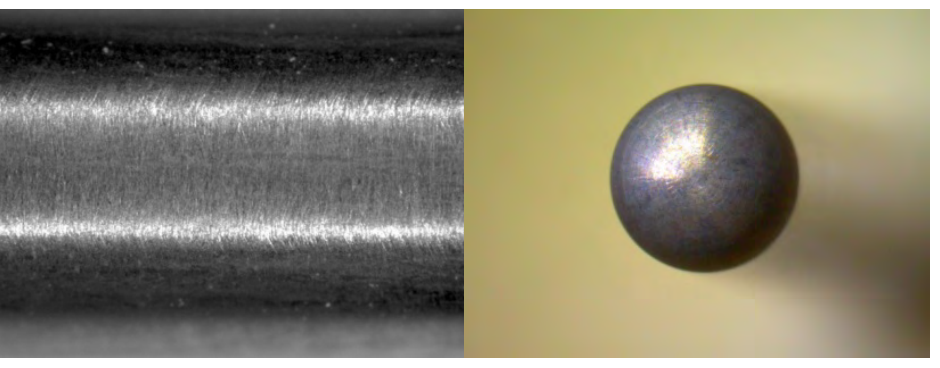

Test 5D

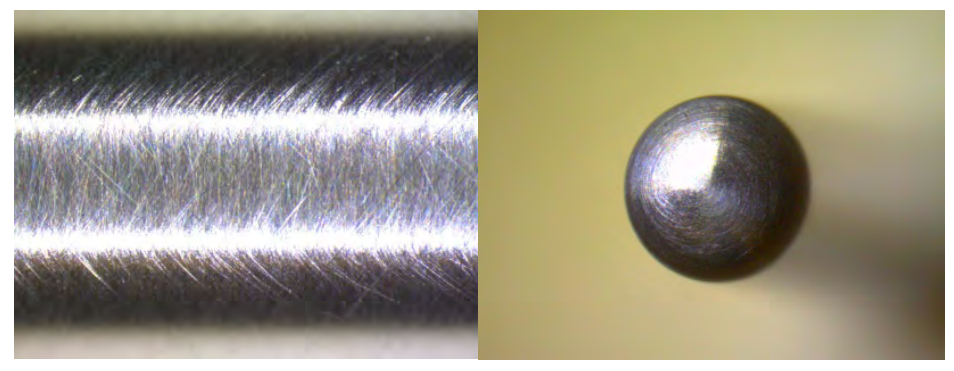

Shank (20X)

Nose (10X) 


\section{Composition of simulant for waste buffering-Test 6}

Test 6

\begin{tabular}{|c|c|c|c|c|}
\hline & 40 & ${ }^{\circ} \mathrm{C}$ & & \\
\hline Volume & 1.4 & $\mathrm{~L}$ & & \\
\hline $\mathrm{pH}$ & 12.62 & & & \\
\hline Simulant Source & Formula & $\begin{array}{c}\text { Molecular } \\
\text { Weight } \\
\text { (g/mol) } \\
\end{array}$ & $\begin{array}{l}\text { Concentration } \\
\text { (M) }\end{array}$ & $\begin{array}{l}\text { weight } \\
\text { required }(g)\end{array}$ \\
\hline Aluminum nitrate, 9-hydrate & $\mathrm{Al}\left(\mathrm{NO}_{3}\right)_{3} \cdot 9 \mathrm{H}_{2} \mathrm{O}$ & 375.0000 & 0.497 & 260.9250 \\
\hline Cadmium nitrate, 4-hydrate & $\mathrm{Cd}\left(\mathrm{NO}_{3}\right)_{2} \cdot 4 \mathrm{H}_{2} \mathrm{O}$ & 308.0000 & 0.000553 & 0.2385 \\
\hline Calcium nitrate, 4-hydrate & $\mathrm{Ca}\left(\mathrm{NO}_{3}\right)_{2} \cdot 4 \mathrm{H}_{2} \mathrm{O}$ & 236.0000 & 0.01 & 3.3040 \\
\hline Cupric nitrate, 2.5 hydrate & $\mathrm{Cu}\left(\mathrm{NO}_{3}\right)_{2} \cdot 2.5 \mathrm{H}_{2} \mathrm{O}$ & 233.0000 & 0.000375 & 0.1223 \\
\hline Ferric nitrate, 9-hydrate & $\mathrm{Fe}\left(\mathrm{NO}_{3}\right)_{3} \cdot 9 \mathrm{H}_{2} \mathrm{O}$ & 404.0000 & 0.000575 & 0.3252 \\
\hline Lanthanum nitrate, 6-hydrate & $\mathrm{La}\left(\mathrm{NO}_{3}\right)_{3} \cdot 6 \mathrm{H}_{2} \mathrm{O}$ & 433.0000 & 0.0000864 & 0.0524 \\
\hline Lead nitrate & $\mathrm{Pb}\left(\mathrm{NO}_{3}\right)_{2}$ & 331.0000 & 0.000656 & 0.3040 \\
\hline $\begin{array}{c}\text { Manganous chloride, 4- } \\
\text { hydrate } \\
\end{array}$ & $\mathrm{MnCl}_{2} \cdot 4 \mathrm{H}_{2} \mathrm{O}$ & 198.0000 & 0.000324 & 0.0898 \\
\hline Nickel nitrate, 6-hydrate & $\mathrm{Ni}\left(\mathrm{NO}_{3}\right)_{2} \cdot 6 \mathrm{H}_{2} \mathrm{O}$ & 291.0000 & 0.00675 & 2.7500 \\
\hline Potassium nitrate & $\mathrm{K}\left(\mathrm{NO}_{3}\right)$ & 101.0000 & 0.0464 & 6.5610 \\
\hline Disodium EDTA & $\mathrm{Na}_{2} \mathrm{C}_{10} \mathrm{H}_{14} \mathrm{O}_{8} .2 \mathrm{H}_{2} \mathrm{O}$ & 372.0000 & 0.048108 & 25.0546 \\
\hline HEDTA & $\mathrm{C}_{10} \mathrm{H}_{18} \mathrm{~N}_{2} \mathrm{O}_{7}$ & 278.0000 & 0.01899 & 7.3909 \\
\hline Sodium gluconate & $\mathrm{C}_{6} \mathrm{H}_{11} \mathrm{O}_{7} \mathrm{Na}$ & 218.0000 & 0.02532 & 7.7277 \\
\hline Citric acid, 1-hydrate & $\mathrm{C}_{6} \mathrm{H}_{8} \mathrm{O}_{7} \cdot \mathrm{H}_{2} \mathrm{O}$ & 210.0000 & 0.111408 & 32.7540 \\
\hline Nitrilotriacetic Acid & $\mathrm{C}_{6} \mathrm{H}_{9} \mathrm{NO}_{6}$ & 191.0000 & 0.007596 & 2.0312 \\
\hline Iminodiacetic Acid & $\mathrm{C}_{4} \mathrm{H}_{7} \mathrm{NO}_{2}$ & 133.0000 & 0.112674 & 20.9799 \\
\hline Sodium chloride & $\mathrm{NaCl}$ & 58.4000 & 0.106 & 8.6666 \\
\hline Sodium sulfate & $\mathrm{Na}_{2} \mathrm{SO}_{4}$ & 142.0000 & 0.128 & 25.4464 \\
\hline Ammonium Chloride & $\mathrm{NH}_{4} \mathrm{Cl}$ & 55.4920 & 0.00498 & 0.3869 \\
\hline Glycolic acid & $\mathrm{C}_{2} \mathrm{H}_{4} \mathrm{O}_{3}$ & 76.1000 & 0.161 & 17.1529 \\
\hline Sodium hydroxide & $\mathrm{NaOH}$ & 40.0000 & 3.00122604 & 168.0687 \\
\hline $\begin{array}{l}\text { Sodium phosphate, 12- } \\
\text { hydrate }\end{array}$ & $\mathrm{Na}_{3} \mathrm{PO}_{4} \cdot 12 \mathrm{H}_{2} \mathrm{O}$ & 380.0000 & 0.0514 & 27.3448 \\
\hline Sodium formate & $\mathrm{Na}\left(\mathrm{CHO}_{2}\right)$ & 68.0000 & 0.233 & 22.1816 \\
\hline Sodium acetate, 3-hydrate & $\mathrm{Na}\left(\mathrm{C}_{2} \mathrm{H}_{3} \mathrm{O}_{2}\right) \cdot 3 \mathrm{H}_{2} \mathrm{O}$ & 136.0000 & 0.0208 & 3.9603 \\
\hline Sodium carbonate & $\mathrm{Na}_{2} \mathrm{CO}_{3}$ & 106.0000 & 1.12 & 166.2080 \\
\hline Sodium nitrate & $\mathrm{NaNO}_{3}$ & 85.0000 & 1.536 & 182.7840 \\
\hline Sodium nitrite & $\mathrm{NaNO}_{2}$ & 69.0000 & 1.95 & 188.3700 \\
\hline
\end{tabular}




\section{Cyclic Potentiodynamic Polarization}

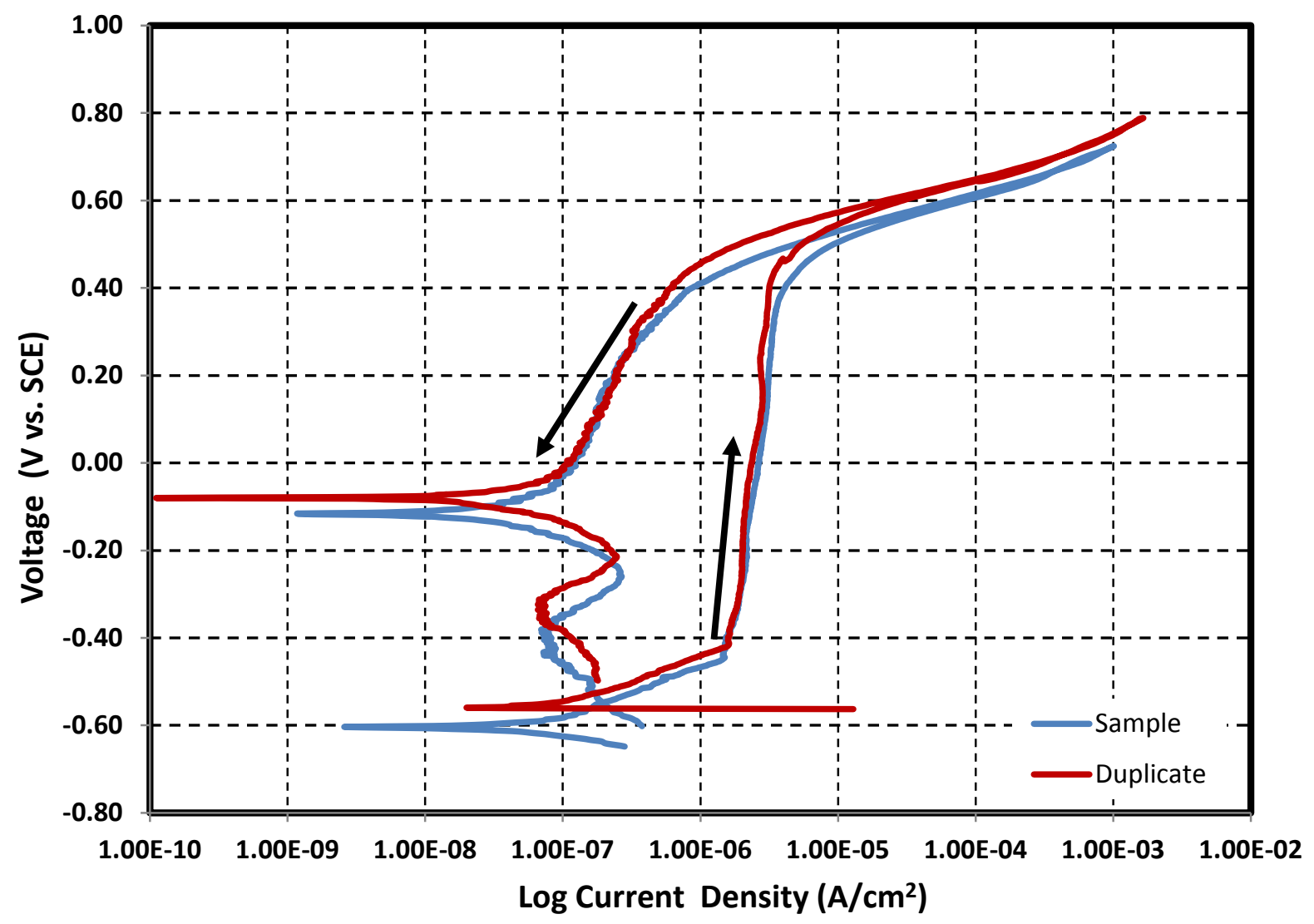

Images of bullet samples after electrochemical tests

Test 6

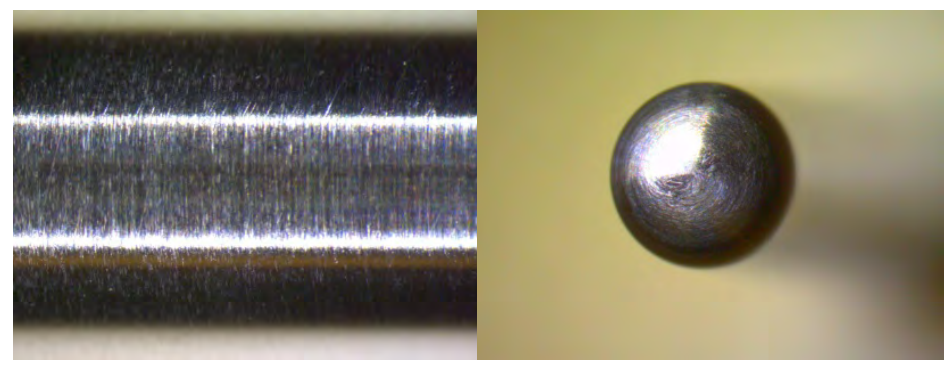

Test 6D

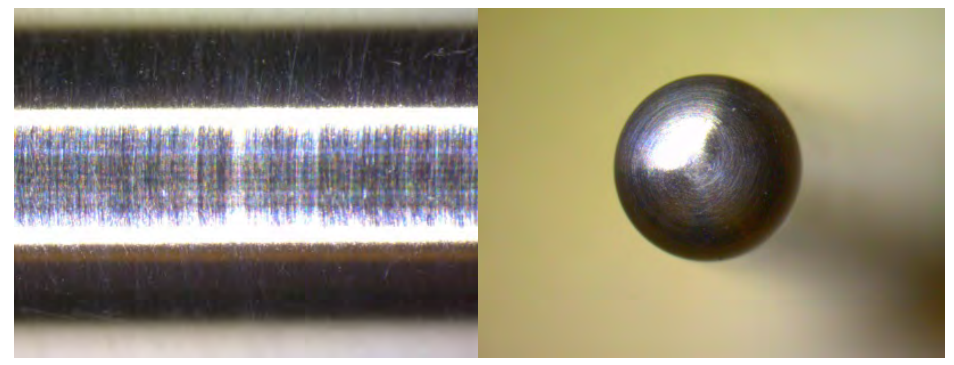

Shank (20X)

Nose (10X) 


\section{Composition of simulant for waste buffering-Test 7}

Test 7

\begin{tabular}{|c|c|c|c|c|}
\hline & 50 & ${ }^{\circ} \mathrm{C}$ & & \\
\hline Volume & 1.4 & L & & \\
\hline $\mathrm{pH}$ & 12.62 & & & \\
\hline Simulant Source & Formula & $\begin{array}{c}\text { Molecular } \\
\text { Weight } \\
\text { (g/mol) } \\
\end{array}$ & $\begin{array}{l}\text { Concentration } \\
\text { (M) }\end{array}$ & $\begin{array}{l}\text { weight } \\
\text { required }(g)\end{array}$ \\
\hline Aluminum nitrate, 9-hydrate & $\mathrm{Al}\left(\mathrm{NO}_{3}\right)_{3} \cdot 9 \mathrm{H}_{2} \mathrm{O}$ & 375.0000 & 0.497 & 260.9250 \\
\hline Cadmium nitrate, 4-hydrate & $\mathrm{Cd}\left(\mathrm{NO}_{3}\right)_{2} \cdot 4 \mathrm{H}_{2} \mathrm{O}$ & 308.0000 & 0.000553 & 0.2385 \\
\hline Calcium nitrate, 4-hydrate & $\mathrm{Ca}\left(\mathrm{NO}_{3}\right)_{2} \cdot 4 \mathrm{H}_{2} \mathrm{O}$ & 236.0000 & 0.01 & 3.3040 \\
\hline Cupric nitrate, 2.5 hydrate & $\mathrm{Cu}\left(\mathrm{NO}_{3}\right)_{2} \cdot 2.5 \mathrm{H}_{2} \mathrm{O}$ & 233.0000 & 0.000375 & 0.1223 \\
\hline Ferric nitrate, 9-hydrate & $\mathrm{Fe}\left(\mathrm{NO}_{3}\right)_{3} \cdot 9 \mathrm{H}_{2} \mathrm{O}$ & 404.0000 & 0.000575 & 0.3252 \\
\hline Lanthanum nitrate, 6-hydrate & $\mathrm{La}\left(\mathrm{NO}_{3}\right)_{3} \cdot 6 \mathrm{H}_{2} \mathrm{O}$ & 433.0000 & 0.0000864 & 0.0524 \\
\hline Lead nitrate & $\mathrm{Pb}\left(\mathrm{NO}_{3}\right)_{2}$ & 331.0000 & 0.000656 & 0.3040 \\
\hline $\begin{array}{c}\text { Manganous chloride, 4- } \\
\text { hydrate }\end{array}$ & $\mathrm{MnCl}_{2} \cdot 4 \mathrm{H}_{2} \mathrm{O}$ & 198.0000 & 0.000324 & 0.0898 \\
\hline Nickel nitrate, 6-hydrate & $\mathrm{Ni}\left(\mathrm{NO}_{3}\right)_{2} \cdot 6 \mathrm{H}_{2} \mathrm{O}$ & 291.0000 & 0.00675 & 2.7500 \\
\hline Potassium nitrate & $\mathrm{K}\left(\mathrm{NO}_{3}\right)$ & 101.0000 & 0.0464 & 6.5610 \\
\hline Disodium EDTA & $\mathrm{Na}_{2} \mathrm{C}_{10} \mathrm{H}_{14} \mathrm{O}_{8} \cdot 2 \mathrm{H}_{2} \mathrm{O}$ & 372.0000 & 0.048108 & 25.0546 \\
\hline HEDTA & $\mathrm{C}_{10} \mathrm{H}_{18} \mathrm{~N}_{2} \mathrm{O}_{7}$ & 278.0000 & 0.01899 & 7.3909 \\
\hline Sodium gluconate & $\mathrm{C}_{6} \mathrm{H}_{11} \mathrm{O}_{7} \mathrm{Na}$ & 218.0000 & 0.02532 & 7.7277 \\
\hline Citric acid, 1-hydrate & $\mathrm{C}_{6} \mathrm{H}_{8} \mathrm{O}_{7} \cdot \mathrm{H}_{2} \mathrm{O}$ & 210.0000 & 0.111408 & 32.7540 \\
\hline Nitrilotriacetic Acid & $\mathrm{C}_{6} \mathrm{H}_{9} \mathrm{NO}_{6}$ & 191.0000 & 0.007596 & 2.0312 \\
\hline Iminodiacetic Acid & $\mathrm{C}_{4} \mathrm{H}_{7} \mathrm{NO}_{2}$ & 133.0000 & 0.112674 & 20.9799 \\
\hline Sodium chloride & $\mathrm{NaCl}$ & 58.4000 & 0.106 & 8.6666 \\
\hline Sodium sulfate & $\mathrm{Na}_{2} \mathrm{SO}_{4}$ & 142.0000 & 0.128 & 25.4464 \\
\hline Ammonium Chloride & $\mathrm{NH}_{4} \mathrm{Cl}$ & 55.4920 & 0.00498 & 0.3869 \\
\hline Glycolic acid & $\mathrm{C}_{2} \mathrm{H}_{4} \mathrm{O}_{3}$ & 76.1000 & 0.161 & 17.1529 \\
\hline Sodium hydroxide & $\mathrm{NaOH}$ & 40.0000 & 3.00122604 & 168.0687 \\
\hline $\begin{array}{l}\text { Sodium phosphate, 12- } \\
\text { hydrate }\end{array}$ & $\mathrm{Na}_{3} \mathrm{PO}_{4} \cdot 12 \mathrm{H}_{2} \mathrm{O}$ & 380.0000 & 0.0514 & 27.3448 \\
\hline Sodium formate & $\mathrm{Na}\left(\mathrm{CHO}_{2}\right)$ & 68.0000 & 0.233 & 22.1816 \\
\hline Sodium acetate, 3-hydrate & $\mathrm{Na}\left(\mathrm{C}_{2} \mathrm{H}_{3} \mathrm{O}_{2}\right) \cdot 3 \mathrm{H}_{2} \mathrm{O}$ & 136.0000 & 0.0208 & 3.9603 \\
\hline Sodium carbonate & $\mathrm{Na}_{2} \mathrm{CO}_{3}$ & 106.0000 & 1.12 & 166.2080 \\
\hline Sodium nitrate & $\mathrm{NaNO}_{3}$ & 85.0000 & 1.536 & 182.7840 \\
\hline Sodium nitrite & $\mathrm{NaNO}_{2}$ & 69.0000 & 1.95 & 188.3700 \\
\hline
\end{tabular}




\section{Cyclic Potentiodynamic Polarization}

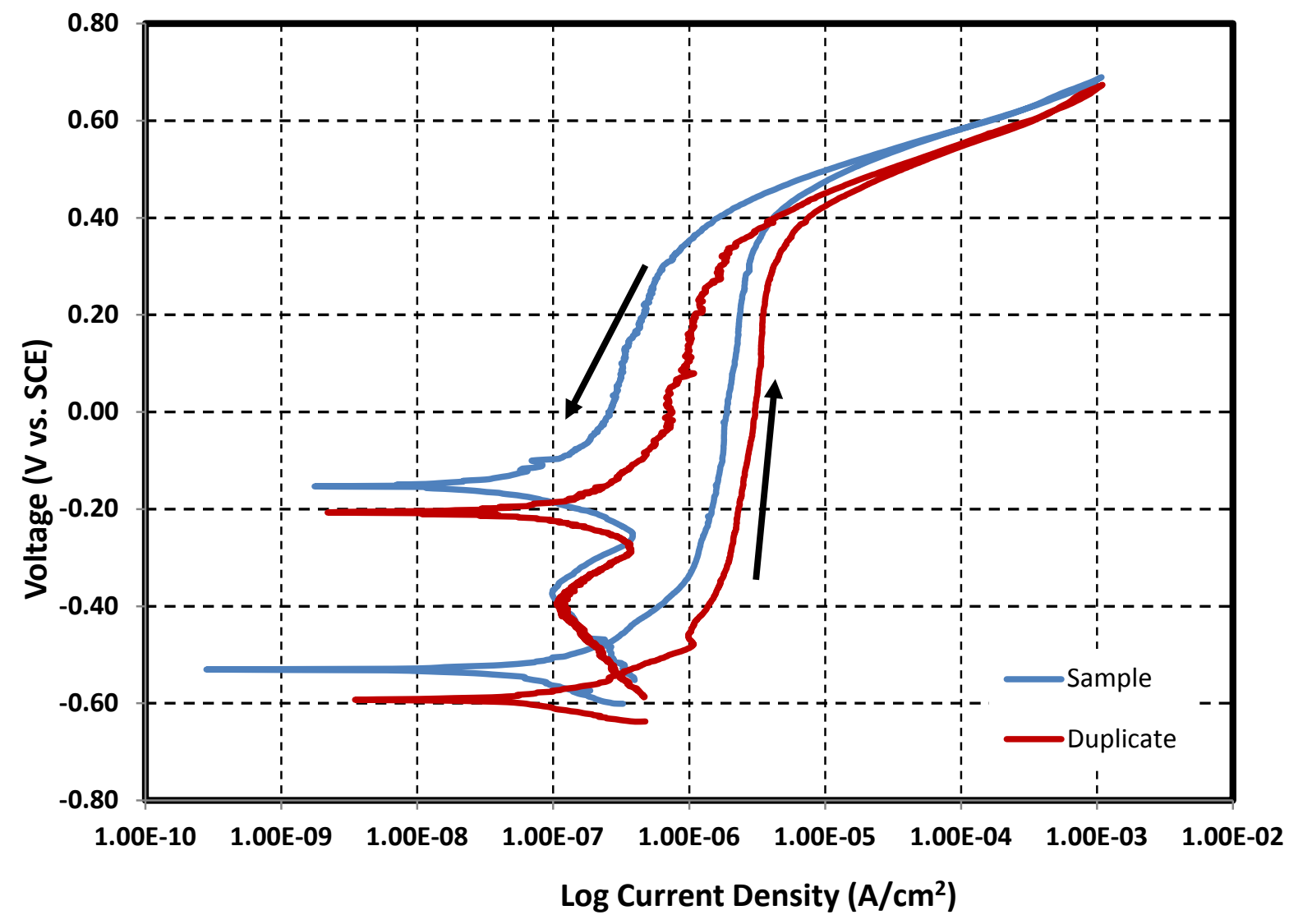

Images of bullet samples after electrochemical tests

Test 7

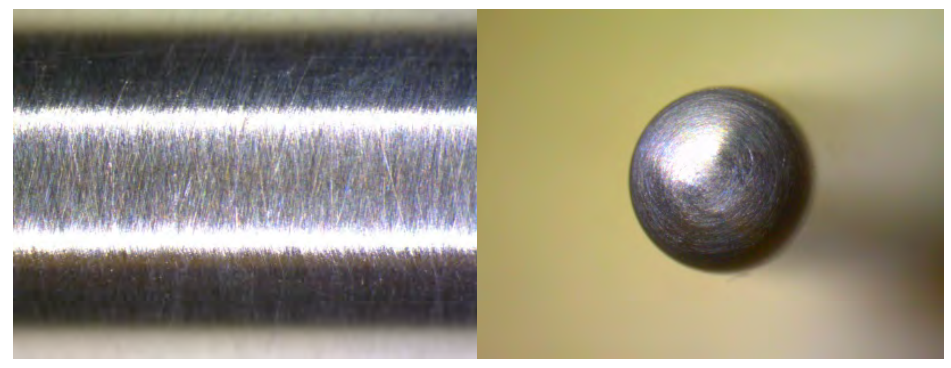

Test 7D

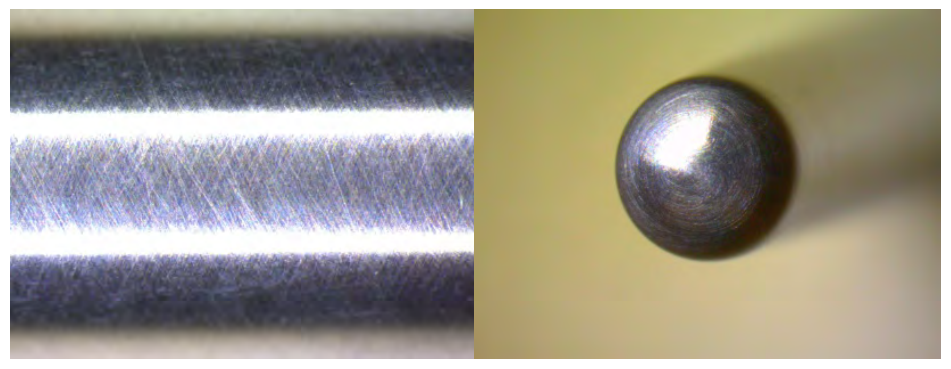

Shank (20X)

Nose (10X) 


\section{Composition of simulant for waste buffering-Test 8}

\section{Test 8}

\begin{tabular}{|c|c|c|c|c|}
\hline $\begin{array}{l}\text { Temperature } \\
\text { Volume } \\
\text { pH }\end{array}$ & $\begin{array}{r}50 \\
1.4 \\
12.00 \\
\end{array}$ & ${ }^{\circ} \mathrm{C}$ & & \\
\hline Simulant Source & Formula & $\begin{array}{c}\text { Molecular } \\
\text { Weight } \\
\text { (g/mol) } \\
\end{array}$ & $\begin{array}{l}\text { Concentration } \\
\text { (M) }\end{array}$ & $\begin{array}{l}\text { weight } \\
\text { required (g) }\end{array}$ \\
\hline Aluminum nitrate, 9-hydrate & $\mathrm{Al}\left(\mathrm{NO}_{3}\right)_{3} \cdot 9 \mathrm{H}_{2} \mathrm{O}$ & 375.0000 & 0.497 & 260.9250 \\
\hline Cadmium nitrate, 4-hydrate & $\mathrm{Cd}\left(\mathrm{NO}_{3}\right)_{2} \cdot 4 \mathrm{H}_{2} \mathrm{O}$ & 308.0000 & 0.000553 & 0.2385 \\
\hline Calcium nitrate, 4-hydrate & $\mathrm{Ca}\left(\mathrm{NO}_{3}\right)_{2} \cdot 4 \mathrm{H}_{2} \mathrm{O}$ & 236.0000 & 0.01 & 3.3040 \\
\hline Cupric nitrate, 2.5 hydrate & $\mathrm{Cu}\left(\mathrm{NO}_{3}\right)_{2} \cdot 2 \cdot 5 \mathrm{H}_{2} \mathrm{O}$ & 233.0000 & 0.000375 & 0.1223 \\
\hline Ferric nitrate, 9-hydrate & $\mathrm{Fe}\left(\mathrm{NO}_{3}\right)_{3} .9 \mathrm{H}_{2} \mathrm{O}$ & 404.0000 & 0.000575 & 0.3252 \\
\hline Lanthanum nitrate, 6-hydrate & $\mathrm{La}\left(\mathrm{NO}_{3}\right)_{3} \cdot 6 \mathrm{H}_{2} \mathrm{O}$ & 433.0000 & 0.0000864 & 0.0524 \\
\hline Lead nitrate & $\mathrm{Pb}\left(\mathrm{NO}_{3}\right)_{2}$ & 331.0000 & 0.000656 & 0.3040 \\
\hline $\begin{array}{c}\text { Manganous chloride, 4- } \\
\text { hydrate }\end{array}$ & $\mathrm{MnCl}_{2} \cdot 4 \mathrm{H}_{2} \mathrm{O}$ & 198.0000 & 0.000324 & 0.0898 \\
\hline Nickel nitrate, 6-hydrate & $\mathrm{Ni}\left(\mathrm{NO}_{3}\right)_{2} \cdot 6 \mathrm{H}_{2} \mathrm{O}$ & 291.0000 & 0.00675 & 2.7500 \\
\hline Potassium nitrate & $\mathrm{K}\left(\mathrm{NO}_{3}\right)$ & 101.0000 & 0.0464 & 6.5610 \\
\hline Disodium EDTA & $\mathrm{Na}_{2} \mathrm{C}_{10} \mathrm{H}_{14} \mathrm{O}_{8} .2 \mathrm{H}_{2} \mathrm{O}$ & 372.0000 & 0.048108 & 25.0546 \\
\hline HEDTA & $\mathrm{C}_{10} \mathrm{H}_{18} \mathrm{~N}_{2} \mathrm{O}_{7}$ & 278.0000 & 0.01899 & 7.3909 \\
\hline Sodium gluconate & $\mathrm{C}_{6} \mathrm{H}_{11} \mathrm{O}_{7} \mathrm{Na}$ & 218.0000 & 0.02532 & 7.7277 \\
\hline Citric acid, 1-hydrate & $\mathrm{C}_{6} \mathrm{H}_{8} \mathrm{O}_{7} \cdot \mathrm{H}_{2} \mathrm{O}$ & 210.0000 & 0.111408 & 32.7540 \\
\hline Nitrilotriacetic Acid & $\mathrm{C}_{6} \mathrm{H}_{9} \mathrm{NO}_{6}$ & 191.0000 & 0.007596 & 2.0312 \\
\hline Iminodiacetic Acid & $\mathrm{C}_{4} \mathrm{H}_{7} \mathrm{NO}_{2}$ & 133.0000 & 0.112674 & 20.9799 \\
\hline Sodium chloride & $\mathrm{NaCl}$ & 58.4000 & 0.106 & 8.6666 \\
\hline Sodium sulfate & $\mathrm{Na}_{2} \mathrm{SO}_{4}$ & 142.0000 & 0.128 & 25.4464 \\
\hline Ammonium Chloride & $\mathrm{NH}_{4} \mathrm{Cl}$ & 55.4920 & 0.00498 & 0.3869 \\
\hline Glycolic acid & $\mathrm{C}_{2} \mathrm{H}_{4} \mathrm{O}_{3}$ & 76.1000 & 0.161 & 17.1529 \\
\hline Sodium hydroxide & $\mathrm{NaOH}$ & 40.0000 & 2.99122604 & 167.5087 \\
\hline $\begin{array}{c}\text { Sodium phosphate, 12- } \\
\text { hydrate }\end{array}$ & $\mathrm{Na}_{3} \mathrm{PO}_{4} 12 \mathrm{H}_{2} \mathrm{O}$ & 380.0000 & 0.0514 & 27.3448 \\
\hline Sodium formate & $\mathrm{Na}\left(\mathrm{CHO}_{2}\right)$ & 68.0000 & 0.233 & 22.1816 \\
\hline Sodium acetate, 3-hydrate & $\mathrm{Na}\left(\mathrm{C}_{2} \mathrm{H}_{3} \mathrm{O}_{2}\right) \cdot 3 \mathrm{H}_{2} \mathrm{O}$ & 136.0000 & 0.0208 & 3.9603 \\
\hline Sodium carbonate & $\mathrm{Na}_{2} \mathrm{CO}_{3}$ & 106.0000 & 1.12 & 166.2080 \\
\hline Sodium nitrate & $\mathrm{NaNO}_{3}$ & 85.0000 & 1.326 & 157.7940 \\
\hline Sodium nitrite & $\mathrm{NaNO}_{2}$ & 69.0000 & 1.94 & 187.4040 \\
\hline
\end{tabular}




\section{Cyclic Potentiodynamic Polarization}

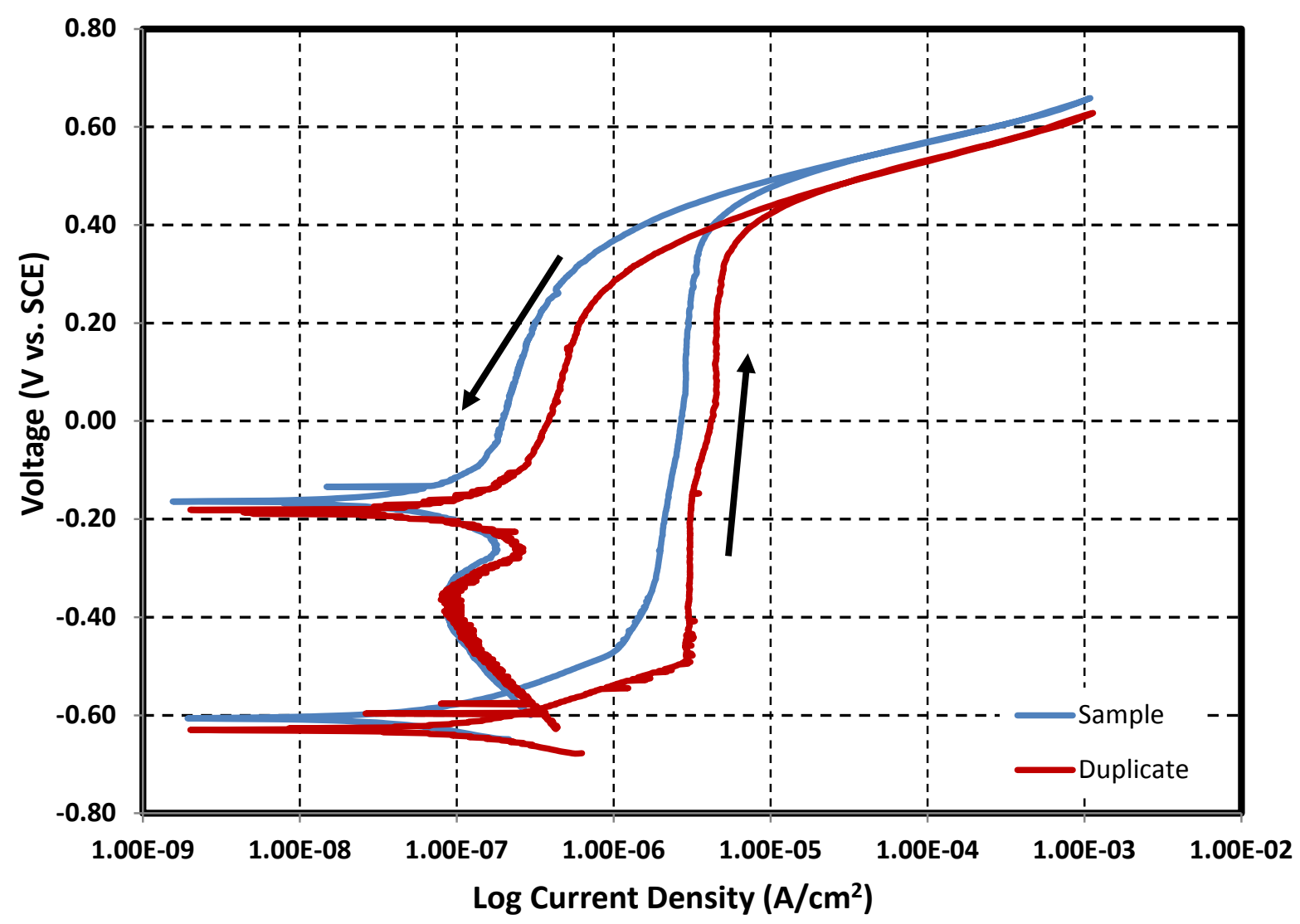

Images of bullet samples after electrochemical tests

Test 8

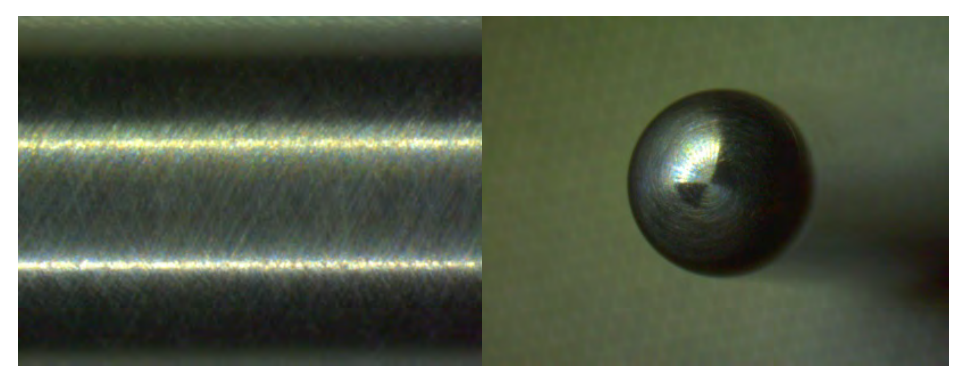

Test 8D

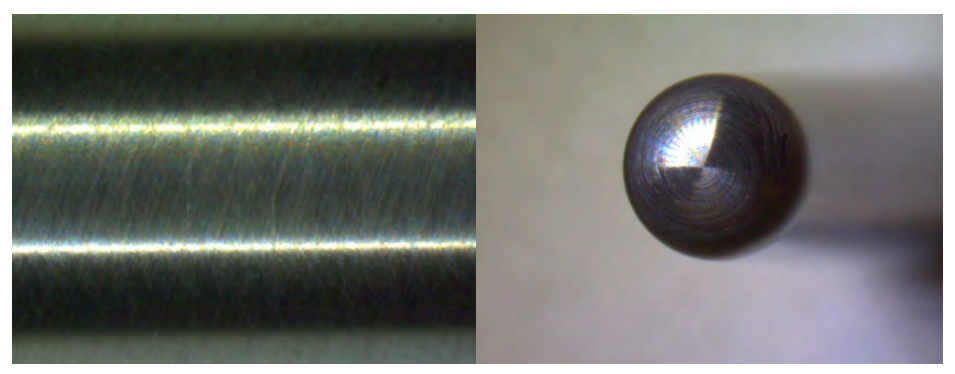

Shank (20X)

Nose (10X) 


\section{Composition of simulant for waste buffering-Test 9}

\section{Test 9}

$\begin{array}{lrl}\text { Temperature } & 30 & { }^{\circ} \mathrm{C} \\ \text { Volume } & 1.4 & \mathrm{~L} \\ \mathrm{pH} & 12.78 & \end{array}$

\begin{tabular}{|c|c|c|c|c|}
\hline Simulant Source & Formula & $\begin{array}{c}\text { Molecular } \\
\text { Weight } \\
\text { (g/mol) }\end{array}$ & $\begin{array}{l}\text { Concentration } \\
\text { (M) }\end{array}$ & $\begin{array}{l}\text { weight } \\
\text { required (g) }\end{array}$ \\
\hline Aluminum nitrate, 9-hydrate & $\mathrm{Al}\left(\mathrm{NO}_{3}\right)_{3} \cdot 9 \mathrm{H}_{2} \mathrm{O}$ & 375.0000 & 0.497 & 260.9250 \\
\hline Cadmium nitrate, 4-hydrate & $\mathrm{Cd}\left(\mathrm{NO}_{3}\right)_{2} \cdot 4 \mathrm{H}_{2} \mathrm{O}$ & 308.0000 & 0.000553 & 0.2385 \\
\hline Calcium nitrate, 4-hydrate & $\mathrm{Ca}\left(\mathrm{NO}_{3}\right)_{2} \cdot 4 \mathrm{H}_{2} \mathrm{O}$ & 236.0000 & 0.01 & 3.3040 \\
\hline Cupric nitrate, 2.5 hydrate & $\mathrm{Cu}\left(\mathrm{NO}_{3}\right)_{2} \cdot 2 \cdot 5 \mathrm{H}_{2} \mathrm{O}$ & 233.0000 & 0.000375 & 0.1223 \\
\hline Ferric nitrate, 9-hydrate & $\mathrm{Fe}\left(\mathrm{NO}_{3}\right)_{3} .9 \mathrm{H}_{2} \mathrm{O}$ & 404.0000 & 0.000575 & 0.3252 \\
\hline Lanthanum nitrate, 6 -hydrate & $\mathrm{La}\left(\mathrm{NO}_{3}\right)_{3} \cdot 6 \mathrm{H}_{2} \mathrm{O}$ & 433.0000 & 0.0000864 & 0.0524 \\
\hline Lead nitrate & $\mathrm{Pb}\left(\mathrm{NO}_{3}\right)_{2}$ & 331.0000 & 0.000656 & 0.3040 \\
\hline $\begin{array}{c}\text { Manganous chloride, 4- } \\
\text { hydrate }\end{array}$ & $\mathrm{MnCl}_{2} \cdot 4 \mathrm{H}_{2} \mathrm{O}$ & 198.0000 & 0.000324 & 0.0898 \\
\hline Nickel nitrate, 6-hydrate & $\mathrm{Ni}\left(\mathrm{NO}_{3}\right)_{2} \cdot 6 \mathrm{H}_{2} \mathrm{O}$ & 291.0000 & 0.00675 & 2.7500 \\
\hline Potassium nitrate & $\mathrm{K}\left(\mathrm{NO}_{3}\right)$ & 101.0000 & 0.0464 & 6.5610 \\
\hline Disodium EDTA & $\mathrm{Na}_{2} \mathrm{C}_{10} \mathrm{H}_{14} \mathrm{O}_{8} .2 \mathrm{H}_{2} \mathrm{O}$ & 372.0000 & 0.048108 & 25.0546 \\
\hline HEDTA & $\mathrm{C}_{10} \mathrm{H}_{18} \mathrm{~N}_{2} \mathrm{O}_{7}$ & 278.0000 & 0.01899 & 7.3909 \\
\hline Sodium gluconate & $\mathrm{C}_{6} \mathrm{H}_{11} \mathrm{O}_{7} \mathrm{Na}$ & 218.0000 & 0.02532 & 7.7277 \\
\hline Citric acid, 1-hydrate & $\mathrm{C}_{6} \mathrm{H}_{8} \mathrm{O}_{7} \cdot \mathrm{H}_{2} \mathrm{O}$ & 210.0000 & 0.111408 & 32.7540 \\
\hline Nitrilotriacetic Acid & $\mathrm{C}_{6} \mathrm{H}_{9} \mathrm{NO}_{6}$ & 191.0000 & 0.007596 & 2.0312 \\
\hline Iminodiacetic Acid & $\mathrm{C}_{4} \mathrm{H}_{7} \mathrm{NO}_{2}$ & 133.0000 & 0.112674 & 20.9799 \\
\hline Sodium chloride & $\mathrm{NaCl}$ & 58.4000 & 0.106 & 8.6666 \\
\hline Sodium sulfate & $\mathrm{Na}_{2} \mathrm{SO}_{4}$ & 142.0000 & 0.128 & 25.4464 \\
\hline Ammonium Chloride & $\mathrm{NH}_{4} \mathrm{Cl}$ & 55.4920 & 0.00498 & 0.3869 \\
\hline Glycolic acid & $\mathrm{C}_{2} \mathrm{H}_{4} \mathrm{O}_{3}$ & 76.1000 & 0.161 & 17.1529 \\
\hline Sodium hydroxide & $\mathrm{NaOH}$ & 40.0000 & 2.98122604 & 166.9487 \\
\hline $\begin{array}{c}\text { Sodium phosphate, 12- } \\
\text { hydrate } \\
\end{array}$ & $\mathrm{Na}_{3} \mathrm{PO}_{4} \cdot 12 \mathrm{H}_{2} \mathrm{O}$ & 380.0000 & 0.0514 & 27.3448 \\
\hline Sodium formate & $\mathrm{Na}\left(\mathrm{CHO}_{2}\right)$ & 68.0000 & 0.233 & 22.1816 \\
\hline Sodium acetate, 3-hydrate & $\mathrm{Na}\left(\mathrm{C}_{2} \mathrm{H}_{3} \mathrm{O}_{2}\right) \cdot 3 \mathrm{H}_{2} \mathrm{O}$ & 136.0000 & 0.0208 & 3.9603 \\
\hline Sodium carbonate & $\mathrm{Na}_{2} \mathrm{CO}_{3}$ & 106.0000 & 1.12 & 166.2080 \\
\hline Sodium nitrate & $\mathrm{NaNO}_{3}$ & 85.0000 & 2.196 & 261.3240 \\
\hline Sodium nitrite & $\mathrm{NaNO}_{2}$ & 69.0000 & 2.24 & 216.3840 \\
\hline
\end{tabular}




\section{Cyclic Potentiodynamic Polarization}

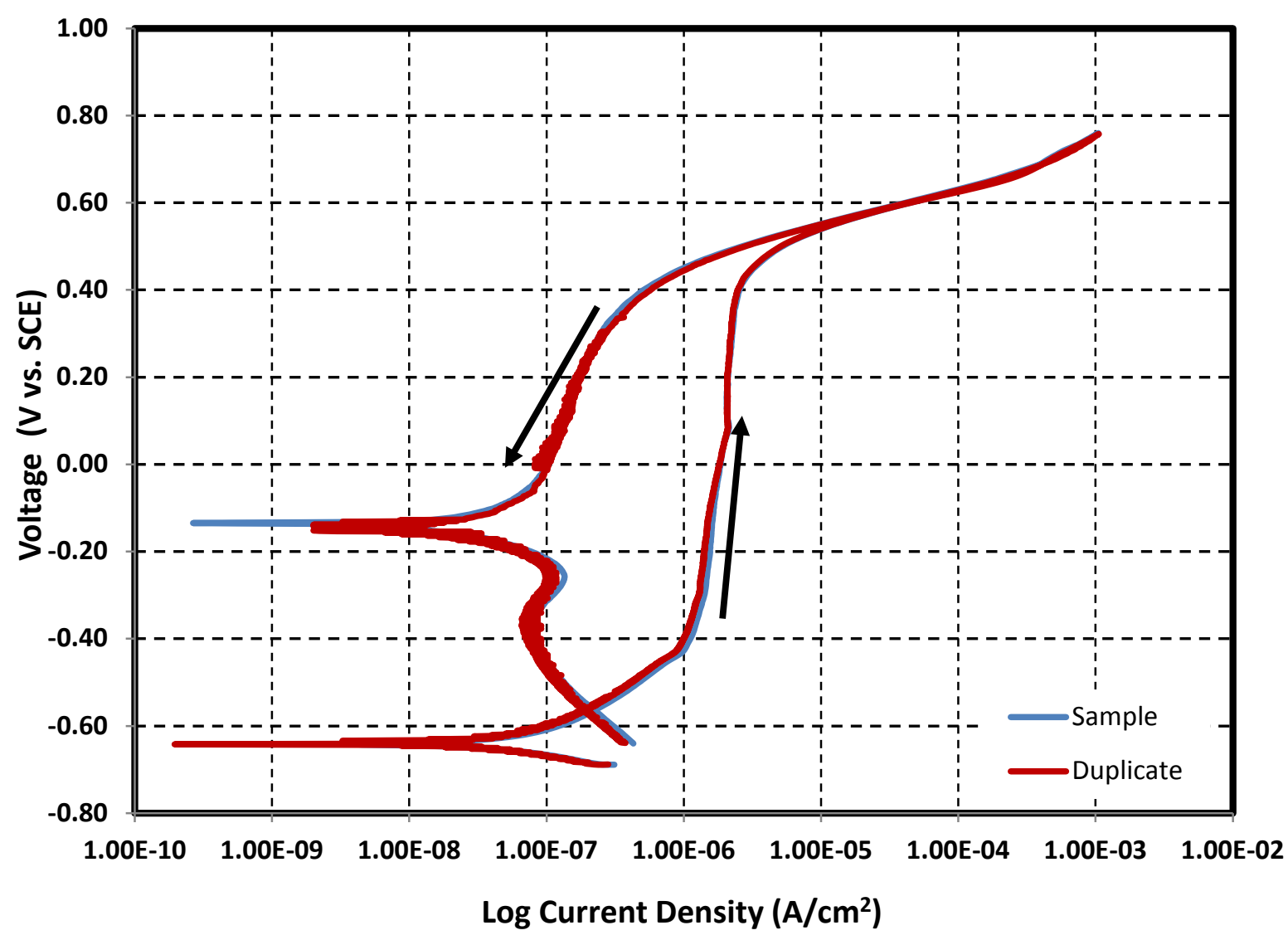

Images of bullet samples after electrochemical tests

Test 9

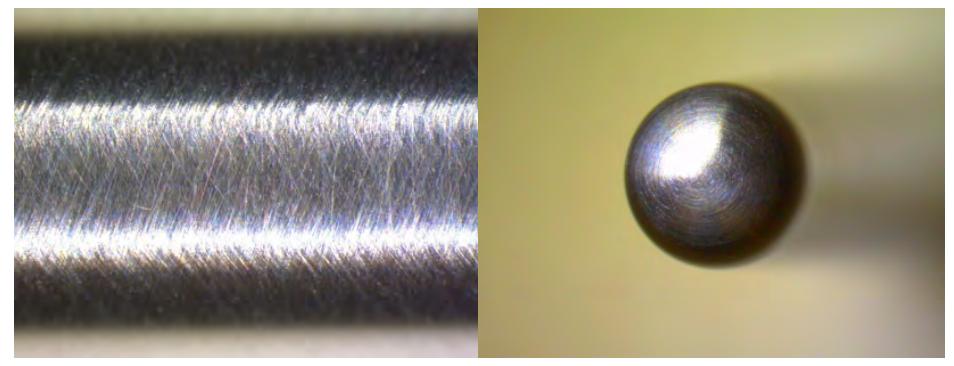

Test 9D

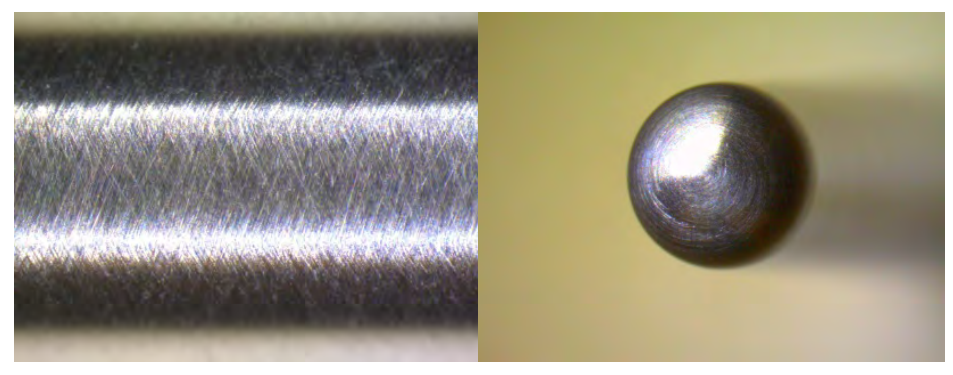

Shank $(20 X)$

Nose (10X) 


\section{Composition of simulant for waste buffering-Test 10}

\section{Test 10}

\begin{tabular}{|c|c|c|c|c|}
\hline $\begin{array}{l}\text { Temperature } \\
\text { Volume } \\
\mathrm{pH}\end{array}$ & $\begin{array}{r}40 \\
1.4 \\
12.78\end{array}$ & $\begin{array}{l}{ }^{\circ} \mathrm{C} \\
\mathrm{L}\end{array}$ & & \\
\hline Simulant Source & Formula & $\begin{array}{c}\text { Molecular } \\
\text { Weight } \\
\text { (g/mol) } \\
\end{array}$ & $\begin{array}{l}\text { Concentration } \\
\text { (M) }\end{array}$ & $\begin{array}{l}\text { weight } \\
\text { required }(g)\end{array}$ \\
\hline Aluminum nitrate, 9-hydrate & $\mathrm{Al}\left(\mathrm{NO}_{3}\right)_{3} \cdot 9 \mathrm{H}_{2} \mathrm{O}$ & 375.0000 & 0.497 & 260.9250 \\
\hline Cadmium nitrate, 4-hydrate & $\mathrm{Cd}\left(\mathrm{NO}_{3}\right)_{2} \cdot 4 \mathrm{H}_{2} \mathrm{O}$ & 308.0000 & 0.000553 & 0.2385 \\
\hline Calcium nitrate, 4-hydrate & $\mathrm{Ca}\left(\mathrm{NO}_{3}\right)_{2} \cdot 4 \mathrm{H}_{2} \mathrm{O}$ & 236.0000 & 0.01 & 3.3040 \\
\hline Cupric nitrate, 2.5 hydrate & $\mathrm{Cu}\left(\mathrm{NO}_{3}\right)_{2} \cdot 2.5 \mathrm{H}_{2} \mathrm{O}$ & 233.0000 & 0.000375 & 0.1223 \\
\hline Ferric nitrate, 9-hydrate & $\mathrm{Fe}\left(\mathrm{NO}_{3}\right)_{3} .9 \mathrm{H}_{2} \mathrm{O}$ & 404.0000 & 0.000575 & 0.3252 \\
\hline Lanthanum nitrate, 6-hydrate & $\mathrm{La}\left(\mathrm{NO}_{3}\right)_{3} \cdot 6 \mathrm{H}_{2} \mathrm{O}$ & 433.0000 & 0.0000864 & 0.0524 \\
\hline Lead nitrate & $\mathrm{Pb}\left(\mathrm{NO}_{3}\right)_{2}$ & 331.0000 & 0.000656 & 0.3040 \\
\hline $\begin{array}{c}\text { Manganous chloride, 4- } \\
\text { hydrate }\end{array}$ & $\mathrm{MnCl}_{2} \cdot 4 \mathrm{H}_{2} \mathrm{O}$ & 198.0000 & 0.000324 & 0.0898 \\
\hline Nickel nitrate, 6-hydrate & $\mathrm{Ni}\left(\mathrm{NO}_{3}\right)_{2} \cdot 6 \mathrm{H}_{2} \mathrm{O}$ & 291.0000 & 0.00675 & 2.7500 \\
\hline Potassium nitrate & $\mathrm{K}\left(\mathrm{NO}_{3}\right)$ & 101.0000 & 0.0464 & 6.5610 \\
\hline Disodium EDTA & $\mathrm{Na}_{2} \mathrm{C}_{10} \mathrm{H}_{14} \mathrm{O}_{8} .2 \mathrm{H}_{2} \mathrm{O}$ & 372.0000 & 0.048108 & 25.0546 \\
\hline HEDTA & $\mathrm{C}_{10} \mathrm{H}_{18} \mathrm{~N}_{2} \mathrm{O}_{7}$ & 278.0000 & 0.01899 & 7.3909 \\
\hline Sodium gluconate & $\mathrm{C}_{6} \mathrm{H}_{11} \mathrm{O}_{7} \mathrm{Na}$ & 218.0000 & 0.02532 & 7.7277 \\
\hline Citric acid, 1-hydrate & $\mathrm{C}_{6} \mathrm{H}_{8} \mathrm{O}_{7} \cdot \mathrm{H}_{2} \mathrm{O}$ & 210.0000 & 0.111408 & 32.7540 \\
\hline Nitrilotriacetic Acid & $\mathrm{C}_{6} \mathrm{H}_{9} \mathrm{NO}_{6}$ & 191.0000 & 0.007596 & 2.0312 \\
\hline Iminodiacetic Acid & $\mathrm{C}_{4} \mathrm{H}_{7} \mathrm{NO}_{2}$ & 133.0000 & 0.112674 & 20.9799 \\
\hline Sodium chloride & $\mathrm{NaCl}$ & 58.4000 & 0.106 & 8.6666 \\
\hline Sodium sulfate & $\mathrm{Na}_{2} \mathrm{SO}_{4}$ & 142.0000 & 0.128 & 25.4464 \\
\hline Ammonium Chloride & $\mathrm{NH}_{4} \mathrm{Cl}$ & 55.4920 & 0.00498 & 0.3869 \\
\hline Glycolic acid & $\mathrm{C}_{2} \mathrm{H}_{4} \mathrm{O}_{3}$ & 76.1000 & 0.161 & 17.1529 \\
\hline Sodium hydroxide & $\mathrm{NaOH}$ & 40.0000 & 2.98122604 & 166.9487 \\
\hline $\begin{array}{c}\text { Sodium phosphate, 12- } \\
\text { hydrate }\end{array}$ & $\mathrm{Na}_{3} \mathrm{PO}_{4} 12 \mathrm{H}_{2} \mathrm{O}$ & 380.0000 & 0.0514 & 27.3448 \\
\hline Sodium formate & $\mathrm{Na}\left(\mathrm{CHO}_{2}\right)$ & 68.0000 & 0.233 & 22.1816 \\
\hline Sodium acetate, 3-hydrate & $\mathrm{Na}\left(\mathrm{C}_{2} \mathrm{H}_{3} \mathrm{O}_{2}\right) \cdot 3 \mathrm{H}_{2} \mathrm{O}$ & 136.0000 & 0.0208 & 3.9603 \\
\hline Sodium carbonate & $\mathrm{Na}_{2} \mathrm{CO}_{3}$ & 106.0000 & 1.12 & 166.2080 \\
\hline Sodium nitrate & $\mathrm{NaNO}_{3}$ & 85.0000 & 2.196 & 261.3240 \\
\hline Sodium nitrite & $\mathrm{NaNO}_{2}$ & 69.0000 & 2.24 & 216.3840 \\
\hline
\end{tabular}




\section{Cyclic Potentiodynamic Polarization}

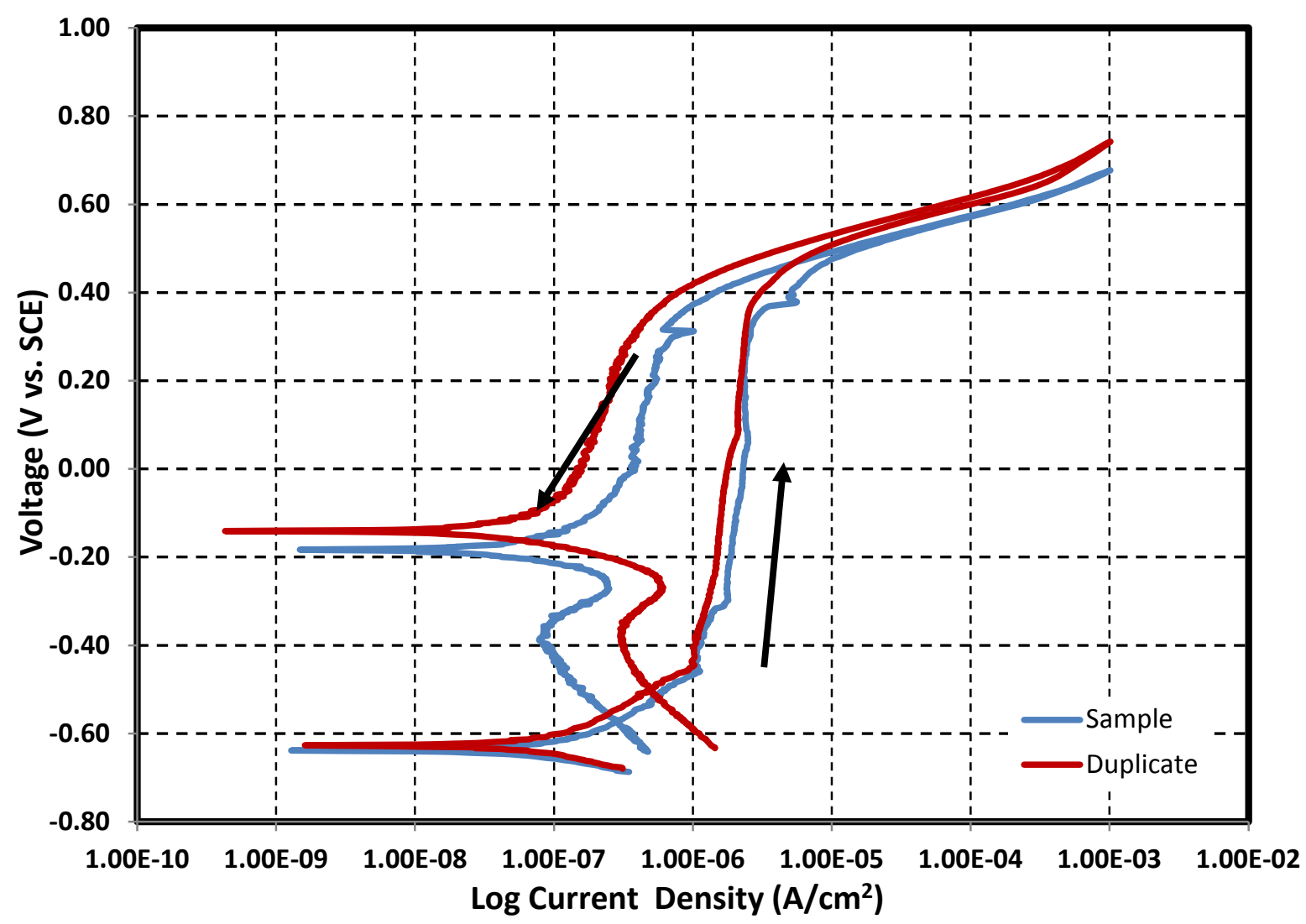

Images of bullet samples after electrochemical tests

Test 10

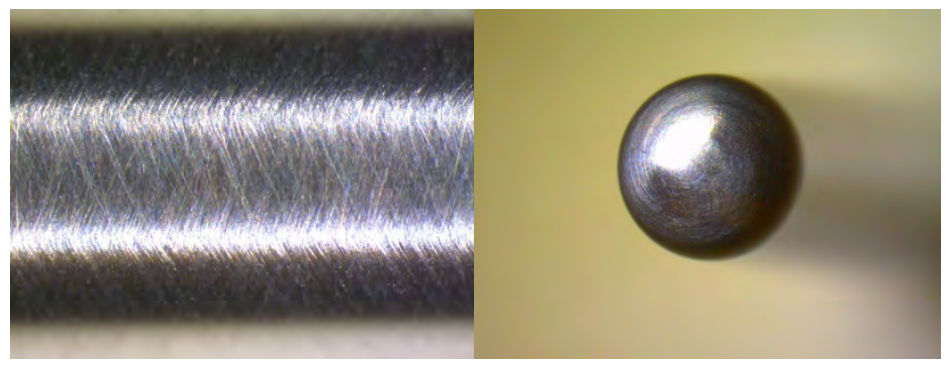

Test 10D

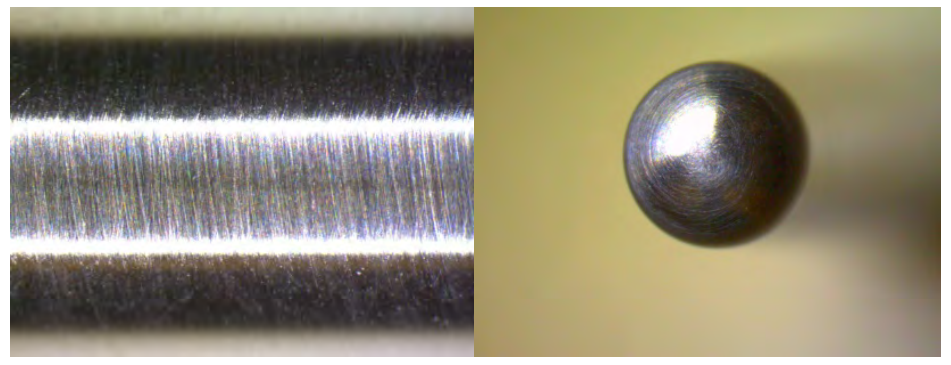

Shank (20X)

Nose (10X) 


\section{Composition of simulant for waste buffering-Test 11}

\section{Test 11}

\begin{tabular}{|c|c|c|c|c|}
\hline $\begin{array}{l}\text { Temperature } \\
\text { Volume } \\
\mathrm{pH}\end{array}$ & $\begin{array}{r}50 \\
1.4 \\
12.78\end{array}$ & $\begin{array}{l}{ }^{\circ} \mathrm{C} \\
\mathrm{L}\end{array}$ & & \\
\hline Simulant Source & Formula & $\begin{array}{c}\text { Molecular } \\
\text { Weight } \\
\text { (g/mol) } \\
\end{array}$ & $\begin{array}{l}\text { Concentration } \\
\text { (M) }\end{array}$ & $\begin{array}{l}\text { weight } \\
\text { required }(g)\end{array}$ \\
\hline Aluminum nitrate, 9-hydrate & $\mathrm{Al}\left(\mathrm{NO}_{3}\right)_{3} \cdot 9 \mathrm{H}_{2} \mathrm{O}$ & 375.0000 & 0.497 & 260.9250 \\
\hline Cadmium nitrate, 4-hydrate & $\mathrm{Cd}\left(\mathrm{NO}_{3}\right)_{2} \cdot 4 \mathrm{H}_{2} \mathrm{O}$ & 308.0000 & 0.000553 & 0.2385 \\
\hline Calcium nitrate, 4-hydrate & $\mathrm{Ca}\left(\mathrm{NO}_{3}\right)_{2} \cdot 4 \mathrm{H}_{2} \mathrm{O}$ & 236.0000 & 0.01 & 3.3040 \\
\hline Cupric nitrate, 2.5 hydrate & $\mathrm{Cu}\left(\mathrm{NO}_{3}\right)_{2} \cdot 2.5 \mathrm{H}_{2} \mathrm{O}$ & 233.0000 & 0.000375 & 0.1223 \\
\hline Ferric nitrate, 9-hydrate & $\mathrm{Fe}\left(\mathrm{NO}_{3}\right)_{3} \cdot 9 \mathrm{H}_{2} \mathrm{O}$ & 404.0000 & 0.000575 & 0.3252 \\
\hline Lanthanum nitrate, 6-hydrate & $\mathrm{La}\left(\mathrm{NO}_{3}\right)_{3} \cdot 6 \mathrm{H}_{2} \mathrm{O}$ & 433.0000 & 0.0000864 & 0.0524 \\
\hline Lead nitrate & $\mathrm{Pb}\left(\mathrm{NO}_{3}\right)_{2}$ & 331.0000 & 0.000656 & 0.3040 \\
\hline $\begin{array}{c}\text { Manganous chloride, 4- } \\
\text { hydrate }\end{array}$ & $\mathrm{MnCl}_{2} \cdot 4 \mathrm{H}_{2} \mathrm{O}$ & 198.0000 & 0.000324 & 0.0898 \\
\hline Nickel nitrate, 6-hydrate & $\mathrm{Ni}\left(\mathrm{NO}_{3}\right)_{2} \cdot 6 \mathrm{H}_{2} \mathrm{O}$ & 291.0000 & 0.00675 & 2.7500 \\
\hline Potassium nitrate & $\mathrm{K}\left(\mathrm{NO}_{3}\right)$ & 101.0000 & 0.0464 & 6.5610 \\
\hline Disodium EDTA & $\mathrm{Na}_{2} \mathrm{C}_{10} \mathrm{H}_{14} \mathrm{O}_{8} .2 \mathrm{H}_{2} \mathrm{O}$ & 372.0000 & 0.048108 & 25.0546 \\
\hline HEDTA & $\mathrm{C}_{10} \mathrm{H}_{18} \mathrm{~N}_{2} \mathrm{O}_{7}$ & 278.0000 & 0.01899 & 7.3909 \\
\hline Sodium gluconate & $\mathrm{C}_{6} \mathrm{H}_{11} \mathrm{O}_{7} \mathrm{Na}$ & 218.0000 & 0.02532 & 7.7277 \\
\hline Citric acid, 1-hydrate & $\mathrm{C}_{6} \mathrm{H}_{8} \mathrm{O}_{7} \cdot \mathrm{H}_{2} \mathrm{O}$ & 210.0000 & 0.111408 & 32.7540 \\
\hline Nitrilotriacetic Acid & $\mathrm{C}_{6} \mathrm{H}_{9} \mathrm{NO}_{6}$ & 191.0000 & 0.007596 & 2.0312 \\
\hline Iminodiacetic Acid & $\mathrm{C}_{4} \mathrm{H}_{7} \mathrm{NO}_{2}$ & 133.0000 & 0.112674 & 20.9799 \\
\hline Sodium chloride & $\mathrm{NaCl}$ & 58.4000 & 0.106 & 8.6666 \\
\hline Sodium sulfate & $\mathrm{Na}_{2} \mathrm{SO}_{4}$ & 142.0000 & 0.128 & 25.4464 \\
\hline Ammonium Chloride & $\mathrm{NH}_{4} \mathrm{Cl}$ & 55.4920 & 0.00498 & 0.3869 \\
\hline Glycolic acid & $\mathrm{C}_{2} \mathrm{H}_{4} \mathrm{O}_{3}$ & 76.1000 & 0.161 & 17.1529 \\
\hline Sodium hydroxide & $\mathrm{NaOH}$ & 40.0000 & 2.98122604 & 166.9487 \\
\hline $\begin{array}{c}\text { Sodium phosphate, 12- } \\
\text { hydrate }\end{array}$ & $\mathrm{Na}_{3} \mathrm{PO}_{4} 12 \mathrm{H}_{2} \mathrm{O}$ & 380.0000 & 0.0514 & 27.3448 \\
\hline Sodium formate & $\mathrm{Na}\left(\mathrm{CHO}_{2}\right)$ & 68.0000 & 0.233 & 22.1816 \\
\hline Sodium acetate, 3-hydrate & $\mathrm{Na}\left(\mathrm{C}_{2} \mathrm{H}_{3} \mathrm{O}_{2}\right) \cdot 3 \mathrm{H}_{2} \mathrm{O}$ & 136.0000 & 0.0208 & 3.9603 \\
\hline Sodium carbonate & $\mathrm{Na}_{2} \mathrm{CO}_{3}$ & 106.0000 & 1.12 & 166.2080 \\
\hline Sodium nitrate & $\mathrm{NaNO}_{3}$ & 85.0000 & 2.196 & 261.3240 \\
\hline Sodium nitrite & $\mathrm{NaNO}_{2}$ & 69.0000 & 2.24 & 216.3840 \\
\hline
\end{tabular}




\section{Cyclic Potentiodynamic Polarization}

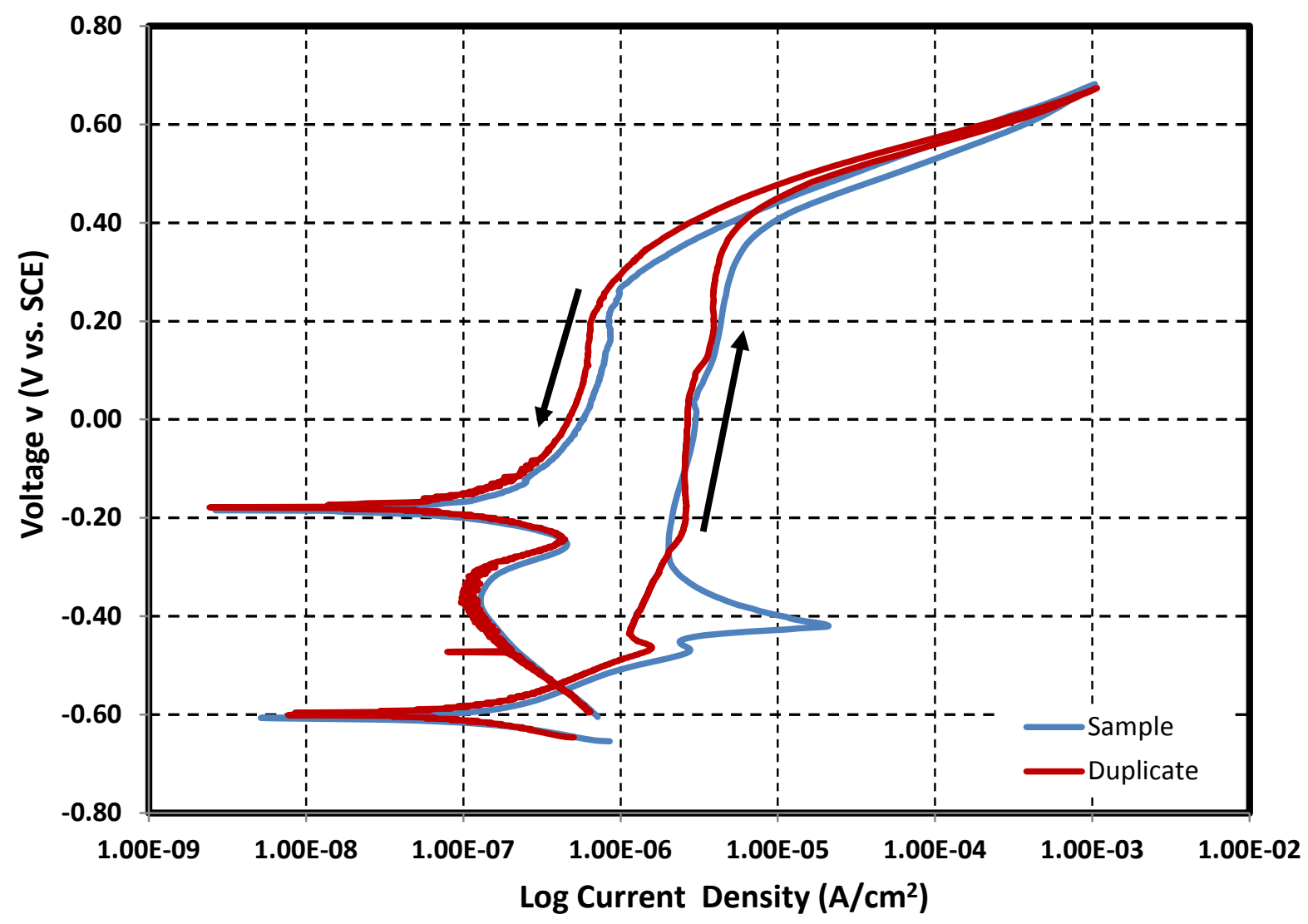

Images of bullet samples after electrochemical tests

Test 11

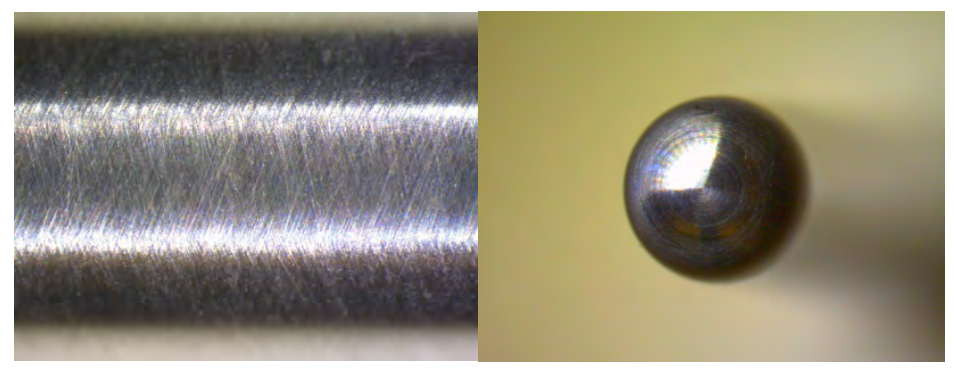

Test 11D

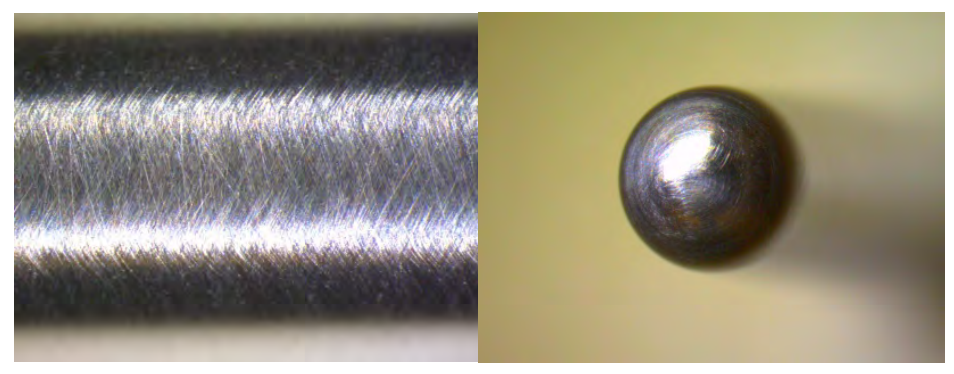

Shank (20X)

Nose (10X) 


\section{Composition of simulant for waste buffering-Test 12}

\section{Test 12}

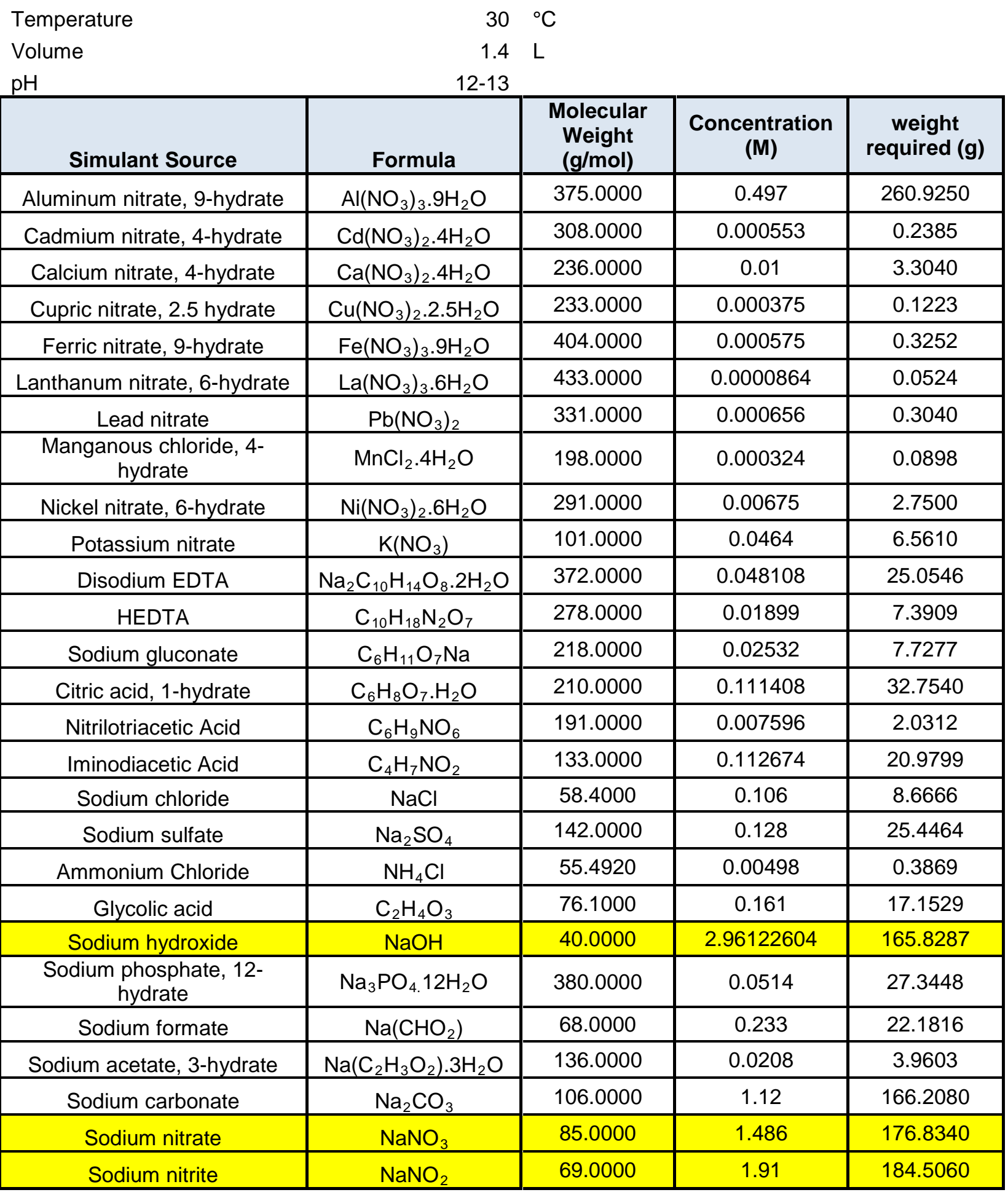




\section{Cyclic Potentiodynamic Polarization}

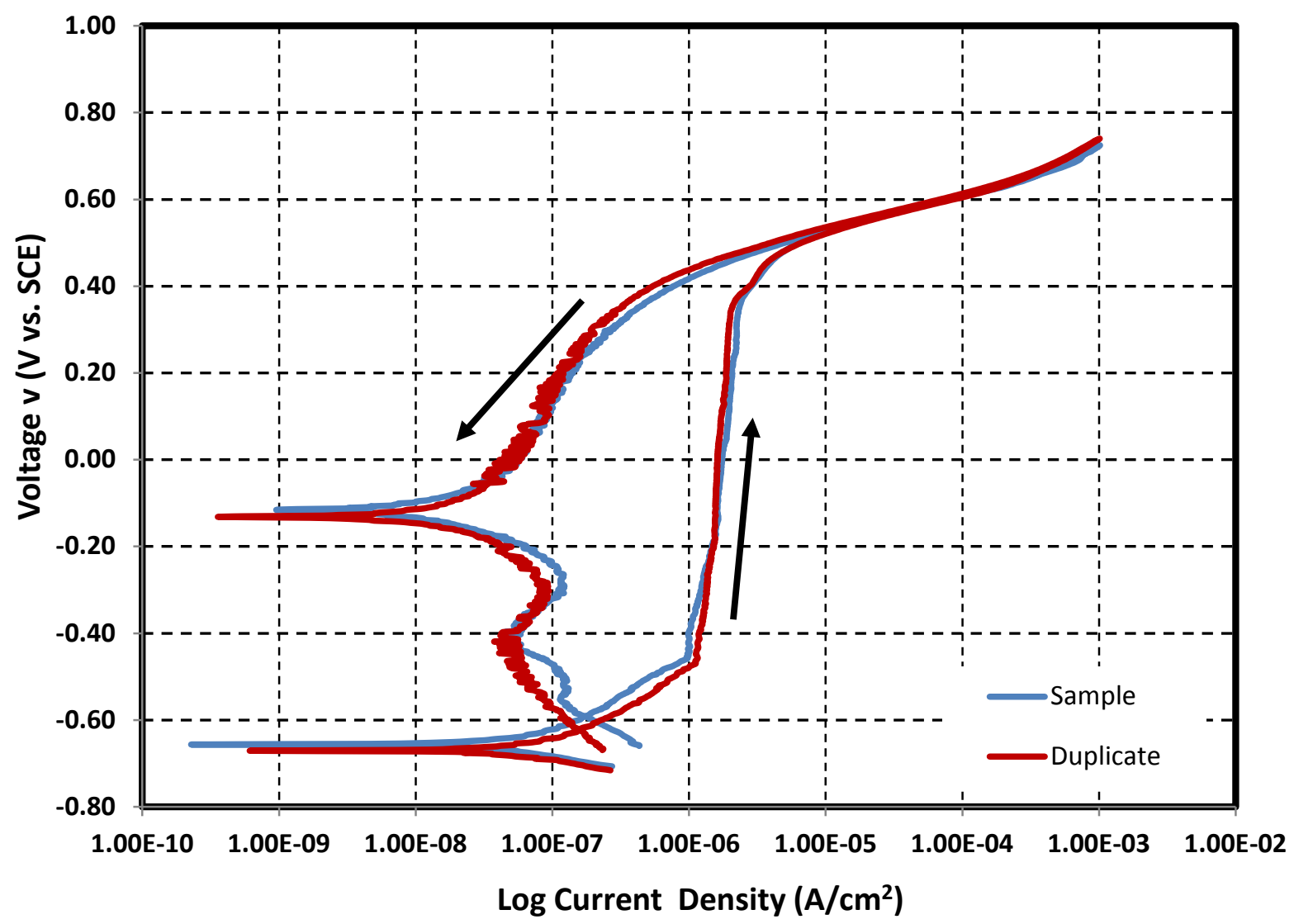

Images of bullet samples after electrochemical tests

Test 12

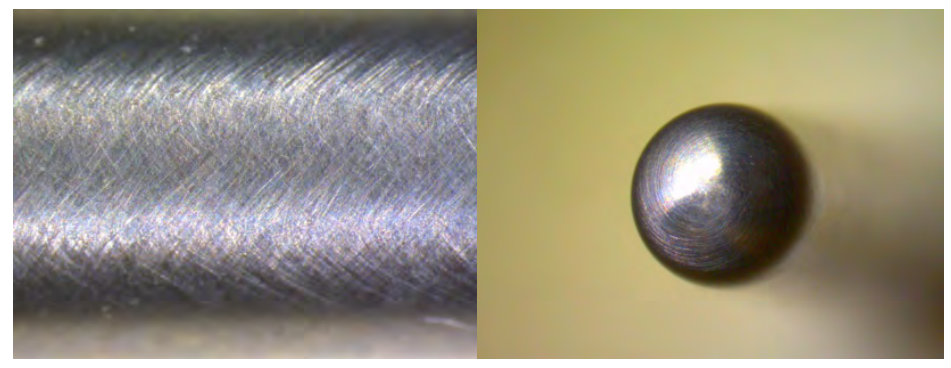

Test 12D

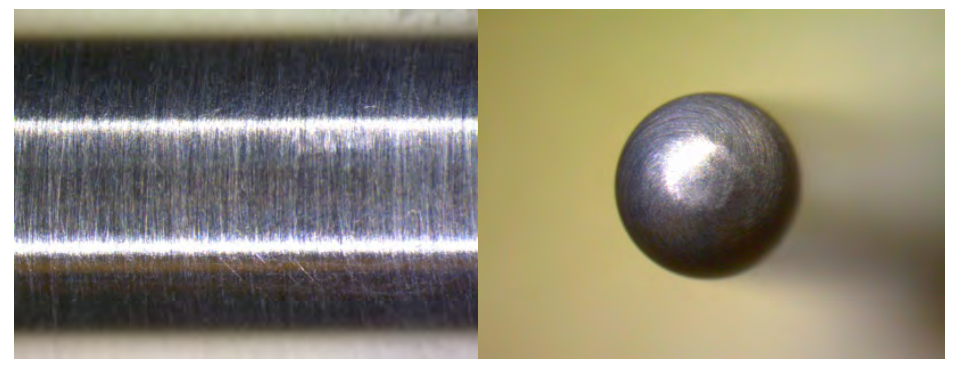

Shank $(20 X)$

Nose (10X) 


\section{Composition of simulant for waste buffering-Test 13}

\section{Test 13}

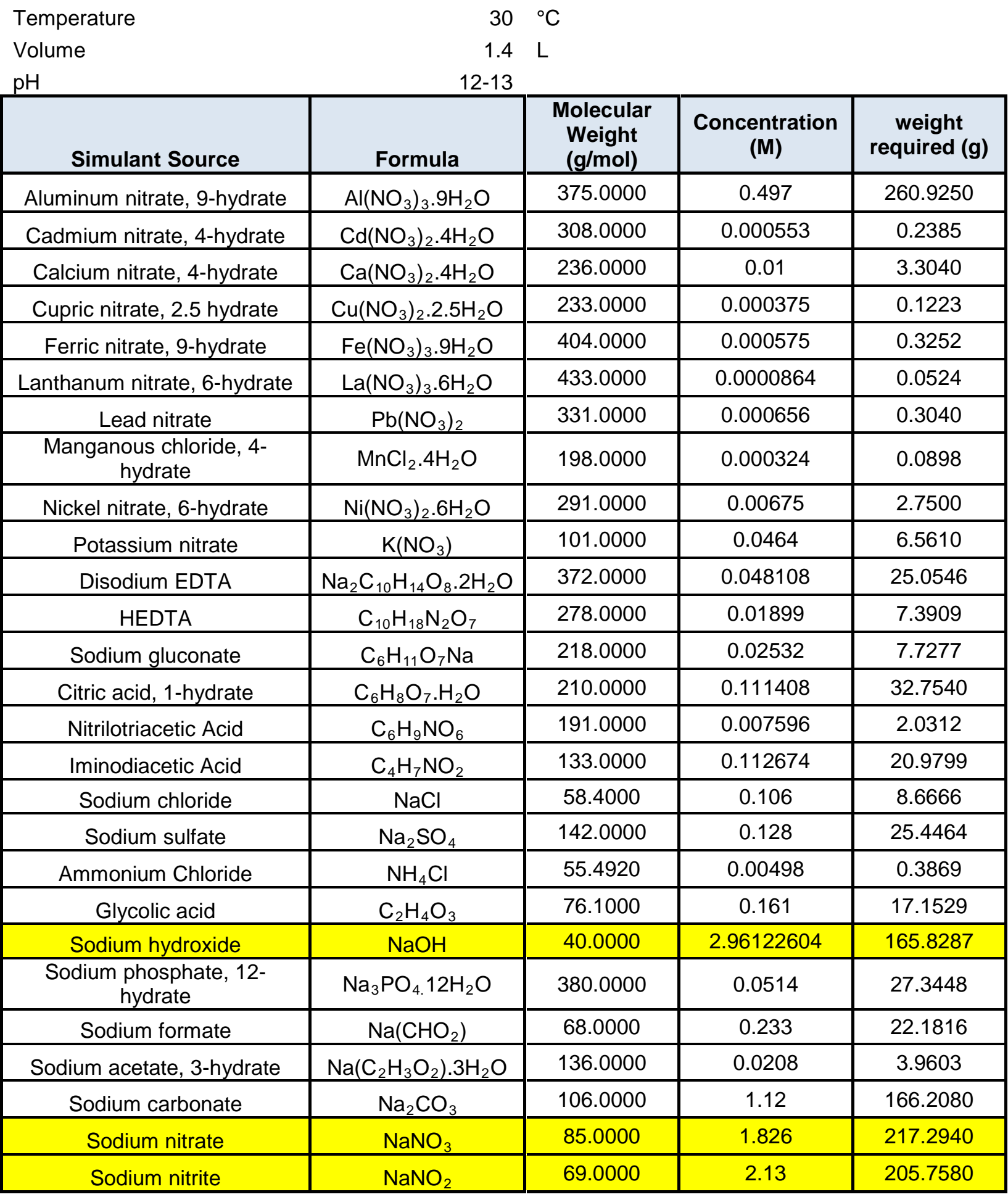




\section{Cyclic Potentiodynamic Polarization}

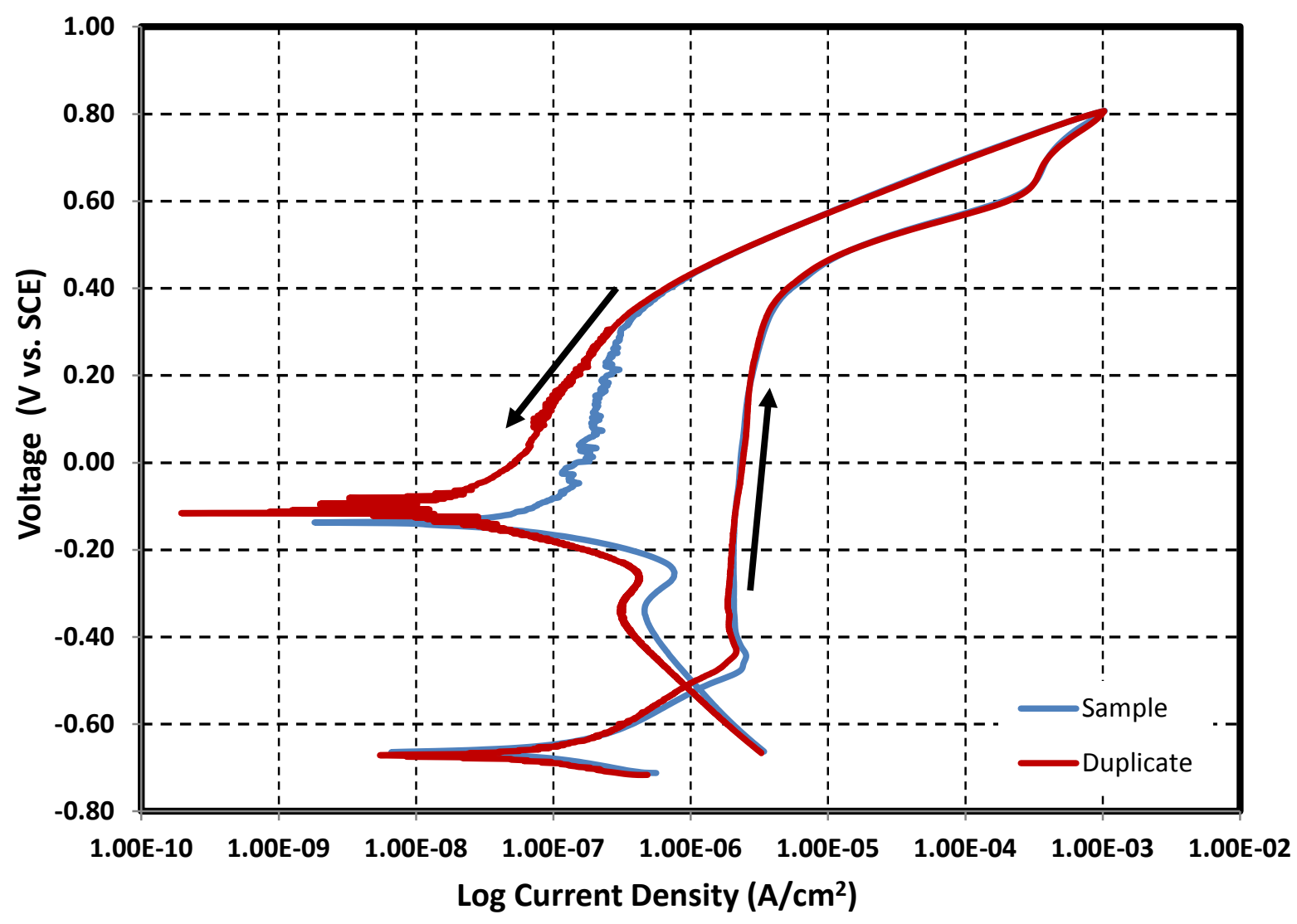

Images of bullet samples after electrochemical tests

Test 13

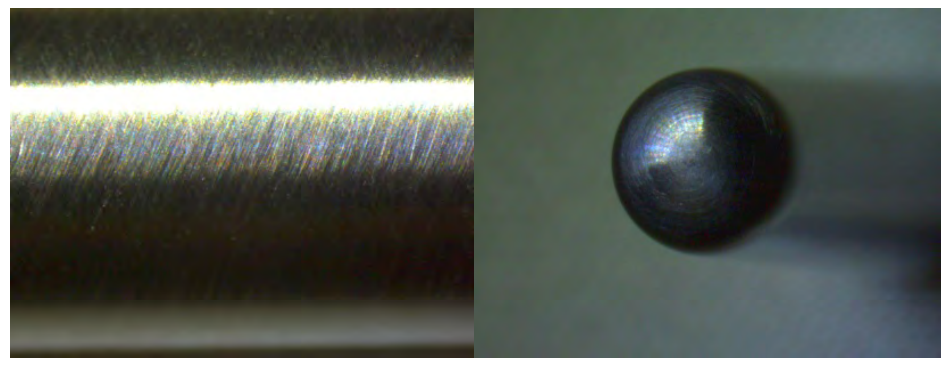

Test 13D

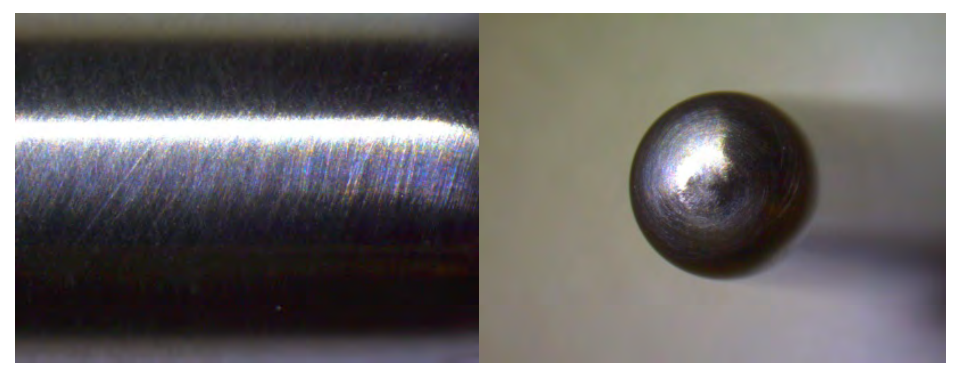

Shank $(20 X)$

Nose (10X) 


\section{Composition of simulant for waste buffering-Test 14}

\section{Test 14}

\begin{tabular}{|c|c|c|c|c|}
\hline $\begin{array}{l}\text { Temperature } \\
\text { Volume } \\
\mathrm{pH}\end{array}$ & $\begin{array}{r}40 \\
1.4 \\
12-13\end{array}$ & $\begin{array}{l}{ }^{\circ} \mathrm{C} \\
\mathrm{L}\end{array}$ & & \\
\hline Simulant Source & Formula & $\begin{array}{c}\text { Molecular } \\
\text { Weight } \\
\text { (g/mol) } \\
\end{array}$ & $\begin{array}{l}\text { Concentration } \\
\text { (M) }\end{array}$ & $\begin{array}{l}\text { weight } \\
\text { required }(g)\end{array}$ \\
\hline Aluminum nitrate, 9-hydrate & $\mathrm{Al}\left(\mathrm{NO}_{3}\right)_{3} \cdot 9 \mathrm{H}_{2} \mathrm{O}$ & 375.0000 & 0.497 & 260.9250 \\
\hline Cadmium nitrate, 4-hydrate & $\mathrm{Cd}\left(\mathrm{NO}_{3}\right)_{2} \cdot 4 \mathrm{H}_{2} \mathrm{O}$ & 308.0000 & 0.000553 & 0.2385 \\
\hline Calcium nitrate, 4-hydrate & $\mathrm{Ca}\left(\mathrm{NO}_{3}\right)_{2} \cdot 4 \mathrm{H}_{2} \mathrm{O}$ & 236.0000 & 0.01 & 3.3040 \\
\hline Cupric nitrate, 2.5 hydrate & $\mathrm{Cu}\left(\mathrm{NO}_{3}\right)_{2} \cdot 2.5 \mathrm{H}_{2} \mathrm{O}$ & 233.0000 & 0.000375 & 0.1223 \\
\hline Ferric nitrate, 9-hydrate & $\mathrm{Fe}\left(\mathrm{NO}_{3}\right)_{3} \cdot 9 \mathrm{H}_{2} \mathrm{O}$ & 404.0000 & 0.000575 & 0.3252 \\
\hline Lanthanum nitrate, 6-hydrate & $\mathrm{La}\left(\mathrm{NO}_{3}\right)_{3} \cdot 6 \mathrm{H}_{2} \mathrm{O}$ & 433.0000 & 0.0000864 & 0.0524 \\
\hline Lead nitrate & $\mathrm{Pb}\left(\mathrm{NO}_{3}\right)_{2}$ & 331.0000 & 0.000656 & 0.3040 \\
\hline $\begin{array}{c}\text { Manganous chloride, 4- } \\
\text { hydrate }\end{array}$ & $\mathrm{MnCl}_{2} \cdot 4 \mathrm{H}_{2} \mathrm{O}$ & 198.0000 & 0.000324 & 0.0898 \\
\hline Nickel nitrate, 6-hydrate & $\mathrm{Ni}\left(\mathrm{NO}_{3}\right)_{2} \cdot 6 \mathrm{H}_{2} \mathrm{O}$ & 291.0000 & 0.00675 & 2.7500 \\
\hline Potassium nitrate & $\mathrm{K}\left(\mathrm{NO}_{3}\right)$ & 101.0000 & 0.0464 & 6.5610 \\
\hline Disodium EDTA & $\mathrm{Na}_{2} \mathrm{C}_{10} \mathrm{H}_{14} \mathrm{O}_{8} .2 \mathrm{H}_{2} \mathrm{O}$ & 372.0000 & 0.048108 & 25.0546 \\
\hline HEDTA & $\mathrm{C}_{10} \mathrm{H}_{18} \mathrm{~N}_{2} \mathrm{O}_{7}$ & 278.0000 & 0.01899 & 7.3909 \\
\hline Sodium gluconate & $\mathrm{C}_{6} \mathrm{H}_{11} \mathrm{O}_{7} \mathrm{Na}$ & 218.0000 & 0.02532 & 7.7277 \\
\hline Citric acid, 1-hydrate & $\mathrm{C}_{6} \mathrm{H}_{8} \mathrm{O}_{7} \cdot \mathrm{H}_{2} \mathrm{O}$ & 210.0000 & 0.111408 & 32.7540 \\
\hline Nitrilotriacetic Acid & $\mathrm{C}_{6} \mathrm{H}_{9} \mathrm{NO}_{6}$ & 191.0000 & 0.007596 & 2.0312 \\
\hline Iminodiacetic Acid & $\mathrm{C}_{4} \mathrm{H}_{7} \mathrm{NO}_{2}$ & 133.0000 & 0.112674 & 20.9799 \\
\hline Sodium chloride & $\mathrm{NaCl}$ & 58.4000 & 0.106 & 8.6666 \\
\hline Sodium sulfate & $\mathrm{Na}_{2} \mathrm{SO}_{4}$ & 142.0000 & 0.128 & 25.4464 \\
\hline Ammonium Chloride & $\mathrm{NH}_{4} \mathrm{Cl}$ & 55.4920 & 0.00498 & 0.3869 \\
\hline Glycolic acid & $\mathrm{C}_{2} \mathrm{H}_{4} \mathrm{O}_{3}$ & 76.1000 & 0.161 & 17.1529 \\
\hline Sodium hydroxide & $\mathrm{NaOH}$ & 40.0000 & 2.96122604 & 165.8287 \\
\hline $\begin{array}{c}\text { Sodium phosphate, 12- } \\
\text { hydrate }\end{array}$ & $\mathrm{Na}_{3} \mathrm{PO}_{4} 12 \mathrm{H}_{2} \mathrm{O}$ & 380.0000 & 0.0514 & 27.3448 \\
\hline Sodium formate & $\mathrm{Na}\left(\mathrm{CHO}_{2}\right)$ & 68.0000 & 0.233 & 22.1816 \\
\hline Sodium acetate, 3-hydrate & $\mathrm{Na}\left(\mathrm{C}_{2} \mathrm{H}_{3} \mathrm{O}_{2}\right) \cdot 3 \mathrm{H}_{2} \mathrm{O}$ & 136.0000 & 0.0208 & 3.9603 \\
\hline Sodium carbonate & $\mathrm{Na}_{2} \mathrm{CO}_{3}$ & 106.0000 & 1.12 & 166.2080 \\
\hline Sodium nitrate & $\mathrm{NaNO}_{3}$ & 85.0000 & 1.486 & 176.8340 \\
\hline Sodium nitrite & $\mathrm{NaNO}_{2}$ & 69.0000 & 1.91 & 184.5060 \\
\hline
\end{tabular}




\section{Cyclic Potentiodynamic Polarization}

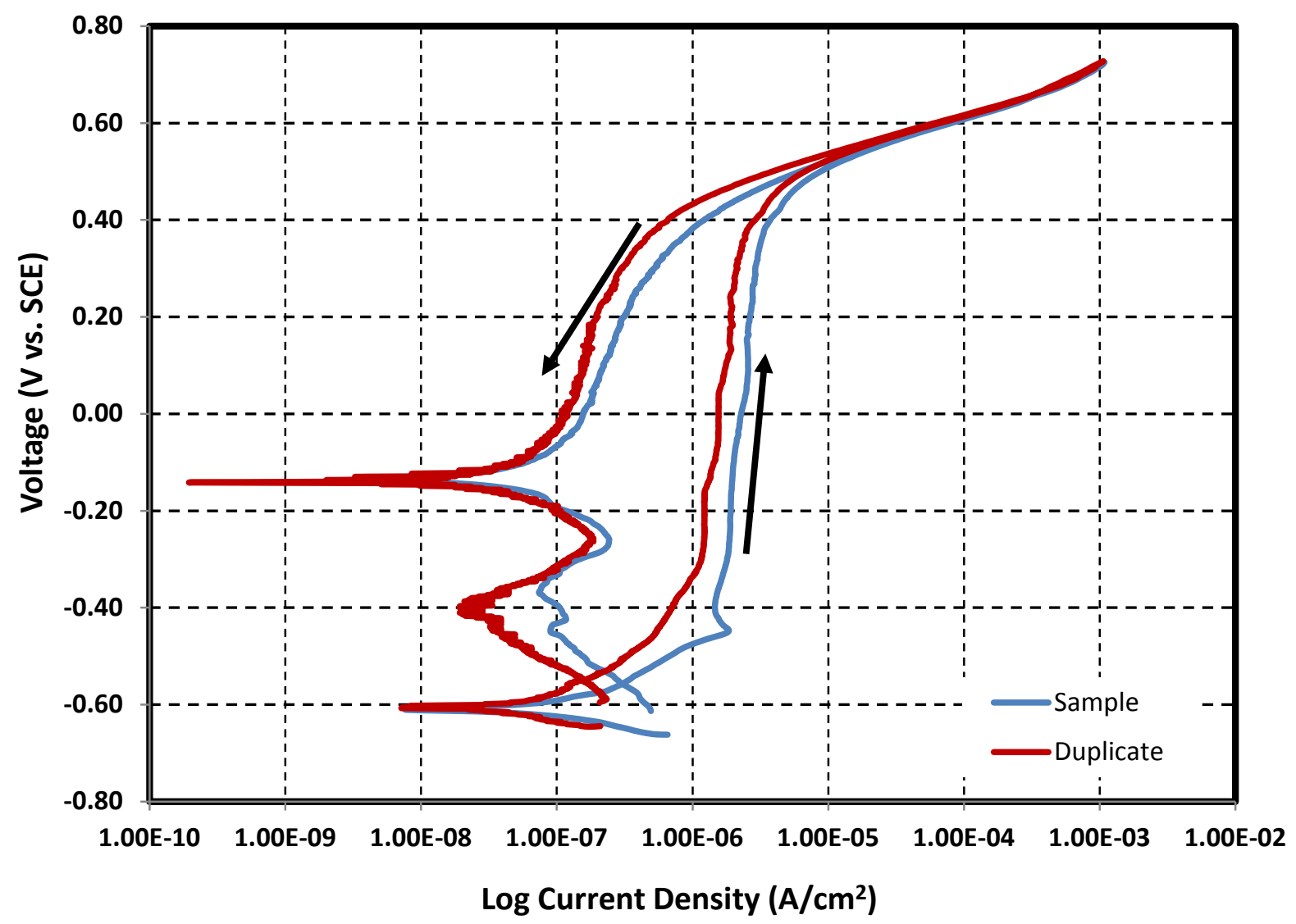

Images of bullet samples after electrochemical tests

Test 14

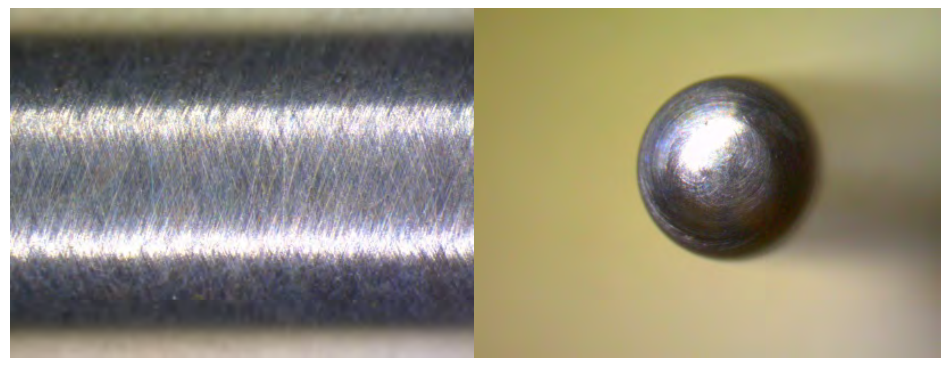

Test 14D

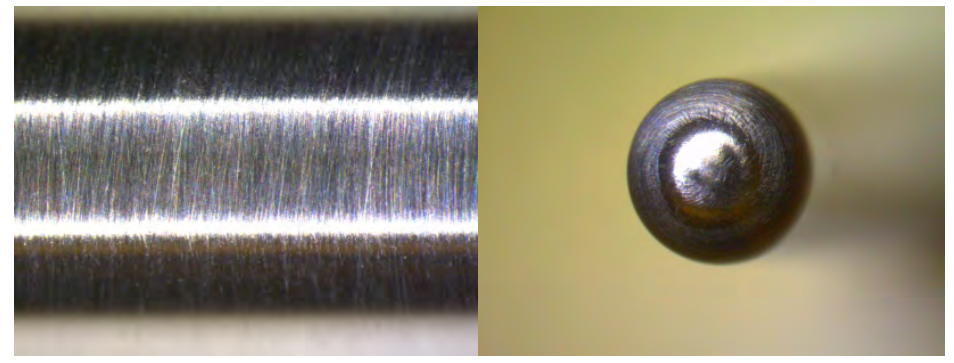

Shank (20X)

Nose (10X) 


\section{Composition of simulant for waste buffering-Test 15}

\section{Test 15}

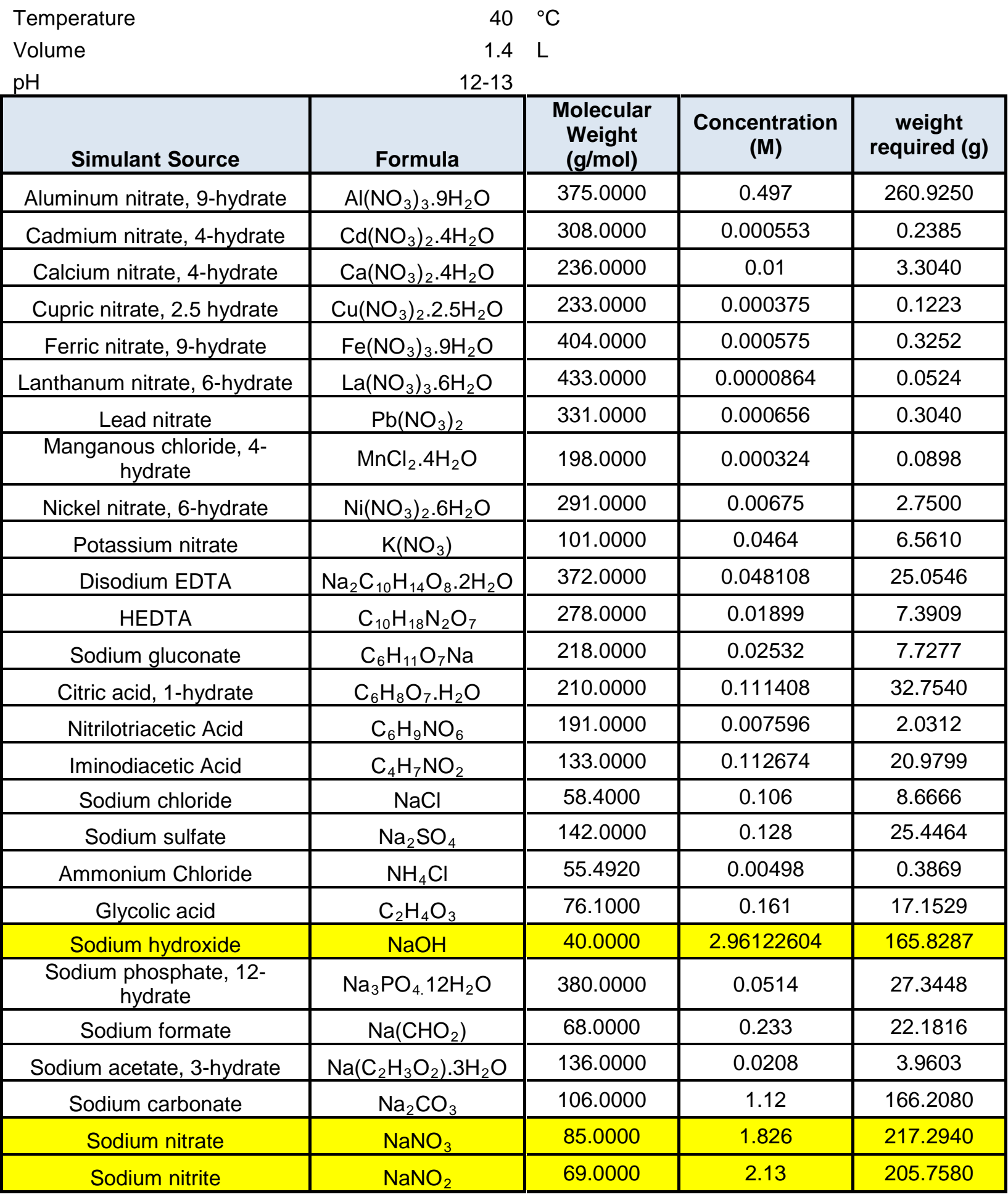




\section{Cyclic Potentiodynamic Polarization}

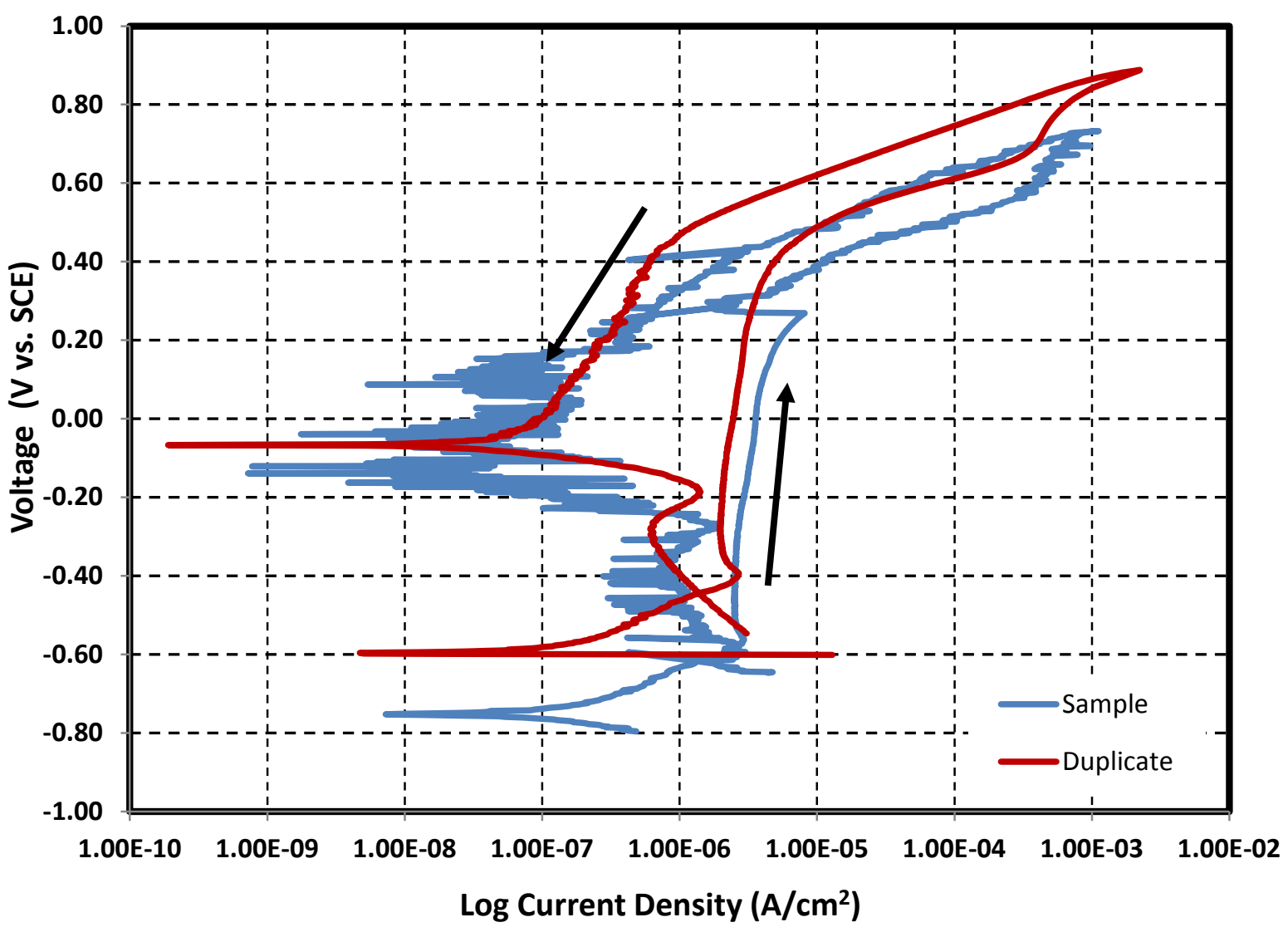

Images of bullet samples after electrochemical tests

Test 15

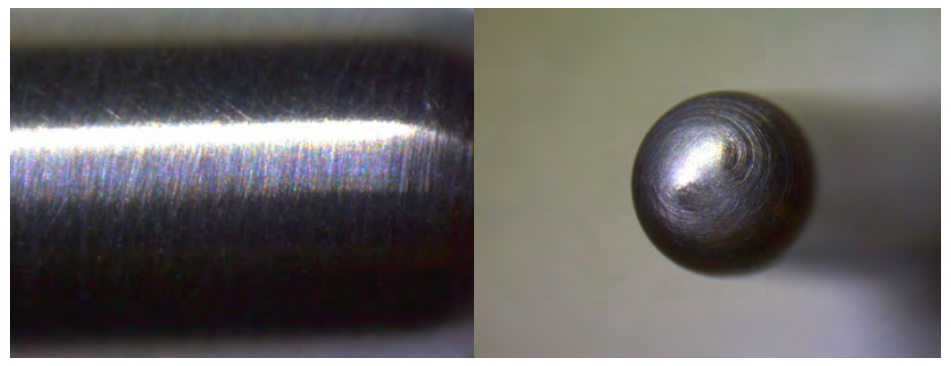

Test 15D

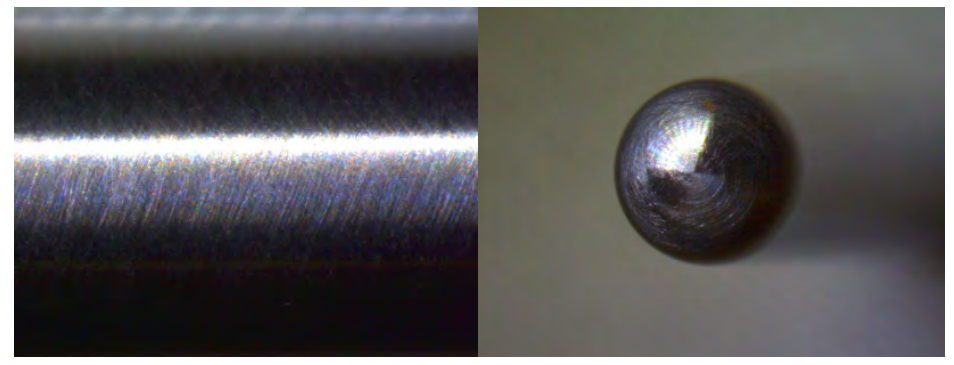

Shank (20X)

Nose (10X) 


\section{Composition of simulant for waste buffering-Test 16}

\section{Test 16}

\begin{tabular}{|c|c|c|c|c|}
\hline $\begin{array}{l}\text { Temperature } \\
\text { Volume } \\
\mathrm{pH}\end{array}$ & $\begin{array}{r}50 \\
1.4 \\
12-13\end{array}$ & $\begin{array}{l}{ }^{\circ} \mathrm{C} \\
\mathrm{L}\end{array}$ & & \\
\hline Simulant Source & Formula & $\begin{array}{c}\text { Molecular } \\
\text { Weight } \\
\text { (g/mol) } \\
\end{array}$ & $\begin{array}{l}\text { Concentration } \\
\text { (M) }\end{array}$ & $\begin{array}{l}\text { weight } \\
\text { required }(g)\end{array}$ \\
\hline Aluminum nitrate, 9-hydrate & $\mathrm{Al}\left(\mathrm{NO}_{3}\right)_{3} \cdot 9 \mathrm{H}_{2} \mathrm{O}$ & 375.0000 & 0.497 & 260.9250 \\
\hline Cadmium nitrate, 4-hydrate & $\mathrm{Cd}\left(\mathrm{NO}_{3}\right)_{2} \cdot 4 \mathrm{H}_{2} \mathrm{O}$ & 308.0000 & 0.000553 & 0.2385 \\
\hline Calcium nitrate, 4-hydrate & $\mathrm{Ca}\left(\mathrm{NO}_{3}\right)_{2} \cdot 4 \mathrm{H}_{2} \mathrm{O}$ & 236.0000 & 0.01 & 3.3040 \\
\hline Cupric nitrate, 2.5 hydrate & $\mathrm{Cu}\left(\mathrm{NO}_{3}\right)_{2} \cdot 2.5 \mathrm{H}_{2} \mathrm{O}$ & 233.0000 & 0.000375 & 0.1223 \\
\hline Ferric nitrate, 9-hydrate & $\mathrm{Fe}\left(\mathrm{NO}_{3}\right)_{3} \cdot 9 \mathrm{H}_{2} \mathrm{O}$ & 404.0000 & 0.000575 & 0.3252 \\
\hline Lanthanum nitrate, 6-hydrate & $\mathrm{La}\left(\mathrm{NO}_{3}\right)_{3} \cdot 6 \mathrm{H}_{2} \mathrm{O}$ & 433.0000 & 0.0000864 & 0.0524 \\
\hline Lead nitrate & $\mathrm{Pb}\left(\mathrm{NO}_{3}\right)_{2}$ & 331.0000 & 0.000656 & 0.3040 \\
\hline $\begin{array}{c}\text { Manganous chloride, 4- } \\
\text { hydrate }\end{array}$ & $\mathrm{MnCl}_{2} \cdot 4 \mathrm{H}_{2} \mathrm{O}$ & 198.0000 & 0.000324 & 0.0898 \\
\hline Nickel nitrate, 6-hydrate & $\mathrm{Ni}\left(\mathrm{NO}_{3}\right)_{2} \cdot 6 \mathrm{H}_{2} \mathrm{O}$ & 291.0000 & 0.00675 & 2.7500 \\
\hline Potassium nitrate & $\mathrm{K}\left(\mathrm{NO}_{3}\right)$ & 101.0000 & 0.0464 & 6.5610 \\
\hline Disodium EDTA & $\mathrm{Na}_{2} \mathrm{C}_{10} \mathrm{H}_{14} \mathrm{O}_{8} .2 \mathrm{H}_{2} \mathrm{O}$ & 372.0000 & 0.048108 & 25.0546 \\
\hline HEDTA & $\mathrm{C}_{10} \mathrm{H}_{18} \mathrm{~N}_{2} \mathrm{O}_{7}$ & 278.0000 & 0.01899 & 7.3909 \\
\hline Sodium gluconate & $\mathrm{C}_{6} \mathrm{H}_{11} \mathrm{O}_{7} \mathrm{Na}$ & 218.0000 & 0.02532 & 7.7277 \\
\hline Citric acid, 1-hydrate & $\mathrm{C}_{6} \mathrm{H}_{8} \mathrm{O}_{7} \cdot \mathrm{H}_{2} \mathrm{O}$ & 210.0000 & 0.111408 & 32.7540 \\
\hline Nitrilotriacetic Acid & $\mathrm{C}_{6} \mathrm{H}_{9} \mathrm{NO}_{6}$ & 191.0000 & 0.007596 & 2.0312 \\
\hline Iminodiacetic Acid & $\mathrm{C}_{4} \mathrm{H}_{7} \mathrm{NO}_{2}$ & 133.0000 & 0.112674 & 20.9799 \\
\hline Sodium chloride & $\mathrm{NaCl}$ & 58.4000 & 0.106 & 8.6666 \\
\hline Sodium sulfate & $\mathrm{Na}_{2} \mathrm{SO}_{4}$ & 142.0000 & 0.128 & 25.4464 \\
\hline Ammonium Chloride & $\mathrm{NH}_{4} \mathrm{Cl}$ & 55.4920 & 0.00498 & 0.3869 \\
\hline Glycolic acid & $\mathrm{C}_{2} \mathrm{H}_{4} \mathrm{O}_{3}$ & 76.1000 & 0.161 & 17.1529 \\
\hline Sodium hydroxide & $\mathrm{NaOH}$ & 40.0000 & 2.96122604 & 165.8287 \\
\hline $\begin{array}{c}\text { Sodium phosphate, 12- } \\
\text { hydrate }\end{array}$ & $\mathrm{Na}_{3} \mathrm{PO}_{4} 12 \mathrm{H}_{2} \mathrm{O}$ & 380.0000 & 0.0514 & 27.3448 \\
\hline Sodium formate & $\mathrm{Na}\left(\mathrm{CHO}_{2}\right)$ & 68.0000 & 0.233 & 22.1816 \\
\hline Sodium acetate, 3-hydrate & $\mathrm{Na}\left(\mathrm{C}_{2} \mathrm{H}_{3} \mathrm{O}_{2}\right) \cdot 3 \mathrm{H}_{2} \mathrm{O}$ & 136.0000 & 0.0208 & 3.9603 \\
\hline Sodium carbonate & $\mathrm{Na}_{2} \mathrm{CO}_{3}$ & 106.0000 & 1.12 & 166.2080 \\
\hline Sodium nitrate & $\mathrm{NaNO}_{3}$ & 85.0000 & 1.486 & 176.8340 \\
\hline Sodium nitrite & $\mathrm{NaNO}_{2}$ & 69.0000 & 1.91 & 184.5060 \\
\hline
\end{tabular}




\section{Cyclic Potentiodynamic Polarization}

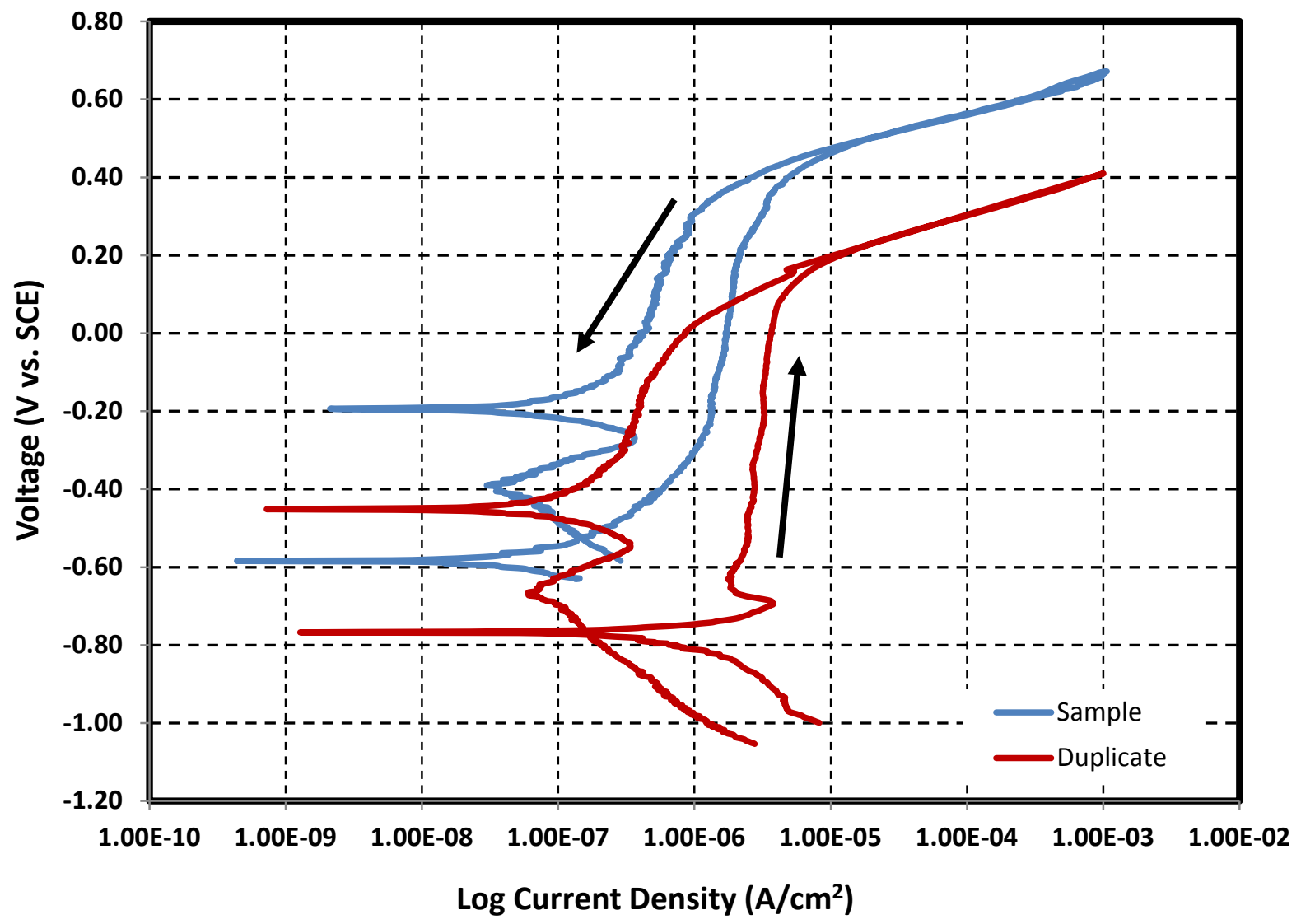

Images of bullet samples after electrochemical tests

Test 16

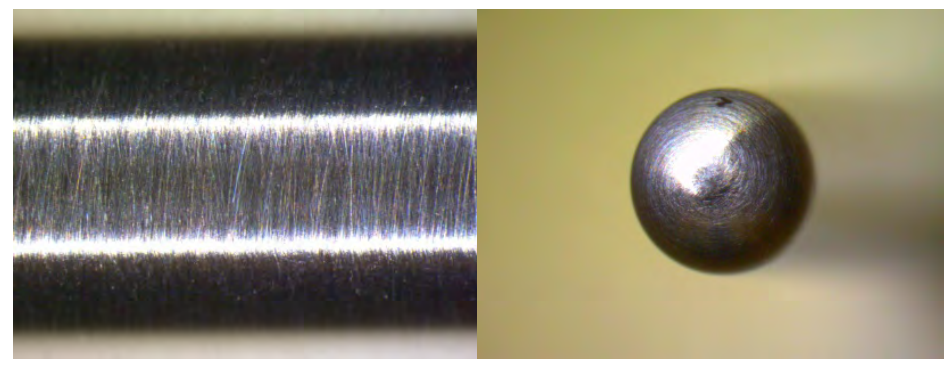

Test 16D

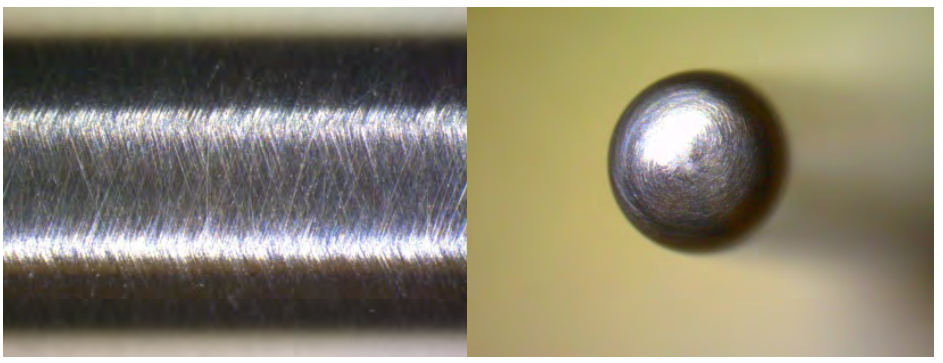

Shank (20X)

Nose (10X) 


\section{Composition of simulant for waste buffering-Test 17}

\section{Test 17}

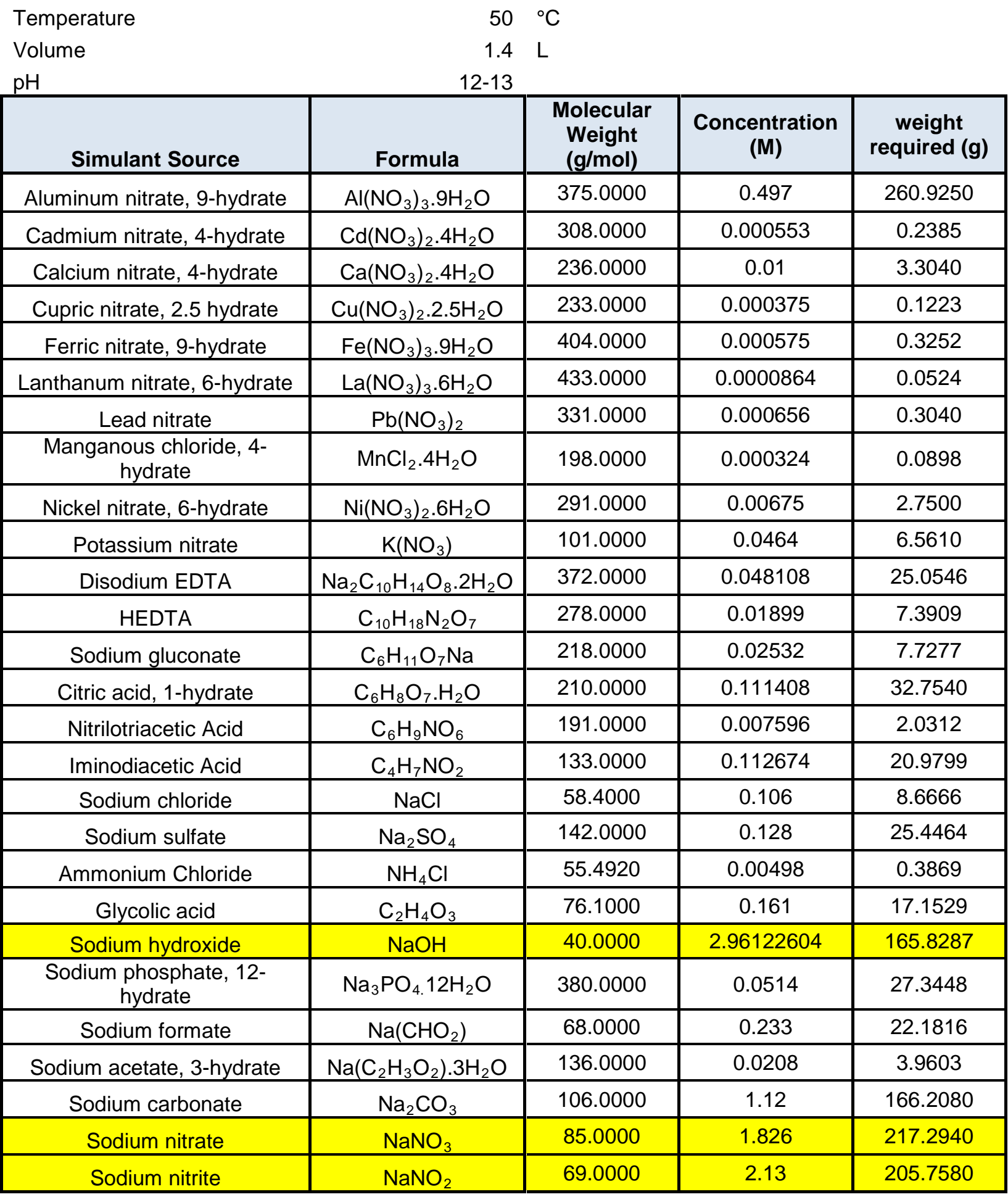




\section{Cyclic Potentiodynamic Polarization}

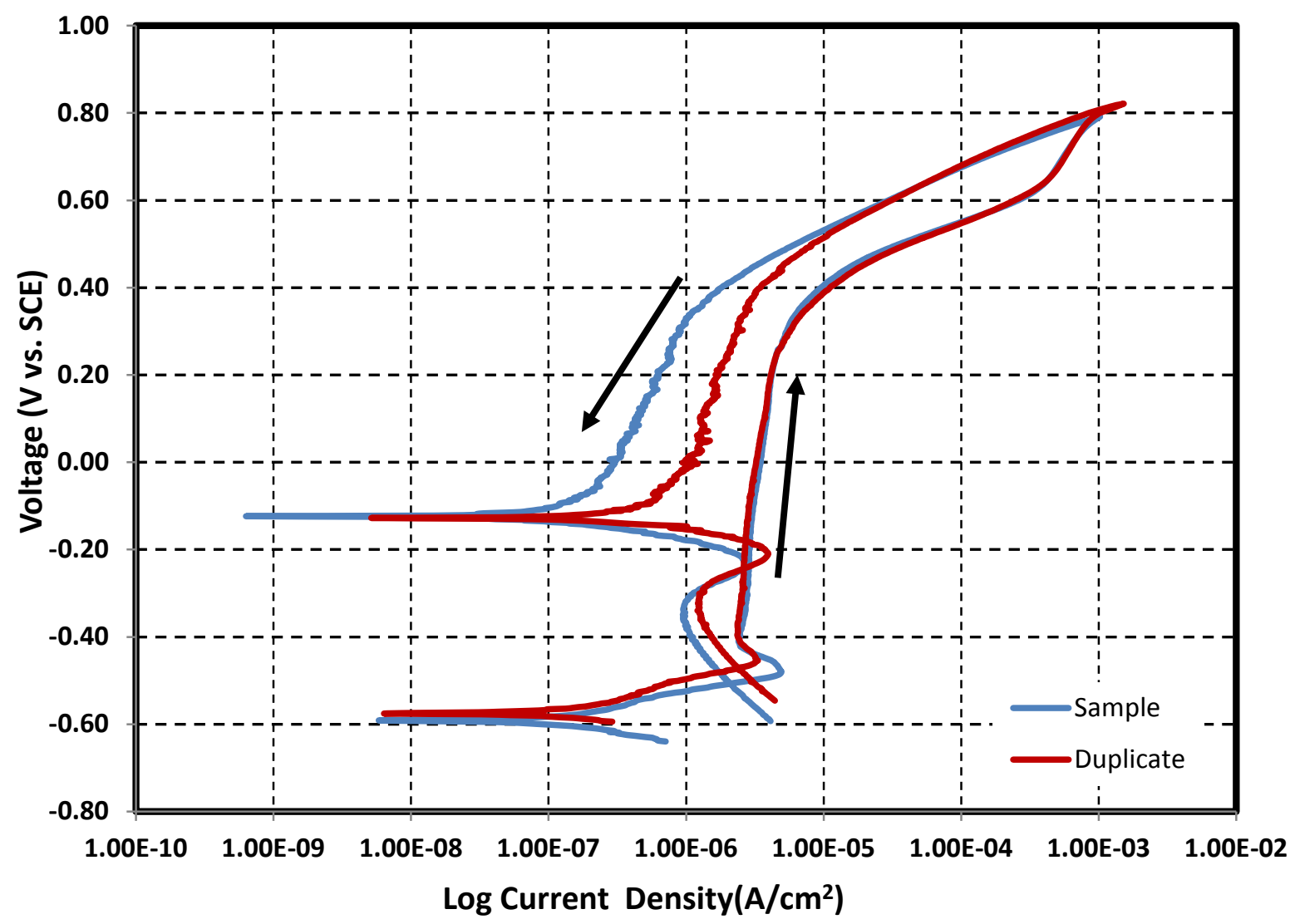

Images of bullet samples after electrochemical tests

Test 17

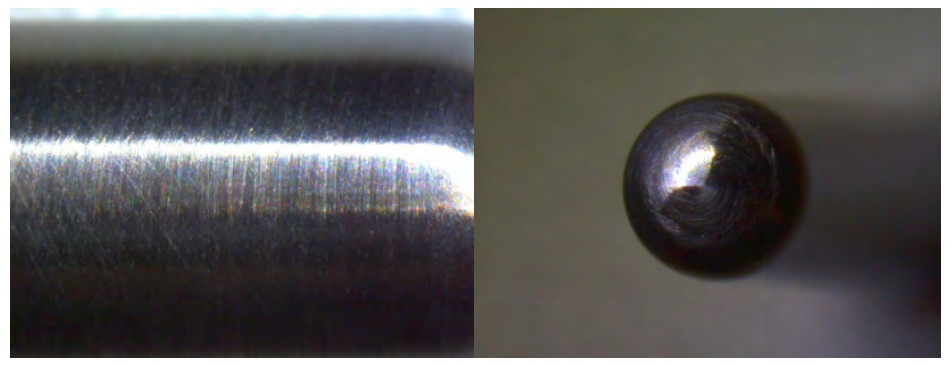

Test 17D

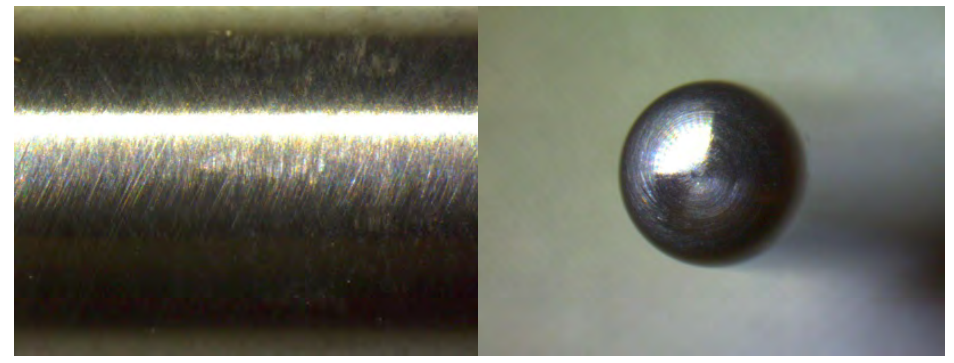

Shank (20X)

Nose (10X) 
Appendix G

Chemical Composition of Simulants used in Pitting Corrosion (Task 4) with Electrochemical Results and After Pictures 


\section{Composition of simulant for pitting corrosion-Test 1}

Reference 10

Test 1

Temperature

$\mathrm{pH}$

Volume
$40{ }^{\circ} \mathrm{C}$

12.50

$1.4 \mathrm{~L}$

\begin{tabular}{|c|c|c|c|c|}
\hline Simulant Source & Formula & $\begin{array}{c}\text { Molecular Weight } \\
\text { (g/mol) }\end{array}$ & $\begin{array}{c}\text { Concentration } \\
\text { (M) }\end{array}$ & $\begin{array}{l}\text { weight required } \\
\text { (g) }\end{array}$ \\
\hline Sodium Carbonate & $\mathrm{Na}_{2} \mathrm{CO}_{3}$ & 106.00 & 0.0173 & 2.5673 \\
\hline Sodium Oxalate & $\mathrm{Na}_{2} \mathrm{C}_{2} \mathrm{O}_{4}$ & 134.00 & 0.0000726 & 0.0136 \\
\hline $\begin{array}{c}\text { Sodium molybdate, } \\
\text { dihydrate }\end{array}$ & $\begin{array}{c}\mathrm{Na}_{2} \mathrm{MoO}_{4} \cdot 2 \mathrm{H} \\
{ }_{2} \mathrm{O}\end{array}$ & 241.95 & 0.00000387 & 0.0013 \\
\hline $\begin{array}{c}\text { Sodium Metasilicate, 5- } \\
\text { hydrate }\end{array}$ & $\begin{array}{c}\mathrm{Na}_{2} \mathrm{SiO}_{3} .5 \mathrm{H}_{2} \\
\mathrm{O}\end{array}$ & 212.14 & 0.00002944 & 0.0087 \\
\hline $\begin{array}{c}\text { Sodium phosphate, 12- } \\
\text { hydrate }\end{array}$ & $\begin{array}{c}\mathrm{Na}_{3} \mathrm{PO}_{4 .} 12 \mathrm{H}_{2} \\
\mathrm{O}\end{array}$ & 380.00 & 0.00008272 & 0.0440 \\
\hline Sodium chloride & $\mathrm{NaCl}$ & 58.40 & 0.0003185 & 0.0260 \\
\hline Sodium Fluoride & $\mathrm{NaF}$ & 41.99 & 0.00015345 & 0.0090 \\
\hline Sodium sulfate & $\mathrm{Na}_{2} \mathrm{SO}_{4}$ & 142.00 & 0.0013674 & 0.2718 \\
\hline Sodium nitrate & $\mathrm{NaNO}_{3}$ & 85.00 & 0.04 & 4.7600 \\
\hline Sodium nitrite & $\mathrm{NaNO}_{2}$ & 69.00 & 0.015 & 1.4490 \\
\hline Sodium Hydroxide & $\mathrm{NaOH}$ & 40.00 & 0.0304 & 1.7024 \\
\hline
\end{tabular}




\section{Cyclic Potentiodynamic Polarization}

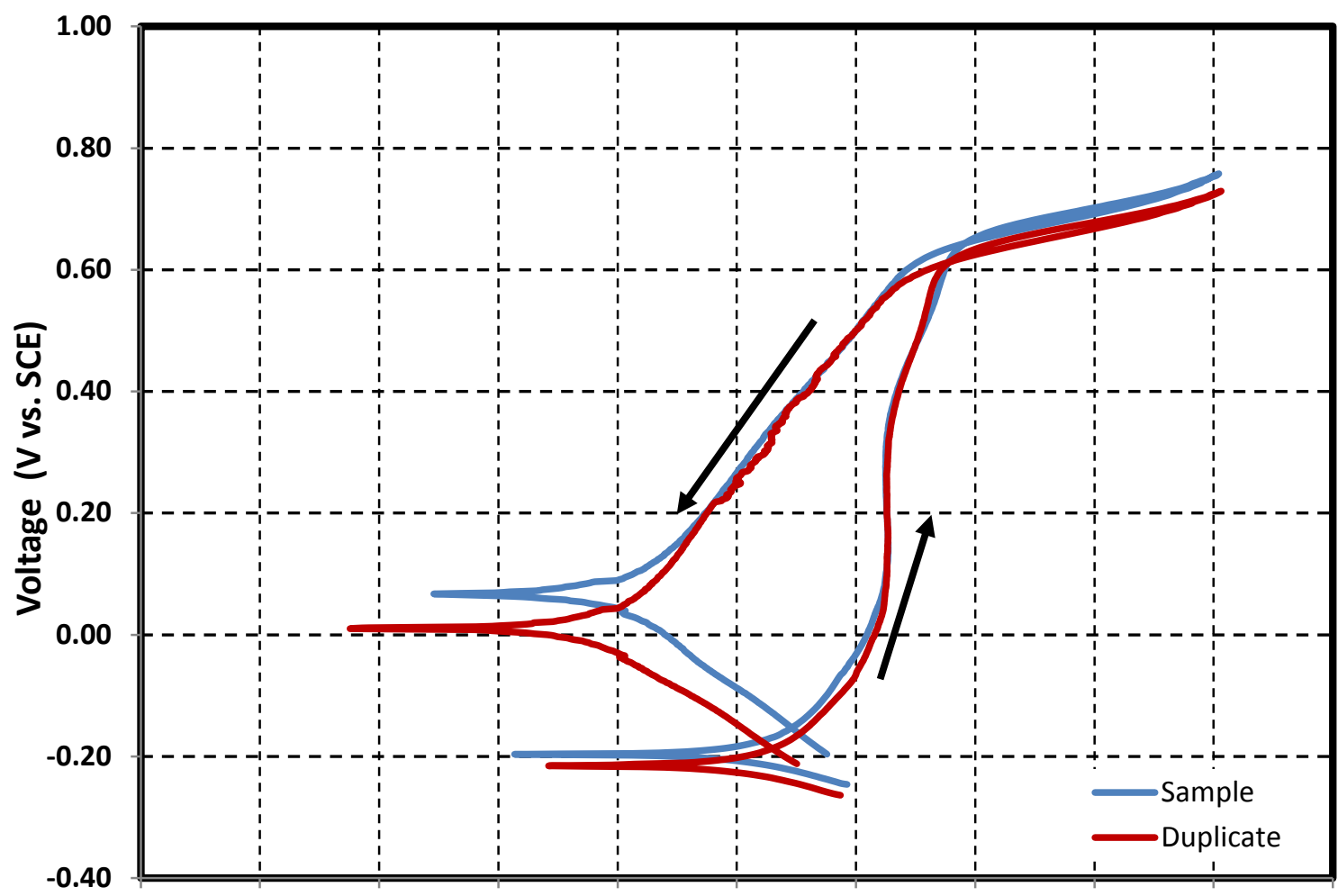

1.00E-12 1.00E-11 1.00E-10 1.00E-09 1.00E-08 1.00E-07 1.00E-06 1.00E-05 1.00E-04 1.00E-03 1.00E-02 Log Current Density $\left(\mathrm{A} / \mathrm{cm}^{2}\right)$

Images of bullet samples after electrochemical tests

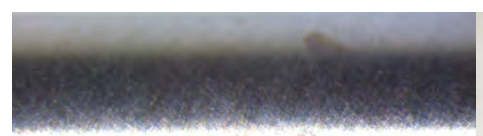

Test 1

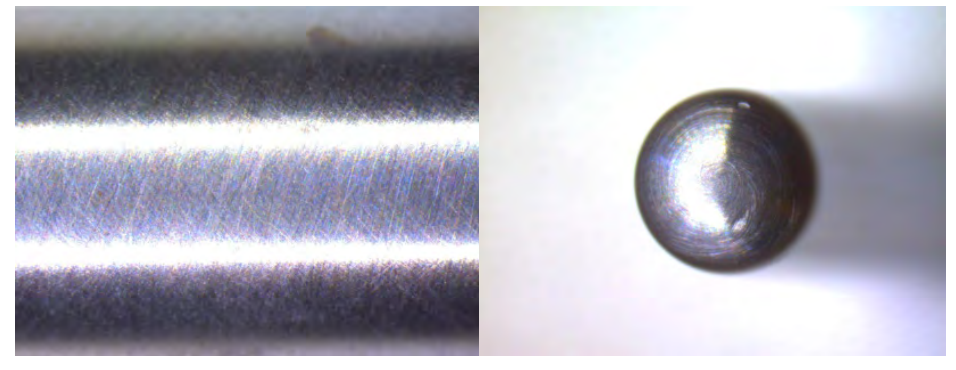

Test 1D

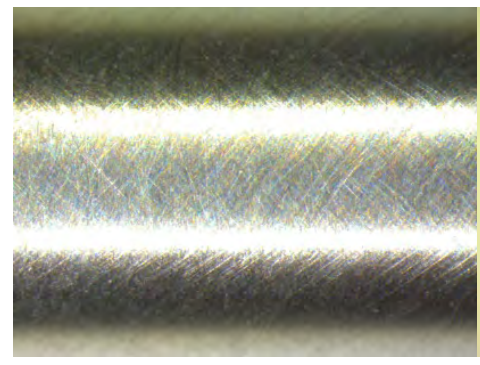

Shank (20X)

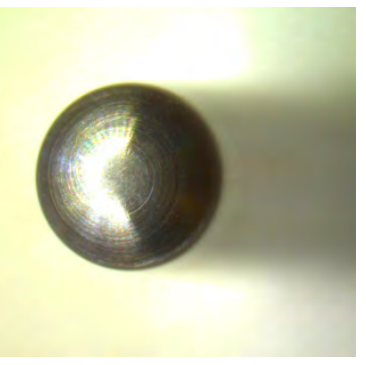

Nose (10X) 


\section{Composition of simulant for pitting corrosion -Test 2}

Reference 19

Test 2
Temperature

$\mathrm{pH}$

Volume
$50{ }^{\circ} \mathrm{C}$

14.50

$1.4 \mathrm{~L}$

\begin{tabular}{|c|c|c|c|c|}
\hline Simulant Source & Formula & $\begin{array}{c}\text { Molecular Weight } \\
(\mathbf{g} / \mathbf{m o l})\end{array}$ & $\begin{array}{c}\text { Concentration } \\
(\mathbf{M})\end{array}$ & $\begin{array}{c}\text { weight required } \\
(\mathbf{g})\end{array}$ \\
\hline Sodium nitrate & $\mathrm{NaNO}_{3}$ & 84.99 & 0.868 & 103.2798 \\
\hline Sodium chloride & $\mathrm{NaCl}$ & 58.44 & 0.046 & 3.7635 \\
\hline Sodium fluoride & $\mathrm{NaF}$ & 42.00 & 0.084 & 4.9392 \\
\hline Sodium nitrite & $\mathrm{NaNO}$ & 1.27 & 122.6820 \\
\hline Sodium hydroxide & $\mathrm{NaOH}$ & 69.00 & 1.243 & 69.6080 \\
\hline Sodium carbonate & $\mathrm{Na}_{2}\left(\mathrm{CO}_{3}\right)$ & 40.00 & 1.118 & 165.9112 \\
\hline Sodium sulfate & $\mathrm{Na}_{2} \mathrm{SO}_{4}$ & 106.00 & 0.028 & 5.5680 \\
\hline Potassium Nitrate & $\mathrm{KNO}_{3}$ & 142.04 & 0.767 & 108.5612 \\
\hline $\begin{array}{c}\text { Sodium phosphate, } 12- \\
\text { hydrate }\end{array}$ & $\mathrm{Na}_{3} \mathrm{PO}_{4} .12 \mathrm{H}_{2}$ & 01.1 & 0.009 & 4.7880 \\
\hline Sodium aluminate & $\mathrm{NaAlO}_{2}$ & 380.00 & 0.288 & 33.0503 \\
\hline
\end{tabular}




\section{Cyclic Potentiodynamic Polarization}

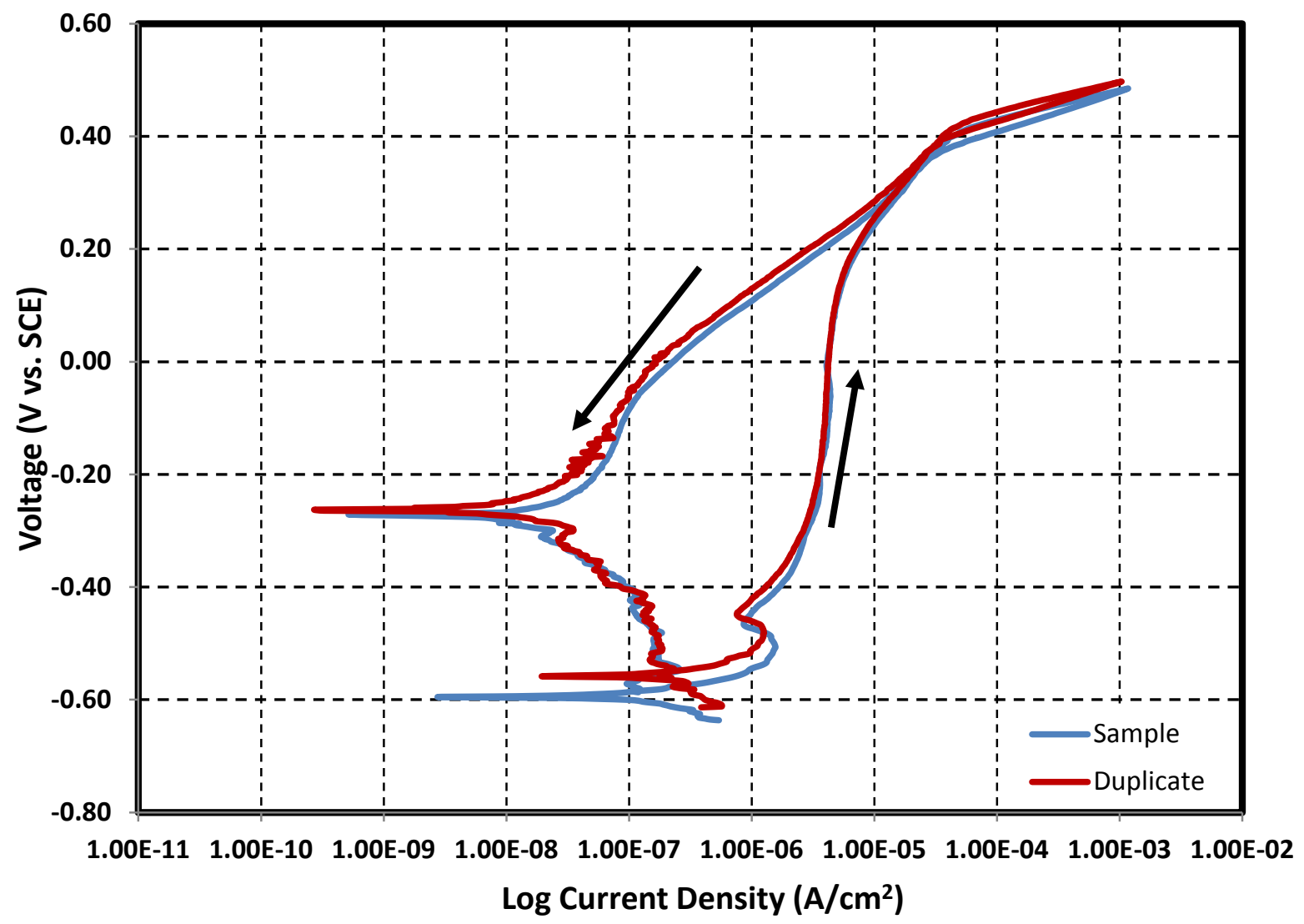

Images of bullet samples after electrochemical tests

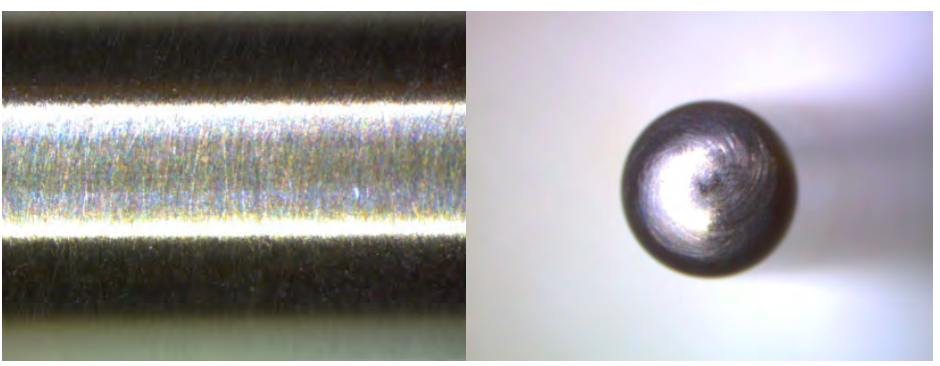

Test 2D

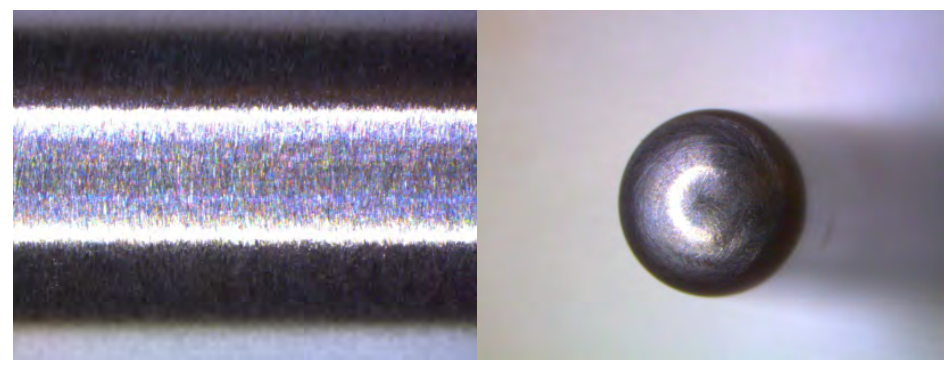

Shank (20X)

Nose (10X) 


\section{Composition of simulant for pitting corrosion -Test 3}

\begin{tabular}{|c|c|c|c|c|}
\hline Reference 17 & Temperature & 50 & ${ }^{\circ} \mathrm{C}$ & \\
\hline Test 3 & $\mathrm{pH}$ & 13.00 & & \\
\hline & Volume & 1.4 & $\mathrm{~L}$ & \\
\hline Simulant Source & Formula & $\begin{array}{l}\text { Molecular } \\
\text { Weight } \\
\text { (g/mol) } \\
\end{array}$ & $\begin{array}{l}\text { Concentration } \\
\text { (M) }\end{array}$ & $\begin{array}{l}\text { weight } \\
\text { required }(g)\end{array}$ \\
\hline Sodium nitrate & $\mathrm{NaNO}_{3}$ & 84.99 & 7 & 832.9020 \\
\hline Sodium nitrite & $\mathrm{NaNO}_{2}$ & 69.00 & 0.1 & 9.6600 \\
\hline Sodium hydroxide & $\mathrm{NaOH}$ & 40.00 & 0.1 & 5.6000 \\
\hline Sodium chloride & $\mathrm{NaCl}$ & 58.44 & 0.1 & 8.1816 \\
\hline Sodium sulfate & $\mathrm{Na}_{2} \mathrm{SO}_{4}$ & 142.04 & 0.1 & 19.8856 \\
\hline Sodium carbonate & $\mathrm{Na}_{2}\left(\mathrm{CO}_{3}\right)$ & 106.00 & 0.1 & 14.8400 \\
\hline Sodium aluminate & $\mathrm{NaAlO}_{2}$ & 81.97 & 0.5 & 57.3790 \\
\hline $\begin{array}{l}\text { Sodium phosphate, 12- } \\
\text { hydrate }\end{array}$ & $\mathrm{Na}_{3} \mathrm{PO}_{4} 12 \mathrm{H}_{2} \mathrm{O}$ & 380.00 & 0.05 & 26.6000 \\
\hline
\end{tabular}




\section{Cyclic Potentiodynamic Polarization}

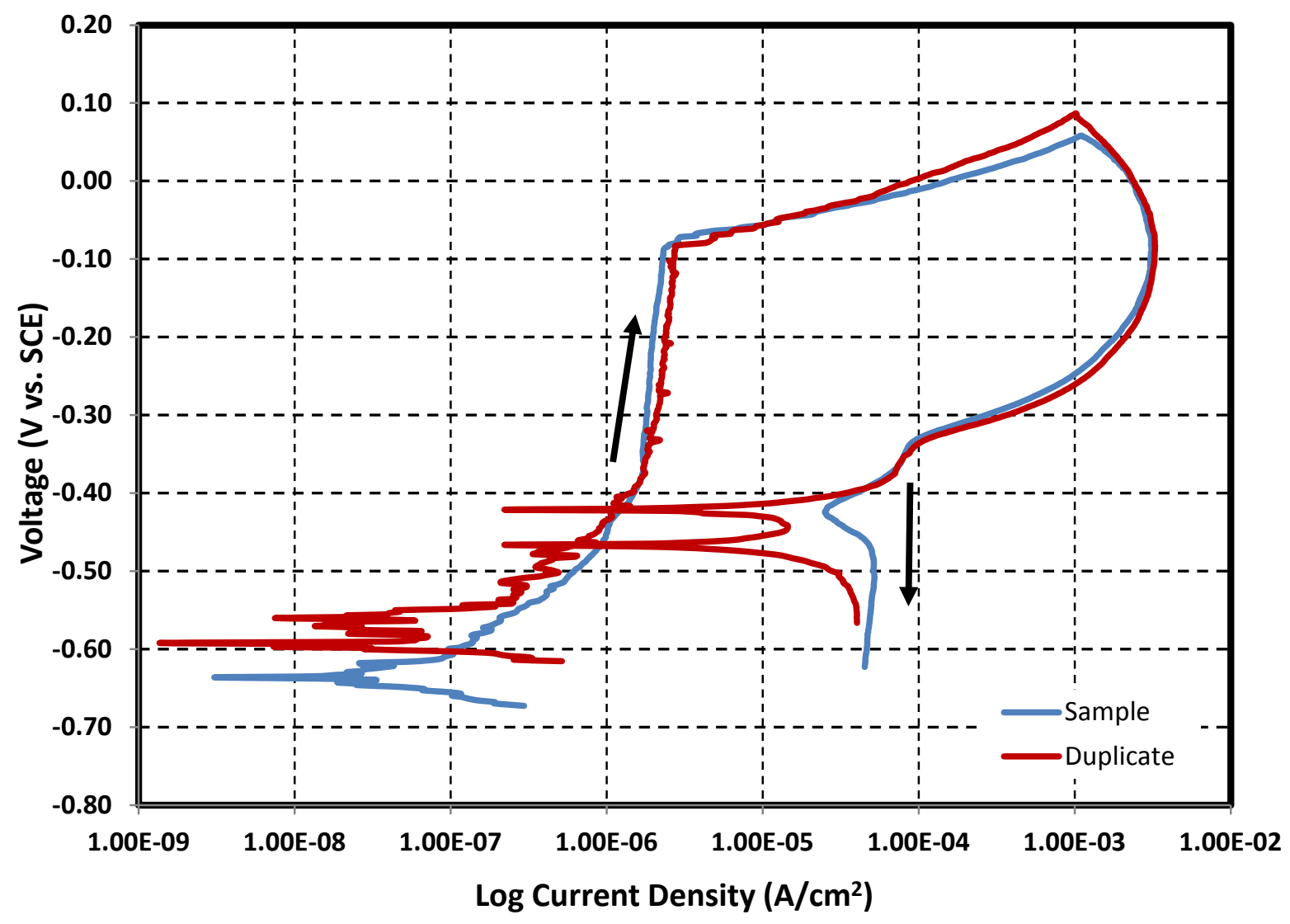

Images of bullet samples after electrochemical tests

Test 3

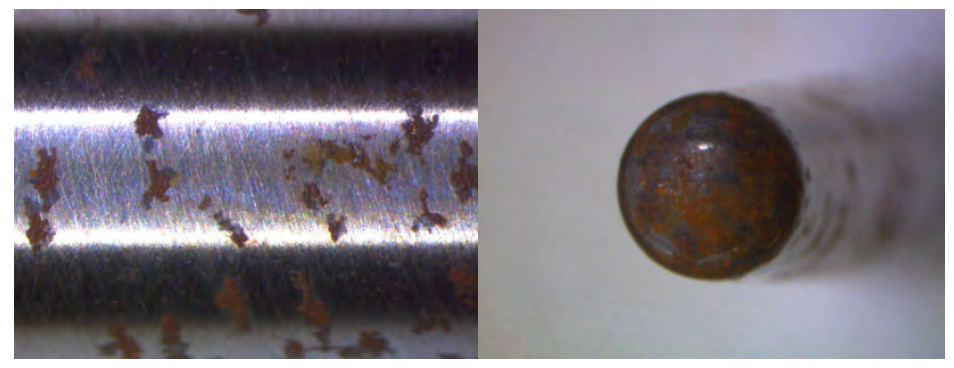

Test 3D

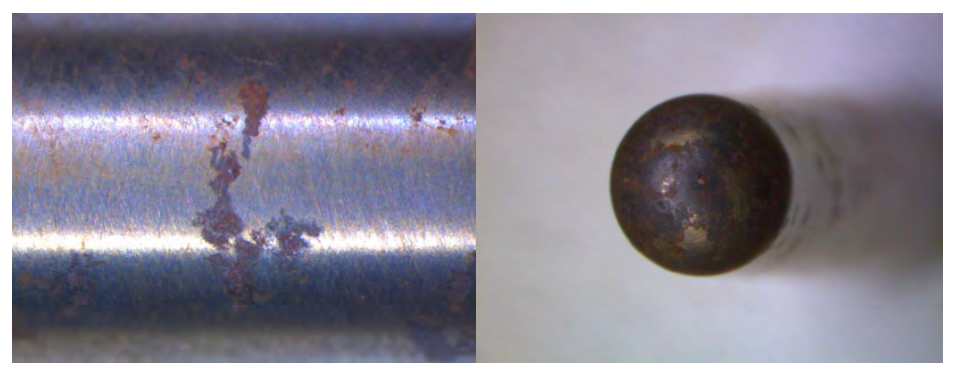

Shank (20X)

Nose (10X) 


\section{Composition of simulant for pitting corrosion -Test 4}

Reference 17

Test 4
Temperature

$\mathrm{pH}$

Volume

\begin{tabular}{|c|c|c|c|c|}
\hline Simulant Source & Formula & $\begin{array}{c}\text { Molecular } \\
\text { Weight } \\
\text { (g/mol) }\end{array}$ & $\begin{array}{c}\text { Concentration } \\
\text { (M) }\end{array}$ & $\begin{array}{c}\text { weight required } \\
\text { (g) }\end{array}$ \\
\hline Sodium nitrate & $\mathrm{NaNO}_{3}$ & 84.99 & 5.5 & 654.4230 \\
\hline Sodium nitrite & $\mathrm{NaNO}_{2}$ & 69.00 & 0.2 & 19.3200 \\
\hline Sodium hydroxide & $\mathrm{NaOH}$ & 40.00 & 0.6 & 33.6000 \\
\hline Sodium chloride & $\mathrm{NaCl}$ & 58.44 & 0.1 & 8.1816 \\
\hline Sodium sulfate & $\mathrm{Na}_{2} \mathrm{SO}_{4}$ & 142.04 & 0.1 & 19.8856 \\
\hline Sodium carbonate & $\mathrm{Na}_{2}\left(\mathrm{CO}_{3}\right)$ & 106.00 & 0.1 & 14.8400 \\
\hline Sodium aluminate & $\mathrm{NaAlO}_{2}$ & 81.97 & 0.5 & 57.3790 \\
\hline $\begin{array}{c}\text { Sodium phosphate, } 12- \\
\text { hydrate }\end{array}$ & $\mathrm{Na}_{3} \mathrm{PO}_{4} 12 \mathrm{H}_{2} \mathrm{O}$ & 380.00 & 0.05 & 26.6000 \\
\hline
\end{tabular}

$50{ }^{\circ} \mathrm{C}$

13.80

1.4 


\section{Cyclic Potentiodynamic Polarization}

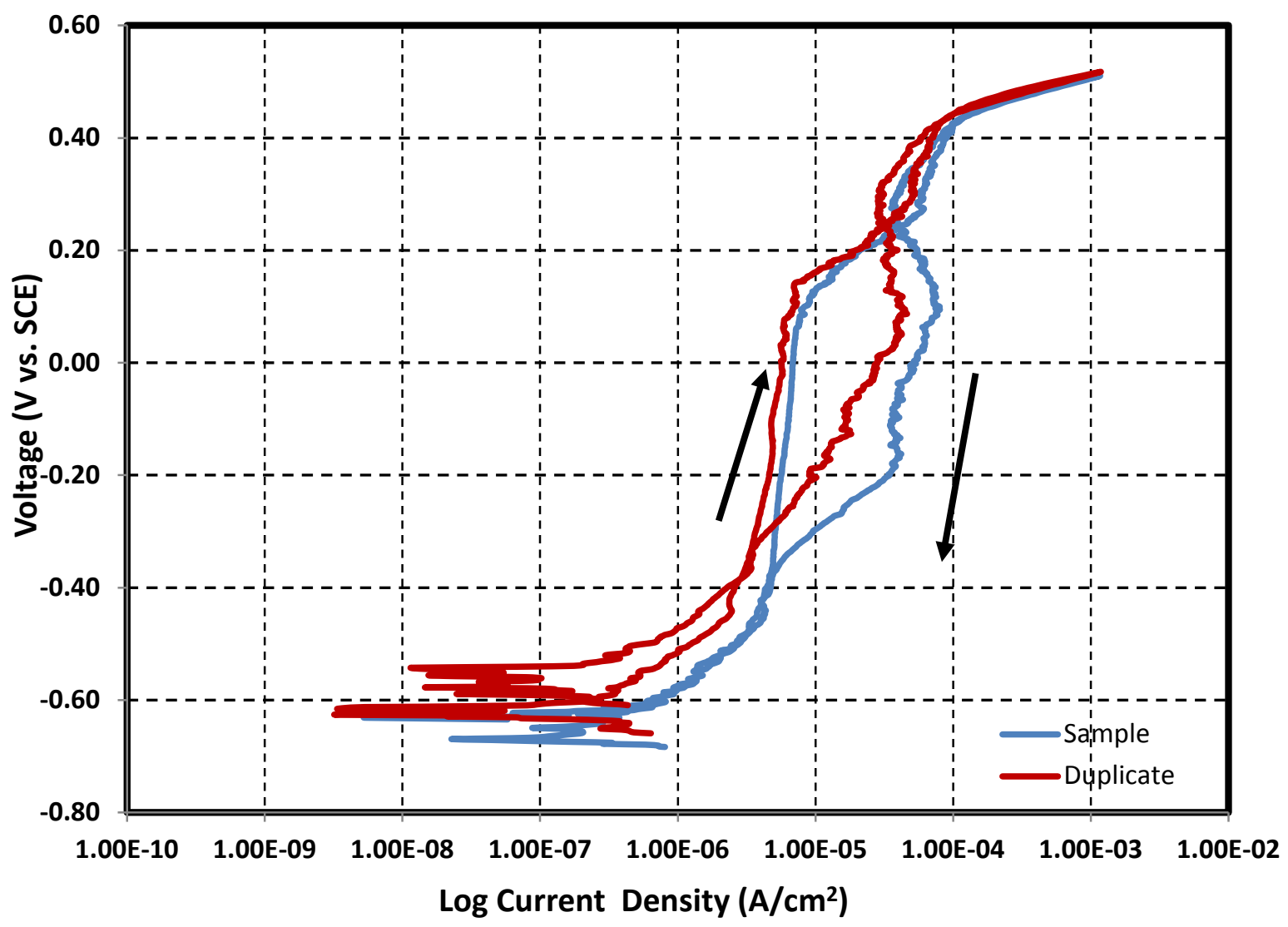

Images of bullet samples after electrochemical tests

Test 4
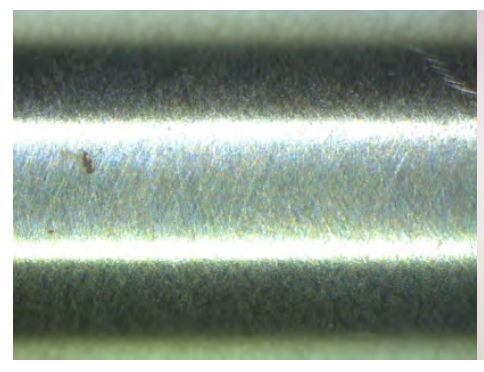

Test 4D

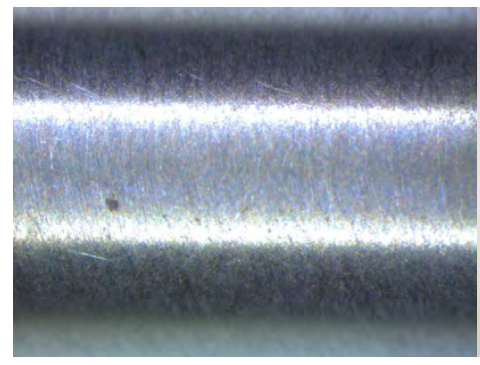

Shank (20X)
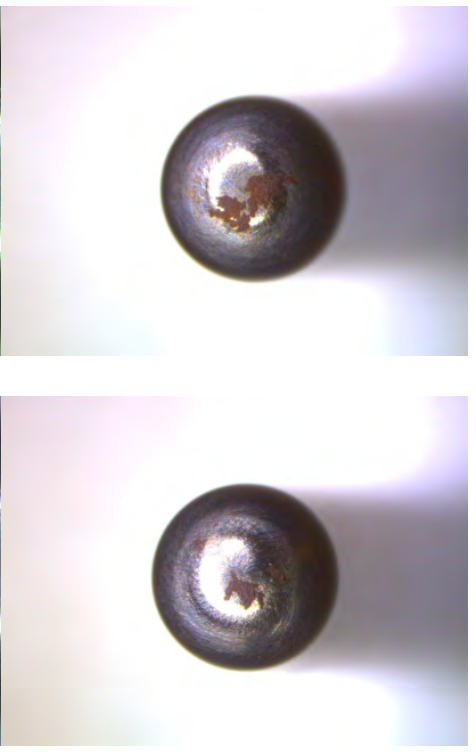

Nose (10X) 


\section{Composition of simulant for pitting corrosion -Test 5}

Reference 10

Test 5
Temperature

$\mathrm{pH}$

Volume

\begin{tabular}{|c|c|c|c|c|}
\hline Simulant Source & Formula & $\begin{array}{c}\text { Molecular } \\
\text { Weight } \\
\text { (g/mol) }\end{array}$ & $\begin{array}{c}\text { Concentration } \\
\text { (M) }\end{array}$ & $\begin{array}{c}\text { weight } \\
\text { required (g) }\end{array}$ \\
\hline Sodium Carbonate & $\mathrm{Na}_{2} \mathrm{CO}_{3}$ & 106.00 & 0.00148 & 0.2196 \\
\hline Sodium Oxalate & $\mathrm{Na}_{2} \mathrm{C}_{2} \mathrm{O}_{4}$ & 134.00 & 0.0000726 & 0.0136 \\
\hline $\begin{array}{c}\text { Sodium molybdate, } \\
\text { dihydrate }\end{array}$ & $\mathrm{Na}_{2} \mathrm{MoO}_{4} \cdot 2 \mathrm{H}_{2} \mathrm{O}$ & 241.95 & 0.00000387 & 0.0013 \\
\hline $\begin{array}{c}\text { Sodium Metasilicate, 5- } \\
\text { hydrate }\end{array}$ & $\mathrm{Na}_{2} \mathrm{SiO}_{3} \cdot 5 \mathrm{H}_{2} \mathrm{O}$ & 212.14 & 0.00002944 & 0.0087 \\
\hline $\begin{array}{c}\text { Sodium phosphate, 12- } \\
\text { hydrate }\end{array}$ & $\mathrm{Na}_{3} \mathrm{PO}_{4} 12 \mathrm{H}_{2} \mathrm{O}$ & 380.00 & 0.00008272 & 0.0440 \\
\hline Sodium chloride & $\mathrm{NaCl}$ & 58.40 & 0.01 & 0.8176 \\
\hline Sodium Fluoride & $\mathrm{NaF}^{2}$ & 41.99 & 0.00015345 & 0.0090 \\
\hline Sodium sulfate & $\mathrm{Na}_{2} \mathrm{SO}_{4}$ & 142.00 & 0.0013674 & 0.2718 \\
\hline Sodium nitrate & $\mathrm{NaNO}_{3}$ & 85.00 & 0.04 & 4.7600 \\
\hline Sodium nitrite & $\mathrm{NaNO}_{2}$ & 69.00 & 0.325 & 31.3950 \\
\hline Sodium Hydroxide & $\mathrm{NaOH}^{2}$ & 40.00 & 0.0304 & 1.7024 \\
\hline
\end{tabular}

$40{ }^{\circ} \mathrm{C}$

12.50 


\section{Cyclic Potentiodynamic Polarization}

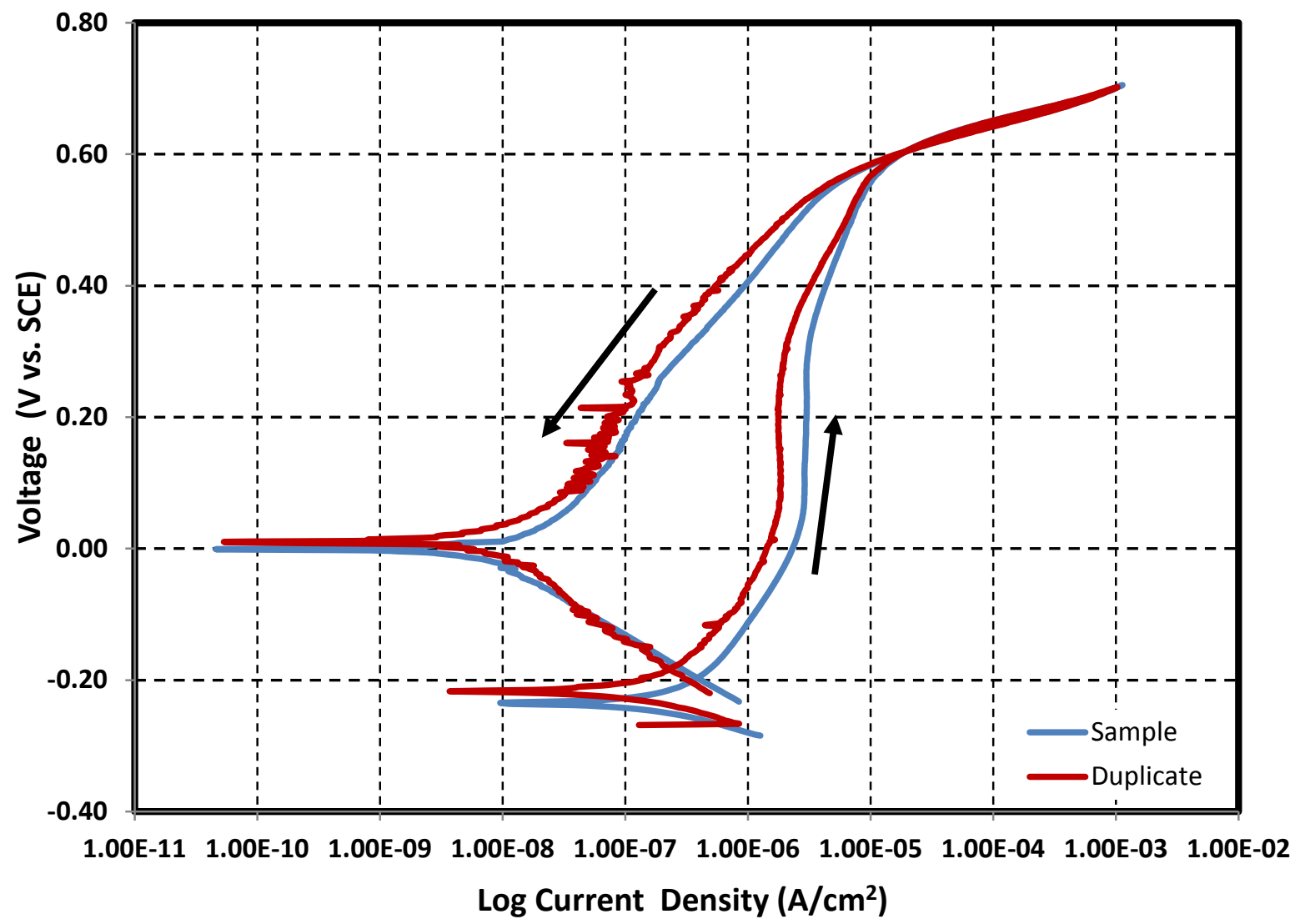

Images of bullet samples after electrochemical tests

Test 5
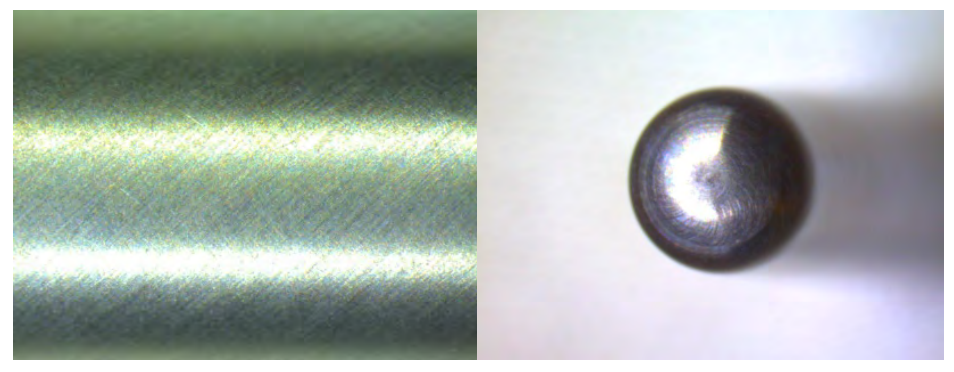

Test 5D

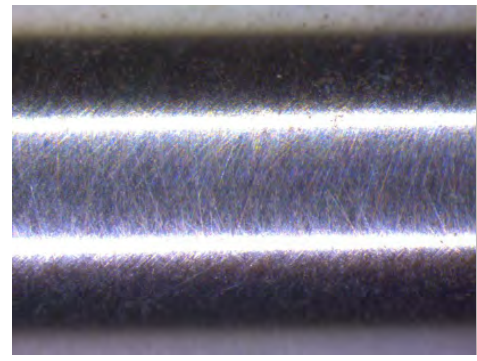

Shank (20X)

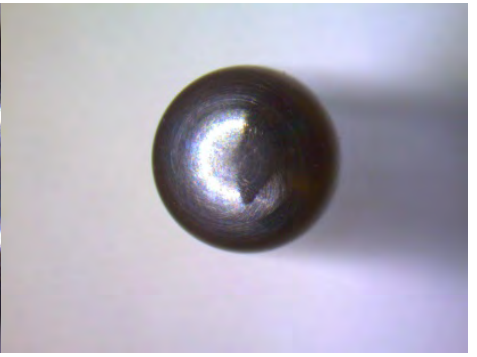

Nose (10X) 


\section{Composition of simulant for pitting corrosion -Test 6}

\begin{tabular}{|c|c|c|c|c|}
\hline \multirow{3}{*}{$\begin{array}{l}\text { Reference } 10 \\
\text { Test } 6\end{array}$} & \multirow{2}{*}{$\begin{array}{l}\text { Temperature } \\
\mathrm{pH}\end{array}$} & & \multicolumn{2}{|l|}{${ }^{\circ} \mathrm{C}$} \\
\hline & & 12.50 & & \\
\hline & Volume & 1.4 & $\mathrm{~L}$ & \\
\hline Simulant Source & Formula & $\begin{array}{l}\text { Molecular } \\
\text { Weight } \\
\text { (g/mol) }\end{array}$ & $\begin{array}{l}\text { Concentration } \\
\text { (M) }\end{array}$ & $\begin{array}{l}\text { weight required } \\
\text { (g) }\end{array}$ \\
\hline Sodium Carbonate & $\mathrm{Na}_{2} \mathrm{CO}_{3}$ & 106.00 & 0.00148 & 0.2196 \\
\hline Sodium Oxalate & $\mathrm{Na}_{2} \mathrm{C}_{2} \mathrm{O}_{4}$ & 134.00 & 0.0000726 & 0.0136 \\
\hline Sodium molybdate, dihydrate & $\mathrm{Na}_{2} \mathrm{MoO}_{4} \cdot 2 \mathrm{H}_{2} \mathrm{O}$ & 241.95 & 0.00000387 & 0.0013 \\
\hline $\begin{array}{l}\text { Sodium Metasilicate, 5- } \\
\text { hydrate }\end{array}$ & $\mathrm{Na}_{2} \mathrm{SiO}_{3} .5 \mathrm{H}_{2} \mathrm{O}$ & 212.14 & 0.00002944 & 0.0087 \\
\hline $\begin{array}{c}\text { Sodium phosphate, 12- } \\
\text { hydrate }\end{array}$ & $\mathrm{Na}_{3} \mathrm{PO}_{4} \cdot 12 \mathrm{H}_{2} \mathrm{O}$ & 380.00 & 0.00008272 & 0.0440 \\
\hline Sodium chloride & $\mathrm{NaCl}$ & 58.40 & 0.025 & 2.0440 \\
\hline Sodium Fluoride & $\mathrm{NaF}$ & 41.99 & 0.00015345 & 0.0090 \\
\hline Sodium sulfate & $\mathrm{Na}_{2} \mathrm{SO}_{4}$ & 142.00 & 0.0013674 & 0.2718 \\
\hline Sodium nitrate & $\mathrm{NaNO}_{3}$ & 85.00 & 0.04 & 4.7600 \\
\hline Sodium nitrite & $\mathrm{NaNO}_{2}$ & 69.00 & 0.9 & 86.9400 \\
\hline Sodium Hydroxide & $\mathrm{NaOH}$ & 40.00 & 0.0304 & 1.7024 \\
\hline
\end{tabular}




\section{Cyclic Potentiodynamic Polarization}

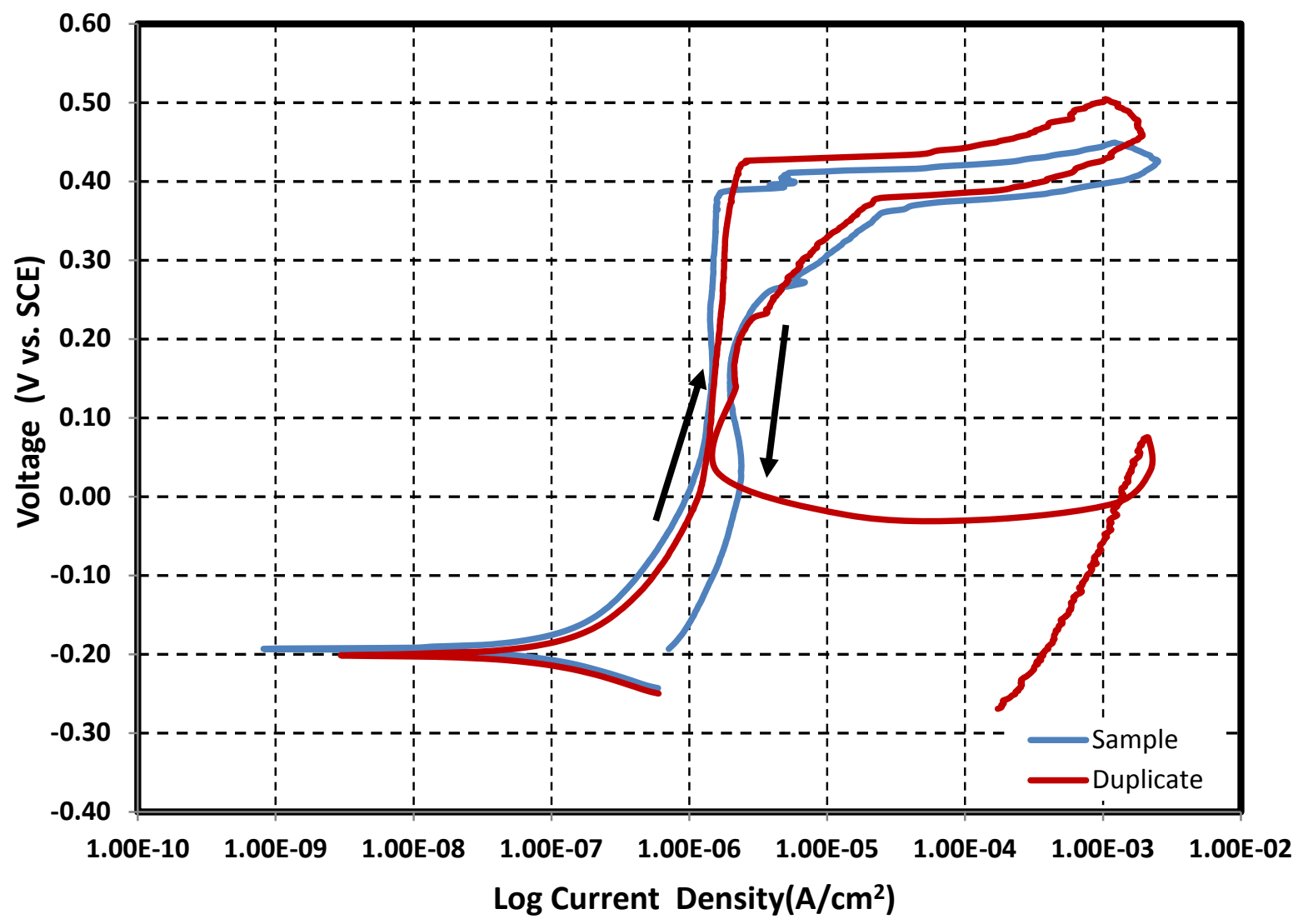

Images of bullet samples after electrochemical tests

Test 6

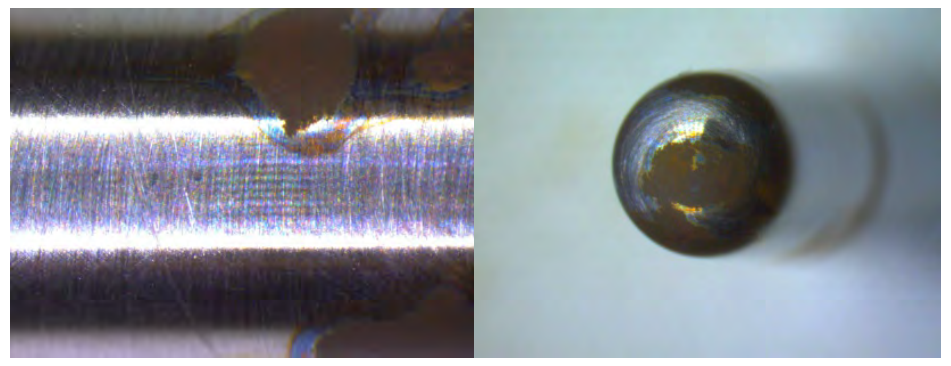

Test 6D

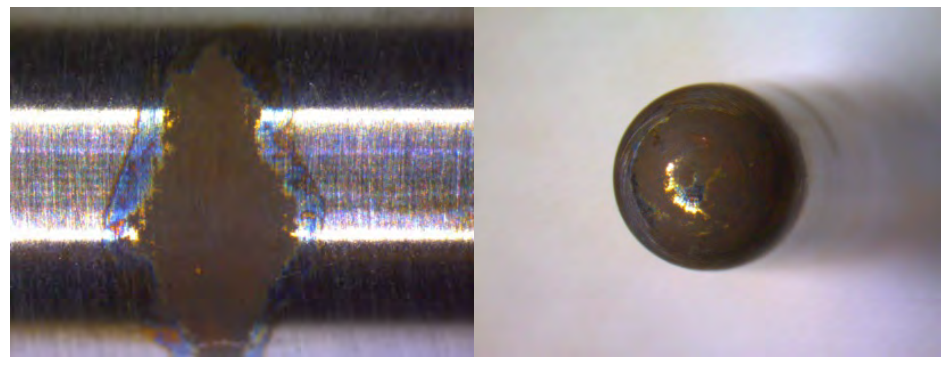

Shank (20X)

Nose (10X) 


\section{Composition of simulant for pitting corrosion -Test 7}

Reference 17

Test 7
Temperature

$\mathrm{pH}$

Volume
$25{ }^{\circ} \mathrm{C}$

12.00

\begin{tabular}{|c|c|c|c|c|}
\hline Simulant Source & Formula & $\begin{array}{c}\text { Molecular } \\
\text { Weight } \\
\text { (g/mol) }\end{array}$ & $\begin{array}{c}\text { Concentration } \\
\text { (M) }\end{array}$ & $\begin{array}{c}\text { weight required } \\
\text { (g) }\end{array}$ \\
\hline Sodium nitrate & $\mathrm{NaNO}_{3}$ & 84.99 & 7 & 832.9020 \\
\hline Sodium nitrite & $\mathrm{NaNO}_{2}$ & 69.00 & 0.01 & 0.9660 \\
\hline Sodium hydroxide & $\mathrm{NaOH}$ & 40.00 & 0.01 & 0.5600 \\
\hline Sodium chloride & $\mathrm{NaCl}$ & 58.44 & 0.1 & 8.1816 \\
\hline Sodium sulfate & $\mathrm{Na}_{2} \mathrm{SO}_{4}$ & 142.04 & 0.1 & 19.8856 \\
\hline Sodium carbonate & $\mathrm{Na}_{2}\left(\mathrm{CO}_{3}\right)$ & 106.00 & 0.1 & 14.8400 \\
\hline Sodium aluminate & $\mathrm{NaAlO}_{2}$ & 81.97 & 0.5 & 57.3790 \\
\hline $\begin{array}{c}\text { Sodium phosphate, } 12- \\
\text { hydrate }\end{array}$ & $\mathrm{Na}_{3} \mathrm{PO}_{4} .12 \mathrm{H}_{2} \mathrm{O}$ & 380.00 & 0.05 & 26.6000 \\
\hline
\end{tabular}




\section{Cyclic Potentiodynamic Polarization}

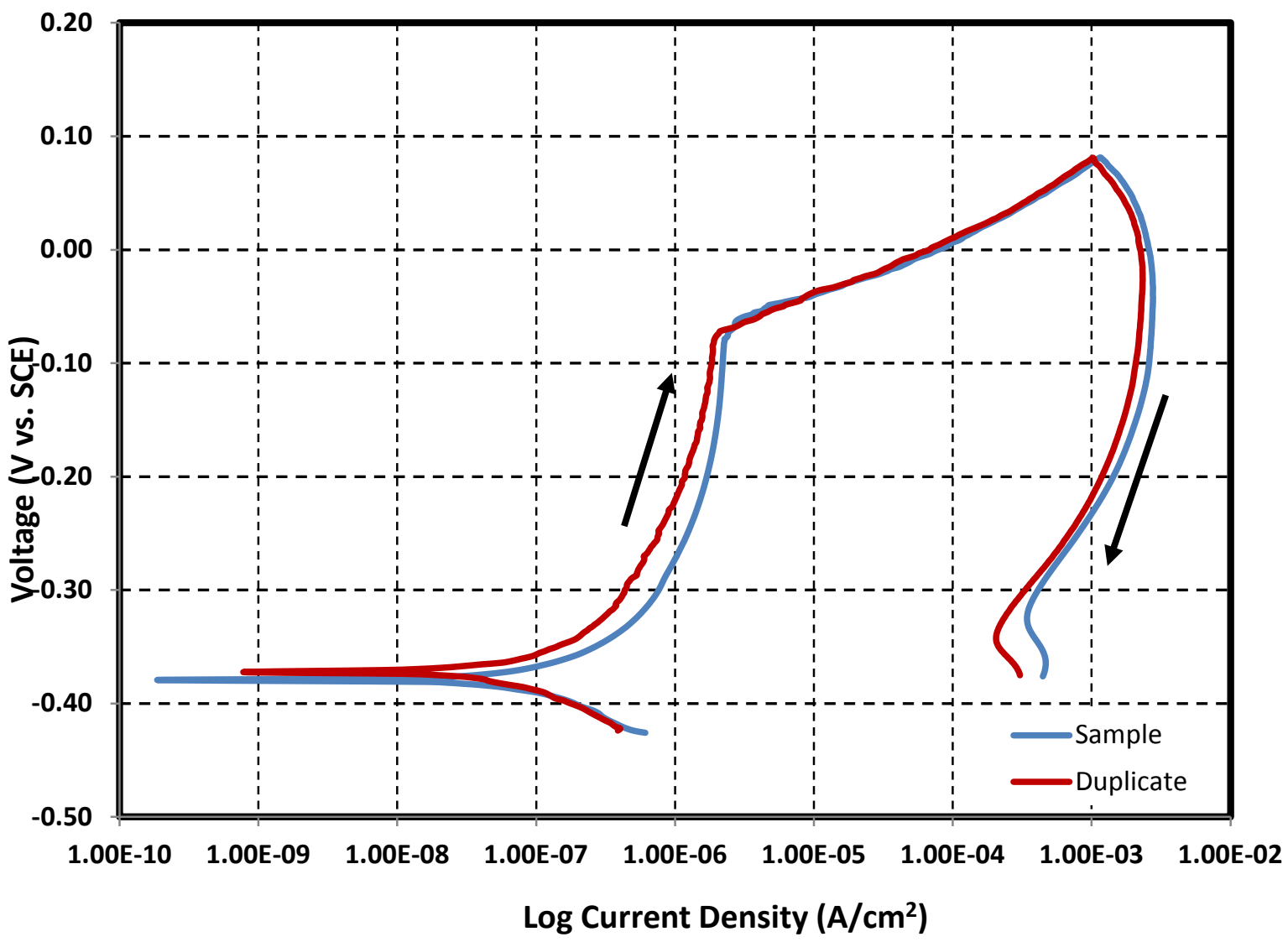

Images of bullet samples after electrochemical tests

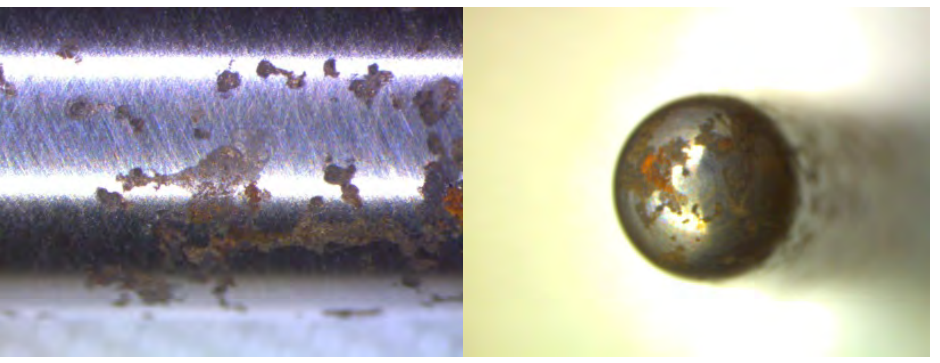

Test 7D

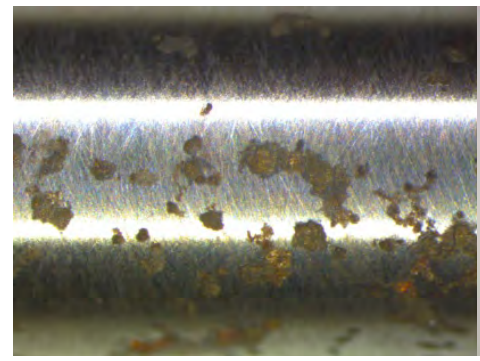

Shank (20X)

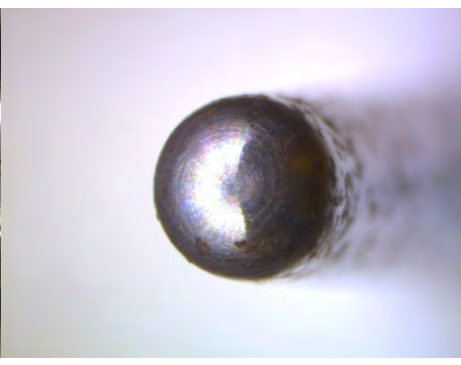

Nose (10X) 


\section{Composition of simulant for pitting corrosion -Test 8}

\section{Reference 10}

Test 8
Temperature

$\mathrm{pH}$

Volume

\begin{tabular}{|c|c|c|c|c|}
\hline Simulant Source & Formula & $\begin{array}{c}\text { Molecular } \\
\text { Weight } \\
\text { (g/mol) }\end{array}$ & $\begin{array}{c}\text { Concentration } \\
\text { (M) }\end{array}$ & $\begin{array}{c}\text { weight } \\
\text { required (g) }\end{array}$ \\
\hline Sodium Carbonate & $\mathrm{Na}_{2} \mathrm{CO}_{3}$ & 106.00 & 0.00148 & 0.2196 \\
\hline Sodium Oxalate & $\mathrm{Na}_{2} \mathrm{C}_{2} \mathrm{O}_{4}$ & 134.00 & 0.0000726 & 0.0136 \\
\hline Sodium molybdate, dihydrate & $\mathrm{Na}_{2} \mathrm{MoO}_{4} .2 \mathrm{H}_{2} \mathrm{O}$ & 241.95 & 0.00000387 & 0.0013 \\
\hline $\begin{array}{c}\text { Sodium Metasilicate, 5- } \\
\text { hydrate }\end{array}$ & $\mathrm{Na}_{2} \mathrm{SiO}_{3} .5 \mathrm{H}_{2} \mathrm{O}$ & 212.14 & 0.00002944 & 0.0087 \\
\hline $\begin{array}{c}\text { Sodium phosphate, 12- } \\
\text { hydrate }\end{array}$ & $\mathrm{Na}_{3} \mathrm{PO}_{4} 12 \mathrm{H}_{2} \mathrm{O}$ & 380.00 & 0.00008272 & 0.0440 \\
\hline Sodium chloride & $\mathrm{NaCl}$ & 58.40 & 0.0003185 & 0.0260 \\
\hline Sodium Fluoride & $\mathrm{NaF}$ & 41.99 & 0.00015345 & 0.0090 \\
\hline Sodium sulfate & $\mathrm{Na} \mathrm{SO}_{4}$ & 142.00 & 0.1 & 19.8800 \\
\hline Sodium nitrate & $\mathrm{NaNO}$ & 85.00 & 0.04 & 4.7600 \\
\hline Sodium nitrite & $\mathrm{NaNO}_{2}$ & 69.00 & 0.12 & 11.5920 \\
\hline Sodium Hydroxide & $\mathrm{NaOH}$ & 40.00 & 0.0304 & 1.7024 \\
\hline
\end{tabular}




\section{Cyclic Potentiodynamic Polarization}

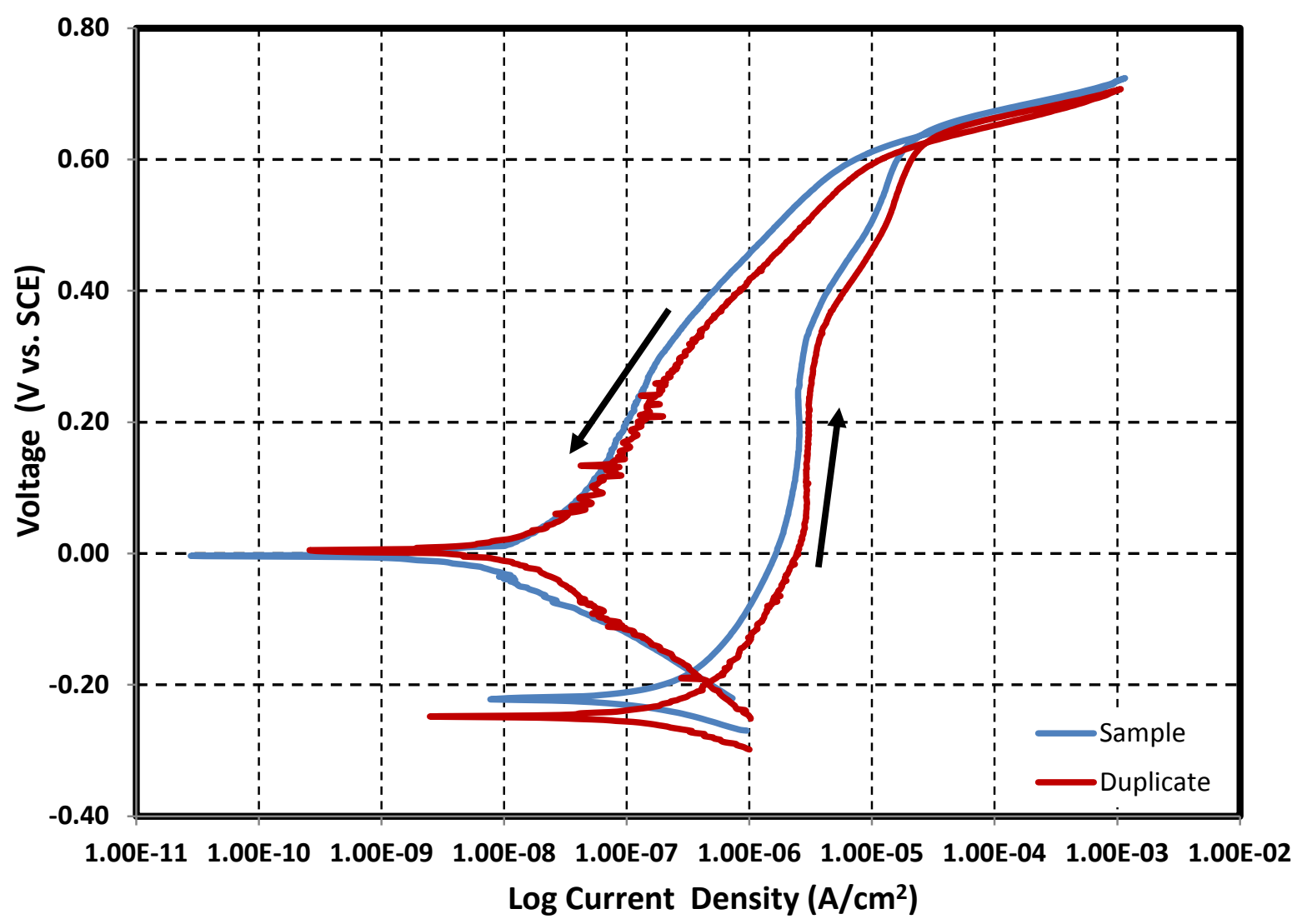

Images of bullet samples after electrochemical tests

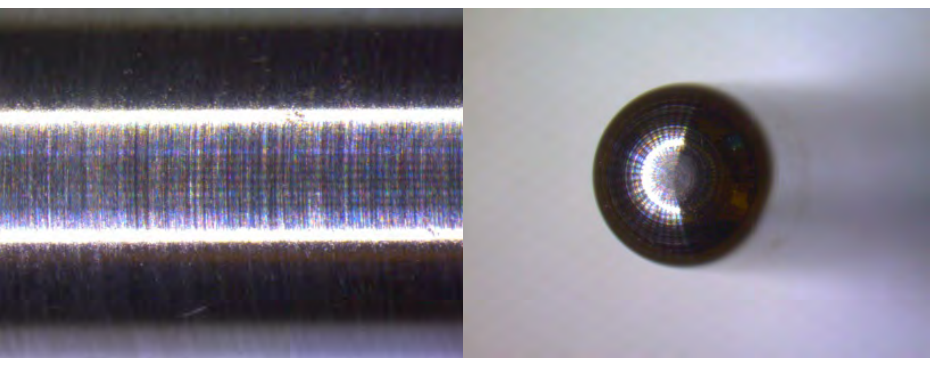

Test 8D

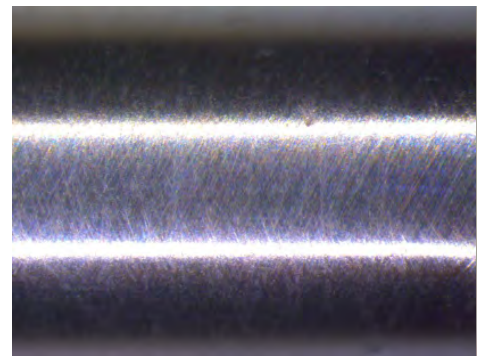

Shank (20X)

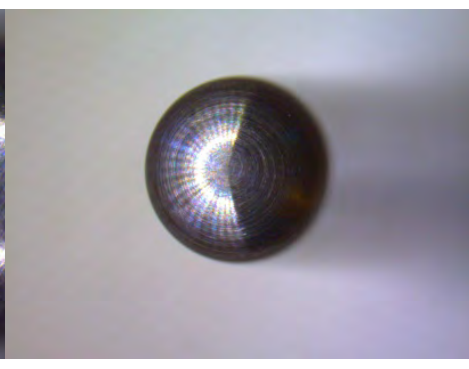

Nose (10X) 


\section{Composition of simulant for pitting corrosion -Test 9}

\begin{tabular}{|c|c|c|c|c|}
\hline \multirow{3}{*}{$\begin{array}{l}\text { Reference } 18 \\
\text { Test } 9\end{array}$} & \multirow{2}{*}{$\begin{array}{l}\text { Temperature } \\
\mathrm{pH}\end{array}$} & 40 & \multicolumn{2}{|l|}{${ }^{\circ} \mathrm{C}$} \\
\hline & & 12.12 & & \\
\hline & Volume & 1.4 & $\mathrm{~L}$ & \\
\hline Simulant Source & Formula & $\begin{array}{l}\text { Molecular } \\
\text { Weight } \\
\text { (g/mol) }\end{array}$ & $\begin{array}{l}\text { Concentration } \\
\text { (M) }\end{array}$ & $\begin{array}{l}\text { weight } \\
\text { required (g) }\end{array}$ \\
\hline Sodium nitrate & $\mathrm{NaNO}_{3}$ & 84.99 & 0.1 & 11.8986 \\
\hline Sodium chloride & $\mathrm{NaCl}$ & 58.44 & 0.4 & 32.7264 \\
\hline Sodium fluoride & $\mathrm{NaF}$ & 42.00 & 0.05 & 2.9400 \\
\hline Sodium nitrite & $\mathrm{NaNO}_{2}$ & 69.00 & 0.5 & 48.3000 \\
\hline Sodium hydroxide & $\mathrm{NaOH}$ & 40.00 & 0.01 & 0.5600 \\
\hline Sodium carbonate & $\mathrm{Na}_{2}\left(\mathrm{CO}_{3}\right)$ & 106.00 & 0.02 & 2.9680 \\
\hline Trisodium citrate dihydrate & $\mathrm{Na}_{3} \mathrm{C}_{6} \mathrm{H}_{5} \mathrm{O}_{7} \cdot 2 \mathrm{H}_{2} \mathrm{O}$ & 294.1000 & 0.6 & 247.0440 \\
\hline Sodium sulfate & $\mathrm{Na}_{2} \mathrm{SO}_{4}$ & 142.04 & 0.1 & 19.8856 \\
\hline $\begin{array}{c}\text { Sodium phosphate, 12- } \\
\text { hydrate }\end{array}$ & $\mathrm{Na}_{3} \mathrm{PO}_{4} \cdot 12 \mathrm{H}_{2} \mathrm{O}$ & 380.00 & 0.0005 & 0.2660 \\
\hline
\end{tabular}




\section{Cyclic Potentiodynamic Polarization}

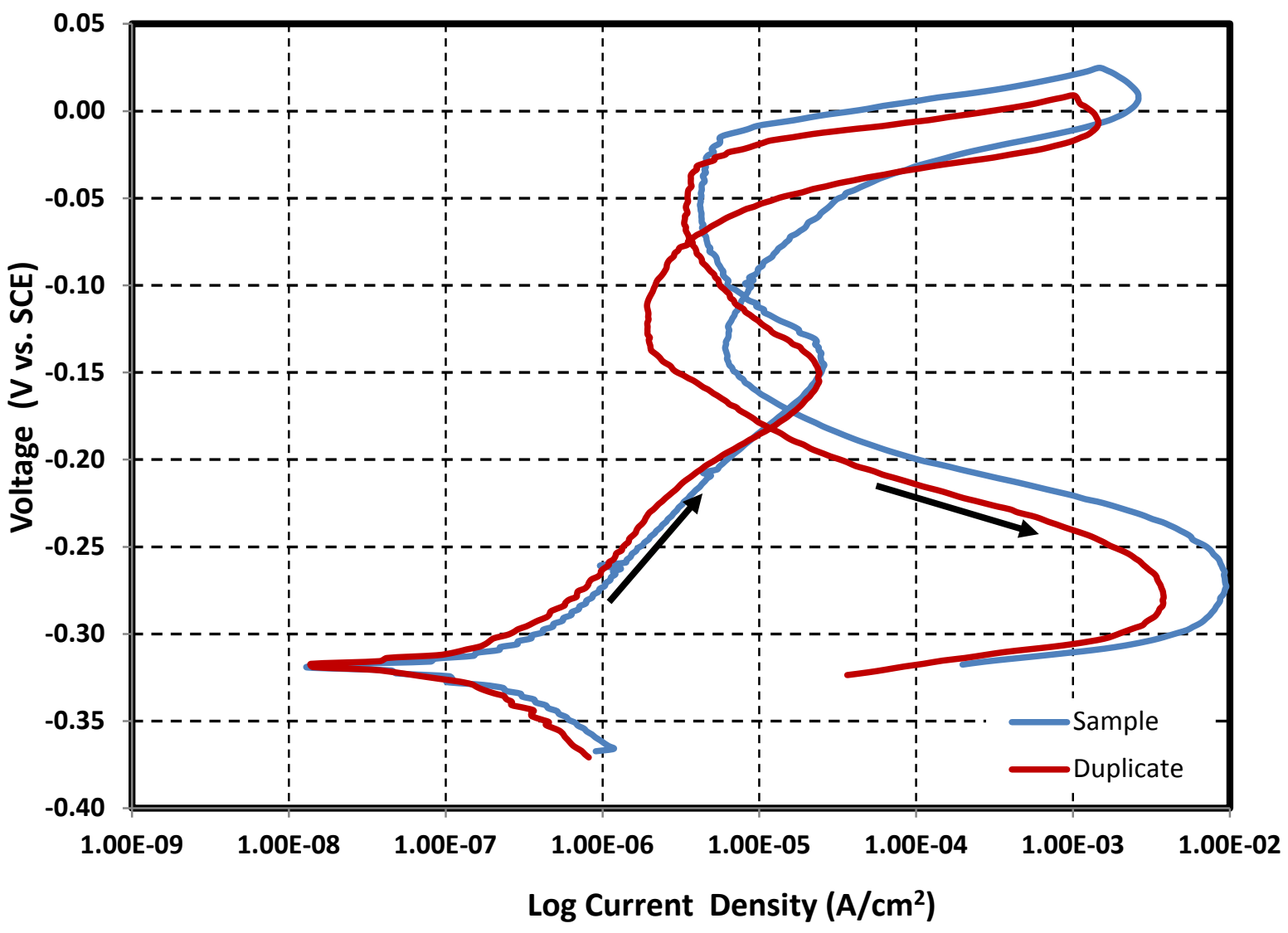

Images of bullet samples after electrochemical tests

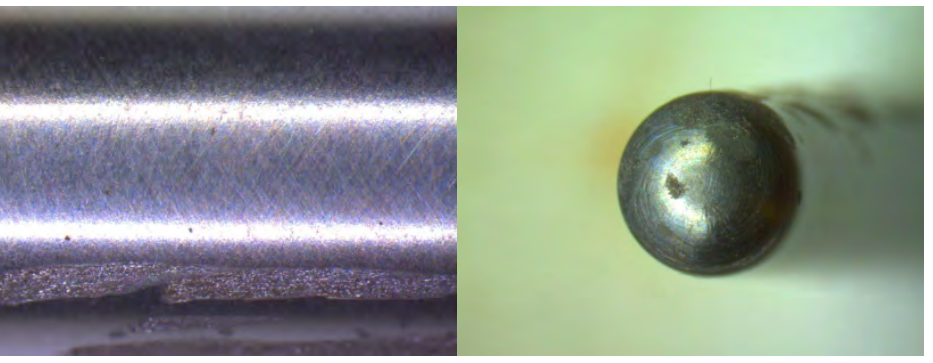

Test 9D

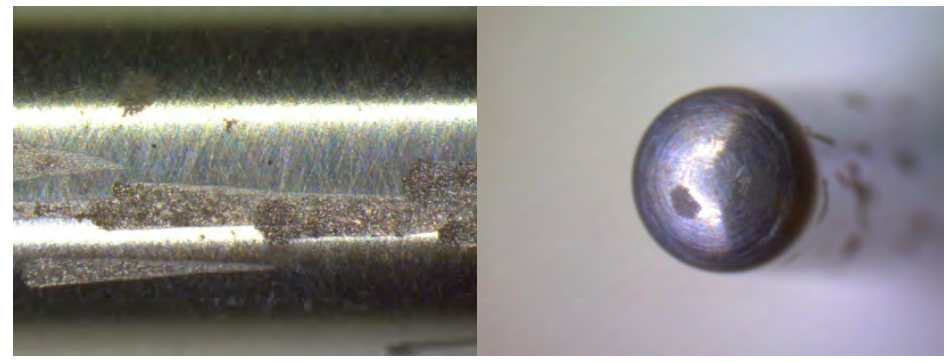

Shank (20X)

Nose (10X) 
Composition of simulant for pitting corrosion -Test 10

\section{Reference 10}

Test 10
Temperature

$\mathrm{pH}$

Volume
$40{ }^{\circ} \mathrm{C}$

12.50

$1.4 \mathrm{~L}$

\begin{tabular}{|c|c|c|c|c|}
\hline Simulant Source & Formula & $\begin{array}{c}\text { Molecular } \\
\text { Weight } \\
\text { (g/mol) }\end{array}$ & $\begin{array}{c}\text { Concentration } \\
\text { (M) }\end{array}$ & $\begin{array}{c}\text { weight required } \\
\text { (g) }\end{array}$ \\
\hline Sodium Carbonate & $\mathrm{Na}_{2} \mathrm{CO}_{3}$ & 106.00 & 0.00148 & 0.2196 \\
\hline Sodium Oxalate & $\mathrm{Na}_{2} \mathrm{C}_{2} \mathrm{O}_{4}$ & 134.00 & 0.0000726 & 0.0136 \\
\hline Sodium molybdate, dihydrate & $\mathrm{Na}_{2} \mathrm{MoO}_{4} \cdot 2 \mathrm{H}_{2} \mathrm{O}$ & 241.95 & 0.00000387 & 0.0013 \\
\hline $\begin{array}{c}\text { Sodium Metasilicate, 5- } \\
\text { hydrate }\end{array}$ & $\mathrm{Na}_{2} \mathrm{SiO}_{3} .5 \mathrm{H}_{2} \mathrm{O}$ & 212.14 & 0.00002944 & 0.0087 \\
\hline $\begin{array}{c}\text { Sodium phosphate, 12- } \\
\text { hydrate }\end{array}$ & $\mathrm{Na}_{3} \mathrm{PO}_{4} 12 \mathrm{H}_{2} \mathrm{O}$ & 380.00 & 0.00008272 & 0.0440 \\
\hline Sodium chloride & $\mathrm{NaCl}$ & 58.40 & 0.0003185 & 0.0260 \\
\hline Sodium Fluoride & $\mathrm{NaF}$ & 41.99 & 0.00015345 & 0.0090 \\
\hline Sodium sulfate & $\mathrm{Na}_{2} \mathrm{SO}_{4}$ & 142.00 & 0.1 & 19.8800 \\
\hline Sodium nitrate & $\mathrm{NaNO}_{3}$ & 85.00 & 0.04 & 4.7600 \\
\hline Sodium nitrite & $\mathrm{NaNO}_{2}$ & 69.00 & 0.18 & 17.3880 \\
\hline Sodium Hydroxide & $\mathrm{NaOH}$ & 40.00 & 0.0304 & 1.7024 \\
\hline
\end{tabular}




\section{Cyclic Potentiodynamic Polarization}

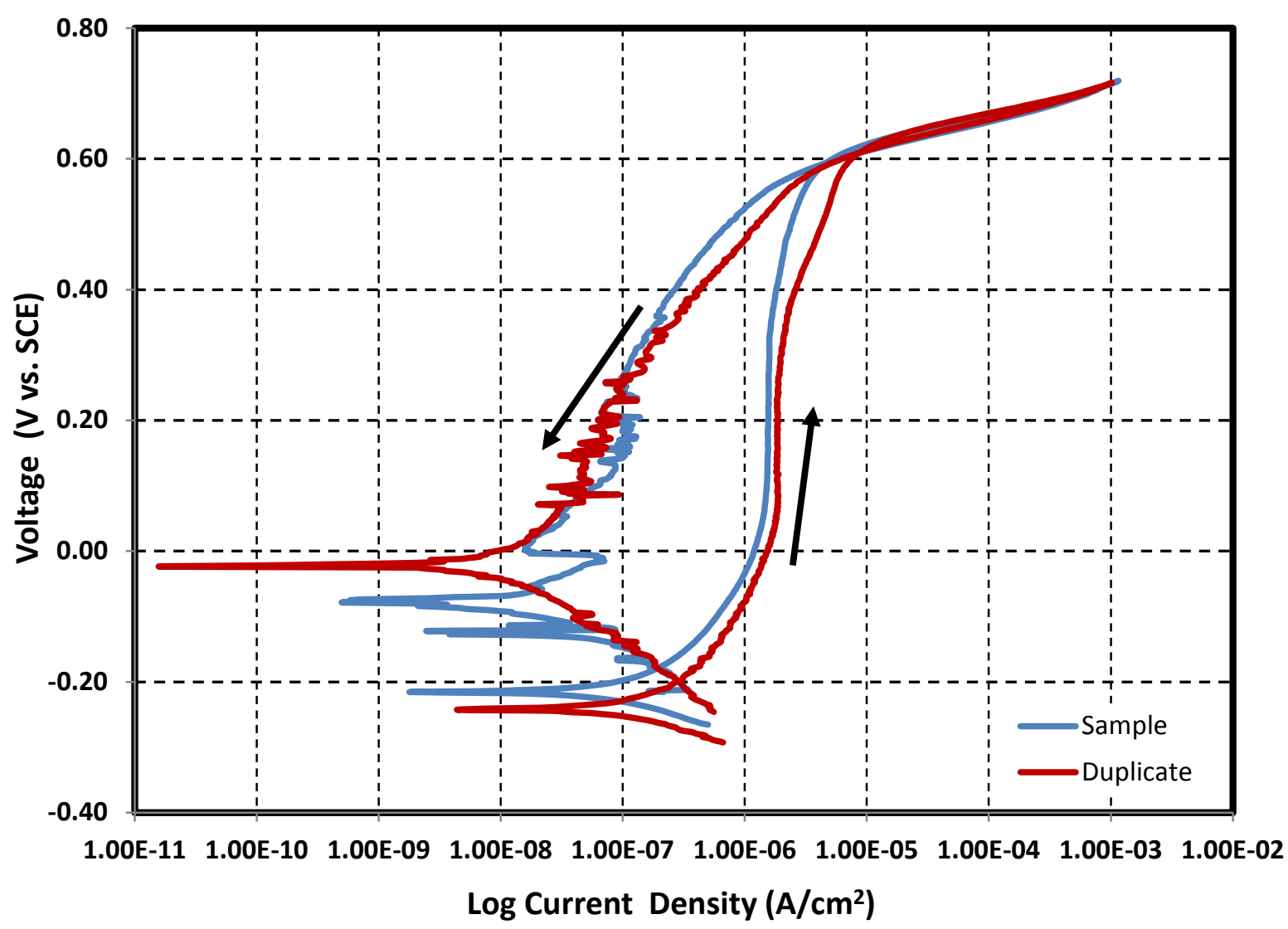

Images of bullet samples after electrochemical tests

Test 10

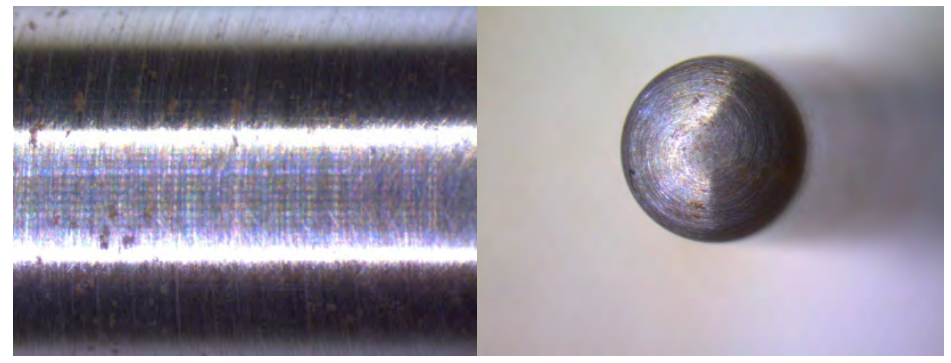

Test 10D

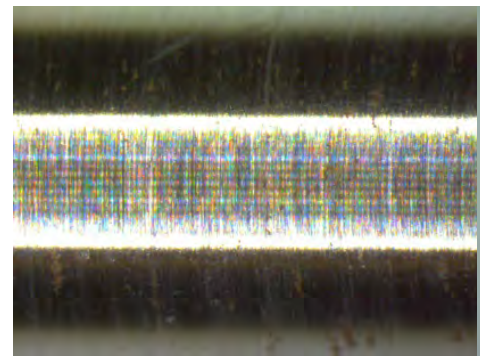

Shank (20X)

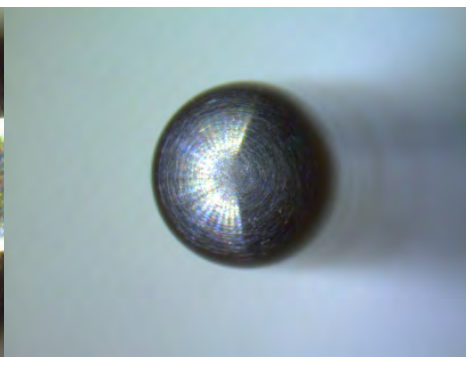

Nose (10X) 
Composition of simulant for pitting corrosion -Test 11

Reference 10

Test 11
Temperature

$\mathrm{pH}$

Volume

\begin{tabular}{|c|c|c|c|c|}
\hline Simulant Source & Formula & $\begin{array}{c}\text { Molecular } \\
\text { Weight } \\
\text { (g/mol) }\end{array}$ & $\begin{array}{c}\text { Concentration } \\
\text { (M) }\end{array}$ & $\begin{array}{c}\text { weight } \\
\text { required (g) }\end{array}$ \\
\hline Sodium Carbonate & $\mathrm{Na}_{2} \mathrm{CO}_{3}$ & 106.00 & 0.0173 & 2.5673 \\
\hline Sodium Oxalate & $\mathrm{Na}_{2} \mathrm{C}_{2} \mathrm{O}_{4}$ & 134.00 & 0.0000726 & 0.0136 \\
\hline Sodium molybdate, dihydrate & $\mathrm{Na}_{2} \mathrm{MoO}_{4} \cdot 2 \mathrm{H}_{2} \mathrm{O}$ & 241.95 & 0.00000387 & 0.0013 \\
\hline $\begin{array}{c}\text { Sodium Metasilicate, 5- } \\
\text { hydrate }\end{array}$ & $\mathrm{Na}_{2} \mathrm{SiO}_{3} \cdot 5 \mathrm{H}_{2} \mathrm{O}$ & 212.14 & 0.00002944 & 0.0087 \\
\hline $\begin{array}{c}\text { Sodium phosphate, 12- } \\
\text { hydrate }\end{array}$ & $\mathrm{Na}_{3} \mathrm{PO}_{4} \cdot \mathrm{1}_{2} \mathrm{H}_{2} \mathrm{O}$ & 380.00 & 0.00008272 & 0.0440 \\
\hline Sodium chloride & $\mathrm{NaCl}$ & 58.40 & 0.0003185 & 0.0260 \\
\hline Sodium Fluoride & $\mathrm{NaF}$ & 41.99 & 0.00015345 & 0.0090 \\
\hline Sodium sulfate & $\mathrm{Na} \mathrm{SO}_{4}$ & 142.00 & 0.02 & 3.9760 \\
\hline Sodium nitrate & $\mathrm{NaNO}$ & 85.00 & 0.04 & 4.7600 \\
\hline Sodium nitrite & $\mathrm{NaNO}$ & 69.00 & 0.04 & 3.8640 \\
\hline Sodium Hydroxide & $\mathrm{NaOH}$ & 40.00 & 0.0304 & 1.7024 \\
\hline
\end{tabular}

$40{ }^{\circ} \mathrm{C}$

12.50

$1.4 \quad \mathrm{~L}$ 


\section{Cyclic Potentiodynamic Polarization}

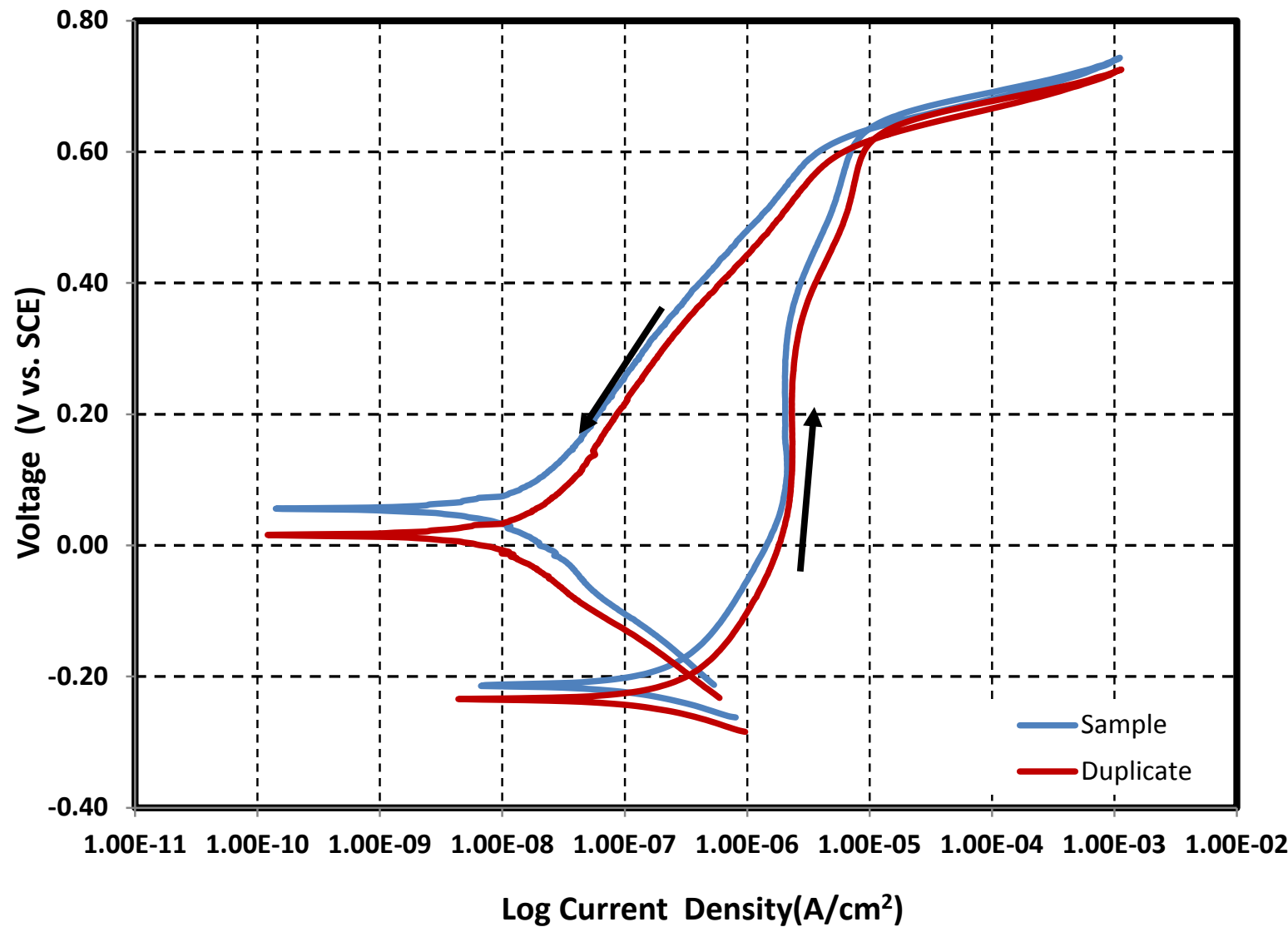

Images of bullet samples after electrochemical tests

Test 11

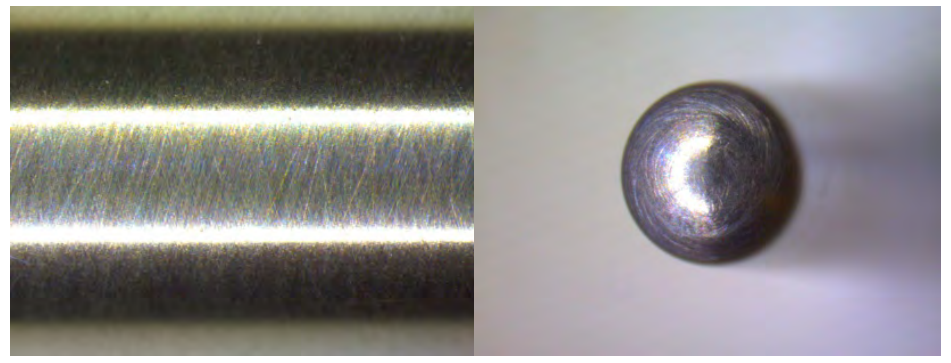

Test 11D

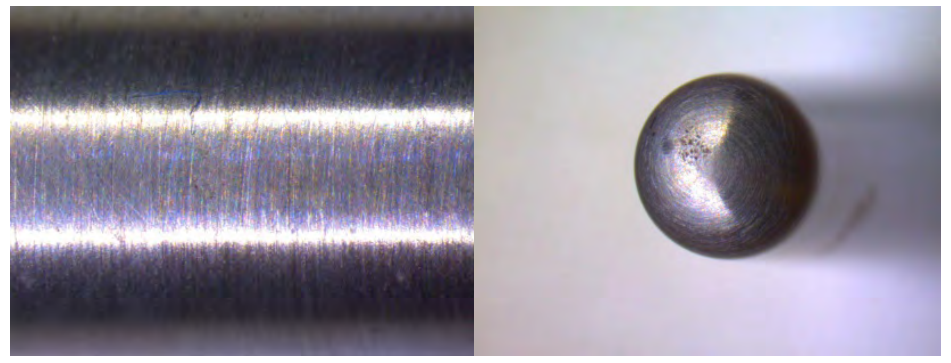

Shank (20X)

Nose (10X) 
Composition of simulant for pitting corrosion -Test 12

Reference 18

Test 12
Temperature

$\mathrm{pH}$
$40{ }^{\circ} \mathrm{C}$

11.70

\begin{tabular}{|c|c|c|c|c|}
\hline Simulant Source & Formula & $\begin{array}{c}\text { Molecular } \\
\text { Weight } \\
\text { (g/mol) }\end{array}$ & $\begin{array}{c}\text { Concentration } \\
\text { (M) }\end{array}$ & $\begin{array}{c}\text { weight } \\
\text { required (g) }\end{array}$ \\
\hline Sodium nitrate & $\mathrm{NaNO}_{3}$ & 84.99 & 0.7 & 83.2902 \\
\hline Sodium chloride & $\mathrm{NaCl}$ & 58.44 & 0.06 & 4.9090 \\
\hline Sodium fluoride & $\mathrm{NaF}$ & 42.00 & 0.02 & 1.1760 \\
\hline Sodium nitrite & $\mathrm{NaNO}_{2}$ & 69.00 & 0.02 & 1.9320 \\
\hline Sodium hydroxide & $\mathrm{NaOH}$ & 40.00 & 0.01 & 0.5600 \\
\hline Sodium carbonate & $\mathrm{Na}_{2}\left(\mathrm{CO}_{3}\right)$ & 106.00 & 0.2 & 29.6800 \\
\hline Trisodium citrate dihydrate & $\mathrm{Na}_{3} \mathrm{C}_{6} \mathrm{H}_{5} \mathrm{O}_{7} .2 \mathrm{H}$ & 294.1000 & 0.04 & 16.4696 \\
\hline Sodium sulfate & $\mathrm{Na}_{2} \mathrm{SO}_{4}$ & 142.04 & 0.1 & 19.8856 \\
\hline Sodium phosphate, $12-$ & $\mathrm{Na}_{3} \mathrm{PO}_{4} 12 \mathrm{H}_{2} \mathrm{O}$ & 380.00 & 0.05 & 26.6000 \\
\hline hydrate & &
\end{tabular}




\section{Cyclic Potentiodynamic Polarization}

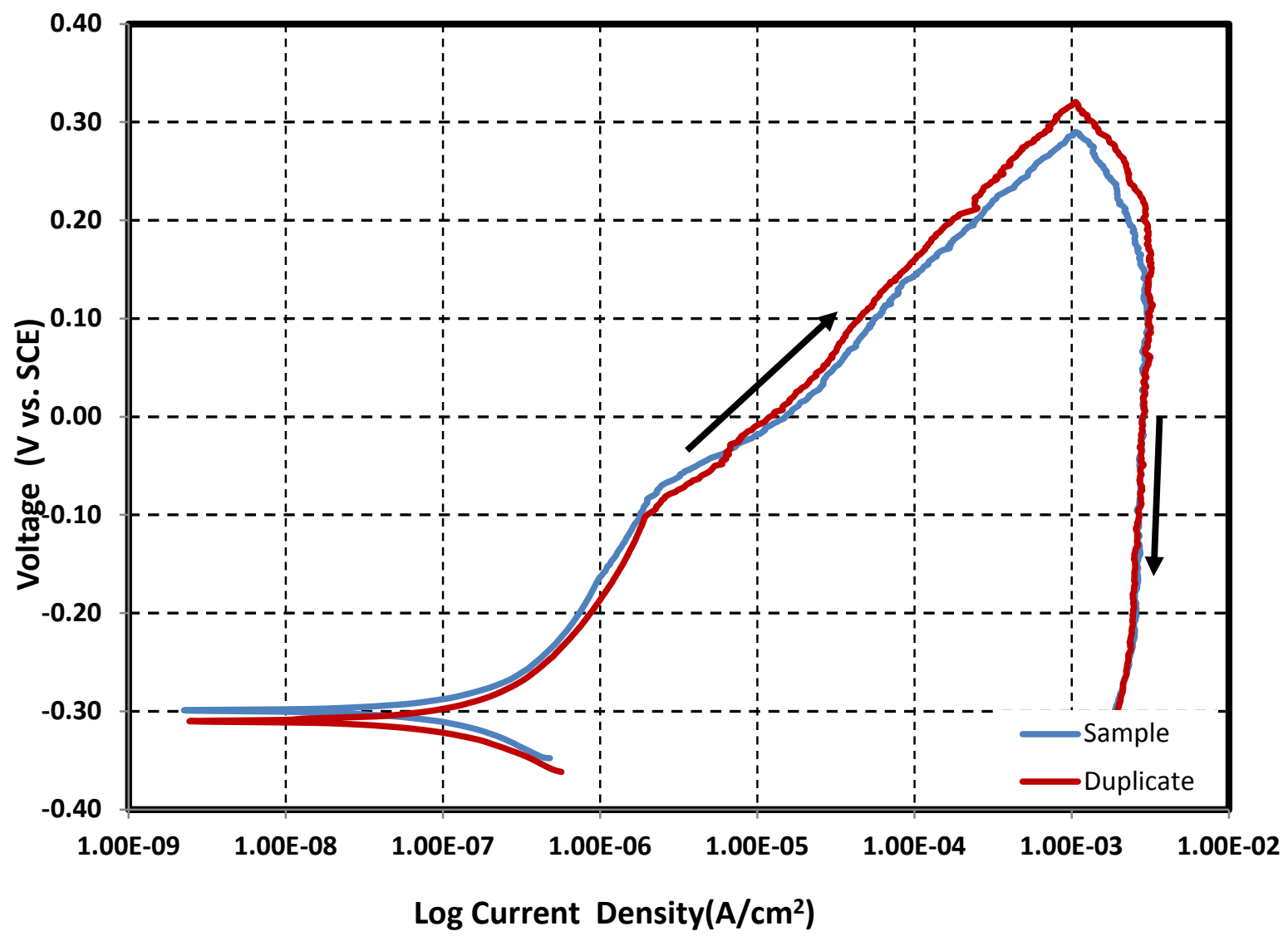

Images of bullet samples after electrochemical tests

Test 12

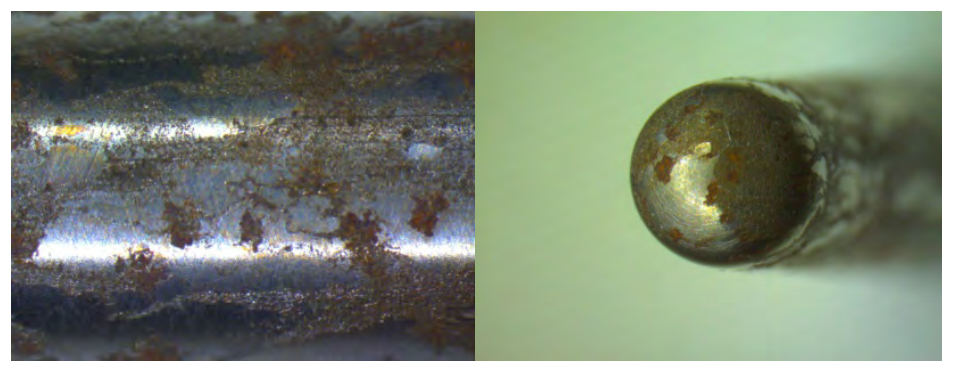

Test 12D

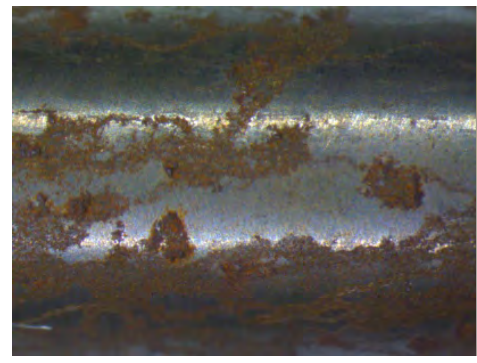

Shank $(20 X)$

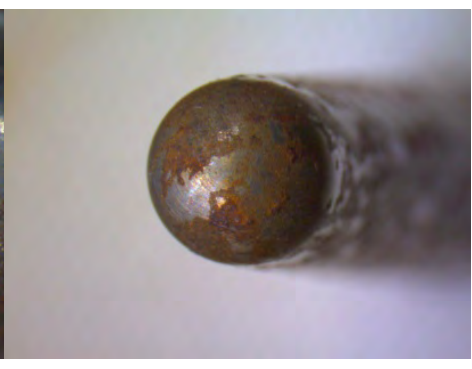

Nose (10X) 
Composition of simulant for pitting corrosion -Test 13

\begin{tabular}{|c|c|c|c|c|}
\hline $\begin{array}{l}\text { Reference } 4 \\
\text { Test } 13\end{array}$ & $\begin{array}{l}\text { Temperature } \\
\mathrm{pH} \\
\text { Volume }\end{array}$ & $\begin{array}{r}40 \\
9.6 \\
1.4\end{array}$ & ${ }^{\circ} \mathrm{C}$ & \\
\hline Simulant Source & Formula & $\begin{array}{l}\text { Molecular } \\
\text { Weight } \\
\text { (g/mol) }\end{array}$ & Concentration (M) & $\begin{array}{l}\text { weight } \\
\text { required (g) }\end{array}$ \\
\hline Aluminum nitrate, 9-hydrate & $\mathrm{Al}\left(\mathrm{NO}_{3}\right)_{3} \cdot 9 \mathrm{H}_{2} \mathrm{O}$ & 375.13 & 0.000159945 & 0.0840 \\
\hline Ferric Nitrate, 9-hydrate & $\mathrm{Fe}\left(\mathrm{NO}_{3}\right)_{3} .9 \mathrm{H}_{2} \mathrm{O}$ & 404.00 & 4.9505E-05 & 0.0280 \\
\hline Sodium hydroxide & $\mathrm{NaOH}$ & 40.00 & 0.0305 & 1.7080 \\
\hline Sodium nitrite & $\mathrm{NaNO}_{2}$ & 69.00 & 0.010028986 & 0.9688 \\
\hline Sodium oxalate & $\mathrm{Na}_{2}\left(\mathrm{C}_{2} \mathrm{O}_{4}\right)$ & 134.00 & 0.002885572 & 0.5413 \\
\hline Sodium sulfate & $\mathrm{Na}_{2} \mathrm{SO}_{4}$ & 142.04 & 0.001980663 & 0.3939 \\
\hline Sodium carbonate & $\mathrm{Na}_{2}\left(\mathrm{CO}_{3}\right)$ & 106.00 & 0.004679245 & 0.6944 \\
\hline Sodium Bicarbonate & $\mathrm{NaHCO}_{3}$ & 84.00 & 0.019333333 & 2.2736 \\
\hline $\begin{array}{c}\text { Sodium phosphate, 12- } \\
\text { hydrate }\end{array}$ & $\mathrm{Na}_{3} \mathrm{PO}_{4} \cdot 12 \mathrm{H}_{2} \mathrm{O}$ & 380.12 & 9.16903E-05 & 0.0488 \\
\hline Calcium Carbonate & $\mathrm{CaCO}_{3}$ & 100.00 & 0.000220133 & 0.0308 \\
\hline Sodium chloride & $\mathrm{NaCl}$ & 58.44 & 0.000382729 & 0.0313 \\
\hline Sodium fluoride & $\mathrm{NaF}$ & 42.00 & 0.000243175 & 0.0143 \\
\hline $\begin{array}{c}\text { Sodium molybdate, } \\
\text { dihydrate }\end{array}$ & $\mathrm{Na}_{2} \mathrm{MoO}_{4} \cdot 2 \mathrm{H}_{2} \mathrm{O}$ & 241.95 & 6.15066E-06 & 0.0021 \\
\hline Manganese Dioxide & $\mathrm{MnO}_{2}$ & 86.94 & 0.000380032 & 0.0463 \\
\hline Nickel nitrate, 6-hydrate & $\mathrm{Ni}\left(\mathrm{NO}_{3}\right)_{2} \cdot 6 \mathrm{H}_{2} \mathrm{O}$ & 290.81 & 0.000173355 & 0.0706 \\
\hline Mercury (II) nitrate & $\mathrm{Hg}\left(\mathrm{NO}_{3}\right)_{2}$ & 342.62 & 0.000146 & 0.0700 \\
\hline Sodium silicate, 9-hydrate & $\mathrm{Na}_{2} \mathrm{SiO}_{3} \cdot 9 \mathrm{H}_{2} \mathrm{O}$ & 284.00 & 5.33803E-05 & 0.0212 \\
\hline Zinc nitrate, 6-hydrate & $\mathrm{Zn}\left(\mathrm{NO}_{3}\right)_{2} \cdot 6 \mathrm{H}_{2} \mathrm{O}$ & 297.49 & 1.37147E-05 & 0.0057 \\
\hline Lead nitrate & $\mathrm{Pb}\left(\mathrm{NO}_{3}\right)_{2}$ & 331.21 & 6.48129E-06 & 0.0030 \\
\hline Cupric nitrate, 2.5 hydrate & $\begin{array}{c}\mathrm{Cu}\left(\mathrm{NO}_{3}\right)_{2} \cdot 2.5 \mathrm{H}_{2} \\
\mathrm{O}\end{array}$ & 233.00 & 8.18312E-06 & 0.0027 \\
\hline
\end{tabular}




\section{Cyclic Potentiodynamic Polarization}

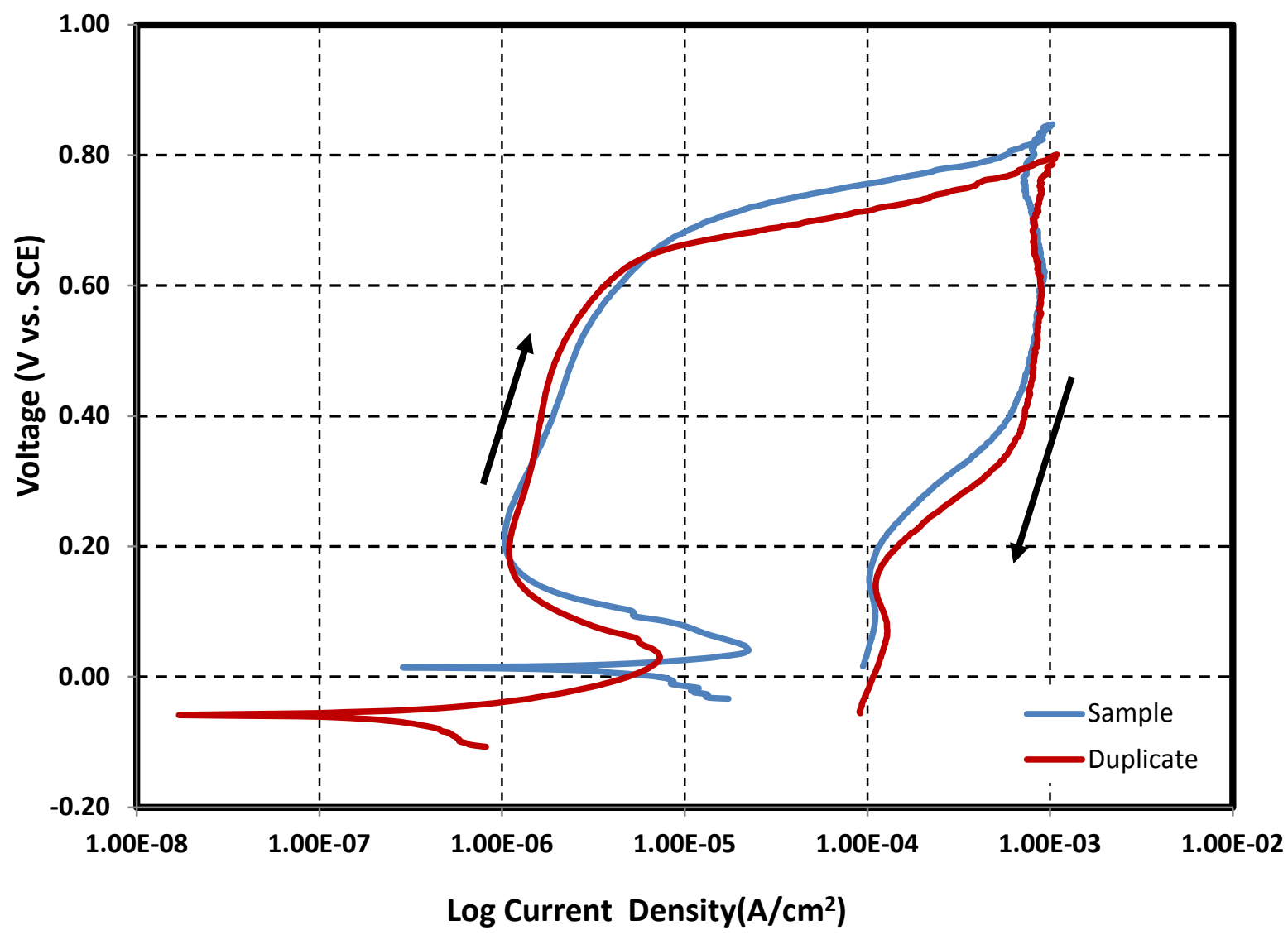

Images of bullet samples after electrochemical tests

Test 13
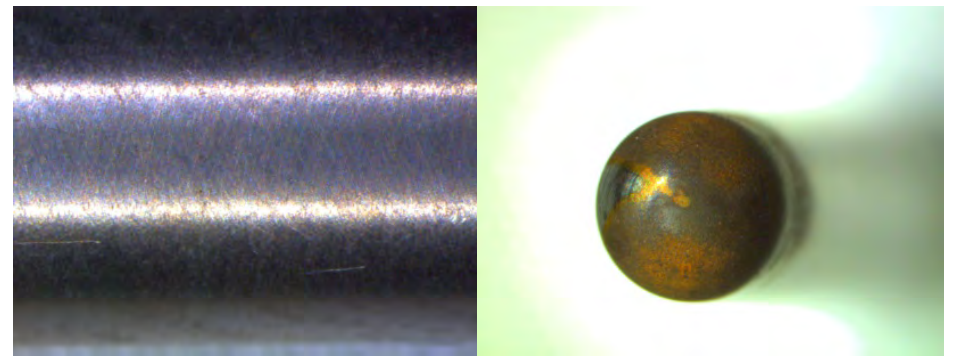

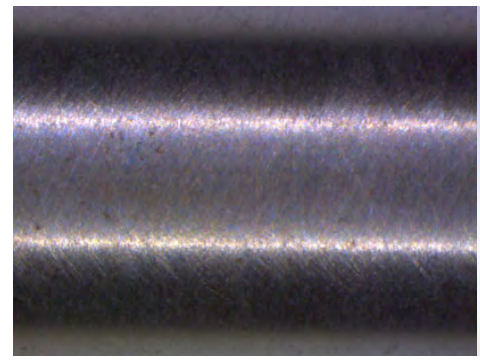

Shank $(20 X)$

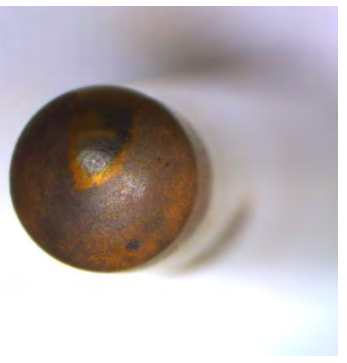

Nose (10X) 


\section{Composition of simulant for pitting corrosion -Test 14}

Reference 1

Test 14
Temperature

$\mathrm{pH}$

Volume
$23{ }^{\circ} \mathrm{C}$

9.73

\begin{tabular}{|c|c|c|c|c|}
\hline Simulant Source & Formula & $\begin{array}{c}\text { Molecular } \\
\text { Weight } \\
\text { (g/mol) }\end{array}$ & $\begin{array}{l}\text { Concentration } \\
\text { (M) }\end{array}$ & $\begin{array}{l}\text { weight } \\
\text { required }(g)\end{array}$ \\
\hline Sodium carbonate & $\mathrm{Na}_{2} \mathrm{CO}_{3}$ & 106.00 & 0.0173 & 2.5673 \\
\hline Sodium bicarbonate & $\mathrm{NaHCO}_{3}$ & 84.01 & 0.0446 & 5.2456 \\
\hline Sodium oxalate & $\mathrm{Na}_{2} \mathrm{C}_{2} \mathrm{O}_{4}$ & 134.00 & 0.000194 & 0.0364 \\
\hline $\begin{array}{c}\text { Sodium molybdate, } \\
\text { dihydrate }\end{array}$ & $\mathrm{Na}_{2} \mathrm{MoO}_{4} \cdot 2 \mathrm{H}_{2} \mathrm{O}$ & 241.95 & 0.0000103 & 0.0035 \\
\hline $\begin{array}{c}\text { Sodium metasilicate, 5- } \\
\text { hydrate }\end{array}$ & $\mathrm{Na}_{2} \mathrm{SiO}_{3} .5 \mathrm{H}_{2} \mathrm{O}$ & 212.14 & 0.0000786 & 0.0233 \\
\hline $\begin{array}{c}\text { Sodium phosphate, 12- } \\
\text { hydrate }\end{array}$ & $\mathrm{Na}_{3} \mathrm{PO}_{4} 12 \mathrm{H}_{2} \mathrm{O}$ & 380.00 & 0.000223 & 0.1186 \\
\hline Sodium chloride & $\mathrm{NaCl}$ & 58.40 & 0.0008635 & 0.0706 \\
\hline Sodium fluoride & $\mathrm{NaF}$ & 41.99 & 0.000413 & 0.0243 \\
\hline Sodium sulfate & $\mathrm{Na}_{2} \mathrm{SO}_{4}$ & 142.00 & 0.00363 & 0.7216 \\
\hline Sodium nitrate & $\mathrm{NaNO}_{3}$ & 85.00 & 0.0546 & 6.4974 \\
\hline Sodium nitrite & $\mathrm{NaNO}_{2}$ & 69.00 & 0.015 & 1.4490 \\
\hline Sodium aluminate & $\mathrm{NaAlO}_{2}$ & 81.97 & 0.00045 & 0.0516 \\
\hline Cobalt nitrate, 6-hydrate & $\mathrm{Co}\left(\mathrm{NO}_{3}\right)_{2} \cdot 6 \mathrm{H}_{2} \mathrm{O}$ & 291.03 & 0.00003 & 0.0122 \\
\hline Nickel nitrate, 6-hydrate & $\mathrm{Ni}\left(\mathrm{NO}_{3}\right)_{2} \cdot 6 \mathrm{H}_{2} \mathrm{O}$ & 291.00 & 0.0015 & 0.6111 \\
\hline Ferric nitrate, 9-hydrate & $\mathrm{Fe}\left(\mathrm{NO}_{3}\right)_{3} .9 \mathrm{H}_{2} \mathrm{O}$ & 404.00 & 0.000248 & 0.1403 \\
\hline Mercury (II) nitrate & $\mathrm{Hg}\left(\mathrm{NO}_{3}\right)_{2}$ & 324.60 & 0.00025 & 0.1136 \\
\hline Cupric nitrate, 2.5 hydrate & $\begin{array}{c}\mathrm{Cu}\left(\mathrm{NO}_{3}\right)_{2} \cdot 2.5 \mathrm{H}_{2} \\
\mathrm{O}\end{array}$ & 233.00 & 0.000043 & 0.0140 \\
\hline Manganese dioxide & $\mathrm{MnO}_{2}$ & 86.94 & 0.00575 & 0.6999 \\
\hline
\end{tabular}




\section{Cyclic Potentiodynamic Polarization}

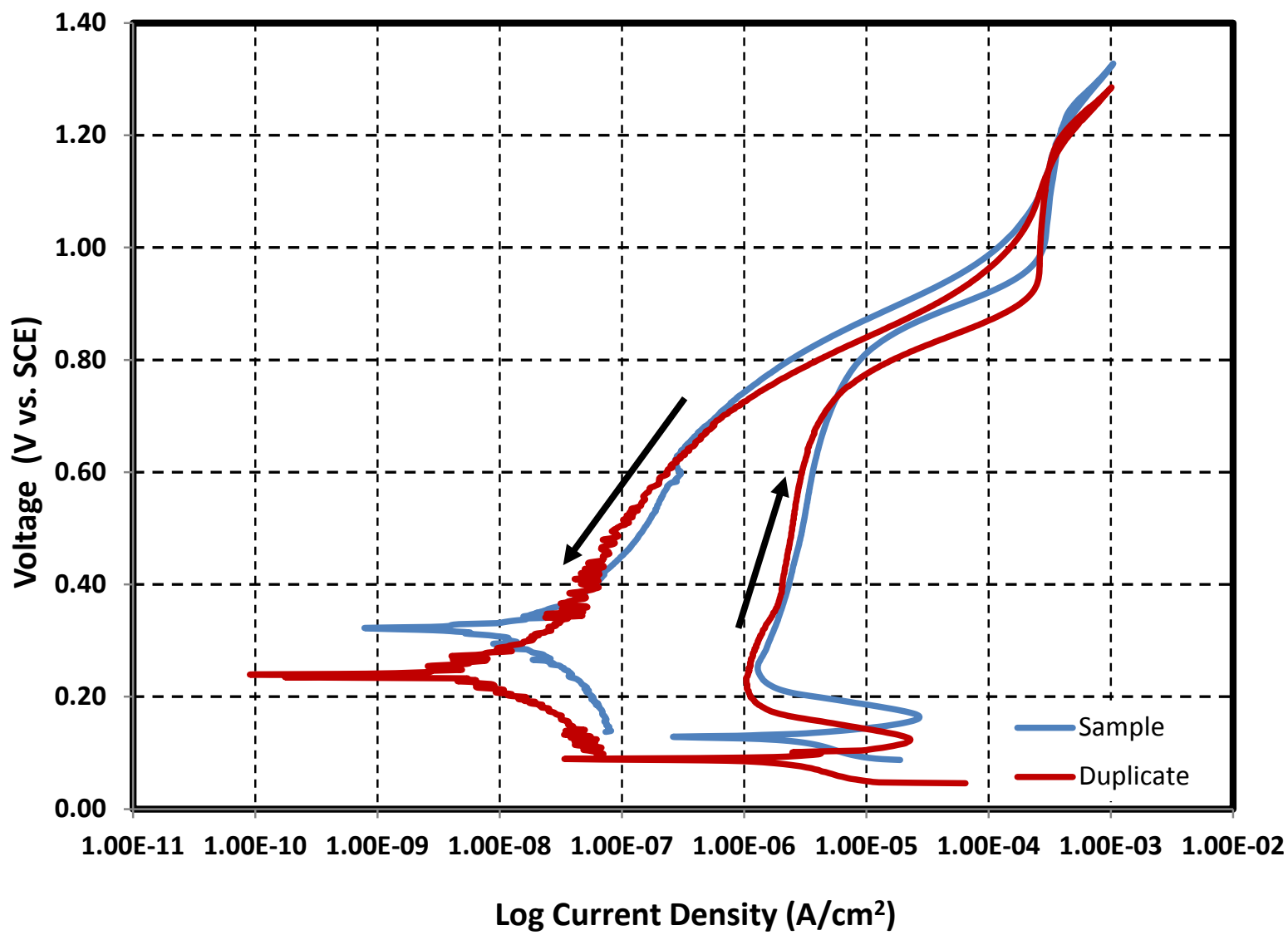

Images of bullet samples after electrochemical tests

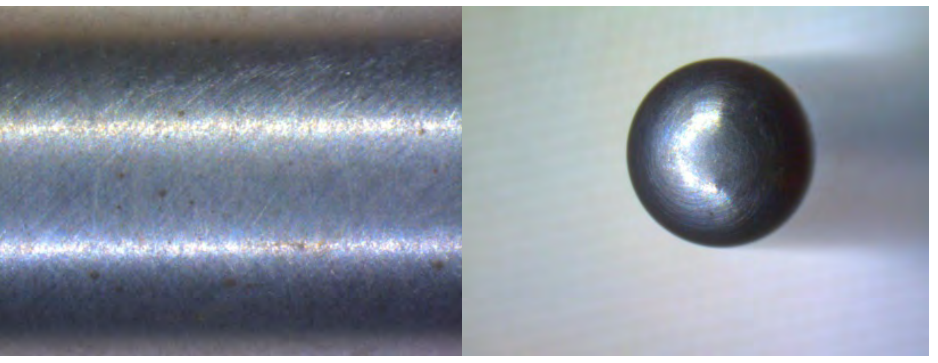

Test 14D

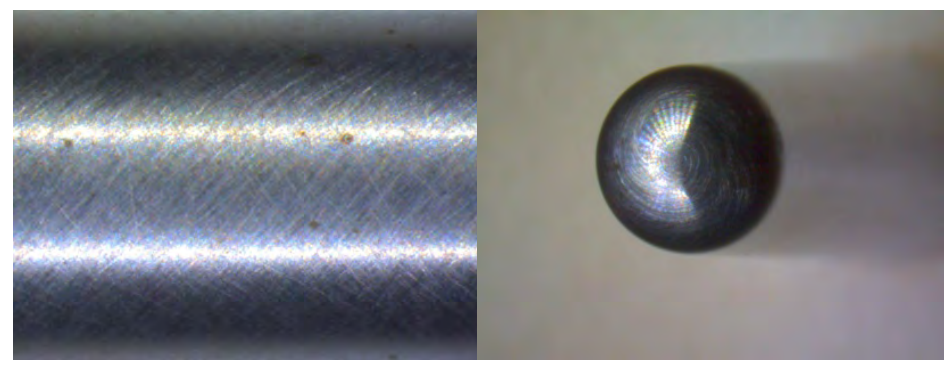

Shank (20X)

Nose (10X) 


\section{Composition of simulant for pitting corrosion -Test 15}

\begin{tabular}{|c|c|c|c|c|}
\hline \multirow[t]{3}{*}{ Reference 1} & \multirow{2}{*}{$\begin{array}{l}\text { Temperature } \\
\mathrm{pH}\end{array}$} & \multirow{2}{*}{$\begin{array}{r}40 \\
9.79\end{array}$} & \multicolumn{2}{|l|}{${ }^{\circ} \mathrm{C}$} \\
\hline & & & \multirow[b]{2}{*}{ L } & \\
\hline & Volume & 1.4 & & \\
\hline Simulant Source & Formula & $\begin{array}{c}\text { Molecular } \\
\text { Weight } \\
\text { (g/mol) }\end{array}$ & $\begin{array}{l}\text { Concentration } \\
\text { (M) }\end{array}$ & $\begin{array}{l}\text { weight } \\
\text { required (g) }\end{array}$ \\
\hline Sodium carbonate & $\mathrm{Na}_{2} \mathrm{CO}_{3}$ & 106.00 & 0.0263 & 3.9029 \\
\hline Sodium bicarbonate & $\mathrm{NaHCO}_{3}$ & 84.01 & 0.0564 & 6.6334 \\
\hline Sodium oxalate & $\mathrm{Na}_{2} \mathrm{C}_{2} \mathrm{O}_{4}$ & 134.00 & 0.000268 & 0.0503 \\
\hline $\begin{array}{c}\text { Sodium molybdate, } \\
\text { dihydrate }\end{array}$ & $\mathrm{Na}_{2} \mathrm{MoO}_{4} \cdot 2 \mathrm{H}_{2} \mathrm{O}$ & 241.95 & 0.0000143 & 0.0048 \\
\hline $\begin{array}{c}\text { Sodium metasilicate, } \\
5 \text {-hydrate }\end{array}$ & $\mathrm{Na}_{2} \mathrm{SiO}_{3} .5 \mathrm{H}_{2} \mathrm{O}$ & 212.14 & 0.000109 & 0.0324 \\
\hline $\begin{array}{c}\text { Sodium phosphate, } \\
12 \text {-hydrate }\end{array}$ & $\mathrm{Na}_{3} \mathrm{PO}_{4} \cdot 12 \mathrm{H}_{2} \mathrm{O}$ & 380.00 & 0.000309 & 0.1644 \\
\hline Sodium chloride & $\mathrm{NaCl}$ & 58.40 & 0.0011875 & 0.0971 \\
\hline Sodium fluoride & $\mathrm{NaF}$ & 41.99 & 0.000573 & 0.0337 \\
\hline Sodium sulfate & $\mathrm{Na}_{2} \mathrm{SO}_{4}$ & 142.00 & 0.00503 & 1.0000 \\
\hline Sodium nitrate & $\mathrm{NaNO}_{3}$ & 85.00 & 0.0758 & 9.0202 \\
\hline Sodium nitrite & $\mathrm{NaNO}_{2}$ & 69.00 & 0.058 & 5.6028 \\
\hline Sodium aluminate & $\mathrm{NaAlO}_{2}$ & 81.97 & 0.00045 & 0.0516 \\
\hline $\begin{array}{c}\text { Cobalt nitrate, 6- } \\
\text { hydrate }\end{array}$ & $\mathrm{Co}\left(\mathrm{NO}_{3}\right)_{2} \cdot 6 \mathrm{H}_{2} \mathrm{O}$ & 291.03 & 0.00003 & 0.0122 \\
\hline $\begin{array}{c}\text { Nickel nitrate, 6- } \\
\text { hydrate }\end{array}$ & $\mathrm{Ni}\left(\mathrm{NO}_{3}\right)_{2} \cdot 6 \mathrm{H}_{2} \mathrm{O}$ & 291.00 & 0.0015 & 0.6111 \\
\hline $\begin{array}{l}\text { Ferric nitrate, 9- } \\
\text { hydrate }\end{array}$ & $\mathrm{Fe}\left(\mathrm{NO}_{3}\right)_{3} \cdot 9 \mathrm{H}_{2} \mathrm{O}$ & 404.00 & 0.000248 & 0.1403 \\
\hline Mercury (II) nitrate & $\mathrm{Hg}\left(\mathrm{NO}_{3}\right)_{2}$ & 324.60 & 0.00025 & 0.1136 \\
\hline $\begin{array}{c}\text { Cupric nitrate, } 2.5 \\
\text { hydrate }\end{array}$ & $\begin{array}{c}\mathrm{Cu}\left(\mathrm{NO}_{3}\right)_{2} \cdot 2.5 \mathrm{H}_{2} \\
\mathrm{O}\end{array}$ & 233.00 & 0.000043 & 0.0140 \\
\hline Manganese dioxide & $\mathrm{MnO}_{2}$ & 86.94 & 0.00575 & 0.6999 \\
\hline
\end{tabular}




\section{Cyclic Potentiodynamic Polarization}

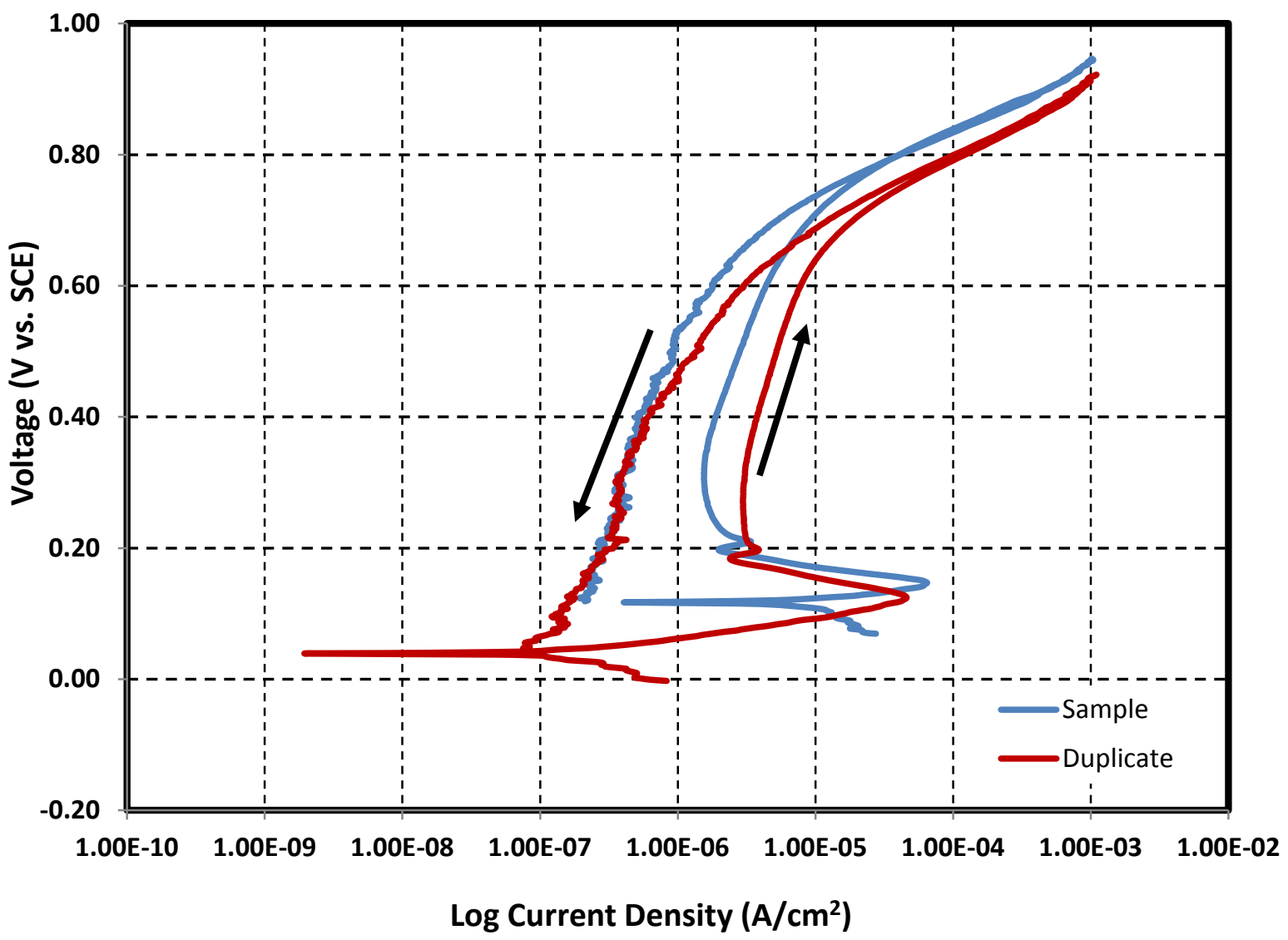

Images of bullet samples after electrochemical tests

Test 15

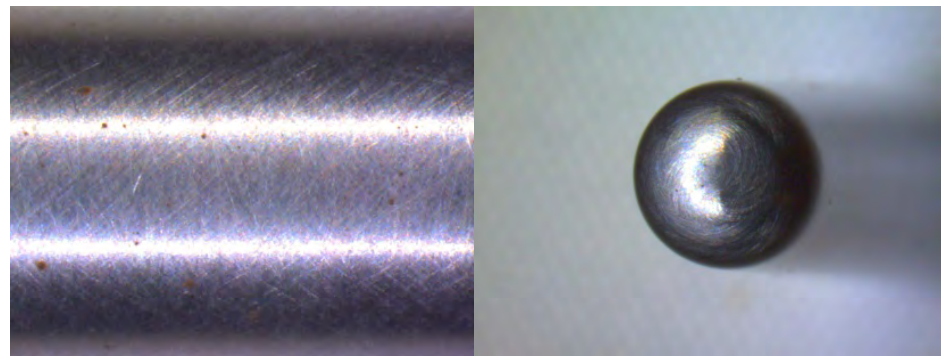

Test 15D

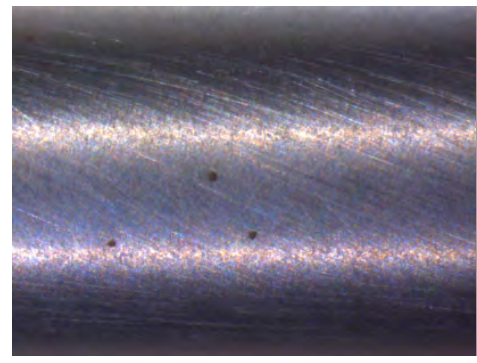

Shank (20X)

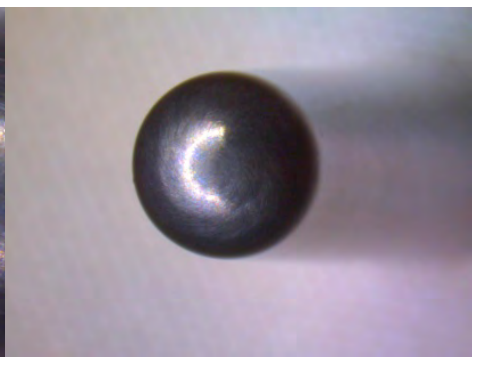

Nose (10X) 
Composition of simulant for pitting corrosion -Test 16

Reference 14

Test 16
Temperature

$\mathrm{pH}$

Volume
$40{ }^{\circ} \mathrm{C}$

10.00

$1.4 \quad \mathrm{~L}$

\begin{tabular}{|c|c|c|c|c|}
\hline Simulant Source & Formula & $\begin{array}{c}\text { Molecular } \\
\text { Weight } \\
\text { (g/mol) }\end{array}$ & $\begin{array}{c}\text { Concentration } \\
\text { (M) }\end{array}$ & $\begin{array}{c}\text { weight } \\
\text { required (g) }\end{array}$ \\
\hline Sodium nitrate & $\mathrm{NaNO}_{3}$ & 84.99 & 0.2 & 23.7972 \\
\hline Sodium nitrite & $\mathrm{NaNO}_{2}$ & 69.00 & 0.15 & 14.4900 \\
\hline Sodium chloride & $\mathrm{NaCl}$ & 58.44 & 0.0037 & 0.3027 \\
\hline Sodium sulfate & $\mathrm{Na}_{2} \mathrm{SO}_{4}$ & 142.04 & 0.0451 & 8.9684 \\
\hline Sodium carbonate & $\mathrm{Na}_{2}\left(\mathrm{CO}_{3}\right)$ & 106.00 & 0.0526 & 7.8058 \\
\hline Sodium bicarbonate & $\mathrm{NaHCO}_{3}$ & 84.00 & 0.098 & 11.5248 \\
\hline
\end{tabular}




\section{Cyclic Potentiodynamic Polarization}

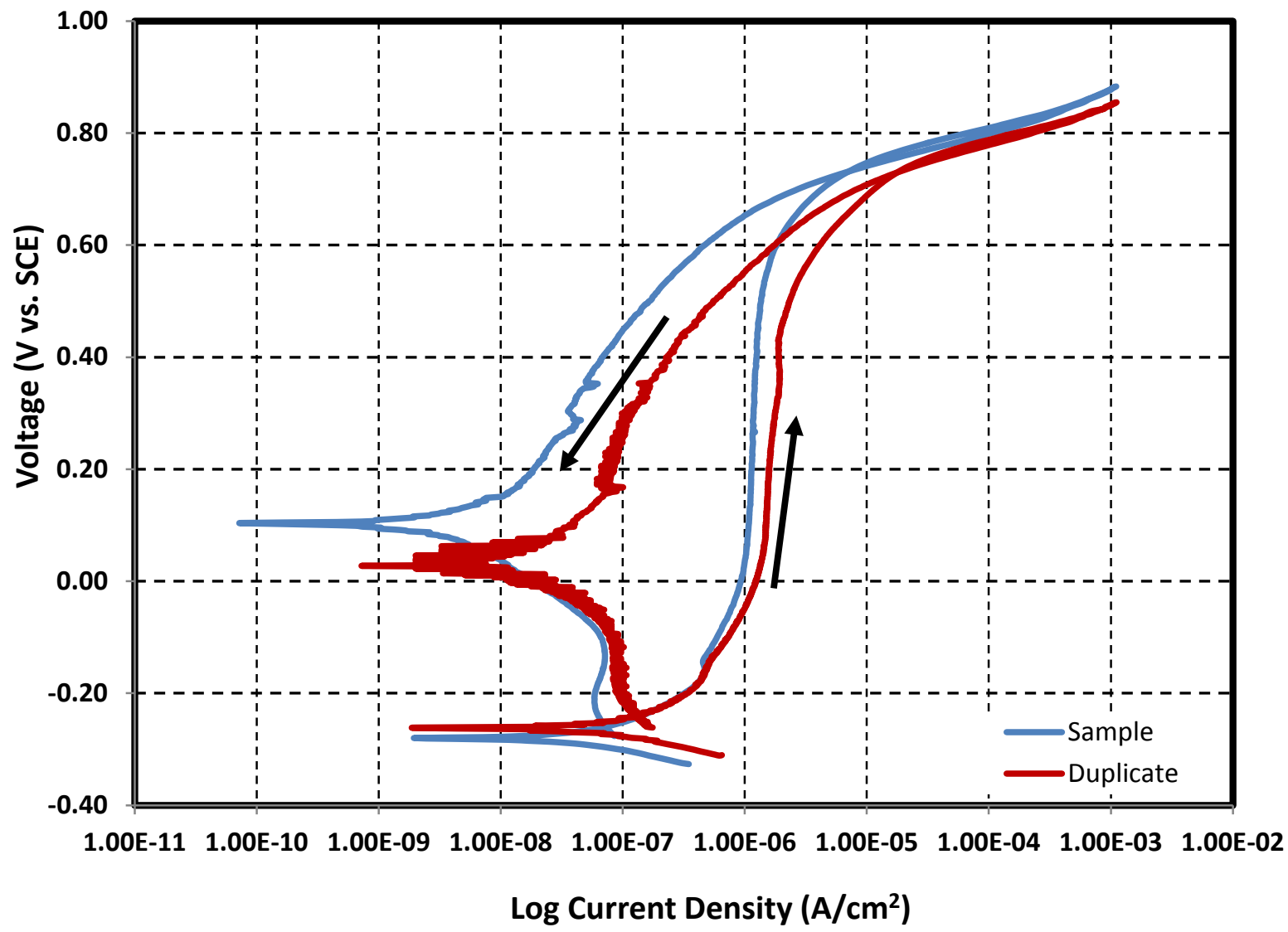

Images of bullet samples after electrochemical tests

Test 16

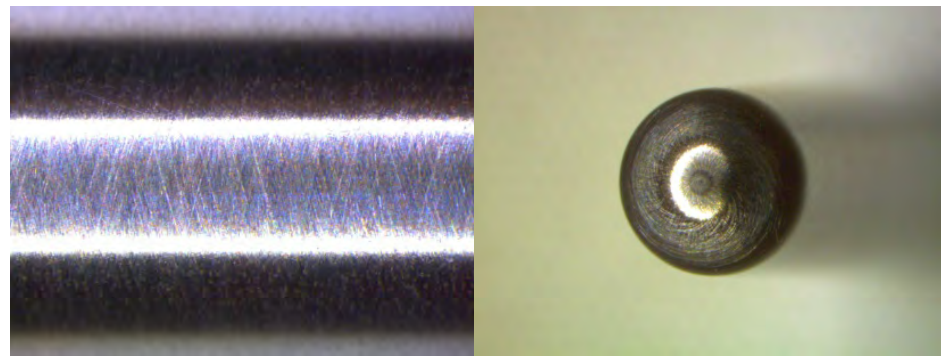

Test 16D
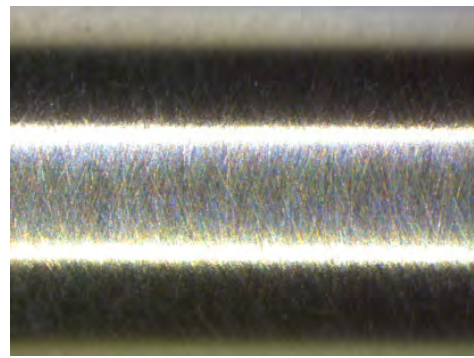

Shank (20X)

Nose (10X) 
Composition of simulant for pitting corrosion -Test 17

Reference 13

Test 17

Temperature

$40{ }^{\circ} \mathrm{C}$

$\mathrm{pH}$

10.00

Volume

\begin{tabular}{|c|c|c|c|c|}
\hline Simulant Source & Formula & $\begin{array}{c}\text { Molecular } \\
\text { Weight } \\
\text { (g/mol) }\end{array}$ & $\begin{array}{c}\text { Concentration } \\
\text { (M) }\end{array}$ & $\begin{array}{c}\text { weight } \\
\text { required (g) }\end{array}$ \\
\hline Sodium nitrate & $\mathrm{NaNO}_{3}$ & 84.99 & 0.9 & 107.0874 \\
\hline Sodium nitrite & $\mathrm{NaNO}_{2}$ & 69.00 & 0.675 & 65.2050 \\
\hline Sodium chloride & $\mathrm{NaCl}$ & 58.44 & 0.0115 & 0.9409 \\
\hline Sodium sulfate & $\mathrm{Na}_{2} \mathrm{SO}_{4}$ & 142.04 & 0.323 & 64.2305 \\
\hline Sodium carbonate & $\mathrm{Na}_{2}\left(\mathrm{CO}_{3}\right)$ & 106.00 & 0.236 & 35.0224 \\
\hline Sodium bicarbonate & $\mathrm{NaHCO}_{3}$ & 84.00 & 0.438 & 51.5088 \\
\hline
\end{tabular}




\section{Cyclic Potentiodynamic Polarization}

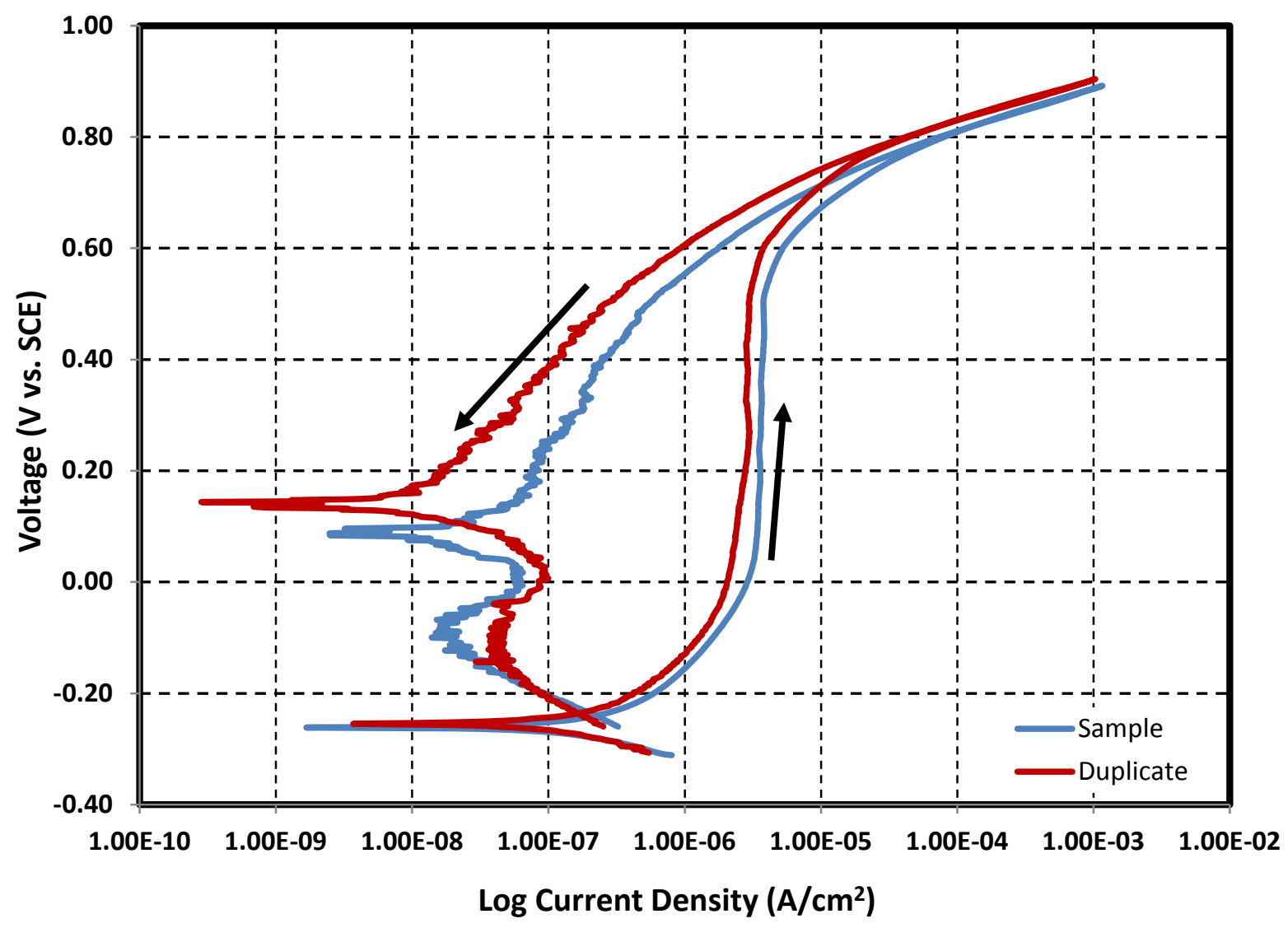

Images of bullet samples after electrochemical tests

Test 17
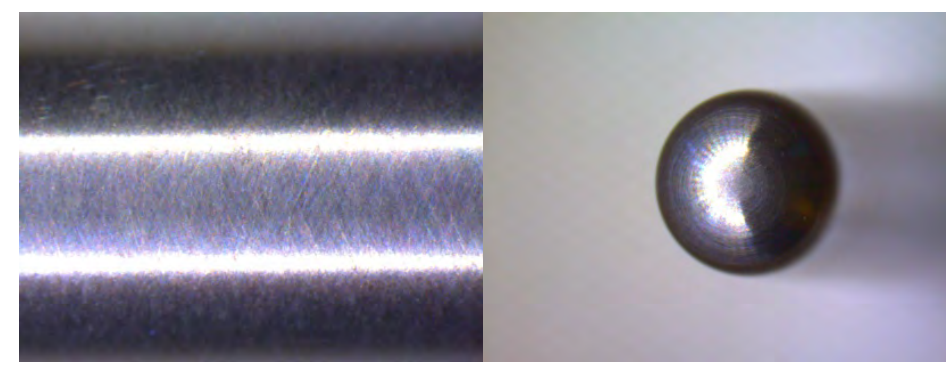

Test 17D

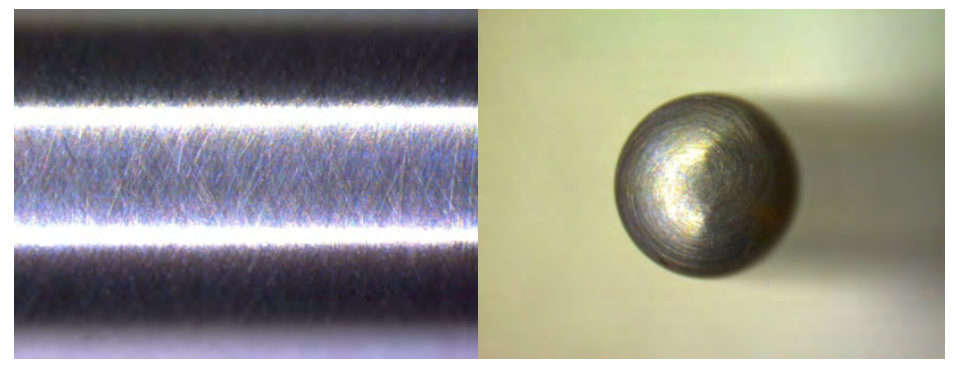

Shank (20X)

Nose (10X) 


\section{Composition of simulant for pitting corrosion -Test 18}

\begin{tabular}{|c|c|c|c|c|}
\hline $\begin{array}{l}\text { Reference } 3 \\
\text { Test } 18\end{array}$ & $\begin{array}{l}\text { Temperature } \\
\mathrm{pH} \\
\text { Volume }\end{array}$ & $\begin{array}{l}40 \\
9.6 \\
1.4\end{array}$ & ${ }^{\circ} \mathrm{C}$ & \\
\hline Simulant Source & Formula & $\begin{array}{l}\text { Molecular } \\
\text { Weight } \\
\text { (g/mol) }\end{array}$ & $\begin{array}{l}\text { Concentration } \\
\text { (M) }\end{array}$ & $\begin{array}{l}\text { weight } \\
\text { required (g) }\end{array}$ \\
\hline Aluminum nitrate, 9-hydrate & $\mathrm{Al}\left(\mathrm{NO}_{3}\right)_{3} \cdot 9 \mathrm{H}_{2} \mathrm{O}$ & 375.13 & 0.014928158 & 7.8400 \\
\hline Ferric Nitrate, 9-hydrate & $\mathrm{Fe}\left(\mathrm{NO}_{3}\right)_{3} .9 \mathrm{H}_{2} \mathrm{O}$ & 404.00 & 0.004945 & 2.7969 \\
\hline Sodium hydroxide & $\mathrm{NaOH}$ & 40.00 & 0.0915 & 5.1240 \\
\hline Sodium nitrate & $\mathrm{NaNO}_{3}$ & 85.00 & 0 & 0 \\
\hline Sodium nitrite & $\mathrm{NaNO}_{2}$ & 69.00 & 0.039971014 & 3.8612 \\
\hline Sodium oxalate & $\mathrm{Na}_{2}\left(\mathrm{C}_{2} \mathrm{O}_{4}\right)$ & 134.00 & 0.008656716 & 1.6240 \\
\hline Sodium sulfate & $\mathrm{Na}_{2} \mathrm{SO}_{4}$ & 142.04 & 0.005941988 & 1.1816 \\
\hline Sodium carbonate & $\mathrm{Na}_{2}\left(\mathrm{CO}_{3}\right)$ & 106.00 & 0.014037736 & 2.0832 \\
\hline Sodium Bicarbonate & $\mathrm{NaHCO}_{3}$ & 84.00 & 0.058 & 6.8208 \\
\hline $\begin{array}{c}\text { Sodium phosphate, 12- } \\
\text { hydrate }\end{array}$ & $\mathrm{Na}_{3} \mathrm{PO}_{4} \cdot 12 \mathrm{H}_{2} \mathrm{O}$ & 380.12 & 0.000275071 & 0.1464 \\
\hline Calcium Carbonate & $\mathrm{CaCO}_{3}$ & 100.00 & 0.0006604 & 0.0925 \\
\hline Sodium chloride & $\mathrm{NaCl}$ & 58.44 & 0.001090008 & 0.0892 \\
\hline Sodium fluoride & $\mathrm{NaF}$ & 42.00 & 0.000729524 & 0.0429 \\
\hline Sodium molybdate, dihydrate & $\mathrm{Na}_{2} \mathrm{MoO}_{4} \cdot 2 \mathrm{H}_{2} \mathrm{O}$ & 241.95 & $1.8452 \mathrm{E}-05$ & 0.0063 \\
\hline Manganese Dioxide & $\mathrm{MnO}_{2}$ & 86.94 & 0.001140097 & 0.1388 \\
\hline Nickel nitrate, 6-hydrate & $\mathrm{Ni}\left(\mathrm{NO}_{3}\right)_{2} \cdot 6 \mathrm{H}_{2} \mathrm{O}$ & 290.81 & 0.000520065 & 0.2117 \\
\hline Mercury (II) nitrate & $\mathrm{Hg}\left(\mathrm{NO}_{3}\right)_{2}$ & 342.62 & 0.00311 & 1.4918 \\
\hline $\begin{array}{c}\text { Sodium metasilicate, 5- } \\
\text { hydrate }\end{array}$ & $\mathrm{Na}_{2} \mathrm{SiO}_{3} .5 \mathrm{H}_{2} \mathrm{O}$ & 212.14 & 0.000160141 & 0.0476 \\
\hline Zinc nitrate, 6-hydrate & $\mathrm{Zn}\left(\mathrm{NO}_{3}\right)_{2} \cdot 6 \mathrm{H}_{2} \mathrm{O}$ & 297.49 & 4.11442E-05 & 0.0171 \\
\hline Lead nitrate & $\mathrm{Pb}\left(\mathrm{NO}_{3}\right)_{2}$ & 331.21 & 1.94439E-05 & 0.0090 \\
\hline Cupric nitrate, 2.5 hydrate & $\mathrm{Cu}\left(\mathrm{NO}_{3}\right)_{2} \cdot 2.5 \mathrm{H}_{2} \mathrm{O}$ & 233.00 & 2.45494E-05 & 0.0080 \\
\hline
\end{tabular}




\section{Cyclic Potentiodynamic Polarization}

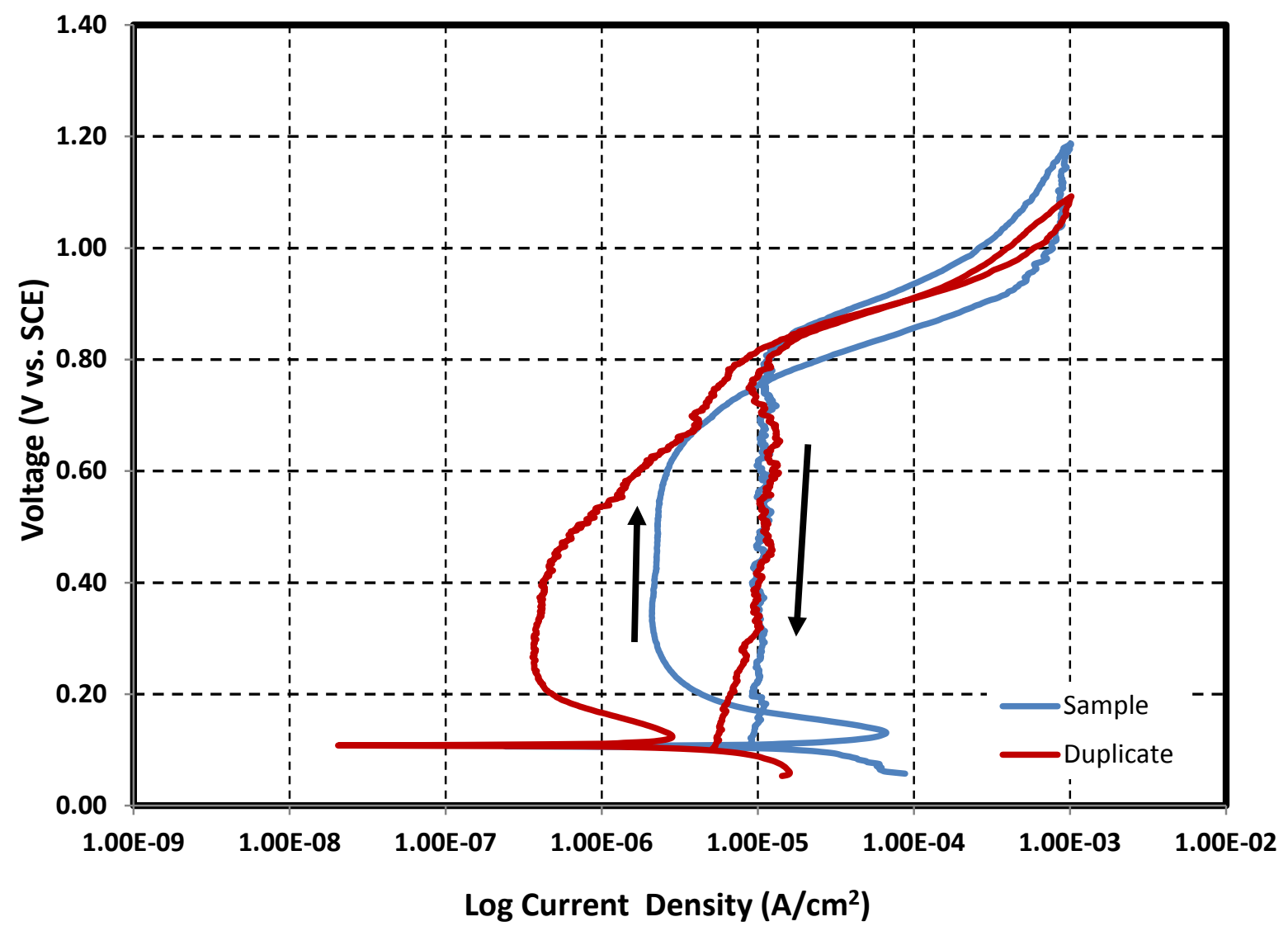

Images of bullet samples after electrochemical tests

Test 18

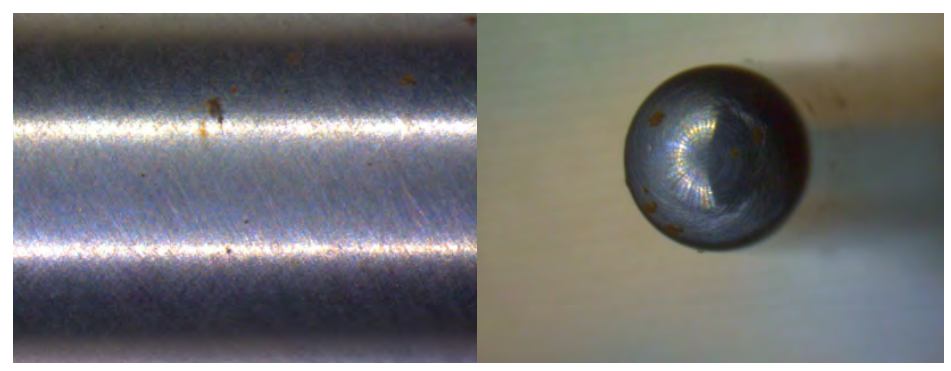

Test 18D

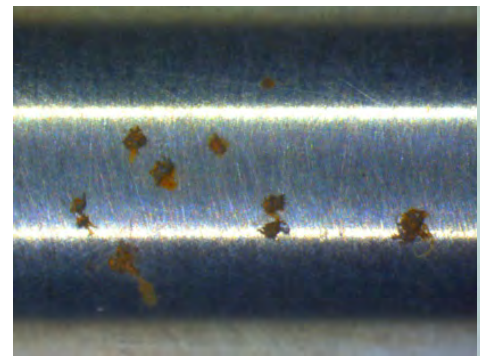

Shank $(20 X)$

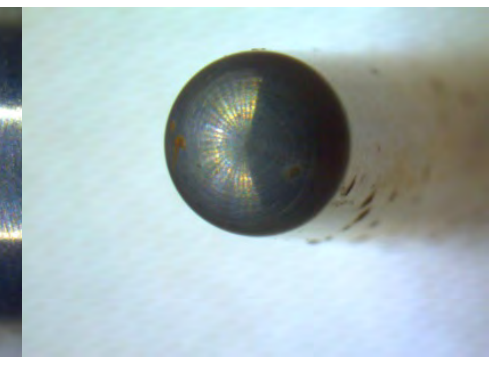

Nose (10X) 
Composition of simulant for pitting corrosion -Test 19

Reference 14

Temperature

$40{ }^{\circ} \mathrm{C}$

Test 19

$\mathrm{pH}$

10.00

Volume

$1.4 \mathrm{~L}$

\begin{tabular}{|c|c|c|c|c|}
\hline Simulant Source & Formula & $\begin{array}{c}\text { Molecular } \\
\text { Weight } \\
\text { (g/mol) }\end{array}$ & $\begin{array}{c}\text { Concentration } \\
\text { (M) }\end{array}$ & $\begin{array}{c}\text { weight } \\
\text { required (g) }\end{array}$ \\
\hline Sodium nitrate & $\mathrm{NaNO}_{3}$ & 84.99 & 0.45 & 53.5437 \\
\hline Sodium nitrite & $\mathrm{NaNO}_{2}$ & 69.00 & 0.225 & 21.7350 \\
\hline Sodium chloride & $\mathrm{NaCl}$ & 58.44 & 0.0051 & 0.4173 \\
\hline Sodium sulfate & $\mathrm{Na}_{2} \mathrm{SO}_{4}$ & 142.04 & 0.0731 & 14.5364 \\
\hline Sodium carbonate & $\mathrm{Na}_{2}\left(\mathrm{CO}_{3}\right)$ & 106.00 & 0.0789 & 11.7088 \\
\hline Sodium bicarbonate & $\mathrm{NaHCO}_{3}$ & 84.00 & 0.147 & 17.2872 \\
\hline
\end{tabular}




\section{Cyclic Potentiodynamic Polarization}

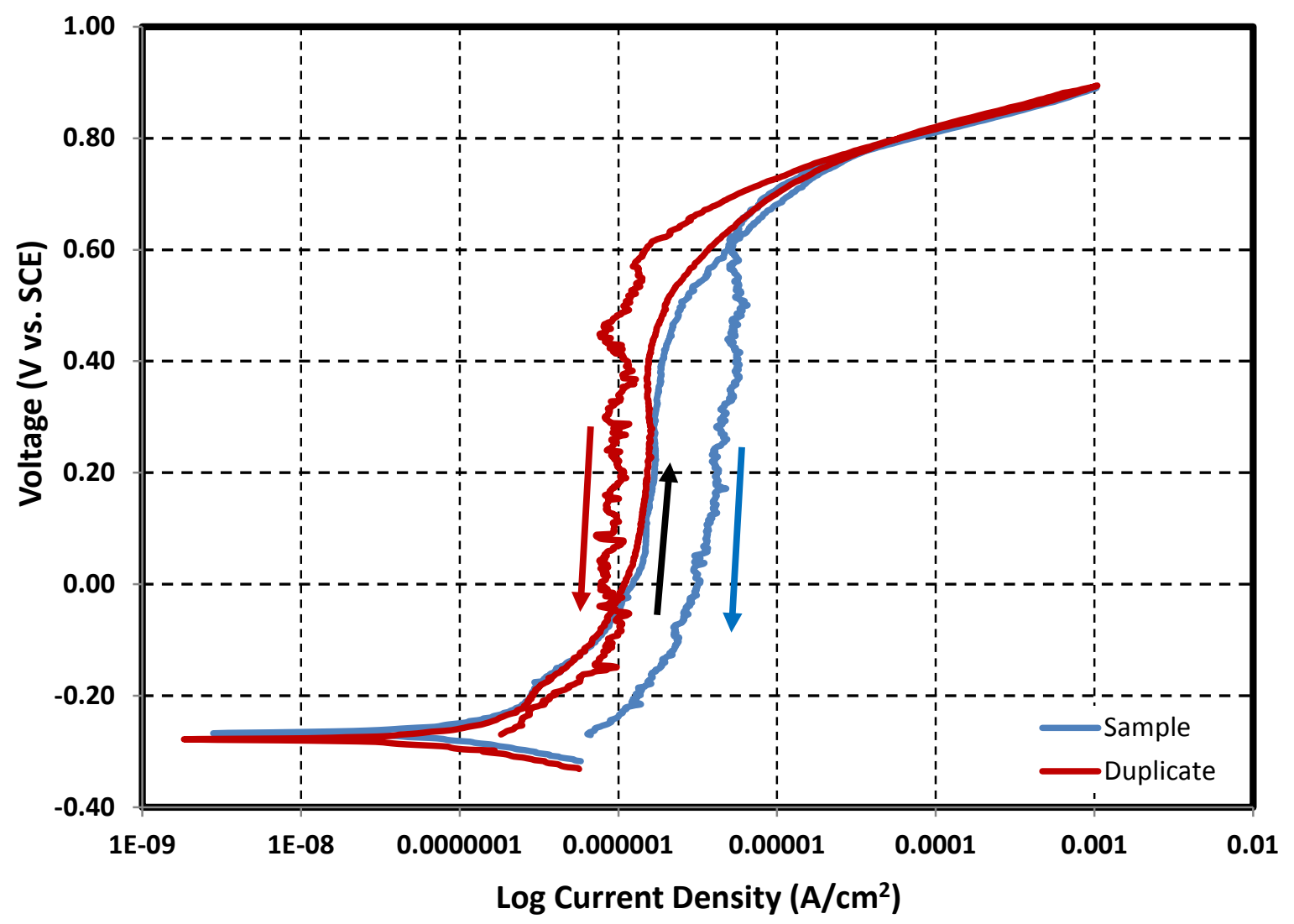

Images of bullet samples after electrochemical tests

Test 19
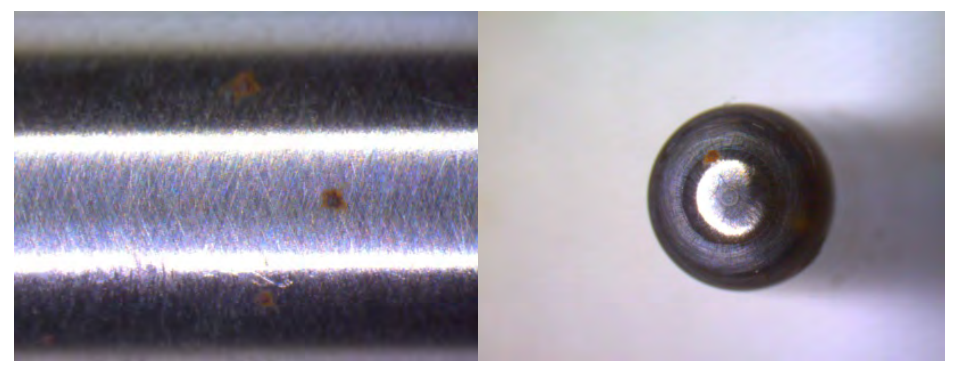

Test 19D
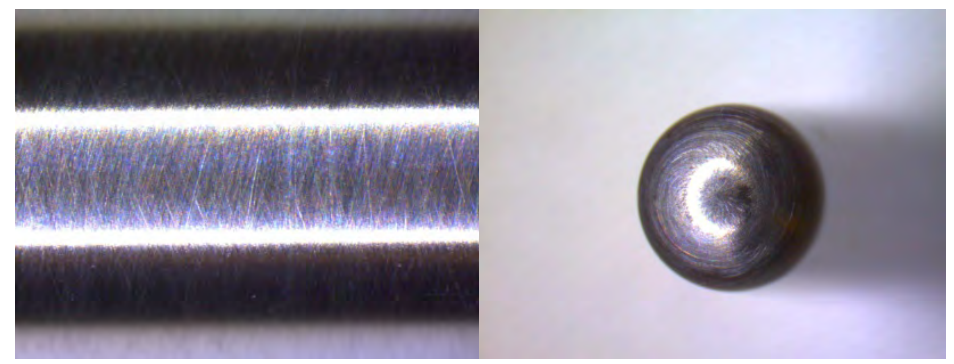

Shank (20X)

Nose (10X) 


\section{Composition of simulant for pitting corrosion -Test 20}

\begin{tabular}{|c|c|c|c|c|}
\hline \multirow{3}{*}{$\begin{array}{l}\text { Reference } 4 \\
\text { Test } 20\end{array}$} & \multirow{2}{*}{$\begin{array}{l}\text { Temperature } \\
\mathrm{pH}\end{array}$} & \multicolumn{2}{|c|}{$40{ }^{\circ} \mathrm{C}$} & \\
\hline & & 9.6 & & \\
\hline & Volume & 1.4 & $\mathrm{~L}$ & \\
\hline Simulant Source & Formula & $\begin{array}{l}\text { Molecular } \\
\text { Weight } \\
\text { (g/mol) }\end{array}$ & $\begin{array}{c}\text { Concentration } \\
\text { (M) }\end{array}$ & $\begin{array}{l}\text { weight } \\
\text { required (g) }\end{array}$ \\
\hline Aluminum nitrate, 9-hydrate & $\mathrm{Al}\left(\mathrm{NO}_{3}\right)_{3} \cdot 9 \mathrm{H}_{2} \mathrm{O}$ & 375.13 & 0.006842073 & 3.5933 \\
\hline Ferric Nitrate, 9-hydrate & $\mathrm{Fe}\left(\mathrm{NO}_{3}\right)_{3} \cdot 9 \mathrm{H}_{2} \mathrm{O}$ & 404.00 & 0.001831683 & 1.0360 \\
\hline Sodium hydroxide & $\mathrm{NaOH}$ & 40.00 & 0.0305 & 1.7080 \\
\hline Sodium nitrate & $\mathrm{NaNO}_{3}$ & 85.00 & 0.001694317 & 0.2016 \\
\hline Sodium nitrite & $\mathrm{NaNO}_{2}$ & 69.00 & 0.20057971 & 19.3760 \\
\hline Sodium oxalate & $\mathrm{Na}_{2}\left(\mathrm{C}_{2} \mathrm{O}_{4}\right)$ & 134.00 & 0.002885572 & 0.5413 \\
\hline Sodium sulfate & $\mathrm{Na}_{2} \mathrm{SO}_{4}$ & 142.04 & 0.130019713 & 25.8552 \\
\hline Sodium carbonate & $\mathrm{Na}_{2}\left(\mathrm{CO}_{3}\right)$ & 106.00 & 0.004679245 & 0.6944 \\
\hline Sodium Bicarbonate & $\mathrm{NaHCO}_{3}$ & 84.00 & 0.019333333 & 2.2736 \\
\hline $\begin{array}{l}\text { Sodium phosphate, } 12- \\
\text { hydrate }\end{array}$ & $\mathrm{Na}_{3} \mathrm{PO}_{4} \cdot 12 \mathrm{H}_{2} \mathrm{O}$ & 380.12 & $9.16903 E-05$ & 0.0488 \\
\hline Calcium Carbonate & $\mathrm{CaCO}_{3}$ & 100.00 & 0.000220133 & 0.0308 \\
\hline Sodium chloride & $\mathrm{NaCl}$ & 58.44 & 0.000382729 & 0.0313 \\
\hline Sodium fluoride & $\mathrm{NaF}$ & 42.00 & 0.000243175 & 0.0143 \\
\hline Sodium molybdate, dihydrate & $\mathrm{Na}_{2} \mathrm{MoO}_{4} \cdot 2 \mathrm{H}_{2} \mathrm{O}$ & 241.95 & $6.15066 \mathrm{E}-06$ & 0.0021 \\
\hline Manganese Dioxide & $\mathrm{MnO}_{2}$ & 86.94 & 0.000380032 & 0.0463 \\
\hline Nickel nitrate, 6-hydrate & $\mathrm{Ni}\left(\mathrm{NO}_{3}\right)_{2} \cdot 6 \mathrm{H}_{2} \mathrm{O}$ & 290.81 & 0.000173355 & 0.0706 \\
\hline Mercury (II) nitrate & $\mathrm{Hg}\left(\mathrm{NO}_{3}\right)_{2}$ & 342.62 & 0.000146 & 0.0700 \\
\hline Sodium silicate, 9-hydrate & $\mathrm{Na}_{2} \mathrm{SiO}_{3} \cdot 9 \mathrm{H}_{2} \mathrm{O}$ & 284.00 & 5.33803E-05 & 0.0212 \\
\hline Zinc nitrate, 6-hydrate & $\mathrm{Zn}\left(\mathrm{NO}_{3}\right)_{2} \cdot 6 \mathrm{H}_{2} \mathrm{O}$ & 297.49 & $1.37147 \mathrm{E}-05$ & 0.0057 \\
\hline Lead nitrate & $\mathrm{Pb}\left(\mathrm{NO}_{3}\right)_{2}$ & 331.21 & $6.48129 \mathrm{E}-06$ & 0.0030 \\
\hline Cupric nitrate, 2.5 hydrate & $\mathrm{Cu}\left(\mathrm{NO}_{3}\right)_{2} \cdot 2 \cdot 5 \mathrm{H}_{2} \mathrm{O}$ & 233.00 & $8.18312 \mathrm{E}-06$ & 0.0027 \\
\hline
\end{tabular}




\section{Cyclic Potentiodynamic Polarization}

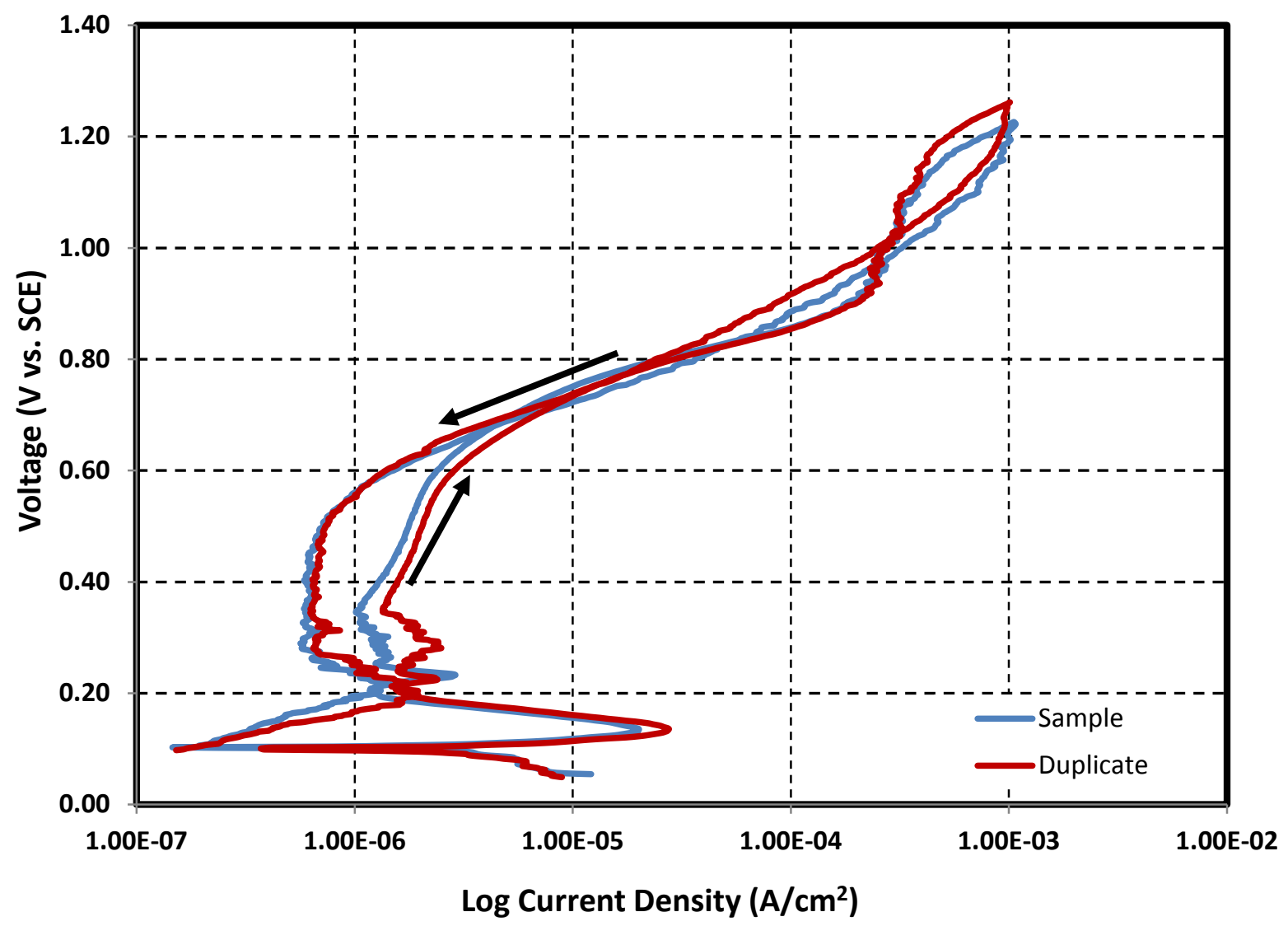

Images of bullet samples after electrochemical tests

Test 20

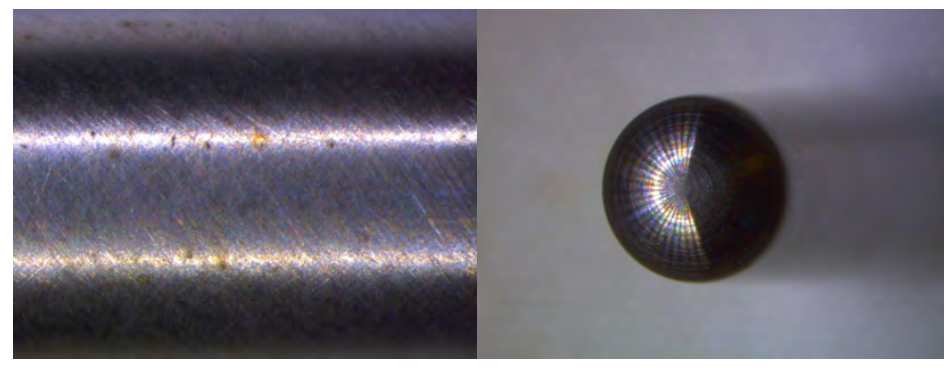

Test 20D

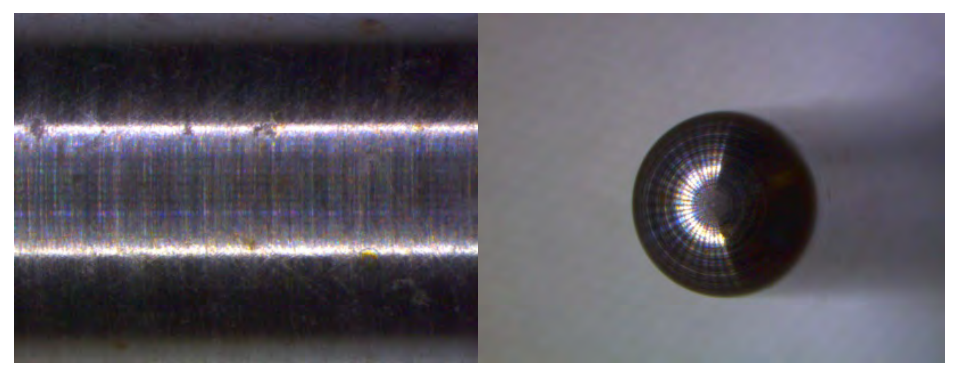

Shank $(20 X)$

Nose (10X) 


\section{Composition of simulant for pitting corrosion - Test 21}

Reference 3

Test 21

\begin{tabular}{|c|c|c|c|c|}
\hline & Volume & 1.4 & $\mathrm{~L}$ & \\
\hline Simulant Source & Formula & $\begin{array}{l}\text { Molecular } \\
\text { Weight } \\
\text { (g/mol) }\end{array}$ & $\begin{array}{l}\text { Concentration } \\
\text { (M) }\end{array}$ & $\begin{array}{l}\text { weight } \\
\text { required }(g)\end{array}$ \\
\hline Aluminum nitrate, 9-hydrate & $\mathrm{Al}\left(\mathrm{NO}_{3}\right)_{3} .9 \mathrm{H}_{2} \mathrm{O}$ & 375.13 & 0.020526218 & 10.7800 \\
\hline Ferric Nitrate, 9-hydrate & $\mathrm{Fe}\left(\mathrm{NO}_{3}\right)_{3} \cdot 9 \mathrm{H}_{2} \mathrm{O}$ & 404.00 & 0.004945 & 2.7969 \\
\hline Sodium hydroxide & $\mathrm{NaOH}$ & 40.00 & 0.0915 & 5.1240 \\
\hline Sodium nitrate & $\mathrm{NaNO}_{3}$ & 85.00 & 0 & 0.0000 \\
\hline Sodium nitrite & $\mathrm{NaNO}_{2}$ & 69.00 & 0.024985507 & 2.4136 \\
\hline Sodium oxalate & $\mathrm{Na}_{2}\left(\mathrm{C}_{2} \mathrm{O}_{4}\right)$ & 134.00 & 0.008656716 & 1.6240 \\
\hline Sodium sulfate & $\mathrm{Na}_{2} \mathrm{SO}_{4}$ & 142.04 & 0.005941988 & 1.1816 \\
\hline Sodium carbonate & $\mathrm{Na}_{2}\left(\mathrm{CO}_{3}\right)$ & 106.00 & 0.014037736 & 2.0832 \\
\hline Sodium Bicarbonate & $\mathrm{NaHCO}_{3}$ & 84.00 & 0.058 & 6.8208 \\
\hline $\begin{array}{l}\text { Sodium phosphate, 12- } \\
\text { hydrate } \\
\end{array}$ & $\mathrm{Na}_{3} \mathrm{PO}_{4} \cdot 12 \mathrm{H}_{2} \mathrm{O}$ & 380.12 & 0.000275071 & 0.1464 \\
\hline Calcium Carbonate & $\mathrm{CaCO}_{3}$ & 100.00 & 0.0006604 & 0.0925 \\
\hline Sodium chloride & $\mathrm{NaCl}$ & 58.44 & 0.001090008 & 0.0892 \\
\hline Sodium fluoride & $\mathrm{NaF}$ & 42.00 & 0.000729524 & 0.0429 \\
\hline Sodium molybdate, dihydrate & $\mathrm{Na}_{2} \mathrm{MoO}_{4} \cdot 2 \mathrm{H}_{2} \mathrm{O}$ & 241.95 & 1.8452E-05 & 0.0063 \\
\hline Manganese Dioxide & $\mathrm{MnO}_{2}$ & 86.94 & 0.001140097 & 0.1388 \\
\hline Nickel nitrate, 6-hydrate & $\mathrm{Ni}\left(\mathrm{NO}_{3}\right)_{2} \cdot 6 \mathrm{H}_{2} \mathrm{O}$ & 290.81 & 0.000520065 & 0.2117 \\
\hline Mercury (II) nitrate & $\mathrm{Hg}\left(\mathrm{NO}_{3}\right)_{2}$ & 342.62 & 0.00311 & 1.4918 \\
\hline $\begin{array}{c}\text { Sodium metasilicate, } 5- \\
\text { hydrate } \\
\end{array}$ & $\mathrm{Na}_{2} \mathrm{SiO}_{3} .5 \mathrm{H}_{2} \mathrm{O}$ & 212.14 & 0.000160141 & 0.0476 \\
\hline Zinc nitrate, 6-hydrate & $\mathrm{Zn}\left(\mathrm{NO}_{3}\right)_{2} \cdot 6 \mathrm{H}_{2} \mathrm{O}$ & 297.49 & 4.11442E-05 & 0.0171 \\
\hline Lead nitrate & $\mathrm{Pb}\left(\mathrm{NO}_{3}\right)_{2}$ & 331.21 & $1.94439 \mathrm{E}-05$ & 0.0090 \\
\hline Cupric nitrate, 2.5 hydrate & $\mathrm{Cu}\left(\mathrm{NO}_{3}\right)_{2} \cdot 2.5 \mathrm{H}_{2} \mathrm{O}$ & 233.00 & 2.45494E-05 & 0.0080 \\
\hline
\end{tabular}




\section{Cyclic Potentiodynamic Polarization}

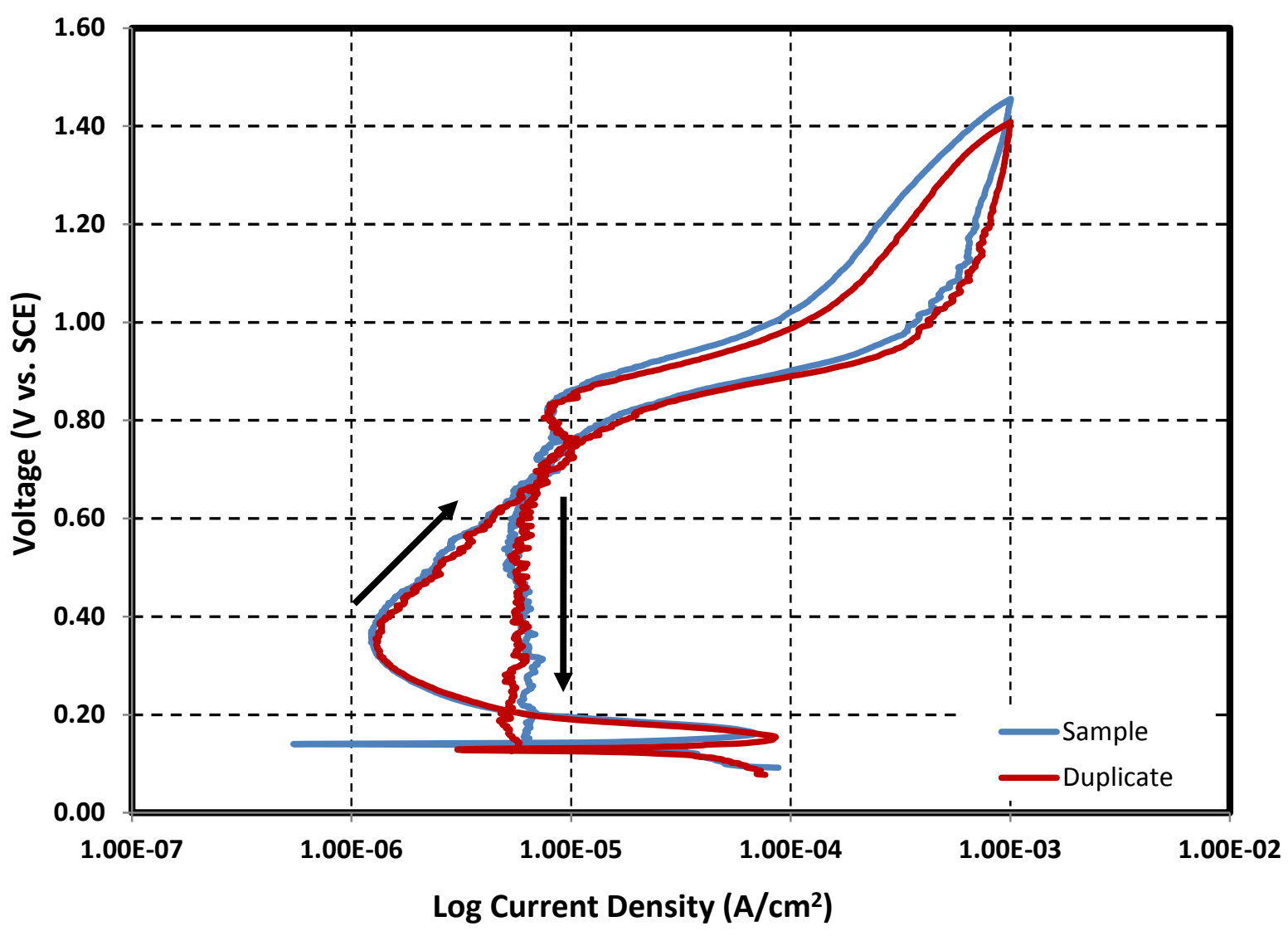

Images of bullet samples after electrochemical tests

Test 21

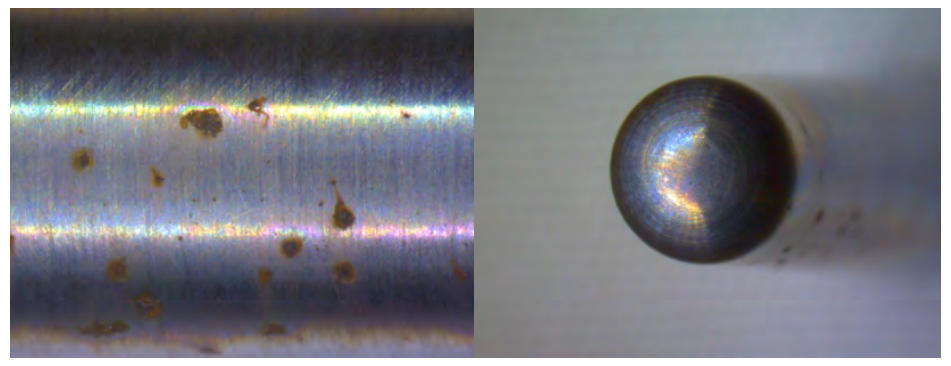

Test 21D

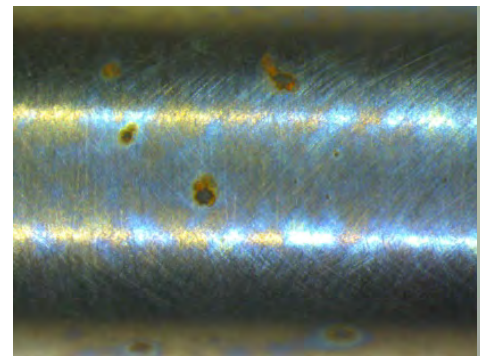

Shank $(20 X)$

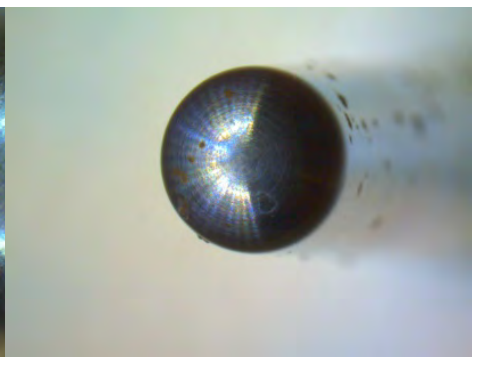

Nose (10X) 


\section{Composition of simulant for pitting corrosion -Test 22}

Reference 3

Test 22
Temperature
$40{ }^{\circ} \mathrm{C}$
$\mathrm{pH}$
9.6
Volume

\begin{tabular}{|c|c|c|c|c|}
\hline Simulant Source & Formula & $\begin{array}{c}\text { Molecular } \\
\text { Weight } \\
\text { (g/mol) }\end{array}$ & $\begin{array}{l}\text { Concentration } \\
\text { (M) }\end{array}$ & $\begin{array}{l}\text { weight } \\
\text { required }(g)\end{array}$ \\
\hline Aluminum nitrate, 9-hydrate & $\mathrm{Al}\left(\mathrm{NO}_{3}\right)_{3} \cdot 9 \mathrm{H}_{2} \mathrm{O}$ & 375.13 & 0.011196119 & 5.8800 \\
\hline Ferric Nitrate, 9-hydrate & $\mathrm{Fe}\left(\mathrm{NO}_{3}\right)_{3} \cdot 9 \mathrm{H}_{2} \mathrm{O}$ & 404.00 & 0.004945 & 2.7969 \\
\hline Sodium hydroxide & $\mathrm{NaOH}$ & 40.00 & 0.0915 & 5.1240 \\
\hline Sodium nitrate & $\mathrm{NaNO}_{3}$ & 85.00 & 0.00701259 & 0.8345 \\
\hline Sodium nitrite & $\mathrm{NaNO}_{2}$ & 69.00 & 0.100289855 & 9.6880 \\
\hline Sodium oxalate & $\mathrm{Na}_{2}\left(\mathrm{C}_{2} \mathrm{O}_{4}\right)$ & 134.00 & 0.008656716 & 1.6240 \\
\hline Sodium sulfate & $\mathrm{Na}_{2} \mathrm{SO}_{4}$ & 142.04 & 0.005941988 & 1.1816 \\
\hline Sodium carbonate & $\mathrm{Na}_{2}\left(\mathrm{CO}_{3}\right)$ & 106.00 & 0.014037736 & 2.0832 \\
\hline Sodium Bicarbonate & $\mathrm{NaHCO}_{3}$ & 84.00 & 0.058 & 6.8208 \\
\hline $\begin{array}{c}\text { Sodium phosphate, 12- } \\
\text { hydrate }\end{array}$ & $\mathrm{Na}_{3} \mathrm{PO}_{4} \cdot 12 \mathrm{H}_{2} \mathrm{O}$ & 380.12 & 0.000275071 & 0.1464 \\
\hline Calcium Carbonate & $\mathrm{CaCO}_{3}$ & 100.00 & 0.0006604 & 0.0925 \\
\hline Sodium chloride & $\mathrm{NaCl}$ & 58.44 & 1.09E-03 & 0.0892 \\
\hline Sodium fluoride & $\mathrm{NaF}$ & 42.00 & 0.000729524 & 0.0429 \\
\hline Sodium molybdate, dihydrate & $\mathrm{Na}_{2} \mathrm{MoO}_{4} \cdot 2 \mathrm{H}_{2} \mathrm{O}$ & 241.95 & 1.8452E-05 & 0.0063 \\
\hline Manganese Dioxide & $\mathrm{MnO}_{2}$ & 86.94 & 0.001140097 & 0.1388 \\
\hline Nickel nitrate, 6-hydrate & $\mathrm{Ni}\left(\mathrm{NO}_{3}\right)_{2} \cdot 6 \mathrm{H}_{2} \mathrm{O}$ & 290.81 & 0.000520065 & 0.2117 \\
\hline Mercury (II) nitrate & $\mathrm{Hg}\left(\mathrm{NO}_{3}\right)_{2}$ & 342.62 & 0.00311 & 1.4918 \\
\hline $\begin{array}{c}\text { Sodium metasilicate, 5- } \\
\text { hydrate }\end{array}$ & $\mathrm{Na}_{2} \mathrm{SiO}_{3} .5 \mathrm{H}_{2} \mathrm{O}$ & 212.14 & 0.000160141 & 0.0476 \\
\hline Zinc nitrate, 6-hydrate & $\mathrm{Zn}\left(\mathrm{NO}_{3}\right)_{2} \cdot 6 \mathrm{H}_{2} \mathrm{O}$ & 297.49 & 4.11442E-05 & 0.0171 \\
\hline Lead nitrate & $\mathrm{Pb}\left(\mathrm{NO}_{3}\right)_{2}$ & 331.21 & 1.94439E-05 & 0.0090 \\
\hline Cupric nitrate, 2.5 hydrate & $\mathrm{Cu}\left(\mathrm{NO}_{3}\right)_{2} \cdot 2.5 \mathrm{H}_{2} \mathrm{O}$ & 233.00 & 2.45494E-05 & 0.0080 \\
\hline
\end{tabular}




\section{Cyclic Potentiodynamic Polarization}

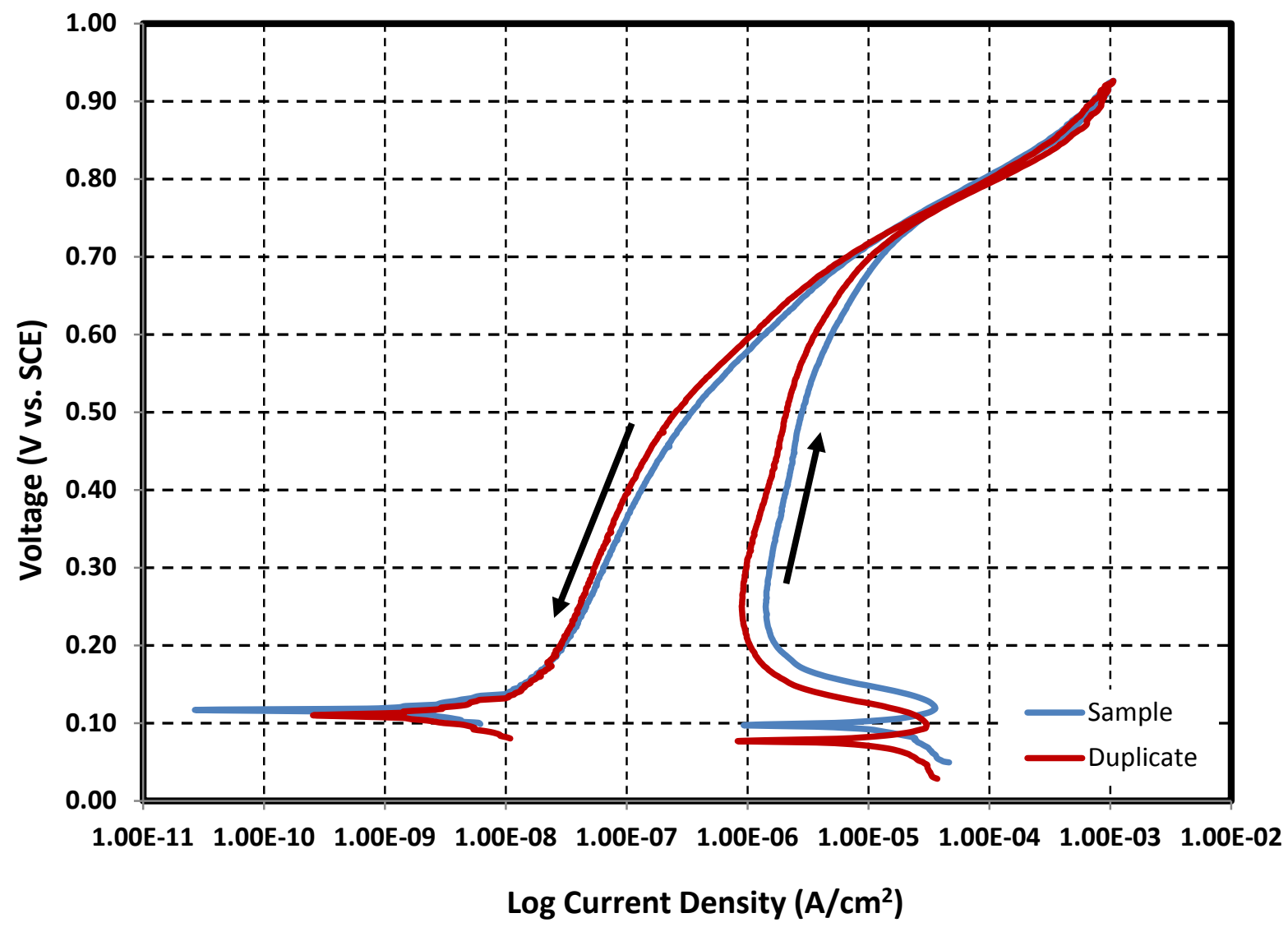

Images of bullet samples after electrochemical tests

Test 22

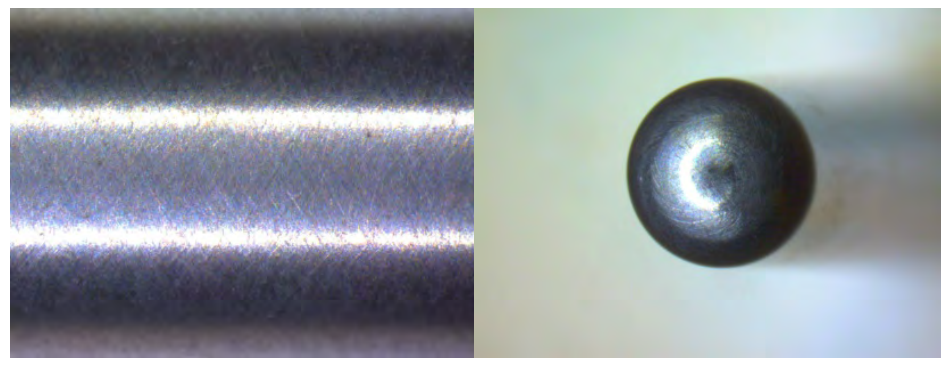

Test 22D

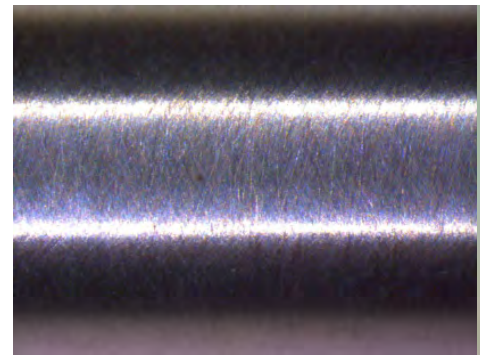

Shank (20X)

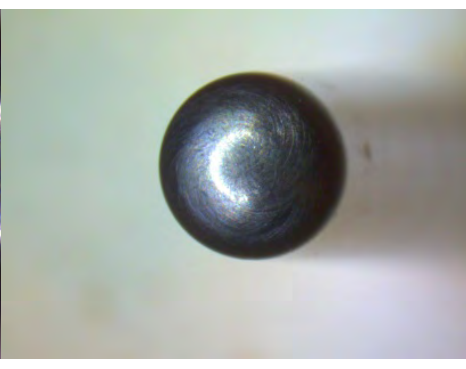

Nose (10X) 


\section{Composition of simulant for pitting corrosion -Test 23}

Reference 14

Test 23
Temperature

$\mathrm{pH}$

Volume
$40{ }^{\circ} \mathrm{C}$

10.00

$1.4 \quad \mathrm{~L}$

\begin{tabular}{|c|c|c|c|c|}
\hline Simulant Source & Formula & $\begin{array}{c}\text { Molecular } \\
\text { Weight } \\
\text { (g/mol) }\end{array}$ & $\begin{array}{c}\text { Concentration } \\
\text { (M) }\end{array}$ & $\begin{array}{c}\text { weight } \\
\text { required (g) }\end{array}$ \\
\hline Sodium nitrate & $\mathrm{NaNO}_{3}$ & 84.99 & 0.9 & 107.0874 \\
\hline Sodium nitrite & $\mathrm{NaNO}_{2}$ & 69.00 & 0.9 & 86.9400 \\
\hline Sodium chloride & $\mathrm{NaCl}$ & 58.44 & 0.0142 & 1.1618 \\
\hline Sodium sulfate & $\mathrm{Na}_{2} \mathrm{SO}_{4}$ & 142.04 & 0.3806 & 75.6846 \\
\hline Sodium carbonate & $\mathrm{Na}_{2}\left(\mathrm{CO}_{3}\right)$ & 106.00 & 0.3158 & 46.8647 \\
\hline Sodium bicarbonate & $\mathrm{NaHCO}_{3}$ & 84.00 & 0.586 & 68.9136 \\
\hline
\end{tabular}




\section{Cyclic Potentiodynamic Polarization}

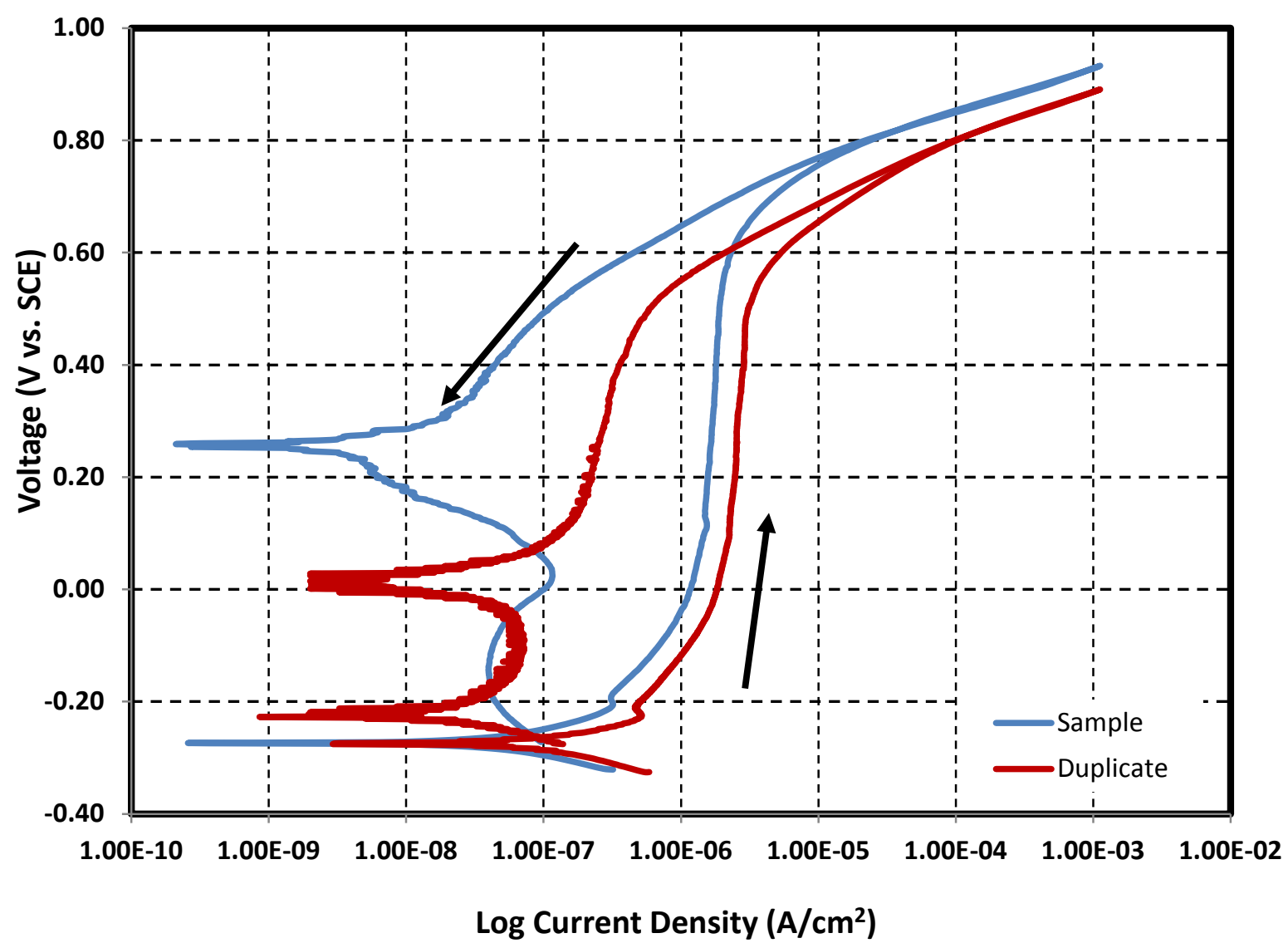

Images of bullet samples after electrochemical tests

Test 23
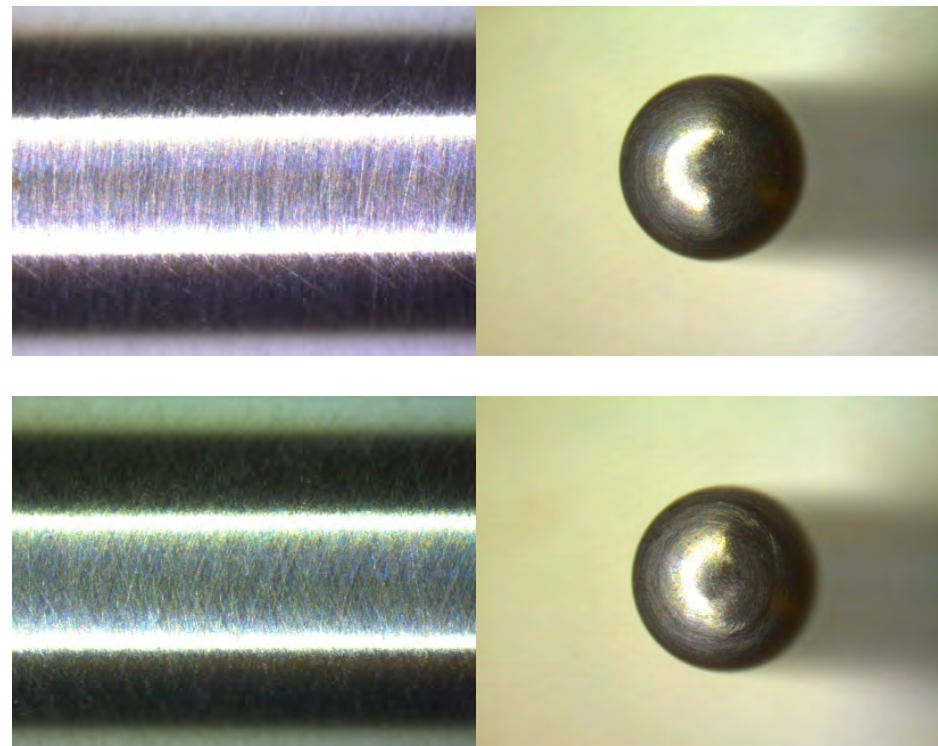

Shank (20X)

Nose (10X) 


\section{Composition of simulant for pitting corrosion -Test 24}

\section{Reference 16}

Test 24

Volume
Temperature

$\mathrm{pH}$

\begin{tabular}{|c|c|c|c|c|}
\hline Simulant Source & Formula & $\begin{array}{c}\text { Molecular } \\
\text { Weight } \\
\text { (g/mol) }\end{array}$ & $\begin{array}{c}\text { Concentration } \\
\text { (M) }\end{array}$ & $\begin{array}{c}\text { weight } \\
\text { required (g) }\end{array}$ \\
\hline Sodium nitrate & $\mathrm{NaNO}_{3}$ & 84.99 & 0.85 & 101.1381 \\
\hline Sodium nitrite & $\mathrm{NaNO}_{2}$ & 69.00 & 0.425 & 41.0550 \\
\hline Sodium chloride & $\mathrm{NaCl}$ & 58.44 & 0.011 & 0.9000 \\
\hline Sodium sulfate & $\mathrm{Na}_{2} \mathrm{SO}_{4}$ & 142.04 & 0.0425 & 8.4514 \\
\hline Sodium carbonate & $\mathrm{Na}_{2}\left(\mathrm{CO}_{3}\right)$ & 106.00 & 0.1491 & 22.1264 \\
\hline Sodium bicarbonate & $\mathrm{NaHCO}_{3}$ & 84.00 & 0.277 & 32.5752 \\
\hline
\end{tabular}

40
10.00

$1.4 \mathrm{~L}$ 


\section{Cyclic Potentiodynamic Polarization}

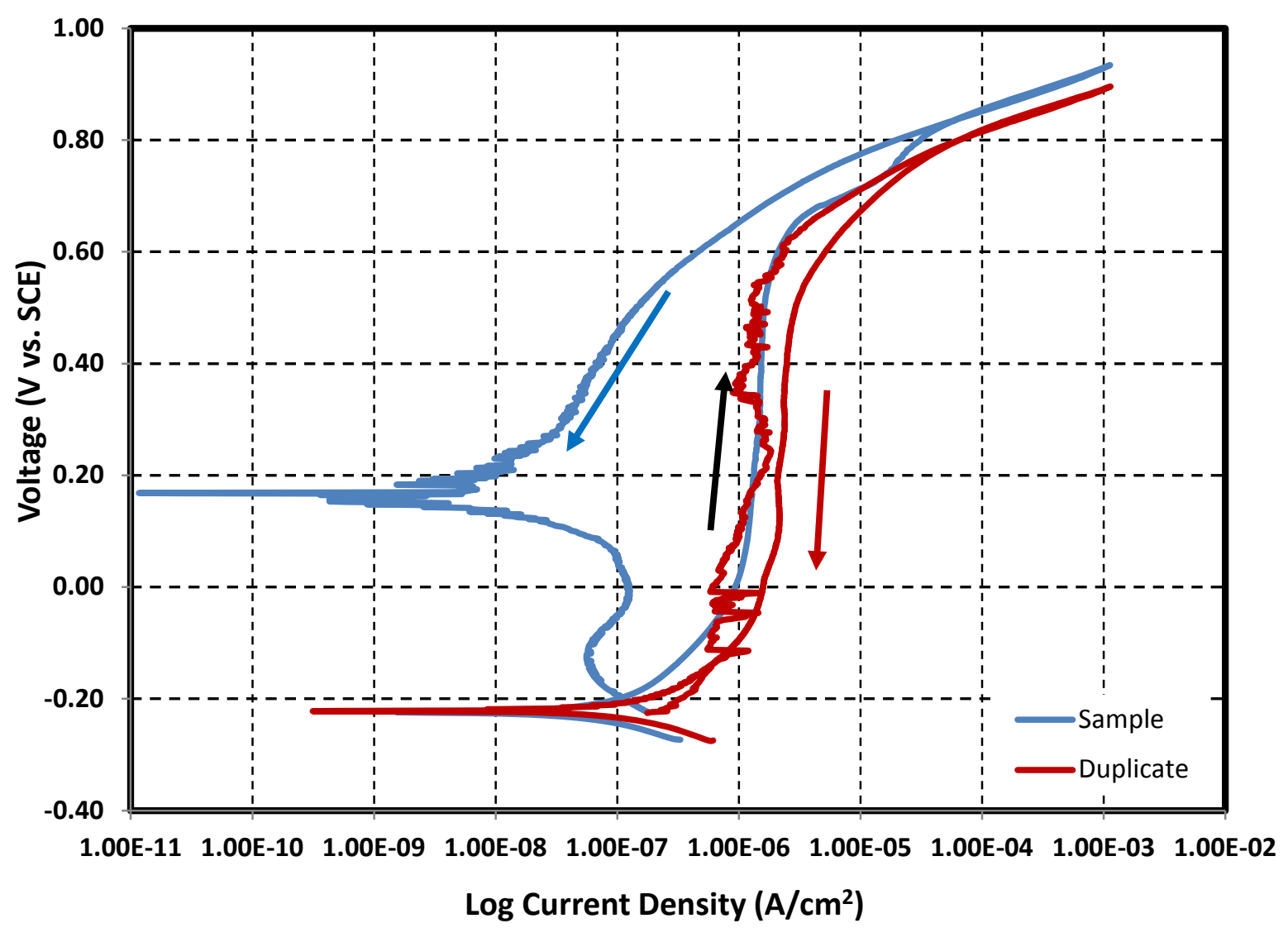

Images of bullet samples after electrochemical tests

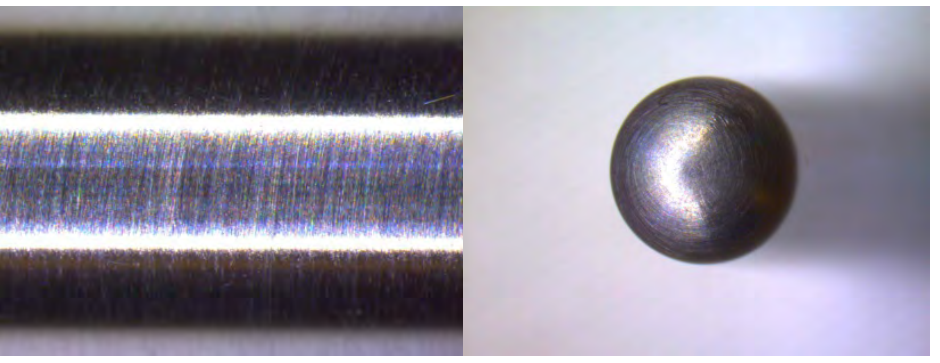

Test 24D

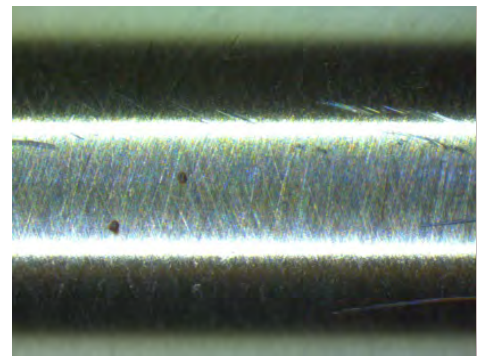

Shank (20X)

Nose (10X) 


\section{Composition of simulant for pitting corrosion -Test 25}

\begin{tabular}{|c|c|c|c|c|}
\hline Reference 1 & Temperature & 40 & ${ }^{\circ} \mathrm{C}$ & \\
\hline Test 25 & $\mathrm{pH}$ & 9.66 & & \\
\hline & Volume & 1.4 & $\mathrm{~L}$ & \\
\hline Simulant Source & Formula & $\begin{array}{l}\text { Molecular } \\
\text { Weight } \\
\text { (g/mol) }\end{array}$ & $\begin{array}{l}\text { Concentration } \\
\text { (M) }\end{array}$ & $\begin{array}{l}\text { weight } \\
\text { required }(g)\end{array}$ \\
\hline Sodium carbonate & $\mathrm{Na}_{2} \mathrm{CO}_{3}$ & 106.00 & 0.0112 & 1.6621 \\
\hline Sodium bicarbonate & $\mathrm{NaHCO}_{3}$ & 84.01 & 0.0353 & 4.1518 \\
\hline Sodium oxalate & $\mathrm{Na}_{2} \mathrm{C}_{2} \mathrm{O}_{4}$ & 134.00 & 0.00014 & 0.0263 \\
\hline Sodium molybdate, dihydrate & $\mathrm{Na}_{2} \mathrm{MoO}_{4} \cdot 2 \mathrm{H}_{2} \mathrm{O}$ & 241.95 & 0.00000744 & 0.0025 \\
\hline $\begin{array}{c}\text { Sodium metasilicate, 5- } \\
\text { hydrate }\end{array}$ & $\mathrm{Na}_{2} \mathrm{SiO}_{3} .5 \mathrm{H}_{2} \mathrm{O}$ & 212.14 & 0.0000567 & 0.0168 \\
\hline $\begin{array}{l}\text { Sodium phosphate, 12- } \\
\text { hydrate }\end{array}$ & $\mathrm{Na}_{3} \mathrm{PO}_{4} 12 \mathrm{H}_{2} \mathrm{O}$ & 380.00 & 0.000161 & 0.0857 \\
\hline Sodium chloride & $\mathrm{NaCl}$ & 58.40 & 0.000596 & 0.0487 \\
\hline Sodium fluoride & $\mathrm{NaF}$ & 41.99 & 0.000298 & 0.0175 \\
\hline Sodium sulfate & $\mathrm{Na}_{2} \mathrm{SO}_{4}$ & 142.00 & 0.00261 & 0.5189 \\
\hline Sodium nitrate & $\mathrm{NaNO}_{3}$ & 85.00 & 0.0394 & 4.6886 \\
\hline Sodium nitrite & $\mathrm{NaNO}_{2}$ & 69.00 & 0.022 & 2.1252 \\
\hline Sodium aluminate & $\mathrm{NaAlO}_{2}$ & 81.97 & 0.00045 & 0.0516 \\
\hline Cobalt nitrate, 6-hydrate & $\mathrm{Co}(\mathrm{NO} 3) 2.6 \mathrm{H} 2 \mathrm{O}$ & 291.03 & 0.00003 & 0.0122 \\
\hline Nickel nitrate, 6-hydrate & $\mathrm{Ni}\left(\mathrm{NO}_{3}\right)_{2} \cdot 6 \mathrm{H}_{2} \mathrm{O}$ & 291.00 & 0.0015 & 0.6111 \\
\hline Ferric nitrate, 9-hydrate & $\mathrm{Fe}\left(\mathrm{NO}_{3}\right)_{3} .9 \mathrm{H}_{2} \mathrm{O}$ & 404.00 & 0.000248 & 0.1403 \\
\hline Mercury (II) nitrate & $\mathrm{Hg}\left(\mathrm{NO}_{3}\right)_{2}$ & 324.60 & 0.00025 & 0.1136 \\
\hline Cupric nitrate, 2.5 hydrate & $\mathrm{Cu}\left(\mathrm{NO}_{3}\right)_{2} \cdot 2.5 \mathrm{H}_{2} \mathrm{O}$ & 233.00 & 0.000043 & 0.0140 \\
\hline Manganese dioxide & $\mathrm{MnO}_{2}$ & 86.94 & 0.00575 & 0.6999 \\
\hline
\end{tabular}




\section{Cyclic Potentiodynamic Polarization}

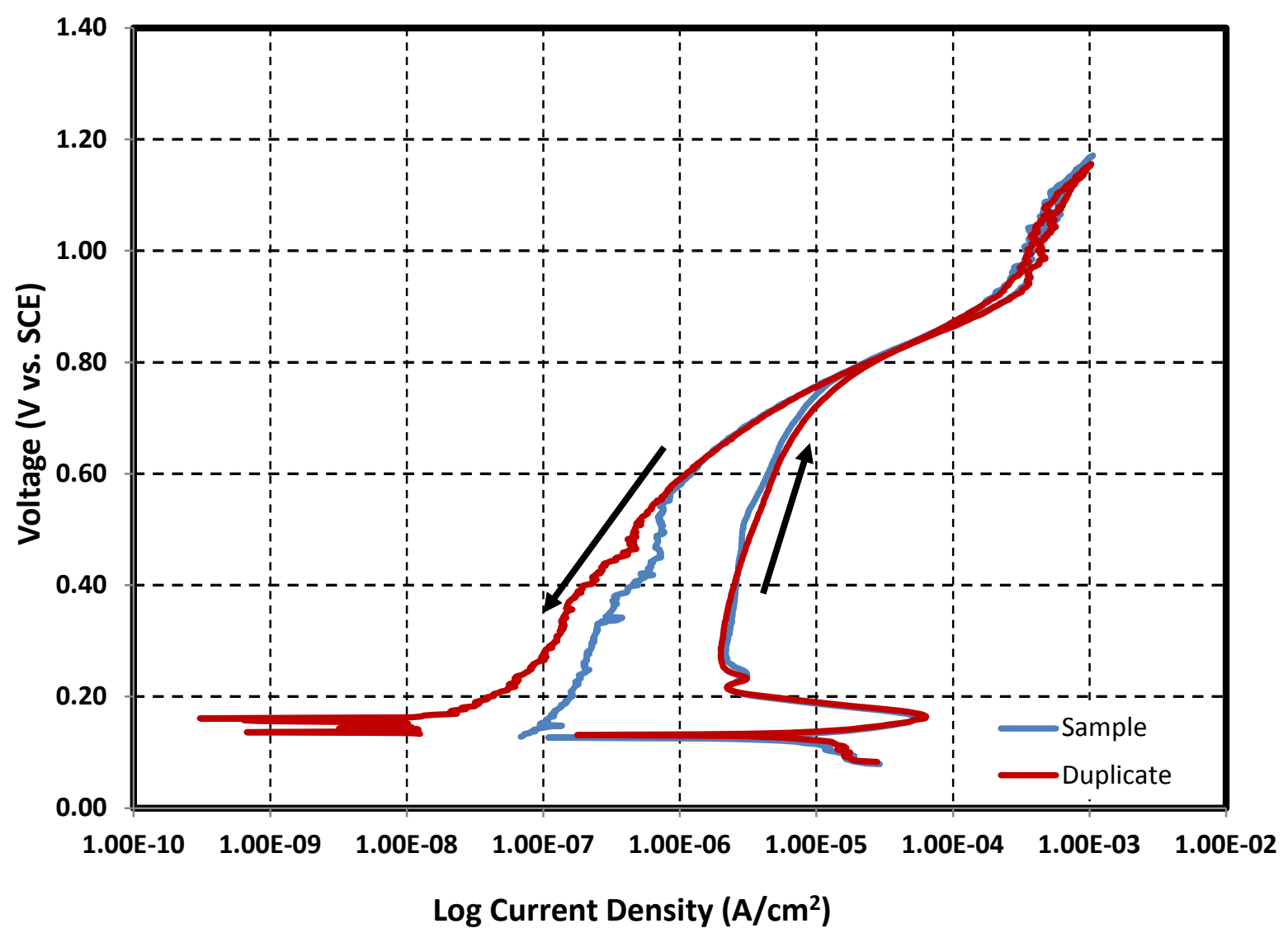

Images of bullet samples after electrochemical tests

Test 25

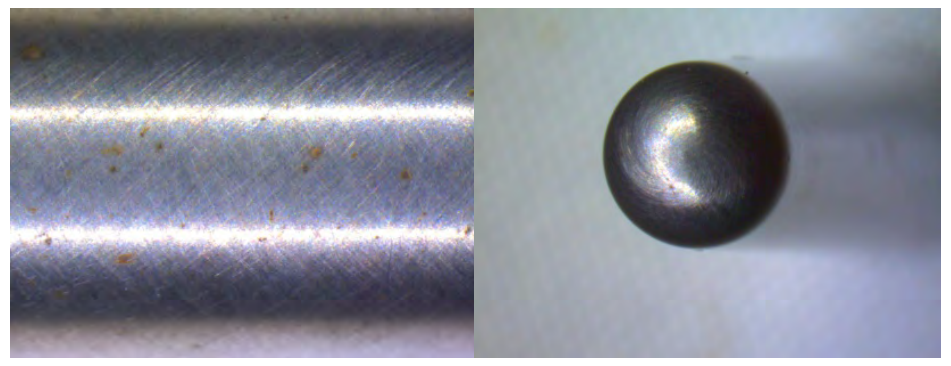

Test 25D

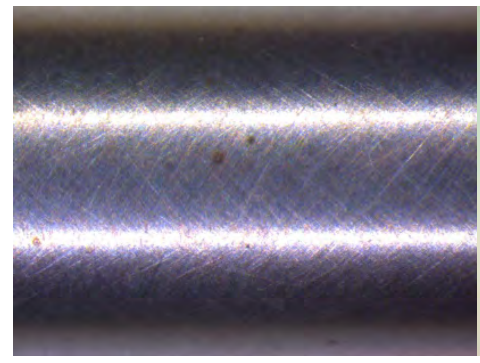

Shank $(20 X)$

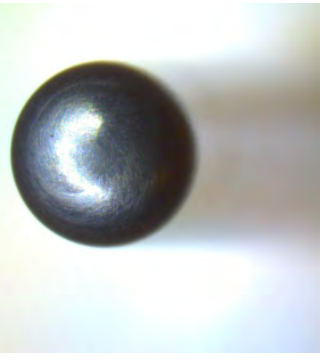

Nose (10X) 


\section{Composition of simulant for pitting corrosion -Test 26}

Reference 1

Test 26
Temperature

$\mathrm{pH}$

Volume
$50{ }^{\circ} \mathrm{C}$

10.06

\begin{tabular}{|c|c|c|c|c|}
\hline & Volume & 1.4 & $\mathrm{~L}$ & \\
\hline Simulant Source & Formula & $\begin{array}{l}\text { Molecular } \\
\text { Weight } \\
\text { (g/mol) }\end{array}$ & $\begin{array}{l}\text { Concentration } \\
\text { (M) }\end{array}$ & $\begin{array}{l}\text { weight } \\
\text { required }(g)\end{array}$ \\
\hline Sodium carbonate & $\mathrm{Na}_{2} \mathrm{CO}_{3}$ & 106.00 & 0.187 & 27.7508 \\
\hline Sodium bicarbonate & $\mathrm{NaHCO}_{3}$ & 84.01 & 0.173 & 20.3472 \\
\hline Sodium oxalate & $\mathrm{Na}_{2} \mathrm{C}_{2} \mathrm{O}_{4}$ & 134.00 & 0.00138 & 0.2589 \\
\hline Sodium molybdate, dihydrate & $\mathrm{Na}_{2} \mathrm{MoO}_{4} \cdot 2 \mathrm{H}_{2} \mathrm{O}$ & 241.95 & 0.0000733 & 0.0248 \\
\hline $\begin{array}{l}\text { Sodium metasilicate, } 5- \\
\text { hydrate }\end{array}$ & $\mathrm{Na}_{2} \mathrm{SiO}_{3} .5 \mathrm{H}_{2} \mathrm{O}$ & 212.14 & 0.000559 & 0.1660 \\
\hline $\begin{array}{l}\text { Sodium phosphate, } 12- \\
\text { hydrate }\end{array}$ & $\mathrm{Na}_{3} \mathrm{PO}_{4} \cdot 12 \mathrm{H}_{2} \mathrm{O}$ & 380.00 & 0.00158 & 0.8406 \\
\hline Sodium chloride & $\mathrm{NaCl}$ & 58.40 & 0.00587 & 0.4799 \\
\hline Sodium fluoride & $\mathrm{NaF}$ & 41.99 & 0.00294 & 0.1728 \\
\hline Sodium sulfate & $\mathrm{Na}_{2} \mathrm{SO}_{4}$ & 142.00 & 0.0258 & 5.1290 \\
\hline Sodium nitrate & $\mathrm{NaNO}_{3}$ & 85.00 & 0.388 & 46.1720 \\
\hline Sodium nitrite & $\mathrm{NaNO}_{2}$ & 69.00 & 0.7 & 67.6200 \\
\hline Sodium aluminate & $\mathrm{NaAlO}_{2}$ & 81.97 & 0.00045 & 0.0516 \\
\hline Cobalt nitrate, 6 -hydrate & $\mathrm{Co}(\mathrm{NO} 3) 2.6 \mathrm{H} 2 \mathrm{O}$ & 291.03 & 0.00003 & 0.0122 \\
\hline Nickel nitrate, 6-hydrate & $\mathrm{Ni}\left(\mathrm{NO}_{3}\right)_{2} \cdot 6 \mathrm{H}_{2} \mathrm{O}$ & 291.00 & 0.0015 & 0.6111 \\
\hline Ferric nitrate, 9-hydrate & $\mathrm{Fe}\left(\mathrm{NO}_{3}\right)_{3} .9 \mathrm{H}_{2} \mathrm{O}$ & 404.00 & 0.000248 & 0.1403 \\
\hline Mercury (II) nitrate & $\mathrm{Hg}\left(\mathrm{NO}_{3}\right)_{2}$ & 324.60 & 0.00025 & 0.1136 \\
\hline Cupric nitrate, 2.5 hydrate & $\mathrm{Cu}\left(\mathrm{NO}_{3}\right)_{2} \cdot 2.5 \mathrm{H}_{2} \mathrm{O}$ & 233.00 & 0.000043 & 0.0140 \\
\hline Manganese dioxide & $\mathrm{MnO}_{2}$ & 86.94 & 0.00575 & 0.6999 \\
\hline
\end{tabular}




\section{Cyclic Potentiodynamic Polarization}

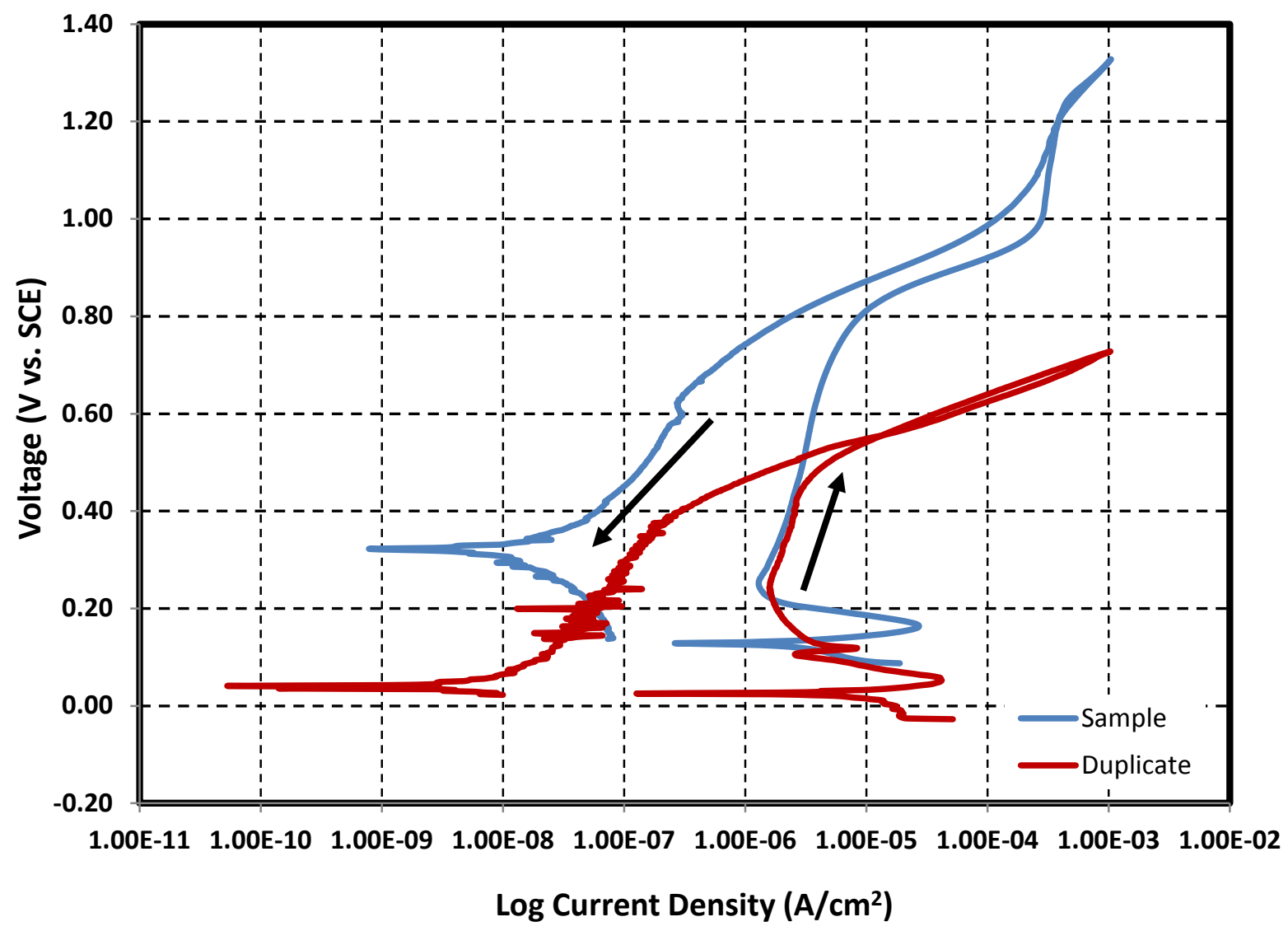

Images of bullet samples after electrochemical tests

Test 26

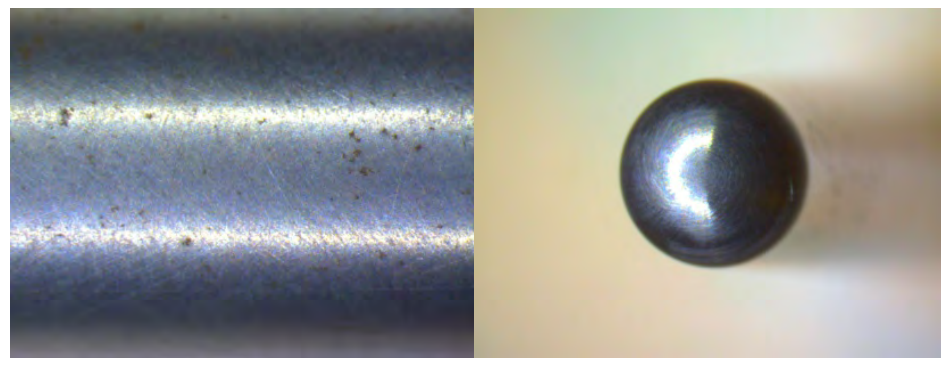

Test 26D

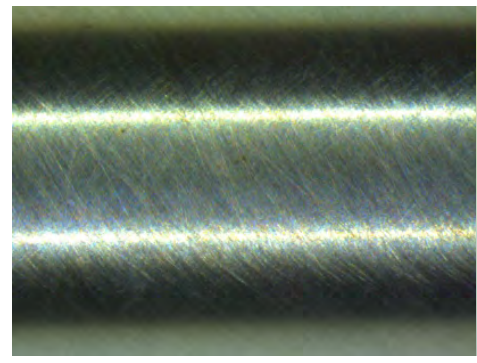

Shank (20X)

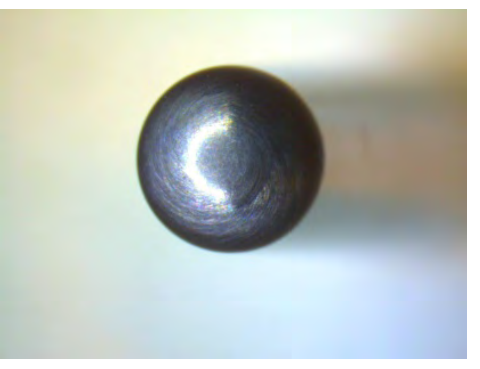

Nose (10X) 


\section{Composition of simulant for pitting corrosion -Test 27}

Reference 14

Test 27

\begin{tabular}{|c|c|c|c|c|}
\hline & Volume & 1. & $\mathrm{~L}$ & \\
\hline Simulant Source & Formula & $\begin{array}{l}\text { Molecular } \\
\text { Weight } \\
\text { (g/mol) }\end{array}$ & $\begin{array}{l}\text { Concentration } \\
\text { (M) }\end{array}$ & $\begin{array}{l}\text { weight } \\
\text { required (g) }\end{array}$ \\
\hline Sodium nitrate & $\mathrm{NaNO}_{3}$ & 84.99 & 0.45 & 53.5437 \\
\hline Sodium nitrite & $\mathrm{NaNO}_{2}$ & 69.00 & 0.113 & 10.9158 \\
\hline Sodium chloride & $\mathrm{NaCl}$ & 58.44 & 0.003 & 0.2454 \\
\hline Sodium sulfate & $\mathrm{Na}_{2} \mathrm{SO}_{4}$ & 142.04 & 0.032 & 6.3634 \\
\hline Sodium carbonate & $\mathrm{Na}_{2}\left(\mathrm{CO}_{3}\right)$ & 106.00 & 0.0395 & 5.8618 \\
\hline Sodium bicarbonate & $\mathrm{NaHCO}_{3}$ & 84.00 & 0.073 & 8.5848 \\
\hline
\end{tabular}




\section{Cyclic Potentiodynamic Polarization}

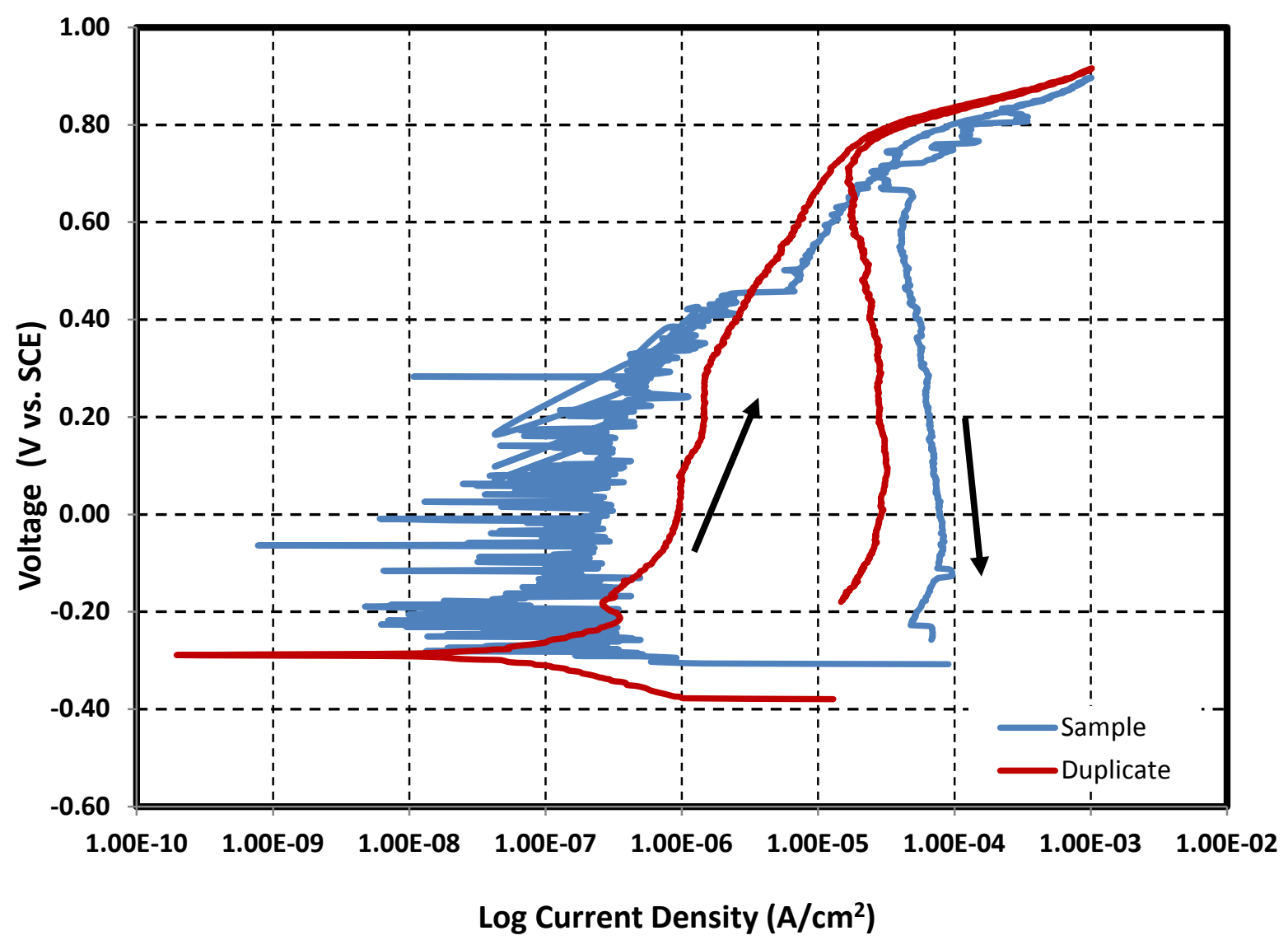

Images of bullet samples after electrochemical tests

Test 27
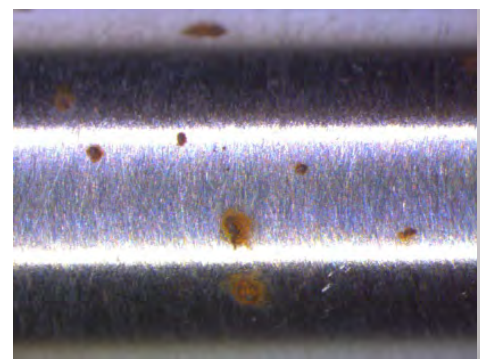

Test 27D

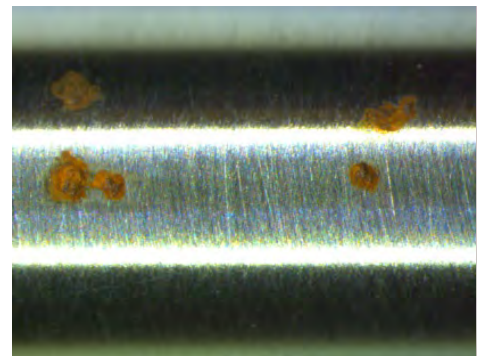

Shank (20X)
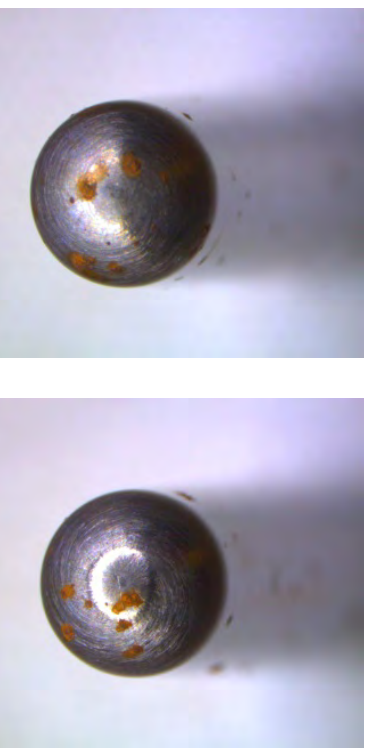

Nose (10X) 


\section{Composition of simulant for pitting corrosion -Test 28}

Reference 16

Test 28
Temperature

$\mathrm{pH}$

Volume

\begin{tabular}{|c|c|c|c|c|}
\hline Simulant Source & Formula & $\begin{array}{c}\text { Molecular } \\
\text { Weight } \\
\text { (g/mol) }\end{array}$ & $\begin{array}{c}\text { Concentration } \\
\text { (M) }\end{array}$ & $\begin{array}{c}\text { weight } \\
\text { required (g) }\end{array}$ \\
\hline Sodium nitrate & $\mathrm{NaNO}_{3}$ & 84.99 & 0.55 & 65.4423 \\
\hline Sodium nitrite & $\mathrm{NaNO}_{2}$ & 69.00 & 0.55 & 53.1300 \\
\hline Sodium chloride & $\mathrm{NaCl}$ & 58.44 & 0.007 & 0.5727 \\
\hline Sodium sulfate & $\mathrm{Na}_{2} \mathrm{SO}_{4}$ & 142.04 & 0.0275 & 5.4685 \\
\hline Sodium carbonate & $\mathrm{Na}_{2}\left(\mathrm{CO}_{3}\right)$ & 106.00 & 0.193 & 28.6412 \\
\hline Sodium bicarbonate & $\mathrm{NaHCO}_{3}$ & 84.00 & 0.358 & 42.1008 \\
\hline
\end{tabular}




\section{Cyclic Potentiodynamic Polarization}

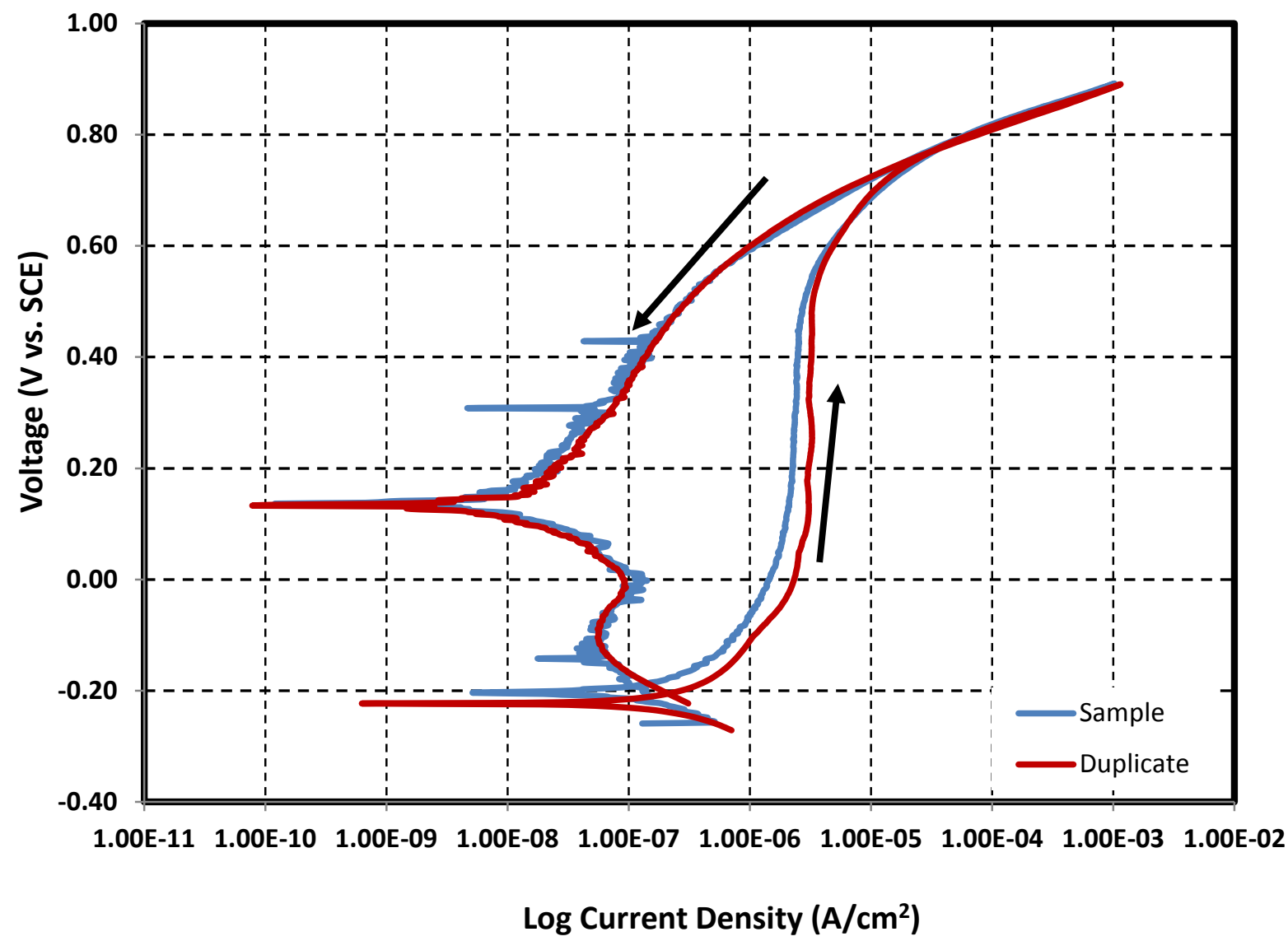

Images of bullet samples after electrochemical tests

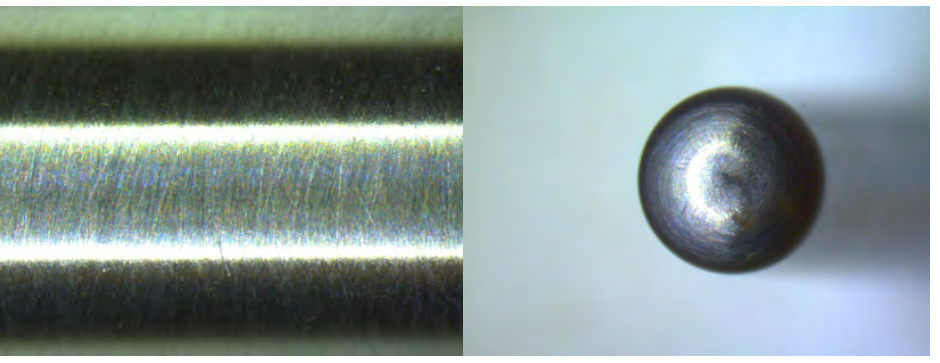

Test 28D

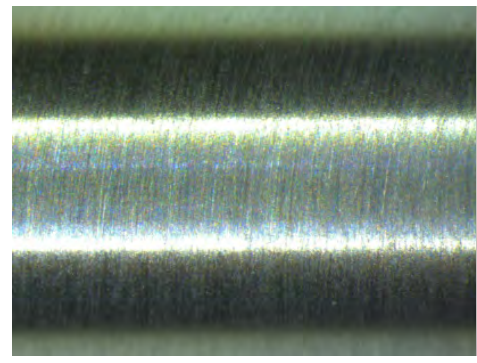

Shank (20X)

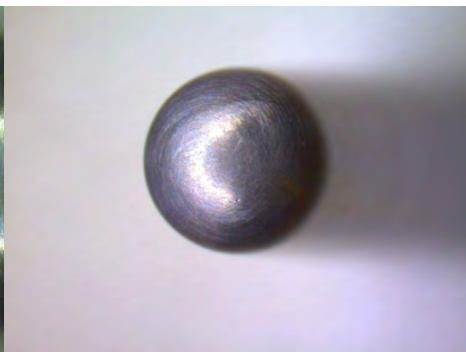

Nose (10X) 


\section{Composition of simulant for pitting corrosion -Test 29}

\begin{tabular}{|c|c|c|c|c|}
\hline \multirow{3}{*}{$\begin{array}{l}\text { Reference } 4 \\
\text { Test } 29\end{array}$} & \multirow{2}{*}{$\begin{array}{l}\text { Temperature } \\
\mathrm{pH}\end{array}$} & \multirow{2}{*}{$\begin{array}{r}40 \\
9.6\end{array}$} & \multicolumn{2}{|l|}{${ }^{\circ} \mathrm{C}$} \\
\hline & & & & \\
\hline & Volume & 1.4 & L & \\
\hline Simulant Source & Formula & $\begin{array}{l}\text { Molecular } \\
\text { Weight } \\
\text { (g/mol) }\end{array}$ & $\begin{array}{l}\text { Concentration } \\
\text { (M) }\end{array}$ & $\begin{array}{l}\text { weight } \\
\text { required }(g)\end{array}$ \\
\hline Aluminum nitrate, 9-hydrate & $\mathrm{Al}\left(\mathrm{NO}_{3}\right)_{3} \cdot 9 \mathrm{H}_{2} \mathrm{O}$ & 375.13 & 0.006842073 & 3.5933 \\
\hline Ferric Nitrate, 9-hydrate & $\mathrm{Fe}\left(\mathrm{NO}_{3}\right)_{3} \cdot 9 \mathrm{H}_{2} \mathrm{O}$ & 404.00 & 0.001831683 & 1.0360 \\
\hline Sodium hydroxide & $\mathrm{NaOH}$ & 40.00 & 0.0305 & 1.7080 \\
\hline Sodium nitrate & $\mathrm{NaNO}_{3}$ & 85.00 & 0.001694317 & 0.2016 \\
\hline Sodium nitrite & $\mathrm{NaNO}_{2}$ & 69.00 & 0.070202899 & 6.7816 \\
\hline Sodium oxalate & $\mathrm{Na}_{2}\left(\mathrm{C}_{2} \mathrm{O}_{4}\right)$ & 134.00 & 0.002885572 & 0.5413 \\
\hline Sodium sulfate & $\mathrm{Na}_{2} \mathrm{SO}_{4}$ & 142.04 & 0.001980663 & 0.3939 \\
\hline Sodium carbonate & $\mathrm{Na}_{2}\left(\mathrm{CO}_{3}\right)$ & 106.00 & 0.004679245 & 0.6944 \\
\hline Sodium Bicarbonate & $\mathrm{NaHCO}_{3}$ & 84.00 & 0.019333333 & 2.2736 \\
\hline $\begin{array}{l}\text { Sodium phosphate, 12- } \\
\text { hydrate }\end{array}$ & $\mathrm{Na}_{3} \mathrm{PO}_{4} \cdot 12 \mathrm{H}_{2} \mathrm{O}$ & 380.12 & 9.16903E-05 & 0.0488 \\
\hline Calcium Carbonate & $\mathrm{CaCO}_{3}$ & 100.00 & 0.000220133 & 0.0308 \\
\hline Sodium chloride & $\mathrm{NaCl}$ & 58.44 & 0.000382729 & 0.0313 \\
\hline Sodium fluoride & $\mathrm{NaF}$ & 42.00 & 0.000243175 & 0.0143 \\
\hline Sodium molybdate, dihydrate & $\mathrm{Na}_{2} \mathrm{MoO}_{4} \cdot 2 \mathrm{H}_{2} \mathrm{O}$ & 241.95 & 6.15066E-06 & 0.0021 \\
\hline Manganese Dioxide & $\mathrm{MnO}_{2}$ & 86.94 & 0.000380032 & 0.0463 \\
\hline Nickel nitrate, 6-hydrate & $\mathrm{Ni}\left(\mathrm{NO}_{3}\right)_{2} \cdot 6 \mathrm{H}_{2} \mathrm{O}$ & 290.81 & 0.000173355 & 0.0706 \\
\hline Mercury (II) nitrate & $\mathrm{Hg}\left(\mathrm{NO}_{3}\right)_{2}$ & 342.62 & 0.000146 & 0.0700 \\
\hline Sodium silicate, 9-hydrate & $\mathrm{Na}_{2} \mathrm{SiO}_{3} .9 \mathrm{H}_{2} \mathrm{O}$ & 284.00 & 5.33803E-05 & 0.0212 \\
\hline Zinc nitrate, 6-hydrate & $\mathrm{Zn}\left(\mathrm{NO}_{3}\right)_{2} \cdot 6 \mathrm{H}_{2} \mathrm{O}$ & 297.49 & 1.37147E-05 & 0.0057 \\
\hline Lead nitrate & $\mathrm{Pb}\left(\mathrm{NO}_{3}\right)_{2}$ & 331.21 & 6.48129E-06 & 0.0030 \\
\hline Cupric nitrate, 2.5 hydrate & $\mathrm{Cu}\left(\mathrm{NO}_{3}\right)_{2} \cdot 2 \cdot 5 \mathrm{H}_{2} \mathrm{O}$ & 233.00 & 8.18312E-06 & 0.0027 \\
\hline
\end{tabular}




\section{Cyclic Potentiodynamic Polarization}

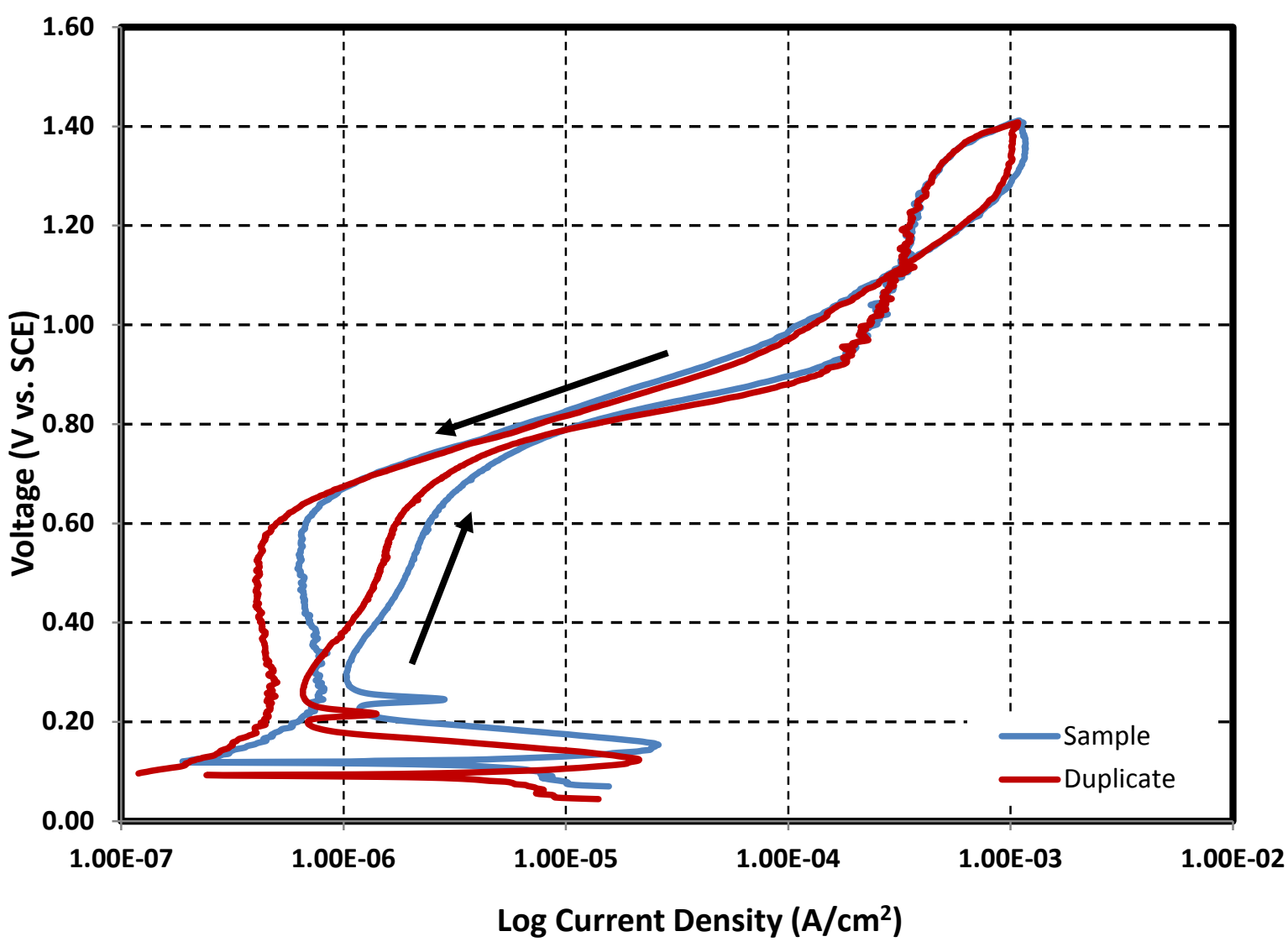

Images of bullet samples after electrochemical tests

Test 29

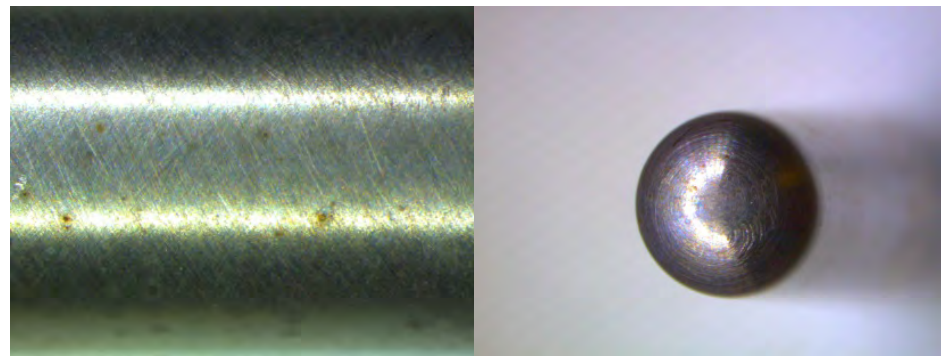

Test 29D

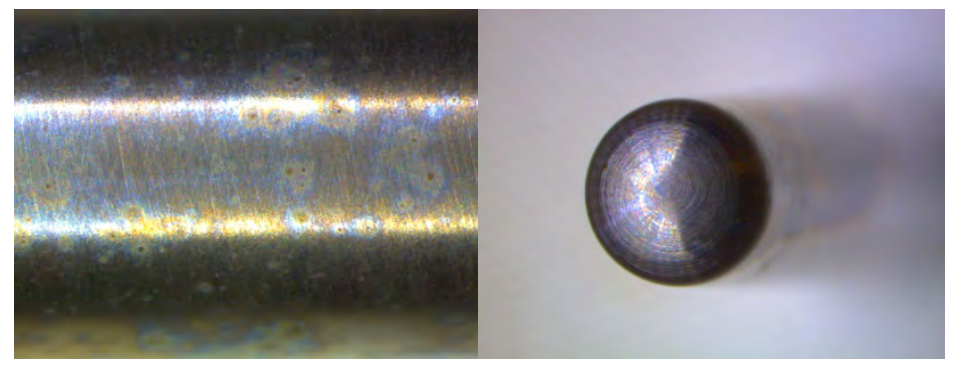

Shank $(20 X)$

Nose (10X) 


\section{Composition of simulant for pitting corrosion -Test 30}

\begin{tabular}{|c|c|c|c|c|}
\hline \multirow{3}{*}{$\begin{array}{l}\text { Reference } 16 \\
\text { Test } 30\end{array}$} & \multirow{2}{*}{$\begin{array}{l}\text { Temperature } \\
\mathrm{pH}\end{array}$} & \multicolumn{2}{|c|}{$40{ }^{\circ} \mathrm{C}$} & \\
\hline & & 10.00 & & \\
\hline & Volume & 1.4 & $\mathrm{~L}$ & \\
\hline Simulant Source & Formula & $\begin{array}{l}\text { Molecular } \\
\text { Weight } \\
\text { (g/mol) } \\
\end{array}$ & $\begin{array}{l}\text { Concentration } \\
\text { (M) }\end{array}$ & $\begin{array}{l}\text { weight } \\
\text { required }(g)\end{array}$ \\
\hline Sodium nitrate & $\mathrm{NaNO}_{3}$ & 84.99 & 0.25 & 29.7465 \\
\hline Sodium nitrite & $\mathrm{NaNO}_{2}$ & 69.00 & 1.8 & 173.8800 \\
\hline Sodium chloride & $\mathrm{NaCl}$ & 58.44 & 0.0175 & 1.4318 \\
\hline Sodium sulfate & $\mathrm{Na}_{2} \mathrm{SO}_{4}$ & 142.04 & 0.0375 & 7.4571 \\
\hline Sodium carbonate & $\mathrm{Na}_{2}\left(\mathrm{CO}_{3}\right)$ & 106.00 & 0.632 & 93.7888 \\
\hline Sodium bicarbonate & $\mathrm{NaHCO}_{3}$ & 84.00 & 1.17 & 137.5920 \\
\hline
\end{tabular}




\section{Cyclic Potentiodynamic Polarization}

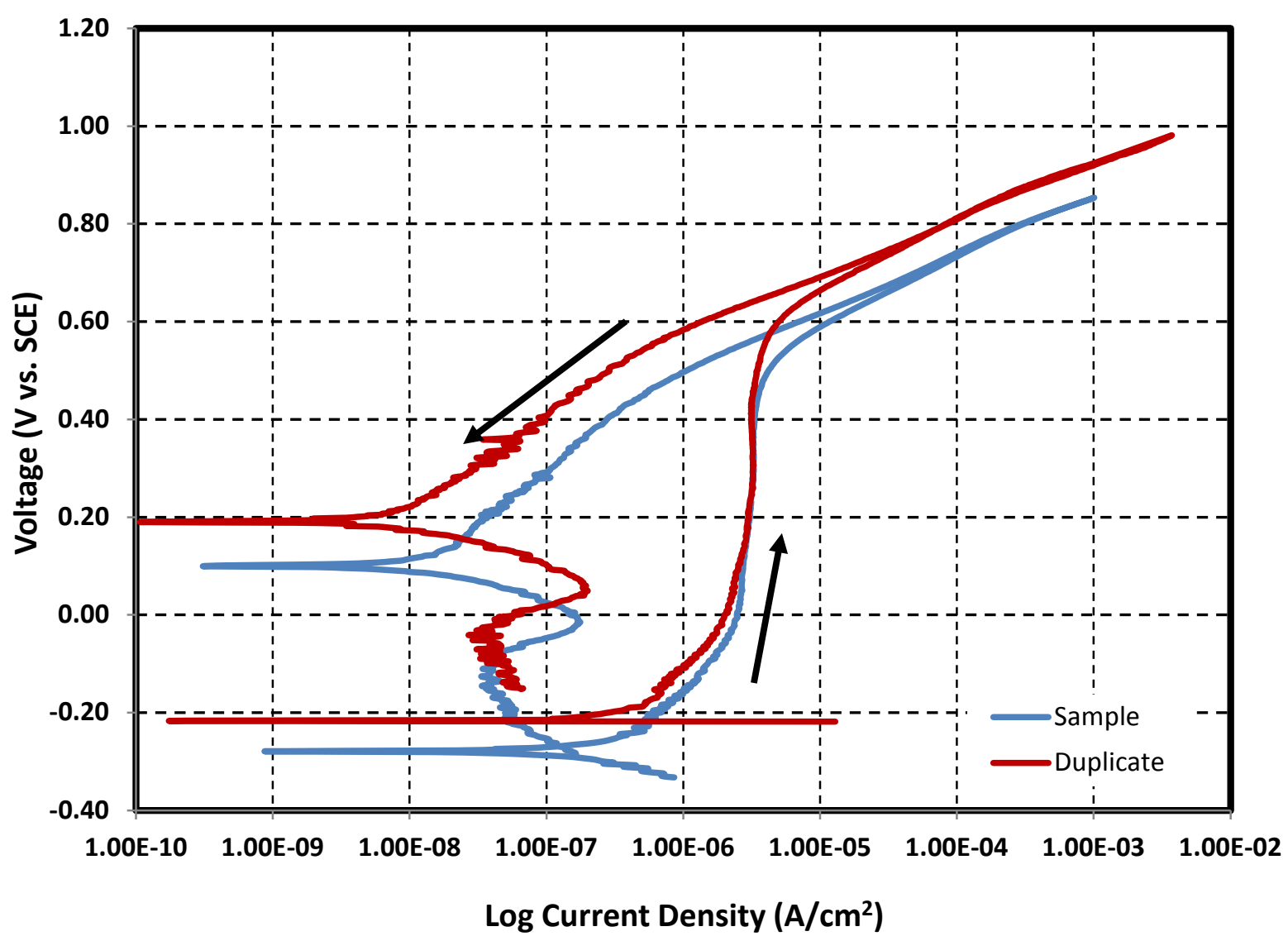

Images of bullet samples after electrochemical tests

Test 30

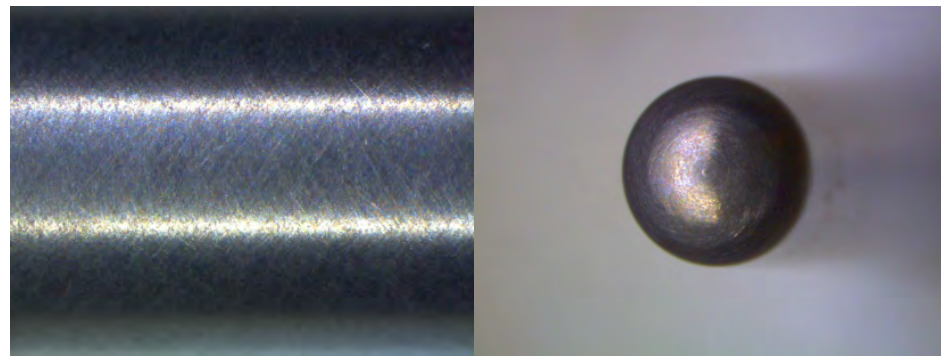

Test 30D

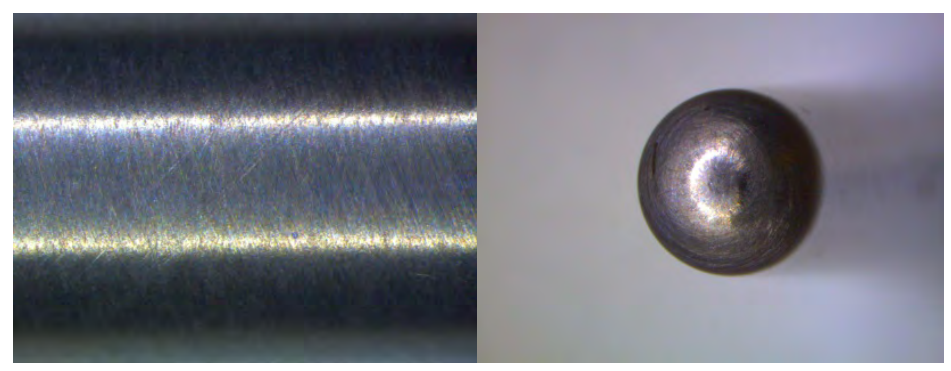

Shank $(20 X)$

Nose (10X) 


\section{Composition of simulant for pitting corrosion -Test 31}

\begin{tabular}{|c|c|c|c|c|}
\hline Reference 3 & Temperature & 40 & ${ }^{\circ} \mathrm{C}$ & \\
\hline Test 31 & $\mathrm{pH}$ & 9.6 & & \\
\hline & Volume & 1.4 & $\mathrm{~L}$ & \\
\hline Simulant Source & Formula & $\begin{array}{l}\text { Molecular } \\
\text { Weight } \\
\text { (g/mol) }\end{array}$ & $\begin{array}{l}\text { Concentration } \\
\text { (M) }\end{array}$ & $\begin{array}{l}\text { weight } \\
\text { required }(g)\end{array}$ \\
\hline Aluminum nitrate, 9-hydrate & $\mathrm{Al}\left(\mathrm{NO}_{3}\right)_{3} \cdot 9 \mathrm{H}_{2} \mathrm{O}$ & 375.13 & 0.014928158 & 7.8400 \\
\hline Ferric Nitrate, 9-hydrate & $\mathrm{Fe}\left(\mathrm{NO}_{3}\right)_{3} \cdot 9 \mathrm{H}_{2} \mathrm{O}$ & 404.00 & 0.004945 & 2.7969 \\
\hline Sodium hydroxide & $\mathrm{NaOH}$ & 40.00 & 0.0915 & 5.1240 \\
\hline Sodium nitrate & $\mathrm{NaNO}_{3}$ & 85.00 & 0 & 0.0000 \\
\hline Sodium nitrite & $\mathrm{NaNO}_{2}$ & 69.00 & 0.06 & 5.7960 \\
\hline Sodium oxalate & $\mathrm{Na}_{2}\left(\mathrm{C}_{2} \mathrm{O}_{4}\right)$ & 134.00 & 0.008656716 & 1.6240 \\
\hline Sodium sulfate & $\mathrm{Na}_{2} \mathrm{SO}_{4}$ & 142.04 & 0.005941988 & 1.1816 \\
\hline Sodium carbonate & $\mathrm{Na}_{2}\left(\mathrm{CO}_{3}\right)$ & 106.00 & 0.014037736 & 2.0832 \\
\hline Sodium Bicarbonate & $\mathrm{NaHCO}_{3}$ & 84.00 & 0.058 & 6.8208 \\
\hline $\begin{array}{l}\text { Sodium phosphate, 12- } \\
\text { hydrate }\end{array}$ & $\mathrm{Na}_{3} \mathrm{PO}_{4} \cdot 12 \mathrm{H}_{2} \mathrm{O}$ & 380.12 & 0.000275071 & 0.1464 \\
\hline Calcium Carbonate & $\mathrm{CaCO}_{3}$ & 100.00 & 0.0006604 & 0.0925 \\
\hline Sodium chloride & $\mathrm{NaCl}$ & 58.44 & $1.09 \mathrm{E}-03$ & 0.0892 \\
\hline Sodium fluoride & $\mathrm{NaF}$ & 42.00 & 0.000729524 & 0.0429 \\
\hline Sodium molybdate, dihydrate & $\mathrm{Na}_{2} \mathrm{MoO}_{4} \cdot 2 \mathrm{H}_{2} \mathrm{O}$ & 241.95 & 1.8452E-05 & 0.0063 \\
\hline Manganese Dioxide & $\mathrm{MnO}_{2}$ & 86.94 & 0.001140097 & 0.1388 \\
\hline Nickel nitrate, 6-hydrate & $\mathrm{Ni}\left(\mathrm{NO}_{3}\right)_{2} \cdot 6 \mathrm{H}_{2} \mathrm{O}$ & 290.81 & 0.000520065 & 0.2117 \\
\hline Mercury (II) nitrate & $\mathrm{Hg}\left(\mathrm{NO}_{3}\right)_{2}$ & 342.62 & 0.00311 & 1.4918 \\
\hline $\begin{array}{c}\text { Sodium metasilicate, 5- } \\
\text { hydrate }\end{array}$ & $\mathrm{Na}_{2} \mathrm{SiO}_{3} .5 \mathrm{H}_{2} \mathrm{O}$ & 212.14 & 0.000160141 & 0.0476 \\
\hline Zinc nitrate, 6-hydrate & $\mathrm{Zn}\left(\mathrm{NO}_{3}\right)_{2} \cdot 6 \mathrm{H}_{2} \mathrm{O}$ & 297.49 & 4.11442E-05 & 0.0171 \\
\hline Lead nitrate & $\mathrm{Pb}\left(\mathrm{NO}_{3}\right)_{2}$ & 331.21 & 1.94439E-05 & 0.0090 \\
\hline Cupric nitrate, 2.5 hydrate & $\mathrm{Cu}\left(\mathrm{NO}_{3}\right)_{2} \cdot 2.5 \mathrm{H}_{2} \mathrm{O}$ & 233.00 & 2.45494E-05 & 0.0080 \\
\hline
\end{tabular}




\section{Cyclic Potentiodynamic Polarization}

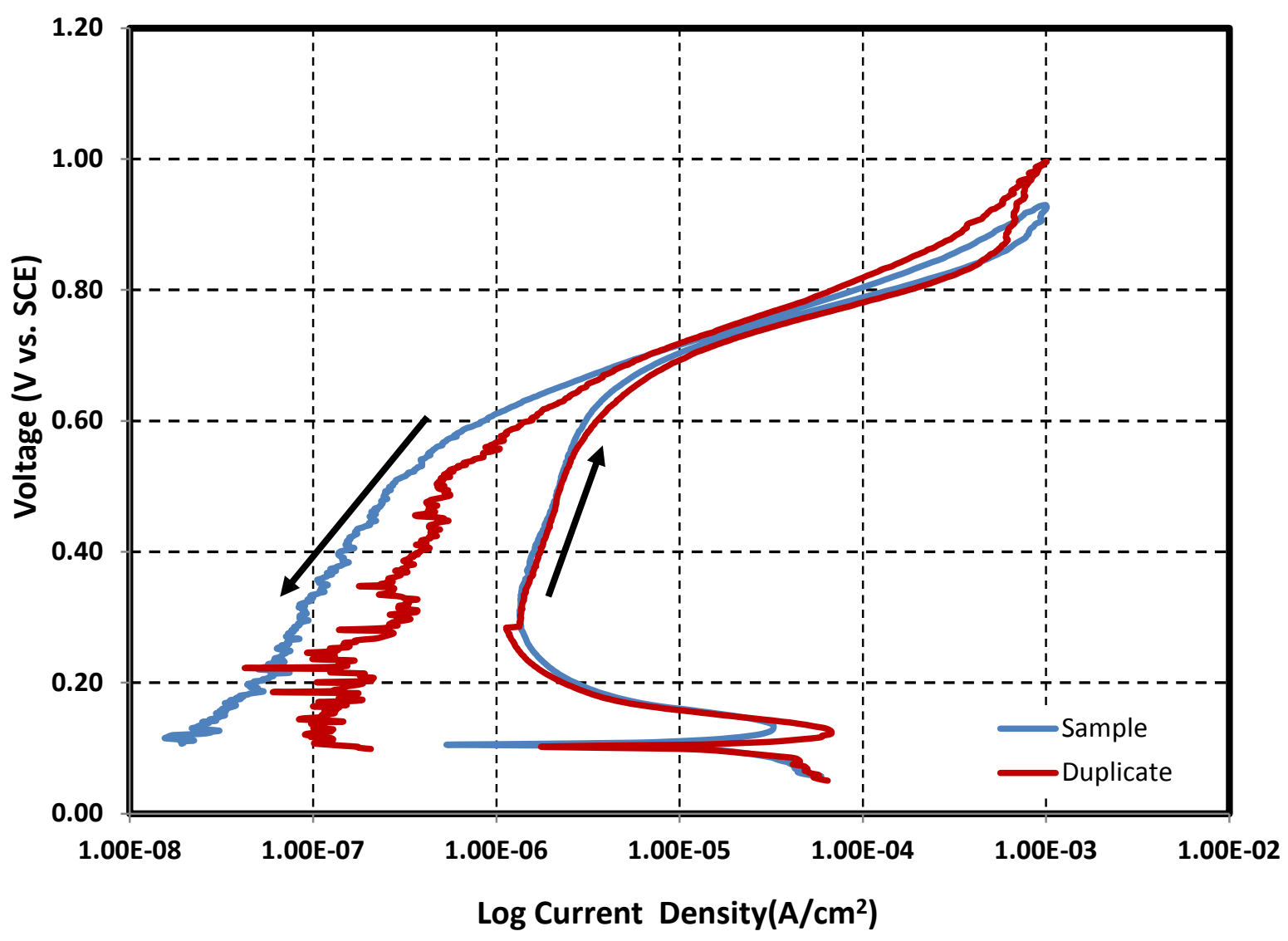

Images of bullet samples after electrochemical tests

Test 31

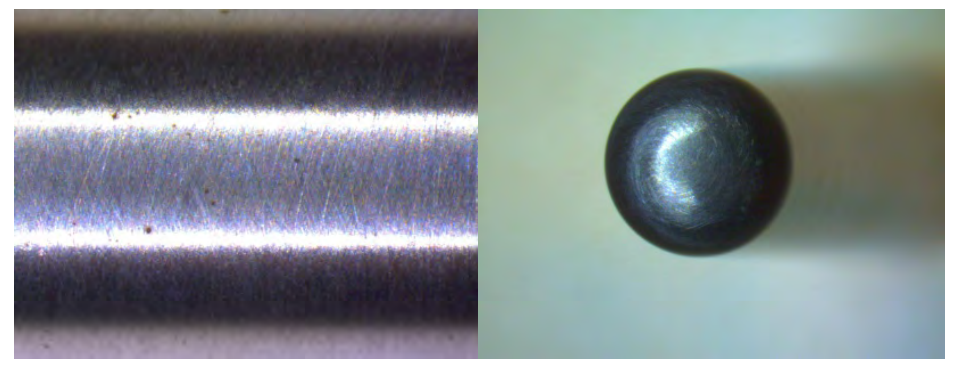

Test 31D

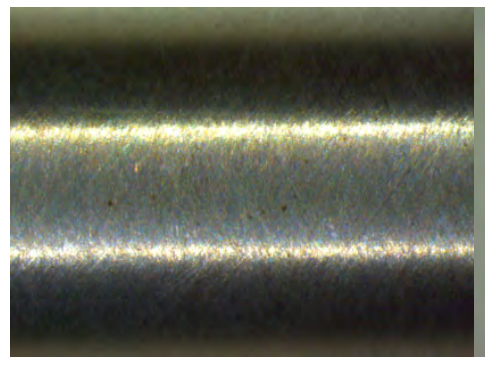

Shank (20X)

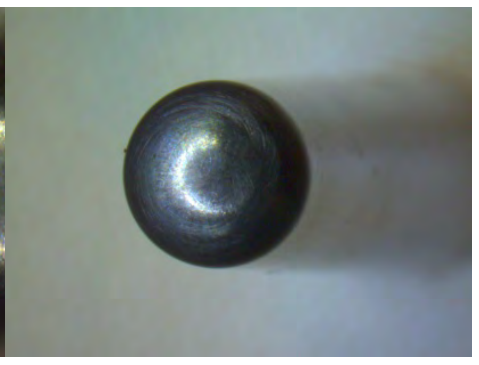

Nose (10X) 


\section{Composition of simulant for pitting corrosion -Test 32}

Reference 12

Test 32
Temperature

$\mathrm{pH}$

Volume
$80{ }^{\circ} \mathrm{C}$

10.18

$1.4 \quad \mathrm{~L}$

\begin{tabular}{|c|c|c|c|c|}
\hline Simulant Source & Formula & $\begin{array}{c}\text { Molecular } \\
\text { Weight } \\
\text { (g/mol) }\end{array}$ & $\begin{array}{c}\text { Concentration } \\
\text { (M) }\end{array}$ & $\begin{array}{c}\text { weight } \\
\text { required (g) }\end{array}$ \\
\hline Sodium nitrate & $\mathrm{NaNO}_{3}$ & 84.99 & 0.0098 & 1.1661 \\
\hline Sodium sulfate & $\mathrm{Na}_{2} \mathrm{SO}_{4}$ & 142.04 & 0.00264 & 0.5250 \\
\hline Sodium lodide & $\mathrm{Nal}$ & 149.89 & 0.00016 & 0.0336 \\
\hline Sodium fluoride & $\mathrm{NaF}$ & 42.00 & 0.00267 & 0.1570 \\
\hline Sodium chloride & $\mathrm{NaCl}$ & 58.44 & 0.0146 & 1.1945 \\
\hline Sodium nitrite & $\mathrm{NaNO}_{2}$ & 69.00 & 0.022 & 2.1252 \\
\hline Sodium carbonate & $\mathrm{Na}_{2}\left(\mathrm{CO}_{3}\right)$ & 106.00 & 0.337 & 50.0108 \\
\hline Sodium bicarbonate & $\mathrm{NaHCO}_{3}$ & 84.00 & 0.243 & 28.5768 \\
\hline
\end{tabular}




\section{Cyclic Potentiodynamic Polarization}

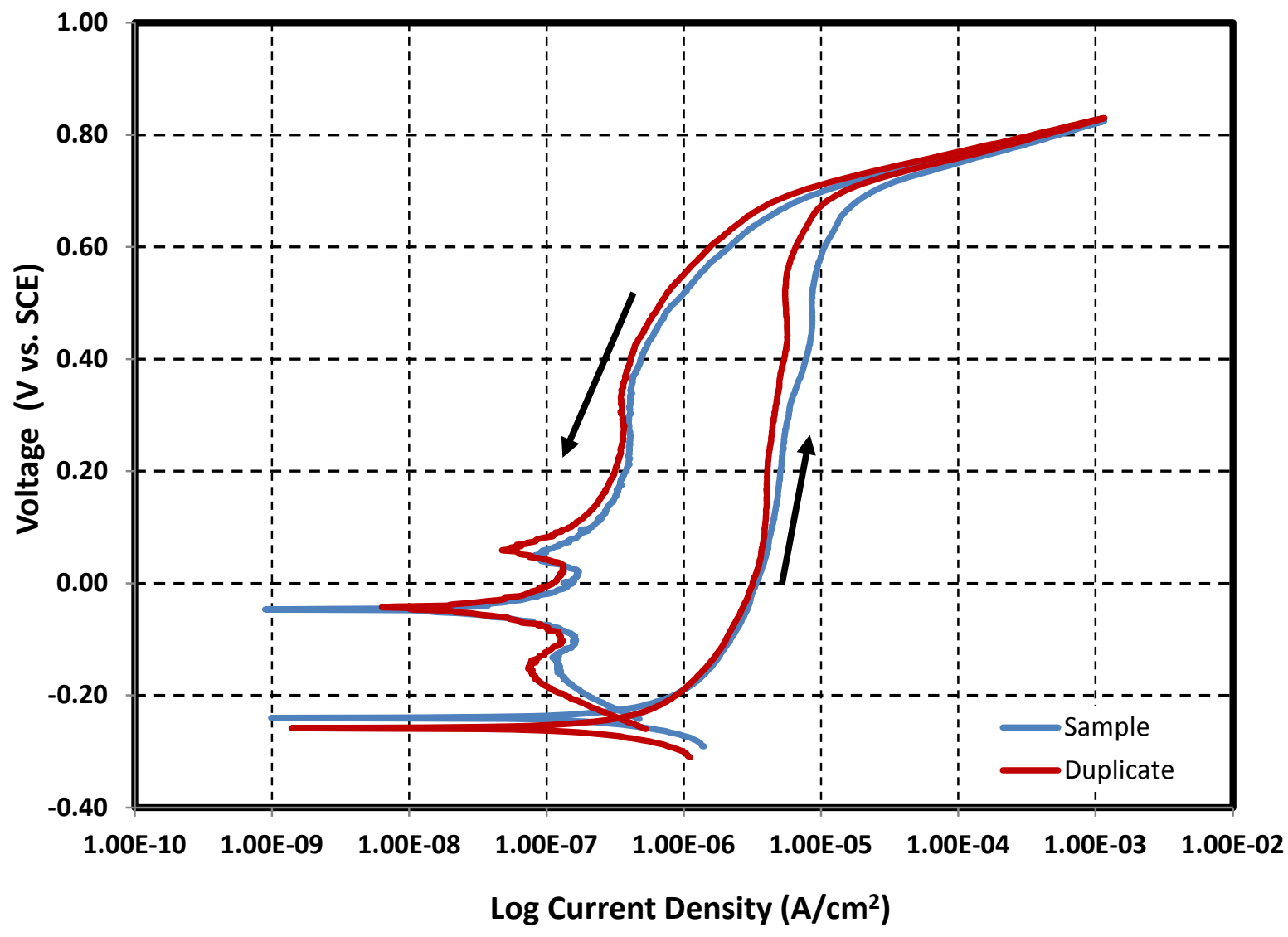

Images of bullet samples after electrochemical tests

Test 32

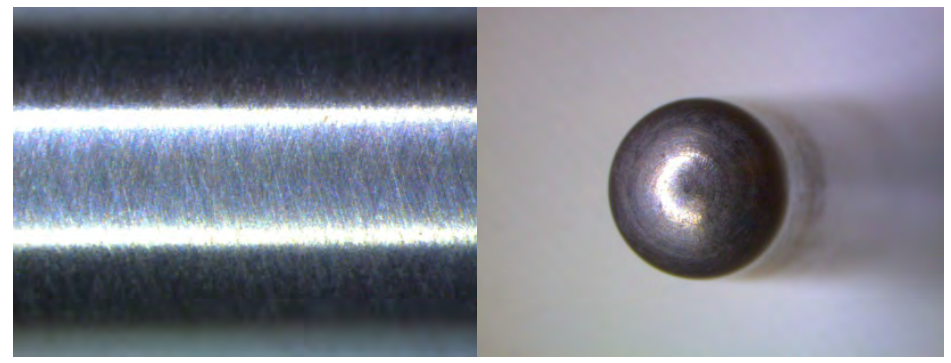

Test 32D

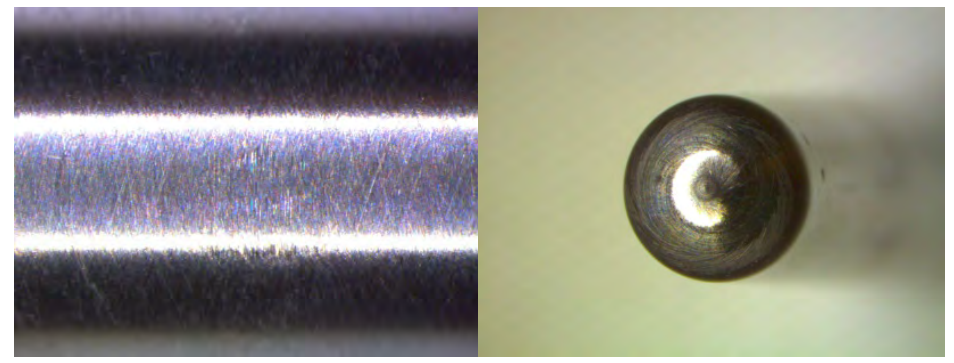

Shank (20X)

Nose (10X) 


\section{Composition of simulant for pitting corrosion -Test 33}

Reference 14

Test 33
Temperature

$\mathrm{pH}$

Volume

\begin{tabular}{|c|c|c|c|c|}
\hline Simulant Source & Formula & $\begin{array}{c}\text { Molecular } \\
\text { Weight } \\
\text { (g/mol) }\end{array}$ & $\begin{array}{c}\text { Concentration } \\
\text { (M) }\end{array}$ & $\begin{array}{c}\text { weight } \\
\text { required (g) }\end{array}$ \\
\hline Sodium nitrate & $\mathrm{NaNO}_{3}$ & 84.99 & 0.8 & 95.1888 \\
\hline Sodium nitrite & $\mathrm{NaNO}_{2}$ & 69.00 & 0.2 & 19.3200 \\
\hline Sodium chloride & $\mathrm{NaCl}$ & 58.44 & 0.0046 & 0.3764 \\
\hline Sodium sulfate & $\mathrm{Na}_{2} \mathrm{SO}_{4}$ & 142.04 & 0.0635 & 12.6274 \\
\hline Sodium carbonate & $\mathrm{Na}_{2}\left(\mathrm{CO}_{3}\right)$ & 106.00 & 0.0702 & 10.4177 \\
\hline Sodium bicarbonate & $\mathrm{NaHCO}_{3}$ & 84.00 & 0.13 & 15.2880 \\
\hline
\end{tabular}




\section{Cyclic Potentiodynamic Polarization}

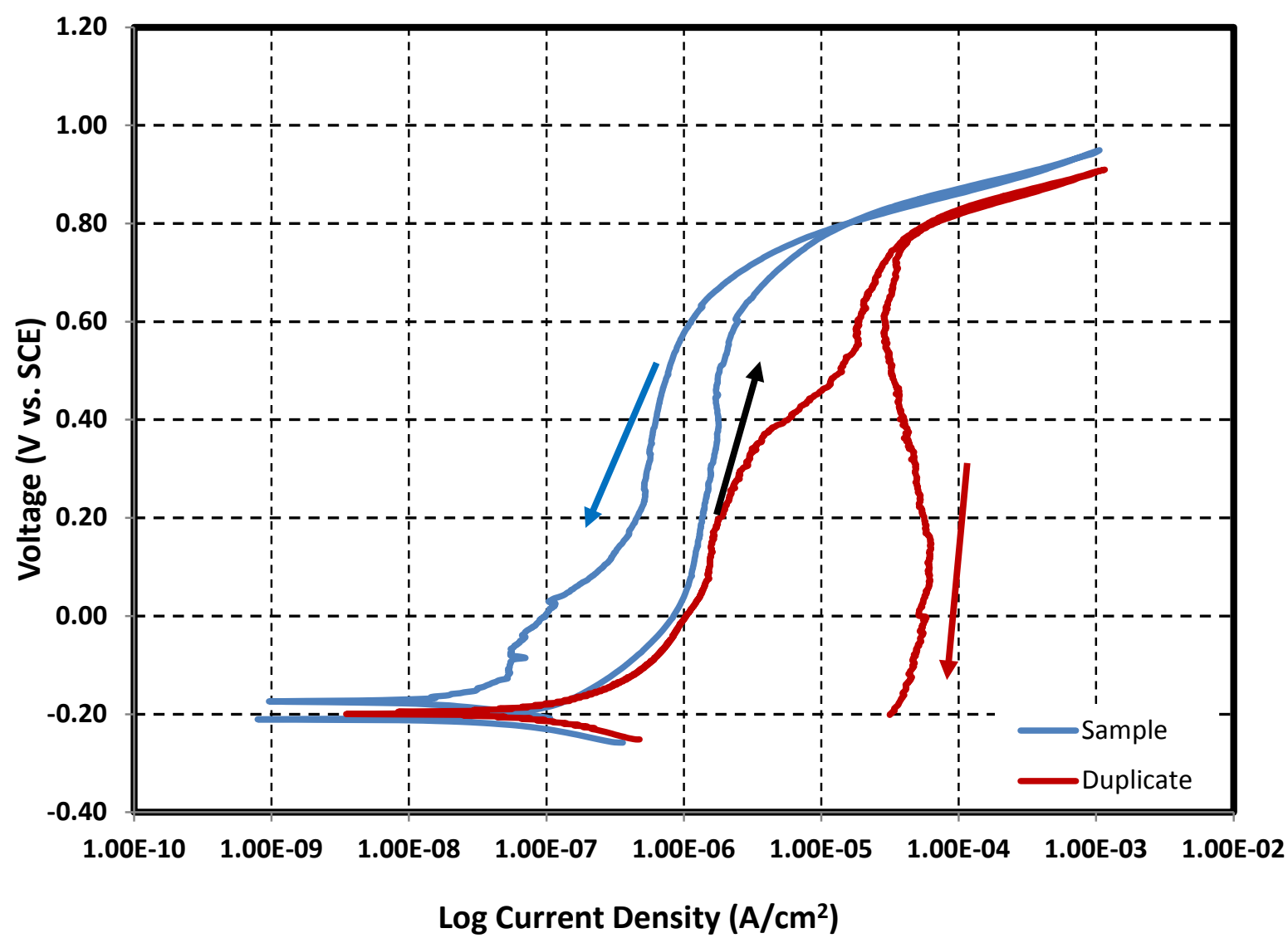

Images of bullet samples after electrochemical tests

Test 33

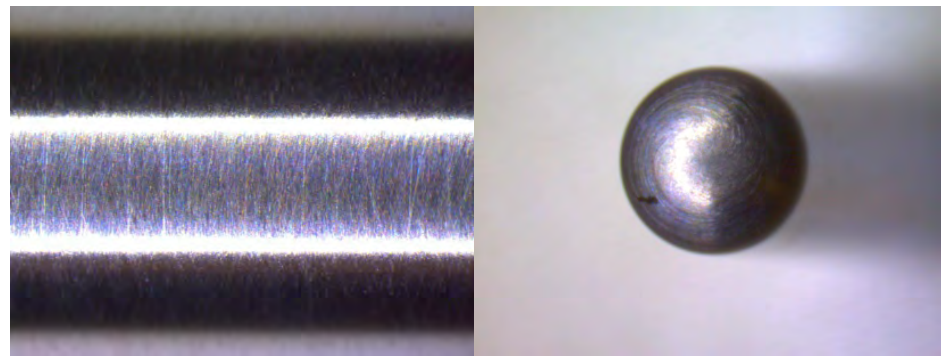

Test 33D

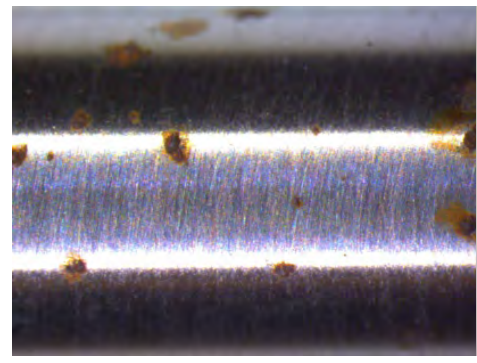

Shank (20X)

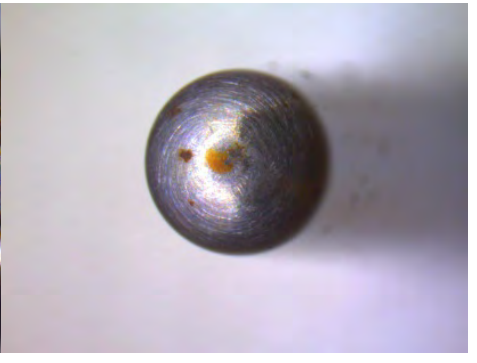

Nose (10X) 


\section{Composition of simulant for pitting corrosion -Test 34}

Reference 19

Test 34
Temperature

$\mathrm{pH}$

Volume
$77{ }^{\circ} \mathrm{C}$

11.00

$1.4 \quad \mathrm{~L}$

\begin{tabular}{|c|c|c|c|c|}
\hline Simulant Source & Formula & $\begin{array}{c}\text { Molecular } \\
\text { Weight } \\
\text { (g/mol) }\end{array}$ & $\begin{array}{c}\text { Concentration } \\
\text { (M) }\end{array}$ & $\begin{array}{c}\text { weight } \\
\text { required (g) }\end{array}$ \\
\hline Sodium nitrate & $\mathrm{NaNO}_{3}$ & 84.99 & 0.002 & 0.2380 \\
\hline Sodium chloride & $\mathrm{NaCl}$ & 58.44 & 0.004 & 0.3273 \\
\hline Sodium fluoride & $\mathrm{NaF}$ & 42.00 & 0.003 & 0.1764 \\
\hline Sodium nitrite & $\mathrm{NaNO}$ & 0.001 & 0.0966 \\
\hline Sodium hydroxide & $\mathrm{NaOH}$ & 40.00 & 0 & 0.0000 \\
\hline Sodium carbonate & $\mathrm{Na}_{2}\left(\mathrm{CO}_{3}\right)$ & 106.00 & 0 & 0.0000 \\
\hline Sodium sulfate & $\mathrm{Na}_{2} \mathrm{SO}_{4}$ & 142.04 & 0.018 & 3.5794 \\
\hline Sodium phosphate, 12- & $\mathrm{Na}_{3} \mathrm{PO}_{4 .} 12 \mathrm{H}_{2} \mathrm{O}$ & 380.00 & 0.012 & 6.3840 \\
\hline hydrate & $\mathrm{NaAlO}_{2}$ & 81.97 & 0.002 & 0.2295 \\
\hline Sodium aluminate & $\mathrm{Na}_{2} \mathrm{C}_{2} \mathrm{O}_{4}$ & 134.00 & 0.033 & 6.1908 \\
\hline Sodium oxalate & $\left.\mathrm{Na}_{2} \mathrm{C}_{2} \mathrm{H}_{3} \mathrm{O}_{2}\right) \cdot 3 \mathrm{H}_{2}$ & 136.0000 & 0.034 & 6.4736 \\
\hline Sodium acetate, 3-hydrate & & & &
\end{tabular}




\section{Cyclic Potentiodynamic Polarization}

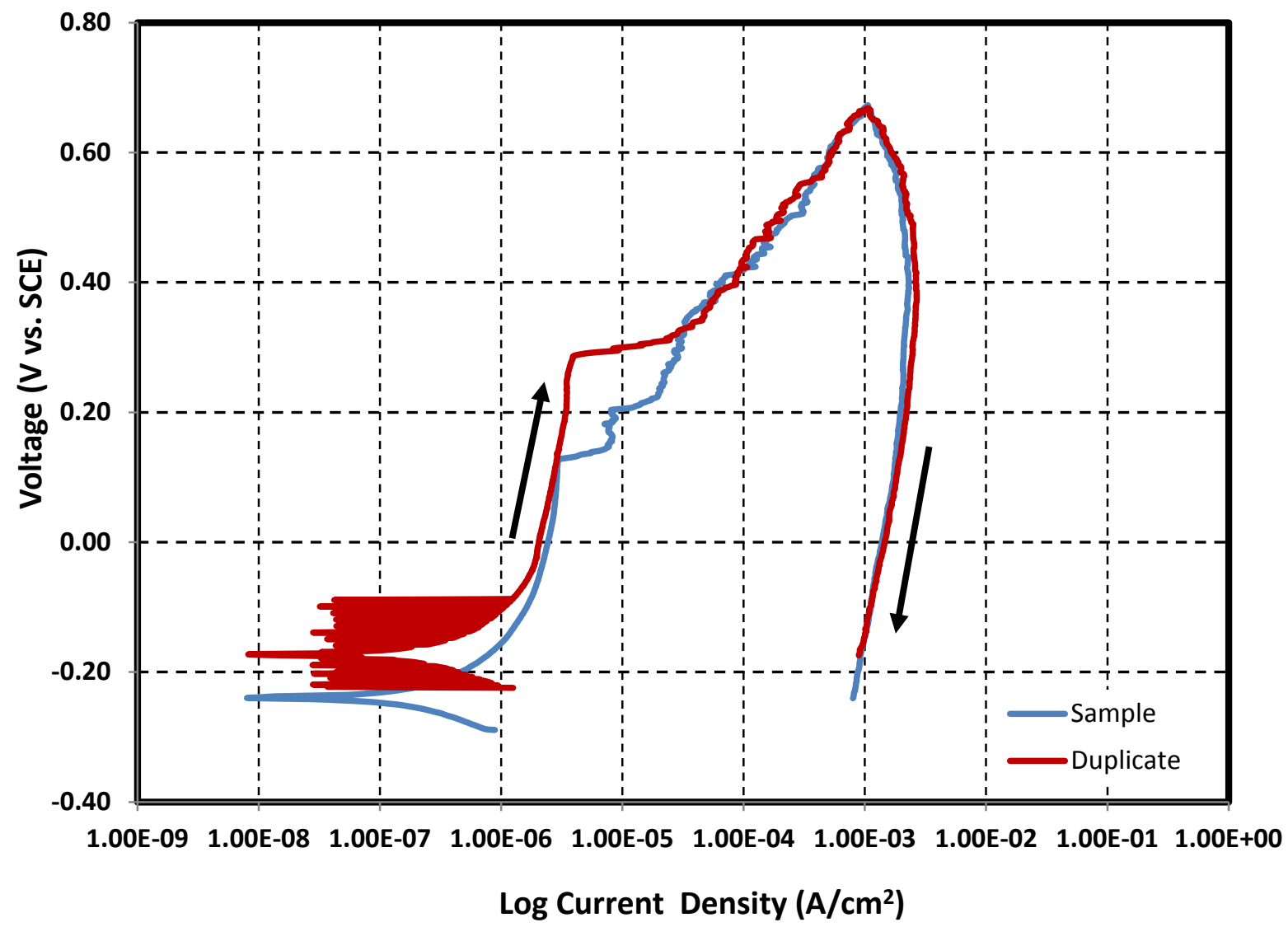

Images of bullet samples after electrochemical tests

Test 34

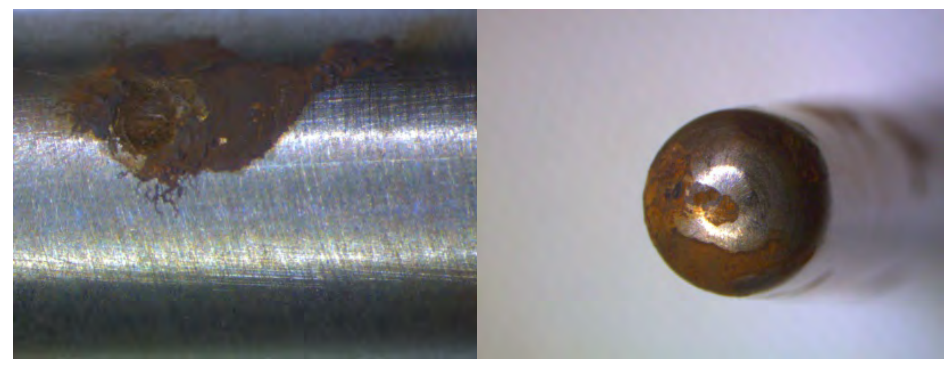

Test 34D

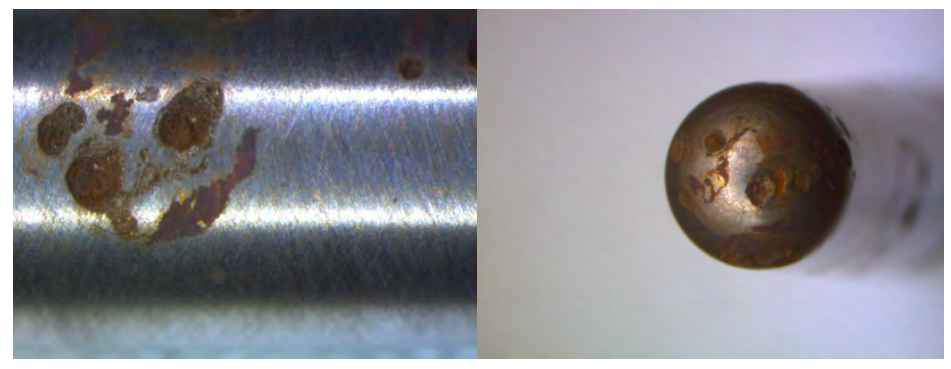

Shank $(20 X)$

Nose (10X) 


\section{Composition of simulant for pitting corrosion -Test 35}

Reference 14

Test 35
Temperature

$\mathrm{pH}$

Volume
$40{ }^{\circ} \mathrm{C}$

10.00

$1.4 \quad \mathrm{~L}$

\begin{tabular}{|c|c|c|c|c|}
\hline Simulant Source & Formula & $\begin{array}{c}\text { Molecular } \\
\text { Weight } \\
\text { (g/mol) }\end{array}$ & $\begin{array}{c}\text { Concentration } \\
\text { (M) }\end{array}$ & $\begin{array}{c}\text { weight required } \\
\text { (g) }\end{array}$ \\
\hline Sodium nitrate & $\mathrm{NaNO}_{3}$ & 84.99 & 0.2 & 23.7972 \\
\hline Sodium nitrite & $\mathrm{NaNO}_{2}$ & 69.00 & 0.1 & 9.6600 \\
\hline Sodium chloride & $\mathrm{NaCl}$ & 58.44 & 0.0028 & 0.2291 \\
\hline Sodium sulfate & $\mathrm{Na}_{2} \mathrm{SO}_{4}$ & 142.04 & 0.0278 & 5.5282 \\
\hline Sodium carbonate & $\mathrm{Na}_{2}\left(\mathrm{CO}_{3}\right)$ & 106.00 & 0.0351 & 5.2088 \\
\hline Sodium bicarbonate & $\mathrm{NaHCO}_{3}$ & 84.00 & 0.065 & 7.6440 \\
\hline
\end{tabular}




\section{Cyclic Potentiodynamic Polarization}

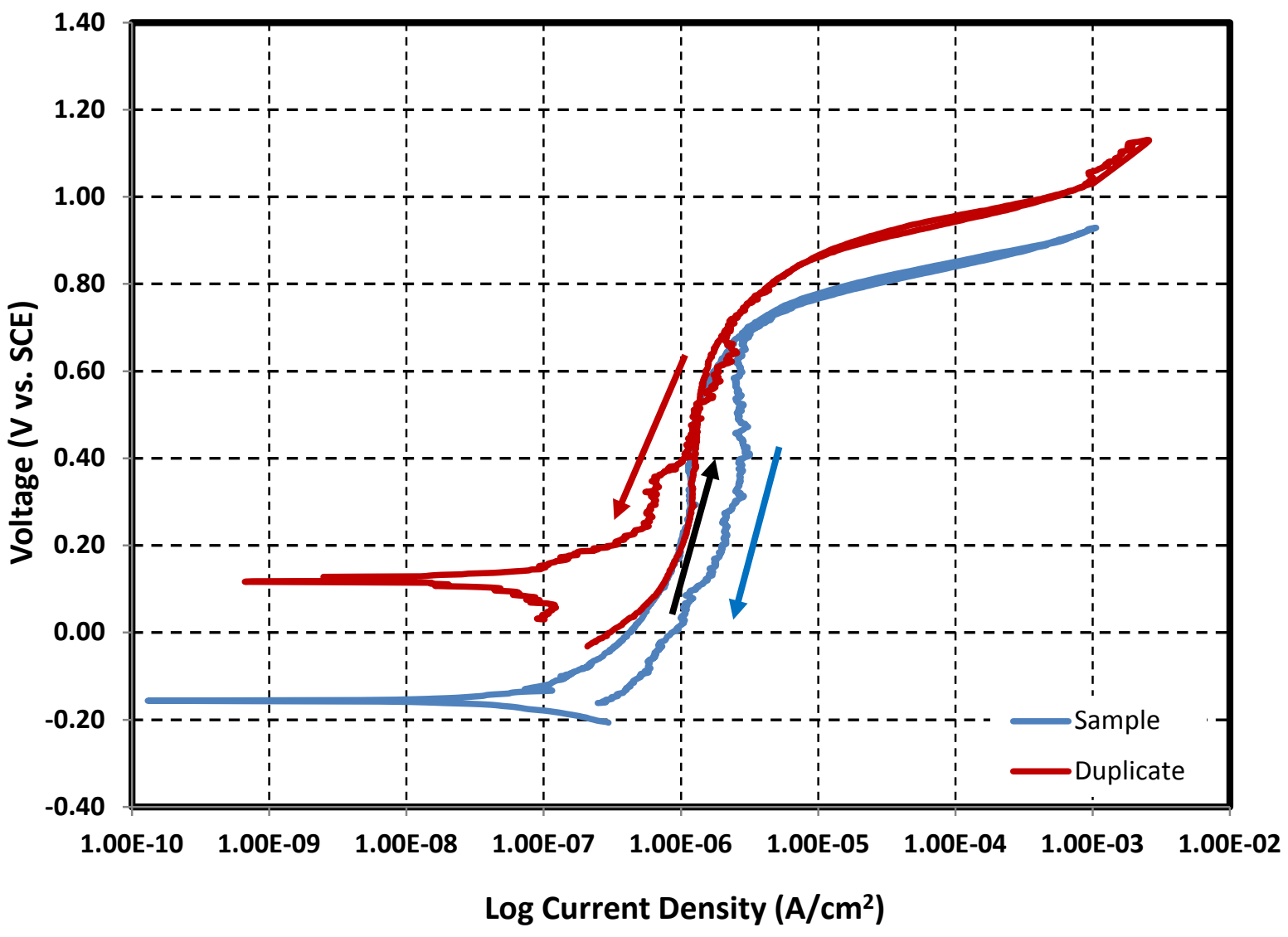

Images of bullet samples after electrochemical tests

Test 35

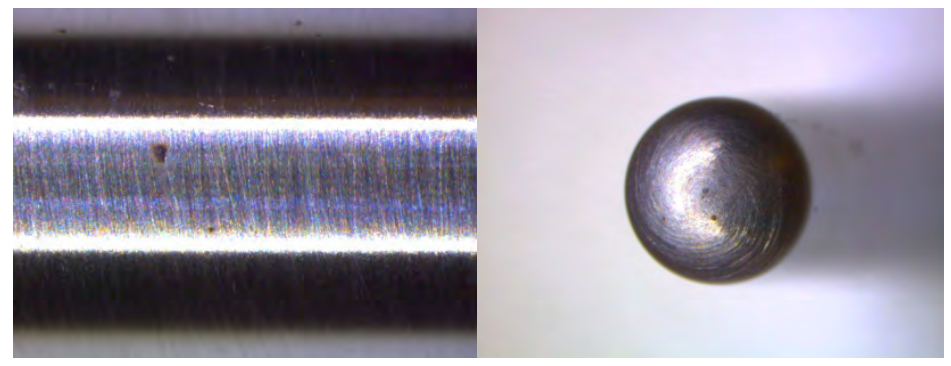

Test 35D

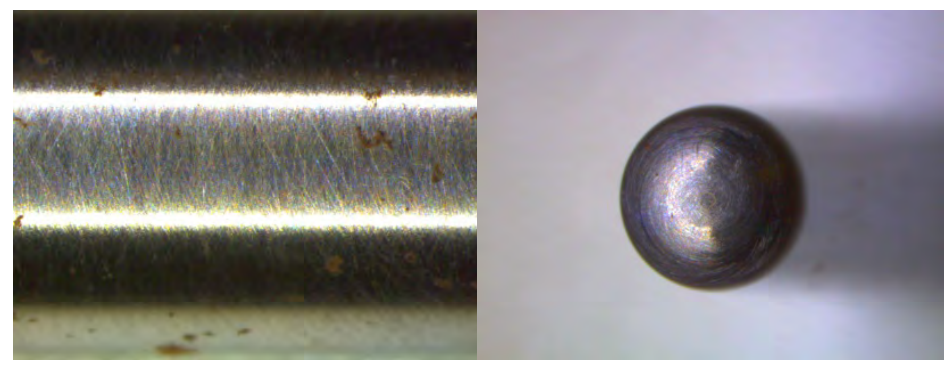

Shank $(20 X)$

Nose (10X) 


\section{Composition of simulant for pitting corrosion -Test 36}

Reference 15

Test 36
Temperature

$\mathrm{pH}$

Volume
$40{ }^{\circ} \mathrm{C}$

10.00

$1.4 \mathrm{~L}$

\begin{tabular}{|c|c|c|c|c|}
\hline Simulant Source & Formula & $\begin{array}{c}\text { Molecular } \\
\text { Weight } \\
\text { (g/mol) }\end{array}$ & $\begin{array}{c}\text { Concentration } \\
\text { (M) }\end{array}$ & $\begin{array}{c}\text { weight } \\
\text { required (g) }\end{array}$ \\
\hline Sodium nitrate & $\mathrm{NaNO}_{3}$ & 84.99 & 0.8 & 95.1888 \\
\hline Sodium nitrite & $\mathrm{NaNO}_{2}$ & 69.00 & 0.5 & 48.3000 \\
\hline Sodium chloride & $\mathrm{NaCl}$ & 58.44 & 0.032 & 2.6181 \\
\hline Sodium sulfate & $\mathrm{Na}_{2} \mathrm{SO}_{4}$ & 142.04 & 0.121 & 24.0616 \\
\hline Sodium carbonate & $\mathrm{Na}_{2}\left(\mathrm{CO}_{3}\right)$ & 106.00 & 0.1754 & 26.0294 \\
\hline Sodium bicarbonate & $\mathrm{NaHCO}_{3}$ & 84.00 & 0.326 & 38.3376 \\
\hline
\end{tabular}




\section{Cyclic Potentiodynamic Polarization}

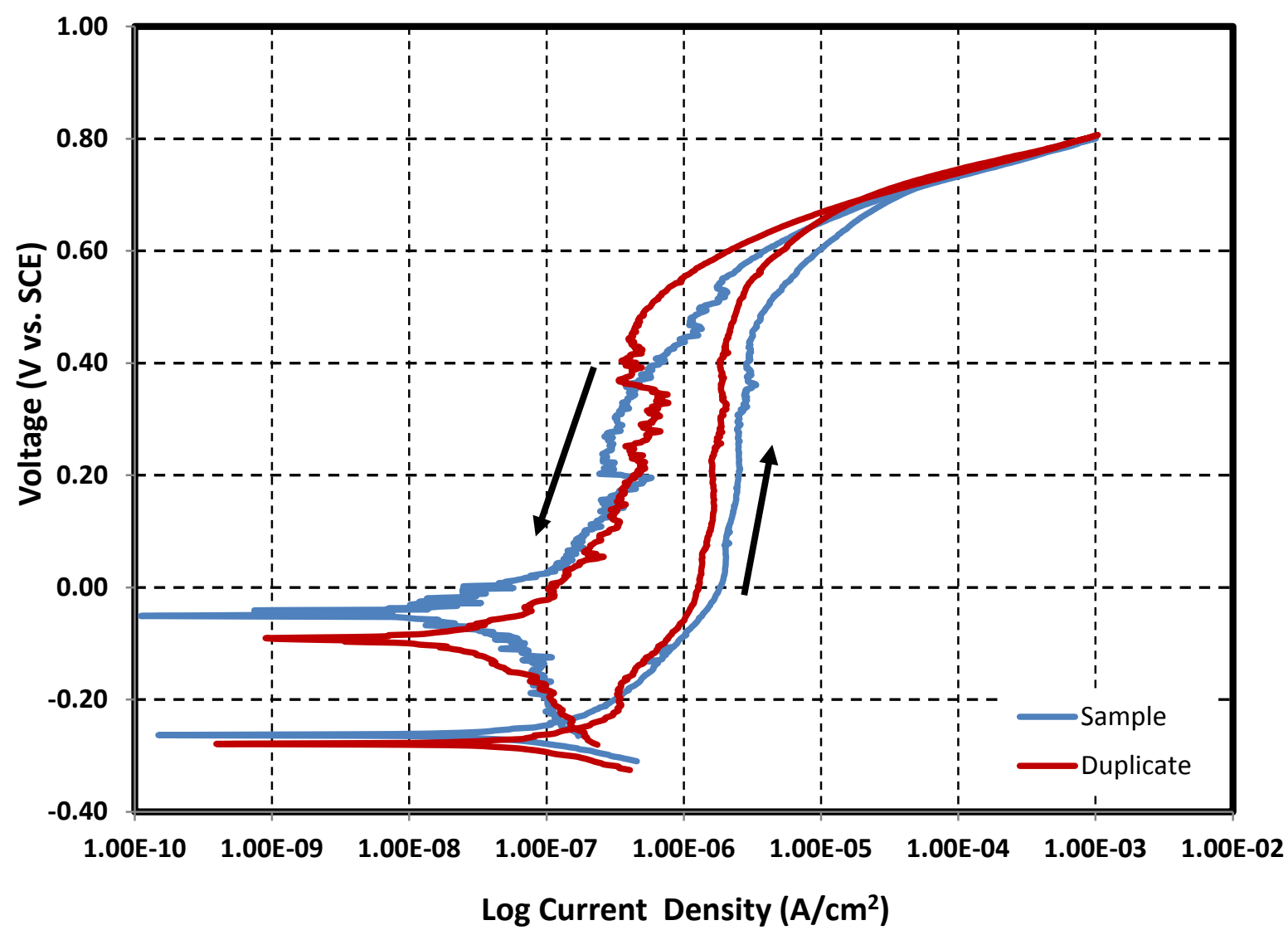

Images of bullet samples after electrochemical tests

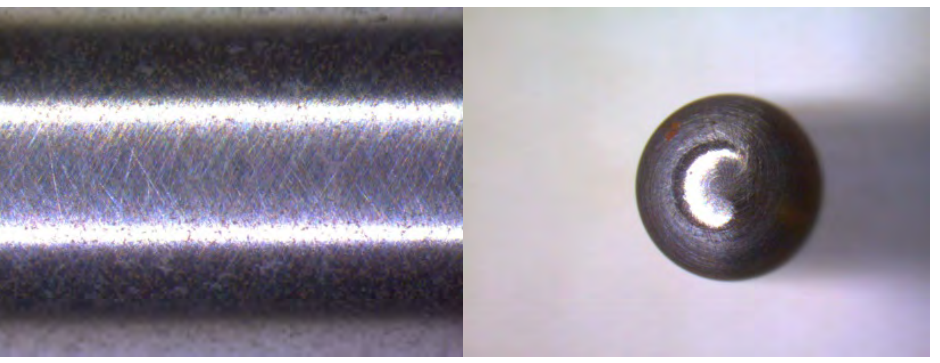

Test 36D

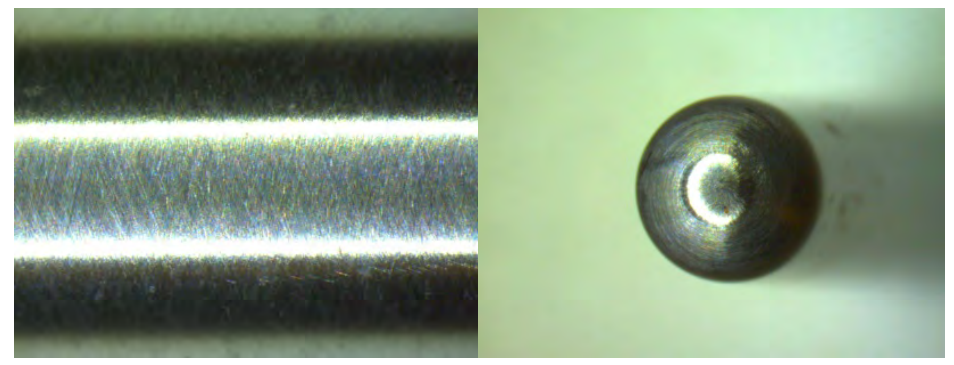

Shank (20X)

Nose (10X) 


\section{Composition of simulant for pitting corrosion -Test 37}

\section{Reference 3}

Test 37

\author{
Temperature \\ $\mathrm{pH}$
}

Volume

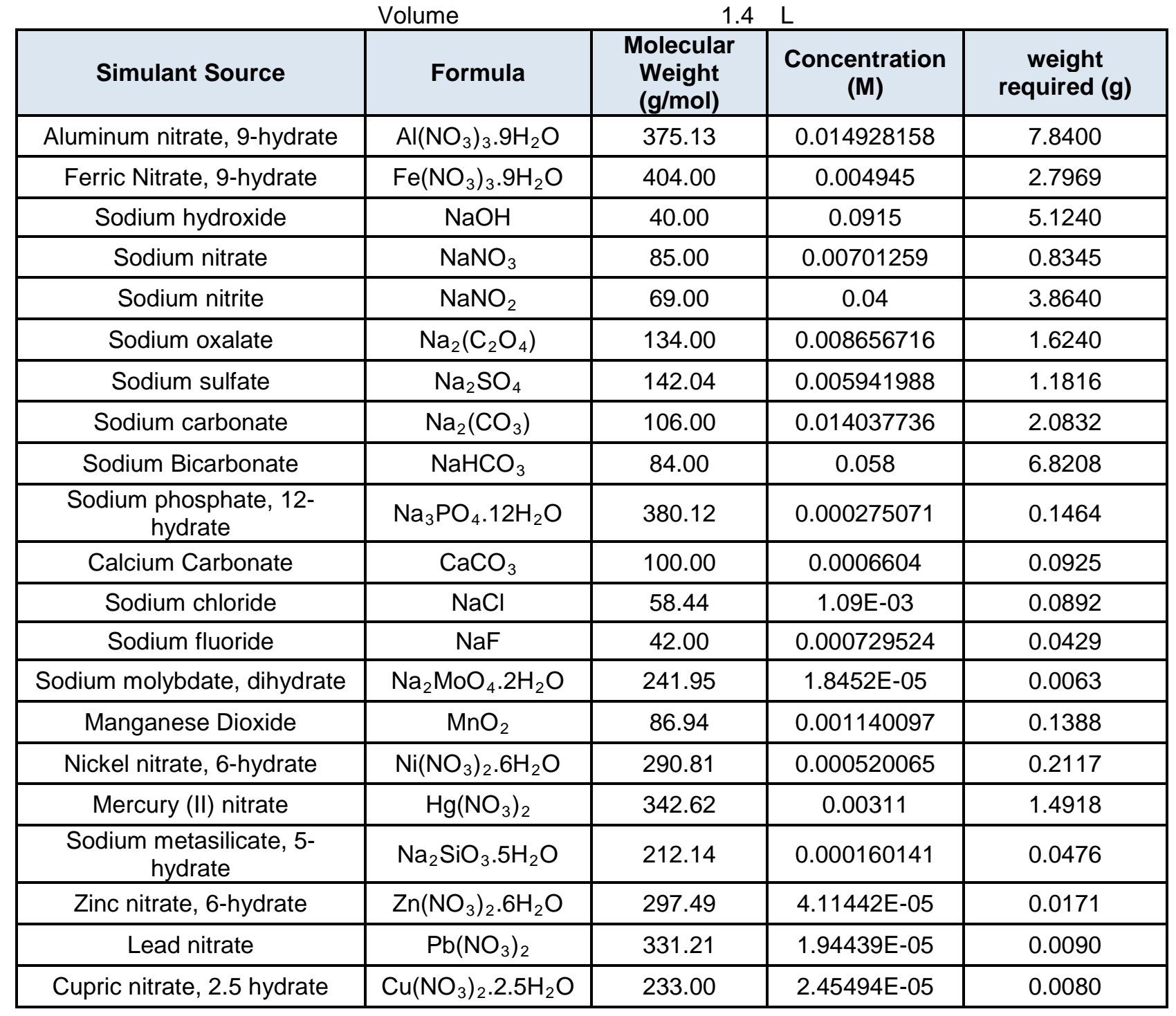




\section{Cyclic Potentiodynamic Polarization}

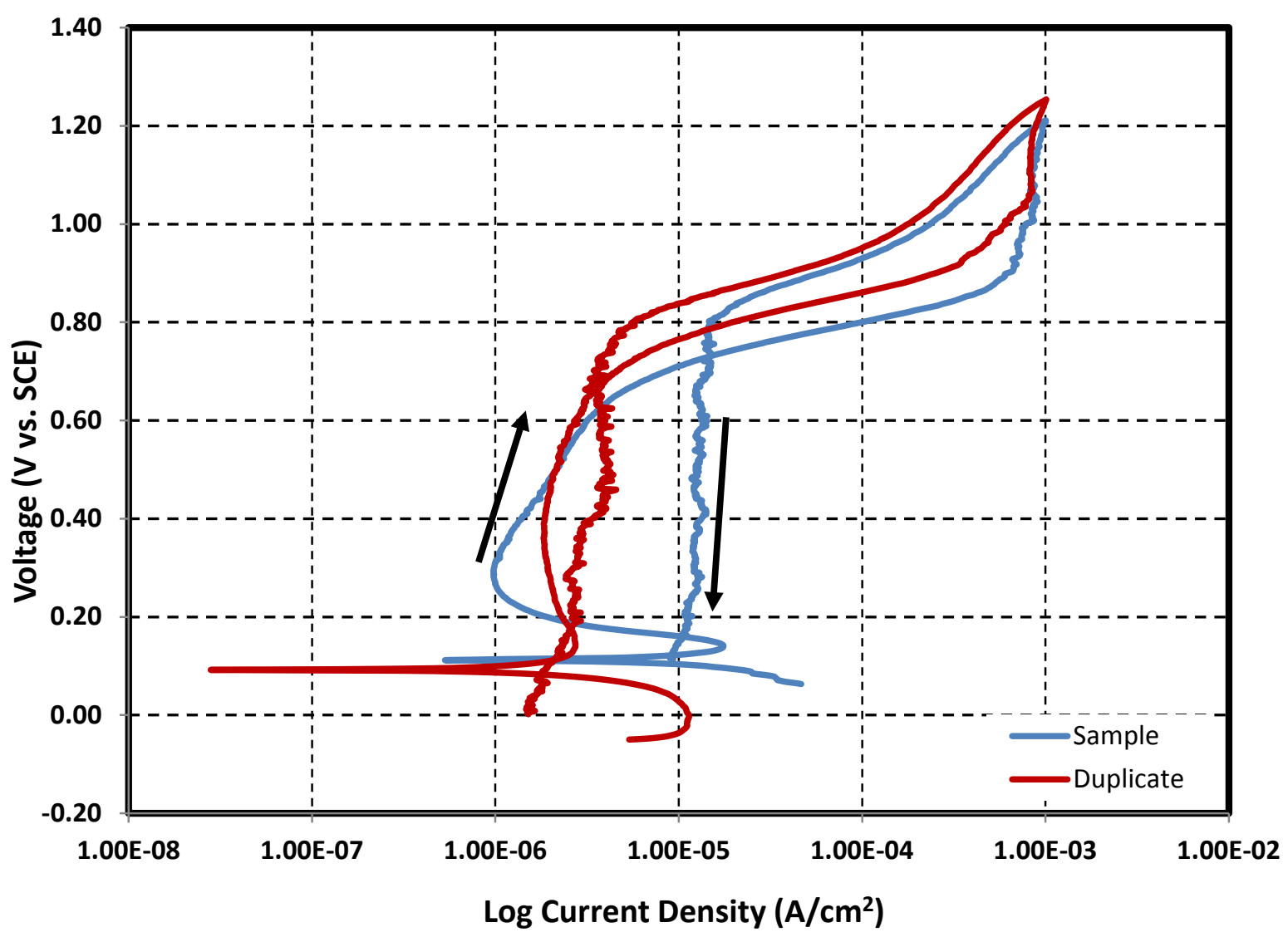

Images of bullet samples after electrochemical tests

Test 37

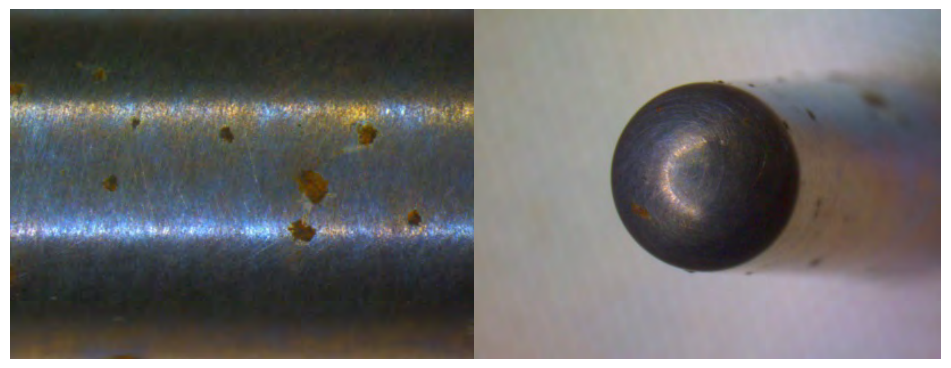

Test 37D

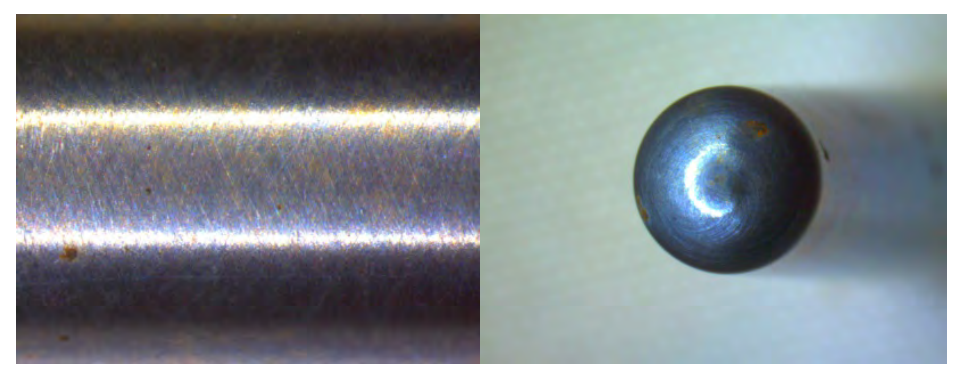

Shank $(20 X)$

Nose (10X) 


\section{Composition of simulant for pitting corrosion -Test 38}

Reference 19

Test 38
Temperature

$\mathrm{pH}$

Volume
$77{ }^{\circ} \mathrm{C}$

11.00

$1.4 \mathrm{~L}$

\begin{tabular}{|c|c|c|c|c|}
\hline Simulant Source & Formula & $\begin{array}{c}\text { Molecular } \\
\text { Weight } \\
\text { (g/mol) }\end{array}$ & $\begin{array}{c}\text { Concentration } \\
\text { (M) }\end{array}$ & $\begin{array}{c}\text { weight } \\
\text { required (g) }\end{array}$ \\
\hline Sodium nitrate & $\mathrm{NaNO}_{3}$ & 84.99 & 0.002 & 0.2380 \\
\hline Sodium chloride & $\mathrm{NaCl}$ & 58.44 & 0.004 & 0.3273 \\
\hline Sodium fluoride & $\mathrm{NaF}$ & 42.00 & 0.003 & 0.1764 \\
\hline Sodium nitrite & $\mathrm{NaNO}$ & 69.00 & 0 & 0.0000 \\
\hline Sodium hydroxide & $\mathrm{NaOH}$ & 40.00 & 0 & 0.0000 \\
\hline Sodium carbonate & $\mathrm{Na}_{2}\left(\mathrm{CO}_{3}\right)$ & 106.00 & 1.028 & 152.5552 \\
\hline Sodium sulfate & $\mathrm{Na}_{2} \mathrm{SO}_{4}$ & 142.04 & 0.018 & 3.5794 \\
\hline Sodium phosphate, 12- & $\mathrm{Na}_{3} \mathrm{PO}_{4} 1_{2} \mathrm{H}_{2} \mathrm{O}$ & 380.00 & 0.012 & 6.3840 \\
\hline hydrate & $\mathrm{NaAlO}_{2}$ & 81.97 & 0.002 & 0.2295 \\
\hline Sodium aluminate & $\mathrm{Na}_{2} \mathrm{C}_{2} \mathrm{O}_{4}$ & 134.00 & 0.033 & 6.1908 \\
\hline Sodium oxalate & $\left.\mathrm{Na}_{2} \mathrm{C}_{2} \mathrm{H}_{3} \mathrm{O}_{2}\right) \cdot 3 \mathrm{H}_{2}$ & 136.0000 & 0.034 & 6.4736 \\
\hline Sodium acetate, 3-hydrate & \multicolumn{2}{|l}{} \\
\hline
\end{tabular}


Cyclic Potentiodynamic Polarization-C

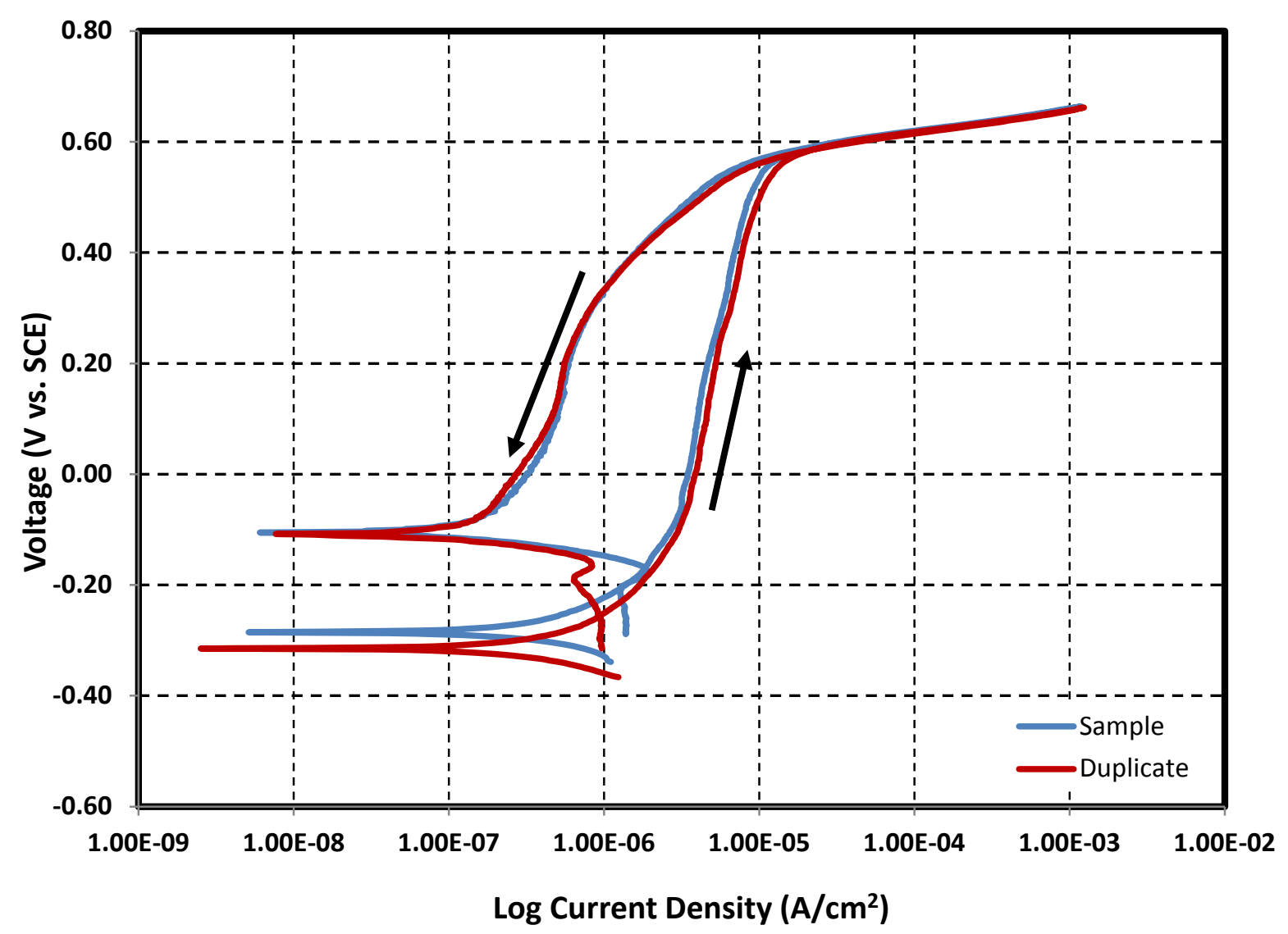

Images of bullet samples after electrochemical tests

Test 38
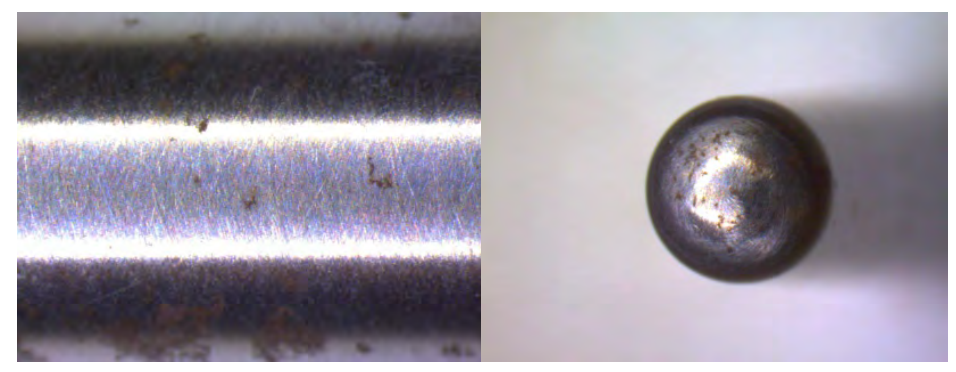

(Pits we not found after the test and they may have developed over time)

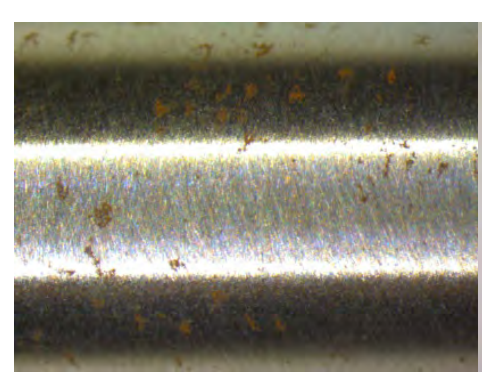

Shank $(20 X)$

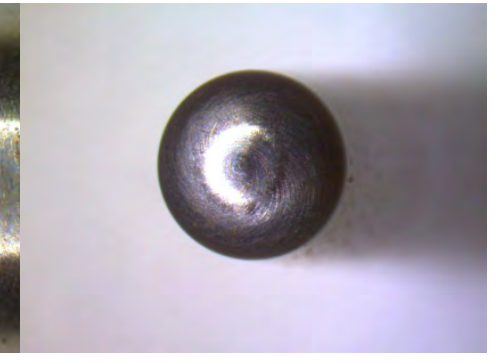

Nose (10X) 


\section{Composition of simulant for pitting corrosion -Test 39}

\begin{tabular}{|c|c|c|c|c|}
\hline Reference 3 & Temperature & 40 & ${ }^{\circ} \mathrm{C}$ & \\
\hline Test 39 & $\mathrm{pH}$ & 9.6 & & \\
\hline & Volume & 1.4 & $\underline{L}$ & \\
\hline Simulant Source & Formula & $\begin{array}{l}\text { Molecular } \\
\text { Weight } \\
\text { (g/mol) } \\
\end{array}$ & $\begin{array}{l}\text { Concentration } \\
\text { (M) }\end{array}$ & $\begin{array}{l}\text { weight } \\
\text { required }(g)\end{array}$ \\
\hline Aluminum nitrate, 9-hydrate & $\mathrm{Al}\left(\mathrm{NO}_{3}\right)_{3} \cdot 9 \mathrm{H}_{2} \mathrm{O}$ & 375.13 & 0.014928158 & 7.8400 \\
\hline Ferric Nitrate, 9-hydrate & $\mathrm{Fe}\left(\mathrm{NO}_{3}\right)_{3} .9 \mathrm{H}_{2} \mathrm{O}$ & 404.00 & 0.004945 & 2.7969 \\
\hline Sodium hydroxide & $\mathrm{NaOH}$ & 40.00 & 0.0915 & 5.1240 \\
\hline Sodium nitrate & $\mathrm{NaNO}_{3}$ & 85.00 & 0.00701259 & 0.8345 \\
\hline Sodium nitrite & $\mathrm{NaNO}_{2}$ & 69.00 & 0.06 & 5.7960 \\
\hline Sodium oxalate & $\mathrm{Na}_{2}\left(\mathrm{C}_{2} \mathrm{O}_{4}\right)$ & 134.00 & 0.008656716 & 1.6240 \\
\hline Sodium sulfate & $\mathrm{Na}_{2} \mathrm{SO}_{4}$ & 142.04 & 0.005941988 & 1.1816 \\
\hline Sodium carbonate & $\mathrm{Na}_{2}\left(\mathrm{CO}_{3}\right)$ & 106.00 & 0.014037736 & 2.0832 \\
\hline Sodium Bicarbonate & $\mathrm{NaHCO}_{3}$ & 84.00 & 0.058 & 6.8208 \\
\hline $\begin{array}{c}\text { Sodium phosphate, 12- } \\
\text { hydrate }\end{array}$ & $\mathrm{Na}_{3} \mathrm{PO}_{4} \cdot 12 \mathrm{H}_{2} \mathrm{O}$ & 380.12 & 0.000275071 & 0.1464 \\
\hline Calcium Carbonate & $\mathrm{CaCO}_{3}$ & 100.00 & 0.0006604 & 0.0925 \\
\hline Sodium chloride & $\mathrm{NaCl}$ & 58.44 & 0.001090008 & 0.0892 \\
\hline Sodium fluoride & $\mathrm{NaF}$ & 42.00 & 0.000729524 & 0.0429 \\
\hline Sodium molybdate, dihydrate & $\mathrm{Na}_{2} \mathrm{MoO}_{4} \cdot 2 \mathrm{H}_{2} \mathrm{O}$ & 241.95 & 1.8452E-05 & 0.0063 \\
\hline Manganese Dioxide & $\mathrm{MnO}_{2}$ & 86.94 & 0.001140097 & 0.1388 \\
\hline Nickel nitrate, 6-hydrate & $\mathrm{Ni}\left(\mathrm{NO}_{3}\right)_{2} \cdot 6 \mathrm{H}_{2} \mathrm{O}$ & 290.81 & 0.000520065 & 0.2117 \\
\hline Mercury (II) nitrate & $\mathrm{Hg}\left(\mathrm{NO}_{3}\right)_{2}$ & 342.62 & 0.00311 & 1.4918 \\
\hline $\begin{array}{l}\text { Sodium metasilicate, 5- } \\
\text { hydrate }\end{array}$ & $\mathrm{Na}_{2} \mathrm{SiO}_{3} .5 \mathrm{H}_{2} \mathrm{O}$ & 212.14 & 0.000160141 & 0.0476 \\
\hline Zinc nitrate, 6-hydrate & $\mathrm{Zn}\left(\mathrm{NO}_{3}\right)_{2} \cdot 6 \mathrm{H}_{2} \mathrm{O}$ & 297.49 & 4.11442E-05 & 0.0171 \\
\hline Lead nitrate & $\mathrm{Pb}\left(\mathrm{NO}_{3}\right)_{2}$ & 331.21 & 1.94439E-05 & 0.0090 \\
\hline Cupric nitrate, 2.5 hydrate & $\mathrm{Cu}\left(\mathrm{NO}_{3}\right)_{2} \cdot 2.5 \mathrm{H}_{2} \mathrm{O}$ & 233.00 & 2.45494E-05 & 0.0080 \\
\hline
\end{tabular}




\section{Cyclic Potentiodynamic Polarization}

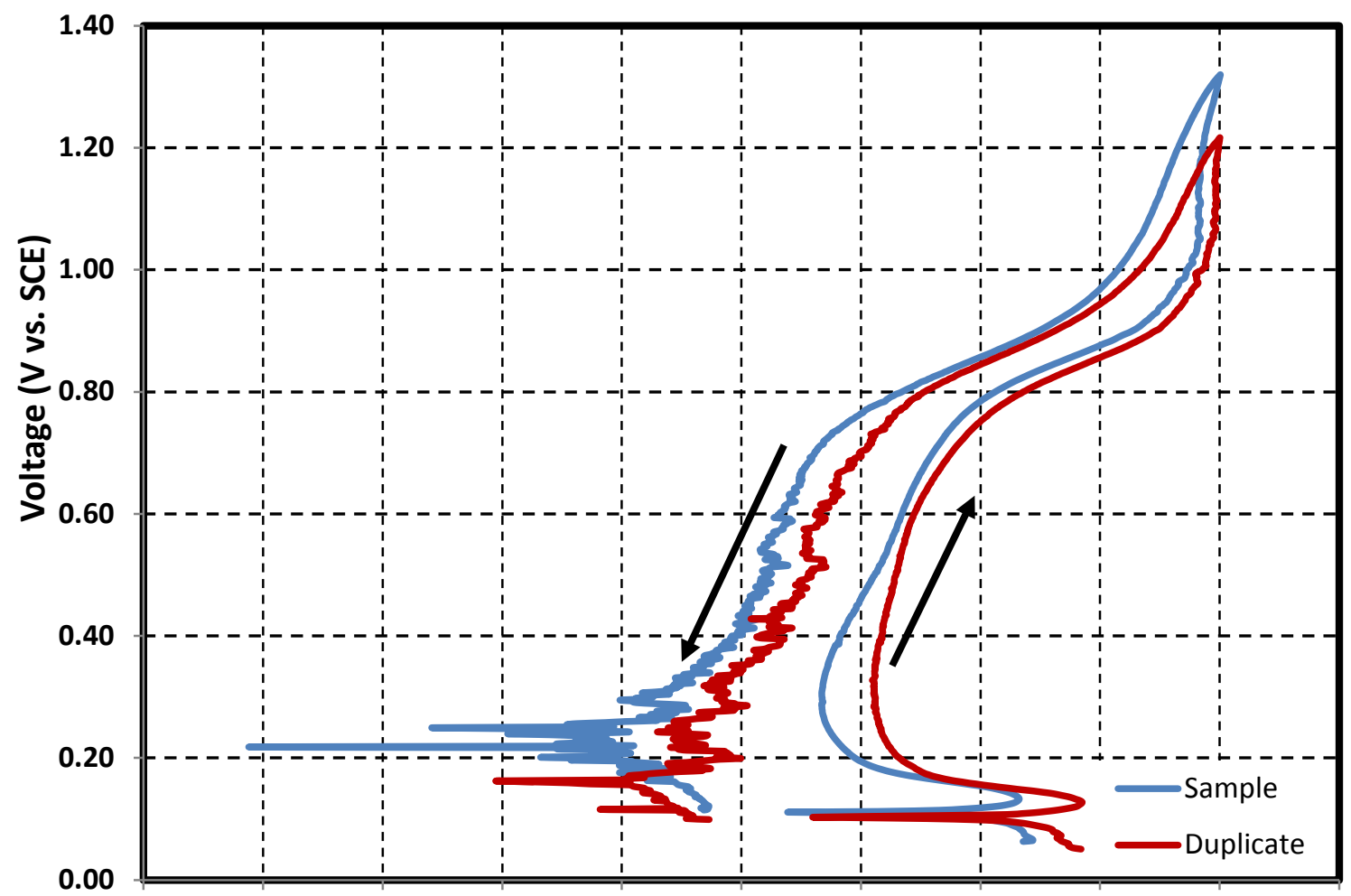

1.00E-12 1.00E-11 1.00E-10 1.00E-09 1.00E-08 1.00E-07 1.00E-06 1.00E-05 1.00E-04 1.00E-03 1.00E-02 Log Current Density $\left(\mathrm{A} / \mathrm{cm}^{2}\right)$

Images of bullet samples after electrochemical tests

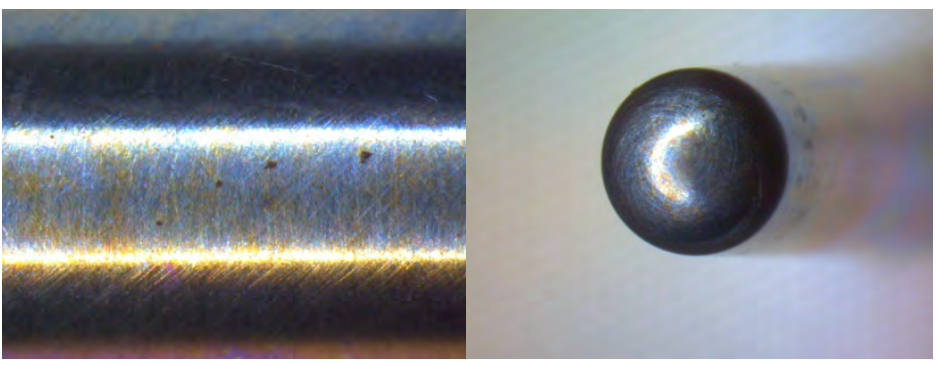

Test 39D

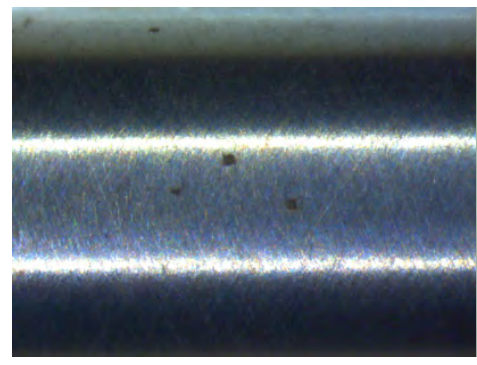

Shank (20X)

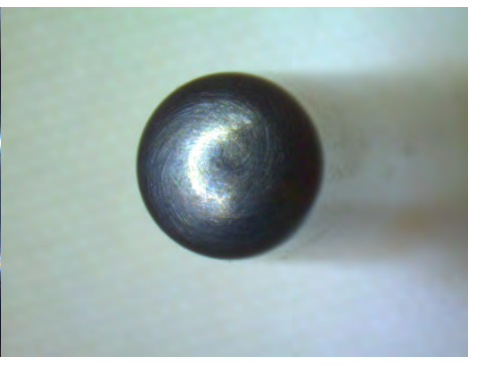

Nose (10X) 


\section{Composition of simulant for pitting corrosion -Test 40}

\begin{tabular}{|c|c|c|c|c|}
\hline \multirow{3}{*}{$\begin{array}{l}\text { Reference } 4 \\
\text { Test } 40\end{array}$} & \multicolumn{2}{|l|}{ Temperature } & \multicolumn{2}{|l|}{${ }^{\circ} \mathrm{C}$} \\
\hline & $\mathrm{pH}$ & 9.6 & & \\
\hline & Volume & 1.4 & $\mathrm{~L}$ & \\
\hline Simulant Source & Formula & $\begin{array}{c}\text { Molecular } \\
\text { Weight } \\
\text { (g/mol) }\end{array}$ & $\begin{array}{c}\text { Concentration } \\
\text { (M) }\end{array}$ & $\begin{array}{l}\text { weight } \\
\text { required (g) }\end{array}$ \\
\hline Aluminum nitrate, 9-hydrate & $\mathrm{Al}\left(\mathrm{NO}_{3}\right)_{3} \cdot 9 \mathrm{H}_{2} \mathrm{O}$ & 375.13 & 0.006842073 & 3.5933 \\
\hline Ferric Nitrate, 9-hydrate & $\mathrm{Fe}\left(\mathrm{NO}_{3}\right)_{3} .9 \mathrm{H}_{2} \mathrm{O}$ & 404.00 & 0.001831683 & 1.0360 \\
\hline Sodium hydroxide & $\mathrm{NaOH}$ & 40.00 & 0.0305 & 1.7080 \\
\hline Sodium nitrate & $\mathrm{NaNO}_{3}$ & 85.00 & 0.001694317 & 0.2016 \\
\hline Sodium nitrite & $\mathrm{NaNO}_{2}$ & 69.00 & 0.020057971 & 1.9376 \\
\hline Sodium oxalate & $\mathrm{Na}_{2}\left(\mathrm{C}_{2} \mathrm{O}_{4}\right)$ & 134.00 & 0.002885572 & 0.5413 \\
\hline Sodium sulfate & $\mathrm{Na}_{2} \mathrm{SO}_{4}$ & 142.04 & 0.030019713 & 5.9696 \\
\hline Sodium carbonate & $\mathrm{Na}_{2}\left(\mathrm{CO}_{3}\right)$ & 106.00 & 0.004679245 & 0.6944 \\
\hline Sodium Bicarbonate & $\mathrm{NaHCO}_{3}$ & 84.00 & 0.019333333 & 2.2736 \\
\hline $\begin{array}{c}\text { Sodium phosphate, } 12- \\
\text { hydrate }\end{array}$ & $\mathrm{Na}_{3} \mathrm{PO}_{4} \cdot 12 \mathrm{H}_{2} \mathrm{O}$ & 380.12 & 9.16903E-05 & 0.0488 \\
\hline Calcium Carbonate & $\mathrm{CaCO}_{3}$ & 100.00 & 0.000220133 & 0.0308 \\
\hline Sodium chloride & $\mathrm{NaCl}$ & 58.44 & 0.000382729 & 0.0313 \\
\hline Sodium fluoride & $\mathrm{NaF}$ & 42.00 & 0.000243175 & 0.0143 \\
\hline Sodium molybdate, dihydrate & $\mathrm{Na}_{2} \mathrm{MoO}_{4} \cdot 2 \mathrm{H}_{2} \mathrm{O}$ & 241.95 & $6.15066 \mathrm{E}-06$ & 0.0021 \\
\hline Manganese Dioxide & $\mathrm{MnO}_{2}$ & 86.94 & 0.000380032 & 0.0463 \\
\hline Nickel nitrate, 6-hydrate & $\mathrm{Ni}\left(\mathrm{NO}_{3}\right)_{2} \cdot 6 \mathrm{H}_{2} \mathrm{O}$ & 290.81 & 0.000173355 & 0.0706 \\
\hline Mercury (II) nitrate & $\mathrm{Hg}\left(\mathrm{NO}_{3}\right)_{2}$ & 342.62 & 0.000146 & 0.0700 \\
\hline $\begin{array}{c}\text { Sodium metasilicate, 5- } \\
\text { hydrate }\end{array}$ & $\mathrm{Na}_{2} \mathrm{SiO}_{3} .5 \mathrm{H}_{2} \mathrm{O}$ & 212.14 & $5.33803 \mathrm{E}-05$ & 0.0159 \\
\hline Zinc nitrate, 6-hydrate & $\mathrm{Zn}\left(\mathrm{NO}_{3}\right)_{2} \cdot 6 \mathrm{H}_{2} \mathrm{O}$ & 297.49 & 1.37147E-05 & 0.0057 \\
\hline Lead nitrate & $\mathrm{Pb}\left(\mathrm{NO}_{3}\right)_{2}$ & 331.21 & 6.48129E-06 & 0.0030 \\
\hline Cupric nitrate, 2.5 hydrate & $\mathrm{Cu}\left(\mathrm{NO}_{3}\right)_{2} \cdot 2 \cdot 5 \mathrm{H}_{2} \mathrm{O}$ & 233.00 & 8.18312E-06 & 0.0027 \\
\hline
\end{tabular}




\section{Cyclic Potentiodynamic Polarization}

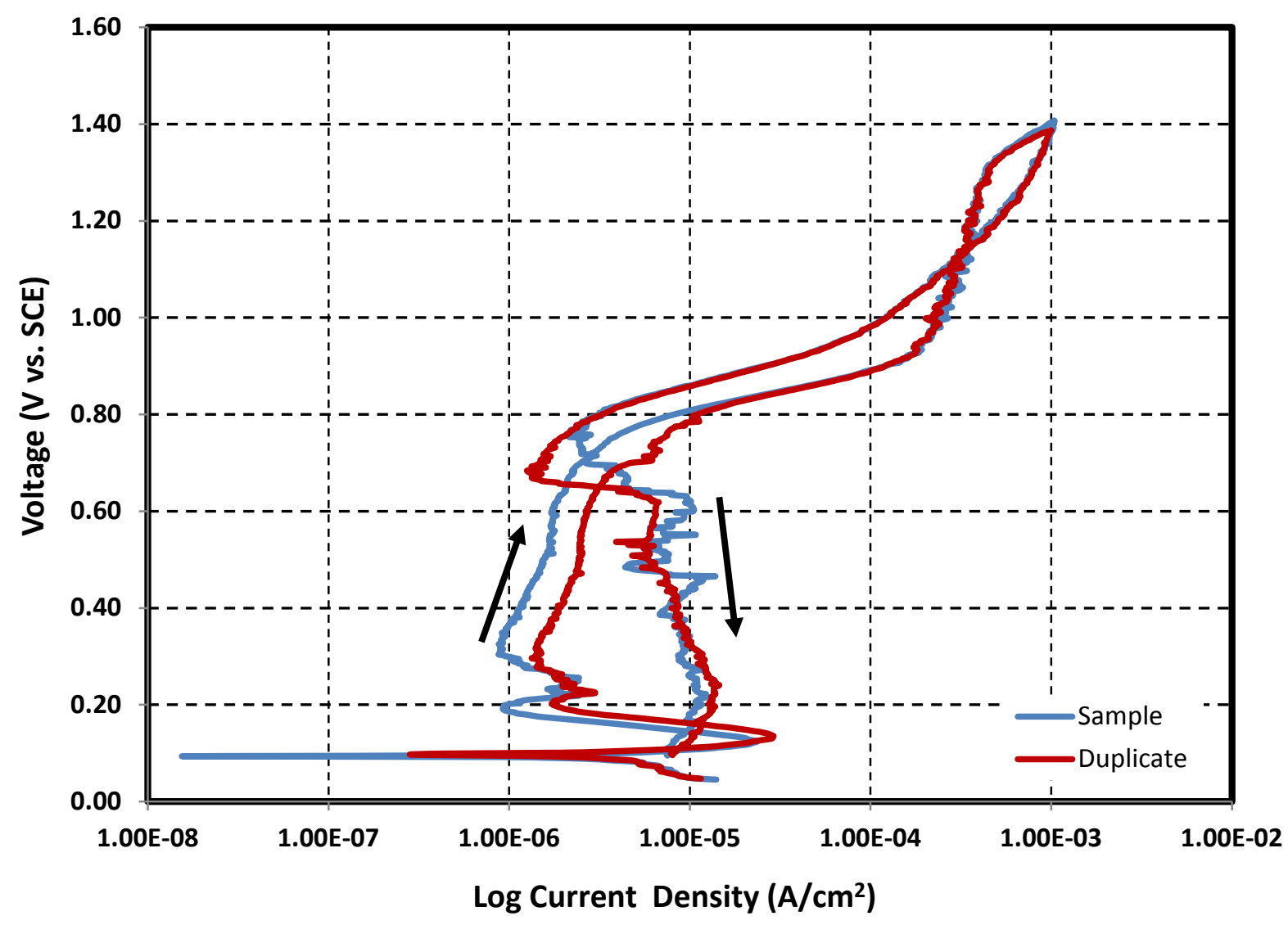

Images of bullet samples after electrochemical tests

Test 40

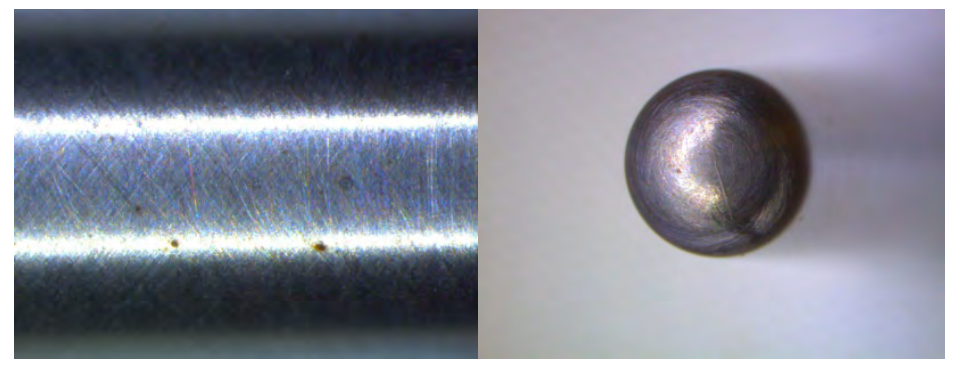

Test 40D

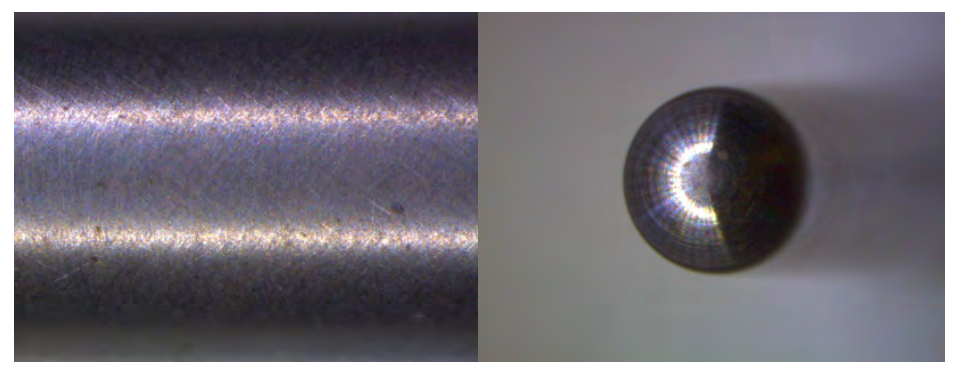

Shank $(20 X)$

Nose (10X) 


\section{Appendix $\mathbf{H}$}

\section{Test Conditions from Previous Electrochemical Results (Task 4)}


Cyclic Potentiodynamic Polarization Parameters, and Sample Geometry and Preparation of Historical Data

\begin{tabular}{|c|c|c|c|c|c|c|c|c|c|c|c|}
\hline Reference & $\begin{array}{c}\text { Potential } \\
\text { Stabilization } \\
\text { (hrs) }\end{array}$ & $\begin{array}{l}\text { Initial } \\
\text { Potential } \\
\text { (V vs. } \\
\text { Ecorr) }\end{array}$ & $\begin{array}{l}\text { Scan } \\
\text { Rate } \\
(\mathrm{mV} / \mathrm{s})\end{array}$ & $\begin{array}{c}\text { Vertex } \\
\text { Threshold } \\
\text { ( } \mathrm{mA} / \mathrm{cm}^{2} \\
\text { or V vs. } \\
\text { ref.) } \\
\end{array}$ & $\begin{array}{l}\text { Final Potential } \\
\text { (V vs. Ecorr) }\end{array}$ & $\begin{array}{l}\text { Sample } \\
\text { Geometry }\end{array}$ & $\begin{array}{c}\text { Surface } \\
\text { Preparation }\end{array}$ & Potentiostat & $\begin{array}{l}\text { Counter } \\
\text { electrode }\end{array}$ & $\begin{array}{l}\text { Reference } \\
\text { Electrode }\end{array}$ & Material \\
\hline 34,35 & 0.5 & -0.05 & 0.166 & $0.9 \mathrm{~V}$ & 0 & $0.625 "$ disk & $\begin{array}{c}600 \text { grit, cleaned } \\
\text { with acetone and } \\
\text { distilled } \mathrm{H}_{2} \mathrm{O} \\
\text { before use. }\end{array}$ & PAR 273 & Graphite & $\begin{array}{l}\text { SCE with } \\
\text { Luggin } \\
\text { Probe filled } \\
\text { with test } \\
\text { solution. }\end{array}$ & A537 \\
\hline 36 & 0.5 & -0.05 & 0.166 & $1.0 \mathrm{~V}$ & 0 & $0.625 "$ disk & $\begin{array}{l}600 \text { grit, cleaned } \\
\text { with acetone and } \\
\text { distilled } \mathrm{H}_{2} \mathrm{O} \\
\text { before use. }\end{array}$ & PAR 273 & Platinum & $\begin{array}{l}\text { SCE with } \\
\text { Luggin } \\
\text { Probe filled } \\
\text { with test } \\
\text { solution. }\end{array}$ & A537 \\
\hline 37 & 0.5 & -0.05 & 0.166 & $1.0 \mathrm{~V}$ & 0 & $0.625 "$ disk & $\begin{array}{l}600 \text { grit, cleaned } \\
\text { with acetone and } \\
\text { distilled } \mathrm{H}_{2} \mathrm{O} \\
\text { before use. }\end{array}$ & PAR 273 & Platinum & $\begin{array}{l}\text { SCE with } \\
\text { Luggin } \\
\text { Probe filled } \\
\text { with test } \\
\text { solution. }\end{array}$ & A537 \\
\hline 38 & 0.5 & -0.05 & 0.166 & $1.0 \mathrm{~V}$ & 0 & $0.625 "$ disk & $\begin{array}{l}600 \text { grit, cleaned } \\
\text { with acetone and } \\
\text { distilled } \mathrm{H}_{2} \mathrm{O} \\
\text { before use. }\end{array}$ & PAR 273 & Platinum & $\begin{array}{l}\text { SCE with } \\
\text { Luggin } \\
\text { Probe filled } \\
\text { with test } \\
\text { solution. }\end{array}$ & A537 \\
\hline 39 & 0.5 & -0.05 & 0.166 & $1.0 \mathrm{~V}$ & 0 & $0.625 "$ disk & $\begin{array}{l}600 \text { grit, cleaned } \\
\text { with acetone and } \\
\text { distilled } \mathrm{H}_{2} \mathrm{O} \\
\text { before use. }\end{array}$ & PAR 273 & Platinum & $\begin{array}{l}\text { SCE with } \\
\text { Luggin } \\
\text { Probe filled } \\
\text { with test } \\
\text { solution. }\end{array}$ & A537 \\
\hline 40 & 0.5 & -0.05 & 0.166 & $1.0 \mathrm{~V}$ & 0 & $0.625 "$ disk & $\begin{array}{l}600 \text { grit, cleaned } \\
\text { with acetone and } \\
\text { distilled } \mathrm{H}_{2} \mathrm{O} \\
\text { before use. }\end{array}$ & PAR 273 & Platinum & $\begin{array}{l}\text { SCE with } \\
\text { Luggin } \\
\text { Probe filled } \\
\text { with test } \\
\text { solution. }\end{array}$ & A537 \\
\hline 41 & 0.5 & -0.05 & 0.166 & $1.0 \mathrm{~V}$ & 0 & $0.625 "$ disk & $\begin{array}{l}600 \text { grit, cleaned } \\
\text { with acetone and } \\
\text { distilled } \mathrm{H}_{2} \mathrm{O} \\
\text { before use. }\end{array}$ & PAR 273 & Platinum & $\begin{array}{l}\text { SCE with } \\
\text { Luggin } \\
\text { Probe filled } \\
\text { with test } \\
\text { solution. }\end{array}$ & A537 \\
\hline
\end{tabular}


Cyclic Potentiodynamic Polarization Parameters, and Sample Geometry and Preparation of Historical Data (continued 1)

\begin{tabular}{|c|c|c|c|c|c|c|c|c|c|c|c|}
\hline Reference & $\begin{array}{c}\text { Potential } \\
\text { Stabilization } \\
\text { (hrs) }\end{array}$ & $\begin{array}{l}\text { Initial } \\
\text { Potential } \\
\text { (V vs. } \\
\text { Ecorr) }\end{array}$ & $\begin{array}{l}\text { Scan } \\
\text { Rate } \\
(\mathrm{mV} / \mathrm{s})\end{array}$ & $\begin{array}{c}\text { Vertex } \\
\text { Threshold } \\
\left(\mathrm{mA} / \mathrm{cm}^{2}\right. \\
\text { or V vs. } \\
\text { ref.) }\end{array}$ & $\begin{array}{l}\text { Final Potential } \\
\text { (V vs. Ecorr) }\end{array}$ & $\begin{array}{l}\text { Sample } \\
\text { Geometry }\end{array}$ & $\begin{array}{c}\text { Surface } \\
\text { Preparation }\end{array}$ & Potentiostat & $\begin{array}{l}\text { Counter } \\
\text { electrode }\end{array}$ & $\begin{array}{l}\text { Reference } \\
\text { Electrode }\end{array}$ & Material \\
\hline 42 & 0.5 & -0.05 & 0.166 & $1.0 \mathrm{~V}$ & 0 & $0.625 "$ disk & $\begin{array}{l}600 \text { grit, cleaned } \\
\text { with acetone and } \\
\text { distilled } \mathrm{H}_{2} \mathrm{O} \\
\text { before use. }\end{array}$ & PAR 273 & Platinum & $\begin{array}{l}\text { SCE with } \\
\text { Luggin } \\
\text { Probe filled } \\
\text { with test } \\
\text { solution. } \\
\end{array}$ & A537 \\
\hline 43 & 0.5 & -0.05 & 0.166 & $1.0 \mathrm{~V}$ & 0 & $0.625 "$ disk & $\begin{array}{l}600 \text { grit, cleaned } \\
\text { with acetone and } \\
\text { distilled } \mathrm{H}_{2} \mathrm{O} \\
\text { before use. }\end{array}$ & PAR 273 & Platinum & $\begin{array}{l}\text { SCE with } \\
\text { Luggin } \\
\text { Probe filled } \\
\text { with test } \\
\text { solution. } \\
\end{array}$ & A537 \\
\hline 44 & 0.5 & -0.05 & 0.166 & $1.0 \mathrm{~V}$ & 0 & $0.625 "$ disk & $\begin{array}{c}600 \text { grit, cleaned } \\
\text { with acetone and } \\
\text { distilled } \mathrm{H}_{2} \mathrm{O} \\
\text { before use. }\end{array}$ & PAR 273 & Platinum & $\begin{array}{l}\text { SCE with } \\
\text { Luggin } \\
\text { Probe filled } \\
\text { with test } \\
\text { solution. } \\
\end{array}$ & A537 \\
\hline 45 & 0.5 & -0.05 & 0.166 & $1.0 \mathrm{~V}$ & 0 & $0.625 "$ disk & $\begin{array}{l}600 \text { grit, cleaned } \\
\text { with acetone and } \\
\text { distilled } \mathrm{H}_{2} \mathrm{O} \\
\text { before use. }\end{array}$ & PAR 273 & Platinum & $\begin{array}{l}\text { SCE with } \\
\text { Luggin } \\
\text { Probe filled } \\
\text { with test } \\
\text { solution. }\end{array}$ & A537 \\
\hline 46 & 2 & -0.1 & 0.5 & $1.2 \mathrm{~V}$ & 0 & $0.625 "$ disk & $\begin{array}{l}800 \text { grit, cleaned } \\
\text { with acetone and } \\
\text { distilled } \mathrm{H}_{2} \mathrm{O} \\
\text { before use. }\end{array}$ & PAR 273 & Graphite & $\begin{array}{l}\text { SCE with } \\
\text { Luggin } \\
\text { Probe filled } \\
\text { with test } \\
\text { solution for } \\
\text { some tests. } \\
\mathrm{Ag} / \mathrm{AgCl} \text { for } \\
\text { other tests. }\end{array}$ & A537 \\
\hline 47 & 2 & -0.1 & 0.5 & $1.2 \mathrm{~V}$ & 0 & $0.625^{\prime \prime}$ disk & $\begin{array}{l}800 \text { grit, cleaned } \\
\text { with acetone and } \\
\text { distilled } \mathrm{H}_{2} \mathrm{O} \\
\text { before use. }\end{array}$ & PAR 273 & Graphite & $\begin{array}{l}\text { SCE with } \\
\text { Luggin } \\
\text { Probe filled } \\
\text { with test } \\
\text { solution for } \\
\text { some tests. } \\
\mathrm{Ag} / \mathrm{AgCl} \text { for } \\
\text { other tests. }\end{array}$ & A537 \\
\hline
\end{tabular}


Cyclic Potentiodynamic Polarization Parameters, and Sample Geometry and Preparation of Historical Data (continued 2)

\begin{tabular}{|c|c|c|c|c|c|c|c|c|c|c|c|}
\hline Reference & $\begin{array}{c}\text { Potential } \\
\text { Stabilization } \\
\text { (hrs) }\end{array}$ & $\begin{array}{l}\text { Initial } \\
\text { Potential } \\
\text { (V vs. } \\
\text { Ecorr) }\end{array}$ & $\begin{array}{l}\text { Scan } \\
\text { Rate } \\
(\mathrm{mV} / \mathrm{s})\end{array}$ & $\begin{array}{c}\text { Vertex } \\
\text { Threshold } \\
\text { ( } \mathrm{mA} / \mathrm{cm}^{2} \\
\text { or V vs. } \\
\text { ref.) }\end{array}$ & $\begin{array}{l}\text { Final Potential } \\
\text { (V vs. Ecorr) }\end{array}$ & $\begin{array}{l}\text { Sample } \\
\text { Geometry }\end{array}$ & $\begin{array}{c}\text { Surface } \\
\text { Preparation }\end{array}$ & Potentiostat & $\begin{array}{l}\text { Counter } \\
\text { electrode }\end{array}$ & $\begin{array}{l}\text { Reference } \\
\text { Electrode }\end{array}$ & Material \\
\hline 48 & 2 & -0.1 & 0.5 & $1.2 \mathrm{~V}$ & 0 & $0.625^{\prime \prime}$ disk & $\begin{array}{l}800 \text { grit, cleaned } \\
\text { with acetone and } \\
\text { distilled } \mathrm{H}_{2} \mathrm{O} \\
\text { before use. }\end{array}$ & PAR 273 & Graphite & $\begin{array}{l}\text { SCE with } \\
\text { Luggin } \\
\text { Probe filled } \\
\text { with test } \\
\text { solution for } \\
\text { some tests. } \\
\mathrm{Ag} / \mathrm{AgCl} \text { for } \\
\text { other tests. }\end{array}$ & A537 \\
\hline 28 & 2.5 & -0.1 & 0.5 & $\begin{array}{l}1.2 \mathrm{~V} \text { or } 1 \\
\mathrm{~mA} / \mathrm{cm}^{2}\end{array}$ & 0 & $0.625 "$ disk & $\begin{array}{l}800 \text { grit, cleaned } \\
\text { with acetone and } \\
\text { distilled } \mathrm{H}_{2} \mathrm{O} \\
\text { before use. }\end{array}$ & PAR 273 & Graphite & $\begin{array}{l}\text { SCE with } \\
\text { Luggin } \\
\text { Probe filled } \\
\text { with test } \\
\text { solution for } \\
\text { some tests. } \\
\mathrm{Ag} / \mathrm{AgCl} \text { for } \\
\text { other tests. }\end{array}$ & A537 \\
\hline 49 & 2 or 168 & -0.05 & 0.5 & $\begin{array}{l}1.0 \mathrm{~V} \text { vs. } \\
\text { OCP }\end{array}$ & 0 & $\begin{array}{l}\text { rectangular } \\
\text { electrode }\end{array}$ & $\begin{array}{l}600 \text { grit, cleaned } \\
\text { with acetone and } \\
\text { distilled } \mathrm{H}_{2} \mathrm{O} \\
\text { before use. }\end{array}$ & PAR 273 & Graphite & $\mathrm{Ag} / \mathrm{AgCl}$ & A537 \\
\hline 50 & NA & -0.15 & 0.167 & $5 \mathrm{~mA} / \mathrm{cm}^{2}$ & $\begin{array}{l}\text { Scan was run } \\
\text { until the } \\
\text { current on the } \\
\text { reverse scan } \\
\text { was less than } \\
\text { the current on } \\
\text { the forward } \\
\text { scan at the } \\
\text { same potential }\end{array}$ & Cylindrical & $\begin{array}{c}600 \text { grit, cleaned } \\
\text { with acetone and } \\
\text { distilled } \mathrm{H}_{2} \mathrm{O} \\
\text { before use. }\end{array}$ & NA & Platinum & $\mathrm{Ag} / \mathrm{AgCl}$ & A537 \\
\hline
\end{tabular}


Cyclic Potentiodynamic Polarization Parameters, and Sample Geometry and Preparation of Historical Data (continued 3)

\begin{tabular}{|c|c|c|c|c|c|c|c|c|c|c|c|}
\hline Reference & $\begin{array}{c}\text { Potential } \\
\text { Stabilization } \\
\text { (hrs) }\end{array}$ & $\begin{array}{l}\text { Initial } \\
\text { Potential } \\
\text { (V vs. } \\
\text { Ecorr) }\end{array}$ & $\begin{array}{l}\text { Scan } \\
\text { Rate } \\
(\mathrm{mV} / \mathrm{s})\end{array}$ & $\begin{array}{c}\text { Vertex } \\
\text { Threshold } \\
\left(\mathrm{mA} / \mathrm{cm}^{2}\right. \\
\text { or V vs. } \\
\text { ref.) }\end{array}$ & $\begin{array}{l}\text { Final Potential } \\
\text { (V vs. Ecorr) }\end{array}$ & $\begin{array}{l}\text { Sample } \\
\text { Geometry }\end{array}$ & $\begin{array}{c}\text { Surface } \\
\text { Preparation }\end{array}$ & Potentiostat & $\begin{array}{l}\text { Counter } \\
\text { electrode }\end{array}$ & $\begin{array}{l}\text { Reference } \\
\text { Electrode }\end{array}$ & Material \\
\hline 51 & 18 & -0.1 & 0.167 & $\begin{array}{l}1.0 \mathrm{~V} \text { vs. } \\
\text { ref or } 1 \\
\mathrm{~mA} / \mathrm{cm}^{2}\end{array}$ & $-0.1 \mathrm{~V}$ & Bullet & $\begin{array}{c}600 \text { grit, } \\
\text { ultrasonically } \\
\text { cleaned with } \\
\text { isopropanol for } \\
5 \text { min., rinsed } \\
\text { with DI, dried } \\
\text { with } \mathrm{N}_{2} \text {. }\end{array}$ & PAR 273 & Platinum & SCE & $\begin{array}{c}\text { Tank Car } \\
\text { Steel }\end{array}$ \\
\hline 52 & 18 & -0.5 & 0.167 & $\begin{array}{l}1.0 \mathrm{~V} \text { vs. } \\
\text { ref or } 1 \\
\mathrm{~mA} / \mathrm{cm}^{2}\end{array}$ & $-0.1 \mathrm{~V}$ & Bullet & $\begin{array}{c}600 \text { grit, } \\
\text { ultrasonically } \\
\text { cleaned with } \\
\text { isopropanol for } \\
5 \text { min., rinsed } \\
\text { with DI, dried } \\
\text { with } \mathrm{N}_{2} \text {. }\end{array}$ & PAR 273 & Platinum & SCE & A537 \\
\hline 53 & 2 & -0.1 & 0.167 & $1 \mathrm{~mA} / \mathrm{cm}^{2}$ & $-0.1 \mathrm{~V}$ & Bullet & $\begin{array}{c}600 \text { grit, } \\
\text { ultrasonically } \\
\text { cleaned with } \\
\text { isopropanol for } \\
5 \text { min., rinsed } \\
\text { with DI, dried } \\
\text { with } \mathrm{N}_{2} \text {. }\end{array}$ & NA & Platinum & SCE & A537 \\
\hline 54 & 2 & -0.1 & 0.167 & $1 \mathrm{~mA} / \mathrm{cm}^{2}$ & $-0.1 \mathrm{~V}$ & Bullet & $\begin{array}{c}600 \text { grit, } \\
\text { ultrasonically } \\
\text { cleaned with } \\
\text { isopropanol for } \\
5 \text { min., rinsed } \\
\text { with DI, dried } \\
\text { with } \mathrm{N}_{2} \text {. }\end{array}$ & NA & Platinum & SCE & A537 \\
\hline
\end{tabular}


Cyclic Potentiodynamic Polarization Conditions, and Chemical Composition and $\mathrm{pH}$ of Historical Data

\begin{tabular}{|c|c|c|c|c|c|c|c|c|c|c|c|c|c|}
\hline Reference & Test & $\begin{array}{c}\text { Temperature } \\
\left({ }^{\circ} \mathrm{C}\right)\end{array}$ & $\begin{array}{l}\text { Carbonate } \\
\text { (M) }\end{array}$ & $\begin{array}{c}\text { Bi- } \\
\text { carbonate } \\
\text { (M) }\end{array}$ & $\begin{array}{l}\text { Citrate } \\
\text { (M) }\end{array}$ & $\begin{array}{l}\text { Phosphate } \\
\text { (M) }\end{array}$ & $\begin{array}{l}\text { Chloride } \\
\text { (M) }\end{array}$ & $\begin{array}{l}\text { Fluoride } \\
\text { (M) }\end{array}$ & $\begin{array}{l}\text { Sulfate } \\
\text { (M) }\end{array}$ & $\begin{array}{l}\text { Nitrate } \\
\text { (M) }\end{array}$ & $\begin{array}{l}\text { Nitrite } \\
\text { (M) }\end{array}$ & $\mathrm{pH}$ & Pit ID* \\
\hline 34,35 & 1 & 23 & 0.0046 & 0.022 & NA & 0.000084 & 0.00031 & 0.00015 & 0.0013 & 0.021 & 0.002 & 9.51 & 2 \\
\hline 34,35 & 2 & 23 & 0.0046 & 0.022 & NA & 0.000084 & 0.00031 & 0.00015 & 0.0013 & 0.021 & 0.004 & 9.51 & 1 \\
\hline 34,35 & 3 & 23 & 0.0046 & 0.022 & NA & 0.000084 & 0.00031 & 0.00015 & 0.0013 & 0.021 & 0.005 & 9.51 & 0 \\
\hline 34,35 & 4 & 23 & 0.0046 & 0.022 & NA & 0.000084 & 0.00031 & 0.00015 & 0.0013 & 0.021 & 0.01 & 9.51 & 0 \\
\hline 34,35 & 5 & 30 & 0.0046 & 0.022 & NA & 0.000084 & 0.00031 & 0.00015 & 0.0013 & 0.021 & 0.002 & 9.51 & 2 \\
\hline 34,35 & 6 & 30 & 0.0046 & 0.022 & NA & 0.000084 & 0.00031 & 0.00015 & 0.0013 & 0.021 & 0.005 & 9.51 & 2 \\
\hline 34,35 & 7 & 30 & 0.0046 & 0.022 & NA & 0.000084 & 0.00031 & 0.00015 & 0.0013 & 0.021 & 0.007 & 9.51 & 2 \\
\hline 34,35 & 8 & 30 & 0.0046 & 0.022 & NA & 0.000084 & 0.00031 & 0.00015 & 0.0013 & 0.021 & 0.01 & 9.51 & 0 \\
\hline 34,35 & 9 & 40 & 0.0046 & 0.022 & NA & 0.000084 & 0.00031 & 0.00015 & 0.0013 & 0.021 & 0.005 & 9.51 & 2 \\
\hline 34,35 & 10 & 40 & 0.0046 & 0.022 & NA & 0.000084 & 0.00031 & 0.00015 & 0.0013 & 0.021 & 0.01 & 9.51 & 1 \\
\hline 34,35 & 11 & 40 & 0.0046 & 0.022 & NA & 0.000084 & 0.00031 & 0.00015 & 0.0013 & 0.021 & 0.015 & 9.51 & 0 \\
\hline 34,35 & 12 & 50 & 0.0046 & 0.022 & NA & 0.000084 & 0.00031 & 0.00015 & 0.0013 & 0.021 & 0.01 & 9.51 & 0 \\
\hline 34,35 & 13 & 50 & 0.0046 & 0.022 & NA & 0.000084 & 0.00031 & 0.00015 & 0.0013 & 0.021 & 0.015 & 9.51 & 1 \\
\hline 34,35 & 14 & 50 & 0.0046 & 0.022 & NA & 0.000084 & 0.00031 & 0.00015 & 0.0013 & 0.021 & 0.02 & 9.51 & 0 \\
\hline 34,35 & 15 & 50 & 0.0046 & 0.022 & $\mathrm{NA}$ & 0.000084 & 0.00031 & 0.00015 & 0.0013 & 0.021 & 0.03 & 9.51 & 0 \\
\hline 34,35 & 16 & 60 & 0.0046 & 0.022 & NA & 0.000084 & 0.00031 & 0.00015 & 0.0013 & 0.021 & 0.1 & 9.51 & 1 \\
\hline 34,35 & 17 & 60 & 0.0046 & 0.022 & NA & 0.000084 & 0.00031 & 0.00015 & 0.0013 & 0.021 & 0.15 & 9.51 & 1 \\
\hline 34,35 & 18 & 60 & 0.0046 & 0.022 & NA & 0.000084 & 0.00031 & 0.00015 & 0.0013 & 0.021 & 0.3 & 9.51 & 0 \\
\hline 34,35 & 19 & 60 & 0.0046 & 0.022 & NA & 0.000084 & 0.00031 & 0.00015 & 0.0013 & 0.021 & 0.4 & 9.51 & 1 \\
\hline 34,35 & 20 & 60 & 0.0046 & 0.022 & NA & 0.000084 & 0.00031 & 0.00015 & 0.0013 & 0.021 & 0.5 & 9.51 & 0 \\
\hline 34,35 & 21 & 40 & 0.0073 & 0.028 & NA & 0.00012 & 0.00043 & 0.00022 & 0.0019 & 0.028 & 0.01 & 9.59 & 2 \\
\hline 34,35 & 22 & 40 & 0.0073 & 0.028 & NA & 0.00012 & 0.00043 & 0.00022 & 0.0019 & 0.028 & 0.014 & 9.59 & 1 \\
\hline 34,35 & 23 & 40 & 0.0073 & 0.028 & NA & 0.00012 & 0.00043 & 0.00022 & 0.0019 & 0.028 & 0.022 & 9.59 & 0 \\
\hline 34,35 & 24 & 40 & 0.011 & 0.035 & NA & 0.00016 & 0.0006 & 0.0003 & 0.0026 & 0.039 & 0.022 & 9.66 & 1 \\
\hline 34,35 & 25 & 40 & 0.011 & 0.035 & NA & 0.00016 & 0.0006 & 0.0003 & 0.0026 & 0.039 & 0.03 & 9.66 & 1 \\
\hline
\end{tabular}


Cyclic Potentiodynamic Polarization Conditions, and Chemical Composition and pH of Historical Data (continued 1)

\begin{tabular}{|c|c|c|c|c|c|c|c|c|c|c|c|c|c|}
\hline Reference & Test & $\begin{array}{l}\text { Temperature } \\
\left({ }^{\circ} \mathrm{C}\right)\end{array}$ & $\begin{array}{l}\text { Carbonate } \\
\text { (M) }\end{array}$ & $\begin{array}{c}\text { Bi- } \\
\text { carbonate } \\
\text { (M) }\end{array}$ & $\begin{array}{l}\text { Citrate } \\
\text { (M) }\end{array}$ & $\begin{array}{l}\text { Phosphate } \\
\text { (M) }\end{array}$ & $\begin{array}{l}\text { Chloride } \\
\text { (M) }\end{array}$ & $\begin{array}{l}\text { Fluoride } \\
\text { (M) }\end{array}$ & $\begin{array}{l}\text { Sulfate } \\
\text { (M) }\end{array}$ & $\begin{array}{l}\text { Nitrate } \\
\text { (M) }\end{array}$ & $\begin{array}{l}\text { Nitrite } \\
\text { (M) }\end{array}$ & $\mathrm{pH}$ & Pit ID* \\
\hline 34,35 & 26 & 40 & 0.011 & 0.035 & $\mathrm{NA}$ & 0.00016 & 0.0006 & 0.0003 & 0.0026 & 0.039 & 0.039 & 9.66 & 0 \\
\hline 34,35 & 27 & 23 & 0.017 & 0.045 & $\mathrm{NA}$ & 0.00022 & 0.00083 & 0.00041 & 0.0036 & 0.055 & 0.005 & 9.73 & 2 \\
\hline 34,35 & 28 & 23 & 0.017 & 0.045 & NA & 0.00022 & 0.00083 & 0.00041 & 0.0036 & 0.055 & 0.009 & 9.73 & 2 \\
\hline 34,35 & 29 & 23 & 0.017 & 0.045 & $\mathrm{NA}$ & 0.00022 & 0.00083 & 0.00041 & 0.0036 & 0.055 & 0.01 & 9.73 & 1 \\
\hline 34,35 & 30 & 23 & 0.017 & 0.045 & NA & 0.00022 & 0.00083 & 0.00041 & 0.0036 & 0.055 & 0.015 & 9.73 & 1 \\
\hline 34,35 & 31 & 23 & 0.017 & 0.045 & $\mathrm{NA}$ & 0.00022 & 0.00083 & 0.00041 & 0.0036 & 0.055 & 0.03 & 9.73 & 0 \\
\hline 34,35 & 32 & 23 & 0.017 & 0.045 & NA & 0.00022 & 0.00083 & 0.00041 & 0.0036 & 0.055 & 0.05 & 9.73 & 0 \\
\hline 34,35 & 33 & 30 & 0.017 & 0.045 & NA & 0.00022 & 0.00083 & 0.00041 & 0.0036 & 0.055 & 0.005 & 9.73 & 2 \\
\hline 34,35 & 34 & 30 & 0.017 & 0.045 & NA & 0.00022 & 0.00083 & 0.00041 & 0.0036 & 0.055 & 0.009 & 9.73 & 2 \\
\hline 34,35 & 35 & 30 & 0.017 & 0.045 & NA & 0.00022 & 0.00083 & 0.00041 & 0.0036 & 0.055 & 0.01 & 9.73 & 2 \\
\hline 34,35 & 36 & 30 & 0.017 & 0.045 & $\mathrm{NA}$ & 0.00022 & 0.00083 & 0.00041 & 0.0036 & 0.055 & 0.015 & 9.73 & 2 \\
\hline 34,35 & 37 & 30 & 0.017 & 0.045 & NA & 0.00022 & 0.00083 & 0.00041 & 0.0036 & 0.055 & 0.03 & 9.73 & 2 \\
\hline 34,35 & 38 & 30 & 0.017 & 0.045 & NA & 0.00022 & 0.00083 & 0.00041 & 0.0036 & 0.055 & 0.042 & 9.73 & 0 \\
\hline 34,35 & 39 & 30 & 0.017 & 0.045 & NA & 0.00022 & 0.00083 & 0.00041 & 0.0036 & 0.055 & 0.05 & 9.73 & 0 \\
\hline 34,35 & 40 & 40 & 0.017 & 0.045 & NA & 0.00022 & 0.00083 & 0.00041 & 0.0036 & 0.055 & 0.02 & 9.73 & 2 \\
\hline 34,35 & 41 & 40 & 0.017 & 0.045 & $\mathrm{NA}$ & 0.00022 & 0.00083 & 0.00041 & 0.0036 & 0.055 & 0.03 & 9.73 & 1 \\
\hline 34,35 & 42 & 40 & 0.017 & 0.045 & NA & 0.00022 & 0.00083 & 0.00041 & 0.0036 & 0.055 & 0.042 & 9.73 & 0 \\
\hline 34,35 & 43 & 40 & 0.017 & 0.045 & NA & 0.00022 & 0.00083 & 0.00041 & 0.0036 & 0.055 & 0.05 & 9.73 & 0 \\
\hline 34,35 & 44 & 40 & 0.017 & 0.045 & $\mathrm{NA}$ & 0.00022 & 0.00083 & 0.00041 & 0.0036 & 0.055 & 0.055 & 9.73 & 0 \\
\hline 34,35 & 45 & 50 & 0.017 & 0.045 & $\mathrm{NA}$ & 0.00022 & 0.00083 & 0.00041 & 0.0036 & 0.055 & 0.042 & 9.73 & 2 \\
\hline 34,35 & 46 & 50 & 0.017 & 0.045 & $\mathrm{NA}$ & 0.00022 & 0.00083 & 0.00041 & 0.0036 & 0.055 & 0.05 & 9.73 & 0 \\
\hline 34,35 & 47 & 50 & 0.017 & 0.045 & NA & 0.00022 & 0.00083 & 0.00041 & 0.0036 & 0.055 & 0.06 & 9.73 & 2 \\
\hline 34,35 & 48 & 50 & 0.017 & 0.045 & NA & 0.00022 & 0.00083 & 0.00041 & 0.0036 & 0.055 & 0.09 & 9.73 & 0 \\
\hline 34,35 & 49 & 50 & 0.017 & 0.045 & $\mathrm{NA}$ & 0.00022 & 0.00083 & 0.00041 & 0.0036 & 0.055 & 0.15 & 9.73 & 2 \\
\hline 34,35 & 50 & 50 & 0.017 & 0.045 & NA & 0.00022 & 0.00083 & 0.00041 & 0.0036 & 0.055 & 0.2 & 9.73 & 2 \\
\hline
\end{tabular}


Cyclic Potentiodynamic Polarization Conditions, and Chemical Composition and pH of Historical Data (continued 2)

\begin{tabular}{|c|c|c|c|c|c|c|c|c|c|c|c|c|c|}
\hline Reference & Test & $\begin{array}{l}\text { Temperature } \\
\left({ }^{\circ} \mathrm{C}\right)\end{array}$ & $\begin{array}{l}\text { Carbonate } \\
\text { (M) }\end{array}$ & $\begin{array}{c}\text { Bi- } \\
\text { carbonate } \\
\text { (M) }\end{array}$ & $\begin{array}{l}\text { Citrate } \\
\text { (M) }\end{array}$ & $\begin{array}{l}\text { Phosphate } \\
\text { (M) }\end{array}$ & $\begin{array}{l}\text { Chloride } \\
\text { (M) }\end{array}$ & $\begin{array}{l}\text { Fluoride } \\
\text { (M) }\end{array}$ & $\begin{array}{l}\text { Sulfate } \\
\text { (M) }\end{array}$ & $\begin{array}{l}\text { Nitrate } \\
\text { (M) }\end{array}$ & $\begin{array}{l}\text { Nitrite } \\
\text { (M) }\end{array}$ & $\mathrm{pH}$ & Pit ID* \\
\hline 34,35 & 51 & 50 & 0.017 & 0.045 & $\mathrm{NA}$ & 0.00022 & 0.00083 & 0.00041 & 0.0036 & 0.055 & 0.25 & 9.73 & 1 \\
\hline 34,35 & 52 & 50 & 0.017 & 0.045 & $\mathrm{NA}$ & 0.00022 & 0.00083 & 0.00041 & 0.0036 & 0.055 & 0.3 & 9.73 & 0 \\
\hline 34,35 & 53 & 50 & 0.017 & 0.045 & NA & 0.00022 & 0.00083 & 0.00041 & 0.0036 & 0.055 & 0.35 & 9.73 & 0 \\
\hline 34,35 & 54 & 60 & 0.017 & 0.045 & $\mathrm{NA}$ & 0.00022 & 0.00083 & 0.00041 & 0.0036 & 0.055 & 0.042 & 9.73 & 2 \\
\hline 34,35 & 55 & 60 & 0.017 & 0.045 & NA & 0.00022 & 0.00083 & 0.00041 & 0.0036 & 0.055 & 0.06 & 9.73 & 1 \\
\hline 34,35 & 56 & 60 & 0.017 & 0.045 & $\mathrm{NA}$ & 0.00022 & 0.00083 & 0.00041 & 0.0036 & 0.055 & 0.09 & 9.73 & 1 \\
\hline 34,35 & 57 & 60 & 0.017 & 0.045 & NA & 0.00022 & 0.00083 & 0.00041 & 0.0036 & 0.055 & 0.13 & 9.73 & 2 \\
\hline 34,35 & 58 & 60 & 0.017 & 0.045 & NA & 0.00022 & 0.00083 & 0.00041 & 0.0036 & 0.055 & 0.3 & 9.73 & 0 \\
\hline 34,35 & 59 & 60 & 0.017 & 0.045 & NA & 0.00022 & 0.00083 & 0.00041 & 0.0036 & 0.055 & 0.35 & 9.73 & 0 \\
\hline 34,35 & 60 & 40 & 0.026 & 0.056 & NA & 0.00031 & 0.0012 & 0.00057 & 0.005 & 0.076 & 0.03 & 9.79 & 2 \\
\hline 34,35 & 61 & 40 & 0.026 & 0.056 & NA & 0.00031 & 0.0012 & 0.00057 & 0.005 & 0.076 & 0.058 & 9.79 & 0 \\
\hline 34,35 & 62 & 40 & 0.026 & 0.056 & NA & 0.00031 & 0.0012 & 0.00057 & 0.005 & 0.076 & 0.075 & 9.79 & 0 \\
\hline 34,35 & 63 & 40 & 0.039 & 0.071 & NA & 0.00043 & 0.0016 & 0.00079 & 0.007 & 0.105 & 0.081 & 9.85 & 1 \\
\hline 34,35 & 64 & 40 & 0.039 & 0.071 & NA & 0.00043 & 0.0016 & 0.00079 & 0.007 & 0.105 & 0.105 & 9.85 & 0 \\
\hline 34,35 & 65 & 23 & 0.058 & 0.089 & NA & 0.00059 & 0.0022 & 0.0011 & 0.01 & 0.146 & 0.01 & 9.91 & 2 \\
\hline 34,35 & 66 & 23 & 0.058 & 0.089 & $\mathrm{NA}$ & 0.00059 & 0.0022 & 0.0011 & 0.01 & 0.146 & 0.03 & 9.91 & 1 \\
\hline 34,35 & 67 & 23 & 0.058 & 0.089 & NA & 0.00059 & 0.0022 & 0.0011 & 0.01 & 0.146 & 0.05 & 9.91 & 0 \\
\hline 34,35 & 68 & 23 & 0.058 & 0.089 & $\mathrm{NA}$ & 0.00059 & 0.0022 & 0.0011 & 0.01 & 0.146 & 0.075 & 9.91 & 0 \\
\hline 34,35 & 69 & 30 & 0.058 & 0.089 & $\mathrm{NA}$ & 0.00059 & 0.0022 & 0.0011 & 0.01 & 0.146 & 0.01 & 9.91 & 2 \\
\hline 34,35 & 70 & 30 & 0.058 & 0.089 & $\mathrm{NA}$ & 0.00059 & 0.0022 & 0.0011 & 0.01 & 0.146 & 0.05 & 9.91 & 1 \\
\hline 34,35 & 71 & 30 & 0.058 & 0.089 & $\mathrm{NA}$ & 0.00059 & 0.0022 & 0.0011 & 0.01 & 0.146 & 0.075 & 9.91 & 0 \\
\hline 34,35 & 72 & 30 & 0.058 & 0.089 & NA & 0.00059 & 0.0022 & 0.0011 & 0.01 & 0.146 & 0.1 & 9.91 & 2 \\
\hline 34,35 & 73 & 30 & 0.058 & 0.089 & NA & 0.00059 & 0.0022 & 0.0011 & 0.01 & 0.146 & 0.15 & 9.91 & 0 \\
\hline 34,35 & 74 & 40 & 0.058 & 0.089 & $\mathrm{NA}$ & 0.00059 & 0.0022 & 0.0011 & 0.01 & 0.146 & 0.085 & 9.91 & 1 \\
\hline 34,35 & 75 & 40 & 0.058 & 0.089 & NA & 0.00059 & 0.0022 & 0.0011 & 0.01 & 0.146 & 0.112 & 9.91 & 0 \\
\hline
\end{tabular}


Cyclic Potentiodynamic Polarization Conditions, and Chemical Composition and pH of Historical Data (continued 3)

\begin{tabular}{|c|c|c|c|c|c|c|c|c|c|c|c|c|c|}
\hline Reference & Test & $\begin{array}{l}\text { Temperature } \\
\left({ }^{\circ} \mathrm{C}\right)\end{array}$ & $\begin{array}{l}\text { Carbonate } \\
\text { (M) }\end{array}$ & $\begin{array}{c}\text { Bi- } \\
\text { carbonate } \\
\text { (M) }\end{array}$ & $\begin{array}{l}\text { Citrate } \\
\text { (M) }\end{array}$ & $\begin{array}{l}\text { Phosphate } \\
\text { (M) }\end{array}$ & $\begin{array}{l}\text { Chloride } \\
\text { (M) }\end{array}$ & $\begin{array}{l}\text { Fluoride } \\
\text { (M) }\end{array}$ & $\begin{array}{l}\text { Sulfate } \\
\text { (M) }\end{array}$ & $\begin{array}{l}\text { Nitrate } \\
\text { (M) }\end{array}$ & $\begin{array}{l}\text { Nitrite } \\
\text { (M) }\end{array}$ & $\mathrm{pH}$ & Pit ID* \\
\hline 34,35 & 76 & 40 & 0.058 & 0.089 & $\mathrm{NA}$ & 0.00059 & 0.0022 & 0.0011 & 0.01 & 0.146 & 0.13 & 9.91 & 0 \\
\hline 34,35 & 77 & 40 & 0.058 & 0.089 & $\mathrm{NA}$ & 0.00059 & 0.0022 & 0.0011 & 0.01 & 0.146 & 0.15 & 9.91 & 0 \\
\hline 34,35 & 78 & 50 & 0.058 & 0.089 & NA & 0.00059 & 0.0022 & 0.0011 & 0.01 & 0.146 & 0.1 & 9.91 & 2 \\
\hline 34,35 & 79 & 50 & 0.058 & 0.089 & $\mathrm{NA}$ & 0.00059 & 0.0022 & 0.0011 & 0.01 & 0.146 & 0.5 & 9.91 & 1 \\
\hline 34,35 & 80 & 50 & 0.058 & 0.089 & NA & 0.00059 & 0.0022 & 0.0011 & 0.01 & 0.146 & 0.75 & 9.91 & 1 \\
\hline 34,35 & 81 & 50 & 0.058 & 0.089 & $\mathrm{NA}$ & 0.00059 & 0.0022 & 0.0011 & 0.01 & 0.146 & 1 & 9.91 & 2 \\
\hline 34,35 & 82 & 50 & 0.058 & 0.089 & NA & 0.00059 & 0.0022 & 0.0011 & 0.01 & 0.146 & 1.5 & 9.91 & 1 \\
\hline 34,35 & 83 & 50 & 0.058 & 0.089 & NA & 0.00059 & 0.0022 & 0.0011 & 0.01 & 0.146 & 2.25 & 9.91 & 0 \\
\hline 34,35 & 84 & 60 & 0.058 & 0.089 & $\mathrm{NA}$ & 0.00059 & 0.0022 & 0.0011 & 0.01 & 0.146 & 0.1 & 9.91 & 2 \\
\hline 34,35 & 85 & 60 & 0.058 & 0.089 & NA & 0.00059 & 0.0022 & 0.0011 & 0.01 & 0.146 & 0.5 & 9.91 & 0 \\
\hline 34,35 & 86 & 60 & 0.058 & 0.089 & NA & 0.00059 & 0.0022 & 0.0011 & 0.01 & 0.146 & 0.75 & 9.91 & 2 \\
\hline 34,35 & 87 & 60 & 0.058 & 0.089 & NA & 0.00059 & 0.0022 & 0.0011 & 0.01 & 0.146 & 1.5 & 9.91 & 1 \\
\hline 34,35 & 88 & 60 & 0.058 & 0.089 & NA & 0.00059 & 0.0022 & 0.0011 & 0.01 & 0.146 & 2.25 & 9.91 & 0 \\
\hline 34,35 & 89 & 60 & 0.058 & 0.089 & $\mathrm{NA}$ & 0.00059 & 0.0022 & 0.0011 & 0.01 & 0.146 & 3 & 9.91 & 0 \\
\hline 34,35 & 90 & 40 & 0.086 & 0.111 & NA & 0.00082 & 0.0031 & 0.0015 & 0.013 & 0.202 & 0.156 & 9.96 & 1 \\
\hline 34,35 & 91 & 40 & 0.086 & 0.111 & $\mathrm{NA}$ & 0.00082 & 0.0031 & 0.0015 & 0.013 & 0.202 & 0.18 & 9.96 & 0 \\
\hline 34,35 & 92 & 40 & 0.13 & 0.139 & NA & 0.0011 & 0.0042 & 0.0021 & 0.019 & 0.28 & 0.216 & 10.01 & 2 \\
\hline 34,35 & 93 & 40 & 0.13 & 0.139 & NA & 0.0011 & 0.0042 & 0.0021 & 0.019 & 0.28 & 0.28 & 10.01 & 0 \\
\hline 34,35 & 94 & 23 & 0.187 & 0.173 & $\mathrm{NA}$ & 0.0016 & 0.0059 & 0.0029 & 0.026 & 0.388 & 0.03 & 10.06 & 1 \\
\hline 34,35 & 95 & 23 & 0.187 & 0.173 & $\mathrm{NA}$ & 0.0016 & 0.0059 & 0.0029 & 0.026 & 0.388 & 0.05 & 10.06 & 0 \\
\hline 34,35 & 96 & 23 & 0.187 & 0.173 & $\mathrm{NA}$ & 0.0016 & 0.0059 & 0.0029 & 0.026 & 0.388 & 0.1 & 10.06 & 0 \\
\hline 34,35 & 97 & 30 & 0.187 & 0.173 & NA & 0.0016 & 0.0059 & 0.0029 & 0.026 & 0.388 & 0.05 & 10.06 & 2 \\
\hline 34,35 & 98 & 30 & 0.187 & 0.173 & NA & 0.0016 & 0.0059 & 0.0029 & 0.026 & 0.388 & 0.1 & 10.06 & 0 \\
\hline 34,35 & 99 & 30 & 0.187 & 0.173 & $\mathrm{NA}$ & 0.0016 & 0.0059 & 0.0029 & 0.026 & 0.388 & 0.1 & 10.06 & 0 \\
\hline 34,35 & 100 & 30 & 0.187 & 0.173 & NA & 0.0016 & 0.0059 & 0.0029 & 0.026 & 0.388 & 0.15 & 10.06 & 0 \\
\hline
\end{tabular}


Cyclic Potentiodynamic Polarization Conditions, and Chemical Composition and pH of Historical Data (continued 4)

\begin{tabular}{|c|c|c|c|c|c|c|c|c|c|c|c|c|c|}
\hline Reference & Test & $\begin{array}{l}\text { Temperature } \\
\left({ }^{\circ} \mathrm{C}\right)\end{array}$ & $\begin{array}{l}\text { Carbonate } \\
\text { (M) }\end{array}$ & $\begin{array}{c}\text { Bi- } \\
\text { carbonate } \\
\text { (M) }\end{array}$ & $\begin{array}{l}\text { Citrate } \\
\text { (M) }\end{array}$ & $\begin{array}{l}\text { Phosphate } \\
\text { (M) }\end{array}$ & $\begin{array}{l}\text { Chloride } \\
\text { (M) }\end{array}$ & $\begin{array}{l}\text { Fluoride } \\
\text { (M) }\end{array}$ & $\begin{array}{l}\text { Sulfate } \\
\text { (M) }\end{array}$ & $\begin{array}{l}\text { Nitrate } \\
\text { (M) }\end{array}$ & $\begin{array}{l}\text { Nitrite } \\
\text { (M) }\end{array}$ & $\mathrm{pH}$ & Pit ID* \\
\hline 34,35 & 101 & 40 & 0.187 & 0.173 & $\mathrm{NA}$ & 0.0016 & 0.0059 & 0.0029 & 0.026 & 0.388 & 0.299 & 10.06 & 1 \\
\hline 34,35 & 102 & 40 & 0.187 & 0.173 & $\mathrm{NA}$ & 0.0016 & 0.0059 & 0.0029 & 0.026 & 0.388 & 0.35 & 10.06 & 1 \\
\hline 34,35 & 103 & 40 & 0.187 & 0.173 & NA & 0.0016 & 0.0059 & 0.0029 & 0.026 & 0.388 & 0.45 & 10.06 & 0 \\
\hline 34,35 & 104 & 50 & 0.187 & 0.173 & $\mathrm{NA}$ & 0.0016 & 0.0059 & 0.0029 & 0.026 & 0.388 & 0.7 & 10.06 & 1 \\
\hline 34,35 & 105 & 50 & 0.187 & 0.173 & NA & 0.0016 & 0.0059 & 0.0029 & 0.026 & 0.388 & 0.8 & 10.06 & 1 \\
\hline 34,35 & 106 & 50 & 0.187 & 0.173 & NA & 0.0016 & 0.0059 & 0.0029 & 0.026 & 0.388 & 1 & 10.06 & 0 \\
\hline 34,35 & 107 & 50 & 0.187 & 0.173 & NA & 0.0016 & 0.0059 & 0.0029 & 0.026 & 0.388 & 1.3 & 10.06 & 0 \\
\hline 34,35 & 108 & 60 & 0.187 & 0.173 & NA & 0.0016 & 0.0059 & 0.0029 & 0.026 & 0.388 & 0.4 & 10.06 & 2 \\
\hline 34,35 & 109 & 60 & 0.187 & 0.173 & $\mathrm{NA}$ & 0.0016 & 0.0059 & 0.0029 & 0.026 & 0.388 & 0.8 & 10.06 & 2 \\
\hline 34,35 & 110 & 60 & 0.187 & 0.173 & NA & 0.0016 & 0.0059 & 0.0029 & 0.026 & 0.388 & 1 & 10.06 & 2 \\
\hline 34,35 & 111 & 60 & 0.187 & 0.173 & NA & 0.0016 & 0.0059 & 0.0029 & 0.026 & 0.388 & 2 & 10.06 & 2 \\
\hline 34,35 & 112 & 60 & 0.187 & 0.173 & NA & 0.0016 & 0.0059 & 0.0029 & 0.026 & 0.388 & 3 & 10.06 & 0 \\
\hline 34,35 & 113 & 40 & 0.272 & 0.216 & NA & 0.0022 & 0.0081 & 0.0041 & 0.036 & 0.538 & 0.415 & 10.12 & 1 \\
\hline 34,35 & 114 & 40 & 0.272 & 0.216 & $\mathrm{NA}$ & 0.0022 & 0.0081 & 0.0041 & 0.036 & 0.538 & 0.5 & 10.12 & 1 \\
\hline 34,35 & 115 & 40 & 0.272 & 0.216 & NA & 0.0022 & 0.0081 & 0.0041 & 0.036 & 0.538 & 0.65 & 10.12 & 0 \\
\hline 34,35 & 116 & 40 & 0.398 & 0.268 & $\mathrm{NA}$ & 0.003 & 0.011 & 0.0056 & 0.059 & 0.746 & 0.3 & 10.17 & 2 \\
\hline 34,35 & 117 & 40 & 0.398 & 0.268 & NA & 0.003 & 0.011 & 0.0056 & 0.059 & 0.746 & 0.4 & 10.17 & 1 \\
\hline 34,35 & 118 & 40 & 0.398 & 0.268 & $\mathrm{NA}$ & 0.003 & 0.011 & 0.0056 & 0.059 & 0.746 & 0.45 & 10.17 & 0 \\
\hline 34,35 & 119 & 40 & 0.398 & 0.268 & $\mathrm{NA}$ & 0.003 & 0.011 & 0.0056 & 0.059 & 0.746 & 0.575 & 10.17 & 0 \\
\hline 34,35 & 120 & 40 & 0.575 & 0.332 & $\mathrm{NA}$ & 0.0042 & 0.016 & 0.0078 & 0.069 & 1.03 & 0.5 & 10.22 & 0 \\
\hline 34,35 & 121 & 40 & 0.575 & 0.332 & $\mathrm{NA}$ & 0.0042 & 0.016 & 0.0078 & 0.069 & 1.03 & 0.798 & 10.22 & 0 \\
\hline 34,35 & 122 & 40 & 0.575 & 0.332 & NA & 0.0042 & 0.016 & 0.0078 & 0.069 & 1.03 & 1.03 & 10.22 & 0 \\
\hline 34,35 & 123 & 40 & 0.831 & 0.409 & NA & 0.0059 & 0.022 & 0.011 & 0.095 & 1.44 & 0.6 & 10.28 & 1 \\
\hline 34,35 & 124 & 40 & 0.831 & 0.409 & $\mathrm{NA}$ & 0.0059 & 0.022 & 0.011 & 0.095 & 1.44 & 1.11 & 10.28 & 0 \\
\hline 36 & 125 & 40 & 0.013 & 0.058 & $\mathrm{NA}$ & 0.00039 & 0.0011 & 0.00073 & 0.0059 & 0.002 & 0.010 & 9.6 & 2 \\
\hline
\end{tabular}


Cyclic Potentiodynamic Polarization Conditions, and Chemical Composition and pH of Historical Data (continued 5)

\begin{tabular}{|c|c|c|c|c|c|c|c|c|c|c|c|c|c|}
\hline Reference & Test & $\begin{array}{l}\text { Temperature } \\
\left({ }^{\circ} \mathrm{C}\right)\end{array}$ & $\begin{array}{l}\text { Carbonate } \\
\text { (M) }\end{array}$ & $\begin{array}{c}\text { Bi- } \\
\text { carbonate } \\
\text { (M) }\end{array}$ & $\begin{array}{l}\text { Citrate } \\
\text { (M) }\end{array}$ & $\begin{array}{l}\text { Phosphate } \\
\text { (M) }\end{array}$ & $\begin{array}{l}\text { Chloride } \\
\text { (M) }\end{array}$ & $\begin{array}{l}\text { Fluoride } \\
\text { (M) }\end{array}$ & $\begin{array}{l}\text { Sulfate } \\
\text { (M) }\end{array}$ & $\begin{array}{l}\text { Nitrate } \\
\text { (M) }\end{array}$ & $\begin{array}{l}\text { Nitrite } \\
\text { (M) }\end{array}$ & $\mathrm{pH}$ & Pit ID* \\
\hline 36 & 126 & 40 & 0.013 & 0.058 & $\mathrm{NA}$ & 0.00039 & 0.0011 & 0.00073 & 0.0059 & 0.002 & 0.015 & 9.6 & 2 \\
\hline 36 & 127 & 40 & 0.013 & 0.058 & $\mathrm{NA}$ & 0.00039 & 0.0011 & 0.00073 & 0.0059 & 0.002 & 0.020 & 9.6 & 2 \\
\hline 36 & 128 & 40 & 0.013 & 0.058 & NA & 0.00039 & 0.0011 & 0.00073 & 0.0059 & 0.002 & 0.025 & 9.6 & 0 \\
\hline 36 & 129 & 40 & 0.013 & 0.058 & $\mathrm{NA}$ & 0.00039 & 0.0011 & 0.00073 & 0.0059 & 0.0085 & 0.015 & 9.6 & 2 \\
\hline 36 & 130 & 40 & 0.013 & 0.058 & NA & 0.00039 & 0.0011 & 0.00073 & 0.0059 & 0.0085 & 0.025 & 9.6 & 1 \\
\hline 36 & 131 & 40 & 0.013 & 0.058 & $\mathrm{NA}$ & 0.00039 & 0.0011 & 0.00073 & 0.0059 & 0.0200 & 0.020 & 9.6 & 1 \\
\hline 36 & 132 & 40 & 0.013 & 0.058 & NA & 0.00039 & 0.0011 & 0.00073 & 0.0059 & 0.0300 & 0.025 & 9.6 & 2 \\
\hline 36 & 133 & 40 & 0.013 & 0.058 & NA & 0.00039 & 0.0011 & 0.00073 & 0.0059 & 0.0400 & 0.025 & 9.6 & 1 \\
\hline 36 & 134 & 40 & 0.013 & 0.058 & $\mathrm{NA}$ & 0.00039 & 0.0011 & 0.00073 & 0.0059 & 0.0500 & 0.025 & 9.6 & 2 \\
\hline 36 & 135 & 40 & 0.013 & 0.058 & NA & 0.00039 & 0.0011 & 0.00073 & 0.0059 & 0.0500 & 0.050 & 9.6 & 0 \\
\hline 36 & 136 & 40 & 0.013 & 0.058 & NA & 0.00039 & 0.0011 & 0.00073 & 0.0059 & 0.0600 & 0.025 & 9.6 & 2 \\
\hline 36 & 137 & 40 & 0.013 & 0.058 & NA & 0.00039 & 0.0011 & 0.00073 & 0.0059 & 0.0600 & 0.040 & 9.6 & 2 \\
\hline 36 & 138 & 40 & 0.013 & 0.058 & NA & 0.00039 & 0.0011 & 0.00073 & 0.0059 & 0.0600 & 0.050 & 9.6 & 1 \\
\hline 36 & 139 & 40 & 0.013 & 0.058 & NA & 0.00039 & 0.0011 & 0.00073 & 0.0059 & 0.0600 & 0.060 & 9.6 & 0 \\
\hline 36 & 140 & 40 & 0.013 & 0.058 & NA & 0.00039 & 0.0011 & 0.00073 & 0.0059 & 0.0600 & 0.120 & 9.6 & 0 \\
\hline 36 & 141 & 40 & 0.013 & 0.058 & $\mathrm{NA}$ & 0.00039 & 0.0011 & 0.00073 & 0.0059 & 0.0600 & 0.220 & 9.6 & 0 \\
\hline 36 & 142 & 40 & 0.013 & 0.058 & NA & 0.00039 & 0.0011 & 0.00073 & 0.0059 & 0.0700 & 0.070 & 9.6 & 0 \\
\hline 36 & 143 & 40 & 0.013 & 0.058 & $\mathrm{NA}$ & 0.00039 & 0.0011 & 0.00073 & 0.0059 & 0.0800 & 0.080 & 9.6 & 0 \\
\hline 36 & 144 & 40 & 0.013 & 0.058 & $\mathrm{NA}$ & 0.00039 & 0.0011 & 0.00073 & 0.0059 & 0.2000 & 0.025 & 9.6 & 2 \\
\hline 36 & 145 & 40 & 0.013 & 0.058 & $\mathrm{NA}$ & 0.00039 & 0.0011 & 0.00073 & 0.0059 & 0.3000 & 0.100 & 9.6 & 1 \\
\hline 36 & 146 & 40 & 0.013 & 0.058 & $\mathrm{NA}$ & 0.00039 & 0.0011 & 0.00073 & 0.0059 & 0.3000 & 0.150 & 9.6 & 0 \\
\hline 36 & 147 & 40 & 0.013 & 0.058 & $\mathrm{NA}$ & 0.00039 & 0.0011 & 0.00073 & 0.0032 & 0.085 & 0.040 & 9.6 & 2 \\
\hline 36 & 148 & 40 & 0.013 & 0.058 & NA & 0.00039 & 0.0011 & 0.00073 & 0.0032 & 0.085 & 0.060 & 9.6 & 0 \\
\hline 36 & 149 & 40 & 0.013 & 0.058 & $\mathrm{NA}$ & 0.00039 & 0.0011 & 0.00073 & 0.0060 & 0.085 & 0.025 & 9.6 & 2 \\
\hline 36 & 150 & 40 & 0.013 & 0.058 & NA & 0.00039 & 0.0011 & 0.00073 & 0.0060 & 0.085 & 0.040 & 9.6 & 2 \\
\hline
\end{tabular}


Cyclic Potentiodynamic Polarization Conditions, and Chemical Composition and pH of Historical Data (continued 6)

\begin{tabular}{|c|c|c|c|c|c|c|c|c|c|c|c|c|c|}
\hline Reference & Test & $\begin{array}{l}\text { Temperature } \\
\left({ }^{\circ} \mathrm{C}\right)\end{array}$ & $\begin{array}{l}\text { Carbonate } \\
\text { (M) }\end{array}$ & $\begin{array}{c}\text { Bi- } \\
\text { carbonate } \\
\text { (M) }\end{array}$ & $\begin{array}{l}\text { Citrate } \\
\text { (M) }\end{array}$ & $\begin{array}{l}\text { Phosphate } \\
\text { (M) }\end{array}$ & $\begin{array}{l}\text { Chloride } \\
\text { (M) }\end{array}$ & $\begin{array}{l}\text { Fluoride } \\
\text { (M) }\end{array}$ & $\begin{array}{l}\text { Sulfate } \\
\text { (M) }\end{array}$ & $\begin{array}{l}\text { Nitrate } \\
\text { (M) }\end{array}$ & $\begin{array}{l}\text { Nitrite } \\
\text { (M) }\end{array}$ & $\mathrm{pH}$ & Pit ID* \\
\hline 36 & 151 & 40 & 0.013 & 0.058 & $\mathrm{NA}$ & 0.00039 & 0.0011 & 0.00073 & 0.0060 & 0.085 & 0.050 & 9.6 & 1 \\
\hline 36 & 152 & 40 & 0.013 & 0.058 & $\mathrm{NA}$ & 0.00039 & 0.0011 & 0.00073 & 0.0060 & 0.085 & 0.060 & 9.6 & 0 \\
\hline 36 & 153 & 40 & 0.013 & 0.058 & NA & 0.00039 & 0.0011 & 0.00073 & 0.0060 & 0.085 & 0.080 & 9.6 & 0 \\
\hline 36 & 154 & 40 & 0.013 & 0.058 & $\mathrm{NA}$ & 0.00039 & 0.0011 & 0.00073 & 0.0060 & 0.085 & 0.100 & 9.6 & 0 \\
\hline 36 & 155 & 40 & 0.013 & 0.058 & NA & 0.00039 & 0.0011 & 0.00073 & 0.0085 & 0.085 & 0.040 & 9.6 & 2 \\
\hline 36 & 156 & 40 & 0.013 & 0.058 & NA & 0.00039 & 0.0011 & 0.00073 & 0.0085 & 0.085 & 0.060 & 9.6 & 0 \\
\hline 36 & 157 & 40 & 0.013 & 0.058 & NA & 0.00039 & 0.0011 & 0.00073 & 0.0085 & 0.085 & 0.100 & 9.6 & 0 \\
\hline 36 & 158 & 40 & 0.013 & 0.058 & NA & 0.00039 & 0.0011 & 0.00073 & 0.0100 & 0.085 & 0.025 & 9.6 & 2 \\
\hline 36 & 159 & 40 & 0.013 & 0.058 & $\mathrm{NA}$ & 0.00039 & 0.0011 & 0.00073 & 0.0120 & 0.085 & 0.025 & 9.6 & 2 \\
\hline 36 & 160 & 40 & 0.013 & 0.058 & NA & 0.00039 & 0.0011 & 0.00073 & 0.0120 & 0.085 & 0.040 & 9.6 & 2 \\
\hline 36 & 161 & 40 & 0.013 & 0.058 & NA & 0.00039 & 0.0011 & 0.00073 & 0.0120 & 0.085 & 0.060 & 9.6 & 0 \\
\hline 36 & 162 & 40 & 0.013 & 0.058 & NA & 0.00039 & 0.0011 & 0.00073 & 0.0170 & 0.085 & 0.025 & 9.6 & 2 \\
\hline 36 & 163 & 40 & 0.013 & 0.058 & NA & 0.00039 & 0.0011 & 0.00073 & 0.0170 & 0.085 & 0.040 & 9.6 & 2 \\
\hline 36 & 164 & 40 & 0.013 & 0.058 & NA & 0.00039 & 0.0011 & 0.00073 & 0.0200 & 0.085 & 0.060 & 9.6 & 1 \\
\hline 36 & 165 & 40 & 0.013 & 0.058 & NA & 0.00039 & 0.0011 & 0.00073 & 0.0300 & 0.085 & 0.070 & 9.6 & 0 \\
\hline 36 & 166 & 40 & 0.013 & 0.058 & $\mathrm{NA}$ & 0.00039 & 0.0011 & 0.00073 & 0.0300 & 0.085 & 0.080 & 9.6 & 0 \\
\hline 36 & 167 & 40 & 0.013 & 0.058 & NA & 0.00039 & 0.0011 & 0.00073 & 0.0400 & 0.085 & 0.070 & 9.6 & 1 \\
\hline 36 & 168 & 40 & 0.013 & 0.058 & NA & 0.00039 & 0.0011 & 0.00073 & 0.0600 & 0.085 & 0.060 & 9.6 & 1 \\
\hline 36 & 169 & 40 & 0.013 & 0.058 & $\mathrm{NA}$ & 0.00039 & 0.0011 & 0.00073 & 0.0700 & 0.085 & 0.100 & 9.6 & 0 \\
\hline 36 & 170 & 40 & 0.013 & 0.058 & $\mathrm{NA}$ & 0.00039 & 0.0011 & 0.00073 & 0.0900 & 0.085 & 0.040 & 9.6 & 2 \\
\hline 36 & 171 & 40 & 0.013 & 0.058 & $\mathrm{NA}$ & 0.00039 & 0.0011 & 0.00073 & 0.0900 & 0.085 & 0.065 & 9.6 & 1 \\
\hline 36 & 172 & 40 & 0.013 & 0.058 & NA & 0.00039 & 0.0011 & 0.00073 & 0.0900 & 0.085 & 0.080 & 9.6 & 1 \\
\hline 36 & 173 & 40 & 0.013 & 0.058 & NA & 0.00039 & 0.0011 & 0.00073 & 0.1800 & 0.085 & 0.080 & 9.6 & 2 \\
\hline 36 & 174 & 40 & 0.013 & 0.058 & $\mathrm{NA}$ & 0.00039 & 0.0011 & 0.00073 & 0.1800 & 0.085 & 0.100 & 9.6 & 2 \\
\hline 36 & 175 & 40 & 0.013 & 0.058 & NA & 0.00039 & 0.0011 & 0.00073 & 0.2000 & 0.085 & 0.120 & 9.6 & 2 \\
\hline
\end{tabular}


Cyclic Potentiodynamic Polarization Conditions, and Chemical Composition and pH of Historical Data (continued 7)

\begin{tabular}{|c|c|c|c|c|c|c|c|c|c|c|c|c|c|}
\hline Reference & Test & $\begin{array}{l}\text { Temperature } \\
\left({ }^{\circ} \mathrm{C}\right)\end{array}$ & $\begin{array}{l}\text { Carbonate } \\
\text { (M) }\end{array}$ & $\begin{array}{c}\text { Bi- } \\
\text { carbonate } \\
\text { (M) }\end{array}$ & $\begin{array}{l}\text { Citrate } \\
\text { (M) }\end{array}$ & $\begin{array}{l}\text { Phosphate } \\
\text { (M) }\end{array}$ & $\begin{array}{l}\text { Chloride } \\
\text { (M) }\end{array}$ & $\begin{array}{l}\text { Fluoride } \\
\text { (M) }\end{array}$ & $\begin{array}{l}\text { Sulfate } \\
\text { (M) }\end{array}$ & $\begin{array}{l}\text { Nitrate } \\
\text { (M) }\end{array}$ & $\begin{array}{l}\text { Nitrite } \\
\text { (M) }\end{array}$ & $\mathrm{pH}$ & Pit ID* \\
\hline 36 & 176 & 40 & 0.013 & 0.058 & $\mathrm{NA}$ & 0.00039 & 0.0011 & 0.00073 & 0.2000 & 0.085 & 0.150 & 9.6 & 1 \\
\hline 36 & 177 & 40 & 0.013 & 0.058 & $\mathrm{NA}$ & 0.00039 & 0.0011 & 0.00073 & 0.2000 & 0.085 & 0.170 & 9.6 & 0 \\
\hline 36 & 178 & 40 & 0.013 & 0.058 & NA & 0.00039 & 0.0003 & 0.00073 & 0.0059 & 0.085 & 0.040 & 9.6 & 1 \\
\hline 36 & 179 & 40 & 0.013 & 0.058 & $\mathrm{NA}$ & 0.00039 & 0.0003 & 0.00073 & 0.0059 & 0.085 & 0.060 & 9.6 & 0 \\
\hline 36 & 180 & 40 & 0.013 & 0.058 & NA & 0.00039 & 0.0011 & 0.00073 & 0.0059 & 0.085 & 0.025 & 9.6 & 2 \\
\hline 36 & 181 & 40 & 0.013 & 0.058 & NA & 0.00039 & 0.0011 & 0.00073 & 0.0059 & 0.085 & 0.040 & 9.6 & 2 \\
\hline 36 & 182 & 40 & 0.013 & 0.058 & NA & 0.00039 & 0.0011 & 0.00073 & 0.0059 & 0.085 & 0.050 & 9.6 & 1 \\
\hline 36 & 183 & 40 & 0.013 & 0.058 & NA & 0.00039 & 0.0011 & 0.00073 & 0.0059 & 0.085 & 0.060 & 9.6 & 0 \\
\hline 36 & 184 & 40 & 0.013 & 0.058 & $\mathrm{NA}$ & 0.00039 & 0.0011 & 0.00073 & 0.0059 & 0.085 & 0.110 & 9.6 & 0 \\
\hline 36 & 185 & 40 & 0.013 & 0.058 & NA & 0.00039 & 0.0015 & 0.00073 & 0.0059 & 0.085 & 0.040 & 9.6 & 2 \\
\hline 36 & 186 & 40 & 0.013 & 0.058 & NA & 0.00039 & 0.0015 & 0.00073 & 0.0059 & 0.085 & 0.060 & 9.6 & 0 \\
\hline 36 & 187 & 40 & 0.013 & 0.058 & NA & 0.00039 & 0.0015 & 0.00073 & 0.0059 & 0.085 & 0.080 & 9.6 & 0 \\
\hline 36 & 188 & 40 & 0.013 & 0.058 & NA & 0.00039 & 0.0015 & 0.00073 & 0.0059 & 0.085 & 0.100 & 9.6 & 0 \\
\hline 36 & 189 & 40 & 0.013 & 0.058 & NA & 0.00039 & 0.0015 & 0.00073 & 0.0059 & 0.085 & 0.110 & 9.6 & 0 \\
\hline 36 & 190 & 40 & 0.013 & 0.058 & NA & 0.00039 & 0.0017 & 0.00073 & 0.0059 & 0.085 & 0.040 & 9.6 & 1 \\
\hline 36 & 191 & 40 & 0.013 & 0.058 & $\mathrm{NA}$ & 0.00039 & 0.0017 & 0.00073 & 0.0059 & 0.085 & 0.060 & 9.6 & 0 \\
\hline 36 & 192 & 40 & 0.013 & 0.058 & NA & 0.00039 & 0.0017 & 0.00073 & 0.0059 & 0.085 & 0.080 & 9.6 & 0 \\
\hline 36 & 193 & 40 & 0.013 & 0.058 & NA & 0.00039 & 0.0017 & 0.00073 & 0.0059 & 0.085 & 0.110 & 9.6 & 0 \\
\hline 36 & 194 & 40 & 0.013 & 0.058 & $\mathrm{NA}$ & 0.00039 & 0.0017 & 0.00073 & 0.0059 & 0.085 & 0.170 & 9.6 & 0 \\
\hline 36 & 195 & 40 & 0.013 & 0.058 & $\mathrm{NA}$ & 0.00039 & 0.0060 & 0.00073 & 0.0059 & 0.085 & 0.065 & 9.6 & 0 \\
\hline 36 & 196 & 40 & 0.013 & 0.058 & $\mathrm{NA}$ & 0.00039 & 0.0075 & 0.00073 & 0.0059 & 0.085 & 0.065 & 9.6 & 0 \\
\hline 36 & 197 & 40 & 0.013 & 0.058 & $\mathrm{NA}$ & 0.00039 & 0.0090 & 0.00073 & 0.0059 & 0.085 & 0.065 & 9.6 & 1 \\
\hline 36 & 198 & 40 & 0.013 & 0.058 & NA & 0.00039 & 0.0100 & 0.00073 & 0.0059 & 0.085 & 0.050 & 9.6 & 2 \\
\hline 36 & 199 & 40 & 0.013 & 0.058 & $\mathrm{NA}$ & 0.00039 & 0.0105 & 0.00073 & 0.0059 & 0.085 & 0.120 & 9.6 & 0 \\
\hline 36 & 200 & 40 & 0.013 & 0.058 & NA & 0.00039 & 0.0110 & 0.00073 & 0.0059 & 0.085 & 0.100 & 9.6 & 2 \\
\hline
\end{tabular}


Cyclic Potentiodynamic Polarization Conditions, and Chemical Composition and pH of Historical Data (continued 8)

\begin{tabular}{|c|c|c|c|c|c|c|c|c|c|c|c|c|c|}
\hline Reference & Test & $\begin{array}{l}\text { Temperature } \\
\left({ }^{\circ} \mathrm{C}\right)\end{array}$ & $\begin{array}{l}\text { Carbonate } \\
\text { (M) }\end{array}$ & $\begin{array}{c}\text { Bi- } \\
\text { carbonate } \\
\text { (M) }\end{array}$ & $\begin{array}{l}\text { Citrate } \\
\text { (M) }\end{array}$ & $\begin{array}{l}\text { Phosphate } \\
\text { (M) }\end{array}$ & $\begin{array}{l}\text { Chloride } \\
\text { (M) }\end{array}$ & $\begin{array}{l}\text { Fluoride } \\
\text { (M) }\end{array}$ & $\begin{array}{l}\text { Sulfate } \\
\text { (M) }\end{array}$ & $\begin{array}{l}\text { Nitrate } \\
\text { (M) }\end{array}$ & $\begin{array}{l}\text { Nitrite } \\
\text { (M) }\end{array}$ & $\mathrm{pH}$ & Pit ID* \\
\hline 36 & 201 & 40 & 0.013 & 0.058 & $\mathrm{NA}$ & 0.00039 & 0.0120 & 0.00073 & 0.0059 & 0.085 & 0.200 & 9.6 & 0 \\
\hline 36 & 202 & 40 & 0.013 & 0.058 & $\mathrm{NA}$ & 0.00039 & 0.0150 & 0.00073 & 0.0059 & 0.085 & 0.300 & 9.6 & 0 \\
\hline 36 & 203 & 40 & 0.013 & 0.058 & NA & 0.00039 & 0.0200 & 0.00073 & 0.0059 & 0.085 & 0.060 & 9.6 & 2 \\
\hline 36 & 204 & 40 & 0.013 & 0.058 & $\mathrm{NA}$ & 0.00039 & 0.0200 & 0.00073 & 0.0059 & 0.085 & 0.200 & 9.6 & 2 \\
\hline 36 & 205 & 40 & 0.013 & 0.058 & NA & 0.00039 & 0.0200 & 0.00073 & 0.0059 & 0.085 & 0.300 & 9.6 & 0 \\
\hline 36 & 206 & 40 & 0.013 & 0.058 & NA & 0.00039 & 0.0220 & 0.00073 & 0.0059 & 0.085 & 0.300 & 9.6 & 2 \\
\hline 36 & 207 & 40 & 0.013 & 0.058 & NA & 0.00039 & 0.0220 & 0.00073 & 0.0059 & 0.085 & 0.350 & 9.6 & 2 \\
\hline 36 & 208 & 40 & 0.013 & 0.058 & NA & 0.00039 & 0.0400 & 0.00073 & 0.0059 & 0.085 & 0.060 & 9.6 & 2 \\
\hline 36 & 209 & 40 & 0.013 & 0.058 & $\mathrm{NA}$ & 0.00039 & 0.0400 & 0.00073 & 0.0059 & 0.085 & 0.100 & 9.6 & 2 \\
\hline 36 & 210 & 40 & 0.013 & 0.058 & NA & 0.00039 & 0.0400 & 0.00073 & 0.0059 & 0.085 & 0.200 & 9.6 & 2 \\
\hline 36 & 211 & 40 & 0.013 & 0.058 & NA & 0.00039 & 0.0011 & 0.00024 & 0.0059 & 0.085 & 0.050 & 9.6 & 1 \\
\hline 36 & 212 & 40 & 0.013 & 0.058 & NA & 0.00039 & 0.0011 & 0.00024 & 0.0059 & 0.085 & 0.060 & 9.6 & 0 \\
\hline 36 & 213 & 40 & 0.013 & 0.058 & NA & 0.00039 & 0.0011 & 0.0005 & 0.0059 & 0.085 & 0.050 & 9.6 & 2 \\
\hline 36 & 214 & 40 & 0.013 & 0.058 & NA & 0.00039 & 0.0011 & 0.00073 & 0.0059 & 0.085 & 0.025 & 9.6 & 2 \\
\hline 36 & 215 & 40 & 0.013 & 0.058 & NA & 0.00039 & 0.0011 & 0.00073 & 0.0059 & 0.085 & 0.040 & 9.6 & 2 \\
\hline 36 & 216 & 40 & 0.013 & 0.058 & $\mathrm{NA}$ & 0.00039 & 0.0011 & 0.00073 & 0.0059 & 0.085 & 0.050 & 9.6 & 1 \\
\hline 36 & 217 & 40 & 0.013 & 0.058 & NA & 0.00039 & 0.0011 & 0.00073 & 0.0059 & 0.085 & 0.060 & 9.6 & 0 \\
\hline 36 & 218 & 40 & 0.013 & 0.058 & NA & 0.00039 & 0.0011 & 0.00073 & 0.0059 & 0.085 & 0.080 & 9.6 & 0 \\
\hline 36 & 219 & 40 & 0.013 & 0.058 & $\mathrm{NA}$ & 0.00039 & 0.0011 & 0.0011 & 0.0059 & 0.085 & 0.060 & 9.6 & 0 \\
\hline 36 & 220 & 40 & 0.013 & 0.058 & $\mathrm{NA}$ & 0.00039 & 0.0011 & 0.0013 & 0.0059 & 0.085 & 0.060 & 9.6 & 1 \\
\hline 36 & 221 & 40 & 0.013 & 0.058 & $\mathrm{NA}$ & 0.00039 & 0.0011 & 0.0013 & 0.0059 & 0.085 & 0.070 & 9.6 & 0 \\
\hline 36 & 222 & 40 & 0.013 & 0.058 & $\mathrm{NA}$ & 0.00039 & 0.0011 & 0.0013 & 0.0059 & 0.085 & 0.080 & 9.6 & 0 \\
\hline 36 & 223 & 40 & 0.013 & 0.058 & NA & 0.00039 & 0.0011 & 0.002 & 0.0059 & 0.085 & 0.065 & 9.6 & 0 \\
\hline 36 & 224 & 40 & 0.013 & 0.058 & $\mathrm{NA}$ & 0.00039 & 0.0011 & 0.004 & 0.0059 & 0.085 & 0.050 & 9.6 & 0 \\
\hline 36 & 225 & 40 & 0.013 & 0.058 & NA & 0.00039 & 0.0011 & 0.004 & 0.0059 & 0.085 & 0.065 & 9.6 & 0 \\
\hline
\end{tabular}


Cyclic Potentiodynamic Polarization Conditions, and Chemical Composition and pH of Historical Data (continued 9)

\begin{tabular}{|c|c|c|c|c|c|c|c|c|c|c|c|c|c|}
\hline Reference & Test & $\begin{array}{l}\text { Temperature } \\
\left({ }^{\circ} \mathrm{C}\right)\end{array}$ & $\begin{array}{l}\text { Carbonate } \\
\text { (M) }\end{array}$ & $\begin{array}{c}\text { Bi- } \\
\text { carbonate } \\
\text { (M) }\end{array}$ & $\begin{array}{l}\text { Citrate } \\
\text { (M) }\end{array}$ & $\begin{array}{l}\text { Phosphate } \\
\text { (M) }\end{array}$ & $\begin{array}{l}\text { Chloride } \\
\text { (M) }\end{array}$ & $\begin{array}{l}\text { Fluoride } \\
\text { (M) }\end{array}$ & $\begin{array}{l}\text { Sulfate } \\
\text { (M) }\end{array}$ & $\begin{array}{l}\text { Nitrate } \\
\text { (M) }\end{array}$ & $\begin{array}{l}\text { Nitrite } \\
\text { (M) }\end{array}$ & $\mathrm{pH}$ & Pit ID* \\
\hline 36 & 226 & 40 & 0.013 & 0.058 & $\mathrm{NA}$ & 0.00039 & 0.0011 & 0.01 & 0.0059 & 0.085 & 0.050 & 9.6 & 2 \\
\hline 36 & 227 & 40 & 0.013 & 0.058 & $\mathrm{NA}$ & 0.00039 & 0.0011 & 0.01 & 0.0059 & 0.085 & 0.065 & 9.6 & 0 \\
\hline 36 & 228 & 40 & 0.013 & 0.058 & NA & 0.00039 & 0.0011 & 0.025 & 0.0059 & 0.085 & 0.065 & 9.6 & 0 \\
\hline 36 & 229 & 40 & 0.013 & 0.058 & $\mathrm{NA}$ & 0.00039 & 0.0011 & 0.03 & 0.0059 & 0.085 & 0.065 & 9.6 & 1 \\
\hline 36 & 230 & 40 & 0.013 & 0.058 & NA & 0.00039 & 0.0011 & 0.04 & 0.0059 & 0.085 & 0.075 & 9.6 & 2 \\
\hline 36 & 231 & 40 & 0.013 & 0.058 & $\mathrm{NA}$ & 0.00039 & 0.0011 & 0.04 & 0.0059 & 0.085 & 0.085 & 9.6 & 0 \\
\hline 36 & 232 & 40 & 0.013 & 0.058 & NA & 0.00039 & 0.0011 & 0.05 & 0.0059 & 0.085 & 0.065 & 9.6 & 2 \\
\hline 36 & 233 & 40 & 0.013 & 0.058 & NA & 0.00039 & 0.0011 & 0.05 & 0.0059 & 0.085 & 0.100 & 9.6 & 0 \\
\hline 36 & 234 & 40 & 0.013 & 0.058 & $\mathrm{NA}$ & 0.00039 & 0.0011 & 0.1 & 0.0059 & 0.085 & 0.110 & 9.6 & 2 \\
\hline 36 & 235 & 40 & 0.013 & 0.058 & NA & 0.00039 & 0.0011 & 0.1 & 0.0059 & 0.085 & 0.120 & 9.6 & 1 \\
\hline 36 & 236 & 40 & 0.013 & 0.058 & NA & 0.00039 & 0.0011 & 0.1 & 0.0059 & 0.085 & 0.150 & 9.6 & 1 \\
\hline 36 & 237 & 40 & 0.013 & 0.058 & NA & 0.00039 & 0.0011 & 0.1 & 0.0059 & 0.085 & 0.170 & 9.6 & 0 \\
\hline 37 & 238 & 40 & 0.0042 & 0.019 & NA & 0.00013 & 0.00036 & 0.00024 & 0.0020 & 0.0008 & 0.0020 & 9.6 & 2 \\
\hline 37 & 239 & 40 & 0.0042 & 0.019 & NA & 0.00013 & 0.00036 & 0.00024 & 0.0020 & 0.0008 & 0.0080 & 9.6 & 1 \\
\hline 37 & 240 & 40 & 0.0042 & 0.019 & $\mathrm{NA}$ & 0.00013 & 0.00036 & 0.00024 & 0.0020 & 0.0008 & 0.0100 & 9.6 & 1 \\
\hline 37 & 241 & 40 & 0.0042 & 0.019 & $\mathrm{NA}$ & 0.00013 & 0.00036 & 0.00024 & 0.0020 & 0.0008 & 0.0110 & 9.6 & 0 \\
\hline 37 & 242 & 40 & 0.0042 & 0.019 & $\mathrm{NA}$ & 0.00013 & 0.00036 & 0.00024 & 0.0020 & 0.0008 & 0.0200 & 9.6 & 0 \\
\hline 37 & 243 & 40 & 0.0042 & 0.019 & $\mathrm{NA}$ & 0.00013 & 0.00036 & 0.00024 & 0.0020 & 0.0040 & 0.0100 & 9.6 & 0 \\
\hline 37 & 244 & 40 & 0.0042 & 0.019 & $\mathrm{NA}$ & 0.00013 & 0.00036 & 0.00024 & 0.0020 & 0.0100 & 0.0100 & 9.6 & 1 \\
\hline 37 & 245 & 40 & 0.0042 & 0.019 & $\mathrm{NA}$ & 0.00013 & 0.00036 & 0.00024 & 0.0020 & 0.0100 & 0.0120 & 9.6 & 0 \\
\hline 37 & 246 & 40 & 0.0042 & 0.019 & $\mathrm{NA}$ & 0.00013 & 0.00036 & 0.00024 & 0.0020 & 0.0100 & 0.0220 & 9.6 & 0 \\
\hline 37 & 247 & 40 & 0.0042 & 0.019 & NA & 0.00013 & 0.00036 & 0.00024 & 0.0020 & 0.0150 & 0.0180 & 9.6 & 0 \\
\hline 37 & 248 & 40 & 0.0042 & 0.019 & NA & 0.00013 & 0.00036 & 0.00024 & 0.0020 & 0.0280 & 0.0100 & 9.6 & 2 \\
\hline 37 & 249 & 40 & 0.0042 & 0.019 & $\mathrm{NA}$ & 0.00013 & 0.00036 & 0.00024 & 0.0020 & 0.0280 & 0.0180 & 9.6 & 1 \\
\hline 37 & 250 & 40 & 0.0042 & 0.019 & NA & 0.00013 & 0.00036 & 0.00024 & 0.0020 & 0.0280 & 0.0280 & 9.6 & 0 \\
\hline
\end{tabular}


Cyclic Potentiodynamic Polarization Conditions, and Chemical Composition and pH of Historical Data (continued 10)

\begin{tabular}{|c|c|c|c|c|c|c|c|c|c|c|c|c|c|}
\hline Reference & Test & $\begin{array}{l}\text { Temperature } \\
\left({ }^{\circ} \mathrm{C}\right)\end{array}$ & $\begin{array}{l}\text { Carbonate } \\
\text { (M) }\end{array}$ & $\begin{array}{c}\text { Bi- } \\
\text { carbonate } \\
\text { (M) }\end{array}$ & $\begin{array}{l}\text { Citrate } \\
\text { (M) }\end{array}$ & $\begin{array}{l}\text { Phosphate } \\
\text { (M) }\end{array}$ & $\begin{array}{l}\text { Chloride } \\
\text { (M) }\end{array}$ & $\begin{array}{l}\text { Fluoride } \\
\text { (M) }\end{array}$ & $\begin{array}{l}\text { Sulfate } \\
\text { (M) }\end{array}$ & $\begin{array}{l}\text { Nitrate } \\
\text { (M) }\end{array}$ & $\begin{array}{l}\text { Nitrite } \\
\text { (M) }\end{array}$ & $\mathrm{pH}$ & Pit ID* \\
\hline 37 & 251 & 40 & 0.0042 & 0.019 & $\mathrm{NA}$ & 0.00013 & 0.00036 & 0.00024 & 0.0020 & 0.0280 & 0.0380 & 9.6 & 0 \\
\hline 37 & 252 & 40 & 0.0042 & 0.019 & $\mathrm{NA}$ & 0.00013 & 0.00036 & 0.00024 & 0.0020 & 0.0500 & 0.0380 & 9.6 & 0 \\
\hline 37 & 253 & 40 & 0.0042 & 0.019 & NA & 0.00013 & 0.00036 & 0.00024 & 0.0020 & 0.0600 & 0.0280 & 9.6 & 1 \\
\hline 37 & 254 & 40 & 0.0042 & 0.019 & $\mathrm{NA}$ & 0.00013 & 0.00036 & 0.00024 & 0.0020 & 0.0600 & 0.0380 & 9.6 & 0 \\
\hline 37 & 255 & 40 & 0.0042 & 0.019 & NA & 0.00013 & 0.00036 & 0.00024 & 0.0020 & 0.0800 & 0.0380 & 9.6 & 0 \\
\hline 37 & 256 & 40 & 0.0042 & 0.019 & $\mathrm{NA}$ & 0.00013 & 0.00036 & 0.00024 & 0.0020 & 0.1000 & 0.0380 & 9.6 & 1 \\
\hline 37 & 257 & 40 & 0.0042 & 0.019 & $\mathrm{NA}$ & 0.00013 & 0.00036 & 0.00024 & 0.0020 & 0.1500 & 0.0680 & 9.6 & 0 \\
\hline 37 & 258 & 40 & 0.0042 & 0.019 & NA & 0.00013 & 0.00036 & 0.00024 & 0.0020 & 0.1500 & 0.0580 & 9.6 & 1 \\
\hline 37 & 259 & 40 & 0.0042 & 0.019 & $\mathrm{NA}$ & 0.00013 & 0.00036 & 0.00024 & 0.0020 & 0.1500 & 0.0680 & 9.6 & 0 \\
\hline 37 & 260 & 40 & 0.0042 & 0.019 & NA & 0.00013 & 0.00036 & 0.00024 & 0.0020 & 0.3000 & 0.0480 & 9.6 & 2 \\
\hline 37 & 261 & 40 & 0.0042 & 0.019 & $\mathrm{NA}$ & 0.00013 & 0.00036 & 0.00024 & 0.0020 & 0.3000 & 0.0680 & 9.6 & 2 \\
\hline 37 & 262 & 40 & 0.0042 & 0.019 & NA & 0.00013 & 0.00036 & 0.00024 & 0.0020 & 0.3000 & 0.0880 & 9.6 & 2 \\
\hline 37 & 263 & 40 & 0.0042 & 0.019 & NA & 0.00013 & 0.00036 & 0.00024 & 0.0020 & 0.3000 & 0.1000 & 9.6 & 0 \\
\hline 37 & 264 & 40 & 0.0042 & 0.019 & NA & 0.00013 & 0.00036 & 0.00024 & 0.0020 & 0.028 & 0.0100 & 9.6 & 2 \\
\hline 37 & 265 & 40 & 0.0042 & 0.019 & $\mathrm{NA}$ & 0.00013 & 0.00036 & 0.00024 & 0.0020 & 0.028 & 0.0200 & 9.6 & 1 \\
\hline 37 & 266 & 40 & 0.0042 & 0.019 & $\mathrm{NA}$ & 0.00013 & 0.00036 & 0.00024 & 0.0020 & 0.028 & 0.0300 & 9.6 & 0 \\
\hline 37 & 267 & 40 & 0.0042 & 0.019 & NA & 0.00013 & 0.00036 & 0.00024 & 0.0020 & 0.028 & 0.0400 & 9.6 & 0 \\
\hline 37 & 268 & 40 & 0.0042 & 0.019 & $\mathrm{NA}$ & 0.00013 & 0.00100 & 0.00024 & 0.0020 & 0.028 & 0.0200 & 9.6 & 1 \\
\hline 37 & 269 & 40 & 0.0042 & 0.019 & $\mathrm{NA}$ & 0.00013 & 0.00100 & 0.00024 & 0.0020 & 0.028 & 0.0300 & 9.6 & 0 \\
\hline 37 & 270 & 40 & 0.0042 & 0.019 & NA & 0.00013 & 0.00200 & 0.00024 & 0.0020 & 0.028 & 0.0300 & 9.6 & 1 \\
\hline 37 & 271 & 40 & 0.0042 & 0.019 & $\mathrm{NA}$ & 0.00013 & 0.00200 & 0.00024 & 0.0020 & 0.028 & 0.0400 & 9.6 & 0 \\
\hline 37 & 272 & 40 & 0.0042 & 0.019 & $\mathrm{NA}$ & 0.00013 & 0.00400 & 0.00024 & 0.0020 & 0.028 & 0.0700 & 9.6 & 0 \\
\hline 37 & 273 & 40 & 0.0042 & 0.019 & $\mathrm{NA}$ & 0.00013 & 0.00400 & 0.00024 & 0.0020 & 0.028 & 0.1000 & 9.6 & 0 \\
\hline 37 & 274 & 40 & 0.0042 & 0.019 & $\mathrm{NA}$ & 0.00013 & 0.00500 & 0.00024 & 0.0020 & 0.028 & 0.0400 & 9.6 & 2 \\
\hline 37 & 275 & 40 & 0.0042 & 0.019 & NA & 0.00013 & 0.00500 & 0.00024 & 0.0020 & 0.028 & 0.0700 & 9.6 & 1 \\
\hline
\end{tabular}


Cyclic Potentiodynamic Polarization Conditions, and Chemical Composition and pH of Historical Data (continued 11)

\begin{tabular}{|c|c|c|c|c|c|c|c|c|c|c|c|c|c|}
\hline Reference & Test & $\begin{array}{l}\text { Temperature } \\
\left({ }^{\circ} \mathrm{C}\right)\end{array}$ & $\begin{array}{l}\text { Carbonate } \\
\text { (M) }\end{array}$ & $\begin{array}{c}\text { Bi- } \\
\text { carbonate } \\
\text { (M) }\end{array}$ & $\begin{array}{l}\text { Citrate } \\
\text { (M) }\end{array}$ & $\begin{array}{l}\text { Phosphate } \\
\text { (M) }\end{array}$ & $\begin{array}{l}\text { Chloride } \\
\text { (M) }\end{array}$ & $\begin{array}{l}\text { Fluoride } \\
\text { (M) }\end{array}$ & $\begin{array}{l}\text { Sulfate } \\
\text { (M) }\end{array}$ & $\begin{array}{l}\text { Nitrate } \\
\text { (M) }\end{array}$ & $\begin{array}{l}\text { Nitrite } \\
\text { (M) }\end{array}$ & $\mathrm{pH}$ & Pit ID* \\
\hline 37 & 276 & 40 & 0.0042 & 0.019 & $\mathrm{NA}$ & 0.00013 & 0.00600 & 0.00024 & 0.0020 & 0.028 & 0.2000 & 9.6 & 0 \\
\hline 37 & 277 & 40 & 0.0042 & 0.019 & $\mathrm{NA}$ & 0.00013 & 0.00800 & 0.00024 & 0.0020 & 0.028 & 0.2000 & 9.6 & 0 \\
\hline 37 & 278 & 40 & 0.0042 & 0.019 & NA & 0.00013 & 0.01000 & 0.00024 & 0.0020 & 0.028 & 0.2000 & 9.6 & 2 \\
\hline 37 & 279 & 40 & 0.0042 & 0.019 & $\mathrm{NA}$ & 0.00013 & 0.01000 & 0.00024 & 0.0020 & 0.028 & 0.2500 & 9.6 & 0 \\
\hline 37 & 280 & 40 & 0.0042 & 0.019 & NA & 0.00013 & 0.00036 & 0.00024 & 0.0020 & 0.0284 & 0.0100 & 9.6 & 2 \\
\hline 37 & 281 & 40 & 0.0042 & 0.019 & $\mathrm{NA}$ & 0.00013 & 0.00036 & 0.00024 & 0.0020 & 0.0284 & 0.0200 & 9.6 & 1 \\
\hline 37 & 282 & 40 & 0.0042 & 0.019 & $\mathrm{NA}$ & 0.00013 & 0.00036 & 0.00024 & 0.0020 & 0.0284 & 0.0300 & 9.6 & 0 \\
\hline 37 & 283 & 40 & 0.0042 & 0.019 & NA & 0.00013 & 0.00036 & 0.00024 & 0.0020 & 0.0284 & 0.0400 & 9.6 & 0 \\
\hline 37 & 284 & 40 & 0.0042 & 0.019 & $\mathrm{NA}$ & 0.00013 & 0.00036 & 0.00024 & 0.0080 & 0.0284 & 0.0300 & 9.6 & 0 \\
\hline 37 & 285 & 40 & 0.0042 & 0.019 & NA & 0.00013 & 0.00036 & 0.00024 & 0.0080 & 0.0284 & 0.0500 & 9.6 & 0 \\
\hline 37 & 286 & 40 & 0.0042 & 0.019 & $\mathrm{NA}$ & 0.00013 & 0.00036 & 0.00024 & 0.0100 & 0.0284 & 0.0200 & 9.6 & 1 \\
\hline 37 & 287 & 40 & 0.0042 & 0.019 & NA & 0.00013 & 0.00036 & 0.00024 & 0.0300 & 0.0284 & 0.0200 & 9.6 & 2 \\
\hline 37 & 288 & 40 & 0.0042 & 0.019 & NA & 0.00013 & 0.00036 & 0.00024 & 0.0300 & 0.0284 & 0.0300 & 9.6 & 2 \\
\hline 37 & 289 & 40 & 0.0042 & 0.019 & NA & 0.00013 & 0.00036 & 0.00024 & 0.0300 & 0.0284 & 0.0350 & 9.6 & 1 \\
\hline 37 & 290 & 40 & 0.0042 & 0.019 & $\mathrm{NA}$ & 0.00013 & 0.00036 & 0.00024 & 0.0300 & 0.0284 & 0.0450 & 9.6 & 0 \\
\hline 37 & 291 & 40 & 0.0042 & 0.019 & $\mathrm{NA}$ & 0.00013 & 0.00036 & 0.00024 & 0.0500 & 0.0284 & 0.0450 & 9.6 & 1 \\
\hline 37 & 292 & 40 & 0.0042 & 0.019 & NA & 0.00013 & 0.00036 & 0.00024 & 0.0600 & 0.0284 & 0.1000 & 9.6 & 0 \\
\hline 37 & 293 & 40 & 0.0042 & 0.019 & $\mathrm{NA}$ & 0.00013 & 0.00036 & 0.00024 & 0.0800 & 0.0284 & 0.0550 & 9.6 & 2 \\
\hline 37 & 294 & 40 & 0.0042 & 0.019 & $\mathrm{NA}$ & 0.00013 & 0.00036 & 0.00024 & 0.0800 & 0.0284 & 0.0750 & 9.6 & 2 \\
\hline 37 & 295 & 40 & 0.0042 & 0.019 & NA & 0.00013 & 0.00036 & 0.00024 & 0.0800 & 0.0284 & 0.1000 & 9.6 & 1 \\
\hline 37 & 296 & 40 & 0.0042 & 0.019 & $\mathrm{NA}$ & 0.00013 & 0.00036 & 0.00024 & 0.1000 & 0.0284 & 0.0450 & 9.6 & 2 \\
\hline 37 & 297 & 40 & 0.0042 & 0.019 & $\mathrm{NA}$ & 0.00013 & 0.00036 & 0.00024 & 0.1300 & 0.0284 & 0.2000 & 9.6 & 0 \\
\hline 37 & 298 & 40 & 0.0042 & 0.019 & $\mathrm{NA}$ & 0.00013 & 0.00036 & 0.00024 & 0.1400 & 0.0284 & 0.2000 & 9.6 & 0 \\
\hline 37 & 299 & 40 & 0.0042 & 0.019 & $\mathrm{NA}$ & 0.00013 & 0.00036 & 0.00024 & 0.1500 & 0.0284 & 0.2000 & 9.6 & 1 \\
\hline 38 & 300 & 40 & 0.13 & 0.58 & NA & 0.00390 & 0.011 & 0.007 & 0.059 & 0.85 & 0.70 & 9.6 & 2 \\
\hline
\end{tabular}


Cyclic Potentiodynamic Polarization Conditions, and Chemical Composition and pH of Historical Data (continued 12)

\begin{tabular}{|c|c|c|c|c|c|c|c|c|c|c|c|c|c|}
\hline Reference & Test & $\begin{array}{c}\text { Temperature } \\
\left({ }^{\circ} \mathrm{C}\right)\end{array}$ & $\begin{array}{l}\text { Carbonate } \\
\text { (M) }\end{array}$ & $\begin{array}{c}\text { Bi- } \\
\text { carbonate } \\
\text { (M) }\end{array}$ & $\begin{array}{l}\text { Citrate } \\
\text { (M) }\end{array}$ & $\begin{array}{l}\text { Phosphate } \\
\text { (M) }\end{array}$ & $\begin{array}{l}\text { Chloride } \\
\text { (M) }\end{array}$ & $\begin{array}{l}\text { Fluoride } \\
\text { (M) }\end{array}$ & $\begin{array}{l}\text { Sulfate } \\
\text { (M) }\end{array}$ & $\begin{array}{l}\text { Nitrate } \\
\text { (M) }\end{array}$ & $\begin{array}{l}\text { Nitrite } \\
\text { (M) }\end{array}$ & $\mathrm{pH}$ & Pit ID* \\
\hline 38 & 301 & 40 & 0.13 & 0.58 & NA & 0.00390 & 0.011 & 0.007 & 0.059 & 0.85 & 0.80 & 9.6 & 2 \\
\hline 38 & 302 & 40 & 0.13 & 0.58 & NA & 0.00390 & 0.011 & 0.007 & 0.059 & 0.85 & 0.90 & 9.6 & 2 \\
\hline 38 & 303 & 40 & 0.13 & 0.58 & NA & 0.00390 & 0.011 & 0.007 & 0.059 & 0.85 & 1.10 & 9.6 & 0 \\
\hline 38 & 304 & 40 & 0.13 & 0.58 & NA & 0.00390 & 0.011 & 0.007 & 0.059 & 0.85 & 0.26 & 10.5 & 2 \\
\hline 38 & 305 & 40 & 0.13 & 0.58 & NA & 0.00390 & 0.011 & 0.007 & 0.059 & 0.85 & 0.26 & 11 & 2 \\
\hline 38 & 306 & 40 & 0.13 & 0.58 & NA & 0.00390 & 0.011 & 0.007 & 0.059 & 0.85 & 0.50 & 11.1 & 2 \\
\hline 38 & 307 & 40 & 0.13 & 0.58 & NA & 0.00390 & 0.011 & 0.007 & 0.059 & 0.85 & 0.70 & 11.1 & 2 \\
\hline 38 & 308 & 40 & 0.13 & 0.58 & NA & 0.00390 & 0.011 & 0.007 & 0.059 & 0.85 & 0.90 & 11.1 & 2 \\
\hline 38 & 309 & 40 & 0.13 & 0.58 & NA & 0.00390 & 0.011 & 0.007 & 0.059 & 0.85 & 0.26 & 11.5 & 2 \\
\hline 38 & 310 & 40 & 0.13 & 0.58 & NA & 0.00390 & 0.011 & 0.007 & 0.059 & 0.85 & 0.50 & 11.5 & 1 \\
\hline 38 & 311 & 40 & 0.13 & 0.58 & NA & 0.00390 & 0.011 & 0.007 & 0.059 & 0.85 & 0.70 & 11.5 & 1 \\
\hline 38 & 312 & 40 & 0.13 & 0.58 & NA & 0.00390 & 0.011 & 0.007 & 0.059 & 0.85 & 0.80 & 11.5 & 0 \\
\hline 38 & 313 & 40 & 0.13 & 0.58 & NA & 0.00390 & 0.011 & 0.007 & 0.059 & 0.85 & 0.26 & 12.2 & 0 \\
\hline 38 & 314 & 40 & 0.13 & 0.58 & NA & 0.00390 & 0.011 & 0.007 & 0.059 & 0.85 & 0.26 & 12.6 & 0 \\
\hline 38 & 315 & 40 & 0.03 & 0.14 & $\mathrm{NA}$ & 0.00098 & 0.0027 & 0.0018 & 0.015 & 0.21 & 0.100 & 9.6 & 2 \\
\hline 38 & 316 & 40 & 0.03 & 0.14 & NA & 0.00098 & 0.0027 & 0.0018 & 0.015 & 0.21 & 0.180 & 9.6 & 0 \\
\hline 38 & 317 & 40 & 0.03 & 0.14 & NA & 0.00098 & 0.0027 & 0.0018 & 0.015 & 0.21 & 0.240 & 9.6 & 0 \\
\hline 38 & 318 & 40 & 0.06 & 0.29 & NA & 0.00195 & 0.0054 & 0.0036 & 0.030 & 0.43 & 0.22 & 9.6 & 1 \\
\hline 38 & 319 & 40 & 0.06 & 0.29 & $\mathrm{NA}$ & 0.00195 & 0.0054 & 0.0036 & 0.030 & 0.43 & 0.28 & 9.6 & 0 \\
\hline 38 & 320 & 40 & 0.06 & 0.29 & NA & 0.00195 & 0.0054 & 0.0036 & 0.030 & 0.43 & 0.42 & 9.6 & 0 \\
\hline 38 & 321 & 40 & 0.06 & 0.29 & NA & 0.00195 & 0.0054 & 0.0036 & 0.030 & 0.43 & 0.56 & 9.6 & 0 \\
\hline 38 & 322 & 40 & 0.09 & 0.43 & NA & 0.00293 & 0.0082 & 0.0055 & 0.045 & 0.64 & 0.32 & 9.6 & 2 \\
\hline 38 & 323 & 40 & 0.09 & 0.43 & NA & 0.00293 & 0.0082 & 0.0055 & 0.045 & 0.64 & 0.45 & 9.6 & 1 \\
\hline 38 & 324 & 40 & 0.09 & 0.43 & NA & 0.00293 & 0.0082 & 0.0055 & 0.045 & 0.64 & 0.60 & 9.6 & 0 \\
\hline 38 & 325 & 40 & 0.09 & 0.43 & NA & 0.00293 & 0.0082 & 0.0055 & 0.045 & 0.64 & 0.82 & 9.6 & 0 \\
\hline
\end{tabular}


Cyclic Potentiodynamic Polarization Conditions, and Chemical Composition and pH of Historical Data (continued 13)

\begin{tabular}{|c|c|c|c|c|c|c|c|c|c|c|c|c|c|}
\hline Reference & Test & $\begin{array}{l}\text { Temperature } \\
\left({ }^{\circ} \mathrm{C}\right)\end{array}$ & $\begin{array}{l}\text { Carbonate } \\
\text { (M) }\end{array}$ & $\begin{array}{c}\text { Bi- } \\
\text { carbonate } \\
\text { (M) }\end{array}$ & $\begin{array}{l}\text { Citrate } \\
\text { (M) }\end{array}$ & $\begin{array}{l}\text { Phosphate } \\
\text { (M) }\end{array}$ & $\begin{array}{l}\text { Chloride } \\
\text { (M) }\end{array}$ & $\begin{array}{l}\text { Fluoride } \\
\text { (M) }\end{array}$ & $\begin{array}{l}\text { Sulfate } \\
\text { (M) }\end{array}$ & $\begin{array}{l}\text { Nitrate } \\
\text { (M) }\end{array}$ & $\begin{array}{l}\text { Nitrite } \\
\text { (M) }\end{array}$ & $\mathrm{pH}$ & Pit ID* \\
\hline 39 & 326 & 60 & 0.06 & 0.29 & $\mathrm{NA}$ & 0.00195 & 0.0054 & 0.0036 & 0.030 & 0.43 & 0.05 & 10.1 & 2 \\
\hline 39 & 327 & 60 & 0.06 & 0.29 & $\mathrm{NA}$ & 0.00195 & 0.0054 & 0.0036 & 0.030 & 0.43 & 0.10 & 10.1 & 2 \\
\hline 39 & 328 & 60 & 0.06 & 0.29 & NA & 0.00195 & 0.0054 & 0.0036 & 0.030 & 0.43 & 0.30 & 10.1 & 2 \\
\hline 39 & 329 & 60 & 0.06 & 0.29 & $\mathrm{NA}$ & 0.00195 & 0.0054 & 0.0036 & 0.030 & 0.43 & 0.40 & 10.1 & 2 \\
\hline 39 & 330 & 60 & 0.06 & 0.29 & NA & 0.00195 & 0.0054 & 0.0036 & 0.030 & 0.43 & 0.50 & 10.1 & 0 \\
\hline 39 & 331 & 60 & 0.06 & 0.29 & $\mathrm{NA}$ & 0.00195 & 0.0054 & 0.0036 & 0.030 & 0.43 & 0.60 & 10.1 & 0 \\
\hline 39 & 332 & 60 & 0.06 & 0.29 & NA & 0.00195 & 0.0054 & 0.0036 & 0.030 & 0.43 & 1.00 & 10.1 & 0 \\
\hline 39 & 333 & 60 & 0.06 & 0.29 & NA & 0.00195 & 0.0054 & 0.0036 & 0.030 & 0.43 & 3.00 & 10.1 & 0 \\
\hline 39 & 334 & 80 & 0.06 & 0.29 & $\mathrm{NA}$ & 0.00195 & 0.0054 & 0.0036 & 0.030 & 0.43 & 0.10 & 10.1 & 2 \\
\hline 39 & 335 & 80 & 0.06 & 0.29 & NA & 0.00195 & 0.0054 & 0.0036 & 0.030 & 0.43 & 0.30 & 10.1 & 2 \\
\hline 39 & 336 & 80 & 0.06 & 0.29 & $\mathrm{NA}$ & 0.00195 & 0.0054 & 0.0036 & 0.030 & 0.43 & 0.35 & 10.1 & 2 \\
\hline 39 & 337 & 80 & 0.06 & 0.29 & NA & 0.00195 & 0.0054 & 0.0036 & 0.030 & 0.43 & 0.75 & 10.1 & 1 \\
\hline 39 & 338 & 80 & 0.06 & 0.29 & NA & 0.00195 & 0.0054 & 0.0036 & 0.030 & 0.43 & 0.90 & 10.1 & 1 \\
\hline 39 & 339 & 80 & 0.06 & 0.29 & NA & 0.00195 & 0.0054 & 0.0036 & 0.030 & 0.43 & 1.00 & 10.1 & 0 \\
\hline 39 & 340 & 80 & 0.06 & 0.29 & $\mathrm{NA}$ & 0.00195 & 0.0054 & 0.0036 & 0.030 & 0.43 & 1.25 & 10.1 & 0 \\
\hline 39 & 341 & 80 & 0.06 & 0.29 & $\mathrm{NA}$ & 0.00195 & 0.0054 & 0.0036 & 0.030 & 0.43 & 3.00 & 10.1 & 0 \\
\hline 39 & 342 & 60 & 0.13 & 0.58 & NA & 0.00390 & 0.011 & 0.007 & 0.059 & 0.85 & 0.10 & 10.2 & 2 \\
\hline 39 & 343 & 60 & 0.13 & 0.58 & $\mathrm{NA}$ & 0.00390 & 0.011 & 0.007 & 0.059 & 0.85 & 0.50 & 10.2 & 0 \\
\hline 39 & 344 & 60 & 0.13 & 0.58 & $\mathrm{NA}$ & 0.00390 & 0.011 & 0.007 & 0.059 & 0.85 & 0.65 & 10.2 & 2 \\
\hline 39 & 345 & 60 & 0.13 & 0.58 & $\mathrm{NA}$ & 0.00390 & 0.011 & 0.007 & 0.059 & 0.85 & 0.80 & 10.2 & 1 \\
\hline 39 & 346 & 60 & 0.13 & 0.58 & $\mathrm{NA}$ & 0.00390 & 0.011 & 0.007 & 0.059 & 0.85 & 1.00 & 10.2 & 0 \\
\hline 39 & 347 & 60 & 0.13 & 0.58 & $\mathrm{NA}$ & 0.00390 & 0.011 & 0.007 & 0.059 & 0.85 & 1.50 & 10.2 & 0 \\
\hline 39 & 348 & 60 & 0.13 & 0.58 & $\mathrm{NA}$ & 0.00390 & 0.011 & 0.007 & 0.059 & 0.85 & 3.00 & 10.2 & 0 \\
\hline 39 & 349 & 80 & 0.13 & 0.58 & $\mathrm{NA}$ & 0.00390 & 0.011 & 0.007 & 0.059 & 0.85 & 0.30 & 10.2 & 2 \\
\hline 39 & 350 & 80 & 0.13 & 0.58 & NA & 0.00390 & 0.011 & 0.007 & 0.059 & 0.85 & 1.00 & 10.2 & 2 \\
\hline
\end{tabular}


Cyclic Potentiodynamic Polarization Conditions, and Chemical Composition and pH of Historical Data (continued 14)

\begin{tabular}{|c|c|c|c|c|c|c|c|c|c|c|c|c|c|}
\hline Reference & Test & $\begin{array}{l}\text { Temperature } \\
\left({ }^{\circ} \mathrm{C}\right)\end{array}$ & $\begin{array}{l}\text { Carbonate } \\
\text { (M) }\end{array}$ & $\begin{array}{c}\text { Bi- } \\
\text { carbonate } \\
\text { (M) }\end{array}$ & $\begin{array}{l}\text { Citrate } \\
\text { (M) }\end{array}$ & $\begin{array}{l}\text { Phosphate } \\
\text { (M) }\end{array}$ & $\begin{array}{l}\text { Chloride } \\
\text { (M) }\end{array}$ & $\begin{array}{l}\text { Fluoride } \\
\text { (M) }\end{array}$ & $\begin{array}{l}\text { Sulfate } \\
\text { (M) }\end{array}$ & $\begin{array}{l}\text { Nitrate } \\
\text { (M) }\end{array}$ & $\begin{array}{l}\text { Nitrite } \\
\text { (M) }\end{array}$ & $\mathrm{pH}$ & Pit ID* \\
\hline 39 & 351 & 80 & 0.13 & 0.58 & $\mathrm{NA}$ & 0.00390 & 0.011 & 0.007 & 0.059 & 0.85 & 1.20 & 10.2 & 1 \\
\hline 39 & 352 & 80 & 0.13 & 0.58 & $\mathrm{NA}$ & 0.00390 & 0.011 & 0.007 & 0.059 & 0.85 & 1.50 & 10.2 & 0 \\
\hline 39 & 353 & 80 & 0.13 & 0.58 & NA & 0.00390 & 0.011 & 0.007 & 0.059 & 0.85 & 2.00 & 10.2 & 0 \\
\hline 39 & 354 & 80 & 0.13 & 0.58 & $\mathrm{NA}$ & 0.00390 & 0.011 & 0.007 & 0.059 & 0.85 & 4.00 & 10.2 & 0 \\
\hline 40 & 355 & 60 & 0.0042 & 0.019 & NA & 0.00013 & 0.00036 & 0.00024 & 0.0020 & 0.028 & 0.0300 & 9.6 & 2 \\
\hline 40 & 356 & 60 & 0.0042 & 0.019 & $\mathrm{NA}$ & 0.00013 & 0.00036 & 0.00024 & 0.0020 & 0.028 & 0.6500 & 9.6 & 0 \\
\hline 40 & 357 & 60 & 0.0042 & 0.019 & $\mathrm{NA}$ & 0.00013 & 0.00036 & 0.00024 & 0.0020 & 0.028 & 0.8000 & 9.6 & 1 \\
\hline 40 & 358 & 60 & 0.0042 & 0.019 & NA & 0.00013 & 0.00036 & 0.00024 & 0.0020 & 0.028 & 1.0000 & 9.6 & 0 \\
\hline 40 & 359 & 80 & 0.0042 & 0.019 & $\mathrm{NA}$ & 0.00013 & 0.00036 & 0.00024 & 0.0020 & 0.028 & 0.0800 & 9.6 & 2 \\
\hline 40 & 360 & 80 & 0.0042 & 0.019 & NA & 0.00013 & 0.00036 & 0.00024 & 0.0020 & 0.028 & 0.1000 & 9.6 & 2 \\
\hline 40 & 361 & 80 & 0.0042 & 0.019 & $\mathrm{NA}$ & 0.00013 & 0.00036 & 0.00024 & 0.0020 & 0.028 & 0.1500 & 9.6 & 2 \\
\hline 40 & 362 & 80 & 0.0042 & 0.019 & NA & 0.00013 & 0.00036 & 0.00024 & 0.0020 & 0.028 & 0.2500 & 9.6 & 2 \\
\hline 40 & 363 & 80 & 0.0042 & 0.019 & NA & 0.00013 & 0.00036 & 0.00024 & 0.0020 & 0.028 & 0.3000 & 9.6 & 2 \\
\hline 40 & 364 & 80 & 0.0042 & 0.019 & NA & 0.00013 & 0.00036 & 0.00024 & 0.0020 & 0.028 & 0.5000 & 9.6 & 2 \\
\hline 40 & 365 & 80 & 0.0042 & 0.019 & $\mathrm{NA}$ & 0.00013 & 0.00036 & 0.00024 & 0.0020 & 0.028 & 1.0000 & 9.6 & 0 \\
\hline 40 & 366 & 80 & 0.0042 & 0.019 & $\mathrm{NA}$ & 0.00013 & 0.00036 & 0.00024 & 0.0020 & 0.028 & 1.5000 & 9.6 & 0 \\
\hline 41 & 367 & 40 & 0.0042 & 0.019 & NA & 0.00013 & 0.00036 & 0.00024 & 0.0020 & 0.028 & 0.0400 & 9.6 & 2 \\
\hline 41 & 368 & 40 & 0.0042 & 0.019 & $\mathrm{NA}$ & 0.00013 & 0.00036 & 0.00024 & 0.0020 & 0.028 & 0.0600 & 9.6 & 1 \\
\hline 41 & 369 & 40 & 0.0042 & 0.019 & $\mathrm{NA}$ & 0.00013 & 0.00036 & 0.00024 & 0.0020 & 0.028 & 0.0700 & 9.6 & 0 \\
\hline 41 & 370 & 50 & 0.0042 & 0.019 & NA & 0.00013 & 0.00036 & 0.00024 & 0.0020 & 0.028 & 0.0700 & 9.6 & 2 \\
\hline 41 & 371 & 50 & 0.0042 & 0.019 & $\mathrm{NA}$ & 0.00013 & 0.00036 & 0.00024 & 0.0020 & 0.028 & 0.0800 & 9.6 & 0 \\
\hline 41 & 372 & 60 & 0.0042 & 0.019 & $\mathrm{NA}$ & 0.00013 & 0.00036 & 0.00024 & 0.0020 & 0.028 & 0.0800 & 9.6 & 2 \\
\hline 41 & 373 & 60 & 0.0042 & 0.019 & NA & 0.00013 & 0.00036 & 0.00024 & 0.0020 & 0.028 & 0.0900 & 9.6 & 2 \\
\hline 41 & 374 & 60 & 0.0042 & 0.019 & $\mathrm{NA}$ & 0.00013 & 0.00036 & 0.00024 & 0.0020 & 0.028 & 0.1000 & 9.6 & 1 \\
\hline 41 & 375 & 60 & 0.0042 & 0.019 & NA & 0.00013 & 0.00036 & 0.00024 & 0.0020 & 0.028 & 0.1200 & 9.6 & 0 \\
\hline
\end{tabular}


Cyclic Potentiodynamic Polarization Conditions, and Chemical Composition and pH of Historical Data (continued 15)

\begin{tabular}{|c|c|c|c|c|c|c|c|c|c|c|c|c|c|}
\hline Reference & Test & $\begin{array}{c}\text { Temperature } \\
\left({ }^{\circ} \mathrm{C}\right)\end{array}$ & $\begin{array}{l}\text { Carbonate } \\
\text { (M) }\end{array}$ & $\begin{array}{c}\text { Bi- } \\
\text { carbonate } \\
\text { (M) }\end{array}$ & $\begin{array}{l}\text { Citrate } \\
\text { (M) }\end{array}$ & $\begin{array}{l}\text { Phosphate } \\
\text { (M) }\end{array}$ & $\begin{array}{l}\text { Chloride } \\
\text { (M) }\end{array}$ & $\begin{array}{l}\text { Fluoride } \\
\text { (M) }\end{array}$ & $\begin{array}{l}\text { Sulfate } \\
\text { (M) }\end{array}$ & $\begin{array}{l}\text { Nitrate } \\
\text { (M) }\end{array}$ & $\begin{array}{l}\text { Nitrite } \\
\text { (M) }\end{array}$ & $\mathrm{pH}$ & Pit ID* \\
\hline 42 & 376 & 2 & 0.0042 & 0.019 & NA & 0.00013 & 0.00036 & 0.00024 & 0.0020 & 0.028 & 0.0050 & 9.45 & 2 \\
\hline 42 & 377 & 2 & 0.0042 & 0.019 & NA & 0.00013 & 0.00036 & 0.00024 & 0.0020 & 0.028 & 0.0085 & 9.45 & 0 \\
\hline 42 & 378 & 23 & 0.0042 & 0.019 & NA & 0.00013 & 0.00036 & 0.00024 & 0.0020 & 0.028 & 0.0050 & 9.45 & 2 \\
\hline 42 & 379 & 23 & 0.0042 & 0.019 & NA & 0.00013 & 0.00036 & 0.00024 & 0.0020 & 0.028 & 0.0085 & 9.45 & 2 \\
\hline 42 & 380 & 23 & 0.0042 & 0.019 & NA & 0.00013 & 0.00036 & 0.00024 & 0.0020 & 0.028 & 0.0120 & 9.45 & 0 \\
\hline 42 & 381 & 30 & 0.0042 & 0.019 & NA & 0.00013 & 0.00036 & 0.00024 & 0.0020 & 0.028 & 0.0050 & 9.45 & 2 \\
\hline 42 & 382 & 30 & 0.0042 & 0.019 & NA & 0.00013 & 0.00036 & 0.00024 & 0.0020 & 0.028 & 0.0085 & 9.45 & 2 \\
\hline 42 & 383 & 30 & 0.0042 & 0.019 & NA & 0.00013 & 0.00036 & 0.00024 & 0.0020 & 0.028 & 0.0120 & 9.45 & 2 \\
\hline 42 & 384 & 30 & 0.0042 & 0.019 & NA & 0.00013 & 0.00036 & 0.00024 & 0.0020 & 0.028 & 0.0200 & 9.45 & 0 \\
\hline 42 & 385 & 40 & 0.0042 & 0.019 & NA & 0.00013 & 0.00036 & 0.00024 & 0.0020 & 0.028 & 0.0085 & 9.45 & 2 \\
\hline 42 & 386 & 40 & 0.0042 & 0.019 & NA & 0.00013 & 0.00036 & 0.00024 & 0.0020 & 0.028 & 0.0300 & 9.45 & 0 \\
\hline 42 & 387 & 50 & 0.0042 & 0.019 & NA & 0.00013 & 0.00036 & 0.00024 & 0.0020 & 0.028 & 0.0085 & 9.45 & 2 \\
\hline 42 & 388 & 50 & 0.0042 & 0.019 & NA & 0.00013 & 0.00036 & 0.00024 & 0.0020 & 0.028 & 0.0120 & 9.45 & 2 \\
\hline 42 & 389 & 50 & 0.0042 & 0.019 & NA & 0.00013 & 0.00036 & 0.00024 & 0.0020 & 0.028 & 0.0300 & 9.45 & 0 \\
\hline 43 & 390 & 40 & 0.0015 & NA & NA & 0.00008 & 0.00032 & 0.000153 & 0.0014 & 0.004 & 0.003 & 12.5 & 2 \\
\hline 43 & 391 & 40 & 0.0015 & NA & NA & 0.00008 & 0.00032 & 0.000153 & 0.0014 & 0.004 & 0.004 & 12.5 & 2 \\
\hline 43 & 392 & 40 & 0.0015 & NA & NA & 0.00008 & 0.00032 & 0.000153 & 0.0014 & 0.004 & 0.008 & 12.5 & 0 \\
\hline 43 & 393 & 40 & 0.0015 & NA & NA & 0.00008 & 0.00032 & 0.000153 & 0.0014 & 0.004 & 0.010 & 12.5 & 0 \\
\hline 43 & 394 & 40 & 0.0015 & NA & NA & 0.00008 & 0.00032 & 0.000153 & 0.0014 & 0.01 & 0.004 & 12.5 & 2 \\
\hline 43 & 395 & 40 & 0.0015 & NA & NA & 0.00008 & 0.00032 & 0.000153 & 0.0014 & 0.01 & 0.007 & 12.5 & 1 \\
\hline 43 & 396 & 40 & 0.0015 & $\mathrm{NA}$ & NA & 0.00008 & 0.00032 & 0.000153 & 0.0014 & 0.01 & 0.008 & 12.5 & 1 \\
\hline 43 & 397 & 40 & 0.0015 & NA & NA & 0.00008 & 0.00032 & 0.000153 & 0.0014 & 0.01 & 0.010 & 12.5 & 0 \\
\hline 43 & 398 & 40 & 0.0015 & $\mathrm{NA}$ & NA & 0.00008 & 0.00032 & 0.000153 & 0.0014 & 0.015 & 0.010 & 12.5 & 2 \\
\hline 43 & 399 & 40 & 0.0015 & NA & NA & 0.00008 & 0.00032 & 0.000153 & 0.0014 & 0.015 & 0.007 & 12.5 & 0 \\
\hline 43 & 400 & 40 & 0.0015 & NA & NA & 0.00008 & 0.00032 & 0.000153 & 0.0014 & 0.015 & 0.010 & 12.5 & 0 \\
\hline
\end{tabular}


Cyclic Potentiodynamic Polarization Conditions, and Chemical Composition and pH of Historical Data (continued 16)

\begin{tabular}{|c|c|c|c|c|c|c|c|c|c|c|c|c|c|}
\hline Reference & Test & $\begin{array}{c}\text { Temperature } \\
\left({ }^{\circ} \mathrm{C}\right)\end{array}$ & $\begin{array}{l}\text { Carbonate } \\
\text { (M) }\end{array}$ & $\begin{array}{c}\text { Bi- } \\
\text { carbonate } \\
\text { (M) }\end{array}$ & $\begin{array}{l}\text { Citrate } \\
\text { (M) }\end{array}$ & $\begin{array}{l}\text { Phosphate } \\
\text { (M) }\end{array}$ & $\begin{array}{l}\text { Chloride } \\
\text { (M) }\end{array}$ & $\begin{array}{l}\text { Fluoride } \\
\text { (M) }\end{array}$ & $\begin{array}{l}\text { Sulfate } \\
\text { (M) }\end{array}$ & $\begin{array}{l}\text { Nitrate } \\
\text { (M) }\end{array}$ & $\begin{array}{l}\text { Nitrite } \\
\text { (M) }\end{array}$ & $\mathrm{pH}$ & Pit ID* \\
\hline 43 & 401 & 40 & 0.0015 & NA & NA & 0.00008 & 0.00032 & 0.000153 & 0.0014 & 0.022 & 0.012 & 12.5 & 1 \\
\hline 43 & 402 & 40 & 0.0015 & NA & NA & 0.00008 & 0.00032 & 0.000153 & 0.0014 & 0.022 & 0.015 & 12.5 & 0 \\
\hline 43 & 403 & 40 & 0.0015 & $\mathrm{NA}$ & NA & 0.00008 & 0.00032 & 0.000153 & 0.0014 & 0.022 & 0.042 & 12.5 & 0 \\
\hline 43 & 404 & 40 & 0.0015 & NA & NA & 0.00008 & 0.00032 & 0.000153 & 0.0014 & 0.04 & 0.015 & 12.5 & 1 \\
\hline 43 & 405 & 40 & 0.0015 & NA & NA & 0.00008 & 0.00032 & 0.000153 & 0.0014 & 0.04 & 0.030 & 12.5 & 1 \\
\hline 43 & 406 & 40 & 0.0015 & NA & NA & 0.00008 & 0.00032 & 0.000153 & 0.0014 & 0.04 & 0.040 & 12.5 & 1 \\
\hline 43 & 407 & 40 & 0.0015 & $\mathrm{NA}$ & NA & 0.00008 & 0.00032 & 0.000153 & 0.0014 & 0.04 & 0.045 & 12.5 & 0 \\
\hline 43 & 408 & 40 & 0.0015 & NA & NA & 0.00008 & 0.00032 & 0.000153 & 0.0014 & 0.04 & 0.050 & 12.5 & 0 \\
\hline 43 & 409 & 40 & 0.0015 & NA & NA & 0.00008 & 0.00032 & 0.000153 & 0.0014 & 0.04 & 0.055 & 12.5 & 0 \\
\hline 43 & 410 & 40 & 0.0015 & NA & NA & 0.00008 & 0.00032 & 0.000153 & 0.0014 & 0.04 & 0.060 & 12.5 & 0 \\
\hline 43 & 411 & 40 & 0.0015 & NA & NA & 0.00008 & 0.00032 & 0.000153 & 0.0014 & 0.04 & 0.200 & 12.5 & 0 \\
\hline 43 & 412 & 40 & 0.0015 & NA & NA & 0.00008 & 0.00032 & 0.000153 & 0.0014 & 0.04 & 0.250 & 12.5 & 0 \\
\hline 43 & 413 & 40 & 0.0015 & NA & NA & 0.00008 & 0.00032 & 0.000153 & 0.0014 & 0.04 & 0.300 & 12.5 & 0 \\
\hline 43 & 414 & 40 & 0.0015 & NA & NA & 0.00008 & 0.00032 & 0.000153 & 0.0014 & 0.11 & 0.050 & 12.5 & 1 \\
\hline 43 & 415 & 40 & 0.0015 & NA & NA & 0.00008 & 0.00032 & 0.000153 & 0.0014 & 0.11 & 0.070 & 12.5 & 0 \\
\hline 43 & 416 & 40 & 0.0015 & NA & NA & 0.00008 & 0.00032 & 0.000153 & 0.0014 & 0.11 & 0.090 & 12.5 & 0 \\
\hline 43 & 417 & 40 & 0.0015 & NA & NA & 0.00008 & 0.00032 & 0.000153 & 0.0014 & 1.00 & 0.700 & 12.5 & 1 \\
\hline 43 & 418 & 40 & 0.0015 & NA & $\mathrm{NA}$ & 0.00008 & 0.00032 & 0.000153 & 0.0014 & 1.00 & 0.800 & 12.5 & 0 \\
\hline 43 & 419 & 40 & 0.0015 & NA & NA & 0.00008 & 0.00032 & 0.000153 & 0.0014 & 5.00 & 2.500 & 12.5 & 0 \\
\hline 43 & 420 & 40 & 0.0015 & $\mathrm{NA}$ & NA & 0.00008 & 0.00032 & 0.000153 & 0.0014 & 5.00 & 3.000 & 12.5 & 0 \\
\hline 43 & 421 & 40 & 0.0015 & NA & NA & 0.00008 & 0.00032 & 0.000153 & 0.0026 & 0.04 & 0.040 & 12.5 & 1 \\
\hline 43 & 422 & 40 & 0.0015 & $\mathrm{NA}$ & NA & 0.00008 & 0.00032 & 0.000153 & 0.0026 & 0.04 & 0.050 & 12.5 & 0 \\
\hline 43 & 423 & 40 & 0.0015 & $\mathrm{NA}$ & $\mathrm{NA}$ & 0.00008 & 0.00032 & 0.000153 & 0.01 & 0.04 & 0.060 & 12.5 & 0 \\
\hline 43 & 424 & 40 & 0.0015 & NA & NA & 0.00008 & 0.00032 & 0.000153 & 0.02 & 0.04 & 0.040 & 12.5 & 2 \\
\hline 43 & 425 & 40 & 0.0015 & $\mathrm{NA}$ & NA & 0.00008 & 0.00032 & 0.000153 & 0.03 & 0.04 & 0.060 & 12.5 & 0 \\
\hline
\end{tabular}


Cyclic Potentiodynamic Polarization Conditions, and Chemical Composition and pH of Historical Data (continued 17)

\begin{tabular}{|c|c|c|c|c|c|c|c|c|c|c|c|c|c|}
\hline Reference & Test & $\begin{array}{l}\text { Temperature } \\
\left({ }^{\circ} \mathrm{C}\right)\end{array}$ & $\begin{array}{l}\text { Carbonate } \\
\text { (M) }\end{array}$ & $\begin{array}{c}\text { Bi- } \\
\text { carbonate } \\
\text { (M) }\end{array}$ & $\begin{array}{l}\text { Citrate } \\
\text { (M) }\end{array}$ & $\begin{array}{l}\text { Phosphate } \\
\text { (M) }\end{array}$ & $\begin{array}{c}\text { Chloride } \\
\text { (M) }\end{array}$ & $\begin{array}{l}\text { Fluoride } \\
\text { (M) }\end{array}$ & $\begin{array}{l}\text { Sulfate } \\
\text { (M) }\end{array}$ & $\begin{array}{l}\text { Nitrate } \\
\text { (M) }\end{array}$ & $\begin{array}{l}\text { Nitrite } \\
\text { (M) }\end{array}$ & $\mathrm{pH}$ & Pit ID* \\
\hline 43 & 426 & 40 & 0.0015 & NA & $\mathrm{NA}$ & 0.00008 & 0.00032 & 0.000153 & 0.04 & 0.04 & 0.060 & 12.5 & 2 \\
\hline 43 & 427 & 40 & 0.0015 & NA & $\mathrm{NA}$ & 0.00008 & 0.00032 & 0.000153 & 0.05 & 0.04 & 0.060 & 12.5 & 2 \\
\hline 43 & 428 & 40 & 0.0015 & NA & NA & 0.00008 & 0.00032 & 0.000153 & 0.07 & 0.04 & 0.100 & 12.5 & 0 \\
\hline 43 & 429 & 40 & 0.0015 & NA & $\mathrm{NA}$ & 0.00008 & 0.00032 & 0.000153 & 0.09 & 0.04 & 0.100 & 12.5 & 1 \\
\hline 43 & 430 & 40 & 0.0015 & NA & NA & 0.00008 & 0.00032 & 0.000153 & 0.1 & 0.04 & 0.120 & 12.5 & 1 \\
\hline 43 & 431 & 40 & 0.0015 & NA & $\mathrm{NA}$ & 0.00008 & 0.00032 & 0.000153 & 0.1 & 0.04 & 0.150 & 12.5 & 1 \\
\hline 43 & 432 & 40 & 0.0015 & NA & NA & 0.00008 & 0.00032 & 0.000153 & 0.1 & 0.04 & 0.180 & 12.5 & 0 \\
\hline 43 & 433 & 40 & 0.0015 & NA & NA & 0.00008 & 0.00032 & 0.000153 & 0.2 & 0.04 & 0.200 & 12.5 & 2 \\
\hline 43 & 434 & 40 & 0.0015 & NA & $\mathrm{NA}$ & 0.00008 & 0.00032 & 0.000153 & 0.2 & 0.04 & 0.250 & 12.5 & 2 \\
\hline 43 & 435 & 40 & 0.0015 & NA & NA & 0.00008 & 0.00032 & 0.000153 & 0.2 & 0.04 & 0.300 & 12.5 & 0 \\
\hline 43 & 436 & 40 & 0.0015 & NA & $\mathrm{NA}$ & 0.00008 & 0.00032 & 0.000153 & 0.0014 & 0.04 & 0.016 & 12.5 & 2 \\
\hline 43 & 437 & 40 & 0.0015 & NA & NA & 0.00008 & 0.00032 & 0.000153 & 0.0014 & 0.04 & 0.030 & 12.5 & 2 \\
\hline 43 & 438 & 40 & 0.0015 & NA & NA & 0.00008 & 0.00032 & 0.000153 & 0.0014 & 0.04 & 0.040 & 12.5 & 2 \\
\hline 43 & 439 & 40 & 0.0015 & NA & NA & 0.00008 & 0.00032 & 0.000153 & 0.0014 & 0.04 & 0.045 & 12.5 & 0 \\
\hline 43 & 440 & 40 & 0.0015 & NA & NA & 0.00008 & 0.00032 & 0.000153 & 0.0014 & 0.04 & 0.050 & 12.5 & 0 \\
\hline 43 & 441 & 40 & 0.0015 & NA & $\mathrm{NA}$ & 0.00008 & 0.00032 & 0.000153 & 0.0014 & 0.04 & 0.055 & 12.5 & 0 \\
\hline 43 & 442 & 40 & 0.0015 & NA & NA & 0.00008 & 0.00032 & 0.000153 & 0.0014 & 0.04 & 0.060 & 12.5 & 0 \\
\hline 43 & 443 & 40 & 0.0015 & NA & $\mathrm{NA}$ & 0.00008 & 0.00032 & 0.000153 & 0.0014 & 0.04 & 0.200 & 12.5 & 0 \\
\hline 43 & 444 & 40 & 0.0015 & NA & $\mathrm{NA}$ & 0.00008 & 0.00032 & 0.000153 & 0.0014 & 0.04 & 0.250 & 12.5 & 0 \\
\hline 43 & 445 & 40 & 0.0015 & NA & NA & 0.00008 & 0.00032 & 0.000153 & 0.0014 & 0.04 & 0.300 & 12.5 & 0 \\
\hline 43 & 446 & 40 & 0.0015 & $\mathrm{NA}$ & $\mathrm{NA}$ & 0.00008 & 0.00060 & 0.000153 & 0.0014 & 0.04 & 0.045 & 12.5 & 0 \\
\hline 43 & 447 & 40 & 0.0015 & NA & $\mathrm{NA}$ & 0.00008 & 0.00100 & 0.000153 & 0.0014 & 0.04 & 0.040 & 12.5 & 2 \\
\hline 43 & 448 & 40 & 0.0015 & NA & NA & 0.00008 & 0.00100 & 0.000153 & 0.0014 & 0.04 & 0.045 & 12.5 & 0 \\
\hline 43 & 449 & 40 & 0.0015 & NA & $\mathrm{NA}$ & 0.00008 & 0.00130 & 0.000153 & 0.0014 & 0.04 & 0.040 & 12.5 & 2 \\
\hline 43 & 450 & 40 & 0.0015 & NA & NA & 0.00008 & 0.00130 & 0.000153 & 0.0014 & 0.04 & 0.045 & 12.5 & 0 \\
\hline
\end{tabular}


Cyclic Potentiodynamic Polarization Conditions, and Chemical Composition and pH of Historical Data (continued 18)

\begin{tabular}{|c|c|c|c|c|c|c|c|c|c|c|c|c|c|}
\hline Reference & Test & $\begin{array}{c}\text { Temperature } \\
\left({ }^{\circ} \mathrm{C}\right)\end{array}$ & $\begin{array}{l}\text { Carbonate } \\
\text { (M) }\end{array}$ & $\begin{array}{c}\text { Bi- } \\
\text { carbonate } \\
\text { (M) }\end{array}$ & $\begin{array}{l}\text { Citrate } \\
\text { (M) }\end{array}$ & $\begin{array}{l}\text { Phosphate } \\
\text { (M) }\end{array}$ & $\begin{array}{l}\text { Chloride } \\
\text { (M) }\end{array}$ & $\begin{array}{l}\text { Fluoride } \\
\text { (M) }\end{array}$ & $\begin{array}{l}\text { Sulfate } \\
\text { (M) }\end{array}$ & $\begin{array}{l}\text { Nitrate } \\
\text { (M) }\end{array}$ & $\begin{array}{l}\text { Nitrite } \\
\text { (M) }\end{array}$ & $\mathrm{pH}$ & Pit ID* \\
\hline 43 & 451 & 40 & 0.0015 & NA & NA & 0.00008 & 0.00140 & 0.000153 & 0.0014 & 0.04 & 0.045 & 12.5 & 0 \\
\hline 43 & 452 & 40 & 0.0015 & NA & NA & 0.00008 & 0.00150 & 0.000153 & 0.0014 & 0.04 & 0.045 & 12.5 & 0 \\
\hline 43 & 453 & 40 & 0.0015 & $\mathrm{NA}$ & NA & 0.00008 & 0.00200 & 0.000153 & 0.0014 & 0.04 & 0.050 & 12.5 & 0 \\
\hline 43 & 454 & 40 & 0.0015 & NA & NA & 0.00008 & 0.00320 & 0.000153 & 0.0014 & 0.04 & 0.045 & 12.5 & 2 \\
\hline 43 & 455 & 40 & 0.0015 & NA & NA & 0.00008 & 0.00320 & 0.000153 & 0.0014 & 0.04 & 0.050 & 12.5 & 2 \\
\hline 43 & 456 & 40 & 0.0015 & NA & NA & 0.00008 & 0.00320 & 0.000153 & 0.0014 & 0.04 & 0.055 & 12.5 & 2 \\
\hline 43 & 457 & 40 & 0.0015 & $\mathrm{NA}$ & NA & 0.00008 & 0.00320 & 0.000153 & 0.0014 & 0.04 & 0.060 & 12.5 & 2 \\
\hline 43 & 458 & 40 & 0.0015 & NA & NA & 0.00008 & 0.00320 & 0.000153 & 0.0014 & 0.04 & 0.070 & 12.5 & 2 \\
\hline 43 & 459 & 40 & 0.0015 & NA & NA & 0.00008 & 0.00320 & 0.000153 & 0.0014 & 0.04 & 0.080 & 12.5 & 0 \\
\hline 43 & 460 & 40 & 0.0015 & NA & NA & 0.00008 & 0.00320 & 0.000153 & 0.0014 & 0.04 & 0.100 & 12.5 & 0 \\
\hline 43 & 461 & 40 & 0.0015 & NA & NA & 0.00008 & 0.00320 & 0.000153 & 0.0014 & 0.04 & 0.105 & 12.5 & 0 \\
\hline 43 & 462 & 40 & 0.0015 & NA & NA & 0.00008 & 0.00320 & 0.000153 & 0.0014 & 0.04 & 0.115 & 12.5 & 0 \\
\hline 43 & 463 & 40 & 0.0015 & NA & NA & 0.00008 & 0.00320 & 0.000153 & 0.0014 & 0.04 & 0.200 & 12.5 & 0 \\
\hline 43 & 464 & 40 & 0.0015 & NA & NA & 0.00008 & 0.00320 & 0.000153 & 0.0014 & 0.04 & 0.250 & 12.5 & 0 \\
\hline 43 & 465 & 40 & 0.0015 & NA & NA & 0.00008 & 0.00320 & 0.000153 & 0.0014 & 0.04 & 0.300 & 12.5 & 0 \\
\hline 43 & 466 & 40 & 0.0015 & NA & NA & 0.00008 & 0.00600 & 0.000153 & 0.0014 & 0.04 & 0.045 & 12.5 & 2 \\
\hline 43 & 467 & 40 & 0.0015 & NA & NA & 0.00008 & 0.00600 & 0.000153 & 0.0014 & 0.04 & 0.065 & 12.5 & 2 \\
\hline 43 & 468 & 40 & 0.0015 & NA & $\mathrm{NA}$ & 0.00008 & 0.00600 & 0.000153 & 0.0014 & 0.04 & 0.080 & 12.5 & 2 \\
\hline 43 & 469 & 40 & 0.0015 & NA & NA & 0.00008 & 0.00600 & 0.000153 & 0.0014 & 0.04 & 0.090 & 12.5 & 2 \\
\hline 43 & 470 & 40 & 0.0015 & $\mathrm{NA}$ & NA & 0.00008 & 0.01000 & 0.000153 & 0.0014 & 0.04 & 0.065 & 12.5 & 2 \\
\hline 43 & 471 & 40 & 0.0015 & NA & NA & 0.00008 & 0.01000 & 0.000153 & 0.0014 & 0.04 & 0.090 & 12.5 & 2 \\
\hline 43 & 472 & 40 & 0.0015 & $\mathrm{NA}$ & NA & 0.00008 & 0.01000 & 0.000153 & 0.0014 & 0.04 & 0.100 & 12.5 & 2 \\
\hline 43 & 473 & 40 & 0.0015 & $\mathrm{NA}$ & $\mathrm{NA}$ & 0.00008 & 0.01000 & 0.000153 & 0.0014 & 0.04 & 0.200 & 12.5 & 2 \\
\hline 43 & 474 & 40 & 0.0015 & NA & NA & 0.00008 & 0.01000 & 0.000153 & 0.0014 & 0.04 & 0.300 & 12.5 & 2 \\
\hline 43 & 475 & 40 & 0.0015 & $\mathrm{NA}$ & NA & 0.00008 & 0.01000 & 0.000153 & 0.0014 & 0.04 & 0.325 & 12.5 & 0 \\
\hline
\end{tabular}


Cyclic Potentiodynamic Polarization Conditions, and Chemical Composition and pH of Historical Data (continued 19)

\begin{tabular}{|c|c|c|c|c|c|c|c|c|c|c|c|c|c|}
\hline Reference & Test & $\begin{array}{c}\text { Temperature } \\
\left({ }^{\circ} \mathrm{C}\right)\end{array}$ & $\begin{array}{l}\text { Carbonate } \\
\text { (M) }\end{array}$ & $\begin{array}{c}\text { Bi- } \\
\text { carbonate } \\
\text { (M) }\end{array}$ & $\begin{array}{l}\text { Citrate } \\
\text { (M) }\end{array}$ & $\begin{array}{l}\text { Phosphate } \\
\text { (M) }\end{array}$ & $\begin{array}{l}\text { Chloride } \\
\text { (M) }\end{array}$ & $\begin{array}{l}\text { Fluoride } \\
\text { (M) }\end{array}$ & $\begin{array}{l}\text { Sulfate } \\
\text { (M) }\end{array}$ & $\begin{array}{l}\text { Nitrate } \\
\text { (M) }\end{array}$ & $\begin{array}{l}\text { Nitrite } \\
\text { (M) }\end{array}$ & $\mathrm{pH}$ & Pit ID* \\
\hline 43 & 476 & 40 & 0.0015 & NA & NA & 0.00008 & 0.01000 & 0.000153 & 0.0014 & 0.04 & 0.350 & 12.5 & 0 \\
\hline 43 & 477 & 40 & 0.0015 & NA & NA & 0.00008 & 0.01000 & 0.000153 & 0.0014 & 0.04 & 0.400 & 12.5 & 0 \\
\hline 43 & 478 & 40 & 0.0015 & $\mathrm{NA}$ & NA & 0.00008 & 0.01000 & 0.000153 & 0.0014 & 0.04 & 0.600 & 12.5 & 0 \\
\hline 43 & 479 & 40 & 0.0015 & NA & NA & 0.00008 & 0.02500 & 0.000153 & 0.0014 & 0.04 & 0.800 & 12.5 & 2 \\
\hline 43 & 480 & 40 & 0.0015 & NA & NA & 0.00008 & 0.02500 & 0.000153 & 0.0014 & 0.04 & 0.900 & 12.5 & 2 \\
\hline 43 & 481 & 40 & 0.0015 & NA & NA & 0.00008 & 0.02500 & 0.000153 & 0.0014 & 0.04 & 1.000 & 12.5 & 2 \\
\hline 43 & 482 & 40 & 0.0015 & $\mathrm{NA}$ & NA & 0.00008 & 0.02500 & 0.000153 & 0.0014 & 0.04 & 1.200 & 12.5 & 0 \\
\hline 43 & 483 & 40 & 0.0015 & NA & NA & 0.00008 & 0.02500 & 0.000153 & 0.0014 & 0.04 & 1.500 & 12.5 & 0 \\
\hline 43 & 484 & 40 & 0.0015 & NA & NA & 0.00008 & 0.02500 & 0.000153 & 0.0014 & 0.04 & 2.000 & 12.5 & 0 \\
\hline 43 & 485 & 40 & 0.0015 & NA & NA & 0.00008 & 0.05000 & 0.000153 & 0.0014 & 0.04 & 1.000 & 12.5 & 2 \\
\hline 43 & 486 & 40 & 0.0015 & NA & NA & 0.00008 & 0.05000 & 0.000153 & 0.0014 & 0.04 & 1.500 & 12.5 & 2 \\
\hline 43 & 487 & 40 & 0.0015 & NA & NA & 0.00008 & 0.05000 & 0.000153 & 0.0014 & 0.04 & 1.750 & 12.5 & 2 \\
\hline 43 & 488 & 40 & 0.0015 & NA & NA & 0.00008 & 0.05000 & 0.000153 & 0.0014 & 0.04 & 2.000 & 12.5 & 2 \\
\hline 43 & 489 & 40 & 0.0015 & NA & NA & 0.00008 & 0.05000 & 0.000153 & 0.0014 & 0.04 & 3.000 & 12.5 & 0 \\
\hline 43 & 490 & 40 & 0.0015 & NA & NA & 0.00008 & 0.05000 & 0.000153 & 0.0014 & 0.04 & 4.000 & 12.5 & 0 \\
\hline 44 & 491 & 40 & 0.5000 & NA & NA & NA & 0.00170 & NA & 0.03 & 0.3 & 0.400 & 9.73 & 1 \\
\hline 44 & 492 & 50 & 0.5000 & NA & NA & NA & 0.00170 & NA & 0.03 & 0.3 & 0.400 & 9.73 & 2 \\
\hline 44 & 493 & 50 & 0.5000 & NA & $\mathrm{NA}$ & NA & 0.00170 & NA & 0.03 & 0.3 & 0.500 & 9.73 & 0 \\
\hline 44 & 494 & 55 & 0.5000 & NA & NA & NA & 0.00170 & NA & 0.03 & 0.3 & 0.700 & 9.73 & 0 \\
\hline 44 & 495 & 60 & 0.5000 & $\mathrm{NA}$ & NA & $\mathrm{NA}$ & 0.00170 & NA & 0.03 & 0.3 & 0.500 & 9.73 & 2 \\
\hline 44 & 496 & 60 & 0.5000 & NA & NA & NA & 0.00170 & NA & 0.03 & 0.3 & 0.750 & 9.73 & 2 \\
\hline 44 & 497 & 60 & 0.5000 & $\mathrm{NA}$ & NA & NA & 0.00170 & NA & 0.03 & 0.3 & 1.250 & 9.73 & 2 \\
\hline 44 & 498 & 60 & 0.5000 & $\mathrm{NA}$ & $\mathrm{NA}$ & NA & 0.00170 & NA & 0.03 & 0.3 & 1.500 & 9.73 & 2 \\
\hline 44 & 499 & 60 & 0.5000 & NA & NA & NA & 0.00170 & NA & 0.03 & 0.3 & 2.000 & 9.73 & 0 \\
\hline 45 & 500 & 40 & 0.1490 & 0.151 & NA & NA & 0.0000 & 0.00267 & 0.00264 & 0.0098 & 0.000 & 10.07 & 2 \\
\hline
\end{tabular}


Cyclic Potentiodynamic Polarization Conditions, and Chemical Composition and pH of Historical Data (continued 20)

\begin{tabular}{|c|c|c|c|c|c|c|c|c|c|c|c|c|c|}
\hline Reference & Test & $\begin{array}{c}\text { Temperature } \\
\left({ }^{\circ} \mathrm{C}\right)\end{array}$ & $\begin{array}{l}\text { Carbonate } \\
\text { (M) }\end{array}$ & $\begin{array}{c}\text { Bi- } \\
\text { carbonate } \\
\text { (M) }\end{array}$ & $\begin{array}{l}\text { Citrate } \\
\text { (M) }\end{array}$ & $\begin{array}{l}\text { Phosphate } \\
\text { (M) }\end{array}$ & $\begin{array}{l}\text { Chloride } \\
\text { (M) }\end{array}$ & $\begin{array}{l}\text { Fluoride } \\
\text { (M) }\end{array}$ & $\begin{array}{l}\text { Sulfate } \\
\text { (M) }\end{array}$ & $\begin{array}{l}\text { Nitrate } \\
\text { (M) }\end{array}$ & $\begin{array}{l}\text { Nitrite } \\
\text { (M) }\end{array}$ & $\mathrm{pH}$ & Pit ID* \\
\hline 45 & 501 & 40 & 0.3370 & 0.243 & NA & NA & 0.0000 & 0.00267 & 0.00264 & 0.0098 & 0.000 & 10.18 & 0 \\
\hline 45 & 502 & 40 & 0.1490 & 0.151 & NA & NA & 0.0009 & 0.00267 & 0.00264 & 0.0098 & 0.022 & 10.07 & 2 \\
\hline 45 & 503 & 70 & 0.1490 & 0.151 & NA & NA & 0.0000 & 0.00267 & 0.00264 & 0.0098 & 0.000 & 10.07 & 2 \\
\hline 45 & 504 & 70 & 0.3370 & 0.243 & NA & NA & 0.0000 & 0.00267 & 0.00264 & 0.0098 & 0.000 & 10.18 & 0 \\
\hline 45 & 505 & 70 & 0.1490 & 0.151 & NA & NA & 0.0009 & 0.00267 & 0.00264 & 0.0098 & 0.022 & 10.07 & 2 \\
\hline 45 & 506 & 70 & 0.3370 & 0.243 & NA & NA & 0.0009 & 0.00267 & 0.00264 & 0.0098 & 0.022 & 10.18 & 0 \\
\hline 45 & 507 & 70 & 0.1490 & 0.151 & NA & NA & 0.0015 & 0.00267 & 0.00264 & 0.0098 & 0.044 & 10.07 & 0 \\
\hline 45 & 508 & 70 & 0.3370 & 0.243 & NA & NA & 0.0015 & 0.00267 & 0.00264 & 0.0098 & 0.045 & 10.18 & 0 \\
\hline 45 & 509 & 70 & 0.4190 & 0.275 & NA & NA & 0.0007 & 0.00267 & 0.00264 & 0.0098 & 0.015 & 10.21 & 0 \\
\hline 45 & 510 & 70 & 0.5180 & 0.311 & NA & NA & 0.0004 & 0.00267 & 0.00264 & 0.0098 & 0.007 & 10.24 & 0 \\
\hline 45 & 511 & 70 & 0.2880 & 0.222 & NA & NA & 0.0009 & 0.00267 & 0.00264 & 0.0098 & 0.022 & 10.16 & 0 \\
\hline 45 & 512 & 70 & 0.2230 & 0.191 & NA & NA & 0.0009 & 0.00267 & 0.00264 & 0.0098 & 0.022 & 10.12 & 0 \\
\hline 45 & 513 & 80 & 0.1490 & 0.151 & NA & NA & 0.0000 & 0.00267 & 0.00264 & 0.0098 & 0.000 & 10.07 & 2 \\
\hline 45 & 514 & 80 & 0.3370 & 0.243 & NA & NA & 0.0000 & 0.00267 & 0.00264 & 0.0098 & 0.000 & 10.18 & 2 \\
\hline 45 & 515 & 80 & 0.1490 & 0.151 & NA & NA & 0.0009 & 0.00267 & 0.00264 & 0.0098 & 0.022 & 10.07 & 2 \\
\hline 45 & 516 & 80 & 0.3370 & 0.243 & NA & NA & 0.0009 & 0.00267 & 0.00264 & 0.0098 & 0.022 & 10.18 & 0 \\
\hline 45 & 517 & 80 & 0.1490 & 0.151 & NA & NA & 0.0015 & 0.00267 & 0.00264 & 0.0098 & 0.044 & 10.07 & 2 \\
\hline 45 & 518 & 80 & 0.3370 & 0.243 & NA & NA & 0.0015 & 0.00267 & 0.00264 & 0.0098 & 0.045 & 10.18 & 0 \\
\hline 46 & 519 & 40 & 0.0050 & 0.009 & NA & NA & 0.0007 & NA & 0.003 & 0.02 & 0.015 & 10.00 & 2 \\
\hline 46 & 520 & 40 & 0.0090 & 0.017 & NA & NA & 0.0010 & NA & 0.006 & 0.05 & 0.025 & 10.00 & 2 \\
\hline 46 & 521 & 40 & 0.0180 & 0.033 & NA & NA & 0.0016 & $\mathrm{NA}$ & 0.015 & 0.05 & 0.050 & 10.00 & 0 \\
\hline 46 & 522 & 40 & 0.0090 & 0.017 & NA & NA & 0.0010 & NA & 0.006 & 0.1 & 0.025 & 10.00 & 2 \\
\hline 46 & 523 & 40 & 0.0260 & 0.048 & $\mathrm{NA}$ & NA & 0.0022 & NA & 0.024 & 0.1 & 0.075 & 10.00 & 2 \\
\hline 46 & 524 & 40 & 0.0440 & 0.082 & NA & NA & 0.0033 & NA & 0.043 & 0.1 & 0.125 & 10.00 & 2 \\
\hline 46 & 525 & 40 & 0.0260 & 0.048 & NA & NA & 0.0022 & NA & 0.024 & 0.15 & 0.075 & 10.00 & 2 \\
\hline
\end{tabular}


Cyclic Potentiodynamic Polarization Conditions, and Chemical Composition and pH of Historical Data (continued 21)

\begin{tabular}{|c|c|c|c|c|c|c|c|c|c|c|c|c|c|}
\hline Reference & Test & $\begin{array}{l}\text { Temperature } \\
\left({ }^{\circ} \mathrm{C}\right)\end{array}$ & $\begin{array}{l}\text { Carbonate } \\
\text { (M) }\end{array}$ & $\begin{array}{c}\text { Bi- } \\
\text { carbonate } \\
\text { (M) }\end{array}$ & $\begin{array}{l}\text { Citrate } \\
\text { (M) }\end{array}$ & $\begin{array}{l}\text { Phosphate } \\
\text { (M) }\end{array}$ & $\begin{array}{l}\text { Chloride } \\
\text { (M) }\end{array}$ & $\begin{array}{l}\text { Fluoride } \\
\text { (M) }\end{array}$ & $\begin{array}{c}\text { Sulfate } \\
\text { (M) }\end{array}$ & $\begin{array}{l}\text { Nitrate } \\
\text { (M) }\end{array}$ & $\begin{array}{l}\text { Nitrite } \\
\text { (M) }\end{array}$ & $\mathrm{pH}$ & Pit ID* \\
\hline 46 & 526 & 40 & 0.0530 & 0.098 & NA & NA & 0.0037 & NA & 0.054 & 0.15 & 0.15 & 10.00 & 0 \\
\hline 46 & 527 & 40 & 0.0180 & 0.033 & NA & NA & 0.0016 & NA & 0.015 & 0.2 & 0.05 & 10.00 & 2 \\
\hline 46 & 528 & 40 & 0.0530 & 0.098 & NA & NA & 0.0037 & NA & 0.054 & 0.2 & 0.15 & 10.00 & 2 \\
\hline 46 & 529 & 40 & 0.0880 & 0.163 & NA & NA & 0.0055 & NA & 0.099 & 0.2 & 0.25 & 10.00 & 2 \\
\hline 46 & 530 & 40 & 0.0440 & 0.082 & NA & NA & 0.0033 & NA & 0.043 & 0.25 & 0.125 & 10.00 & 2 \\
\hline 46 & 531 & 40 & 0.0880 & 0.163 & NA & NA & 0.0055 & NA & 0.099 & 0.25 & 0.25 & 10.00 & 2 \\
\hline 46 & 532 & 40 & 0.0260 & 0.048 & NA & NA & 0.0022 & NA & 0.024 & 0.3 & 0.075 & 10.00 & 2 \\
\hline 46 & 533 & 40 & 0.0790 & 0.147 & NA & NA & 0.0051 & NA & 0.087 & 0.3 & 0.225 & 10.00 & 0 \\
\hline 46 & 534 & 40 & 0.1310 & 0.243 & NA & NA & 0.0074 & NA & 0.16 & 0.3 & 0.375 & 10.00 & 2 \\
\hline 46 & 535 & 40 & 0.0610 & 0.113 & NA & NA & 0.0042 & NA & 0.065 & 0.35 & 0.175 & 10.00 & 2 \\
\hline 46 & 536 & 40 & 0.1230 & 0.228 & NA & NA & 0.0070 & NA & 0.148 & 0.35 & 0.35 & 10.00 & 2 \\
\hline 46 & 537 & 40 & 0.0350 & 0.065 & NA & NA & 0.0028 & NA & 0.033 & 0.4 & 0.1 & 10.00 & 0 \\
\hline 46 & 538 & 40 & 0.1050 & 0.195 & NA & NA & 0.0063 & NA & 0.123 & 0.4 & 0.3 & 10.00 & 2 \\
\hline 46 & 539 & 40 & 0.1750 & 0.325 & NA & NA & 0.0092 & NA & 0.226 & 0.4 & 0.5 & 10.00 & 2 \\
\hline 46 & 540 & 40 & 0.0790 & 0.147 & NA & NA & 0.0051 & NA & 0.087 & 0.45 & 0.225 & 10.00 & 2 \\
\hline 46 & 541 & 40 & 0.1580 & 0.293 & NA & NA & 0.0085 & NA & 0.199 & 0.45 & 0.45 & 10.00 & 2 \\
\hline 46 & 542 & 40 & 0.0440 & 0.082 & $\mathrm{NA}$ & $\mathrm{NA}$ & 0.0033 & NA & 0.043 & 0.5 & 0.125 & 10.00 & 2 \\
\hline 46 & 543 & 40 & 0.1310 & 0.243 & NA & NA & 0.0074 & NA & 0.16 & 0.5 & 0.375 & 10.00 & 2 \\
\hline 46 & 544 & 40 & 0.2190 & 0.407 & NA & NA & 0.0109 & NA & 0.294 & 0.5 & 0.625 & 10.00 & 0 \\
\hline 46 & 545 & 40 & 0.0960 & 0.178 & NA & NA & 0.0059 & NA & 0.11 & 0.55 & 0.275 & 10.00 & 2 \\
\hline 46 & 546 & 40 & 0.1930 & 0.358 & NA & NA & 0.0099 & NA & 0.253 & 0.55 & 0.55 & 10.00 & 0 \\
\hline 46 & 547 & 40 & 0.0530 & 0.098 & NA & NA & 0.0037 & NA & 0.054 & 0.6 & 0.15 & 10.00 & 2 \\
\hline 46 & 548 & 40 & 0.1580 & 0.293 & NA & NA & 0.0085 & NA & 0.199 & 0.6 & 0.45 & 10.00 & 2 \\
\hline 46 & 549 & 40 & 0.2630 & 0.488 & $\mathrm{NA}$ & NA & 0.0124 & NA & 0.366 & 0.6 & 0.75 & 10.00 & 0 \\
\hline 46 & 550 & 40 & 0.1140 & 0.212 & NA & NA & 0.0067 & NA & 0.135 & 0.65 & 0.325 & 10.00 & 0 \\
\hline
\end{tabular}


Cyclic Potentiodynamic Polarization Conditions, and Chemical Composition and pH of Historical Data (continued 22)

\begin{tabular}{|c|c|c|c|c|c|c|c|c|c|c|c|c|c|}
\hline Reference & Test & $\begin{array}{c}\text { Temperature } \\
\left({ }^{\circ} \mathrm{C}\right)\end{array}$ & $\begin{array}{l}\text { Carbonate } \\
\text { (M) }\end{array}$ & $\begin{array}{c}\text { Bi- } \\
\text { carbonate } \\
\text { (M) }\end{array}$ & $\begin{array}{l}\text { Citrate } \\
\text { (M) }\end{array}$ & $\begin{array}{l}\text { Phosphate } \\
\text { (M) }\end{array}$ & $\begin{array}{l}\text { Chloride } \\
\text { (M) }\end{array}$ & $\begin{array}{l}\text { Fluoride } \\
\text { (M) }\end{array}$ & $\begin{array}{l}\text { Sulfate } \\
\text { (M) }\end{array}$ & $\begin{array}{l}\text { Nitrate } \\
\text { (M) }\end{array}$ & $\begin{array}{l}\text { Nitrite } \\
\text { (M) }\end{array}$ & $\mathrm{pH}$ & Pit ID* \\
\hline 46 & 551 & 40 & 0.2280 & 0.423 & NA & NA & 0.0112 & NA & 0.308 & 0.65 & 0.65 & 10.00 & 0 \\
\hline 46 & 552 & 40 & 0.0610 & 0.113 & NA & NA & 0.0042 & NA & 0.065 & 0.7 & 0.175 & 10.00 & 0 \\
\hline 46 & 553 & 40 & 0.1840 & 0.342 & NA & NA & 0.0095 & NA & 0.239 & 0.7 & 0.525 & 10.00 & 2 \\
\hline 46 & 554 & 40 & 0.3060 & 0.568 & NA & NA & 0.0140 & NA & 0.439 & 0.7 & 0.875 & 10.00 & 0 \\
\hline 46 & 555 & 40 & 0.1310 & 0.243 & NA & NA & 0.0074 & NA & 0.16 & 0.75 & 0.375 & 10.00 & 2 \\
\hline 46 & 556 & 40 & 0.2630 & 0.488 & NA & NA & 0.0124 & NA & 0.366 & 0.75 & 0.75 & 10.00 & 2 \\
\hline 46 & 557 & 40 & 0.0700 & 0.130 & NA & NA & 0.0046 & NA & 0.076 & 0.8 & 0.2 & 10.00 & 2 \\
\hline 46 & 558 & 40 & 0.2100 & 0.390 & NA & NA & 0.0105 & NA & 0.28 & 0.8 & 0.6 & 10.00 & 2 \\
\hline 46 & 559 & 40 & 0.3500 & 0.650 & NA & NA & 0.0154 & NA & 0.515 & 0.8 & 1 & 10.00 & 0 \\
\hline 46 & 560 & 40 & 0.1490 & 0.277 & NA & NA & 0.0081 & NA & 0.186 & 0.85 & 0.425 & 10.00 & 2 \\
\hline 46 & 561 & 40 & 0.2980 & 0.553 & NA & NA & 0.0137 & NA & 0.424 & 0.85 & 0.85 & 10.00 & 0 \\
\hline 46 & 562 & 40 & 0.0790 & 0.147 & NA & NA & 0.0051 & NA & 0.087 & 0.9 & 0.225 & 10.00 & 0 \\
\hline 46 & 563 & 40 & 0.2360 & 0.438 & NA & NA & 0.0115 & NA & 0.186 & 0.9 & 0.675 & 10.00 & 2 \\
\hline 46 & 564 & 40 & 0.3940 & 0.732 & NA & NA & 0.0168 & NA & 0.593 & 0.9 & 1.125 & 10.00 & 2 \\
\hline 46 & 565 & 40 & 0.1660 & 0.308 & NA & NA & 0.0088 & NA & 0.212 & 0.95 & 0.475 & 10.00 & 0 \\
\hline 46 & 566 & 40 & 0.3330 & 0.618 & NA & NA & 0.0148 & NA & 0.485 & 0.95 & 0.95 & 10.00 & 2 \\
\hline 46 & 567 & 40 & 0.0880 & 0.163 & NA & NA & 0.0055 & NA & 0.099 & 1 & 0.25 & 10.00 & 2 \\
\hline 46 & 568 & 40 & 0.2630 & 0.488 & NA & NA & 0.0124 & NA & 0.366 & 1 & 0.75 & 10.00 & 0 \\
\hline 46 & 569 & 40 & 0.4380 & 0.813 & NA & NA & 0.0182 & NA & 0.672 & 1 & 1.25 & 10.00 & 0 \\
\hline 47 & 570 & 40 & 0.0035 & 0.007 & NA & NA & 0.0005 & NA & 0.0018 & 0.02 & 0.010 & 10.00 & 2 \\
\hline 47 & 571 & 40 & 0.0053 & 0.010 & NA & NA & 0.0007 & NA & 0.0029 & 0.02 & 0.015 & 10.00 & 2 \\
\hline 47 & 572 & 40 & 0.0070 & 0.013 & NA & NA & 0.0008 & NA & 0.0041 & 0.02 & 0.020 & 10.00 & 2 \\
\hline 47 & 573 & 40 & 0.0044 & 0.008 & NA & NA & 0.0006 & NA & 0.0023 & 0.05 & 0.013 & 10.00 & 2 \\
\hline 47 & 574 & 40 & 0.0088 & 0.016 & NA & NA & 0.0010 & NA & 0.0053 & 0.05 & 0.025 & 10.00 & 2 \\
\hline 47 & 575 & 40 & 0.0132 & 0.024 & NA & NA & 0.0013 & NA & 0.0087 & 0.05 & 0.038 & 10.00 & 2 \\
\hline
\end{tabular}


Cyclic Potentiodynamic Polarization Conditions, and Chemical Composition and pH of Historical Data (continued 23)

\begin{tabular}{|c|c|c|c|c|c|c|c|c|c|c|c|c|c|}
\hline Reference & Test & $\begin{array}{l}\text { Temperature } \\
\left({ }^{\circ} \mathrm{C}\right)\end{array}$ & $\begin{array}{l}\text { Carbonate } \\
\text { (M) }\end{array}$ & $\begin{array}{c}\text { Bi- } \\
\text { carbonate } \\
\text { (M) }\end{array}$ & $\begin{array}{l}\text { Citrate } \\
\text { (M) }\end{array}$ & $\begin{array}{l}\text { Phosphate } \\
\text { (M) }\end{array}$ & $\begin{array}{l}\text { Chloride } \\
\text { (M) }\end{array}$ & $\begin{array}{l}\text { Fluoride } \\
\text { (M) }\end{array}$ & $\begin{array}{l}\text { Sulfate } \\
\text { (M) }\end{array}$ & $\begin{array}{l}\text { Nitrate } \\
\text { (M) }\end{array}$ & $\begin{array}{l}\text { Nitrite } \\
\text { (M) }\end{array}$ & $\mathrm{pH}$ & Pit ID* \\
\hline 47 & 576 & 40 & 0.0175 & 0.033 & $\mathrm{NA}$ & $\mathrm{NA}$ & 0.0016 & NA & 0.0122 & 0.05 & 0.050 & 10.00 & 2 \\
\hline 47 & 577 & 40 & 0.0219 & 0.041 & $\mathrm{NA}$ & $\mathrm{NA}$ & 0.0019 & NA & 0.0159 & 0.05 & 0.063 & 10.00 & 0 \\
\hline 47 & 578 & 40 & 0.0088 & 0.016 & NA & NA & 0.0010 & NA & 0.0053 & 0.1 & 0.025 & 10.00 & 2 \\
\hline 47 & 579 & 40 & 0.0175 & 0.033 & $\mathrm{NA}$ & $\mathrm{NA}$ & 0.0016 & NA & 0.0122 & 0.1 & 0.050 & 10.00 & 2 \\
\hline 47 & 580 & 40 & 0.0263 & 0.049 & NA & NA & 0.0022 & NA & 0.0198 & 0.1 & 0.075 & 10.00 & 2 \\
\hline 47 & 581 & 40 & 0.0351 & 0.065 & NA & NA & 0.0028 & NA & 0.0278 & 0.1 & 0.100 & 10.00 & 2 \\
\hline 47 & 582 & 40 & 0.0439 & 0.081 & NA & NA & 0.0033 & NA & 0.0363 & 0.1 & 0.125 & 10.00 & 2 \\
\hline 47 & 583 & 40 & 0.0132 & 0.024 & NA & NA & 0.0013 & NA & 0.0087 & 0.15 & 0.038 & 10.00 & 2 \\
\hline 47 & 584 & 40 & 0.0263 & 0.049 & $\mathrm{NA}$ & $\mathrm{NA}$ & 0.0022 & NA & 0.0198 & 0.15 & 0.075 & 10.00 & 2 \\
\hline 47 & 585 & 40 & 0.0395 & 0.073 & NA & NA & 0.0030 & NA & 0.0320 & 0.15 & 0.113 & 10.00 & 2 \\
\hline 47 & 586 & 40 & 0.0526 & 0.098 & NA & NA & 0.0037 & NA & 0.0451 & 0.15 & 0.150 & 10.00 & 0 \\
\hline 47 & 587 & 40 & 0.0658 & 0.122 & NA & NA & 0.0044 & NA & 0.0588 & 0.15 & 0.188 & 10.00 & 2 \\
\hline 47 & 588 & 40 & 0.0175 & 0.033 & NA & NA & 0.0016 & NA & 0.0122 & 0.2 & 0.050 & 10.00 & 2 \\
\hline 47 & 589 & 40 & 0.0351 & 0.065 & NA & NA & 0.0028 & NA & 0.0278 & 0.2 & 0.100 & 10.00 & 2 \\
\hline 47 & 590 & 40 & 0.0526 & 0.098 & NA & NA & 0.0037 & NA & 0.0451 & 0.2 & 0.150 & 10.00 & 2 \\
\hline 47 & 591 & 40 & 0.0702 & 0.130 & $\mathrm{NA}$ & $\mathrm{NA}$ & 0.0046 & NA & 0.0635 & 0.2 & 0.200 & 10.00 & 2 \\
\hline 47 & 592 & 40 & 0.0877 & 0.163 & NA & $\mathrm{NA}$ & 0.0055 & NA & 0.0828 & 0.2 & 0.250 & 10.00 & 2 \\
\hline 47 & 593 & 40 & 0.0219 & 0.041 & $\mathrm{NA}$ & $\mathrm{NA}$ & 0.0019 & NA & 0.0159 & 0.25 & 0.063 & 10.00 & 2 \\
\hline 47 & 594 & 40 & 0.0439 & 0.081 & $\mathrm{NA}$ & $\mathrm{NA}$ & 0.0033 & NA & 0.0363 & 0.25 & 0.125 & 10.00 & 2 \\
\hline 47 & 595 & 40 & 0.0658 & 0.122 & $\mathrm{NA}$ & $\mathrm{NA}$ & 0.0044 & NA & 0.0588 & 0.25 & 0.188 & 10.00 & 2 \\
\hline 47 & 596 & 40 & 0.0877 & 0.163 & $\mathrm{NA}$ & $\mathrm{NA}$ & 0.0055 & $\mathrm{NA}$ & 0.0828 & 0.25 & 0.250 & 10.00 & 2 \\
\hline 47 & 597 & 40 & 0.1096 & 0.204 & NA & NA & 0.0065 & NA & 0.1080 & 0.25 & 0.313 & 10.00 & 2 \\
\hline 47 & 598 & 40 & 0.0263 & 0.049 & NA & NA & 0.0022 & NA & 0.0198 & 0.3 & 0.075 & 10.00 & 2 \\
\hline 47 & 599 & 40 & 0.0526 & 0.098 & $\mathrm{NA}$ & $\mathrm{NA}$ & 0.0037 & NA & 0.0451 & 0.3 & 0.150 & 10.00 & 2 \\
\hline 47 & 600 & 40 & 0.0789 & 0.147 & NA & NA & 0.0051 & NA & 0.0731 & 0.3 & 0.225 & 10.00 & 0 \\
\hline
\end{tabular}


Cyclic Potentiodynamic Polarization Conditions, and Chemical Composition and pH of Historical Data (continued 24)

\begin{tabular}{|c|c|c|c|c|c|c|c|c|c|c|c|c|c|}
\hline Reference & Test & $\begin{array}{c}\text { Temperature } \\
\left({ }^{\circ} \mathrm{C}\right)\end{array}$ & $\begin{array}{l}\text { Carbonate } \\
\text { (M) }\end{array}$ & $\begin{array}{c}\text { Bi- } \\
\text { carbonate } \\
\text { (M) }\end{array}$ & $\begin{array}{l}\text { Citrate } \\
\text { (M) }\end{array}$ & $\begin{array}{l}\text { Phosphate } \\
\text { (M) }\end{array}$ & $\begin{array}{l}\text { Chloride } \\
\text { (M) }\end{array}$ & $\begin{array}{l}\text { Fluoride } \\
\text { (M) }\end{array}$ & $\begin{array}{l}\text { Sulfate } \\
\text { (M) }\end{array}$ & $\begin{array}{l}\text { Nitrate } \\
\text { (M) }\end{array}$ & $\begin{array}{l}\text { Nitrite } \\
\text { (M) }\end{array}$ & $\mathrm{pH}$ & Pit ID* \\
\hline 47 & 601 & 40 & 0.1053 & 0.195 & NA & NA & 0.0063 & NA & 0.1029 & 0.3 & 0.300 & 10.00 & 2 \\
\hline 47 & 602 & 40 & 0.1316 & 0.244 & NA & NA & 0.0074 & NA & 0.1342 & 0.3 & 0.375 & 10.00 & 2 \\
\hline 47 & 603 & 40 & 0.0307 & 0.057 & NA & NA & 0.0025 & NA & 0.0237 & 0.35 & 0.088 & 10.00 & 2 \\
\hline 47 & 604 & 40 & 0.0614 & 0.114 & NA & NA & 0.0042 & NA & 0.0542 & 0.35 & 0.175 & 10.00 & 2 \\
\hline 47 & 605 & 40 & 0.0921 & 0.171 & NA & NA & 0.0057 & NA & 0.0878 & 0.35 & 0.263 & 10.00 & 2 \\
\hline 47 & 606 & 40 & 0.1228 & 0.228 & NA & NA & 0.0070 & NA & 0.1236 & 0.35 & 0.350 & 10.00 & 2 \\
\hline 47 & 607 & 40 & 0.1535 & 0.285 & NA & NA & 0.0083 & NA & 0.1613 & 0.35 & 0.438 & 10.00 & 2 \\
\hline 47 & 608 & 40 & 0.0351 & 0.065 & NA & NA & 0.0028 & NA & 0.0278 & 0.4 & 0.100 & 10.00 & 0 \\
\hline 47 & 609 & 40 & 0.0702 & 0.130 & NA & NA & 0.0046 & NA & 0.0635 & 0.4 & 0.200 & 10.00 & 2 \\
\hline 47 & 610 & 40 & 0.1053 & 0.195 & NA & NA & 0.0063 & NA & 0.1029 & 0.4 & 0.300 & 10.00 & 2 \\
\hline 47 & 611 & 40 & 0.1404 & 0.261 & NA & NA & 0.0078 & NA & 0.1449 & 0.4 & 0.400 & 10.00 & 2 \\
\hline 47 & 612 & 40 & 0.1754 & 0.326 & NA & NA & 0.0092 & NA & 0.1891 & 0.4 & 0.500 & 10.00 & 2 \\
\hline 47 & 613 & 40 & 0.0395 & 0.073 & NA & NA & 0.0030 & NA & 0.0320 & 0.45 & 0.113 & 10.00 & 2 \\
\hline 47 & 614 & 40 & 0.0789 & 0.147 & NA & NA & 0.0051 & NA & 0.0731 & 0.45 & 0.225 & 10.00 & 2 \\
\hline 47 & 615 & 40 & 0.1184 & 0.220 & NA & NA & 0.0069 & NA & 0.1184 & 0.45 & 0.338 & 10.00 & 2 \\
\hline 47 & 616 & 40 & 0.1579 & 0.293 & NA & NA & 0.0085 & NA & 0.1668 & 0.45 & 0.450 & 10.00 & 2 \\
\hline 47 & 617 & 40 & 0.1974 & 0.367 & NA & NA & 0.0100 & NA & 0.2175 & 0.45 & 0.563 & 10.00 & 2 \\
\hline 47 & 618 & 40 & 0.0439 & 0.081 & NA & NA & 0.0033 & NA & 0.0363 & 0.5 & 0.125 & 10.00 & 2 \\
\hline 47 & 619 & 40 & 0.0877 & 0.163 & NA & NA & 0.0055 & NA & 0.0828 & 0.5 & 0.250 & 10.00 & 2 \\
\hline 47 & 620 & 40 & 0.1316 & 0.244 & NA & NA & 0.0074 & NA & 0.1342 & 0.5 & 0.375 & 10.00 & 2 \\
\hline 47 & 621 & 40 & 0.1754 & 0.326 & NA & NA & 0.0092 & NA & 0.1891 & 0.5 & 0.500 & 10.00 & 2 \\
\hline 47 & 622 & 40 & 0.2193 & 0.407 & NA & NA & 0.0109 & NA & 0.2466 & 0.5 & 0.625 & 10.00 & 0 \\
\hline 47 & 623 & 40 & 0.0482 & 0.090 & NA & NA & 0.0035 & NA & 0.0407 & 0.55 & 0.138 & 10.00 & 2 \\
\hline 47 & 624 & 40 & 0.0965 & 0.179 & NA & NA & 0.0059 & NA & 0.0928 & 0.55 & 0.275 & 10.00 & 2 \\
\hline 47 & 625 & 40 & 0.1447 & 0.269 & NA & NA & 0.0080 & NA & 0.1504 & 0.55 & 0.413 & 10.00 & 2 \\
\hline
\end{tabular}


Cyclic Potentiodynamic Polarization Conditions, and Chemical Composition and pH of Historical Data (continued 25)

\begin{tabular}{|c|c|c|c|c|c|c|c|c|c|c|c|c|c|}
\hline Reference & Test & $\begin{array}{l}\text { Temperature } \\
\left({ }^{\circ} \mathrm{C}\right)\end{array}$ & $\begin{array}{l}\text { Carbonate } \\
\text { (M) }\end{array}$ & $\begin{array}{c}\text { Bi- } \\
\text { carbonate } \\
\text { (M) }\end{array}$ & $\begin{array}{l}\text { Citrate } \\
\text { (M) }\end{array}$ & $\begin{array}{l}\text { Phosphate } \\
\text { (M) }\end{array}$ & $\begin{array}{l}\text { Chloride } \\
\text { (M) }\end{array}$ & $\begin{array}{l}\text { Fluoride } \\
\text { (M) }\end{array}$ & $\begin{array}{c}\text { Sulfate } \\
\text { (M) }\end{array}$ & $\begin{array}{l}\text { Nitrate } \\
\text { (M) }\end{array}$ & $\begin{array}{l}\text { Nitrite } \\
\text { (M) }\end{array}$ & $\mathrm{pH}$ & Pit ID* \\
\hline 47 & 626 & 40 & 0.1930 & 0.358 & NA & NA & 0.0099 & NA & 0.2118 & 0.55 & 0.550 & 10.00 & 0 \\
\hline 47 & 627 & 40 & 0.2412 & 0.448 & NA & NA & 0.0117 & NA & 0.2762 & 0.55 & 0.688 & 10.00 & 0 \\
\hline 47 & 628 & 40 & 0.0526 & 0.098 & NA & NA & 0.0037 & NA & 0.0451 & 0.6 & 0.150 & 10.00 & 2 \\
\hline 47 & 629 & 40 & 0.1053 & 0.195 & NA & NA & 0.0063 & NA & 0.1029 & 0.6 & 0.300 & 10.00 & 2 \\
\hline 47 & 630 & 40 & 0.1579 & 0.293 & NA & NA & 0.0085 & NA & 0.1668 & 0.6 & 0.450 & 10.00 & 2 \\
\hline 47 & 631 & 40 & 0.2105 & 0.391 & NA & NA & 0.0105 & NA & 0.2349 & 0.6 & 0.600 & 10.00 & 0 \\
\hline 47 & 632 & 40 & 0.2632 & 0.489 & NA & NA & 0.0124 & NA & 0.3063 & 0.6 & 0.750 & 10.00 & 0 \\
\hline 47 & 633 & 40 & 0.0570 & 0.106 & NA & NA & 0.0040 & NA & 0.0496 & 0.65 & 0.163 & 10.00 & 2 \\
\hline 47 & 634 & 40 & 0.1140 & 0.212 & NA & NA & 0.0067 & NA & 0.1132 & 0.65 & 0.325 & 10.00 & 0 \\
\hline 47 & 635 & 40 & 0.1711 & 0.318 & NA & NA & 0.0090 & NA & 0.1834 & 0.65 & 0.488 & 10.00 & 0 \\
\hline 47 & 636 & 40 & 0.2281 & 0.424 & NA & NA & 0.0112 & NA & 0.2584 & 0.65 & 0.650 & 10.00 & 0 \\
\hline 47 & 637 & 40 & 0.2851 & 0.529 & NA & NA & 0.0132 & NA & 0.3370 & 0.65 & 0.813 & 10.00 & 0 \\
\hline 47 & 638 & 40 & 0.0614 & 0.114 & NA & NA & 0.0042 & NA & 0.0542 & 0.7 & 0.175 & 10.00 & 0 \\
\hline 47 & 639 & 40 & 0.1228 & 0.228 & NA & NA & 0.0070 & NA & 0.1236 & 0.7 & 0.350 & 10.00 & 2 \\
\hline 47 & 640 & 40 & 0.1842 & 0.342 & NA & NA & 0.0095 & NA & 0.2004 & 0.7 & 0.525 & 10.00 & 2 \\
\hline 47 & 641 & 40 & 0.2456 & 0.456 & NA & NA & 0.0118 & NA & 0.2822 & 0.7 & 0.700 & 10.00 & 0 \\
\hline 47 & 642 & 40 & 0.3070 & 0.570 & $\mathrm{NA}$ & $\mathrm{NA}$ & 0.0140 & NA & 0.3681 & 0.7 & 0.875 & 10.00 & 0 \\
\hline 47 & 643 & 40 & 0.0658 & 0.122 & NA & NA & 0.0044 & NA & 0.0588 & 0.75 & 0.188 & 10.00 & 2 \\
\hline 47 & 644 & 40 & 0.1316 & 0.244 & NA & NA & 0.0074 & NA & 0.1342 & 0.75 & 0.375 & 10.00 & 2 \\
\hline 47 & 645 & 40 & 0.1974 & 0.367 & NA & NA & 0.0100 & NA & 0.2175 & 0.75 & 0.563 & 10.00 & 2 \\
\hline 47 & 646 & 40 & 0.2632 & 0.489 & NA & NA & 0.0124 & NA & 0.3063 & 0.75 & 0.750 & 10.00 & 2 \\
\hline 47 & 647 & 40 & 0.3289 & 0.611 & NA & NA & 0.0147 & NA & 0.3996 & 0.75 & 0.938 & 10.00 & 0 \\
\hline 47 & 648 & 40 & 0.0702 & 0.130 & NA & NA & 0.0046 & NA & 0.0635 & 0.8 & 0.200 & 10.00 & 2 \\
\hline 47 & 649 & 40 & 0.1404 & 0.261 & $\mathrm{NA}$ & NA & 0.0078 & NA & 0.1449 & 0.8 & 0.400 & 10.00 & 0 \\
\hline 47 & 650 & 40 & 0.2105 & 0.391 & NA & NA & 0.0105 & NA & 0.2349 & 0.8 & 0.600 & 10.00 & 2 \\
\hline
\end{tabular}


Cyclic Potentiodynamic Polarization Conditions, and Chemical Composition and pH of Historical Data (continued 26)

\begin{tabular}{|c|c|c|c|c|c|c|c|c|c|c|c|c|c|}
\hline Reference & Test & $\begin{array}{l}\text { Temperature } \\
\left({ }^{\circ} \mathrm{C}\right)\end{array}$ & $\begin{array}{l}\text { Carbonate } \\
\text { (M) }\end{array}$ & $\begin{array}{c}\text { Bi- } \\
\text { carbonate } \\
\text { (M) }\end{array}$ & $\begin{array}{l}\text { Citrate } \\
\text { (M) }\end{array}$ & $\begin{array}{l}\text { Phosphate } \\
\text { (M) }\end{array}$ & $\begin{array}{l}\text { Chloride } \\
\text { (M) }\end{array}$ & $\begin{array}{l}\text { Fluoride } \\
\text { (M) }\end{array}$ & $\begin{array}{c}\text { Sulfate } \\
\text { (M) }\end{array}$ & $\begin{array}{l}\text { Nitrate } \\
\text { (M) }\end{array}$ & $\begin{array}{l}\text { Nitrite } \\
\text { (M) }\end{array}$ & $\mathrm{pH}$ & Pit ID* \\
\hline 47 & 651 & 40 & 0.2807 & 0.521 & NA & NA & 0.0131 & NA & 0.3308 & 0.8 & 0.800 & 10.00 & 0 \\
\hline 47 & 652 & 40 & 0.3509 & 0.652 & NA & NA & 0.0154 & NA & 0.4315 & 0.8 & 1.000 & 10.00 & 0 \\
\hline 47 & 653 & 40 & 0.0746 & 0.138 & NA & NA & 0.0049 & NA & 0.0683 & 0.85 & 0.213 & 10.00 & 2 \\
\hline 47 & 654 & 40 & 0.1491 & 0.277 & NA & NA & 0.0081 & NA & 0.1558 & 0.85 & 0.425 & 10.00 & 2 \\
\hline 47 & 655 & 40 & 0.2237 & 0.415 & NA & NA & 0.0110 & NA & 0.2525 & 0.85 & 0.638 & 10.00 & 2 \\
\hline 47 & 656 & 40 & 0.2982 & 0.554 & NA & NA & 0.0137 & NA & 0.3556 & 0.85 & 0.850 & 10.00 & 0 \\
\hline 47 & 657 & 40 & 0.3728 & 0.692 & NA & NA & 0.0161 & NA & 0.4638 & 0.85 & 1.063 & 10.00 & 0 \\
\hline 47 & 658 & 40 & 0.0789 & 0.147 & NA & NA & 0.0051 & NA & 0.0731 & 0.9 & 0.225 & 10.00 & 2 \\
\hline 47 & 659 & 40 & 0.1579 & 0.293 & NA & NA & 0.0085 & NA & 0.1668 & 0.9 & 0.450 & 10.00 & 2 \\
\hline 47 & 660 & 40 & 0.2368 & 0.440 & NA & NA & 0.0115 & NA & 0.2702 & 0.9 & 0.675 & 10.00 & 2 \\
\hline 47 & 661 & 40 & 0.3158 & 0.586 & NA & NA & 0.0142 & NA & 0.3806 & 0.9 & 0.900 & 10.00 & 0 \\
\hline 47 & 662 & 40 & 0.3947 & 0.733 & NA & NA & 0.0168 & NA & 0.4964 & 0.9 & 1.125 & 10.00 & 0 \\
\hline 47 & 663 & 40 & 0.0833 & 0.155 & NA & NA & 0.0053 & NA & 0.0779 & 0.95 & 0.238 & 10.00 & 2 \\
\hline 47 & 664 & 40 & 0.1667 & 0.310 & NA & NA & 0.0088 & NA & 0.1779 & 0.95 & 0.475 & 10.00 & 0 \\
\hline 47 & 665 & 40 & 0.2500 & 0.464 & NA & NA & 0.0120 & NA & 0.2882 & 0.95 & 0.713 & 10.00 & 0 \\
\hline 47 & 666 & 40 & 0.3333 & 0.619 & NA & NA & 0.0148 & NA & 0.4059 & 0.95 & 0.950 & 10.00 & 2 \\
\hline 47 & 667 & 40 & 0.4167 & 0.774 & $\mathrm{NA}$ & $\mathrm{NA}$ & 0.0175 & NA & 0.5294 & 0.95 & 1.188 & 10.00 & 0 \\
\hline 47 & 668 & 40 & 0.0877 & 0.163 & NA & NA & 0.0055 & NA & 0.0828 & 1 & 0.250 & 10.00 & 2 \\
\hline 47 & 669 & 40 & 0.1754 & 0.326 & NA & NA & 0.0092 & NA & 0.1891 & 1 & 0.500 & 10.00 & 0 \\
\hline 47 & 670 & 40 & 0.2632 & 0.489 & NA & NA & 0.0124 & NA & 0.3063 & 1 & 0.750 & 10.00 & 0 \\
\hline 47 & 671 & 40 & 0.3509 & 0.652 & NA & NA & 0.0154 & NA & 0.4315 & 1 & 1.000 & 10.00 & 0 \\
\hline 47 & 672 & 40 & 0.4386 & 0.815 & NA & NA & 0.0182 & NA & 0.5628 & 1 & 1.250 & 10.00 & 0 \\
\hline 48 & 673 & 40 & 0.3509 & 0.652 & NA & NA & 0.015 & NA & 0.43 & 0.6 & 1 & 10.00 & 0 \\
\hline 48 & 674 & 40 & 0.3509 & 0.652 & $\mathrm{NA}$ & NA & 0.015 & NA & 0.43 & 0.65 & 1 & 10.00 & 0 \\
\hline 48 & 675 & 40 & 0.3509 & 0.652 & NA & NA & 0.015 & NA & 0.43 & 0.7 & 1 & 10.00 & 0 \\
\hline
\end{tabular}


Cyclic Potentiodynamic Polarization Conditions, and Chemical Composition and pH of Historical Data (continued 27)

\begin{tabular}{|c|c|c|c|c|c|c|c|c|c|c|c|c|c|}
\hline Reference & Test & $\begin{array}{l}\text { Temperature } \\
\left({ }^{\circ} \mathrm{C}\right)\end{array}$ & $\begin{array}{l}\text { Carbonate } \\
\text { (M) }\end{array}$ & $\begin{array}{c}\text { Bi- } \\
\text { carbonate } \\
\text { (M) }\end{array}$ & $\begin{array}{l}\text { Citrate } \\
\text { (M) }\end{array}$ & $\begin{array}{l}\text { Phosphate } \\
\text { (M) }\end{array}$ & $\begin{array}{l}\text { Chloride } \\
\text { (M) }\end{array}$ & $\begin{array}{l}\text { Fluoride } \\
\text { (M) }\end{array}$ & $\begin{array}{c}\text { Sulfate } \\
\text { (M) }\end{array}$ & $\begin{array}{l}\text { Nitrate } \\
\text { (M) }\end{array}$ & $\begin{array}{l}\text { Nitrite } \\
\text { (M) }\end{array}$ & $\mathrm{pH}$ & Pit ID* \\
\hline 48 & 676 & 40 & 0.3509 & 0.652 & NA & NA & 0.015 & NA & 0.43 & 0.75 & 1 & 10.00 & 0 \\
\hline 48 & 677 & 40 & 0.3509 & 0.652 & NA & NA & 0.015 & NA & 0.43 & 0.8 & 1 & 10.00 & 0 \\
\hline 48 & 678 & 40 & 0.3509 & 0.652 & NA & NA & 0.015 & NA & 0.43 & 0.85 & 1 & 10.00 & 0 \\
\hline 48 & 679 & 40 & 0.3509 & 0.652 & NA & NA & 0.015 & NA & 0.43 & 0.9 & 1 & 10.00 & 0 \\
\hline 48 & 680 & 40 & 0.3509 & 0.652 & NA & NA & 0.015 & NA & 0.43 & 0.95 & 1 & 10.00 & 0 \\
\hline 48 & 681 & 40 & 0.3509 & 0.652 & NA & NA & 0.015 & NA & 0.43 & 1 & 1 & 10.00 & 0 \\
\hline 48 & 682 & 40 & 0.1754 & 0.326 & NA & NA & 0.002 & NA & 0.025 & 0.2 & 0.5 & 10.00 & 0 \\
\hline 48 & 683 & 40 & 0.2105 & 0.391 & NA & NA & 0.002 & NA & 0.025 & 0.2 & 0.6 & 10.00 & 0 \\
\hline 48 & 684 & 40 & 0.1754 & 0.326 & NA & NA & 0.005 & NA & 0.055 & 0.4 & 0.5 & 10.00 & 0 \\
\hline 48 & 685 & 40 & 0.2105 & 0.391 & NA & NA & 0.005 & NA & 0.055 & 0.4 & 0.6 & 10.00 & 0 \\
\hline 48 & 686 & 40 & 0.2632 & 0.489 & NA & NA & 0.005 & NA & 0.055 & 0.4 & 0.75 & 10.00 & 0 \\
\hline 48 & 687 & 40 & 0.2105 & 0.391 & NA & NA & 0.025 & NA & 0.09 & 0.6 & 0.6 & 10.00 & 0 \\
\hline 48 & 688 & 40 & 0.2632 & 0.489 & NA & NA & 0.025 & NA & 0.09 & 0.6 & 0.75 & 10.00 & 0 \\
\hline 48 & 689 & 40 & 0.1754 & 0.326 & NA & NA & 0.032 & NA & 0.121 & 0.8 & 0.5 & 10.00 & 2 \\
\hline 48 & 690 & 40 & 0.2105 & 0.391 & $\mathrm{NA}$ & $\mathrm{NA}$ & 0.032 & NA & 0.121 & 0.8 & 0.6 & 10.00 & 1 \\
\hline 48 & 691 & 40 & 0.2632 & 0.489 & NA & NA & 0.032 & NA & 0.121 & 0.8 & 0.75 & 10.00 & 0 \\
\hline 28 & 692 & 40 & 0.0175 & 0.033 & NA & NA & 0.0005 & NA & 0.015 & 0.1 & 0.05 & 10.00 & 2 \\
\hline 28 & 693 & 40 & 0.0439 & 0.081 & NA & $\mathrm{NA}$ & 0.00125 & NA & 0.0375 & 0.25 & 0.125 & 10.00 & 0 \\
\hline 28 & 694 & 40 & 0.0702 & 0.130 & NA & NA & 0.002 & NA & 0.06 & 0.4 & 0.2 & 10.00 & 2 \\
\hline 28 & 695 & 40 & 0.0965 & 0.179 & NA & NA & 0.00275 & NA & 0.0825 & 0.55 & 0.275 & 10.00 & 0 \\
\hline 28 & 696 & 40 & 0.1228 & 0.228 & NA & NA & 0.0035 & NA & 0.105 & 0.7 & 0.35 & 10.00 & 0 \\
\hline 28 & 697 & 40 & 0.1491 & 0.277 & NA & NA & 0.00425 & NA & 0.1275 & 0.85 & 0.425 & 10.00 & 0 \\
\hline 28 & 698 & 40 & 0.1754 & 0.326 & NA & NA & 0.005 & NA & 0.15 & 1 & 0.5 & 10.00 & 0 \\
\hline 28 & 699 & 40 & 0.2105 & 0.391 & $\mathrm{NA}$ & NA & 0.006 & NA & 0.18 & 1.2 & 0.6 & 10.00 & 0 \\
\hline 28 & 700 & 40 & 0.0351 & 0.065 & NA & NA & 0.0005 & NA & 0.015 & 0.1 & 0.1 & 10.00 & 0 \\
\hline
\end{tabular}


Cyclic Potentiodynamic Polarization Conditions, and Chemical Composition and pH of Historical Data (continued 28)

\begin{tabular}{|c|c|c|c|c|c|c|c|c|c|c|c|c|c|}
\hline Reference & Test & $\begin{array}{l}\text { Temperature } \\
\left({ }^{\circ} \mathrm{C}\right)\end{array}$ & $\begin{array}{l}\text { Carbonate } \\
\text { (M) }\end{array}$ & $\begin{array}{c}\text { Bi- } \\
\text { carbonate } \\
\text { (M) }\end{array}$ & $\begin{array}{l}\text { Citrate } \\
\text { (M) }\end{array}$ & $\begin{array}{l}\text { Phosphate } \\
\text { (M) }\end{array}$ & $\begin{array}{c}\text { Chloride } \\
\text { (M) }\end{array}$ & $\begin{array}{l}\text { Fluoride } \\
\text { (M) }\end{array}$ & $\begin{array}{l}\text { Sulfate } \\
\text { (M) }\end{array}$ & $\begin{array}{l}\text { Nitrate } \\
\text { (M) }\end{array}$ & $\begin{array}{l}\text { Nitrite } \\
\text { (M) }\end{array}$ & $\mathrm{pH}$ & Pit ID* \\
\hline 28 & 701 & 40 & 0.0877 & 0.163 & $\mathrm{NA}$ & $\mathrm{NA}$ & 0.00125 & NA & 0.0375 & 0.25 & 0.25 & 10.00 & 0 \\
\hline 28 & 702 & 40 & 0.1404 & 0.261 & $\mathrm{NA}$ & $\mathrm{NA}$ & 0.002 & NA & 0.06 & 0.4 & 0.4 & 10.00 & 0 \\
\hline 28 & 703 & 40 & 0.1930 & 0.358 & NA & NA & 0.00275 & NA & 0.0825 & 0.55 & 0.55 & 10.00 & 0 \\
\hline 28 & 704 & 40 & 0.2456 & 0.456 & $\mathrm{NA}$ & NA & 0.0035 & NA & 0.105 & 0.7 & 0.7 & 10.00 & 0 \\
\hline 28 & 705 & 40 & 0.2982 & 0.554 & NA & NA & 0.00425 & NA & 0.1275 & 0.85 & 0.85 & 10.00 & 0 \\
\hline 28 & 706 & 40 & 0.3509 & 0.652 & $\mathrm{NA}$ & $\mathrm{NA}$ & 0.005 & NA & 0.15 & 1 & 1 & 10.00 & 0 \\
\hline 28 & 707 & 40 & 0.4211 & 0.782 & NA & NA & 0.006 & NA & 0.18 & 1.2 & 1.2 & 10.00 & 0 \\
\hline 28 & 708 & 40 & 0.0526 & 0.098 & NA & NA & 0.0005 & NA & 0.015 & 0.1 & 0.15 & 10.00 & 0 \\
\hline 28 & 709 & 40 & 0.1316 & 0.244 & $\mathrm{NA}$ & $\mathrm{NA}$ & 0.00125 & NA & 0.0375 & 0.25 & 0.375 & 10.00 & 0 \\
\hline 28 & 710 & 40 & 0.2105 & 0.391 & NA & NA & 0.002 & NA & 0.06 & 0.4 & 0.6 & 10.00 & 0 \\
\hline 28 & 711 & 40 & 0.2895 & 0.538 & $\mathrm{NA}$ & $\mathrm{NA}$ & 0.00275 & NA & 0.0825 & 0.55 & 0.825 & 10.00 & 0 \\
\hline 28 & 712 & 40 & 0.3684 & 0.684 & NA & NA & 0.0035 & NA & 0.105 & 0.7 & 1.05 & 10.00 & 0 \\
\hline 28 & 713 & 40 & 0.4474 & 0.831 & NA & NA & 0.00425 & NA & 0.1275 & 0.85 & 1.275 & 10.00 & 0 \\
\hline 28 & 714 & 40 & 0.5263 & 0.977 & NA & NA & 0.005 & NA & 0.15 & 1 & 1.5 & 10.00 & 0 \\
\hline 28 & 715 & 40 & 0.6316 & 1.173 & NA & NA & 0.006 & NA & 0.18 & 1.2 & 1.8 & 10.00 & 0 \\
\hline 28 & 716 & 40 & 0.0175 & 0.033 & $\mathrm{NA}$ & $\mathrm{NA}$ & 0.007 & NA & 0.015 & 0.1 & 0.05 & 10.00 & 2 \\
\hline 28 & 717 & 40 & 0.0439 & 0.081 & NA & $\mathrm{NA}$ & 0.0175 & NA & 0.0375 & 0.25 & 0.125 & 10.00 & 2 \\
\hline 28 & 718 & 40 & 0.0702 & 0.130 & $\mathrm{NA}$ & $\mathrm{NA}$ & 0.028 & NA & 0.06 & 0.4 & 0.2 & 10.00 & 2 \\
\hline 28 & 719 & 40 & 0.0965 & 0.179 & $\mathrm{NA}$ & $\mathrm{NA}$ & 0.0385 & $\mathrm{NA}$ & 0.0825 & 0.55 & 0.275 & 10.00 & 2 \\
\hline 28 & 720 & 40 & 0.1228 & 0.228 & $\mathrm{NA}$ & NA & 0.049 & NA & 0.105 & 0.7 & 0.35 & 10.00 & 2 \\
\hline 28 & 721 & 40 & 0.1491 & 0.277 & NA & NA & 0.0595 & NA & 0.1275 & 0.85 & 0.425 & 10.00 & 2 \\
\hline 28 & 722 & 40 & 0.1754 & 0.326 & NA & NA & 0.07 & NA & 0.15 & 1 & 0.5 & 10.00 & 2 \\
\hline 28 & 723 & 40 & 0.2105 & 0.391 & NA & NA & 0.084 & NA & 0.18 & 1.2 & 0.6 & 10.00 & 2 \\
\hline 28 & 724 & 40 & 0.0351 & 0.065 & $\mathrm{NA}$ & $\mathrm{NA}$ & 0.007 & NA & 0.015 & 0.1 & 0.1 & 10.00 & 2 \\
\hline 28 & 725 & 40 & 0.0877 & 0.163 & NA & NA & 0.0175 & NA & 0.0375 & 0.25 & 0.25 & 10.00 & 2 \\
\hline
\end{tabular}


Cyclic Potentiodynamic Polarization Conditions, and Chemical Composition and pH of Historical Data (continued 29)

\begin{tabular}{|c|c|c|c|c|c|c|c|c|c|c|c|c|c|}
\hline Reference & Test & $\begin{array}{l}\text { Temperature } \\
\left({ }^{\circ} \mathrm{C}\right)\end{array}$ & $\begin{array}{l}\text { Carbonate } \\
\text { (M) }\end{array}$ & $\begin{array}{c}\text { Bi- } \\
\text { carbonate } \\
\text { (M) }\end{array}$ & $\begin{array}{l}\text { Citrate } \\
\text { (M) }\end{array}$ & $\begin{array}{l}\text { Phosphate } \\
\text { (M) }\end{array}$ & $\begin{array}{l}\text { Chloride } \\
\text { (M) }\end{array}$ & $\begin{array}{l}\text { Fluoride } \\
\text { (M) }\end{array}$ & $\begin{array}{c}\text { Sulfate } \\
\text { (M) }\end{array}$ & $\begin{array}{l}\text { Nitrate } \\
\text { (M) }\end{array}$ & $\begin{array}{l}\text { Nitrite } \\
\text { (M) }\end{array}$ & $\mathrm{pH}$ & Pit ID* \\
\hline 28 & 726 & 40 & 0.1404 & 0.261 & NA & NA & 0.028 & NA & 0.06 & 0.4 & 0.4 & 10.00 & 2 \\
\hline 28 & 727 & 40 & 0.1930 & 0.358 & NA & NA & 0.0385 & NA & 0.0825 & 0.55 & 0.55 & 10.00 & 0 \\
\hline 28 & 728 & 40 & 0.2456 & 0.456 & NA & NA & 0.049 & NA & 0.105 & 0.7 & 0.7 & 10.00 & 0 \\
\hline 28 & 729 & 40 & 0.2982 & 0.554 & NA & NA & 0.0595 & NA & 0.1275 & 0.85 & 0.85 & 10.00 & 0 \\
\hline 28 & 730 & 40 & 0.3509 & 0.652 & NA & NA & 0.07 & NA & 0.15 & 1 & 1 & 10.00 & 0 \\
\hline 28 & 731 & 40 & 0.4211 & 0.782 & $\mathrm{NA}$ & $\mathrm{NA}$ & 0.084 & NA & 0.18 & 1.2 & 1.2 & 10.00 & 0 \\
\hline 28 & 732 & 40 & 0.0526 & 0.098 & NA & NA & 0.007 & NA & 0.015 & 0.1 & 0.15 & 10.00 & 0 \\
\hline 28 & 733 & 40 & 0.1316 & 0.244 & NA & NA & 0.0175 & NA & 0.0375 & 0.25 & 0.375 & 10.00 & 0 \\
\hline 28 & 734 & 40 & 0.2105 & 0.391 & NA & NA & 0.028 & NA & 0.06 & 0.4 & 0.6 & 10.00 & 0 \\
\hline 28 & 735 & 40 & 0.2895 & 0.538 & NA & NA & 0.0385 & NA & 0.0825 & 0.55 & 0.825 & 10.00 & 0 \\
\hline 28 & 736 & 40 & 0.3684 & 0.684 & NA & NA & 0.049 & NA & 0.105 & 0.7 & 1.05 & 10.00 & 0 \\
\hline 28 & 737 & 40 & 0.4474 & 0.831 & $\mathrm{NA}$ & NA & 0.0595 & NA & 0.1275 & 0.85 & 1.275 & 10.00 & 0 \\
\hline 28 & 738 & 40 & 0.5263 & 0.977 & NA & NA & 0.07 & NA & 0.15 & 1 & 1.5 & 10.00 & 0 \\
\hline 28 & 739 & 40 & 0.6316 & 1.173 & NA & NA & 0.084 & NA & 0.18 & 1.2 & 1.8 & 10.00 & 0 \\
\hline 28 & 740 & 40 & 0.0175 & 0.033 & $\mathrm{NA}$ & NA & 0.00125 & NA & 0.005 & 0.1 & 0.05 & 10.00 & 2 \\
\hline 28 & 741 & 40 & 0.0439 & 0.081 & NA & NA & 0.003125 & NA & 0.0125 & 0.25 & 0.125 & 10.00 & 2 \\
\hline 28 & 742 & 40 & 0.0702 & 0.130 & $\mathrm{NA}$ & $\mathrm{NA}$ & 0.005 & NA & 0.02 & 0.4 & 0.2 & 10.00 & 2 \\
\hline 28 & 743 & 40 & 0.0965 & 0.179 & NA & $\mathrm{NA}$ & 0.006875 & NA & 0.0275 & 0.55 & 0.275 & 10.00 & 2 \\
\hline 28 & 744 & 40 & 0.1228 & 0.228 & NA & NA & 0.00875 & NA & 0.035 & 0.7 & 0.35 & 10.00 & 2 \\
\hline 28 & 745 & 40 & 0.1491 & 0.277 & NA & NA & 0.010625 & NA & 0.0425 & 0.85 & 0.425 & 10.00 & 2 \\
\hline 28 & 746 & 40 & 0.1754 & 0.326 & NA & NA & 0.0125 & NA & 0.05 & 1 & 0.5 & 10.00 & 0 \\
\hline 28 & 747 & 40 & 0.2105 & 0.391 & NA & NA & 0.015 & $\mathrm{NA}$ & 0.06 & 1.2 & 0.6 & 10.00 & 2 \\
\hline 28 & 748 & 40 & 0.0351 & 0.065 & NA & NA & 0.00125 & NA & 0.005 & 0.1 & 0.1 & 10.00 & 2 \\
\hline 28 & 749 & 40 & 0.0877 & 0.163 & $\mathrm{NA}$ & $\mathrm{NA}$ & 0.003125 & NA & 0.0125 & 0.25 & 0.25 & 10.00 & 2 \\
\hline 28 & 750 & 40 & 0.1404 & 0.261 & NA & NA & 0.005 & $\mathrm{NA}$ & 0.02 & 0.4 & 0.4 & 10.00 & 2 \\
\hline
\end{tabular}


Cyclic Potentiodynamic Polarization Conditions, and Chemical Composition and pH of Historical Data (continued 30)

\begin{tabular}{|c|c|c|c|c|c|c|c|c|c|c|c|c|c|}
\hline Reference & Test & $\begin{array}{c}\text { Temperature } \\
\left({ }^{\circ} \mathrm{C}\right)\end{array}$ & $\begin{array}{l}\text { Carbonate } \\
\text { (M) }\end{array}$ & $\begin{array}{c}\text { Bi- } \\
\text { carbonate } \\
\text { (M) }\end{array}$ & $\begin{array}{l}\text { Citrate } \\
\text { (M) }\end{array}$ & $\begin{array}{l}\text { Phosphate } \\
\text { (M) }\end{array}$ & $\begin{array}{l}\text { Chloride } \\
\text { (M) }\end{array}$ & $\begin{array}{l}\text { Fluoride } \\
\text { (M) }\end{array}$ & $\begin{array}{l}\text { Sulfate } \\
\text { (M) }\end{array}$ & $\begin{array}{l}\text { Nitrate } \\
\text { (M) }\end{array}$ & $\begin{array}{l}\text { Nitrite } \\
\text { (M) }\end{array}$ & $\mathrm{pH}$ & Pit ID* \\
\hline 28 & 751 & 40 & 0.1930 & 0.358 & NA & NA & 0.006875 & NA & 0.0275 & 0.55 & 0.55 & 10.00 & 0 \\
\hline 28 & 752 & 40 & 0.2456 & 0.456 & NA & NA & 0.00875 & NA & 0.035 & 0.7 & 0.7 & 10.00 & 0 \\
\hline 28 & 753 & 40 & 0.2982 & 0.554 & NA & NA & 0.010625 & NA & 0.0425 & 0.85 & 0.85 & 10.00 & 0 \\
\hline 28 & 754 & 40 & 0.3509 & 0.652 & NA & NA & 0.0125 & NA & 0.05 & 1 & 1 & 10.00 & 0 \\
\hline 28 & 755 & 40 & 0.4211 & 0.782 & NA & NA & 0.015 & NA & 0.06 & 1.2 & 1.2 & 10.00 & 0 \\
\hline 28 & 756 & 40 & 0.0526 & 0.098 & NA & NA & 0.00125 & NA & 0.005 & 0.1 & 0.15 & 10.00 & 0 \\
\hline 28 & 757 & 40 & 0.1316 & 0.244 & NA & NA & 0.003125 & NA & 0.0125 & 0.25 & 0.375 & 10.00 & 2 \\
\hline 28 & 758 & 40 & 0.2105 & 0.391 & $\mathrm{NA}$ & NA & 0.005 & NA & 0.02 & 0.4 & 0.6 & 10.00 & 0 \\
\hline 28 & 759 & 40 & 0.2895 & 0.538 & NA & NA & 0.006875 & NA & 0.0275 & 0.55 & 0.825 & 10.00 & 0 \\
\hline 28 & 760 & 40 & 0.3684 & 0.684 & NA & NA & 0.00875 & NA & 0.035 & 0.7 & 1.05 & 10.00 & 0 \\
\hline 28 & 761 & 40 & 0.4474 & 0.831 & NA & NA & 0.010625 & NA & 0.0425 & 0.85 & 1.275 & 10.00 & 0 \\
\hline 28 & 762 & 40 & 0.5263 & 0.977 & NA & NA & 0.0125 & NA & 0.05 & 1 & 1.5 & 10.00 & 0 \\
\hline 28 & 763 & 40 & 0.6316 & 1.173 & NA & NA & 0.015 & NA & 0.06 & 1.2 & 1.8 & 10.00 & 0 \\
\hline 28 & 764 & 40 & 0.0175 & 0.033 & NA & NA & 0.00125 & NA & 0.05 & 0.1 & 0.05 & 10.00 & 2 \\
\hline 28 & 765 & 40 & 0.0439 & 0.081 & $\mathrm{NA}$ & NA & 0.003125 & NA & 0.125 & 0.25 & 0.125 & 10.00 & 2 \\
\hline 28 & 766 & 40 & 0.0702 & 0.130 & NA & NA & 0.005 & NA & 0.2 & 0.4 & 0.2 & 10.00 & 2 \\
\hline 28 & 767 & 40 & 0.0965 & 0.179 & NA & NA & 0.006875 & NA & 0.275 & 0.55 & 0.275 & 10.00 & 2 \\
\hline 28 & 768 & 40 & 0.1228 & 0.228 & NA & NA & 0.00875 & NA & 0.35 & 0.7 & 0.35 & 10.00 & 0 \\
\hline 28 & 769 & 40 & 0.1491 & 0.277 & $\mathrm{NA}$ & NA & 0.010625 & NA & 0.425 & 0.85 & 0.425 & 10.00 & 0 \\
\hline 28 & 770 & 40 & 0.1754 & 0.326 & NA & NA & 0.0125 & NA & 0.5 & 1 & 0.5 & 10.00 & 2 \\
\hline 28 & 771 & 40 & 0.2105 & 0.391 & NA & NA & 0.015 & NA & 0.6 & 1.2 & 0.6 & 10.00 & 0 \\
\hline 28 & 772 & 40 & 0.0351 & 0.065 & NA & NA & 0.00125 & NA & 0.05 & 0.1 & 0.1 & 10.00 & 2 \\
\hline 28 & 773 & 40 & 0.0877 & 0.163 & NA & NA & 0.003125 & NA & 0.125 & 0.25 & 0.25 & 10.00 & 0 \\
\hline 28 & 774 & 40 & 0.1404 & 0.261 & NA & $\mathrm{NA}$ & 0.005 & NA & 0.2 & 0.4 & 0.4 & 10.00 & 0 \\
\hline 28 & 775 & 40 & 0.1930 & 0.358 & NA & NA & 0.006875 & NA & 0.275 & 0.55 & 0.55 & 10.00 & 0 \\
\hline
\end{tabular}


Cyclic Potentiodynamic Polarization Conditions, and Chemical Composition and pH of Historical Data (continued 31)

\begin{tabular}{|c|c|c|c|c|c|c|c|c|c|c|c|c|c|}
\hline Reference & Test & $\begin{array}{l}\text { Temperature } \\
\left({ }^{\circ} \mathrm{C}\right)\end{array}$ & $\begin{array}{l}\text { Carbonate } \\
\text { (M) }\end{array}$ & $\begin{array}{c}\text { Bi- } \\
\text { carbonate } \\
\text { (M) }\end{array}$ & $\begin{array}{l}\text { Citrate } \\
\text { (M) }\end{array}$ & $\begin{array}{l}\text { Phosphate } \\
\text { (M) }\end{array}$ & $\begin{array}{l}\text { Chloride } \\
\text { (M) }\end{array}$ & $\begin{array}{l}\text { Fluoride } \\
\text { (M) }\end{array}$ & $\begin{array}{c}\text { Sulfate } \\
\text { (M) }\end{array}$ & $\begin{array}{l}\text { Nitrate } \\
\text { (M) }\end{array}$ & $\begin{array}{l}\text { Nitrite } \\
\text { (M) }\end{array}$ & $\mathrm{pH}$ & Pit ID* \\
\hline 28 & 776 & 40 & 0.2456 & 0.456 & NA & NA & 0.00875 & NA & 0.35 & 0.7 & 0.7 & 10.00 & 0 \\
\hline 28 & 777 & 40 & 0.2982 & 0.554 & NA & NA & 0.010625 & NA & 0.425 & 0.85 & 0.85 & 10.00 & 0 \\
\hline 28 & 778 & 40 & 0.3509 & 0.652 & NA & NA & 0.0125 & NA & 0.5 & 1 & 1 & 10.00 & 0 \\
\hline 28 & 779 & 40 & 0.4211 & 0.782 & NA & NA & 0.015 & NA & 0.6 & 1.2 & 1.2 & 10.00 & 2 \\
\hline 28 & 780 & 40 & 0.0526 & 0.098 & NA & NA & 0.00125 & NA & 0.05 & 0.1 & 0.15 & 10.00 & 0 \\
\hline 28 & 781 & 40 & 0.1316 & 0.244 & $\mathrm{NA}$ & $\mathrm{NA}$ & 0.003125 & NA & 0.125 & 0.25 & 0.375 & 10.00 & 0 \\
\hline 28 & 782 & 40 & 0.2105 & 0.391 & NA & NA & 0.005 & NA & 0.2 & 0.4 & 0.6 & 10.00 & 0 \\
\hline 28 & 783 & 40 & 0.2895 & 0.538 & NA & NA & 0.006875 & NA & 0.275 & 0.55 & 0.825 & 10.00 & 0 \\
\hline 28 & 784 & 40 & 0.3684 & 0.684 & NA & NA & 0.00875 & NA & 0.35 & 0.7 & 1.05 & 10.00 & 0 \\
\hline 28 & 785 & 40 & 0.4474 & 0.831 & NA & NA & 0.010625 & NA & 0.425 & 0.85 & 1.275 & 10.00 & 0 \\
\hline 28 & 786 & 40 & 0.5263 & 0.977 & NA & NA & 0.0125 & NA & 0.5 & 1 & 1.5 & 10.00 & 0 \\
\hline 28 & 787 & 40 & 0.6316 & 1.173 & $\mathrm{NA}$ & NA & 0.015 & NA & 0.6 & 1.2 & 1.8 & 10.00 & 0 \\
\hline 28 & 788 & 40 & 0.6316 & 1.173 & NA & NA & 0.007 & NA & 0.015 & 0.1 & 1.8 & 10.00 & 0 \\
\hline 28 & 789 & 40 & 0.6316 & 1.173 & NA & NA & 0.0175 & NA & 0.0375 & 0.25 & 1.8 & 10.00 & 0 \\
\hline 28 & 790 & 40 & 0.6316 & 1.173 & $\mathrm{NA}$ & NA & 0.028 & NA & 0.06 & 0.4 & 1.8 & 10.00 & 0 \\
\hline 28 & 791 & 40 & 0.6316 & 1.173 & NA & NA & 0.0385 & NA & 0.0825 & 0.55 & 1.8 & 10.00 & 0 \\
\hline 28 & 792 & 40 & 0.6316 & 1.173 & $\mathrm{NA}$ & $\mathrm{NA}$ & 0.049 & NA & 0.105 & 0.7 & 1.8 & 10.00 & 0 \\
\hline 28 & 793 & 40 & 0.6316 & 1.173 & NA & $\mathrm{NA}$ & 0.0595 & NA & 0.1275 & 0.85 & 1.8 & 10.00 & 0 \\
\hline 28 & 794 & 40 & 0.6316 & 1.173 & NA & NA & 0.07 & NA & 0.15 & 1 & 1.8 & 10.00 & 0 \\
\hline 28 & 795 & 40 & 0.6316 & 1.173 & NA & NA & 0.084 & NA & 0.18 & 1.2 & 1.8 & 10.00 & 0 \\
\hline 49 & 796 & 25 & 0.1000 & NA & NA & 0.05000 & 0.1 & NA & 0.1 & 5.5 & 0.01 & 12.00 & 2 \\
\hline 49 & 797 & 25 & 0.1000 & NA & NA & 0.05000 & 0.1 & $\mathrm{NA}$ & 0.1 & 5.5 & 0.1 & 13.00 & 2 \\
\hline 49 & 798 & 25 & 0.1000 & NA & NA & 0.05000 & 0.1 & NA & 0.1 & 5.5 & 0.2 & 13.80 & 0 \\
\hline 49 & 799 & 25 & 0.1000 & NA & $\mathrm{NA}$ & 0.05000 & 0.1 & NA & 0.1 & 7 & 0.01 & 12.00 & 2 \\
\hline 49 & 800 & 25 & 0.1000 & NA & NA & 0.05000 & 0.1 & $\mathrm{NA}$ & 0.1 & 7 & 0.1 & 13.00 & 2 \\
\hline
\end{tabular}


Cyclic Potentiodynamic Polarization Conditions, and Chemical Composition and pH of Historical Data (continued 32)

\begin{tabular}{|c|c|c|c|c|c|c|c|c|c|c|c|c|c|}
\hline Reference & Test & $\begin{array}{c}\text { Temperature } \\
\left({ }^{\circ} \mathrm{C}\right)\end{array}$ & $\begin{array}{l}\text { Carbonate } \\
\text { (M) }\end{array}$ & $\begin{array}{c}\text { Bi- } \\
\text { carbonate } \\
\text { (M) }\end{array}$ & $\begin{array}{l}\text { Citrate } \\
\text { (M) }\end{array}$ & $\begin{array}{l}\text { Phosphate } \\
\text { (M) }\end{array}$ & $\begin{array}{l}\text { Chloride } \\
\text { (M) }\end{array}$ & $\begin{array}{l}\text { Fluoride } \\
\text { (M) }\end{array}$ & $\begin{array}{l}\text { Sulfate } \\
\text { (M) }\end{array}$ & $\begin{array}{l}\text { Nitrate } \\
\text { (M) }\end{array}$ & $\begin{array}{l}\text { Nitrite } \\
\text { (M) }\end{array}$ & $\mathrm{pH}$ & Pit ID* \\
\hline 49 & 801 & 25 & 0.1000 & NA & NA & 0.05000 & 0.1 & NA & 0.1 & 7 & 0.2 & 13.80 & 0 \\
\hline 49 & 802 & 50 & 0.1000 & NA & NA & 0.05000 & 0.1 & NA & 0.1 & 5.5 & 0.01 & 12.00 & 2 \\
\hline 49 & 803 & 50 & 0.1000 & NA & NA & 0.05000 & 0.1 & NA & 0.1 & 5.5 & 0.1 & 13.00 & 2 \\
\hline 49 & 804 & 50 & 0.1000 & NA & NA & 0.05000 & 0.1 & NA & 0.1 & 5.5 & 0.2 & 13.80 & 0 \\
\hline 49 & 805 & 50 & 0.1000 & NA & NA & 0.05000 & 0.1 & NA & 0.1 & 7 & 0.01 & 12.00 & 2 \\
\hline 49 & 806 & 50 & 0.1000 & NA & NA & 0.05000 & 0.1 & NA & 0.1 & 7 & 0.1 & 13.00 & 2 \\
\hline 49 & 807 & 50 & 0.1000 & NA & NA & 0.05000 & 0.1 & NA & 0.1 & 7 & 0.2 & 13.80 & 0 \\
\hline 49 & 808 & 50 & 0.1000 & NA & $\mathrm{NA}$ & 0.05000 & 0.1 & NA & 0.1 & 8.5 & 0.01 & 12.00 & 2 \\
\hline 49 & 809 & 50 & 0.1000 & NA & NA & 0.05000 & 0.1 & NA & 0.1 & 8.5 & 0.1 & 13.00 & 2 \\
\hline 49 & 810 & 50 & 0.1000 & NA & NA & 0.05000 & 0.1 & NA & 0.1 & 8.5 & 0.2 & 13.80 & 0 \\
\hline 49 & 811 & 25 & 0.1000 & NA & NA & 0.05000 & 0.1 & NA & 0.1 & 4.5 & 0.01 & 12.00 & 2 \\
\hline 49 & 812 & 25 & 0.1000 & NA & NA & 0.05000 & 0.1 & NA & 0.1 & 4.5 & 0.1 & 13.00 & 2 \\
\hline 49 & 813 & 50 & 0.1000 & NA & NA & 0.05000 & 0.1 & NA & 0.1 & 4.5 & 0.01 & 12.00 & 2 \\
\hline 49 & 814 & 50 & 0.1000 & NA & NA & 0.05000 & 0.1 & NA & 0.1 & 4.5 & 0.1 & 13.00 & 2 \\
\hline 50 & 815 & 40 & 0.2000 & NA & 0.6 & 0.00050 & 0.01 & 0.003 & 0.1 & 0.1 & 0.5 & 11.57 & 0 \\
\hline 50 & 816 & 40 & 0.2000 & NA & 0.04 & 0.00050 & 0.01 & 0.003 & 0.005 & 5 & 0.02 & 11.36 & 2 \\
\hline 50 & 817 & 40 & 0.0200 & NA & 0.6 & 0.05000 & 0.01 & 0.003 & 0.005 & 5 & 0.5 & 10.67 & 0 \\
\hline 50 & 818 & 40 & 0.2000 & NA & 0.04 & 0.05000 & 0.01 & 0.05 & 0.005 & 0.1 & 0.5 & 11.45 & 0 \\
\hline 50 & 819 & 40 & 0.0200 & NA & 0.6 & 0.00050 & 0.01 & 0.05 & 0.1 & 5 & 0.02 & 10.79 & 2 \\
\hline 50 & 820 & 40 & 0.0200 & NA & 0.04 & 0.00050 & 0.06 & 0.05 & 0.1 & 0.1 & 0.02 & 11.08 & 2 \\
\hline 50 & 821 & 40 & 0.0200 & NA & 0.04 & 0.00050 & 0.06 & 0.05 & 0.1 & 5 & 0.5 & 10.35 & 2 \\
\hline 50 & 822 & 40 & 0.0200 & $\mathrm{NA}$ & 0.6 & 0.00050 & 0.4 & 0.003 & 0.005 & 0.1 & 0.02 & 10.84 & 2 \\
\hline 50 & 823 & 40 & 0.2000 & NA & 0.04 & 0.05000 & 0.4 & 0.003 & 0.1 & 0.1 & 0.5 & 11.30 & 2 \\
\hline 50 & 824 & 40 & 0.2000 & NA & 0.6 & 0.00050 & 0.4 & 0.003 & 0.005 & 5 & 0.5 & 11.47 & 2 \\
\hline 50 & 825 & 40 & 0.0200 & NA & 0.04 & 0.00050 & 0.4 & 0.02 & 0.1 & 0.1 & 0.1 & 10.77 & 2 \\
\hline
\end{tabular}


Cyclic Potentiodynamic Polarization Conditions, and Chemical Composition and pH of Historical Data (continued 33)

\begin{tabular}{|c|c|c|c|c|c|c|c|c|c|c|c|c|c|}
\hline Reference & Test & $\begin{array}{c}\text { Temperature } \\
\left({ }^{\circ} \mathrm{C}\right)\end{array}$ & $\begin{array}{l}\text { Carbonate } \\
\text { (M) }\end{array}$ & $\begin{array}{c}\text { Bi- } \\
\text { carbonate } \\
\text { (M) }\end{array}$ & $\begin{array}{l}\text { Citrate } \\
\text { (M) }\end{array}$ & $\begin{array}{l}\text { Phosphate } \\
\text { (M) }\end{array}$ & $\begin{array}{l}\text { Chloride } \\
\text { (M) }\end{array}$ & $\begin{array}{l}\text { Fluoride } \\
\text { (M) }\end{array}$ & $\begin{array}{l}\text { Sulfate } \\
\text { (M) }\end{array}$ & $\begin{array}{l}\text { Nitrate } \\
\text { (M) }\end{array}$ & $\begin{array}{l}\text { Nitrite } \\
\text { (M) }\end{array}$ & $\mathrm{pH}$ & Pit ID* \\
\hline 50 & 826 & 40 & 0.2000 & NA & 0.04 & 0.05000 & 0.4 & 0.05 & 0.1 & 5 & 0.02 & 10.85 & 2 \\
\hline 50 & 827 & 40 & 0.0200 & NA & 0.04 & 0.05000 & 0.01 & 0.003 & 0.1 & 0.1 & 0.1 & 11.87 & 0 \\
\hline 50 & 828 & 40 & 0.2000 & NA & 0.6 & 0.00050 & 0.01 & 0.02 & 0.1 & 5 & 0.5 & 12.27 & 0 \\
\hline 50 & 829 & 40 & 0.2000 & NA & 0.04 & 0.05000 & 0.01 & 0.05 & 0.005 & 0.7 & 0.1 & 11.68 & 2 \\
\hline 50 & 830 & 40 & 0.2000 & NA & 0.04 & 0.05000 & 0.06 & 0.02 & 0.1 & 0.7 & 0.02 & 11.70 & 2 \\
\hline 50 & 831 & 40 & 0.0200 & NA & 0.6 & 0.00050 & 0.4 & 0.05 & 0.005 & 0.1 & 0.5 & 12.12 & 2 \\
\hline 50 & 832 & 40 & 0.0200 & NA & 0.6 & 0.00050 & 0.01 & 0.003 & 0.005 & 0.7 & 0.1 & 13.15 & 0 \\
\hline 50 & 833 & 40 & 0.2000 & NA & 0.04 & 0.00050 & 0.01 & 0.003 & 0.1 & 5 & 0.5 & 13.11 & 0 \\
\hline 50 & 834 & 40 & 0.0200 & NA & 0.04 & 0.05000 & 0.01 & 0.02 & 0.005 & 0.1 & 0.02 & 13.06 & 0 \\
\hline 50 & 835 & 40 & 0.0200 & NA & 0.04 & 0.00050 & 0.01 & 0.05 & 0.1 & 0.1 & 0.5 & 13.03 & 0 \\
\hline 50 & 836 & 40 & 0.2000 & NA & 0.6 & 0.05000 & 0.01 & 0.05 & 0.005 & 5 & 0.02 & 13.20 & 2 \\
\hline 50 & 837 & 40 & 0.2000 & NA & 0.6 & 0.05000 & 0.06 & 0.02 & 0.005 & 0.1 & 0.1 & 13.15 & 0 \\
\hline 50 & 838 & 40 & 0.2000 & NA & 0.6 & 0.00050 & 0.4 & 0.003 & 0.1 & 0.1 & 0.02 & 13.17 & 2 \\
\hline 50 & 839 & 40 & 0.0200 & NA & 0.04 & 0.00050 & 0.4 & 0.003 & 0.005 & 0.1 & 0.5 & 12.98 & 2 \\
\hline 50 & 840 & 40 & 0.0200 & NA & 0.6 & 0.05000 & 0.4 & 0.003 & 0.1 & 5 & 0.02 & 13.09 & 2 \\
\hline 50 & 841 & 40 & 0.0200 & NA & 0.04 & 0.05000 & 0.4 & 0.02 & 0.005 & 5 & 0.5 & 12.98 & 2 \\
\hline 50 & 842 & 40 & 0.0200 & NA & 0.04 & 0.05000 & 0.4 & 0.05 & 0.1 & 0.1 & 0.02 & 12.92 & 2 \\
\hline 50 & 843 & 40 & 0.2000 & NA & 0.6 & 0.05000 & 0.4 & 0.05 & 0.1 & 0.7 & 0.5 & 13.03 & 2 \\
\hline 50 & 844 & 40 & 0.2000 & NA & 0.04 & 0.00050 & 0.4 & 0.05 & 0.005 & 5 & 0.02 & 13.07 & 2 \\
\hline 51 & 845 & 50 & 1.0280 & NA & NA & 0.01200 & 0.004 & 0.003 & 0.018 & 0.002 & 0.001 & 11.00 & 0 \\
\hline 51 & 846 & 77 & 1.0280 & NA & NA & 0.01200 & 0.004 & 0.003 & 0.018 & 0.002 & 0.001 & 11.00 & 0 \\
\hline 51 & 847 & 50 & 1.0280 & NA & NA & 0.01200 & 0.004 & 0.003 & 0.018 & 0.002 & 0.001 & 11.00 & 0 \\
\hline 51 & 848 & 77 & 1.0280 & NA & NA & 0.01200 & 0.004 & 0.003 & 0.018 & 0.002 & 0.001 & 11.00 & 0 \\
\hline 51 & 849 & 50 & 1.0280 & NA & NA & 0.01200 & 0.004 & 0.003 & 0.018 & 0.002 & 0.001 & 11.00 & 0 \\
\hline 51 & 850 & 77 & 1.0280 & NA & NA & 0.01200 & 0.004 & 0.003 & 0.018 & 0.002 & 0.001 & 11.00 & 0 \\
\hline
\end{tabular}


Cyclic Potentiodynamic Polarization Conditions, and Chemical Composition and pH of Historical Data (continued 34)

\begin{tabular}{|c|c|c|c|c|c|c|c|c|c|c|c|c|c|}
\hline Reference & Test & $\begin{array}{c}\text { Temperature } \\
\left({ }^{\circ} \mathrm{C}\right)\end{array}$ & $\begin{array}{l}\text { Carbonate } \\
\text { (M) }\end{array}$ & $\begin{array}{c}\text { Bi- } \\
\text { carbonate } \\
\text { (M) }\end{array}$ & $\begin{array}{l}\text { Citrate } \\
\text { (M) }\end{array}$ & $\begin{array}{l}\text { Phosphate } \\
\text { (M) }\end{array}$ & $\begin{array}{l}\text { Chloride } \\
\text { (M) }\end{array}$ & $\begin{array}{l}\text { Fluoride } \\
\text { (M) }\end{array}$ & $\begin{array}{l}\text { Sulfate } \\
\text { (M) }\end{array}$ & $\begin{array}{l}\text { Nitrate } \\
\text { (M) }\end{array}$ & $\begin{array}{l}\text { Nitrite } \\
\text { (M) }\end{array}$ & $\mathrm{pH}$ & Pit ID* \\
\hline 51 & 851 & 77 & 0.0000 & NA & NA & 0.01200 & 0.004 & 0.003 & 0.018 & 0.002 & 0.001 & 11.00 & 2 \\
\hline 51 & 852 & 77 & 0.0000 & NA & NA & 0.01200 & 0.004 & 0.003 & 0.018 & 0.002 & 0.001 & 11.00 & 2 \\
\hline 51 & 853 & 77 & 1.0280 & NA & NA & 0.01200 & 0.004 & 0.003 & 0.018 & 0.002 & 0 & 11.00 & 0 \\
\hline 51 & 854 & 77 & 1.0280 & NA & NA & 0.01200 & 0.004 & 0.003 & 0.018 & 1.967 & 0.001 & 11.00 & 2 \\
\hline 51 & 855 & 50 & 0.4700 & NA & NA & 0.00900 & 0.046 & 0.084 & 0.028 & 2.13 & 0.98 & $14+$ & 0 \\
\hline 51 & 856 & 77 & 0.4700 & NA & NA & 0.00900 & 0.046 & 0.084 & 0.028 & 2.13 & 0.98 & $14+$ & 0 \\
\hline 51 & 857 & 77 & 0.4700 & NA & NA & 0.00900 & 0.046 & 0.084 & 0.028 & 2.13 & 0.49 & $14+$ & 0 \\
\hline 51 & 858 & 77 & 0.4700 & NA & NA & 0.00900 & 0.046 & 0.084 & 0.028 & 2.13 & 0.49 & $14+$ & 0 \\
\hline 51 & 859 & 77 & 1.0000 & NA & NA & 0.00900 & 0.046 & 0.084 & 0.028 & 0.21 & 0.049 & 13.6 & 0 \\
\hline 51 & 860 & 50 & 1.1180 & NA & NA & 0.00900 & 0.046 & 0.084 & 0.028 & 1.635 & 1.27 & $14+$ & 0 \\
\hline 51 & 861 & 77 & 0.9350 & 0.203 & NA & 0.01200 & 0.004 & 0.003 & 0.018 & 0.002 & 0.001 & 11 & 0 \\
\hline 51 & 862 & 77 & 0.9350 & 0.203 & NA & 0.01200 & 0.004 & 0.003 & 0.018 & 0.002 & 0.001 & 11 & 0 \\
\hline 51 & 863 & 77 & 0.9350 & 0.203 & NA & 0.01200 & 0.004 & 0.003 & 0.018 & 0.002 & 0.001 & 11 & 0 \\
\hline 51 & 864 & 77 & 0.9350 & 0.203 & NA & 0.01200 & 0.004 & 0.003 & 0.018 & 0.002 & 0.001 & 11 & 0 \\
\hline 51 & 865 & 77 & 1.2420 & NA & $\mathrm{NA}$ & 0.01100 & 0.043 & 0.079 & 0.027 & 1.532 & 1.2 & 14 & 0 \\
\hline 51 & 866 & 50 & 0.4770 & NA & NA & 0.00900 & 0.046 & 0.084 & 0.028 & 1.967 & 0.938 & $14+$ & 0 \\
\hline 51 & 867 & 50 & 0.4770 & NA & NA & 0.00900 & 0.046 & 0.084 & 0.028 & 1.967 & 0.938 & $14+$ & 0 \\
\hline 51 & 868 & 50 & 0.0000 & NA & NA & 0.00900 & 0.046 & 0.084 & 0.028 & 1.967 & 0.938 & $14+$ & 0 \\
\hline 51 & 869 & 50 & 0.4770 & NA & $\mathrm{NA}$ & 0.00900 & 0.046 & 0.084 & 0.028 & 1.967 & 0 & 14 & 0 \\
\hline 51 & 870 & 50 & 0.0000 & NA & NA & 0.00900 & 0.046 & 0.084 & 0.028 & 1.967 & 0 & 14 & 0 \\
\hline 51 & 871 & 50 & 0.0000 & NA & NA & 0.00900 & 0.046 & 0.084 & 0.028 & 1.967 & 0.938 & 14 & 0 \\
\hline 51 & 872 & 50 & 0.4770 & $\mathrm{NA}$ & NA & 0.00900 & 0.046 & 0.084 & 0.028 & 1.967 & 0.938 & $14+$ & 0 \\
\hline 51 & 873 & 50 & 0.4770 & NA & NA & 0.00900 & 0.046 & 0.084 & 0.028 & 1.967 & 0.938 & $14+$ & 0 \\
\hline 51 & 874 & 50 & 0.2010 & NA & NA & 0.05000 & 0.018 & 0.014 & 0.02 & 1.33 & 0.205 & 13 & 0 \\
\hline 52 & 875 & 50 & 1.4000 & NA & NA & 0.00120 & 0.1 & 0.0069 & 0.086 & 3.7 & 1.2 & 7 & 2 \\
\hline
\end{tabular}


Cyclic Potentiodynamic Polarization Conditions, and Chemical Composition and pH of Historical Data (continued 35)

\begin{tabular}{|c|c|c|c|c|c|c|c|c|c|c|c|c|c|}
\hline Reference & Test & $\begin{array}{l}\text { Temperature } \\
\left({ }^{\circ} \mathrm{C}\right)\end{array}$ & $\begin{array}{l}\text { Carbonate } \\
\text { (M) }\end{array}$ & $\begin{array}{c}\text { Bi- } \\
\text { carbonate } \\
\text { (M) }\end{array}$ & $\begin{array}{l}\text { Citrate } \\
\text { (M) }\end{array}$ & $\begin{array}{l}\text { Phosphate } \\
\text { (M) }\end{array}$ & $\begin{array}{l}\text { Chloride } \\
\text { (M) }\end{array}$ & $\begin{array}{l}\text { Fluoride } \\
\text { (M) }\end{array}$ & $\begin{array}{c}\text { Sulfate } \\
\text { (M) }\end{array}$ & $\begin{array}{l}\text { Nitrate } \\
\text { (M) }\end{array}$ & $\begin{array}{l}\text { Nitrite } \\
\text { (M) }\end{array}$ & $\mathrm{pH}$ & Pit ID* \\
\hline 52 & 876 & 50 & 1.4000 & NA & NA & 0.00120 & 0.1 & 0.0069 & 0.086 & 3.7 & 1.2 & 9.5 & 2 \\
\hline 52 & 877 & 50 & 1.4000 & $\mathrm{NA}$ & $\mathrm{NA}$ & 0.00120 & 0.1 & 0.0069 & 0.086 & 3.7 & 1.2 & 10 & 2 \\
\hline 52 & 878 & 50 & 1.4000 & NA & NA & 0.00120 & 0.1 & 0.0069 & 0.086 & 3.7 & 1.2 & 11 & 0 \\
\hline 52 & 879 & 50 & 1.4000 & NA & NA & 0.00120 & 0.1 & 0.0069 & 0.086 & 3.7 & 1.2 & 12 & 0 \\
\hline 52 & 880 & 50 & 1.4000 & NA & NA & 0.00120 & 0.1 & 0.0069 & 0.086 & 3.7 & 1.2 & 13 & 0 \\
\hline 52 & 881 & 50 & 1.4000 & NA & $\mathrm{NA}$ & 0.00120 & 0.1 & 0.0069 & 0.086 & 3.7 & 1.2 & 13.5 & 0 \\
\hline 52 & 882 & 50 & 1.4000 & NA & NA & 0.00120 & 0.1 & 0.0069 & 0.086 & 3.7 & 0 & 11 & 2 \\
\hline 52 & 883 & 50 & 1.4000 & NA & NA & 0.00120 & 0.1 & 0.0069 & 0.086 & 3.7 & 0.35 & 11 & 2 \\
\hline 52 & 884 & 50 & 1.4000 & NA & NA & 0.00120 & 0.1 & 0.0069 & 0.086 & 3.7 & 0.875 & 11 & 2 \\
\hline 52 & 885 & 50 & 1.4000 & NA & NA & 0.00120 & 0.1 & 0.0069 & 0.086 & 3.7 & 1.035 & 11 & 2 \\
\hline 52 & 886 & 50 & 1.4000 & NA & NA & 0.00120 & 0.1 & 0.0069 & 0.086 & 2.4 & 2.3 & 10 & 0 \\
\hline 52 & 887 & 50 & 1.4000 & NA & $\mathrm{NA}$ & 0.00120 & 0.1 & 0.0069 & 0.086 & 3.7 & 3.5 & 11 & 0 \\
\hline 52 & 888 & 50 & 1.4000 & NA & NA & 0.00120 & 0.1 & 0.0069 & 0.086 & 3.7 & 7 & 11 & 0 \\
\hline 52 & 889 & 50 & 1.4000 & NA & NA & 0.00120 & 0.1 & 0.0069 & 0.086 & 1.5 & 0.75 & 11 & 0 \\
\hline 52 & 890 & 50 & 1.4000 & NA & $\mathrm{NA}$ & 0.00120 & 0.1 & 0.0069 & 0.086 & 3.7 & 3.5 & 11 & 0 \\
\hline 52 & 891 & 50 & 1.4000 & NA & NA & 0.00120 & 0.1 & 0.0069 & 0.086 & 1.5 & 1.5 & 11 & 0 \\
\hline 52 & 892 & 50 & 1.4000 & NA & $\mathrm{NA}$ & 0.00120 & 0.05 & 0.0069 & 0.086 & 3.7 & 1.2 & 11 & 0 \\
\hline 52 & 893 & 50 & 1.4000 & NA & NA & 0.00120 & 0.2 & 0.0069 & 0.086 & 3.7 & 1.2 & 11 & 2 \\
\hline 52 & 894 & 50 & 1.4000 & NA & NA & 0.00120 & 0.2 & 0.0069 & 0.086 & 3.7 & 1.2 & 11 & 2 \\
\hline 53 & 895 & 50 & 1.1400 & NA & 0.088 & 0.05800 & 0.1 & NA & 0.14 & 3 & 1 & $13+$ & 2 \\
\hline 53 & 896 & 40 & 1.1400 & NA & 0.088 & 0.05800 & 0.1 & NA & 0.14 & 3 & 1 & $13+$ & 2 \\
\hline 53 & 897 & 30 & 1.1400 & NA & 0.088 & 0.05800 & 0.1 & $\mathrm{NA}$ & 0.14 & 3 & 1 & $13+$ & 2 \\
\hline 53 & 898 & 50 & 1.1400 & $\mathrm{NA}$ & 0.088 & 0.05800 & 0.1 & NA & 0.14 & 3 & 1 & $13+$ & 2 \\
\hline 53 & 899 & 50 & 1.1400 & NA & 0.088 & 0.05800 & 0.1 & NA & 0.14 & 3 & 1 & 10.3 & 2 \\
\hline 53 & 900 & 50 & 1.1400 & NA & 0.088 & 0.05800 & 0.1 & NA & 0.14 & 2.9 & 1.94 & $13+$ & 2 \\
\hline
\end{tabular}


Cyclic Potentiodynamic Polarization Conditions, and Chemical Composition and pH of Historical Data (continued 36)

\begin{tabular}{|c|c|c|c|c|c|c|c|c|c|c|c|c|c|}
\hline Reference & Test & $\begin{array}{c}\text { Temperature } \\
\left({ }^{\circ} \mathrm{C}\right)\end{array}$ & $\begin{array}{l}\text { Carbonate } \\
\text { (M) }\end{array}$ & $\begin{array}{l}\text { Bi- } \\
\text { carbonate } \\
\text { (M) }\end{array}$ & $\begin{array}{l}\text { Citrate } \\
\text { (M) }\end{array}$ & $\begin{array}{l}\text { Phosphate } \\
\text { (M) }\end{array}$ & $\begin{array}{l}\text { Chloride } \\
\text { (M) }\end{array}$ & $\begin{array}{l}\text { Fluoride } \\
\text { (M) }\end{array}$ & $\begin{array}{l}\text { Sulfate } \\
\text { (M) }\end{array}$ & $\begin{array}{l}\text { Nitrate } \\
\text { (M) }\end{array}$ & $\begin{array}{l}\text { Nitrite } \\
\text { (M) }\end{array}$ & $\mathrm{pH}$ & Pit ID* \\
\hline 53 & 901 & 30 & 1.1400 & NA & 0.088 & 0.05800 & 0.1 & NA & 0.14 & 3.2 & 1.9 & $13+$ & 2 \\
\hline 53 & 902 & 30 & 1.1400 & NA & 0.088 & 0.05800 & 0.1 & NA & 0.14 & 3.2 & 1.9 & $13+$ & 2 \\
\hline 53 & 903 & 30 & 1.1400 & NA & 0.088 & 0.05800 & 0.1 & NA & 0.14 & 3.2 & 1.9 & $13+$ & 2 \\
\hline 54 & 904 & 45 & NA & NA & NA & $\mathrm{NA}$ & NA & NA & NA & 0.91 & 0.68 & 11 & 2 \\
\hline 54 & 905 & 45 & NA & NA & NA & NA & NA & NA & NA & 0.91 & 0.68 & 11.5 & 2 \\
\hline 54 & 906 & 50 & NA & NA & NA & NA & NA & NA & NA & 0.91 & 0.68 & 11.5 & 2 \\
\hline 54 & 907 & 50 & NA & NA & $\mathrm{NA}$ & NA & NA & NA & NA & 0.91 & 0.68 & 12 & 2 \\
\hline 54 & 908 & 55 & NA & NA & NA & NA & NA & NA & NA & 0.91 & 0.68 & 11.5 & 2 \\
\hline 54 & 909 & 55 & NA & NA & NA & NA & NA & NA & NA & 0.91 & 0.68 & 12 & 2 \\
\hline 54 & 910 & 60 & $\mathrm{NA}$ & NA & NA & NA & NA & NA & $\mathrm{NA}$ & 0.91 & 0.68 & 12 & 2 \\
\hline 54 & 911 & 60 & NA & NA & NA & NA & NA & NA & NA & 0.91 & 0.68 & 12.5 & 0 \\
\hline 54 & 912 & 40 & NA & NA & NA & NA & NA & NA & NA & 0.88 & 0.86 & 11.5 & 0 \\
\hline 54 & 913 & 40 & NA & NA & NA & NA & NA & NA & NA & 0.88 & 0.86 & 12 & 0 \\
\hline 54 & 914 & 50 & NA & NA & NA & NA & NA & NA & NA & 0.88 & 0.86 & 11 & 2 \\
\hline 54 & 915 & 50 & NA & NA & NA & NA & NA & NA & NA & 0.88 & 0.86 & 11.5 & 0 \\
\hline 54 & 916 & 50 & NA & NA & NA & NA & NA & NA & NA & 0.88 & 0.86 & 12 & 0 \\
\hline 54 & 917 & 55 & NA & NA & NA & NA & NA & NA & NA & 0.88 & 0.86 & 11 & 2 \\
\hline 54 & 918 & 55 & NA & NA & NA & NA & NA & NA & NA & 0.88 & 0.86 & 11.5 & 0 \\
\hline 54 & 919 & 55 & NA & NA & NA & NA & NA & NA & NA & 0.88 & 0.86 & 12 & 0 \\
\hline 54 & 920 & 60 & NA & NA & NA & NA & NA & NA & NA & 0.88 & 0.86 & 12 & 0 \\
\hline
\end{tabular}

*The Pit ID corresponds to number of tests that pits were observed for a total of two tests. This means:

Pit ID of 0: none of the two tests performed showed pits.

Pit ID of 1: only one of the two tests performed showed pits.

Pit ID of 2: the two tests performed showed pits. 